University of Louisville

ThinkIR: The University of Louisville's Institutional Repository

Electronic Theses and Dissertations

$12-2014$

\title{
Triggered release of small molecules from solid supports using heat or an applied magnetic field.
}

\author{
Ralph Jacob Knipp \\ University of Louisville
}

Follow this and additional works at: https://ir.library.louisville.edu/etd

Part of the Chemistry Commons

\section{Recommended Citation}

Knipp, Ralph Jacob, "Triggered release of small molecules from solid supports using heat or an applied magnetic field." (2014). Electronic Theses and Dissertations. Paper 1756.

https://doi.org/10.18297/etd/1756

This Doctoral Dissertation is brought to you for free and open access by ThinkIR: The University of Louisville's Institutional Repository. It has been accepted for inclusion in Electronic Theses and Dissertations by an authorized administrator of ThinkIR: The University of Louisville's Institutional Repository. This title appears here courtesy of the author, who has retained all other copyrights. For more information, please contact thinkir@louisville.edu. 


\title{
TRIGGERED RELEASE OF SMALL MOLECULES FROM SOLID SUPPORTS USING HEAT OR AN APPLIED MAGNETIC FIELD
}

\author{
By \\ Ralph Jacob Knipp \\ B.S., Ohio Dominican University, 2007 \\ M.S., University of Louisville, 2012 \\ A Dissertation \\ Submitted to the Faculty of the \\ College of Arts \& Sciences of the University of Louisville \\ in Partial Satisfaction of the Requirements \\ for the Degree of
}

Doctor of Philosophy

Department of Chemistry

University of Louisville

Louisville, Kentucky

December 2014 



\title{
TRIGGERED RELEASE OF SMALL MOLECULES FROM SOLID SUPPORTS USING HEAT OR AN APPLIED MAGNETIC FIELD
}

\author{
By
}

\author{
Ralph Jacob Knipp \\ B.S., Ohio Dominican University, 2007 \\ M.S., University of Louisville, 2012
}

A Dissertation Approved on

November 19, 2014

By the following Dissertation Committee:

Dissertation Director: Dr. Michael H. Nantz

Dr. Christopher T. Burns

Dr. Palaniappan Sethu

Dr. Francis P. Zamborini 


\section{DEDICATION}

This dissertation is dedicated to my loving and supportive wife

Andrea Michelle Knipp,

and my wonderful children

Cooper Elliott Knipp

and

Libby Jane Knipp 


\section{ACKNOWLEDGEMENTS}

I consider myself very lucky to have worked with Professor Michael H. Nantz as my advisor during my tenure at the University of Louisville. Any question or problem that I brought before him was immediately met with a torrent of well-contemplated ideas. But his brilliance was not limited to the research lab. Professor Nantz is a fantastic presenter and lecturer due to his ability to capture the audience's attention and provide thoughtful explanations in a way that best reflects the importance of the issue at hand.

Dr. Nantz is also a natural leader who engenders respect while being fair and supportive. He always knew when he needed to push me to ensure that I reached my full potential.

I would also like to thank my committee members Professor Christopher Burns, Professor Francis Zamborini and Professor Palaniappan Sethu for their advice and direction throughout my graduate career.

During my time at the University of Louisville I was in several collaborations that provided me with the opportunity to work with researchers from disciplines outside of chemistry. I was lucky enough to 
work with Professor Xiao-An Fu (University of Louisville, Department of Chemical Engineering), Professor Palaniappan Sethu (University of Alabama, Department of Cardiovascular Disease), Professor Ralf Schirrmacher (University of Alberta, Department of Oncology) and Dr. Anu Puri (National Cancer Institute, Frederick, MD).

One of the best aspects of spending so many hours working in the lab was the friendship that I built with my group members. Xuan Huang and Souvik Biswas were always willing to offer advice and direction during my early days in the Nantz Lab. There is no doubt in my mind that I could never have made it through graduate school without the music, YouTube videos, hysterical lunches and thought-provoking conversations that I enjoyed with Sébastien Laulhé and Stephanie Mattingly. I have also become great friends with my younger lab mates Sadakatali Gori, Sara Biladeau, and Mumiye Ogunwale, as well as our post-doc Raju Mandapati. I would also like to thank the great undergraduate students Joseph Riddle, Ben Neltner, Olivetta Uradu, and Kristopher Nelson whom I trained.

I would like to thank the School of Interdisciplinary and Graduate Studies, the Institute for Molecular Diversity and Drug Design $\left(\mathrm{IMD}^{3}\right)$, and the Office of Technology Transfer for their financial support and their belief in my research. 
I also feel indebted to my high school teachers Jerry Rismiller and Nicholas Palmer who enthusiastically taught and awakened my desire to pursue a career in chemistry. I also want to thank my undergraduate advisor Professor A. J. C. Lewis Hogarth who provided me with a firm scientific foundation on which to build my career.

I want to expres my gratitude also to my parents Ralph and Donna Knipp, my father- and mother-in-law Gregg and Reneé Dyke, my brother and his wife Caleb and Madoka Knipp, and my brother- and sister-in-law Aaron and Katherine Dyke. Their love, support and encouragement helped me reach my educational goals and emboldened me to persevere through the high and low points of graduate school.

Finally, my wife Andrea Knipp and my children Cooper Elliott and Libby Jane were my loudest cheerleaders. I thank them for enduring my absence through the long days and late nights, my frustration when my research was not going well and my stress as deadlines approached. Throughout my time at the University of Louisville they have believed in my aspirations and never ceased to build me up with words of encouragement. 


\title{
ABSTRACT
}

\section{TRIGGERED RELEASE OF SMALL MOLECULES FROM SOLID SUPPORTS USING HEAT OR AN}

\author{
APPLIED MAGNETIC FIELD \\ Ralph Jacob Knipp
}

November 19, 2014

The reaction of an aminooxy moiety $\left(\mathrm{RONH}_{2}\right)$ with an aldehyde or ketone carbonyl, an oximation reaction, results in the formation of robust oxime ether linkages. Oximation is a form of click chemistry and is chemoselective, occurs in a variety of solvents including water, and produces high yields with little to no purification.

We were inspired to exploit the advantages of oximation reactions by attaching aminooxy-functionalized molecules to solid supports, thus allowing us to employ aminooxy chemistry in ways that cannot be achieved using solution phase. Chapter 1 provides a review of aminooxy chemistry, its advantages over similar reactions and its multitude of applications. Chapter 2 describes the use of aminium aminooxy salts fixed to a silicon microreactor to enable the capture of volatile aldehydes and ketones from exhaled breath for early detection of lung cancer. Since only carbonyl compounds were 
retained and thereby concentrated, the accurate measurements of scarce metabolite cancer markers were realized. Use of an acid-scavenging polymer transformed the aminium salt adducts into a volatile adducts, thus enabling analyses by both highresolution mass spectrometry and gas chromatography.

Chapter 3 details our investigation into the use of thermally-induced intramolecular cyclization as a release mechanism. A kinetic study on the cyclization rates of a panel of thermally-labile linkers led to a demonstration involving such an aminooxyfunctionalized linker covalently bonded within a poly(dimethylsiloxane) microchannel. After capture of an aldehyde-functionalized fluorophore that had been passed through the microchannel, the intramolecular cyclization release mechanism was induced by gentle warming to liberate the fluorescent oxime ether adduct. In Chapter 4 we applied our thermally-labile linkers to iron oxide nanoparticles in an effort to design an externally controlled drug delivery system. $\mathrm{Fe}_{3} \mathrm{O}_{4}$ nanoparticles generate heat when subjected to an alternating magnetic field (AMF), thus eliminating the need for an in vivo conventional heat source. During our investigation of AMF-induced release we discovered the unprecedented hydrolysis of robust chemical functionalities that occurs only at the nanoparticle-liquid interface. This discovery was exploited in the development of a delivery system capable of releasing oxime ether adducts.

In conclusion, this thesis describes novel innovations that can be immediately applied in a multitude of disciplines, from analytics to diagnoses and to drug delivery. 


\section{TABLE OF CONTENTS}

DEDICATION

ACKNOWLEDGMENTS

iii

ABSTRACT

LIST OF TABLES

iv

LIST OF FIGURES

vii

LIST OF SCHEMES

xii

xiii

xviii

CHAPTER 1 ADVANCEMENTS IN OXIMATION: 1

APPLICATION OF AMINOOXY

CHEMISTRY AND ATTACHMENT TO

SOLID SUPPORTS

1.1. Introduction

1.2. Chemoselective Ligation 10

1.3. Solid-Supported Aminooxy Chemistry 15

1.4. Use of Aminooxy Chemistry in Nanotechnology 17

1.5. Iron Oxide Nanoparticles 20

1.6. Use of Iron Oxide Nanoparticles to Generate Heat 22

1.7. Conclusion 26

CHAPTER 2 CHEMOSELECTIVE CAPTURE OF 30 VOLATILE ALDEHYDES AND KETONES

2.1. Introduction 31

2.2. Introduction of AMAH 38

2.3. Results and Discussion $\quad 40$

2.4. Conclusion 51

2.5. Future Directions 52 
INTRAMOLECULAR CYCLIZATION AS A MECHANISM FOR SUBSTRATE RELEASE

3.1. Introduction 56

3.2. Initial Concept 58

3.3. Second Generation 63

3.3.1. Cyclization Panel 66

3.3.2. Syntheses 67

3.4. Cyclization study $\quad 73$

3.5. Microchannel Application $\quad 79$

3.6. Conclusion 82

CHAPTER 4 ALTERNATING MAGNETIC FIELD-INDUCED 84 SUBSTRATE RELEASE FROM IRON OXIDE NANOPARTICLES

4.1. Introduction 85

4.2. Results and Discussion $\quad 88$

4.2.1. Initial Loading 88

4.2.2. Formation of Covalent Bond to NP 98

4.2.3. Acidic Boc-deprotection on NPs 104

4.2.4. AMF vs. Non-AMF Induced Release: Hydrolysis 107

4.2.5. Suppression of Hydrolysis 114

4.2.6. Commencement of $\mathrm{SiO}_{2} @ \mathrm{Fe}_{3} \mathrm{O}_{4} \mathrm{NPs} \quad 117$

4.2.7. Monodispersed $\mathrm{SiO}_{2} @ \mathrm{Fe}_{3} \mathrm{O}_{4}$ Nanoparticles 123

4.2.8. AMF-Induced Hydrolysis 133

4.3. Conclusion 142

4.4. Future Directions 143

CHAPTER 5 EXPERIMENTAL PROCEDURES 148

5.1. General Statement 151

5.2. Experimental Procedures of Chapter 2

5.3. Experimental Procedures of Chapter $3 \quad 162$

5.4. Experimental Procedures of Chapter $4 \quad 198$ 
REFERENCES

R.1. Chapter 1 References 227

R.2. Chapter 2 References 230

R.3. Chapter 3 References 235

R.4. Chapter 4 References 238

R.5. Chapter 5 References 244

APPENDIX A: SPECTRA 245

APPENDIX B: LIST OF PUBLICATIONS 291

CURRICULUM VITAE 306 


\section{LIST OF TABLES}

TABLE

PAGE

1.1 Comparison of the aminooxy functionality with a primary amine. 4

1.2 Yields of hydrazone formation of nitrobenzoxadiazole hydrazine and 8 4-nitrobenzaldehyde with varied anthranilic acid catalysts at $\mathrm{pH}$ 7.46.

1.3 Yields of hydrazone formation of nitrobenzoxadiazole hydrazine and 10 4-nitrobenzaldehyde with varied arylamine/acid catalysts at $\mathrm{pH}$ 7.4.

1.4 Oxime coupling with Fmoc-Glu-Glu-Gly-Gly-H on aminooxyterminated core-shell gold NPs.

2.1 Analytical procedures for the diagnosis of lung cancer.

2.2 Mean advantages and disadvantages of non-invasive diagnostic tools detecting biomarkers of pulmonary inflammation in smoking subjects.

2.3 Current technology for the diagnosis of lung cancer using VOCs.

2.4 Tabulated relationship between VOC capture efficiency and AMAH/VOC molar ratio.

2.5 Retention times of AMA- $\mathrm{C}_{1}-\mathrm{C}_{7}$ adducts. 46

2.6 Quantitative comparison of FT-ICR-MS and GC-MS analyses of a 48 breath sample.

3.1 Hydrosilylation of allylamine with methyldiethoxysilane in the presence of various catalysts.

3.2 Heat-induced release of alcohol 25. 


\section{LIST OF FIGURES}

1.1 Generic examples of the four click chemistry classes of chemical transformations.

1.2 Resonance forms of oximes, hydrazones and imines. 6

1.3 Mechanistic considerations for acid-induced hydrolysis of oximes and 7 hydrazones.

1.4 Chemoselective ligation reactions used in the convergent assembly of 11 biopolymers and their mimetics, and in the modification of biopolymers and cells.

1.5 Chemical structure of the tetravalent RGD ligand RAFT conjugated 13 to a fluorescent or radioactive label.

1.6 Schematic representation of the structures of the ST-based lipopeptide 14 vaccines with oxime ether and thioether linkages.

1.7 Multilayer deposition of PPV supported aldehyde and aminooxy substrates on derivatized glass via chemoselective ligation.

1.8 Final functionalization of polymer-coated iron oxide NPs with an amine terminated fragment of urokinase-type plasminogen activator.

1.9 Magnetic NPs respond to AMF through Néel relaxation or Brownian 23 relaxation.

1.10 Thermotherapy treatment of the pelvic region after intratumoral injection of magnetic NPs using the AMF applicator MFH 300F.

1.11 Chapter 3 summary.

7


2.6 Graphical relationship between VOC capture efficiency and AMAH/

VOC molar ratio.

2.7 GC-MS chromatogram of reference AMA-carbonyl adducts.

2.8 Qualitative comparison of FT-ICR-MS spectrum and GC-MS chromatogram of VOCs from a healthy, non- smoking subject.

2.9 Analyses of a healthy non-smoker control, smoker control and lung cancer subject via FT-ICR-MS and GC-MS.

2.10 Structures of ATM and ADMH.

2.11 Time course for derivatization of $12 \alpha$-hydroxy-3-oxo-5 $\beta$-chloanoic acid with $O$-(2-anthrylmethyl)hydroxylamine.

3.1 General structure of drug or prodrug delivery systems involving electron cascade reactions, rapid intramolecular cyclizations or a hybrid of intramolecular cyclization and electron cascade reactions.

3.2 Heat-induced cyclization via lactamization or carbamate formation.

3.3 Proposed alterations to molecule published by Ojima and Vidal.

$3.4 \quad{ }^{1} \mathrm{H}$ and ${ }^{13} \mathrm{C}$ NMR spectra confirming the synthesis of $\mathbf{5}$.

3.5 Retrosynthetic analysis of $\mathbf{2}$ reveals a path for simplification involving an $\omega$-amino acid starting material.

3.6 $\quad{ }^{1} \mathrm{H}$ NMR spectrum of the hydrosilylated ester 14.3.

3.7 Cyclization precursors ( $\mathrm{R}=2$-(9-anthracenyl)ethyl).

3.8 ${ }^{1} \mathrm{H}$ NMR of ester $\mathbf{1 4 . 3}$ showing successful Boc-protection, $N$-allylation and esterification with 2-(9-anthracenyl)ethanol.

3.9 ${ }^{13} \mathrm{C} \mathrm{NMR}\left(\mathrm{CDCl}_{3}\right)$ spectra of ester 14.3, amide 14.4 and Bocprotected carbonate $\mathbf{7 . 2}$ emphasizing the effects of carbonyl functionality on ${ }^{13} \mathrm{C}$ chemical shift and confirming molecular structure.

$3.10{ }^{1} \mathrm{H}$ NMR spectrum of ester 22 after complete bis- $\alpha$-mehylation. $\quad 73$

3.11 Percent release of $\mathbf{2 5}$ from indicated substrates in $\mathrm{MeOH}$ at $55{ }^{\circ} \mathrm{C}$. $\quad 75$

$3.12{ }^{1} \mathrm{H}$ NMR spectra of ester 6.1 before heating and after thermally- $\quad 78$ induced intramolecular cyclization.

3.13 Schematic of poly(dimethylsiloxane) microchannel.

3.14 Fluorescence microscopy image of FITC-derived substrate within the microchannel and same field of view showing microchannel fluorescence after heating.

4.1 Liposomal, mesoporous silica and polymeric iron oxide drug delivery platforms that use AMF-induced heat as a mechanism for controlled drug relesase. 
4.2 Drug release mechanism for acid-labile hydrazone linkages and photolabile $o$-nitrobenzyl linkages.

4.3 AMF-mediated intramolecular cyclization results in release of ROH. $\quad 88$

4.4 Cyclization precursors ( $\mathrm{R}=2$-(9-anthracenyl)ethyl). $\quad 89$

4.5 Phosphonic acid/phosphonate, chlorosilane and alkoxysilane 90 functionalities used to form moderate to strong bonds to $\mathrm{Fe}_{3} \mathrm{O}_{4} \mathrm{NPs}$.

4.6 Effectiveness of silane bonding to various substrates. 91

4.7 Hydrolytic deposition of silanes. 92

4.8 FT-IR spectrum of $\mathrm{Fe}_{3} \mathrm{O}_{4}$ NPs functionalized via an $\mathrm{EtOH}: \mathrm{H}_{2} \mathrm{O}$ route 94 with linker 4.3 obtained using ATR.

4.9 Examples of hydrolysis and condensation of alkoxysilanes with 96 surface hydroxyl groups in the presence of water to afford a monolayer, a bilayer and an uncontrolled and disorderly polymeric shell.

4.10 FT-IR spectrum of $\mathrm{Fe}_{3} \mathrm{O}_{4}$ NPs functionalized via an anhydrous route 97 with linker 4.3 obtained using ATR.

4.11 595 Amp AMF and non-AMF induced release of fluorophore from $\sim 4 \quad 99$ $\mathrm{mg}$ of 20-30 nm $\mathrm{Fe}_{3} \mathrm{O}_{4}$ NPs functionalized with linker 4.3 in 2:1 PBS:MeCN at $\mathrm{pH} 7.4$.

4.12 Possible explanations for non-AMF induced release of fluorophore. $\quad 100$

4.13 Linker 4.3, capable of intramolecular cyclization, and $\mathbf{1 0}$ with a 101 methylene instead of a nucleophilic amine, rendering it incapable of intramolecular cyclization. Linker $\mathbf{1 0}$ was used to probe the origin of the observed non-AMF induced release.

4.14 595 Amp AMF and non-AMF induced release of fluorophore from 3 102 mg of 20-30 nm $\mathrm{Fe}_{3} \mathrm{O}_{4}$ NPs functionalized with linker 10 in 2:1 PBS:MeCN at $\mathrm{pH} 7.4$.

4.15 595 Amp AMF and non-AMF induced release of fluorophore from 3 103 $\mathrm{mg}$ of 20-30 $\mathrm{nm} \mathrm{Fe}{ }_{3} \mathrm{O}_{4} \mathrm{NPs}$ functionalized with linker 10 in 2:1 PBS:MeCN at $\mathrm{pH}$ 7.4. With and without heat refers to the curing process of the functionalized NPs. Without heat NPs were cured under vacuum and with heat NPs were cured at $110^{\circ} \mathrm{C}$ for 24 hour at atmospheric pressure.

4.16 Fluorescent signal of $\mathbf{1 3}$ functionalized with FITC via a thiourea linkage in $\mathrm{MeCN}$.

4.17 595 Amp AMF and non-AMF induced release of fluorophore from $\sim 7 \quad 107$ $\mathrm{mg}$ of 16 in $1.5 \mathrm{~mL} \mathrm{2:1} \mathrm{PBS:MeCN}$ at $\mathrm{pH}$ 7.4. The AMF-induced release is the result of what remained on the NPs following the incubation period of 30 minutes at room temperature in $1.5 \mathrm{~mL} 2: 1$ PBS:MeCN at $\mathrm{pH} 7.4$. 
4.18 ${ }^{13} \mathrm{C}$ NMR spectra confirming the structures of 17.1-3.

$4.19200 \mathrm{amp}$ AMF induced release of fluorophore from $\sim 6 \mathrm{mg}$ of 18.1-3 111 in $0.75 \mathrm{~mL} \mathrm{2:1} \mathrm{PBS:MeCN}$ at $\mathrm{pH} 7.4$.

4.20 Release of fluorophore from $\sim 6 \mathrm{mg}$ of $\mathbf{1 8 . 1 - 3}$ in $0.75 \mathrm{~mL} \mathrm{2:1}$ PBS:MeCN at $\mathrm{pH} 7.4$ incubated at $37^{\circ} \mathrm{C}$ for $20 \mathrm{~h}$.

4.21 Proposed mechanism for $\mathrm{Fe}_{3} \mathrm{O}_{4} \mathrm{NP}$ accelerated hydrolysis of esters, carbonates and carbamates under mild conditions.

4.22 Non-triggered (i.e., no AMF application) hydrolysis of $\sim 7 \mathrm{mg} \mathbf{1 8 . 2}$ compared with the suppressed hydrolysis of 19.1-2 and 20 at $37^{\circ} \mathrm{C}$ in 2:1 PBS:MeCN pH 7.4.

4.23 AMF-induced release of anthracene fluorophore from NPs 21 and $22 . \quad 120$

4.24 Procedure used to obtain sample for MALDI-TOF analysis and verification of released substrate.

4.25 MALDI-TOF spectrum using a 2,5-dihydroxybenzoic acid matrix of the radical carbocation 2-(9-anthracenyl)ethanol released from 22.

4.26 HAADF Z-contrast STEM image of aggregated $\mathrm{Fe}_{3} \mathrm{O}_{4} \mathrm{NPs}$ with a thick silica shell surrounding them.

4.27 DLS of $\mathrm{SiO}_{2} @ \mathrm{Fe}_{3} \mathrm{O}_{4} \mathrm{NPs}$ in EtOH showing the hydrodynamic diameter at $310 \mathrm{~nm}$ with a polydispersity of 0.23 .

4.28 TEM image of $\mathrm{SiO}_{2} @ \mathrm{Fe}_{3} \mathrm{O}_{4}$ NPs synthesized from EMG 304 showing excessive aggregation.

4.29 Superconducting quantum interference device (SQUID) measurement of $\mathrm{SiO}_{2} @ \mathrm{Fe}_{3} \mathrm{O}_{4}$ NPs prepared from EMG 304.

4.30 TEM image of $\mathrm{SiO}_{2} @ \mathrm{Fe}_{3} \mathrm{O}_{4}$ NPs synthesized from EMG 304 with a particle size distribution of $24 \pm 6 \mathrm{~nm}$ and a silica shell thickness of 6$7 \mathrm{~nm}$.

4.31 DLS of $\mathrm{SiO}_{2} @ \mathrm{Fe}_{3} \mathrm{O}_{4}$ NPs in Millipore water showing the hydrodynamic diameter at $103 \mathrm{~nm}$ with a polydispersity of 0.17 .

4.32 AMF-induced heating/cooling of 2:1 PBS:acetonitrile, $\mathrm{SiO}_{2} @ \mathrm{Fe}_{3} \mathrm{O}_{4}$ NPs in 2:1 PBS:acetonitrile, and AMF 5 min on/off sequence.

4.33 AMF-triggered hydrolysis of pendant functionality.

4.34 TEM image of $\mathrm{AO} @ \mathrm{SiO}_{2} @ \mathrm{Fe}_{3} \mathrm{O}_{4} \mathrm{NPs}$ with a particle size distribution $(30.4 \pm 0.8 \mathrm{~nm})$ resulting in a $\sim 3 \mathrm{~nm}$ increase in the NP radius.

4.35 DLS of $\mathrm{AO} @ \mathrm{SiO}_{2} @ \mathrm{Fe}_{3} \mathrm{O}_{4}$ NPs in Millipore water showing the hydrodynamic diameter at $96 \mathrm{~nm}$ with a polydispersity of 0.16 .

4.36 AMF-induced release of 27 from FL@ $\mathrm{SiO}_{2} @ \mathrm{Fe}_{3} \mathrm{O}_{4}$.

4.37 MALDI-TOF spectra of the radical carbocation of the anthracenyl oxime ether of $\mathbf{2 3}$ and $\mathbf{2 7}$ after AMF-induced hydrolysis and release from $\mathrm{FL} @ \mathrm{SiO}_{2} @ \mathrm{Fe}_{3} \mathrm{O}_{4} \mathrm{NPs}(\mathbf{2 5})$. 
4.38 AMF-induced release, as determined by fluorescence measurements, of 27 from $\mathrm{FL} @ \mathrm{SiO}_{2} @ \mathrm{Fe}_{3} \mathrm{O}_{4} \mathrm{NPs}$ in 2:1 PBS:acetonitrile at AMF amperages of 100.7, 300.2 or 501.6.

4.39 AMF-induced release of 27 from FL@ $@ \mathrm{SiO}_{2} @ \mathrm{Fe}_{3} \mathrm{O}_{4} \mathrm{NPs}$ during 2 minute or 5 minute AMF pulses at 501.6 amps versus incubation at $37^{\circ} \mathrm{C}$.

4.40 TEM image of $\mathrm{Au} @ \mathrm{Fe}_{3} \mathrm{O}_{4} \mathrm{NPs}$.

4.41 SQUID measuments of $\mathrm{Au} @ \mathrm{Fe}_{3} \mathrm{O}_{4}$ NPs showing superparamagnetism. 


\section{LIST OF SCHEMES}

1.1 Reaction of an aminooxy with an aldehyde or ketone to form an oxime ether.

1.2 Intermediates in the nucleophilic catalysis of oximation and hydrazonation by aniline at $\mathrm{pH} 4-5$ as proposed by Thygesen et al.

1.3 Site-specific immobilization of peptides and proteins on a biosensor 16 chip surface using aniline-catalyzed oximation.

1.4 Synthesis of aminooxy-terminated core-shell gold NPs via Trim deprotection and oximation of a peptide, glycopeptide and glycan.

1.5 Preparation of lipid-coated $\mathrm{Fe}_{3} \mathrm{O}_{4}$ NPs using oximation to attach hydrophobic chains.

1.6 Installation of an aminooxy moiety onto core-shell $\mathrm{Fe}_{3} \mathrm{O}_{4}-\mathrm{SiO}_{2} \mathrm{NPs} \quad 20$ for subsequent chemoselective reaction with glycopeptides.

1.7 Chapter 2 Summary.

2.1 AMAH oximation covalently traps aldehydes and ketones in the microreactor.

2.2 Use of AMAH for both FT-ICR-MS and GC-MS analyses.

3.1 Synthesis of $\mathbf{2}$.

3.2 Modification of carbonyl functionality from carboxylic acid to a carbonate or carbamate.

3.3 Synthesis of amino esters 6 and amide 9.

3.4 Synthesis of amino carbonates 7.

3.5 Synthesis of amino carbamate $\mathbf{8}$.

3.6 Synthesis of gem-dimethylated ester $\mathbf{1 0}$. 72

3.7 Synthesis of gem-dimethylated carbonate 11.

$\begin{array}{lll}3.8 & \text { Heat-induced release of anthracene probes. } & 74\end{array}$

3.9 Poly(dimethylsiloxane) microchannel functionalization and heat- 80 induced release study.

3.10 Preparation of the aldehydic FITC moiety. 
4.1 Boc-deprotection of a $2^{\circ}$ amine bound to a 20-30 nm $\mathrm{Fe}_{3} \mathrm{O}_{4}$ NP under 104 acidic conditions.

4.2 Loading of 14 onto 20-30 $\mathrm{nm} \mathrm{Fe}_{3} \mathrm{O}_{4}$ NPs and Boc-deprotection of 15106 under acidic conditions to afford a thermally labile linker.

4.3 Short chain linkers bound to $\mathrm{Fe}_{3} \mathrm{O}_{4}$ NPs to test hydrolytic stability of 109 ester, carbonate and carbamate functionalities.

4.4 Examples from current literature of the hydrolysis of an ester, a carbonate and a carbamate.

4.5 Attempts to mask the iron oxide NP surface by coating the functionalized NPs with polysaccharides or coating the iron oxide core with a silica shell.

4.6 Synthesis of NP-carbonate linker conjugates with anthracenyl fluorophore.

4.7 Illustration of the microemulsion coating mechanism of $\mathrm{SiO}_{2}$ on the surface of $\mathrm{Fe}_{3} \mathrm{O}_{4}$-oleate NPs.

4.8 Synthesis of linkers for substrate release via AMF-induced hydrolysis.

4.9 Linkers designed for release via AMF-induced hydrolysis bound to $\mathrm{SiO}_{2} @ \mathrm{Fe}_{3} \mathrm{O}_{4} \mathrm{NPs}$.

4.10 AMF-induced carbamate hydrolysis.

4.11 Synthesis of $\mathrm{Au} @ \mathrm{Fe}_{3} \mathrm{O}_{4} \mathrm{NPs}$.

4.12 Synthesis of carbonate linker 28 for attachment to $\mathrm{Au} @ \mathrm{Fe}_{3} \mathrm{O}_{4}$ NPs. 


\section{CHAPTER 1}

\section{ADVANCEMENTS IN OXIMATION: APPLICATION OF AMINOOXY CHEMISTRY AND ATTACHMENT TO SOLID SUPPORTS}

\subsection{INTRODUCTION}

1.2. CHEMOSELECTIVE LIGATION

1.3. SOLID-SUPPORTED AMINOOXY CHEMISTRY

1.4. USE OF AMINOOXY CHEMISTRY IN NANOTECHNOLOGY

1.5. IRON OXIDE NANOPARTICLES

1.6. USE OF IRON OXIDE NANOPARTICLES TO GENERATE HEAT

1.7. CONCLUSION 


\subsection{INTRODUCTION}

Aminooxy chemistry is one of the true forms of click chemistry as described by Nobel laureate K. Barry Sharpless in 2001. ${ }^{1}$ Sharpless recommended to follow Nature's lead, namely to synthesize substances by joining small segments together through heteroatom linkages $(\mathrm{C}-\mathrm{X}-\mathrm{C})$ as opposed to focusing on carbon-carbon bond generation as the key assembly process. In order for a reaction to be considered click chemistry, it must be modular, wide in scope, have high yields, generate only inoffensive byproducts that can be removed without chromatography, use only readily available starting materials and reagents and be carried out either without solvents or with solvents such as water.

With such stringent specifications, only a handful of reactions can be labeled as click chemistry. Click chemistry reactions can be divided into four different classes: 1) cycloadditions of unsaturated species, especially 1,3-dipolar cycloaddition reactions, but also the Diels-Alder family of transformations; 2) additions to carbon-carbon multiple bonds, particularly oxidative cases such as epoxidation and aziridination; 3) carbonyl chemistry of the "non-aldol" type, such as formation of oxime ethers, hydrazones and amides; and 4) nucleophilic substitution chemistry of the $\mathrm{S}_{\mathrm{N}} 2$ type, such as ring-opening reactions of strained heterocyclic electrophiles (i.e., epoxides and aziridines). ${ }^{1}$ Examples of these reactions can be seen in Figure 1.1. 


\section{Cycloadditions of unsaturated species}

Azide-Alkyne Cycloadditions

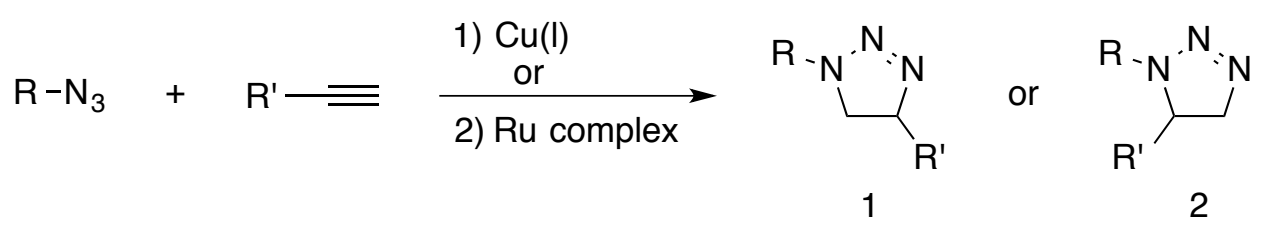

2. Additions to carbon-carbon multiple bonds

Olefin Epoxidation

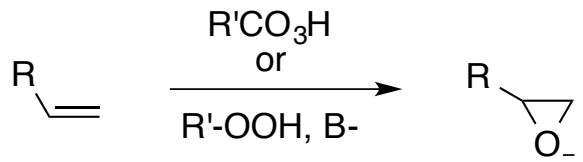

\section{Carbonyl chemistry of the "non-aldol" type}

Aminooxy-Carbonyl Oximation

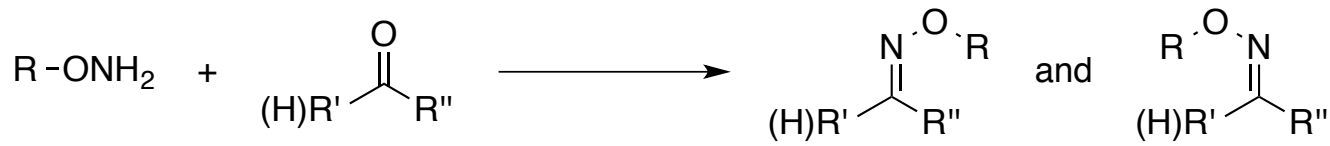

\section{Ring opening reaction of strained hetercycles}

Epoxide ring-opening<smiles>[R]NCC(O)([10IH])CN[R]</smiles>

Figure 1.1. Generic examples of the four click chemistry classes of chemical transformations.

Though the azide-alkyne cycloaddition is one of the most popular forms of click chemistry, our lab has focused on the development of carbonyl chemistry of the "nonaldol" type, specifically aminooxy chemistry. Aminooxy chemistry involves the reaction of an aminooxy group with an aldehyde or ketone to form an oxime ether derivative 
(Scheme 1.1). The aminooxy nitrogen exhibits very different properties from the nitrogen of an amine moiety. Table 1.1 highlights a few of these major differences.

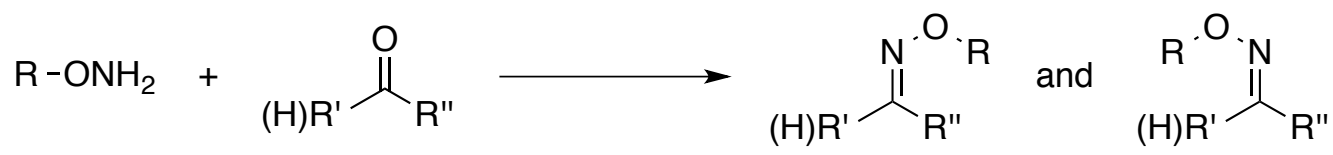

Scheme 1.1. Reaction of an aminooxy with an aldehyde or ketone to form an oxime ether.

Table 1.1. Comparison of the aminooxy functionality with a primary amine.

\begin{tabular}{|c|c|}
\hline Aminooxy & Primary Amine \\
\hline $\begin{array}{r}\mathrm{R}-\mathrm{ONH}_{2} \stackrel{\mathrm{H}^{+}}{\longrightarrow} \underset{\mathrm{R}-\mathrm{ONH}_{3}^{+}}{\mathrm{pKa}=5-6}\end{array}$ & $\begin{aligned} \mathrm{R}-\mathrm{NH}_{2} \stackrel{\mathrm{H}^{+}}{\longrightarrow} & \mathrm{R}-\stackrel{+}{\mathrm{N}} \mathrm{H}_{3} \\
& \mathrm{pKa}=9-10\end{aligned}$ \\
\hline $\begin{array}{l}\text { At room temperature and neutral } \mathrm{pH} \text {, the } \\
\text { aminooxy group reacts chemoselectively } \\
\text { with aldehydes and ketones to form the } E / Z \\
\text { isomeric oxime ethers. }\end{array}$ & $\begin{array}{l}\text { At room temperature and neutral } \mathrm{pH} \text {, a } \\
\text { primary amine reacts with aldehydes, } \\
\text { ketones to form imines and with esters to } \\
\text { form secondary amides. }\end{array}$ \\
\hline 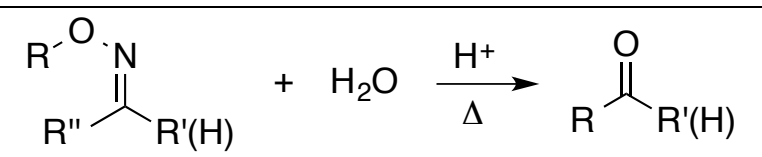 & 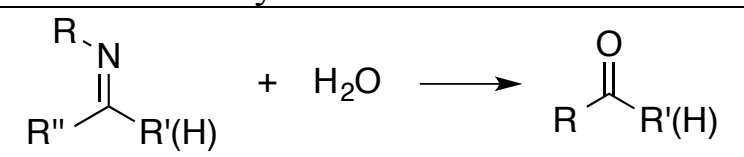 \\
\hline $\begin{array}{l}\text { Hydrolysis of the oxime ether linkage } \\
\text { cannot be achieved by water alone and } \\
\text { results in the restoration of the active } \\
\text { aminooxy group. }\end{array}$ & $\begin{array}{l}\text { Hydrolysis of the imine linkage is readily } \\
\text { achieved under aqueous conditions and } \\
\text { results in the restoration of the active } \\
\text { primary amine. }\end{array}$ \\
\hline
\end{tabular}

The stark difference between the aminooxy group and the primary amine stems from the presence of the oxygen adjacent to the nitrogen (i.e., in an $\alpha$-position), a phenomenon termed the $\alpha$-effect. ${ }^{2}$ Edwards and Pearson ${ }^{3}$ first introduced the $\alpha$-effect in 1962 to explain the enhanced nucleophilicity of a certain group of nucleophiles. The presence of an electronegative atom with one or more unshared pairs of electrons in an $\alpha$ - 
position to the nucleophilic atom was the one common feature among this class of nucleophiles. Edwards and Pearson postulated that the unshared electrons in the $\alpha$ position help to stabilize a positive charge on the nucleophilic atom, thus allowing the nucleophilic atom to more freely bond to electrophiles.

A major contributing factor to the chemoselectivity of the aminooxy nitrogen is the electronegativity of the $\alpha$-oxygen. This is most evident by observing the $\mathrm{p} K_{\mathrm{a}}$ difference between a protonated aminooxy $\left(\mathrm{RONH}_{3}{ }^{+}\right)$having a $\mathrm{p} K_{\mathrm{a}}$ of $5-6^{4}$ and a primary aminium $\left(\mathrm{RNH}_{3}{ }^{+}\right)$having a $K_{\mathrm{a}}$ of 9-10. Hydrazine, another molecule/functional group subject to the $\alpha$-effect, has a $\mathrm{p} K_{\mathrm{a}}$ of 8 when protonated. Similar to an aminooxy group, hydrazine reacts with aldehydes and ketones to form stable hydrazones, but it is not chemoselective like the aminooxy group in that it will react with esters, phthalimides and anhydrides. The hydrolytic stability of oximes and hydrazones, in contrast with imines, is also due to the $\alpha$-effect. As can be seen in Figure 1.2, the lone pair of electrons $\alpha$ to the $\mathrm{sp}^{2}$ hybridized nitrogen allow for resonance stabilization by increasing the negative charge density at $\mathrm{C} 1$, thus reducing its electrophilicity. ${ }^{5}$ When the resonance forms of an oxime or hydrazone are compared to the resonance forms of an imine it is easy to understand why imines are susceptible to hydrolysis. Imine electron density is primarily located at the nitrogen atom, making $\mathrm{C} 1$ electrophilic and prone to nucleophilic attack by water. In addition, Wiberg and Glaser ${ }^{6}$ proposed another explanation for the hydrolytic stability of oximes ethers and hydrazones. They predict that the reduction in lone-pair repulsion achieved by going from an aminooxy and hydrazine to respective oxime ether and hydrazone leads to this stability. 


\section{Oximes and Hydrazones}

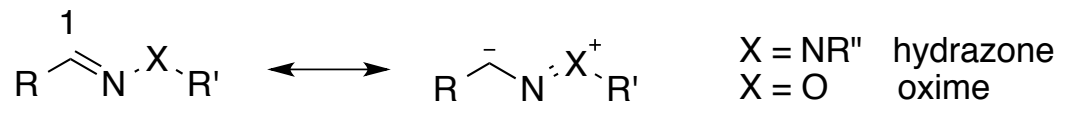

Imines

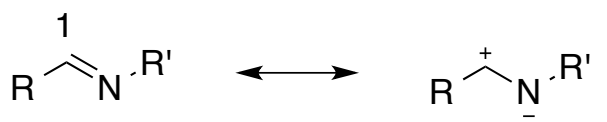

Figure 1.2. Resonance forms of oximes, hydrazones and imines.

Resonance in oximes or hydrazones increases the negative charge density at $\mathrm{C} 1$ and imparts hydrolytic stability.

Though oximes and hydrazones are hydrolytically stable in an aqueous environment, hydrolysis can still be achieved. Figure 1.3 shows the mechanism of the acidic hydrolysis of oximes and hydrazones to restore the aminooxy and hydrazine along with the starting aldehyde. It is well known that the hydrazone bond is more susceptible to acidic hydrolysis than its oxime counterpart. As was noted by Kalia and Raines, ${ }^{5}$ the difference in hydrolytic stability is due to the electronegativity of the atom $\alpha$ to the nucleophilic atom in the preceding aminooxy or hydrazine (atom X, Figure 1.3). For the oxime, the increased electronegativity of an oxygen atom $\left(\chi_{\mathrm{O}}=3.5^{7}\right)$ in the $\mathrm{X}$ position (Figure 1.3), $\alpha$ to the nitrogen, reduces the probability of the formation of III and increases the likelihood of forming II. This resonance makes the convenient hydrolysis of oxime ethers require heat with $\mathrm{HCl}$ or $\mathrm{HClO}_{4}$ at $\mathrm{pH} 0-4$. Hydrazones, due to the reduced electronegativity of a nitrogen atom $\left(\chi_{\mathrm{N}}=3.0\right)$ in the $\mathrm{X}$ position (Figure 1.3), can undergo hydrolysis under more mildly acidic conditions. The hydrazone functionality 
makes the formation of III much more likely, thus perpetuating the acidic hydrolysis to give the hydrazine and starting aldehyde.

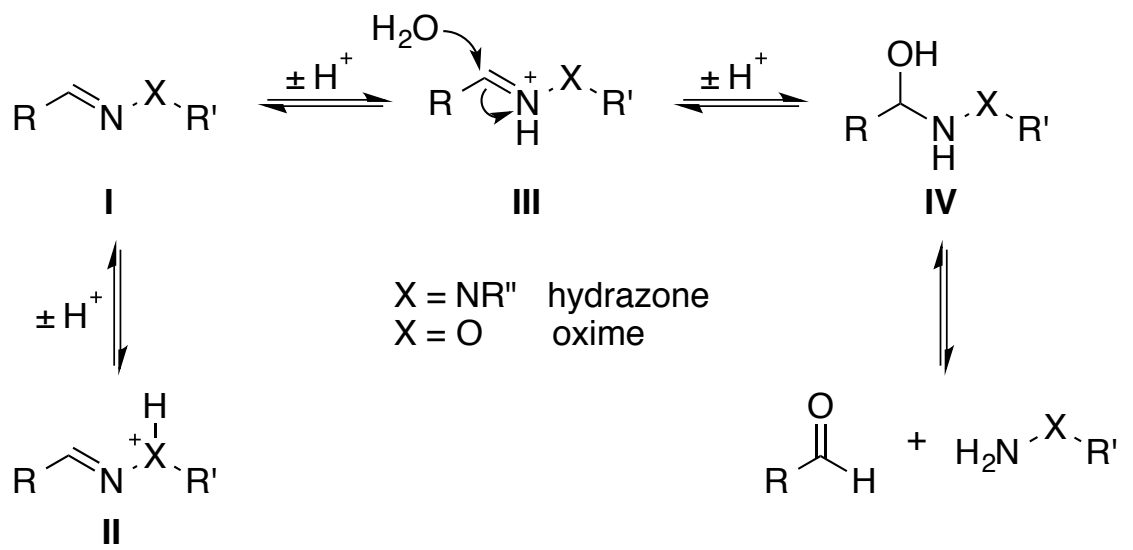

Figure 1.3. Mechanistic considerations for acid-induced hydrolysis of oximes and hydrazones.

The ability of the aminooxy functionality to chemoselectively bond to aldehydes and ketones to form oxime ethers makes it attractive for a variety of applications. Though aminooxy groups are usually quite reactive and can react to completion very rapidly, there are some oximation reactions that occur very slowly. Numerous different catalysts have been studied to hasten this process. Crisalli and $\mathrm{Kool}^{8}$ report a panel of anthranilic acids for the nucleophilic catalysis of oximation and hydrazonation (Table 1.2). Aniline is the central focal point of the majority of nucleophilic catalysts for oximation and hydrazonation. ${ }^{9}$ Aniline catalyzes the reaction by first forming an iminium ion, then the nucleophilic amine of an aminooxy or hydrazine functionality can 
Table 1.2. Yields of hydrazone formation of nitrobenzoxadiazole hydrazine and 4-nitrobenzaldehyde with varied anthranilic acid catalysts at $\mathrm{pH} 7.4 .^{8}$

\begin{tabular}{|c|c|c|c|c|}
\hline Entry & Catalyst & Structure & $\begin{array}{c}\text { Conversion } \\
(2 \mathrm{~h})\end{array}$ & $\begin{array}{c}\text { Relative } \\
\text { Yield }\end{array}$ \\
\hline 1 & no catalyst & & $0.7 \pm 0.2 \%$ & 1.0 \\
\hline 2 & aniline & & $10.4 \pm 1.3 \%$ & 14 \\
\hline 3 & anthranilic acid & & $21.5 \pm 0.8 \%$ & 29 \\
\hline 4 & 4-methylanthranilic acid & & $28.4 \pm 4.4 \%$ & 38 \\
\hline 5 & 5-methylanthranilic acid & & $32.6 \pm 0.5 \%$ & 44 \\
\hline 6 & 3-amino-2-naphthoic acid & & $31.5 \pm 1.5 \%$ & 43 \\
\hline 7 & 4-methoxyanthranilic acid & & $21.7 \pm 2.2 \%$ & 29 \\
\hline 8 & 4,5-dimethoxyanthranilic acid & & $37.1 \pm 2.5 \%$ & 50 \\
\hline 9 & 5-methoxyanthranilic acid & & $55.2 \pm 1.1 \%$ & 75 \\
\hline 10 & 4,6'-bianthranilic acid & & $57.8 \pm 7.3 \%$ & 78 \\
\hline
\end{tabular}

attack the unstable iminium ion in aqueous conditions to form the stable oxime or hydrazone, respectively (Scheme 1.2). ${ }^{10}$ This process, the attack of a nucleophilic 


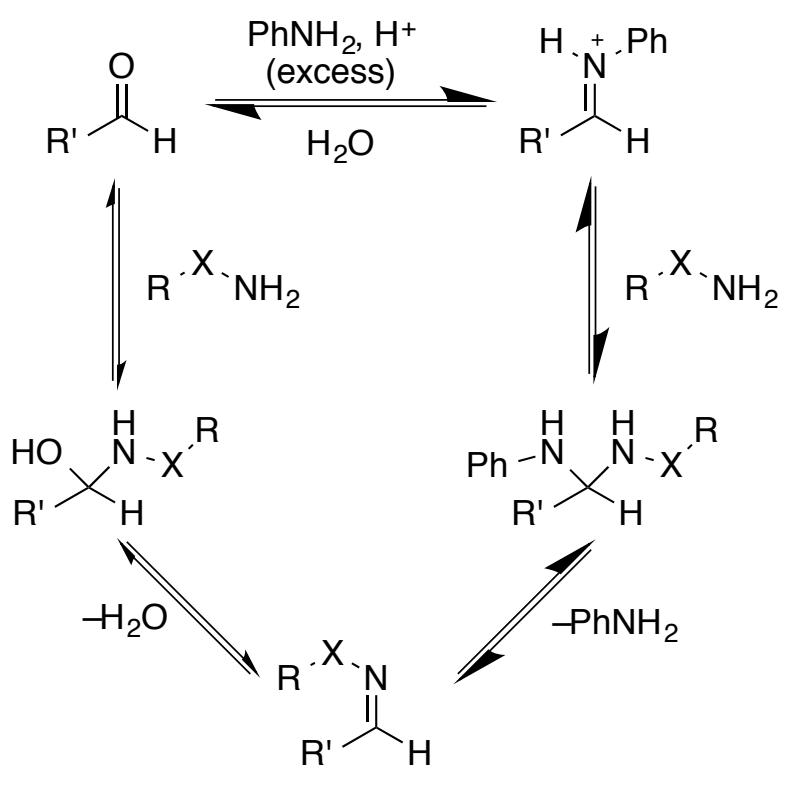

Scheme 1.2. Intermediates in the nucleophilic catalysis of oximation $(\mathrm{X}=\mathrm{O})$ and hydrazonation $(\mathrm{X}=\mathrm{NR}$ ”) by aniline at $\mathrm{pH}$ 4-5 as proposed by Thygesen et al..$^{10}$

primary amine on an aldehyde or ketone under acidic conditions to form the susceptible iminium ion, can be compared to a reductive amination reaction. ${ }^{11}$ The enhancement of oximation and hydrazonation reactions under acidic conditions was displayed in Crisalli and Kool's ${ }^{8}$ catalytic studies, as can be seen in Table 1.3. It should also be emphasized that all reported catalysts for oximation and hydrazonation reactions are water-soluble and the water solubility of the catalyst is oftentimes a deciding factor for its promise. ${ }^{12}$ 
Table 1.3. Yields of hydrazone formation of nitrobenzoxadiazole hydrazine and 4-nitrobenzaldehyde with varied arylamine/acid catalysts at $\mathrm{pH} 7.4 .^{8}$

\begin{tabular}{cccc} 
Entry & Catalyst & Conversion (2 h) & Relative Yield \\
\hline \hline 1 & no catalyst & $0.7 \pm 0.2 \%$ & 1.0 \\
2 & aniline & $10.4 \pm 1.3 \%$ & 14 \\
3 & anthranilic acid & $21.5 \pm 0.8 \%$ & 29 \\
4 & anthranilonitrile & $0.8 \pm 0.1 \%$ & 1.0 \\
5 & anthranilamide & $1.6 \pm 0.1 \%$ & 2.1 \\
6 & ethyl anthranilate & $1.5 \pm 0.3 \%$ & 2.1 \\
7 & benzoic acid & $1.8 \pm 0.4 \%$ & 2.4 \\
8 & benzoic acid + aniline 1:1 & $18.1 \pm 1.9 \%$ & 25 \\
9 & 3-aminobenzoic acid & $14.3 \pm 1.2 \%$ & 19 \\
10 & 4-aminobenzoic acid & $7.4 \pm 0.4 \%$ & 10 \\
11 & 3,5-diaminobenzoic acid & $34.7 \pm 2.4 \%$ & 47
\end{tabular}

\subsection{CHEMOSELECTIVE LIGATION}

Most organic chemistry transformations cannot be performed in aqueous conditions, thus making the application of organic chemistry techniques to biological systems, such as cells, challenging. Due to this difficulty, researchers interested in common biological processes, such as ligation, are constrained and forced to work with a limited number of reactions. ${ }^{13}$ Figure 1.4 shows the limited catalog of chemoselective reactions available for ligation or other modifications of cells. The hydrolytic stability, chemoselectivity and overall robustness of the aminooxy linkage make it an excellent candidate for such biological applications. 
A

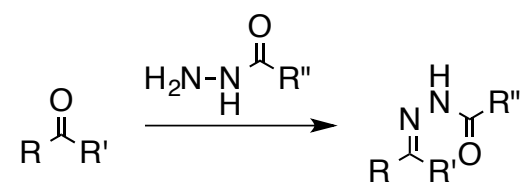

C

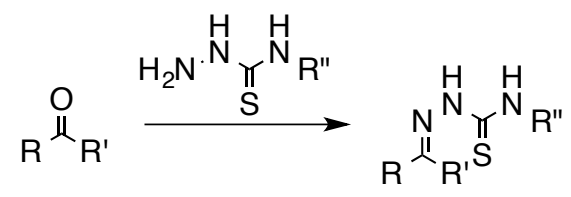

B

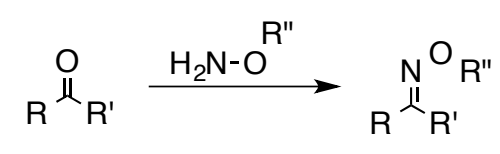

D

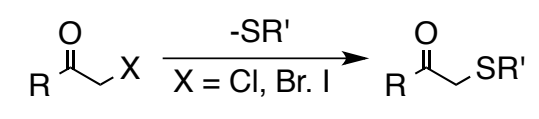

$\mathbf{F}$

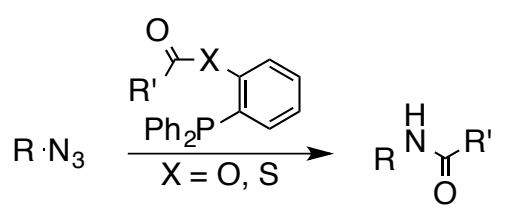

G

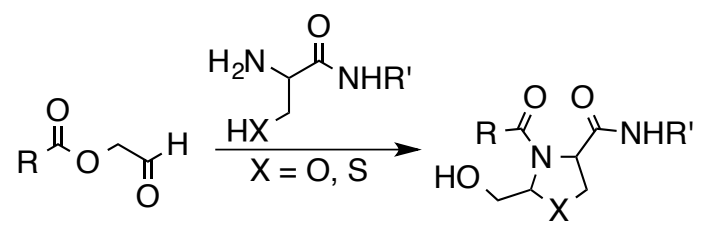

H

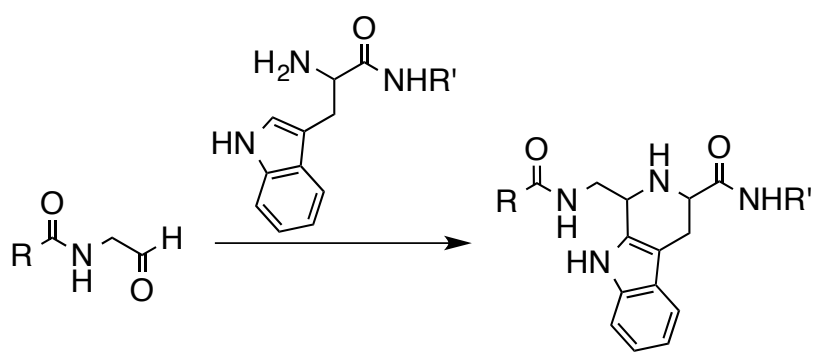

I

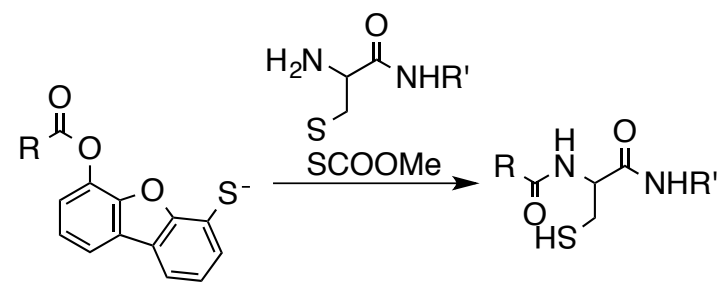

\section{J}

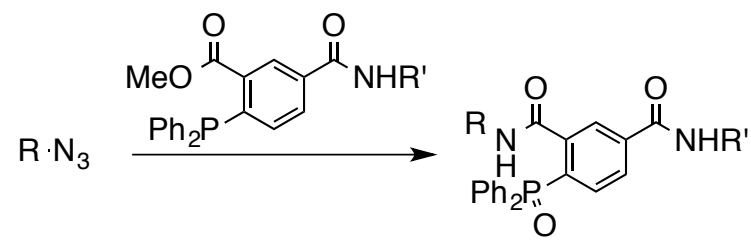

Figure 1.4. Chemoselective ligation reactions used in the convergent assembly of biopolymers and their mimetics, and in the modification of biopolymers and cells. The products of the reactions are (A) N-acylhydrazones, (B) oxime ethers, (C) thiosemicarbazones, (D) thioethers, (E) native amide bonds, (F) simple amide bonds, (G) pseudoproline linkages, $(H)$ tetrahydro- $\beta$-carbolines, (I) native amide bonds after reduction of disulfide bonds, and (J) amide phosphine oxide. ${ }^{14}$ 
Pascal Dumy's lab has developed a tetrameric cRGD containing nano-probe by covalently linking four peptides to the cyclic decapeptide scaffold regioselectively addressable functionalized template (RAFT). ${ }^{15}$ Dumy et al. used aminooxy ligation to ensure chemoselectivity, versatility and allow for covalent attachment without the use of coupling agents. But the most influential reason that oximation was the chosen ligation technique was that the oxime bond afforded robust and stable linkages for both in vitro and in vivo applications. This technology, now commercially available under the name AngioLone $^{\mathrm{TM}}$, can be linked with a fluorescent dye or with radioactive contrast agents for use in the surgical removal of tumors. The $\alpha_{v} \beta_{3}$ integrin is overexpressed on the surface of endothelial cells ${ }^{16}$ and several tumor cell types. ${ }^{17}$ RGD is a known recognition motif and thus allows the AngioLone ${ }^{\mathrm{TM}}$ to have a high tumor-targeting efficacy. ${ }^{18}$ As a proof of concept for upcoming human clinical trials, Coll et al. applied AngioLone ${ }^{\mathrm{TM}}$ for the removal of a mesenchymal tumor, a feline fibrosarcoma, where it was found to be effective in the accurate, safe and complete removal of the tumors in real surgical conditions. As can be seen in Figure 1.5, the cluster of $\alpha_{v} \beta_{3}$ ligands are bound to the decapeptide RAFT through oxime ether bonds. 


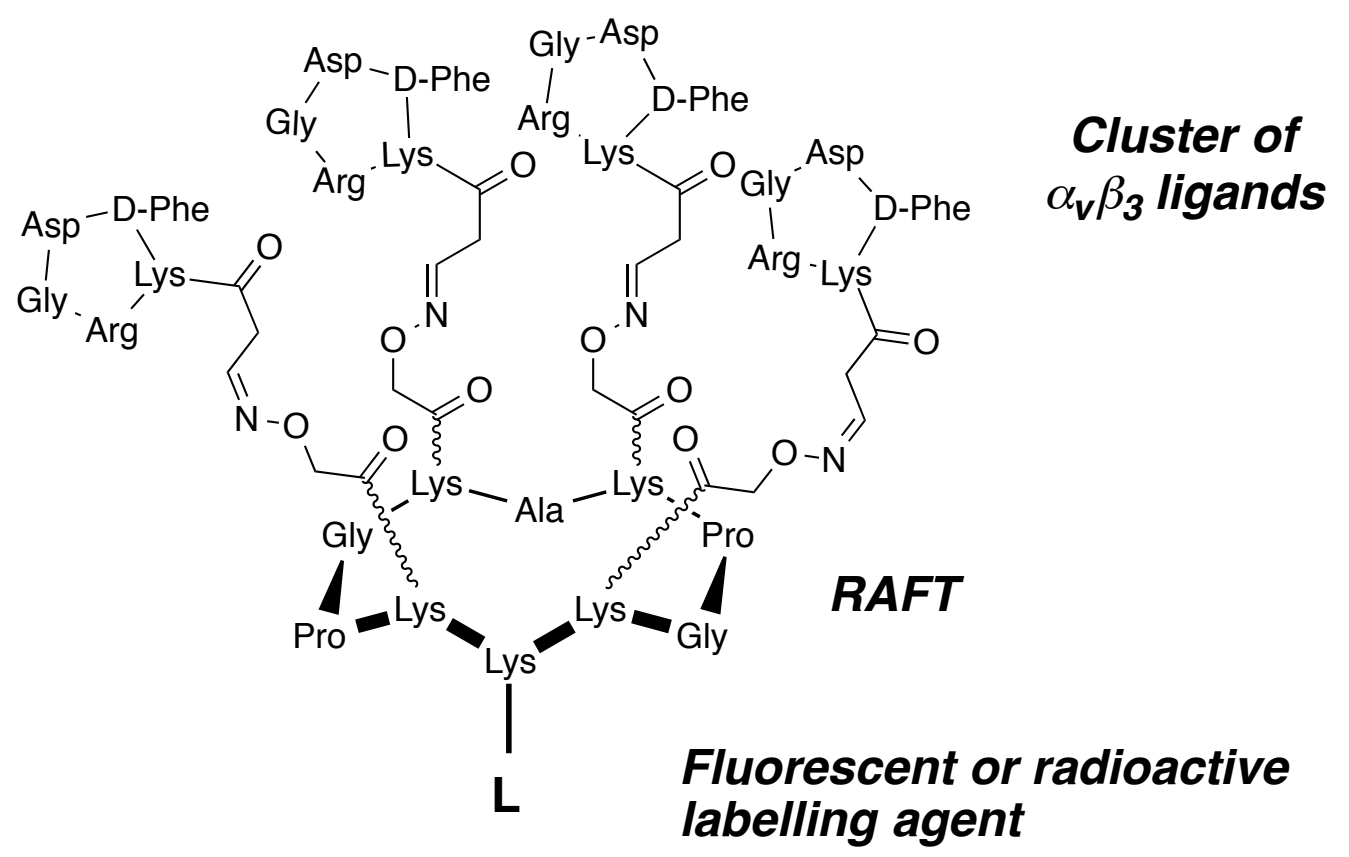

Figure 1.5. Chemical structure of the tetravalent RGD ligand RAFT conjugated to a fluorescent or radioactive label. ${ }^{19}$

Zeng et al. ${ }^{20}$ used oxime ether and thioether linkages to build a synthetic lipopeptide-based self-adjuvanting vaccine that can elicit neutralizing antibodies against heat-stable enterotoxin from enterotoxigenic Escherichia coli. They found that the thioether vaccine showed residual toxicity in suckling mice while the oxime ether versions expressed no cytotoxicity. Though both the thioether and oxime ether vaccine produced specific anti-ST antibodies, only the oxime ether vaccine, specifically the $\mathrm{ST}^{\mathrm{N}}$ oxime-P2C- $\mathrm{T}_{\mathrm{H}}$ vaccine, generated antibodies capable of neutralizing the virus when administered by the mucosal (intranasal) route. This finding suggests that the chemical linkage affects the efficacy of the antibodies. Figure 1.6 shows the three different vaccines that Zeng and coworkers synthesized. 

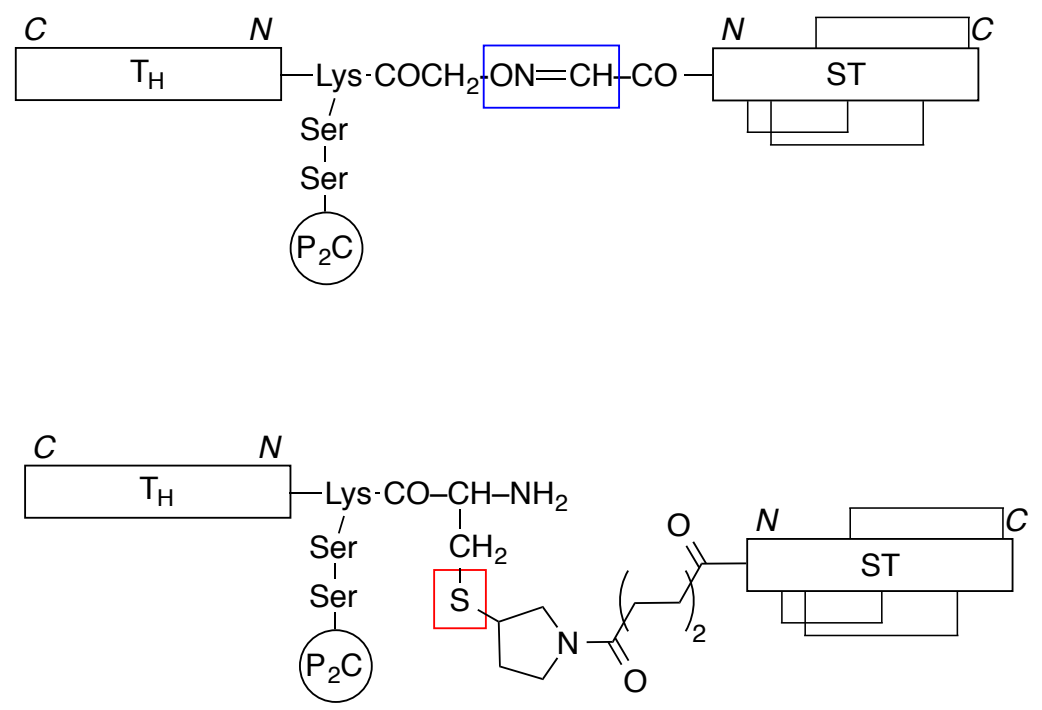

ST ${ }^{N}$-thioether-P2C- $T_{H}$

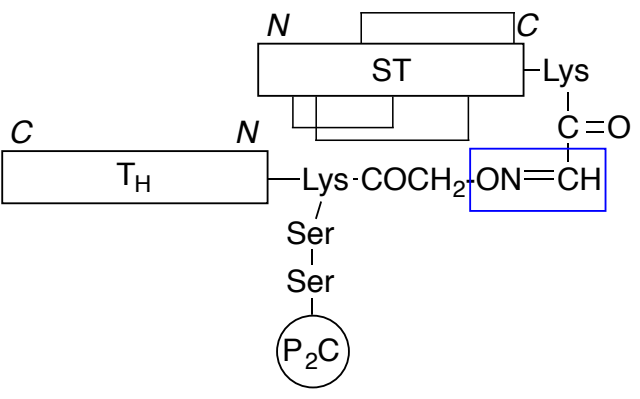

STC-oxime-P2C-T

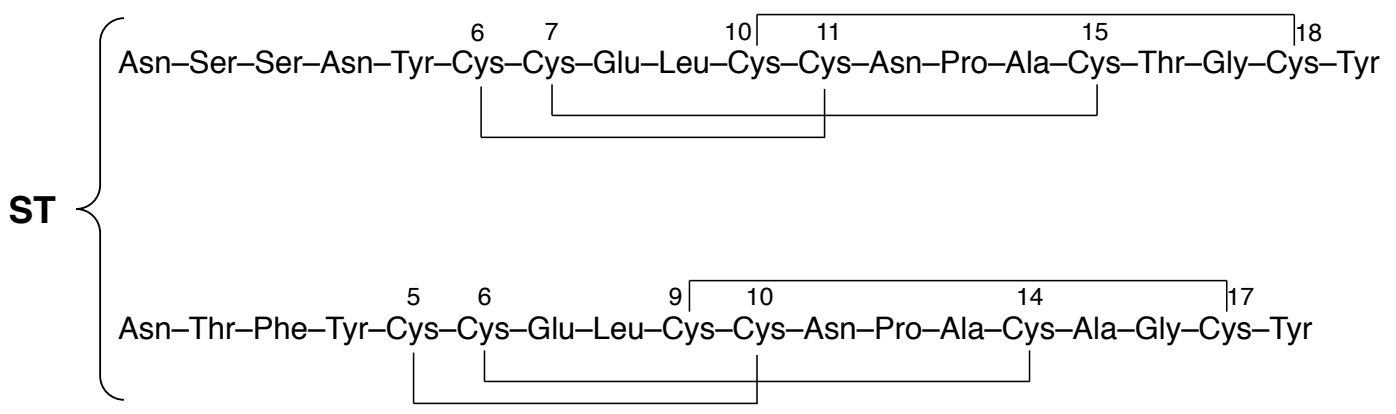

Figure 1.6. Schematic representation of the structures of the ST-based lipopeptide vaccines with oxime ether and thioether linkages. $T_{H}$ refers to the helper $\mathrm{T}$ cell epitope, $\mathrm{C}$ and $\mathrm{N}$ refer to the $\mathrm{N}$-terminus and $\mathrm{C}$-terminus, respectively, of peptide sequences and $\mathrm{P} 2 \mathrm{C}$ refers to $S$-[2,3bis(palmitoyloxy)propyl]cysteine. ${ }^{20}$ 


\subsection{SOLID-SUPPORTED AMINOOXY CHEMISTRY}

Oxime bonds are also used extensively for solid support surface modification. Lepens et $a l .{ }^{21}$ modified surface plasmon resonance (SPR) chips with aminooxy groups using a newly developed bifunctional linker. After the carboxylic acid groups on the SPR chip surface were activated with 1-ethyl-3-(3-dimethylaminopropyl) carbodiimide (EDC) and $\mathrm{N}$-hydroxysuccinimide (NHS), a solution of a Boc-protected aminooxy derivative was injected. To quench the unreacted activated esters on the SPR chip a solution of ethanolamine was injected. Following cleavage of the Boc protecting group, $\mathrm{N}$-terminal aldehyde or ketone functionality was introduced either by oxidation of $\mathrm{N}$ terminal amino acids with pyridoxal 5'-phosphate $(\mathrm{R}=\mathrm{R}$ ' = amino acid side chain $)$ or by oxidation of $N$-terminal serine $\left(\mathrm{R}^{\prime}=\mathrm{H}, \mathrm{R}^{\prime \prime}=\mathrm{CH}_{2} \mathrm{OH}\right)$ or threonine $\left(\mathrm{R}^{\prime}=\mathrm{H}, \mathrm{R}^{\prime \prime}=\right.$ $\left.\mathrm{CH}\left(\mathrm{CH}_{3}\right) \mathrm{OH}\right)$ residues using $\mathrm{NaIO}_{4}$. These functional groups allowed for the covalent retention of the modified proteins on the SPR chip (Scheme 1.3). The process was catalyzed with aniline and, consistent with previously discussed findings, Lepens et al. found that the oximation reaction occurred most rapidly under acidic conditions at $\mathrm{pH}$ 4.5. 


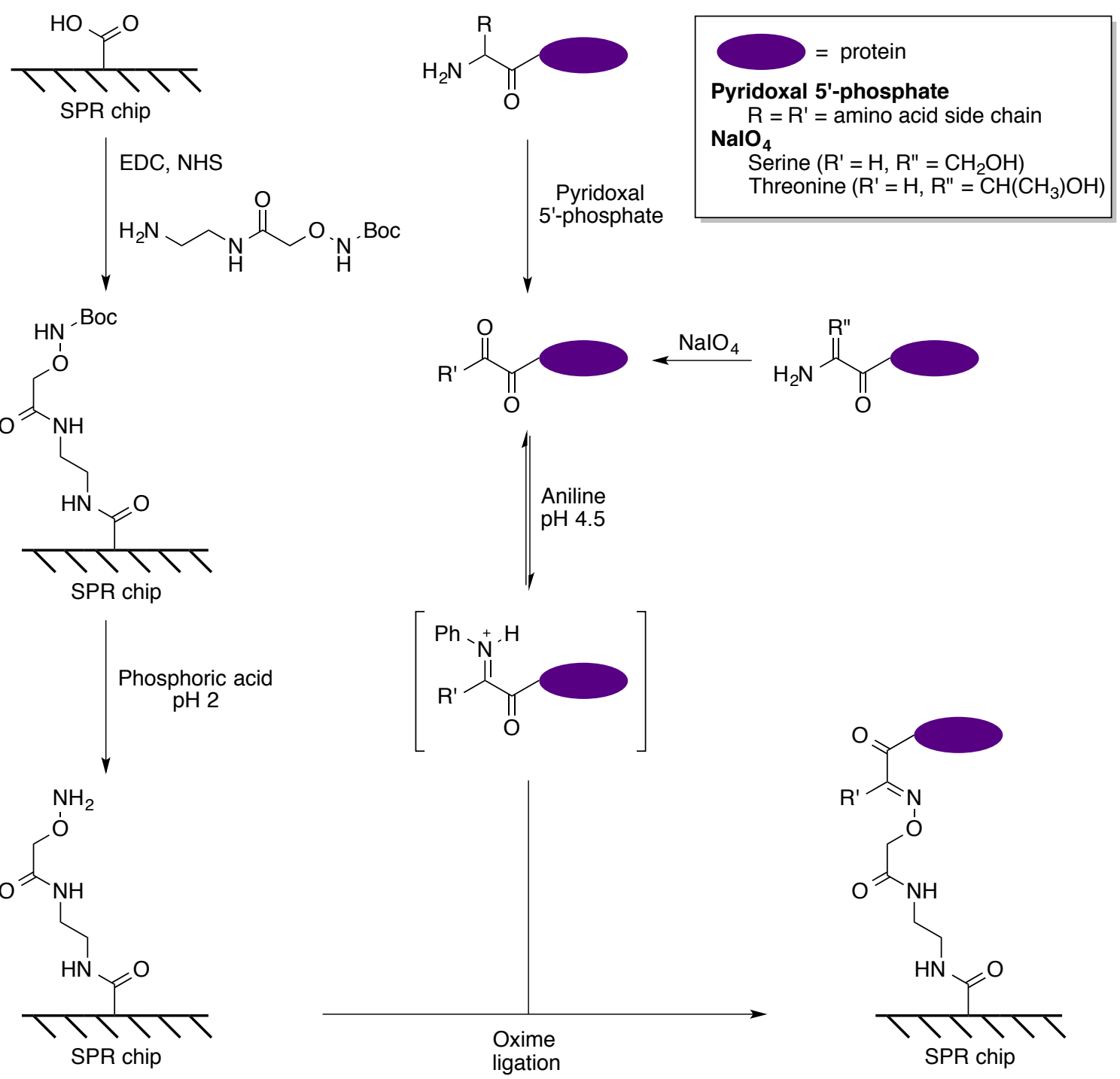

Scheme 1.3. Site-specific immobilization of peptides and proteins on a biosensor chip surface using aniline-catalyzed oximation. ${ }^{21}$

Another example of solid-supported aminooxy chemistry was shown by Chan et $a l .^{22}$ in a layer-by-layer approach to immobilize polymeric films onto glass. Glass substrates derivatized with aldehyde groups were dipped into a solution of aminooxy substituted poly(phenylenevinylene) (PPV) in methylene chloride and were allowed to sit for 4 hours. Sonication was used to remove unbound polymer. The coated plates could then be alternatingly immersed in aldehyde and aminooxy functionalized PVP to form the 
multilayer films (Figure 1.7). This technology provided nanostructured thin films with potential in a range of applications from light-emitting diodes to the coating for biomedical devices. ${ }^{23}$

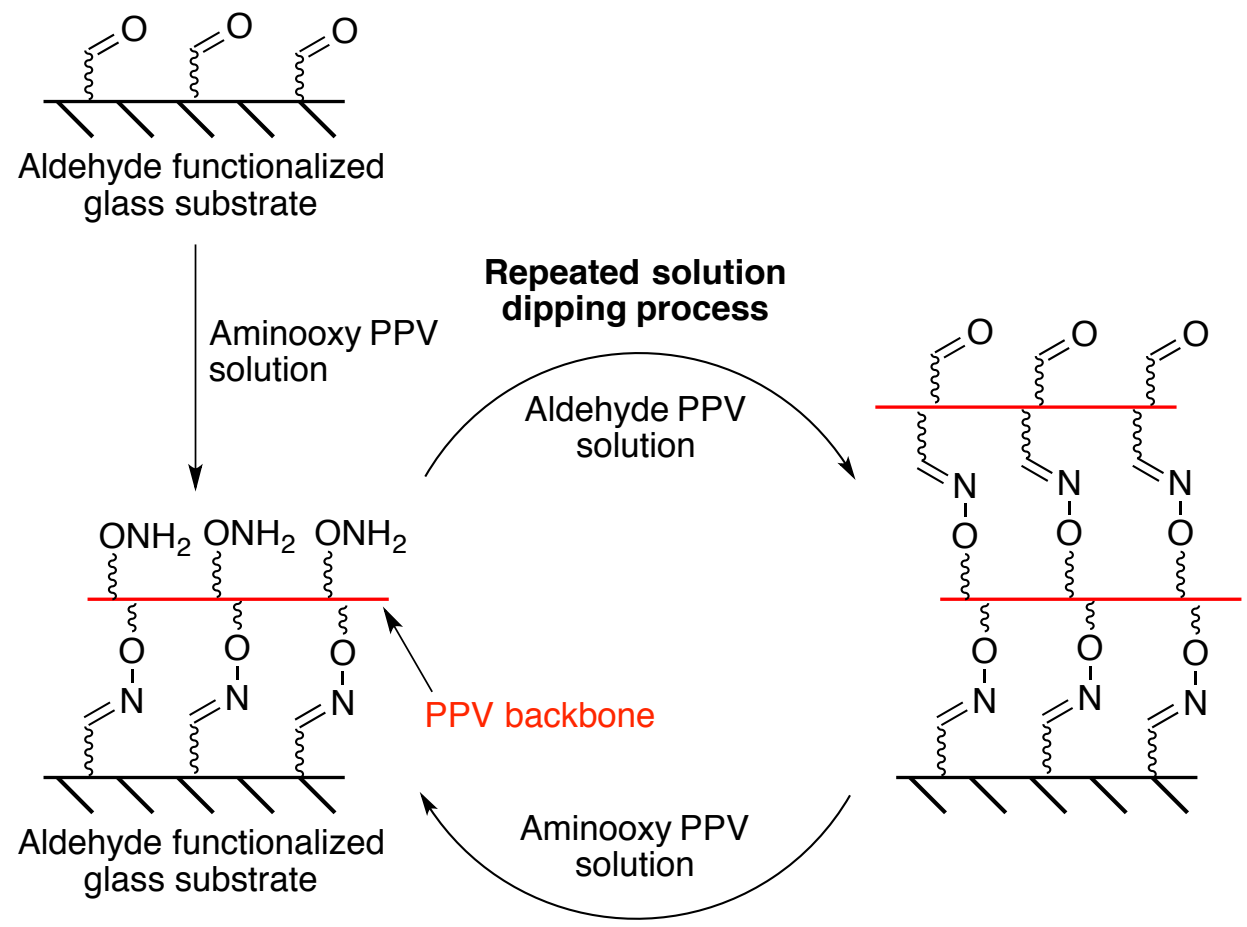

Figure 1.7. Multilayer deposition of PPV supported aldehyde and aminooxy substrates on derivatized glass via chemoselective ligation. ${ }^{22}$

\subsection{USE OF AMINOOXY CHEMISTRY IN NANOTECHNOLOGY}

The click assembly of aminooxy compounds with aldehyde and ketone substrates has also been employed in the popular field of nanotechnology. Using gold nanoparticles (NPs), Thygesen et al. ${ }^{24}$ synthesized glyconanoparticles from unprotected reducing glycans and glycopeptide aldehydes. To do this, a protected aminooxy-thiol linker was bound to a gold NP through a gold-thiol bond. Following deprotection, the active aminooxy was reacted with unmodified reducing glycans via a single chemoselective 
reaction to attach them through an oxime ether bond to the surface of the gold NPs (Table 1.4). The oximation was performed in phosphate buffer solution (PBS) without catalysis and all of the glycans contained a fluorenylmethyloxycarbonyl (Fmoc) protecting group to allow for spectrophotometric quantification of the aminooxy NP capture efficiency (Scheme 1.4).

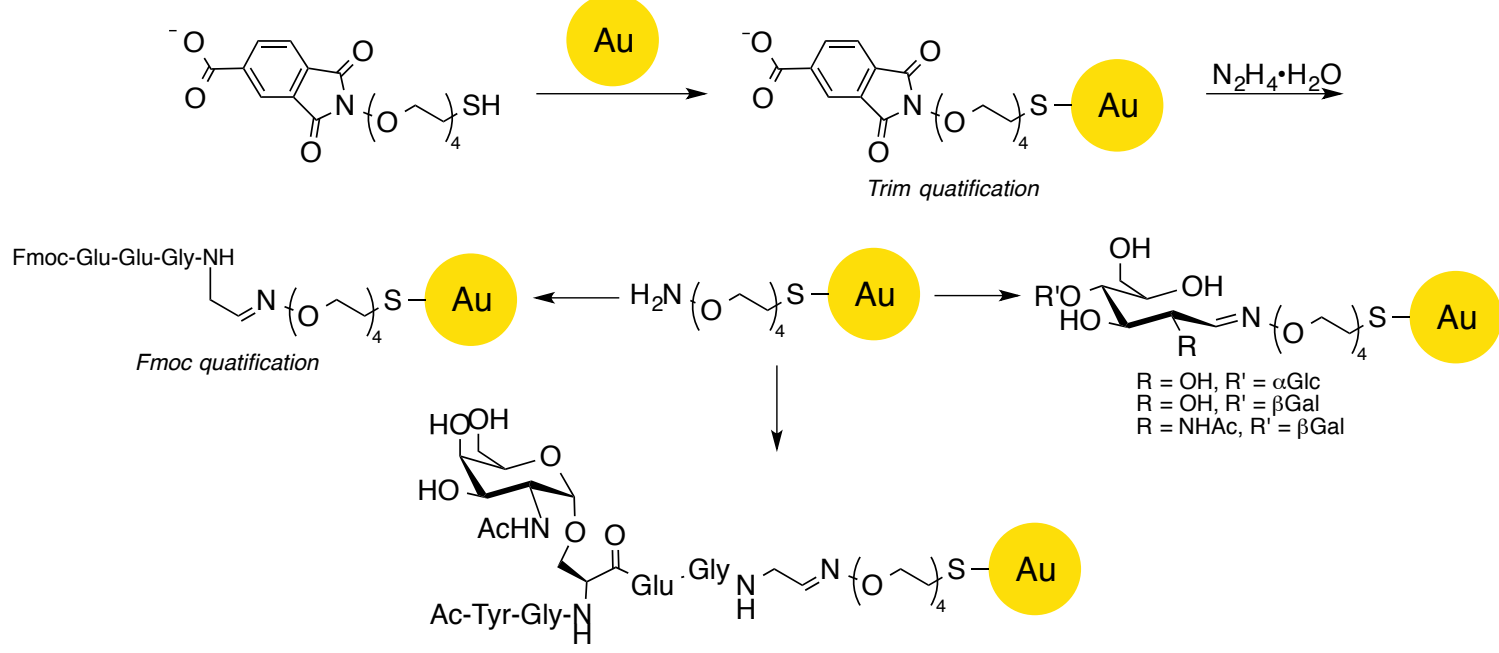

Scheme 1.4. Synthesis of aminooxy-terminated core-shell gold NPs via Trim deprotection and oximation of a peptide, glycopeptide and glycan. Trim quantification gave an aminooxy loading of $\sim 435$ reactive linkers/gold NP. ${ }^{24}$

Table 1.4. Oxime coupling with Fmoc-Glu-Glu-Gly-Gly-H on aminooxyterminated core-shell gold NPs. ${ }^{24}$

\begin{tabular}{|c|c|c|c|c|}
\hline Entry & Conditions & $\begin{array}{c}\text { Ligand density } \\
\text { (peptides/Au NP) }\end{array}$ & $\begin{array}{c}\text { Surface coverage } \\
\left(\text { peptides } / \mathrm{nm}^{2}\right)\end{array}$ & Yield $(\%)^{a}$ \\
\hline 1 & $25^{\circ} \mathrm{C}, 16 \mathrm{~h}$ & 157 & 0.32 & 36 \\
\hline 2 & $60^{\circ} \mathrm{C}^{b}, 1 \mathrm{~h}$ & 162 & 0.33 & 37 \\
\hline 3 & $40^{\circ} \mathrm{C}, 16 \mathrm{~h}$ & 427 & 0.86 & 98 \\
\hline \multicolumn{5}{|c|}{$\begin{array}{l}{ }^{a} \text { Calculated from Fmoc quantification relative to trimellitoyl (Trim) } \\
\text { quantification. } \\
b \text { By microwave heating. }\end{array}$} \\
\hline
\end{tabular}


Biswas and coworkers ${ }^{25}$ used iron oxide, specifically $\mathrm{Fe}_{3} \mathrm{O}_{4}$, NPs as a solid support for quaternary ammonium lipids to serve as a magnetic transfection vector (Scheme 1.5). The ammonium salt allowed for electrostatic attraction between the negatively charged surface for the NPs and a cationic polar head group of the lipid. By using click ligation to attach the hydrophobic chain a variety of lipids could be easily bound to the NPs to aid in finding the most efficient lipid for transfection. In addition, the magnetic NPs allow for in vivo direction and collection of the coated NPs at the treatment site.

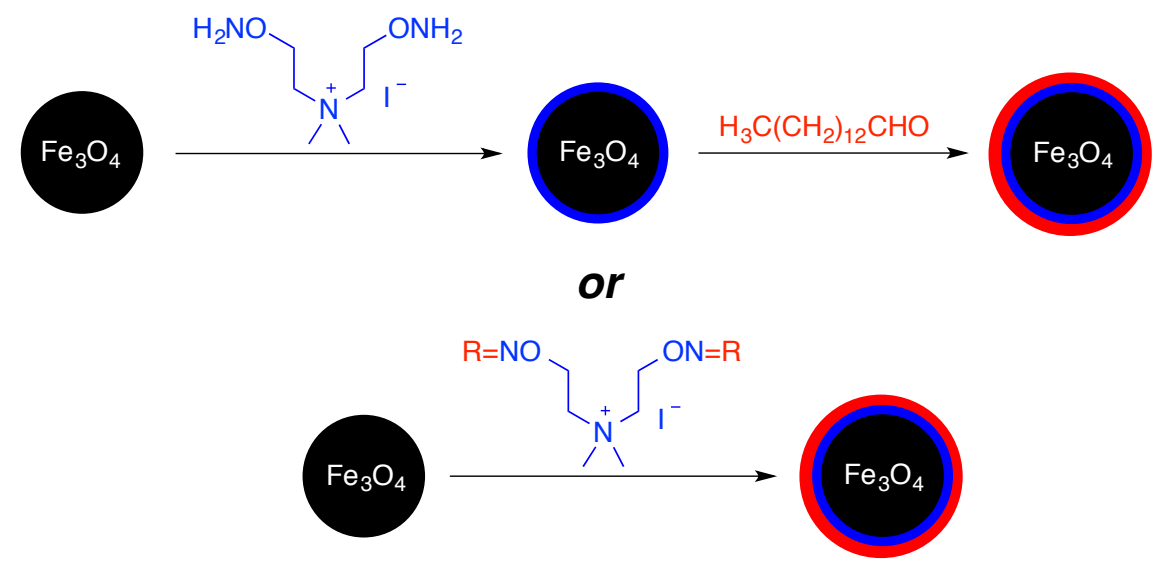

Scheme 1.5. Preparation of lipid-coated $\mathrm{Fe}_{3} \mathrm{O}_{4}$ NPs using oximation to attach hydrophobic chains. ${ }^{25}$

Zhang et al. ${ }^{26}$ used core-shell $\mathrm{Fe}_{3} \mathrm{O}_{4}-\mathrm{SiO}_{2} \mathrm{NPs}$ to covalently attach an aminooxy linker to the NP surface. Using alkoxysilane chemistry, a Boc-protected was bound to the NP surface followed by deprotection using trifluoroacetic acid (TFA) to unmask the reactive aminooxy (Scheme 1.6). Zhang and coworkers used the functionalized NPs to chemoselectively capture glycopeptides via oximation in the presence of non-oxidized peptides. This method is particularly attractive due to the magnetic properties of the iron oxide NPs. After the glycopeptides are covalently bound to the NPs, the loaded iron NPs 
can be rapidly magnetically separated from the mixture.

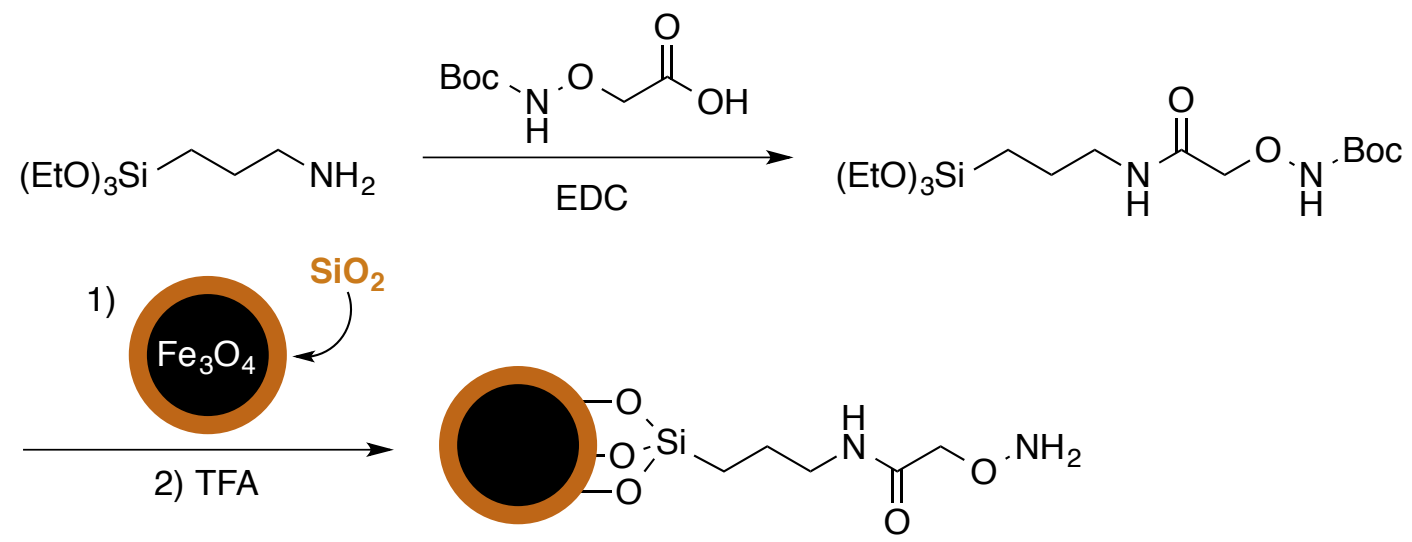

Scheme 1.6. Installation of an aminooxy moiety onto core-shell $\mathrm{Fe}_{3} \mathrm{O}_{4}-\mathrm{SiO}_{2}$

NPs for subsequent chemoselective reaction with glycopeptides. ${ }^{26}$

\subsection{IRON OXIDE NANOPARTICLES}

As is apparent from the aforementioned examples, iron oxide NPs can readily serve as an advantageous solid support. Iron oxide NPs are ferromagnetic in both the form of $\mathrm{Fe}_{3} \mathrm{O}_{4}$ or the more oxidized version $\mathrm{Fe}_{2} \mathrm{O}_{3} .{ }^{27}$ This magnetism allows the NPs to be easily removed from suspensions with the use of a magnet. The magnetism of the NPs also allows enables applications as magnetic resonance imaging (MRI) contrast agents. ${ }^{28}$ In this capacity, the magnetic NPs greatly enhance the sensitivity of MRI, a technique that is often coupled with another imaging method, such as positron emission tomography (PET), to account for its low sensitivity. Huang et al. ${ }^{29}$ have reported such a coupling of MRI using NPs with PET for increased sensitivity.

Localization within the body of the iron oxide NPs in the area of interest is an important part of effectively utilizing the magnetic NPs as a contrast agent. To achieve spatial and temporal orientation of NPs, surface functionalization is generally required. 
Yang et al. ${ }^{30}$ synthesized receptor-targeted NPs for in vivo imaging of breast cancer. The NP surface was functionalized with a peptide containing a fragment of a urokinase-type plasminogen activator (uPA) (Figure 1.8). The bound uPA ligand directed the NPs to uPA receptors that were overexpressed in breast cancer tissues. The benefit of using a magnetic NP-based contrast agent over a small molecule contrast agent is that the small molecule agents have a relatively short lifespan $(<30$ minutes $)$, thus resulting in degradation prior to accumulation at the tumor site. ${ }^{31}$ Polymer-coated iron oxide NPbased contrast agents have been shown to have over eight hours of plasma retention time. $^{32}$ This helps to ensure that the NPs reach and bind to the tumor cells. Yang and coworkers ${ }^{30}$ report that the NPs remained stable in vivo and in intracellular environments for over forty-eight hours during the imaging experiments. The Food and Drug Administration (FDA) have already approved use of iron oxide NPs as an MRI contrast agent due to biological compatibility and an agreeable toxicity profile. 


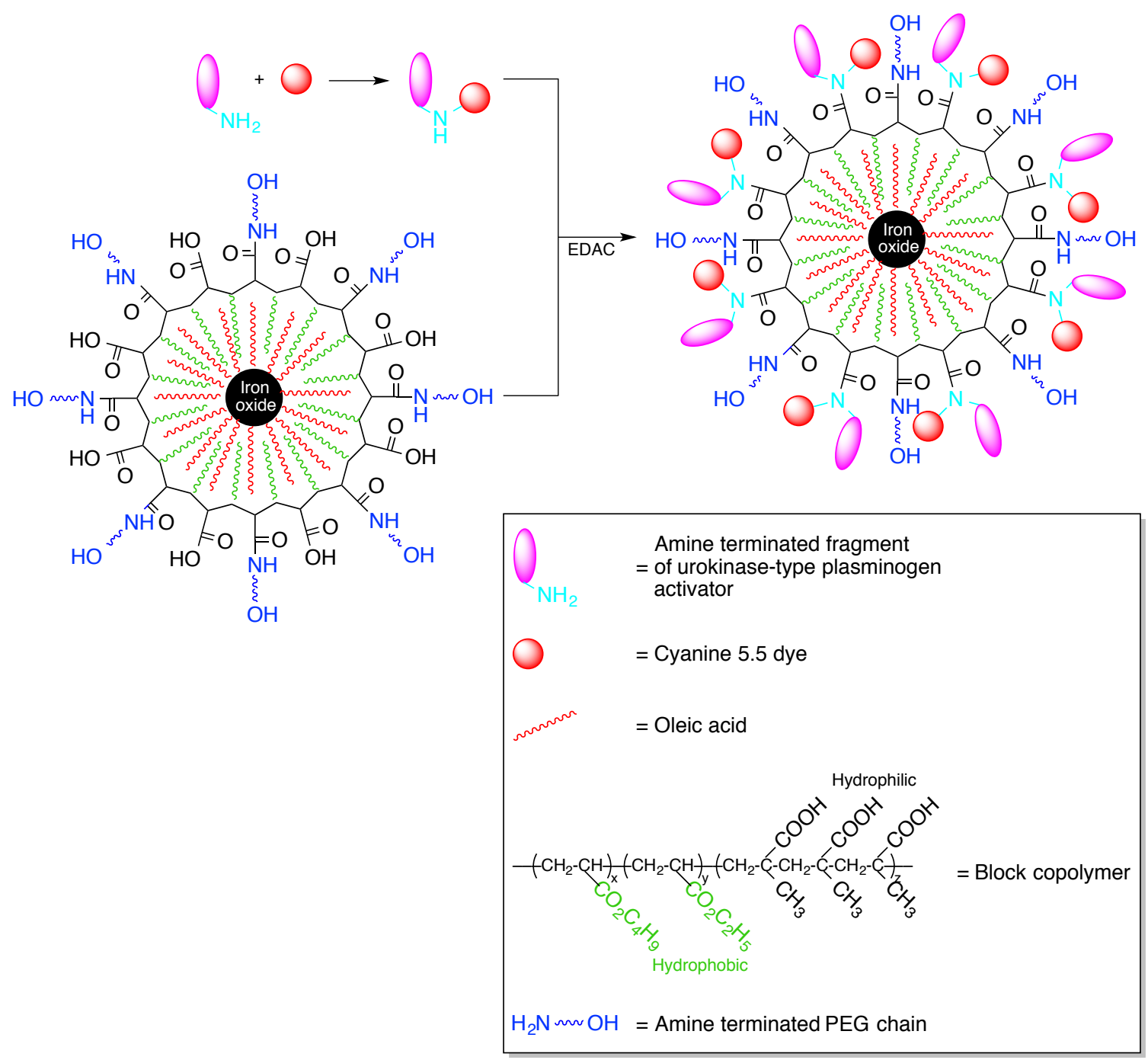

Figure 1.8. Final functionalization of polymer-coated iron oxide NPs with an amine terminated fragment of urokinase-type plasminogen activator to enable binding of the NP to breast cancer cells, and cyanine 5.5 dye to aid in fluorescent imaging of the NP location. ${ }^{30,33}$

\subsection{USE OF IRON OXIDE NANOPARTICLES TO GENERATE HEAT}

One of the more novel ways to exploit the magnetic properties of iron oxide NPs is to use them as a source of heat. When the magnetic NPs are subjected to an alternating magnetic field (AMF) the NPs generate heat as they attempt to realign their magnetic pole with that of the AMF. The NPs transform the energy from the AMF into heat via 
two mechanisms: 1) through internal dipole rotation or Néel relaxation, and 2) by physical particle rotation called Brownian relaxation as can be seen in Figure $1.9 .^{27}$ In either case, there are internal and external factors that prevent the magnetic dipole of the NP from realigning with the AMF, thus resulting in the production and release of heat. The dominant form of relaxation is largely thought to follow the mechanism with the shortest relaxation time. Small nanoparticles tend to be dominated by Néel relaxation while in larger particles the Brownian relaxation prevails.
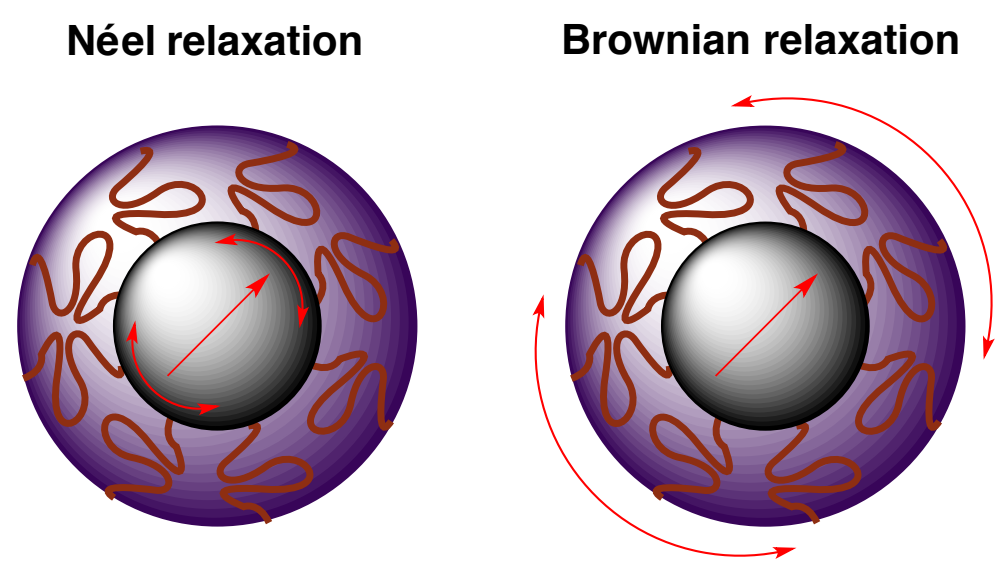

Figure 1.9. Magnetic NPs respond to AMF through either internal dipole rotation, Néel relaxation, or through physical particle rotation called Brownian relaxation. The straight arrow shows the alignment of the internal magnetic dipole and the arched arrows show whether the dipole or the NP is rotating. ${ }^{27}$

When the iron oxide NPs are $<20 \mathrm{~nm}$ in diameter they can begin to be superparamagnetic. ${ }^{34}$ Superparamagnetism is a form of magnetism where the magnetization can randomly flip direction under the influence of temperature. ${ }^{35}$ The time between the reorientation of the magnetic dipole is the Néel relaxation. In the absence of 
an external magnetic field the superparamagnetic particles appear to show no magnetism. This phenomenon is due to the time used to measure the reorientation of the magnetization being much longer than the Néel relaxation. The aforementioned attributes, in addition to other variations such as alteration of size, ${ }^{36}$ shape $^{37}$ and functionality, ${ }^{38}$ make iron oxide NPs an attractive solid support with tunable magnetic properties.

One application of AMF-induced iron oxide NP heating is localized hyperthermia for cancer abolition. Hyperthermia treatment first involves localization of iron oxide NPs $\left(\mathrm{Fe}_{3} \mathrm{O}_{4}\right.$ or $\left.\gamma-\mathrm{Fe}_{2} \mathrm{O}_{3}\right)$ in a tumor and then AMF exposure to elevate the local, cellular temperature to at least $40-47{ }^{\circ} \mathrm{C} .{ }^{39}$ These elevated temperatures result in cell death. Alternate methods for inducing hyperthermia are based on the application of microwaves. ${ }^{40}$ But the use of microwaves has been limited due to the inability to control temperatures and the ineptitude to minimize collateral damage such as exposure to radiation and a lack of selectiveness that causes extensive damage to the surrounding healthy tissue. Treatment of iron oxide NPs with an AMF also has the advantage of using an alternating current (AC) magnetic field with a significantly lower frequency $\left(10^{5}\right.$ $\mathrm{Hz}$ ) than its microwave counterpart at nearly $10^{15} \mathrm{~Hz}$. Operation at a frequency of $10^{5} \mathrm{~Hz}$ results in negligible damage to the living tissue. Figure 1.10 shows an $\mathrm{MFH} 300 \mathrm{~F}^{\star} \mathrm{AMF}$ applicator instrument (MagForce Nanotechnologies, AG, Berlin, Germany) that is currently being used to treat patients via hyperthermia. ${ }^{39}$ 


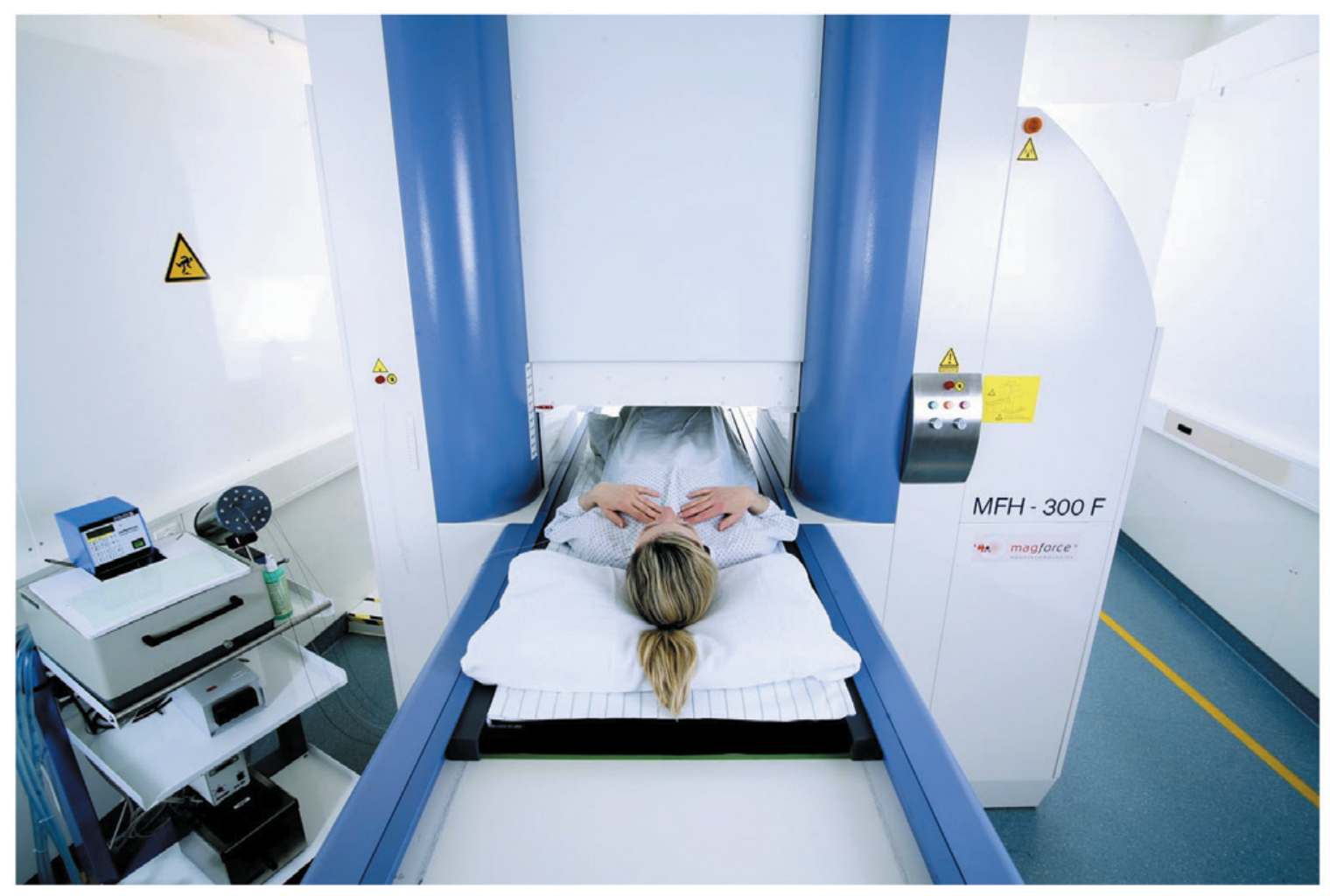

Figure 1.10. Thermotherapy treatment of the pelvic region after intratumoral injection of magnetic NPs using the AMF applicator MFH $300 \mathrm{~F}^{\oplus} \cdot 39$

AMF-induced hyperthermia is also frequently used in conjunction with chemotherapy to increase the efficacy of an administered drug. This multimodal approach circumvents many of the limitations of conventional chemotherapy and hyperthermia. In chemotherapy, aggressive cytotoxic drugs kill both cancerous and healthy cells without discrimination and can cause systemic toxicity and decreased drug concentrations in the tumor. ${ }^{41}$ Hyperthermia suffers from insufficient temperature increase in the tumor, poor temperature distribution and risk of damage to surrounding organs due to overheating. ${ }^{42}$ Aoki et $a l .{ }^{43}$ reported a significant increase in drug efficacy when malignant gliomas were treated with liposomal adriamycin, a chemotherapeutic 
drug, in addition to mild hyperthermia at $40-43{ }^{\circ} \mathrm{C}$. Purushotham and Ramanujan ${ }^{44}$ found that when doxorubicin (Dox) was loaded into poly- $n$-isopropylacrylamide coated iron oxide (12 nm diameter $\mathrm{Fe}_{3} \mathrm{O}_{4}$ or $23 \mathrm{~nm}$ diameter $\gamma-\mathrm{Fe}_{2} \mathrm{O}_{3}$ ) NPs the in vitro tumor cells were far more responsive to treatment when compared to in vitro tumor cells treated with only Dox or hyperthermia. The efficacy of Dox was increased enough that the therapeutic effect obtained with a normal dosing could be matched by using Dox/magnetic NPs with doses as low as $20 \%-50 \%$ of the standard dosing. Using the combination of Dox and AMF-induced hyperthermia with magnetic NPs, Alexiou et al. ${ }^{45}$ found that the same chemotherapeutic result could be obtained using a dose of as low as to $20 \%-50 \%$ of the standard dosing of Dox.

\subsection{CONCLUSION}

It is clear from the above examples that aminooxy chemistry, and the resultant oxime ethers formed on chemoselective reaction of an aminooxy moiety with an aldehyde or ketone carbonyl, are of great value for biological applications. The aminooxy groups also provide the versatility to react readily in a variety of solvents, importantly water, while either in solution or bound to a solid support. Chapter 2 presents a solid-support application of a new aminooxy-based reagent, 4-(2aminooxyethyl)-morpholin-4-ium chloride (AMAH). Relying on both electrostatic and hydrogen-bonding interations, a silicon microreactor can be loaded with AMAH for chemoselective, covalent capture and concentration of volatile aldehydes and ketones in exhaled breath (Scheme 1.7). This application has great potential in the early diagnosis of lung cancer. AMAH-adducts can be analyzed via Fourier transform-ion cyclotron 
resonance (FT-ICR) mass spectrometry (MS) as well as gas chromatography (GC) MS. By utilizing both forms of analysis, volatile organic compounds (VOCs) can be quantified and the conformational isomers, indistinguishable by FT-ICR-MS, can be accurately assigned. We developed a novel method to benefit from the cationic aminium salt using FT-ICR-MS while being able to neutralize the salt and directly inject the VOC adduct solution for GC-MS analysis without the use of salts or a liquid-liquid extraction.

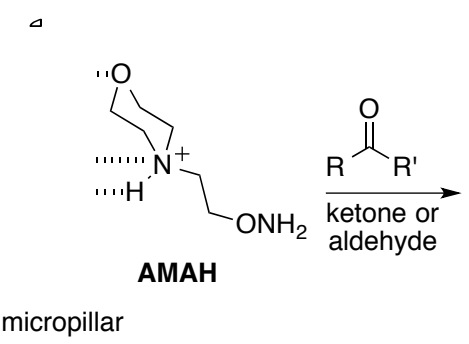

Scheme 1.7. Chapter 2 Summary: AMAH oximation covalently traps aldehydes and ketones in the microreactor.

Chapter 3 describes the synthesis of a panel of thermally labile linkers engineered to undergo intramolecular cyclization on heating. One goal of this work is application to a polydimethylsiloxane microchannel (Figure 1.11) for use in combination with aminooxy chemistry to capture aldehyde and ketone metabolites passing through the microchannel. After preparing the linker panel, each substrate was analyzed to determine any thermal responsiveness. By design, heating is intended to promote addition of a nucleophilic amine, positioned along a connecting chain, to an electrophilic carbonyl to form varioussized heterocyclic rings. To tune the thermal responsiveness of the linker different functional groups including esters, amides, carbonates and carbamates were inserted 
around the electrophilic carbonyl. We also tuned the cyclization rate by altering the ring size from five to seven and employing gem-dimethylation. After an optimal linker was established, we installed an aminooxy group and then bound the assembly to the interior walls of a microchannel for carbonyl capture applications.

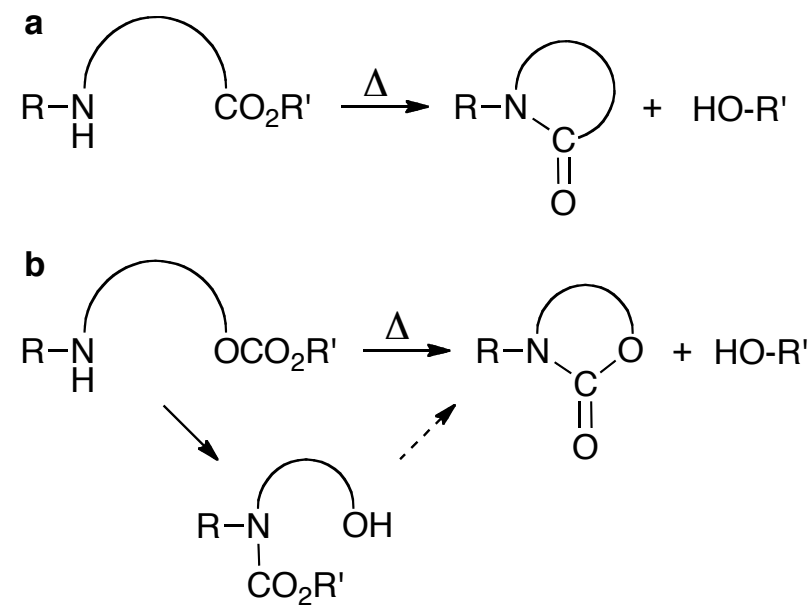

Figure 1.11. Chapter 3 Summary: Heatinduced cyclization via (a) lactamization or (b) carbamate formation.

Chapter 4 describes our efforts to engineer an AMF-induced iron oxide NP drug delivery system, one that involves a covalently NP-bound drug as opposed to a NPassociated drug (Figure 1.12). This work possessed many hurdles including formation of a covalent bond between the iron oxide NP and the organic linker, modification of the surface properties of the NPs, reduction of NP agglomeration as well as the discovery of a phenomenon that had not yet been reported. Though originally expecting to utilize our thermally labile linkers for drug delivery, we discovered a not-yet-reported release mechanism that appears to be the result of an AMF-induced hydrolysis. We were able to 
hydrolyze robust, i.e. carbonate and carbamate, functionalities at room temperature, neutral $\mathrm{pH}$ and in less than 45 minutes.

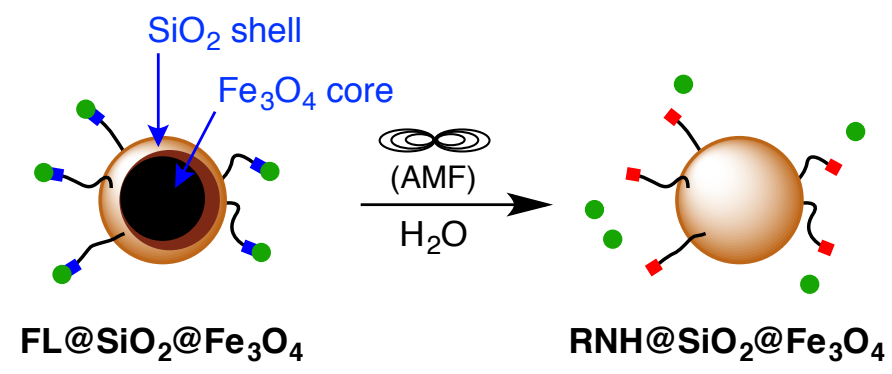

Figure 1.12. Chapter 4 Summary: AMF-triggered hydrolysis of pendant functionality (FL = fluorophore, •; carbamate moiety, $n$; terminal amine residue, $n)$.

Chapter 5 presents the experimental procedures for all of the chemistry described in Chapters 2, 3 and 4. Select ${ }^{1} \mathrm{H}$ and ${ }^{13} \mathrm{C}$ NMR spectra as well as other select spectroscopic data of synthesized molecules and NPs are provided in Appendix A. 
CHAPTER 2

CHEMOSELECTIVE CAPTURE OF

VOLATILE ALDEHYDES AND KETONES

2.1. INTRODUCTION

2.2. INTRODUCTION OF AMAH

2.3. RESULTS AND DISCUSSION

2.4. CONCLUSION

2.5. FUTURE DIRECTIONS 


\subsection{INTRODUCTION}

In 2011 , there were 98,000 men and 77,000 women who developed lung cancer. ${ }^{1}$ Only $\sim 18 \%$ of these people will survive beyond five years. ${ }^{1}$ However, if the spread of the lung cancer can be minimized at the time of diagnosis and quickly treated, the fiveyear survival rate increases to $54 \% .{ }^{1}$ Thus, the early detection of lung cancer is of critical importance. Currently, the common methods of diagnosing lung cancer include, but are not limited to, bronchoscopy, PET scan or needle biopsy, all of which are after having completed a CT scan. All of these methods are either invasive or subject the patient to a substantial dose of radiation. Due to the aforementioned side effects, these methods of diagnosis are usually used only when the cancer has become symptomatic. Lung cancer is largely asymptomatic in its early stages. Screening is the only systematic means of finding tumors at the surgically curable stage. ${ }^{2}$ Therefore, it is easy to appreciate the need for a non-invasive method to accurately diagnosis lung cancer in its early stages.

There are many minimally or non-invasive methods being designed and tested for clinical application in the early detection of lung cancer. Some of these methods include the cytology of the sputum, ${ }^{3,4}$ detection of circulating cancer biomarkers, ${ }^{5,6}$ determination of blood proteomic patterns, ${ }^{7,8}$ chest tomography, ${ }^{9,10}$ and whole body magnetic resonance imaging (wbMRI). ${ }^{11}$ But all of these techniques suffer from serious drawbacks. Specifically, sputum cytology regularly provides false negatives and, therefore, fails to detect numerous early-stage cases of lung cancer. ${ }^{12}$ Circulating cancer biomarkers, when used alone, have not shown promise as a screening test and it is not yet clear which panel of tests will afford the necessary sensitivity and specificity. ${ }^{13,14}$ Blood proteomic patterns are difficult to establish, though FT-ICR has aided in the advancement of this 
technology. ${ }^{5,6}$ Computed tomography (CT) scans, despite subjecting the patient to high doses of X-ray radiation, can accurately detect nodules as small as $1 \mathrm{~mm}$ in diameter, but they also show many false positives if not confirmed by positron emission tomography (PET), that lead to unnecessary biopsies. ${ }^{15}$ Table 2.1 outlines the different methods of analysis, the procedure for the collection of a sample, the invasiveness and the method of diagnosis of lung cancer. 
Table 2.1. Analytical procedures for the diagnosis of lung cancer.

\begin{tabular}{|c|c|c|c|}
\hline $\begin{array}{c}\text { Method of } \\
\text { Analysis } \\
\end{array}$ & Collection of Sample & $\begin{array}{c}\text { Invasive/ } \\
\text { Non-Invasive } \\
\end{array}$ & Method of Diagnosis \\
\hline $\begin{array}{l}\text { Cytology of } \\
\text { the sputum }\end{array}$ & $\begin{array}{l}\text { - Subject coughs up } \\
\text { mucus } \\
\text { - Subject inhales } \\
\text { saline followed by } \\
\text { coughing } \\
\text { - Bronchoscopy }\end{array}$ & $\begin{array}{c}\text { Non-Invasive } \\
\text { (coughing) } \\
\text { Invasive } \\
\text { (bronchoscopy) }\end{array}$ & $\begin{array}{l}\text { Cells are observed } \\
\text { under microscope to } \\
\text { determine if } \\
\text { abnormalities are } \\
\text { carcinogenic }\end{array}$ \\
\hline $\begin{array}{l}\text { Circulating } \\
\text { tumor } \\
\text { biomarkers }\end{array}$ & $\begin{array}{l}\text { - Tissue collection } \\
\text { - Draw blood }\end{array}$ & Invasive & $\begin{array}{l}\text { Tissue biopsy/blood } \\
\text { analysis looking for } \\
\text { known cancer derived } \\
\text { metabolites }\end{array}$ \\
\hline $\begin{array}{l}\text { Blood } \\
\text { proteomic } \\
\text { patterns }\end{array}$ & $\begin{array}{l}\text { - Draw blood } \\
\text { - Lung cancer tissue } \\
\text { - Serum } \\
\text { - Plasma } \\
\text { - Pleural effusions }\end{array}$ & Invasive & $\begin{array}{l}\text { MALDI-TOF or FT- } \\
\text { ICR-MS used to scan } \\
\text { for known proteins } \\
\text { derived from cancer }\end{array}$ \\
\hline $\begin{array}{l}\text { Chest } \\
\text { tomography }\end{array}$ & $\begin{array}{l}\text { Spiral CT scan of } \\
\text { chest }\end{array}$ & Non-Invasive & $\begin{array}{l}\text { CT image shows } \\
\text { visible nodules in } \\
\text { lung }\end{array}$ \\
\hline $\begin{array}{l}\text { Whole body } \\
\text { magnetic } \\
\text { resonance } \\
\text { imaging } \\
\text { (wbMRI) }\end{array}$ & wbMRI & Non-Invasive & $\begin{array}{l}\text { Used in partnership } \\
\text { with diffusion } \\
\text { weighted imaging } \\
\text { (DWI) and apparent } \\
\text { diffusion coefficient } \\
\text { (ADC) to determine } \\
\text { metabolic and } \\
\text { morphological } \\
\text { deviations }\end{array}$ \\
\hline
\end{tabular}


The analysis of volatile organic compounds (VOCs) in exhaled human breath is another area of focus for non-invasive early diagnosis of lung cancer. In 1971, Pauling et al. ${ }^{16}$ reported that human breath is comprised of over 1000 VOCs. Beginning in 1985, O'Neill and coworkers ${ }^{17,18}$ identified 28 VOCs from exhaled breath as candidate markers of lung cancer, including hexane, methylpentane, $o$-toluidine, and aniline. In addition, altered lipid-peroxidation activity in patients with lung cancer was observed in the form of over-oxidized VOC metabolites. ${ }^{19,20}$ But since most VOCs occur at picomolar concentrations, advanced techniques must be employed to effectively capture and concentrate the VOCs prior to analysis. ${ }^{21}$

The development of devices to perform the capture and subsequent concentration of the VOCs in exhaled breath has received much attention. One of the most simplistic (and sensational) methods of diagnosing lung cancer from exhaled breath involves the use of canines for scent detection. First proposed by Williams and Pembroke in $1989,{ }^{22}$ the hypothesis for this method of detection is based on the idea that dogs are known to possess superior olfactory systems capable of detecting VOCs at parts-per-trillion levels from complex mixtures. ${ }^{23}$ Application of five dogs (both genders) for canine scent detection in a double-blinded testing of 28 lung cancer patients and 6 breast cancer patients against 17 controls found that the dogs had an overall sensitivity of 0.99 (95\% confidence) and overall specificity of 0.99 ( $95 \%$ confidence) when compared to biopsyconfirmed conventional diagnosis. ${ }^{2}$ Despite the tremendous sensitivity and specificity of the dogs, there are significant drawbacks to this idea, largely due to the variables involved with a living species. 
Other, more conventional, forms of non-invasive lung cancer diagnoses include the aforementioned induced sputum, exhaled nitric oxide, exhaled breath condensate and the electronic nose. The advantages and disadvantages of each non-invasive diagnostic method are summarized in Table 2.2. In particular, the electronic nose possesses great potential in its ability to accurately identify specific VOC profiles in exhaled breath. But the electronic nose is comprised of a series of electronic sensors of different composition and relies heavily on complex algorithms to give rise to the so-called "breathprint." ${ }^{24}$

Analysis of VOCs that are a direct result of the oxidative stress inherent in lung cancer, usually in the form of aldehydes and ketones, is another area that has great potential. Table 2.3 shows common targeted VOC metabolites along with the method of detection, concentration threshold and whether the technology has been tested with humans. 
Table 2.2. Mean advantages and disadvantages of non-invasive diagnostic tools detecting biomarkers of pulmonary inflammation in smoking subjects. ${ }^{24}$

\begin{tabular}{|c|c|c|c|c|}
\hline $\begin{array}{c}\text { Diagnostic } \\
\text { Tools }\end{array}$ & Biomarkers & Advantages & Disadvantages & Conclusions \\
\hline $\begin{array}{l}\text { Induced } \\
\text { Sputum }\end{array}$ & $\begin{array}{l}\text { Airway } \\
\text { cellularity } \\
\text { Inflammatory } \\
\text { mediators in } \\
\text { supernatants }\end{array}$ & $\begin{array}{l}\text { Easier and more } \\
\text { comfortable } \\
\text { than } \\
\text { bronchoalveolar } \\
\text { lavage or } \\
\text { bronchial } \\
\text { biopsy }\end{array}$ & $\begin{array}{l}\text { Not completely } \\
\text { non-invasive; } \\
\text { may induce } \\
\text { bronchospasm }\end{array}$ & $\begin{array}{l}\text { Partially } \\
\text { conflicting } \\
\text { data; need large } \\
\text { longitudinal } \\
\text { studies }\end{array}$ \\
\hline $\begin{array}{l}\text { Exhaled } \\
\text { Nitric Oxide }\end{array}$ & $\begin{array}{l}\text { Nitric oxide in } \\
\text { exhaled air }\end{array}$ & $\begin{array}{l}\text { Completely } \\
\text { non-invasive; } \\
\text { repeatable }\end{array}$ & $\begin{array}{l}\text { Many } \\
\text { confounding } \\
\text { factors such as } \\
\text { meals, asthma, } \\
\text { respiratory } \\
\text { infections }\end{array}$ & $\begin{array}{l}\text { May play an } \\
\text { important role } \\
\text { in smoking } \\
\text { cessation } \\
\text { programs }\end{array}$ \\
\hline $\begin{array}{l}\text { Exhaled } \\
\text { Breath } \\
\text { Condensate }\end{array}$ & $\begin{array}{l}\text { Many } \\
\text { inflammatory } \\
\text { markers in } \\
\text { exhaled air }\end{array}$ & $\begin{array}{l}\text { Completely } \\
\text { non-invasive; } \\
\text { repeatable }\end{array}$ & $\begin{array}{l}\text { Not } \\
\text { standardized }\end{array}$ & $\begin{array}{l}\text { Smokers show } \\
\text { an increased } \\
\text { oxidative stress } \\
\text { and a lower pH } \\
\text { than non- } \\
\text { smokers }\end{array}$ \\
\hline $\begin{array}{l}\text { Electronic } \\
\text { Nose }\end{array}$ & $\begin{array}{l}\text { Able to identify } \\
\text { specific VOC } \\
\text { profiles in } \\
\text { exhaled breath }\end{array}$ & $\begin{array}{l}\text { Completely } \\
\text { non-invasive; } \\
\text { repeatable }\end{array}$ & $\begin{array}{l}\text { Not } \\
\text { standardized }\end{array}$ & $\begin{array}{l}\text { Discriminate } \\
\text { between } \\
\text { healthy non- } \\
\text { smokers and } \\
\text { healthy } \\
\text { smokers }\end{array}$ \\
\hline
\end{tabular}


Table 2.3. Current technology for the diagnosis of lung cancer using VOCs. ${ }^{25}$

\begin{tabular}{|c|c|c|c|c|c|c|}
\hline $\begin{array}{l}\text { Primary } \\
\text { Target }\end{array}$ & $\begin{array}{l}\text { Sensor } \\
\text { Technology }\end{array}$ & Sensing Materials & $\begin{array}{l}\text { Human } \\
\text { Tests }\end{array}$ & $\begin{array}{l}\text { Limit Of } \\
\text { Detection }\end{array}$ & $\begin{array}{l}\text { Sample } \\
\text { Treatment }\end{array}$ & Reference \\
\hline $\begin{array}{l}\text { Volatile } \\
\text { thiols }\end{array}$ & Colorimetry & Iodine & Yes & $\begin{array}{l}0.05 \mu \mathrm{g} \mathrm{L}^{-1} \text { of } \\
\mathrm{H}_{2} \mathrm{~S}\end{array}$ & No & 26 \\
\hline $\begin{array}{l}\text { Volatile } \\
\text { thiols }\end{array}$ & Chemiresistor & $\begin{array}{l}\text { Au nanoparticles } \\
\text { decorated with } \\
\text { polyaniline }\end{array}$ & No & $\begin{array}{l}1 \mathrm{mM} \text { of } \mathrm{H}_{2} \mathrm{~S} \\
\text { and } \mathrm{CH}_{3} \mathrm{SH}\end{array}$ & No & 27 \\
\hline $\begin{array}{l}\text { Volatile } \\
\text { thiols }\end{array}$ & Fiber optic & $\begin{array}{l}\text { Monoamine oxidase } \\
\text { A \& optical O sensor }\end{array}$ & Yes & $200 \mathrm{ppb}$ & No & 28 \\
\hline Acetone & Chemiresistor & $\mathrm{Si}: \mathrm{WO}_{3}$ & Yes & $20 \mathrm{ppb}$ & No & 29 \\
\hline Acetone & Chemiresistor & Chitosan & No & $0.1 \mathrm{ppm}$ & No & 30 \\
\hline Acetone & Chemiresistor & $\mathrm{In}_{2} \mathrm{O}_{3} \& \mathrm{Pt}-\mathrm{In}_{2} \mathrm{O}_{3}$ & No & $<1 \mathrm{ppm}$ & No & 31 \\
\hline Acetone & Chemiresistor & $\begin{array}{l}\text { Hemitubes of Pt- } \\
\mathrm{WO}_{3}\end{array}$ & No & $120 \mathrm{ppb}$ & No & 32 \\
\hline Acetone & $\begin{array}{l}\text { Direct optical } \\
\text { spectroscopy }\end{array}$ & $\begin{array}{l}\text { Cavity ringdown } \\
\text { spectroscopy }\end{array}$ & Yes & $130 \mathrm{ppb}$ & Yes & 33 \\
\hline $\begin{array}{l}\text { Hydrogen } \\
\text { peroxide }\end{array}$ & ChemFET & $\begin{array}{l}\text { Os-PVP containing } \\
\text { peroxidase }\end{array}$ & No & $0.8 \mu \mathrm{M}$ & $\begin{array}{l}\text { Breath } \\
\text { condensate }\end{array}$ & 34 \\
\hline $\begin{array}{l}\text { Hydrogen } \\
\text { peroxide }\end{array}$ & Amperometric & $\begin{array}{l}\text { Pt electrode with } \\
\text { agarose membrane }\end{array}$ & Yes & $50 \mathrm{ppb}$ & No & 35 \\
\hline $\begin{array}{l}\text { Hydrogen } \\
\text { peroxide }\end{array}$ & Potentiometric & $\begin{array}{l}\text { Prussian blue solid } \\
\text { state salt }\end{array}$ & Yes & $\begin{array}{l}0.1 \mu \mathrm{M} \text { in } \\
\text { aerosol }\end{array}$ & No & 36 \\
\hline $\begin{array}{l}\text { Nitric } \\
\text { oxide }\end{array}$ & Potentiometric & YSZ & Yes & $5 \mathrm{ppb}$ & $\begin{array}{l}\text { Water } \\
\text { removal }\end{array}$ & 37 \\
\hline $\begin{array}{l}\text { Nitric } \\
\text { oxide }\end{array}$ & Chemiresistor & $\begin{array}{l}\text { PEDOT:PSS coated } \\
\text { nanofibrous } \mathrm{TiO}_{2}\end{array}$ & No & $6 \mathrm{ppb}$ & No & 38 \\
\hline $\begin{array}{l}\text { Nitric } \\
\text { oxide }\end{array}$ & $\begin{array}{l}\text { Direct optical } \\
\text { spectroscopy }\end{array}$ & $\begin{array}{l}\text { Tunable diode laser } \\
\text { absorption } \\
\text { spectroscopy }\end{array}$ & Yes & $11 \mathrm{ppb}$ & No & 39 \\
\hline $\begin{array}{l}\text { Nitric } \\
\text { oxide }\end{array}$ & $\begin{array}{l}\text { Direct optical } \\
\text { spectroscopy }\end{array}$ & $\begin{array}{l}\text { Quantum cascade } \\
\text { laser and cavity } \\
\text { spectroscopy }\end{array}$ & Yes & $4 \mathrm{ppb}$ & No & 40 \\
\hline $\begin{array}{l}\text { Nitric } \\
\text { Acid }\end{array}$ & Chemiresistor & $\begin{array}{l}\text { Chemically } \\
\text { functionalized } \\
\text { carbon nanotubes }\end{array}$ & No & $5 \mathrm{ppb}$ & $\begin{array}{l}\mathrm{CO}_{2} \\
\text { removal }\end{array}$ & 41 \\
\hline $\begin{array}{l}\text { Sleep } \\
\text { apnea }\end{array}$ & Chemiresistor & $\begin{array}{l}\text { Multi-wall carbon } \\
\text { nanotubes }\end{array}$ & Yes & $\begin{array}{l}\text { Less than } 6 \\
\text { breaths/minute }\end{array}$ & No & 42 \\
\hline Ammonia & Chemiresistor & $\mathrm{H}_{2} \mathrm{SO}_{4}$ solution & Yes & $<18 \mathrm{ppb}$ & No & 43 \\
\hline Ammonia & Chemiresistor & $\mathrm{MoO}_{3}$ & No & $<1 \mathrm{ppm}$ & No & 44 \\
\hline Ammonia & Optical & $\begin{array}{l}\text { TFE membrane and } \\
\text { pH dye }\end{array}$ & Yes & $50 \mathrm{ppb}$ & No & 45 \\
\hline $\begin{array}{l}\text { Carbon } \\
\text { dioxide }\end{array}$ & Optical & $\begin{array}{l}{\left[\mathrm{CH}_{3}\left(\mathrm{CH}_{2}\right)_{7}\right]_{4} \mathrm{~N}(\mathrm{OH})} \\
\text { and } \mathrm{pH} \text { dyes }\end{array}$ & No & $0.25 \%$ & No & 46 \\
\hline $\begin{array}{l}\text { Carbon } \\
\text { dioxide }\end{array}$ & Optical & $\begin{array}{l}{\left[\mathrm{CH}_{3}\left(\mathrm{CH}_{2}\right)_{7}\right]_{4} \mathrm{~N}(\mathrm{OH})} \\
\text { and } \mathrm{pH} \text { dyes }\end{array}$ & No & $<5 \%$ & No & 47 \\
\hline $\begin{array}{l}\text { Influenza } \\
\text { virus }\end{array}$ & Chemiresistor & $\begin{array}{l}\text { Antibody coated } \\
\text { silicon nanowire }\end{array}$ & Yes & $<1 \mathrm{pg} \mathrm{mL}^{-1}$ & $\begin{array}{l}\text { Dilution of } \\
\text { condensed } \\
\text { breath }\end{array}$ & 48 \\
\hline $\begin{array}{l}\text { Influenza } \\
\text { virus }\end{array}$ & $\begin{array}{l}\text { Dynamic light } \\
\text { scattering }\end{array}$ & $\begin{array}{l}\text { Antibody coated } \\
\text { gold nanoparticles }\end{array}$ & No & $\begin{array}{l}\text { 8.6 TCID } \\
\mathrm{mL}^{-1}\end{array}$ & No & 49 \\
\hline Drink test & Potentiometry & $\begin{array}{l}\text { Alcohol oxidase and } \\
\text { aldehyde } \\
\text { dehydrogenase }\end{array}$ & Yes & $\begin{array}{l}\text { Ethanol } \\
<1 \text { ppm } \\
\text { Acetaldehyde } \\
<0.1 \text { ppm }\end{array}$ & No & 50 \\
\hline
\end{tabular}




\subsection{AMAH: A VERSATILE REAGENT FOR ANALYSIS OF VOCS}

Our attempt to develop a non-invasive method for early detection of lung cancer also focuses on the capture and identification of carbonyl metabolites. My group colleague, Dr. Souvik Biswas, ${ }^{51}$ had developed the reagent $N$-(2-(aminooxy)ethyl)$N, N, N$-trimethylammonium iodide (ATM) to chemoselectively capture volatile aldehydes and ketones from exhaled human breath and ambient air. ${ }^{52,53,54,55}$ Several carbonyl compounds in exhaled breath previously had been identified as lung cancer markers. ${ }^{52,56,}$ 57, 58, 59, 60,61 Reaction of ATM with VOCs was achieved by first coating ATM on the surface of micropillars within a silicon microreactor. The microreactor (or, more precisely, a preconcentrator) contains thousands of micropillars that function to distribute gas flow through channels to provide surface area for efficient capture of the VOCs by the reactive coating. Aldehydes and ketones from exhaled breath are selectively and covalently preconcentrated in the microreactor using a click chemistry reaction (oximation). The resultant ATM-carbonyl adducts are eluted from the microreactor using a small volume $(c a .50 \mu \mathrm{L})$ of methanol, and analysis of the breath analytes then is performed using rapid direct-infusion Fourier transform-ion cyclotron resonance (FTICR) mass spectrometry (MS) to determine the concentrations of exhaled carbonyl VOCs.

Though convenient for rapid identification of biomarkers, FT-ICR-MS is often incapable of distinguishing structural isomers (e.g., constitutional isomers), especially as compound molecular weights increase. One method for confirming an isomer assignment is to use gas chromatography-mass spectrometry (GC-MS) in conjunction with a reference standard of the compound. Unfortunately, the adducts of ATM are not 
suitable for analysis by GC-MS since ATM is non-volatile as a consequence of its quaternary ammonium moiety. The use of ammonium ions to enhance the MS signal intensity at low concentrations is a well-established technique,${ }^{62}$ but since such ions typically are non-volatile a new strategy is required to conjunctively use the speed and accuracy of FT-ICR-MS with the isomeric differentiation afforded by GC-MS. We developed an approach to address this challenge by introducing a versatile aminooxy reagent, 4-(2-aminooxyethyl)-morpholin-4-ium chloride (AMAH, Scheme 2.1). AMAH functions as a chemoselective probe as a consequence of the aminooxy reactivity, and it contains a titratable ammonium salt for enhancing [+]-ion electrospray MS analysis. Moreover, AMAH-carbonyl adducts can be made volatile for analysis by GC using a straightforward basification procedure. By applying AMAH onto the micropillars of the silicon microreactor, the dual capability of AMAH can be exploited through detection and structural assignment of carbonyl VOC adducts from exhaled breath using both FTICR-MS and GC-MS.

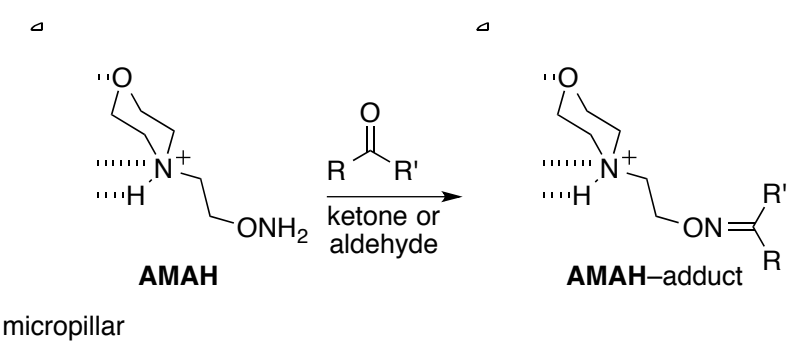

Scheme 2.1. AMAH oximation covalently traps aldehydes and ketones in the microreactor. 


\subsection{RESULTS AND DISCUSSION}

ATM was specifically designed with several features that would optimize analyses of its adducts by FT-ICR-MS. The quaternary ammonium salt allowed for electrostatic interaction between ATM and the negatively charged silica surface of the micropillars (Scheme 2.1). Secondly, the aminooxy moiety allowed for the rapid, chemoselective capture of gaseous aldehyde and ketone metabolites in exhaled breath samples with $>90 \%$ capture efficiency. ${ }^{55}$ Finally, the permanent positive charge of the quaternary ammonium salt enhanced the signal intensity of the metabolite adducts at low concentrations when analyzed by FT-ICR-MS. ${ }^{62}$ In addition to cost considerations, a limitation of analyzing ATM-adducts using FT-ICR-MS is the inability to differentiate constitutional isomers for a given molecular mass. MS-MS techniques can be applied to address this issue; however, use of a more widely available and affordable analytical platform, such as GC-MS, would improve the accessibility of the microreactor approach for identifying metabolites. Unfortunately, GC-MS cannot be used to analyze ATMcarbonyl metabolite adducts since these are non-volatile. To address this issue, we developed a second-generation reagent that allows for both FT-ICR-MS and GC-MS identification with isomeric accuracy.

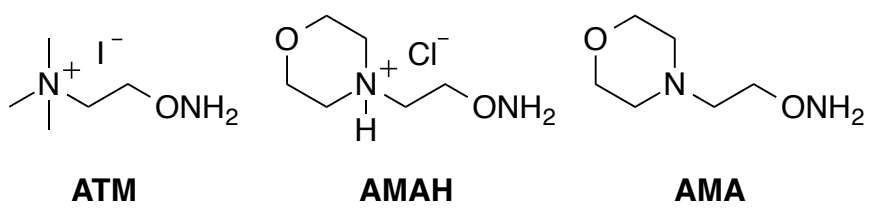

Figure 2.1. Structures of ATM, AMAH and AMA. 
By slightly expanding the structural framework of ATM, we engineered secondgeneration reagent, AMAH (Figure 2.1), that features a titratable aminium nitrogen instead of a quaternary ammonium salt. The morpholino bridge, as opposed to $\mathrm{N}, \mathrm{N}$ dimethyl, imparts an order of magnitude greater acidity for ease of basification, a subsequent key step, to generate neutral species, such as AMA or neutral AMA-carbonyl adducts. ${ }^{63}$ Upon introduction to the microreactor, the aminium salt of AMAH electrostatically bonds to the silica surface of the micropillars while the morpholino oxygen and aminic proton also provide hydrogen bonding opportunities with surface silanols to improve the availability of the aminooxy moiety for carbonyl capture. Finally, the tertiary ammonium ion also enhances the MS signal intensity for analyses using FTICR-MS so that high mass accuracy can be determined for even low abundance carbonyl metabolites.

The key advantage in using AMAH over ATM for metabolite identification is its ability to be volatilized through a basification procedure, thus making it applicable for both FT-ICR-MS and GC-MS analyses. To ensure that the basification of AMAHcarbonyl adducts proceeds to give an analyte mixture that can be injected directly into a GC instrument, we developed a salt-free neutralization procedure that does not require liquid-liquid extraction or other lengthy handling processes (Scheme 2.2). First, the eluted methanolic AMAH-carbonyl adduct mixture can be directly analyzed by FT-ICRMS. An aliquot can then be prepared for GC-MS analysis by reaction with poly(4vinylpyridine) (PVP), an acid scavenging polymer (Figure 2.2). We were gratified to 


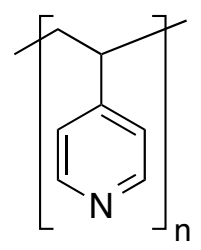

Figure 2.2. Structure of poly(4vinylpyridine) (PVP).

find that when a $40 \mu \mathrm{L}$ sample of AMAH-adducts in methanol was added to $2 \mathrm{mg}$ of PVP followed by shaking for 30 seconds, complete neutralization of the adducts was achieved. Efficient neutralization using this procedure was confirmed by NMR spectroscopy (Figure 2.3). When the acetone- $d_{6}$ adduct of AMAH is treated with PVP, the chemical shifts of the morpholino methylene protons coalesce into broad singlets, at $\delta$ 3.95 and $\delta 3.37 \mathrm{ppm}$ for the methylene signals adjacent to oxygen and nitrogen, respectively, as a result of the greater conformational flexibility of the AMA-adduct. This procedure is particularly appealing in that after basification using PVP the polymer quickly sediments to allow convenient aliquot sampling for direct GC injection.

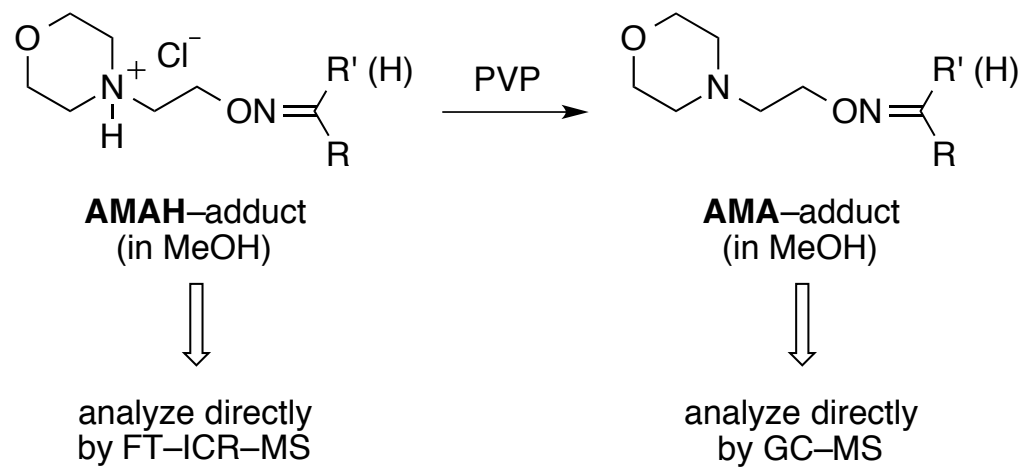

Scheme 2.2. Use of AMAH for both FT-ICR-MS and GC-MS analyses. PVP $=$ poly(4-vinylpyridine) 

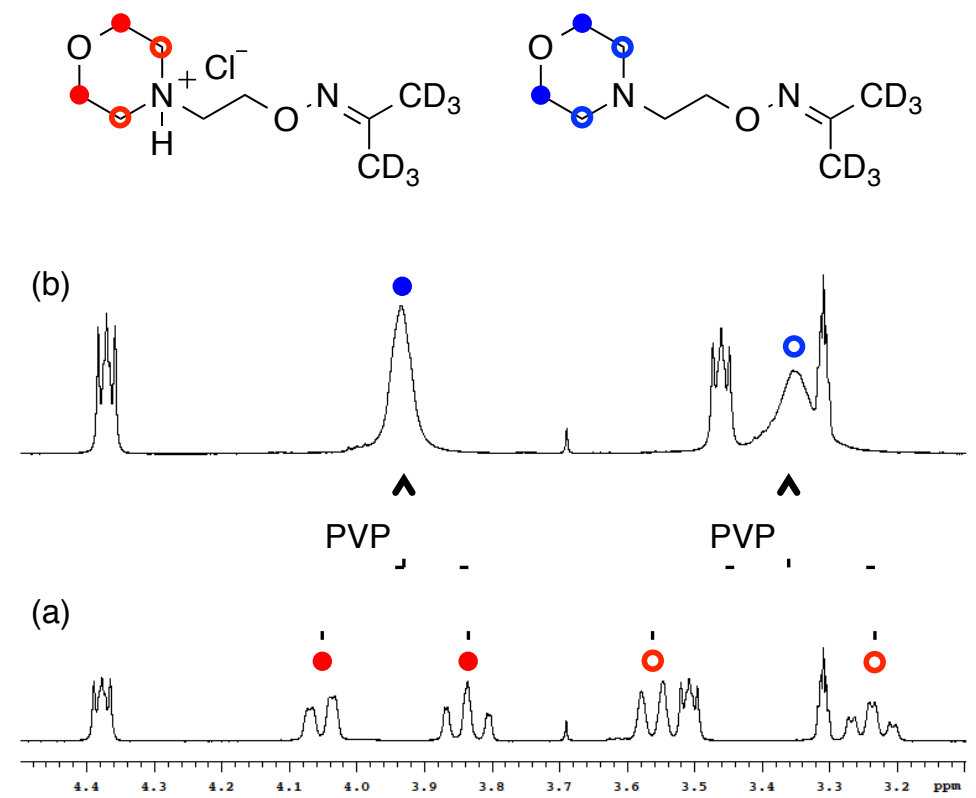

Figure 2.3. $400 \mathrm{MHz}{ }^{1} \mathrm{H}$ NMR $\left(\mathrm{CD}_{3} \mathrm{OD}\right)$ of (a) AMAH-acetone- $d_{6}$ adduct and (b) AMA-acetone$d_{6}$ after treatment with PVP.

To examine the reactivity of AMAH with volatile carbonyl compounds, we performed a calibration experiment with acetaldehyde, acetone and 2-butanone in a closed chip. Each carbonyl $\left(7.67 \times 10^{-7}\right.$ to $\left.7.67 \times 10^{-10} \mathrm{~mol}\right)$ was injected into a 1 liter Tedlar bag and connected to the preconcentration setup (Figure 2.4). A vacuum pump

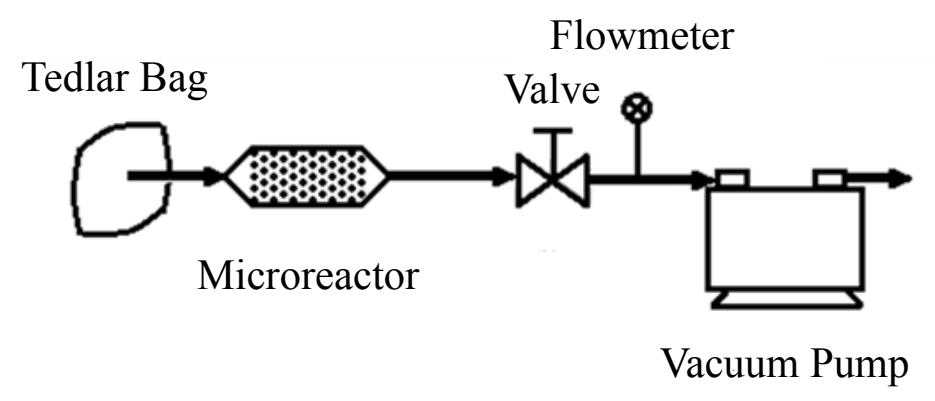

Figure 2.4. Schematic flow diagram of the preconcentration set-up. 
pulled the gaseous contents of the bag through the AMAH-functionalized, closed microreactor (Figure 2.5 ) at a rate of $3.5 \mathrm{~mL} / \mathrm{min}$. After the Tedlar bag had been fully evacuated, the microreactor was removed from the preconcentration assembly and the analytes were washed free from the chip using methanol. A solution of AMAH-

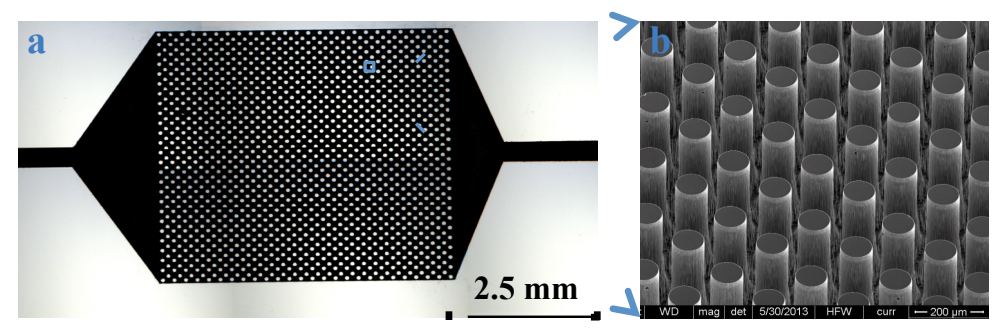

Figure 2.5. Silicon microreactor for the capture of carbonyl VOCs in exhaled breath. (a) Optical micrograph of the microreactor before bonding with a glass wafer. (b) SEM micrograph of the micropillar array within the microreactor.

acetone- $d_{6}$ adduct was added to the analyte solution to serve as an internal standard. For both aldehydes and ketones, the linear capture efficiency of AMAH peaks at a $\sim 91 \%$ capture rate at a $10^{3} \mathrm{AMAH} /$ analytes molar ratio (Figure 2.6, Table 2.4). The reason that the highest capture efficiency occurred at higher AMAH/analyte molar ratios is due to the elevated reaction probability for the capture of aldehyde and ketone VOCs. Due to the low concentrations of carbonyl VOCs in exhaled breath, 180-800 ppbv for acetone, AMAH should capture nearly all of VOCs of interest and provide an accurate concentration of each metabolite. ${ }^{64,65}$ Having confirmed that AMAH was highly reactive 


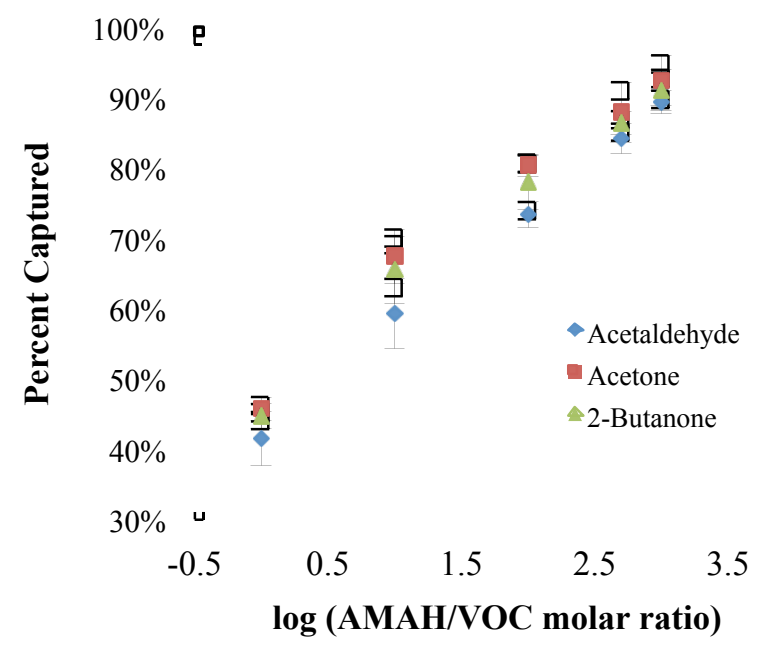

Figure 2.6. Graphical relationship between VOC capture efficiency and AMAH/VOC molar ratio.

Table 2.4. Tabulated relationship between VOC capture efficiency and $\mathrm{AMAH} / \mathrm{VOC}$ molar ratio.

\begin{tabular}{|c|c|c|c|c|}
\hline \multirow{2}{*}{\multicolumn{2}{|c|}{$\begin{array}{cc}\text { AMAH/VOC } & \log (\mathrm{AMAH} / \\
\text { molar ratio } & \begin{array}{c}\text { VOC molar } \\
\text { ratio) }\end{array} \\
\end{array}$}} & \multicolumn{3}{|c|}{ Capture Efficiency ( $\% \pm$ standard error) } \\
\hline & & Acetaldehyde & Acetone & 2-Butanone \\
\hline 1 & 0 & $41.5 \pm 1.7$ & $45.8 \pm 1.8$ & $44.7 \pm 3.8$ \\
\hline 10 & 1 & $59.3 \pm 3.9$ & $67.4 \pm 4.8$ & $65.5 \pm 4.9$ \\
\hline 100 & 2 & $73.3 \pm 1.6$ & $80.3 \pm 3.8$ & $78.0 \pm 1.8$ \\
\hline 500 & 2.7 & $84.1 \pm 4.3$ & $87.9 \pm 1.7$ & $86.4 \pm 2.2$ \\
\hline 1000 & 3 & $89.3 \pm 3.6$ & $92.4 \pm 2.9$ & $91.1 \pm 1.6$ \\
\hline
\end{tabular}

and applicable for both FT-ICR-MS and GC-MS, we analyzed a panel of AMA- $\mathrm{C}_{1}-\mathrm{C}_{5}$ aldehyde and ketone adducts (Figure 2.7, Table 2.5) on GC-MS to establish reference retention times for the adducts. 


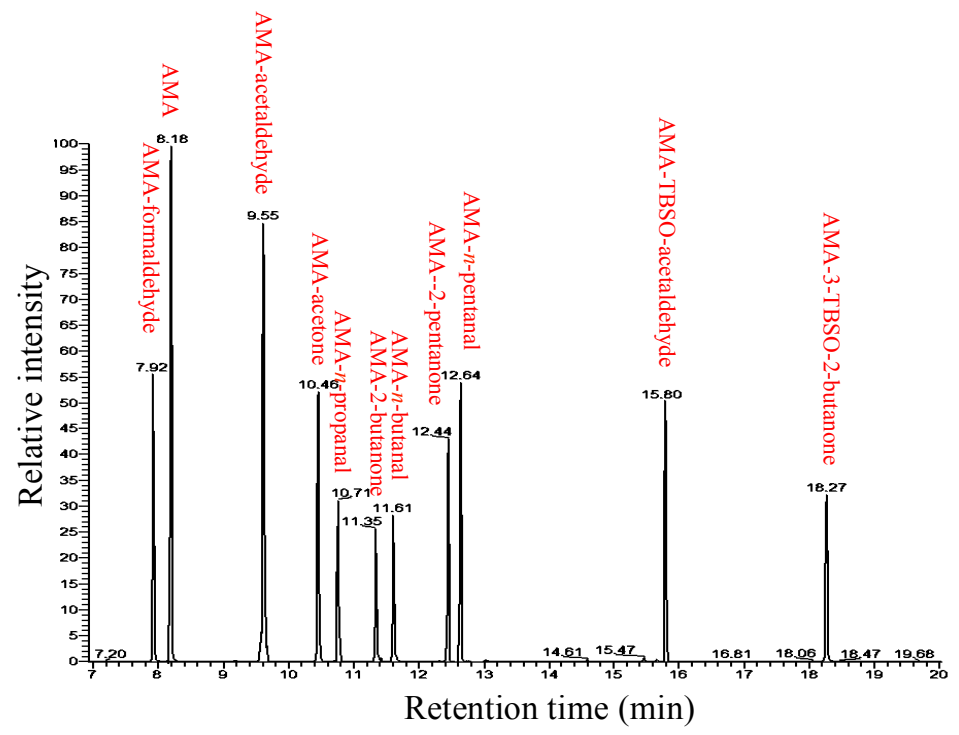

Figure 2.7. GC-MS chromatogram of reference AMA-carbonyl adducts; TBSO $=t$ butyldimethylsilyloxy.

Table 2.5. Retention times of $\mathrm{AMA}-\mathrm{C}_{1}-\mathrm{C}_{7}$ adducts.

\begin{tabular}{lc|lc}
\hline Adduct & $\boldsymbol{t}_{\mathbf{R}}(\mathbf{m i n})$ & \multicolumn{1}{l}{ Adduct } & $\boldsymbol{t}_{\mathbf{R}}(\mathbf{m i n})$ \\
\hline $\begin{array}{l}\text { AMA- } \\
\text { formaldehyde }\end{array}$ & 7.92 & AMA- $n$-butanal & 11.61 \\
AMA & 8.18 & AMA-2-pentanone & 12.44 \\
$\begin{array}{l}\text { AMA- } \\
\text { acetaldehyde }\end{array}$ & 9.55 & AMA-n-pentanal & 12.64 \\
$\begin{array}{l}\text { AMA-acetone } \\
\text { AMA-n-propanal }\end{array}$ & 10.71 & $\begin{array}{l}\text { AMA-TBSO- } \\
\text { acetaldehyde }\end{array}$ & $\begin{array}{l}\text { AMA-3-TBSO-2- } \\
\text { butanone }\end{array}$ \\
$\begin{array}{l}\text { AMA-2-butanone } \\
{ }^{a} \text { TBSO }=t \text {-butyldimethylsilyloxy }\end{array}$ & 11.35
\end{tabular}


Following calibration, the closed chip was replaced by an open chip to allow a patient to blow directly onto the AMAH-functionalized microreactor through a straw. Subjects blew into a vial containing the microreactor for 15 minutes. Upon completion, methanol was used to elute the AMAH-adducts from the microreactor, and the collected eluent then was directly analyzed by FT-ICR-MS. Figure 2.8 shows a FT-ICR-MS spectrum of a healthy non-smoking subject. An aliquot $(c a .35 \mu \mathrm{L})$ of the methanol AMAH-adduct mixture also was added to PVP. After neutralization, the PVP was

(a)

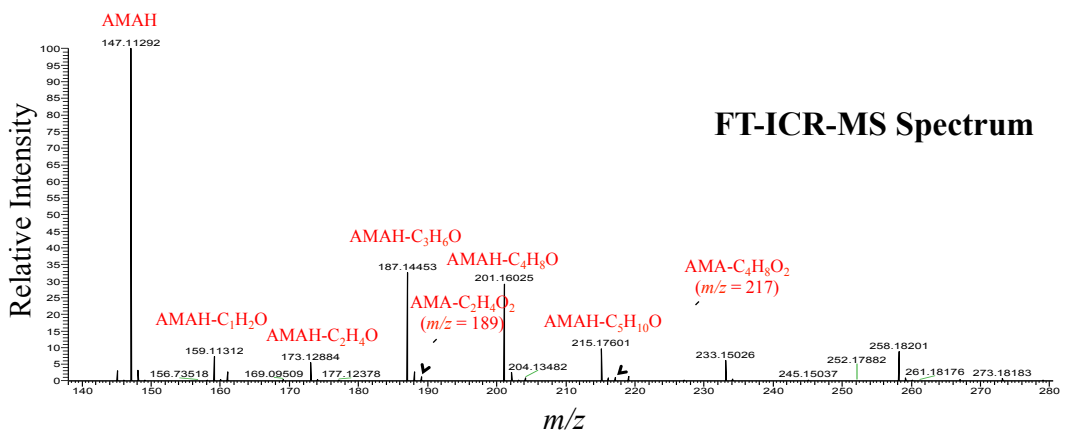

(b)

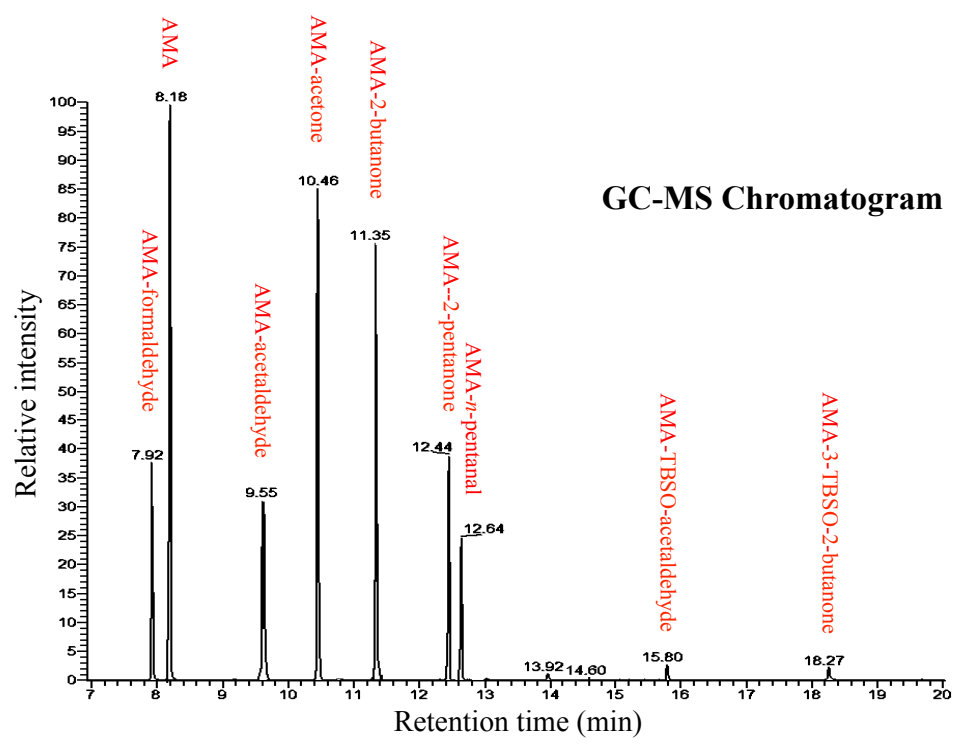

Figure 2.8. Qualitative comparison of (a) FT-ICR-

MS spectrum and (b) GC-MS chromatogram of

VOCs from a healthy, non-smoking subject. 
filtered and the methanol filtrate was evaporated. To the residue was added a 1:1 mixture of acetonitrile: $N$-tert-butyldimethylsilyl- $N$-methyltrifluoroacetamide (MTBSTFA) (v/v) $(30 \mu \mathrm{L})$. MTBSTFA was used to produce volatile and stable tert-butyldimethylsilyl (TBS) derivatives of hydroxyl groups for GC analysis. MTBSTFA was used over TBSCl because its reaction byproducts are volatile and neutral, thus minimizing the risk of degrading other VOCs. The resultant silylated AMA-adduct mixture was analyzed by GC-MS. With results from both the FT-ICR-MS and GC-MS analyses in hand, we compared the abundance of captured carbonyls by calculating the ratio of the abundance of an analyte to the sum of the abundance of all analytes (Table 2.6). The results indicate that the carbonyl ratios based on GC-MS analysis were consistent with the ratios calculated using the FT-ICR-MS results with a $2.6 \%$ variance as the largest difference (entry 1).

Table 2.6. Quantitative comparison of FT-ICR-MS and GC-MS analyses of a breath sample. ${ }^{a}$

\begin{tabular}{clcc}
\hline Entry & \multicolumn{1}{c}{ Carbonyl VOC } & GC-MS $^{b}$ & FT-ICR-MS $^{b}$ \\
\hline 1 & $\mathrm{CH}_{2} \mathrm{O}$ (formaldehyde) & 0.128 & 0.154 \\
2 & $\mathrm{C}_{2} \mathrm{H}_{4} \mathrm{O}$ (acetaldehyde) & 0.109 & 0.099 \\
3 & $\mathrm{C}_{3} \mathrm{H}_{6} \mathrm{O}$ (acetone) & 0.281 & 0.290 \\
4 & $\mathrm{C}_{4} \mathrm{H}_{8} \mathrm{O}$ (2-butanone) & 0.260 & 0.254 \\
5 & $\mathrm{C}_{5} \mathrm{H}_{10} \mathrm{O}$ (2-pentanone, & 0.193 & 0.173 \\
& $n$-pentanal) & & \\
6 & $\mathrm{C}_{2} \mathrm{H}_{4} \mathrm{O}_{2}$ (hydroxyl- & 0.015 & 0.017 \\
& acetaldehyde) & & \\
7 & $\mathrm{C}_{4} \mathrm{H}_{8} \mathrm{O}_{2}$ (3-hydroxy- & 0.011 & 0.013 \\
& 2-butanone) & & \\
\hline
\end{tabular}

${ }^{a}$ Breath sample from a healthy, non-smoker;

${ }^{b}$ Analyte contribution to total (ratio of analyte abundance/sum of the abundance of all analytes). 
With the knowledge that AMAH can be used in conjunction with either GC-MS or FT-ICR-MS to analyze these high abundance carbonyl compounds in exhaled breath, Dr Fu working with Dr. Michael Bousamra, University of Louisville, Brown Cancer Center, examined samples from more than 30 non-smoker controls, 30 smoker controls and 50 patients diagnosed with lung cancer to determine differences in their exhaled breath. Each subject blew into a vial containing an AMAH-functionalized open chip for 15 minutes. Then, the AMAH-adducts were washed from the microreactor with methanol and injected for FT-ICR MS analysis (Figure 2.9a). Finally, an aliquot was neutralized, silylated and then analyzed by GC-MS. Figure 2.9a shows typical FT-ICR-MS spectra of a non-smoker control, a smoker control and a lung cancer patient. Figure $2.9 \mathrm{~b}$ shows typical GC-MS chromatograms of a non-smoker control, a smoker control and a lung cancer patient. 


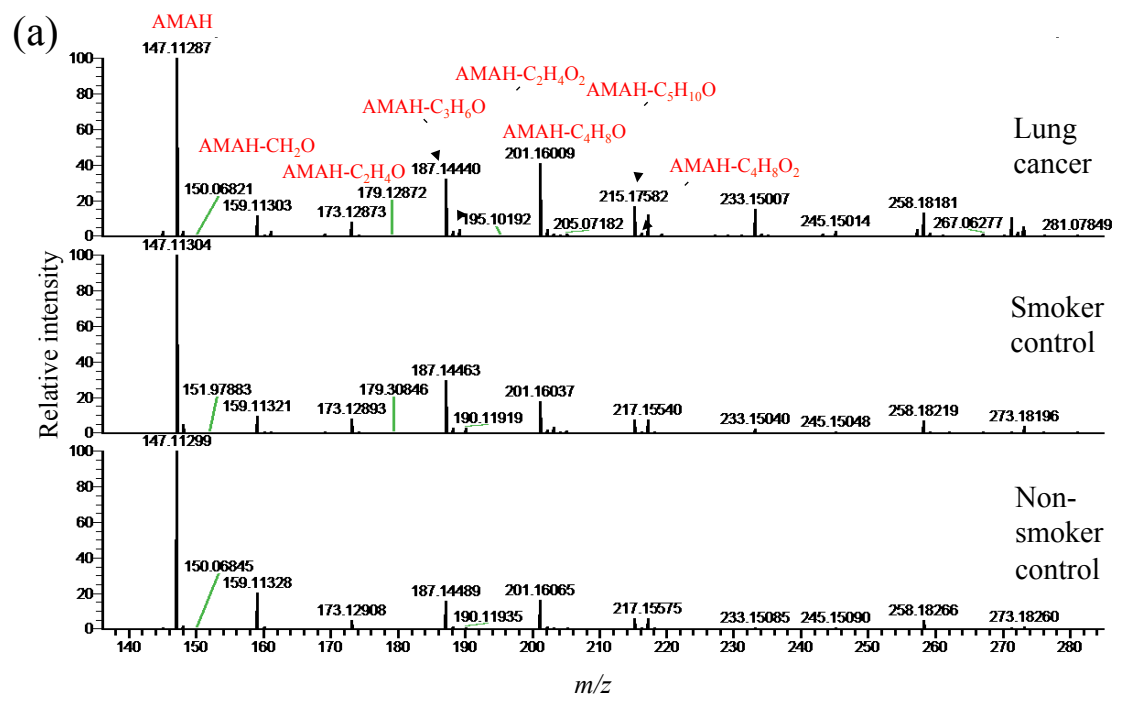

(b)

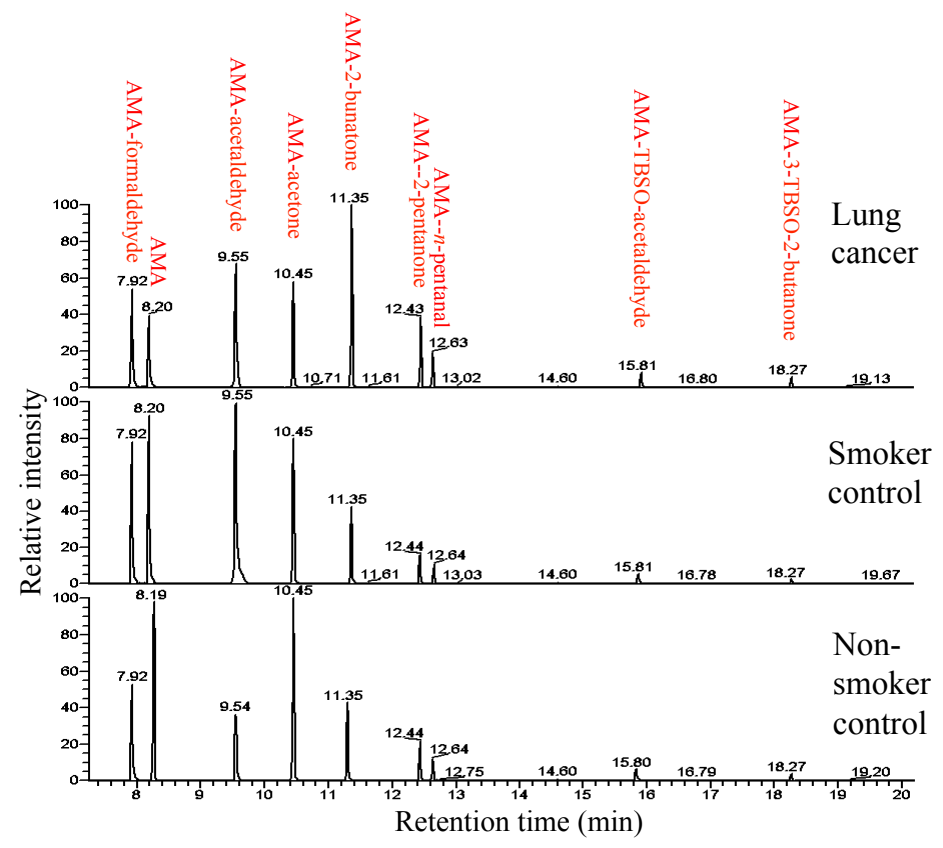

Figure 2.9. Analyses of a healthy non-smoker control, smoker control and lung cancer subject via (a) FT-ICR-MS and (b) GC-MS. 
The FT-ICR-MS and GC-MS spectra both show that the smoker control and the lung cancer subject have considerably more carbonyl VOCs than the non-smoker control. For example, $n$-propanal and 2-pentanone are conspicuous in the lung cancer subject. Figure 2.8a and 2.8b also show a higher abundance of 2-butanone and $n$-pentanal in the exhaled breath of the lung cancer patient. These compounds have been reported as lung cancer markers. $^{52,57,58,60}$ Hydroxyacetaldehyde and 3-hydroxy-2-butanone also have been identified as lung cancer markers in our previous work on closed chip microreactors. ${ }^{52}$ Whereas breath analysis for detection of lung cancer using GC-MS has great potential, these results are only a preliminary indication of the potential of AMAH. In this regard, a major advantage in using AMAH/AMA is the ability to accurately determine marker molecular formulae as well as to detect the isomeric differences by GC-MS that FT-ICR-MS does not provide. For example, 2-pentanone and pentanal are constitutional isomers that would register as a single $m / z$ on FT-ICR-MS, but the AMA adducts of these VOCs are readily separated by GC-MS to reveal an abundance of one over the other. In addition, due to the chemoselectivity of aminooxy compounds for reacting with aldehydes and ketones, ${ }^{66}$ interference of other abundant volatiles in exhaled human breath, such as $\mathrm{O}_{2}, \mathrm{CO}_{2}, \mathrm{H}_{2} \mathrm{O}$ and numerous VOCs, is avoided and this greatly facilitates carbonyl compound calibration as well as peak identification.

\subsection{CONCLUSION}

This thesis research resulted in the development of a second-generation ammonium aminooxy probe, AMAH, to efficiently capture trace aldehyde and ketone VOCs from exhaled breath when applied to a silicon microreactor containing thousands of 
micropillars. The AMAH-coated microreactor chemoselectively and covalently concentrates carbonyl VOCs through an oximation reaction. The resultant AMAHadducts can be readily rinsed from the microreactor by elution with cold methanol and then directly analyzed by FT-ICR-MS. The positive charge of the AMAH-VOC adducts enhances sensitivity for the high mass accuracy and ultra-high resolution of FT-ICR-MS to enable determination of molecular formulae. Also developed was a protocol whereby the AMAH-VOC adduct solution is added to a small amount of the acid-scavenging polymer PVP to produce volatile AMA-VOC adducts that can be analyzed by GC-MS. This versatile attribute of AMAH enables the identification and quantification of isomeric metabolites using GC-MS. The combination of a titratable aminium reagent with the PVP neutralization protocol constitutes a general approach that may be applicable for the development of other chemoselective probes.

\subsection{FUTURE DIRECTIONS}

To expand the use of this technology, I have also synthesized ADMH, an aminium version of ATM, as can be seen in Figure 2.10. The synthesis of ADMH was similar to that of ATM. A Mitsunobu reaction with $N, N$-dimethylethanolamine was performed to afford the phthalimide protected aminooxy. The phthalimide was cleaved via hydrazinolysis with methylhydrazine and the resulting ADM was distilled using a

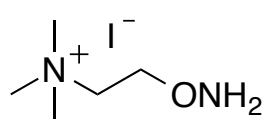

ATM

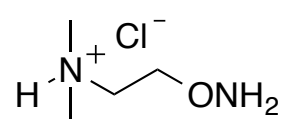

ADMH

Figure 2.10. Structures of ATM and ADMH. 
Kügelrohr apparatus. Due to the reactive nature of the aminooxy terminus, the ${ }^{1} \mathrm{H}$ NMR spectrum revealed that $c a .48 \%$ of the product had reacted and formed an oxime ether adduct. To circumvent this problem, the partially reacted material was hydrolyzed under acidic conditions (6 $\mathrm{M} \mathrm{HCl}$, reflux, $22 \mathrm{~h}$ ) to unmask the aminooxy moiety and to provide the ADMH amminic proton needed to reduce volatility. The reactivity of ADMH and its application to both FT-ICR-MS and GC-MS analyses are currently being investigated by Dr. Xiao-An Fu and collaborators.

In addition, I have pursued initial efforts to prepare an aminooxy reagent that could be used for the analysis and detection of VOC adducts via HPLC-MS. Our goal in designing this molecule was to have a non-volatile aminooxy molecule that was sufficiently reactive to efficiently capture and covalently retain VOCs from exhaled breath while also providing a convenient and sensitive method of detection, such as by HPLC. We chose to use a fluorescent detector with the HPLC to ensure that we would be able to quantify the VOC oxime ether adducts at nanomolar concentrations. When searching for a fluorophore, we found a paper by Goto et al. ${ }^{67}$ that specifically addressed the formation of sensitive fluorescence labeling reagents for HPLC of carbonyl compounds. Figure 2.11 shows a time course to exemplify the oximation of 2 with a ketone (ca. 12 $\alpha$-hydroxy-3-oxo-5 $\beta$-chloanoic acid). Though Goto only reported the reactivity of $\mathbf{2}$, he noted that there was no difference in the reactivity between the anthracene-aminooxy molecules. 


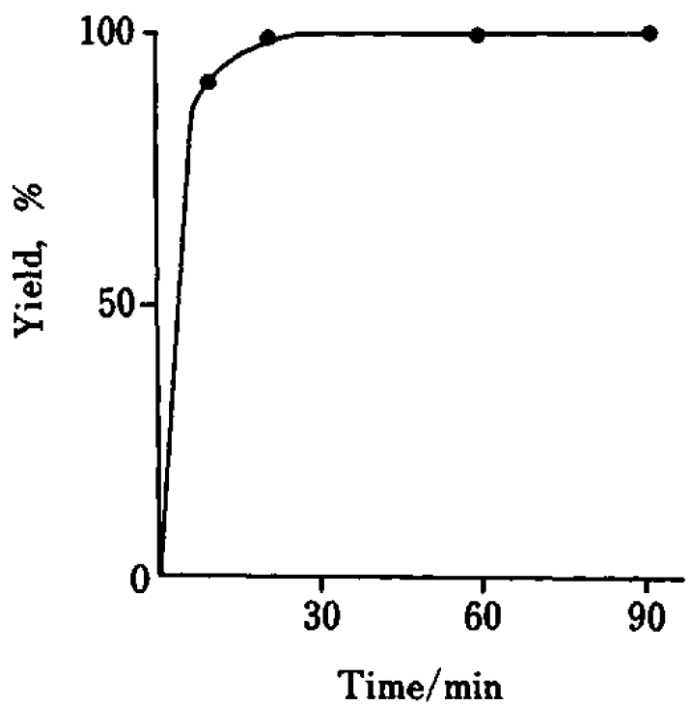

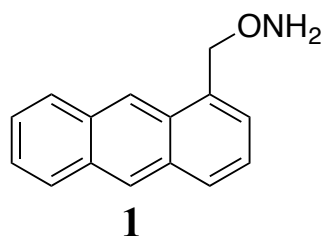

Goto et al.

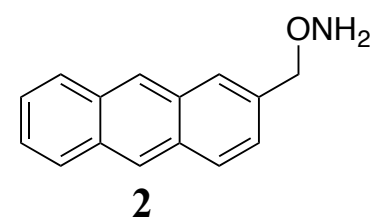

Goto et al.

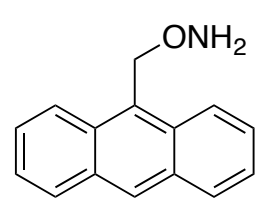

AAn

Knipp

Figure 2.11. Time course for derivatization of $12 \alpha$-hydroxy-3-oxo-5 $\beta$ chloanoic acid with $O$-(2-anthrylmethyl)hydroxylamine (2) ${ }^{67}$

With this information in-hand, we synthesized AAn (Figure 2.11) from 9anthracenemethanol. Briefly, the alcohol was reacted with $N$-hydroxyphthalimide under Mitsunobu conditions to afford the phthalimide-protected aminooxy that was deprotected with hydrazine monohydrate to give AAn. Though initial results are encouraging, the Fu lab is still in the early stages of testing AAn and comparing its reactivity with ATM and AMAH. 


\section{CHAPTER 3}

THERMALLY-INDUCED INTRAMOLECULAR CYCLIZATION AS A MECHANISM FOR SUBSTRATE RELEASE

\subsection{INTRODUCTION}

3.2. INITIAL CONCEPT

3.3. SECOND GENERATION

3.3.1. Cyclization Panel

3.3.2. Syntheses

3.4. CYCLIZATION STUDY

3.5. MICROCHANNEL APPLICATION

3.6. CONCLUSION 


\subsection{INTRODUCTION}

Intramolecular cyclization is a well known chemical process often exploited in synthesis, not only for target molecule synthesis but also for diverse applications in many fields, such as materials science and biochemistry. Examples include carbon-carbon bond formation via intramolecular Diels-Alder reactions ${ }^{1}$ as well as catalyzed cyclizations ${ }^{2}$ and formation of heterocyclic rings such as lactones, ${ }^{3}$ lactams, ${ }^{4}$ and oxazolidinones. ${ }^{5}$ Though intramolecular cyclization is usually utilized as a means to ring formation, it can also be used as a mode of substrate release. The intramolecular cyclizations that focused more so on substrate release typically were engineered in this manner to function as mechanisms for drug delivery. ${ }^{6,7,8,9}$ In these cases, the released substrate is either a phenol $\left(\mathrm{R}^{\prime}=\mathrm{Ar}\right)$ or an aniline derivative (Figure $\left.3.1 \mathrm{~b}\right)$ so that the intramolecular cyclization occurs rapidly at physiological temperature, ${ }^{10}$ generally with a half-life from minutes to one hour. ${ }^{11}$ Intramolecular cyclizations of this type also have been used to unmask aromatic hydroxyl,${ }^{12}$ amine ${ }^{13}$ or thiol ${ }^{14}$ moieties as mechanisms to initiate electron cascade reactions or for release of polymer-bound drugs (Figure 3.1). ${ }^{15}$ Again the focus was to use the intramolecular cyclization for rapid drug release at physiological temperature, and this required R' to be aromatic. Few examples have used

this approach to release non-aromatic alcohols, ${ }^{16,17}$ the most common being for the delivery of 5-halo deoxyuridine analogs. ${ }^{18,19,20}$ 
$\mathbf{a}$
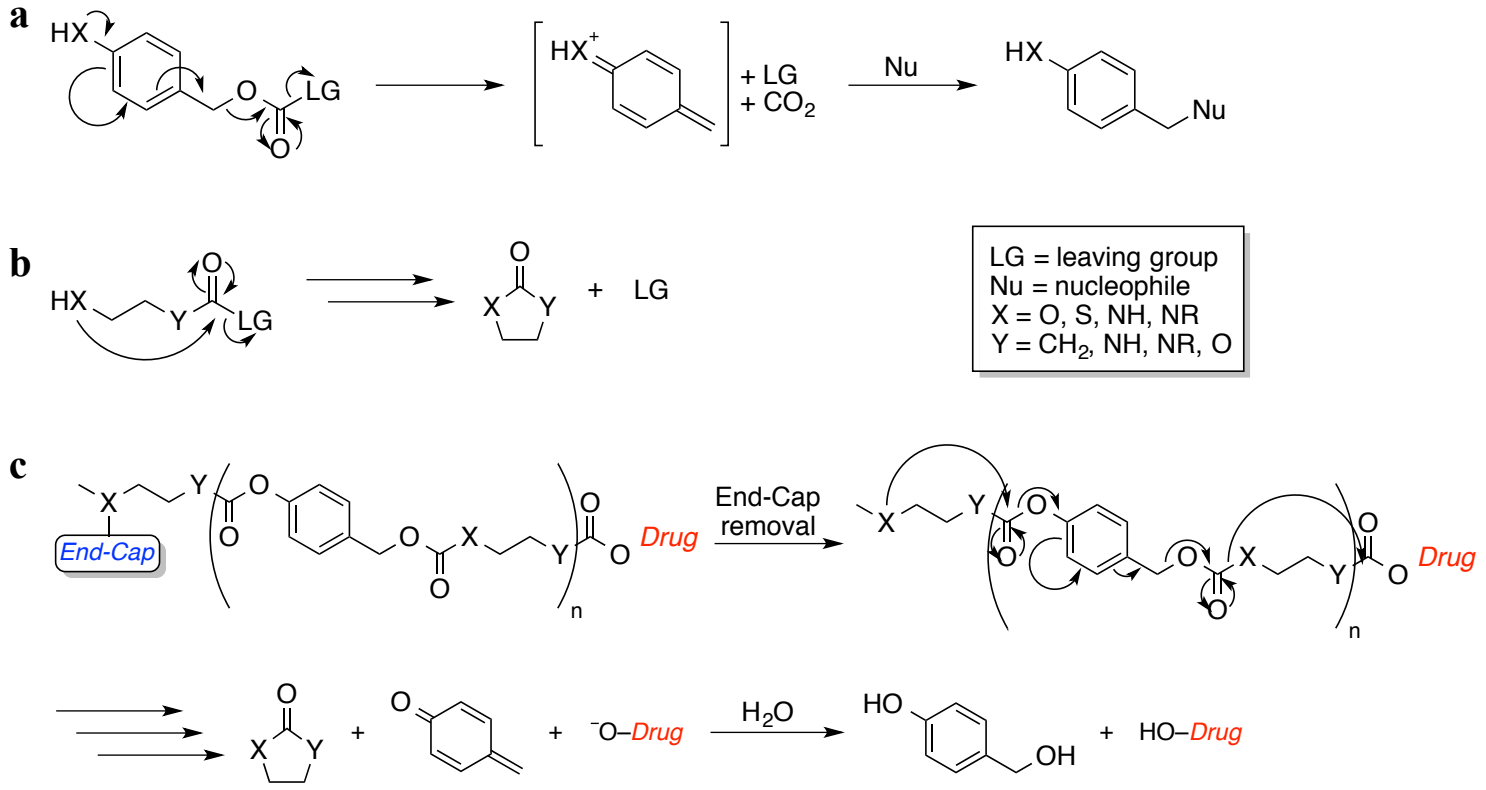

Figure 3.1. General structure of drug or prodrug delivery systems involving (a) electron cascade reactions, (b) rapid intramolecular cyclizations or (c) a hybrid of intramolecular cyclization and electron cascade reactions. ${ }^{11}$

Our interest in control over heat-induced intramolecular cyclization as a mechanism for substrate release led us to investigate linkers that would expel HO-R' (Figure 3.2) at temperatures above $37{ }^{\circ} \mathrm{C}$, such as in response to an externally triggered local hyperthermia. In addition to the release of alcoholic substrates, thermally induced intramolecular cyclization could also be used to release any carbonyl agent by fitting R' with an aminooxy group. 

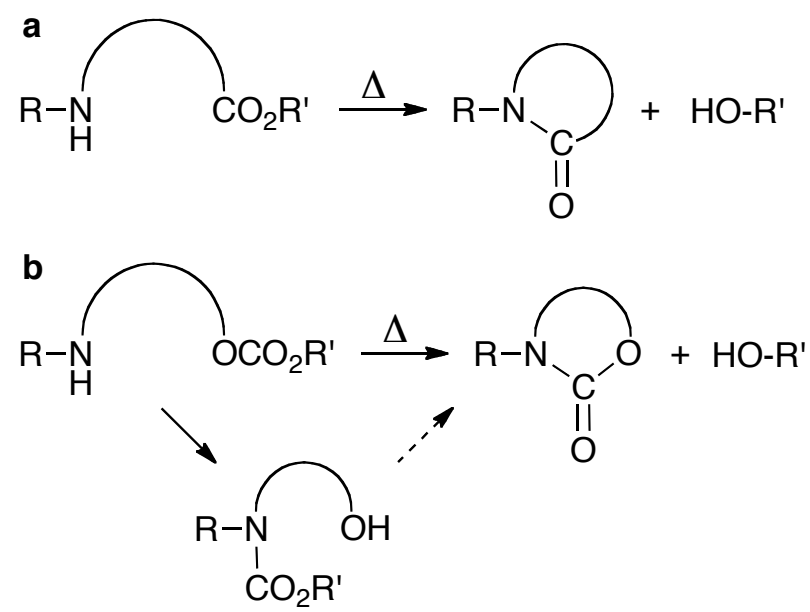

Figure 3.2. Heat-induced cyclization via (a) lactamization or (b) carbamate formation.

\subsection{FIRST GENERATION}

My first generation attempts at developing this strategy were motivated by a report published in Organometallics by Ojima and $\mathrm{Vidal}^{21}$ that described the synthesis and reactivity of amine $\mathbf{1}$ (Figure 3.3 ). We envisioned that the hydroxyl group could readily be transformed into the requisite, electrophilic carbonyl needed for the cyclization trigger, as in $\mathbf{2}$ (Figure 3.3). Aside from this attribute, $\mathbf{2}$ would also afford an alkoxysilane group that could allow the linker to be covalently bound to a variety of solid supports containing surface hydroxyl groups. ${ }^{22,23}$ Finally, the intramolecular cyclization could occur in response to heat. Thermal induction was desired because the cyclization rate could be tuned to occur at a range of temperatures, allowing for the technology to be adjusted for the application. In order to tune the thermal responsiveness of $\mathbf{2}$, the butyl group could be modified to increase or decrease steric bulk. In addition, the primary alcohol of 1 would allow the subsequent carbonyl to be an ester or a carbonate. This 
functional group variation from ester to carbonate would allow for accelerated or retarded cyclization, respectively.

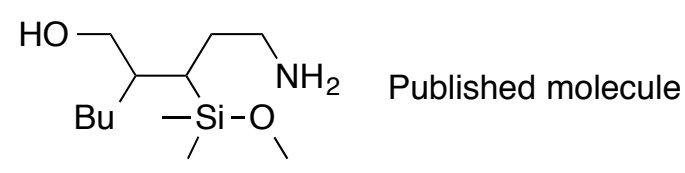

1

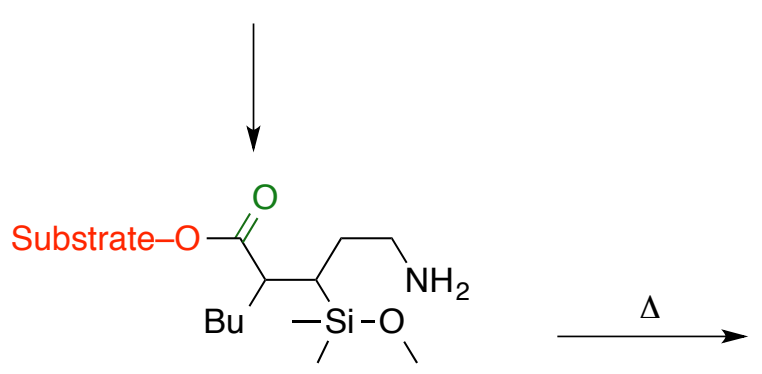

2

Target molecule

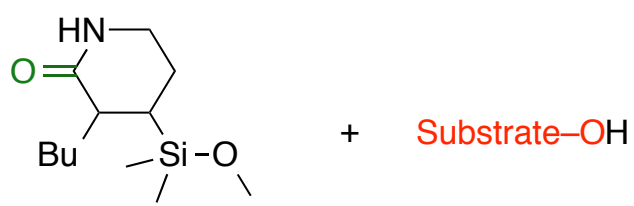

3

Cyclized lactam

Released alcoholic substrate

Figure 3.3. Proposed alterations to molecule published by Ojima and Vidal. ${ }^{21}$ The target molecule would cyclize when heated to form the lactam. 

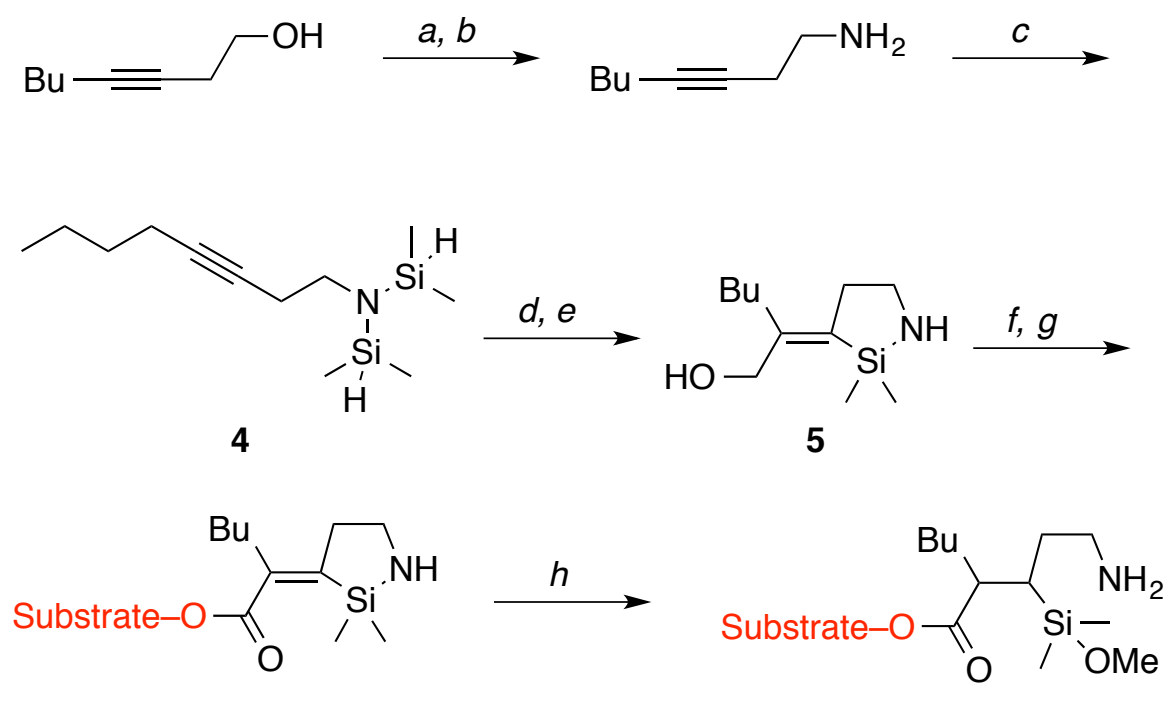

2

Scheme 3.1. Synthesis of 2. Conditions: a. Phthalimide, DIAD, $\mathrm{PPh}_{3}$, THF, $0{ }^{\circ} \mathrm{C}, 87 \%$; b. $\mathrm{N}_{2} \mathrm{H}_{4} \cdot \mathrm{H}_{2} \mathrm{O}, \mathrm{CH}_{2} \mathrm{Cl}_{2}, 56 \%$; c. $n$-BuLi, chlorodimethylsilane, THF, $-78{ }^{\circ} \mathrm{C}, 73 \%$; $d$. cat. $\mathrm{Rh}_{4}(\mathrm{CO})_{12}$, $\mathrm{CO}(10$ atm), toluene, $60{ }^{\circ} \mathrm{C} ; e . \mathrm{NaBH}_{4}, \mathrm{MeOH}$, toluene, $66 \%$ over 2 steps; $f$. [O]; g. Substrate-OH; $h$. cat. $\mathrm{Pd} / \mathrm{C}, \mathrm{H}_{2}, \mathrm{MeOH}$.

Starting with 3-octyn-1-ol (Scheme 3.1), a two-step functional group transformation using Mitsunobu conditions to add a phthalimide followed by hydrazinolysis yielded oct3-yn-1-amine in $49 \%$ overall yield. The primary amine was then bis-silylated by reaction with chlorodimethylsilane. Subsequent $\mathrm{Rh}_{4}(\mathrm{CO})_{12}$-catalyzed intramolecular silylformylation of the resident alkyne moiety under 10 atm $\mathrm{CO}$ followed by immediate reaction with methanolic $\mathrm{NaBH}_{4}$ afforded the stable azasilacyclopentane $\mathbf{5}$ in good yield. The results of the reaction were supported by the downfield position of the methylene protons and carbon of 1 (Figure 3.4) that was added in the reaction of $\mathrm{CO}$ with the alkyne bond. Further verification was found in the ${ }^{13} \mathrm{C}$ NMR spectrum with carbon 2 and 3 
(Figure 3.4) in the olefinic region. The presence of the exo-alkylene group of $\mathbf{5}$ is postulated to stabilize the $\mathrm{Si}-\mathrm{N}$ bond against hydrolysis. This unique stability would allow for further modification of $\mathbf{5}$ while affording the ability to restore the characteristic hydrolytic susceptibility of $\mathrm{Si}-\mathrm{N}$ bonds by subsequent hydrogenation. On hydrogenation of the exomethylene (and thus removal of its stabilizing influence) in dry methanol, the $\mathrm{Si}-\mathrm{N}$ bond would be expected to immediately hydrolyze and form the target methoxysilane (e.g., as in 2, Figure 3.3). 

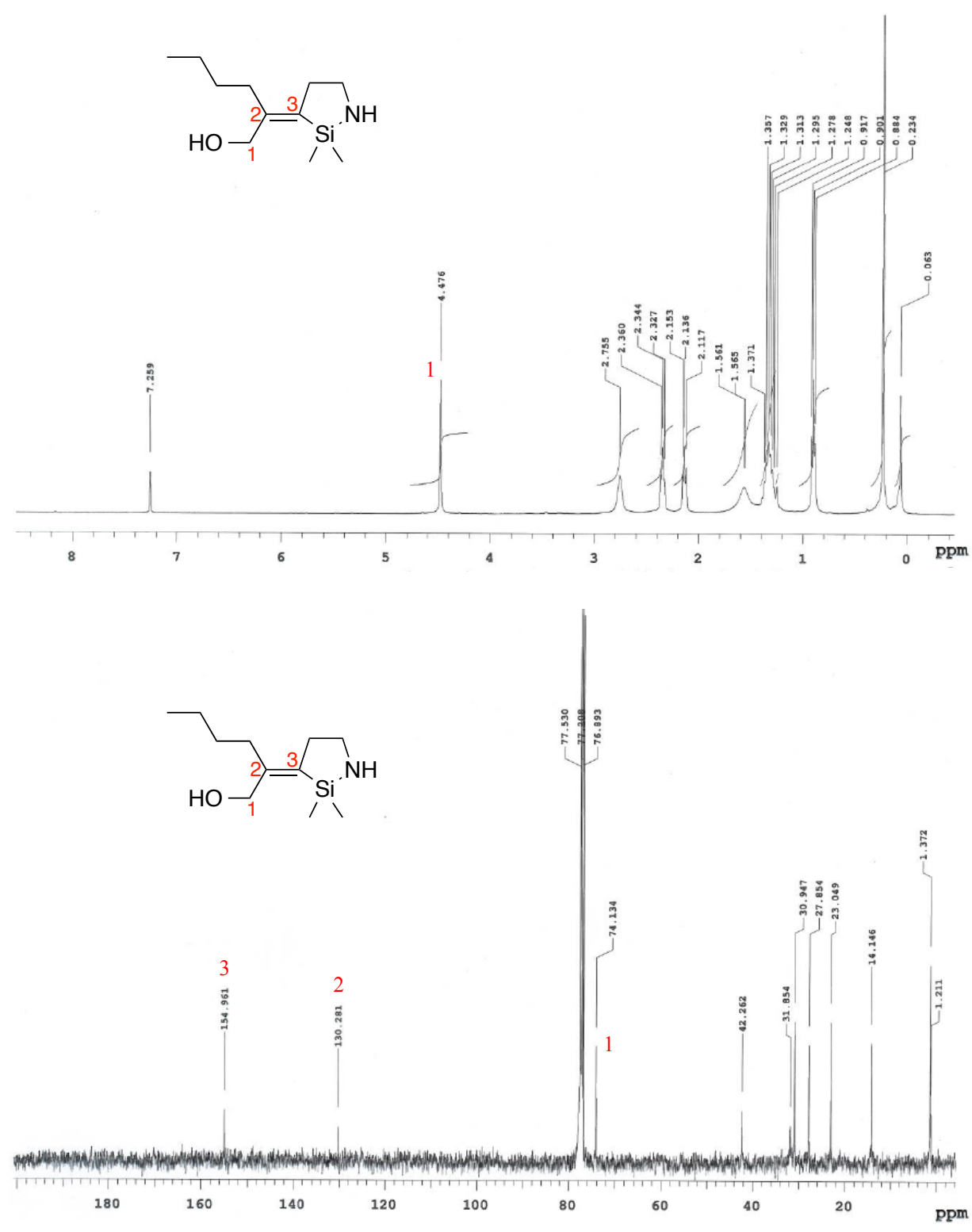

Figure 3.4. ${ }^{1} \mathrm{H}$ and ${ }^{13} \mathrm{C}$ NMR spectra $\left(\mathrm{CDCl}_{3}\right)$ confirming the synthesis of 5 (Scheme 3.1).

Despite our synthetic success, we ceased our pursuits of $\mathbf{2}$ after obtaining $\mathbf{5}$ due to concerns about the synthetic pathway. The first challenge was the hydrolytic susceptibility of $\mathbf{4}$ and the second concern was the safety of using $\mathrm{CO}$ gas at $10 \mathrm{~atm}$ of pressure at elevated temperatures. Throughout this process the scalability of our 
syntheses was always a goal; therefore, a new target molecule was deemed necessary due to the finesse and potential dangers associated with the aforementioned concerns.

\subsection{SECOND GENERATION}

To create a less cumbersome synthetic approach while still maintaining the goal for development of a thermally induced intramolecular cyclization we reflected on the positive and negative aspects of the route involving 2 . On doing so, the linearity of the amine with the carbonyl became evident and made us realize that an $\omega$-amino acid could be used as a readily available starting material (Figure 3.5).

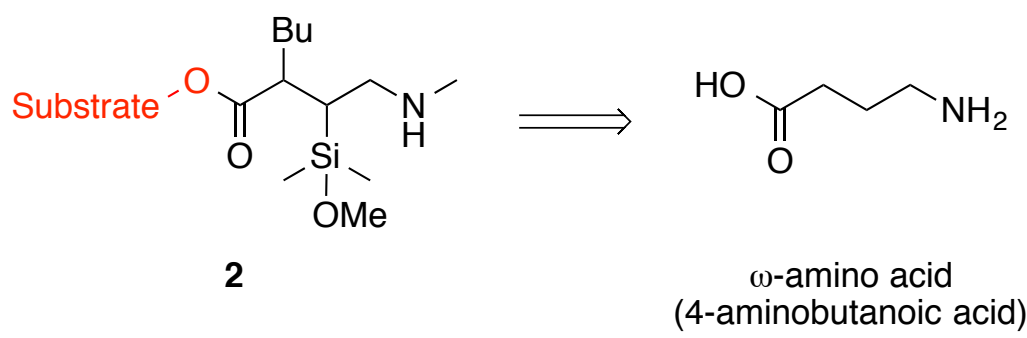

Figure 3.5. Retrosynthetic analysis of $\mathbf{2}$ reveals a path for simplification involving an $\omega$-amino acid starting material.

In addition to availability, there were other advantages to modifying the synthetic procedure to start with an $\omega$-amino acid. First, the distance between the nucleophilic amine and electrophilic carbonyl was no longer fixed at four carbons. This is of great importance because it allows for tuning the thermal responsiveness by adjusting the size of the heterocyclic ring that would be formed, from an expected rapid 5-membered ring formation to a slower forming 7-membered ring. Second, an oxidation step to form the carbonyl was no longer necessary. 
But there were also new synthetic challenges in using an $\omega$-amino acid starting material. First, we would have to devise a way to incorporate an alkoxysilane into the linker. The second challenge was that the preexisting carbonyl was only advantageous for esterification. In order to modify the functionality around the carbonyl we would have to reduce the esterified or acidic carboxyl group to form a primary alcohol. The alcohol could then be used in the formation of a carbonate or undergo a functional group transformation to an amine for eventual carbamate formation (Scheme 3.2).

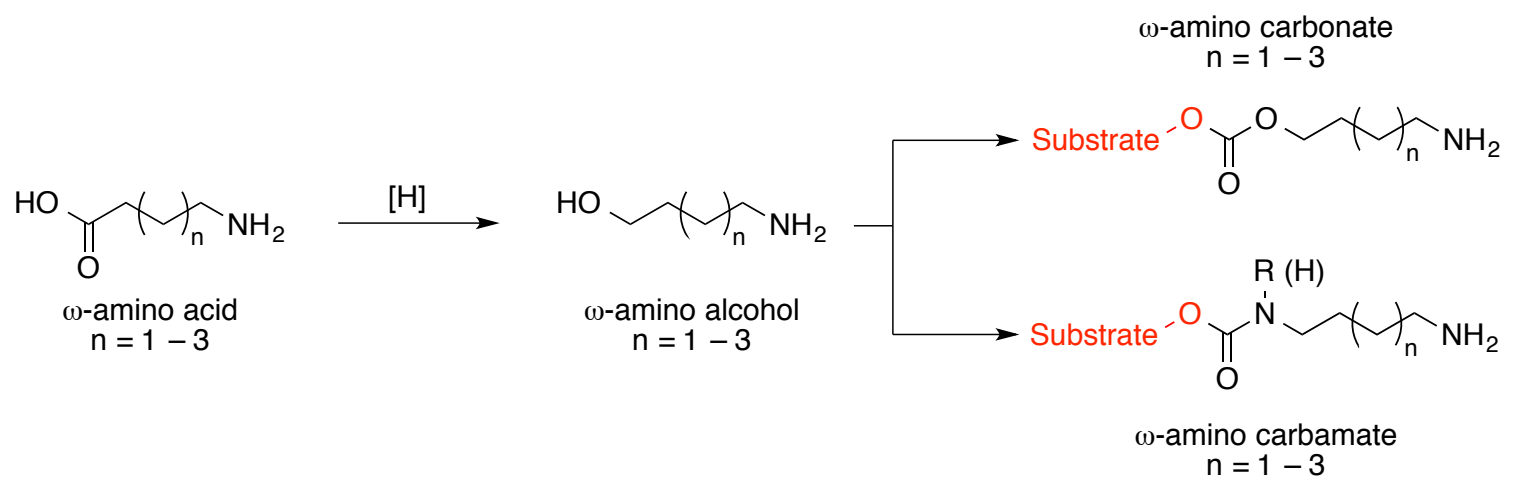

Scheme 3.2. Modification of carbonyl functionality from carboxylic acid to a carbonate or carbamate.

The first issue that we addressed was the incorporation of an alkoxysilane into the linker motif. A convenient location for attachment of an alkoxysilane group is to use the amine. In doing so, we gain nucleophilicity by forming a secondary amine. Furthermore, this approach would not be expected to deter the desired intramolecular cyclization steric hindrance issues are minimal. We therefore decided to alkylate the primary amine with an allyl group to subsequently use the unsaturation as a means for incorporating the alkoxysilane through hydrosilylation methodology. Following a report by Sabourault et 
$a l .{ }^{24}$ we used $\mathrm{PtO}_{2}$ as a catalyst to add triethoxysilane in the $\gamma$ position. As can be seen from the ${ }^{1} \mathrm{H}$ NMR spectrum in Figure 3.6, the disappearance of the olefinic peaks (red), the collapse of the methylenes surrounding the Boc-protected nitrogen into one broad peak (blue) and the appearance of an upfield peak at $0.57 \mathrm{ppm}$ (green) all support the effectiveness of the hydrosilylation. Despite the option of other hydrosilylation catalysts (Table 3.1), $\mathrm{PtO}_{2}$ was an affordable option that would enable us to use a minimal amount due to its high $\mathrm{w} / \mathrm{w}$ percentage of Pt. In addition, the catalyst could be recovered and reused, thus making $\mathrm{PtO}_{2}$ applicable for industrial reactions.

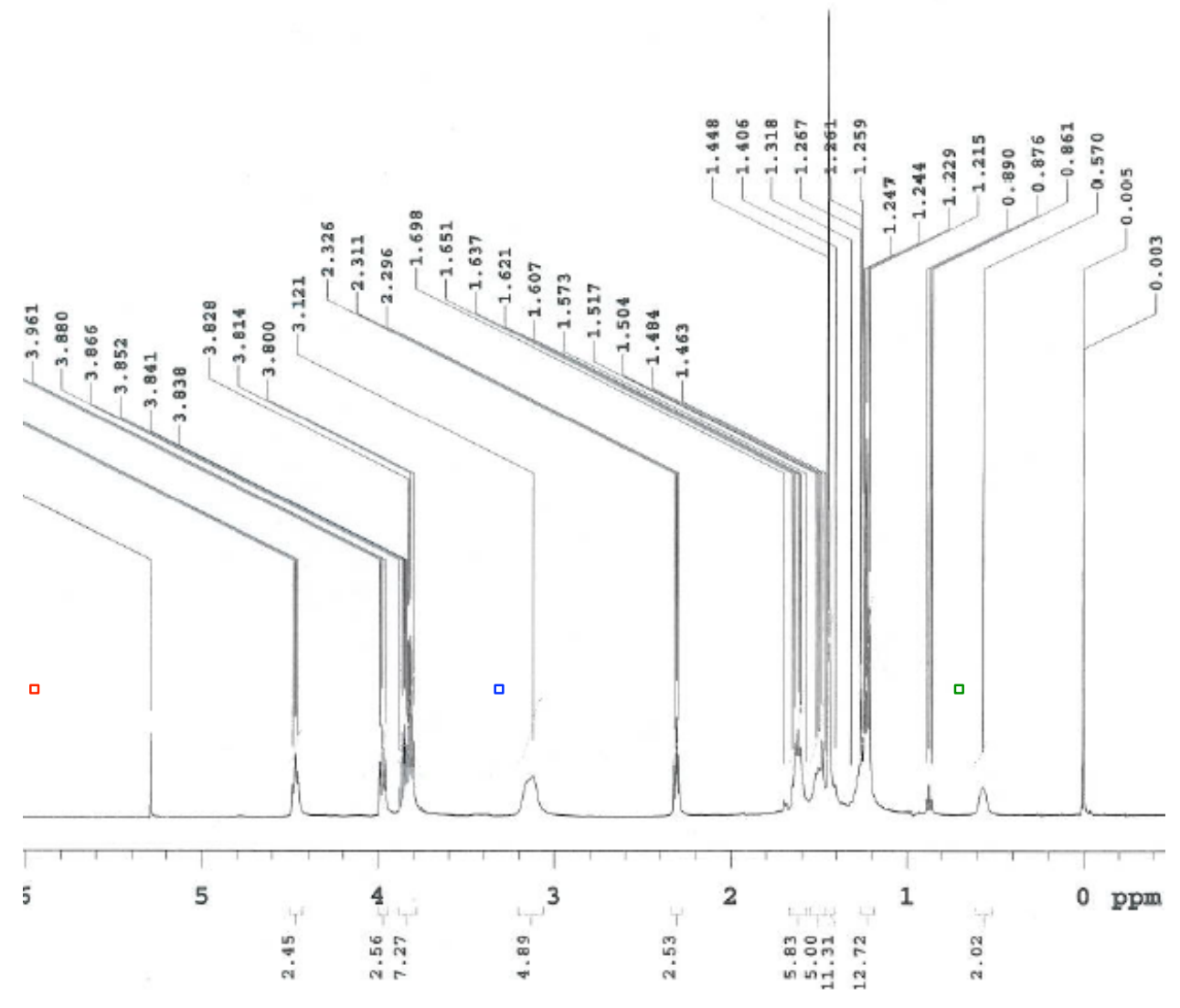

Figure 3.6. ${ }^{1} \mathrm{H} \mathrm{NMR}$ spectrum $\left(\mathrm{CDCl}_{3}\right)$ of the hydrosilylated ester 14.3. 
Table 3.1. Hydrosilylation of allylamine with methyldiethoxysilane in the presence of various catalysts. $^{24}$

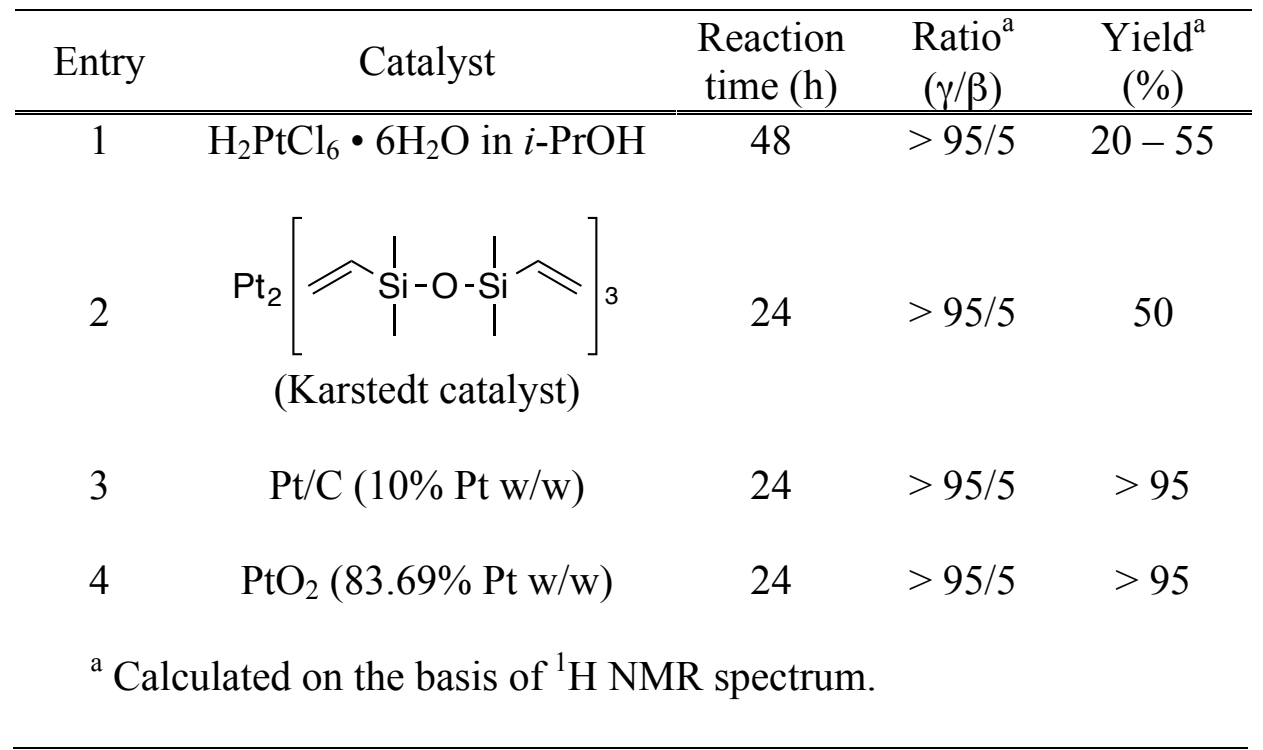

We now turned our attention to studying how the functionality around the carbonyl and the heterocyclic ring size affected the rate of cyclization. To do this, we prepared a panel of amino carbonyl substrates to determine factors that would minimize the rate of cyclization at $37^{\circ} \mathrm{C}$ while allowing cyclization to proceed at higher temperature (Figure 3.6).

\subsubsection{Cyclization Panel (Figure 3.7)}

All substrates were prepared to contain an $\mathrm{N}$-allyl moiety for convenient functionalization, such as hydrosilylation or thiol incorporation, for eventual covalent attachment to various supports. To quantify release of the 'payload' (e.g., HO-R' in 6-8 and 10-11, $\mathrm{H}_{2} \mathrm{~N}-\mathrm{R}^{\prime}$ in 9) on heating, $\mathrm{R}^{\prime}$ was selected as a 9-substituted anthracene for ease of UV and fluorescent measurements. When using the commercially available 9anthracenemethanol, we found that the acidic Boc-deprotection step would also 
hydrolyze the fluorophore. This issue was circumvented by synthesizing the homologous 2-(9-anthracenyl)ethanol. In addition to the cyclization ring size (italics, Figure 3.6), we<smiles>C=CCNCCCCC(=O)OCC</smiles>

6.1: $\mathrm{n}=1$ (5-ring)

6.2: $\mathrm{n}=2$ (6-ring)

6.3: $\mathrm{n}=3(7$-ring)<smiles>C=CCNCCNC(=O)OCC</smiles>

8: (5-ring)<smiles>C=CCNCCOC(=O)OCC</smiles>

7.1: $\mathrm{n}=1$ (5-ring)

7.2: $\mathrm{n}=2$ (6-ring)<smiles>C=CCNCCCC(=O)NP</smiles>

9: (5-ring)<smiles>[R7]OC(=O)[Y]CC(C)(C)CCNCC=C</smiles>

10: $\mathrm{Y}=\mathrm{CH}_{2}$ (7-ring)

11: $Y=O$ (7-ring)

Figure 3.7. Cyclization precursors $(\mathrm{R}=2$ (9-anthracenyl)ethyl). Ring size after intramolecular cyclization given in parentheses.

varied the carbonyl functionality to include ester, carbonate, carbamate and amide examples. The influence of gem-dimethylation, as in seven-membered ring precursors ester 10 and carbonate 11, also was examined as a means to enhance intramolecular cyclization via the Thorpe-Ingold effect. ${ }^{25}$

\subsubsection{Syntheses (Schemes 3.3-3.7)}

$N$-allylation ${ }^{26}$ of commercial Boc-protected amino acids $\mathbf{1 2}$ followed by carbodiimide-mediated esterification with 2-(9-anthracenyl)ethanol ${ }^{27}$ and Bocdeprotection furnished amino esters 6.1-3 (Figure 3.8, Scheme 3.3). 


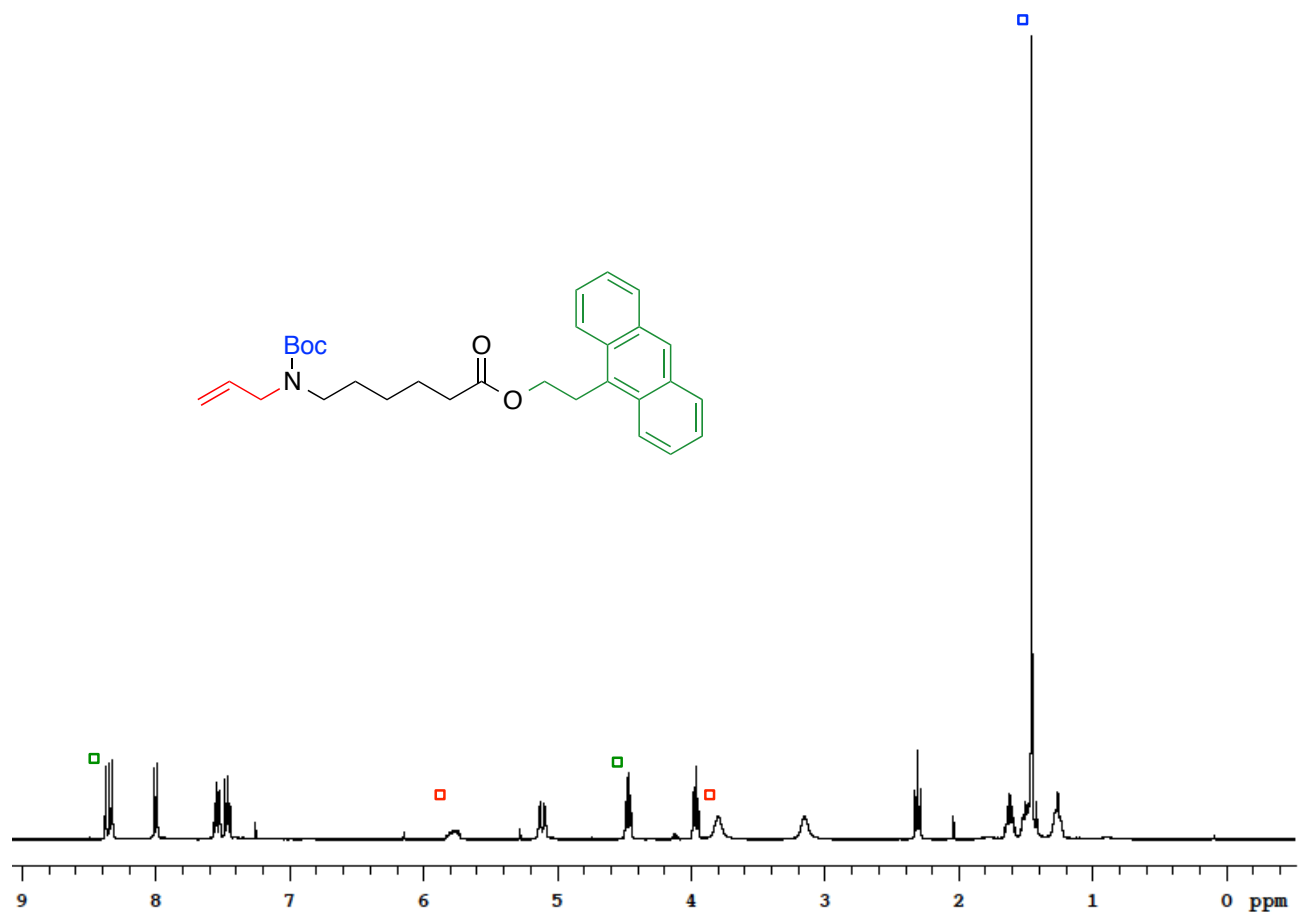

Figure 3.8. ${ }^{1} \mathrm{H}$ NMR $\left(\mathrm{CDCl}_{3}\right)$ of ester 14.3 showing successful Bocprotection, $N$-allylation and esterification with 2-(9-anthracenyl)ethanol.

Condensation of amino acid $\mathbf{1 3 . 1}$ with 2-(9-anthracenyl)ethanamine ${ }^{28}$ gave the amino amide substrate 9. In similar fashion, $N$-allylation of Boc-protected alcohols $\mathbf{1 5}$ (Scheme 3.4), after necessary silylation and desilylation steps, gave alcohols $\mathbf{1 6}$. The carbonate moiety was installed by first forming the corresponding acyl imidazole intermediates 17. Reaction with anthracenyl ethanol under basic conditions ${ }^{21}$ then gave amino carbonates 7.1-2. Though the ${ }^{1} \mathrm{H}$ NMR spectra were similar for these compounds, the ${ }^{13} \mathrm{C}$ NMR spectra allowed for confirmation of the functionality around the carbonyl (Figure 3.9). 


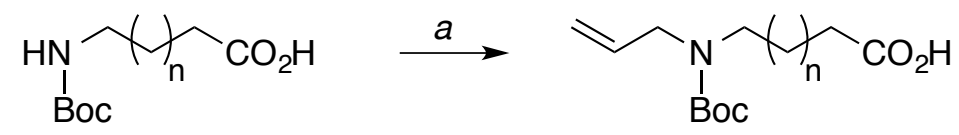

12.1-3

13.1: $\mathrm{n}=1$

13.2: $\mathrm{n}=2$

13.3: $\mathrm{n}=3$

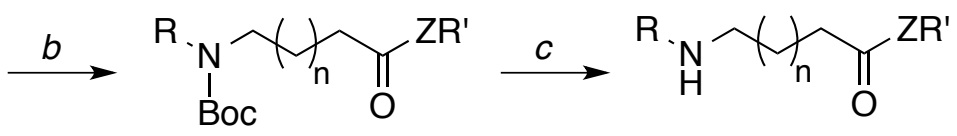

14.1-3: $R=$ allyl, $Z=O$

6.1: $\mathrm{n}=1, \mathrm{Z}=\mathrm{O}$

14.4: $\mathrm{R}=$ allyl, $\mathrm{Z}=\mathrm{NH}$

6.2: $n=2, Z=0$

$\mathrm{R}^{\prime}=$ 2-(9-anthracenyl)ethyl

6.3: $n=3, Z=0$

9: $n=1, Z=N H$

Scheme 3.3. Synthesis of amino esters 6 and amide 9.

Conditions: $a$. allyl bromide, $\mathrm{NaH}$, THF, $0{ }^{\circ} \mathrm{C}$ to $\mathrm{rt}, 81-$

$98 \%$; b. 2-(9-anthracenyl)ethanol (or 2-(9anthracenyl)ethanamine), DIC, cat. DMAP, $\mathrm{CH}_{2} \mathrm{Cl}_{2}, 12 \mathrm{~h}$, $59-97 \%$; c. TFA, $\mathrm{CH}_{2} \mathrm{Cl}_{2}, 0{ }^{\circ} \mathrm{C}, 1 \mathrm{~h}, 72-100 \%$

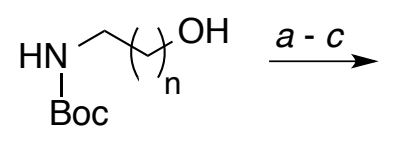

15.1-2

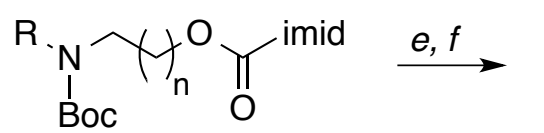

\section{1-2}

$\mathrm{R}=$ allyl

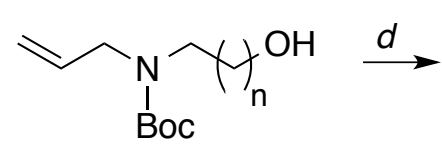

16.1-2<smiles>[R]NCC1CCCC1OC(=O)O[Tl]</smiles>

7.1: $\mathrm{n}=1$

7.2: $\mathrm{n}=2$

R': 2-(9-anthracenyl)ethyl

Scheme 3.4. Synthesis of amino carbonates 7. Conditions:

a. TBSCl, $\mathrm{Et}_{3} \mathrm{~N}$, imidazole, $\mathrm{CH}_{2} \mathrm{Cl}_{2}, 0{ }^{\circ} \mathrm{C}$ to $\mathrm{rt}, 95-98 \% ; b$. allyl bromide, $\mathrm{NaH}, \mathrm{THF}, 0{ }^{\circ} \mathrm{C}$ to $\mathrm{rt}, 77-84 \%$; $c$. TBAF, THF, $0{ }^{\circ} \mathrm{C}$ to $\mathrm{rt}, 90-95 \%$; $d$ (imid $)_{2} \mathrm{C}=\mathrm{O},(i \text {-Pr })_{2} \mathrm{NEt}$, $\mathrm{CH}_{2} \mathrm{Cl}_{2}, 0{ }^{\circ} \mathrm{C}$ to $\mathrm{rt}, 93-95 \%$; e. 2-(9-anthracenyl)ethanol, $\mathrm{KOH}$, toluene, $60{ }^{\circ} \mathrm{C}, 34-45 \%$; $f$. TFA, $\mathrm{CH}_{2} \mathrm{Cl}_{2}, 0{ }^{\circ} \mathrm{C}, 1 \mathrm{~h}$, 96-100\%. 


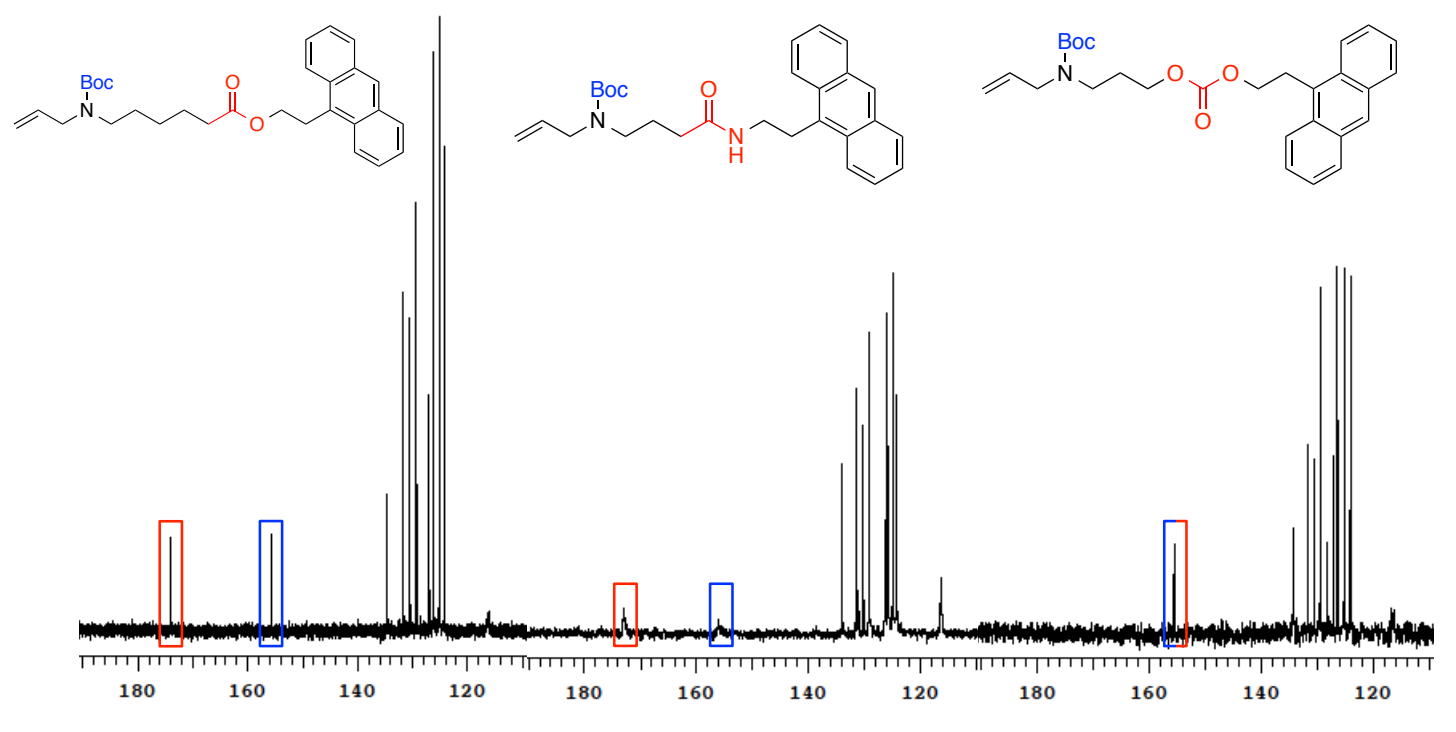

Figure 3.9. ${ }^{13} \mathrm{C} \mathrm{NMR}\left(\mathrm{CDCl}_{3}\right)$ spectra of ester $\mathbf{1 4 . 3}(155.7$ and $173.9 \mathrm{ppm})$, amide 14.4 (156.3 and 173.1) and Boc-protected carbonate 7.2 (155.5 and 155.6 ppm) emphasizing the effects of carbonyl functionality on ${ }^{13} \mathrm{C}$ chemical shift and confirming molecular structure.

Chloroformate acylation of $N$-allyl- $N$-Boc-ethanediamine provided a convenient route for synthesis of the amino carbamate substrate $\mathbf{8}$ (Scheme 3.5). 


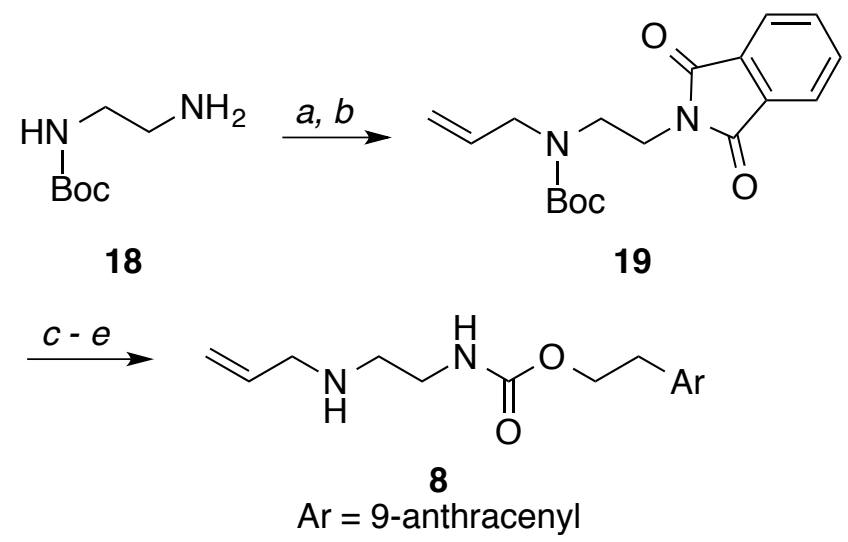

Scheme 3.5. Synthesis of amino carbamate 8. Conditions:

a. phthalic anhydride, toluene, reflux, $77 \%$; $b$. allyl bromide, $\mathrm{NaH}$, THF, $0{ }^{\circ} \mathrm{C}$ to $\mathrm{rt}, 58 \%$; $c$. hydrazine monohydrate, 2:1 $\mathrm{CH}_{2} \mathrm{Cl}_{2}: \mathrm{EtOH}, 0{ }^{\circ} \mathrm{C}$ to $\mathrm{rt}, 92 \%$; $d$. $\mathrm{ArCH}_{2} \mathrm{CH}_{2} \mathrm{OC}(\mathrm{O}) \mathrm{Cl}, \mathrm{Et}_{3} \mathrm{~N}, \mathrm{CH}_{2} \mathrm{Cl}_{2}, 0{ }^{\circ} \mathrm{C}$ to rt, $12 \mathrm{~h}, 41 \%$; $e$. TFA, $\mathrm{CH}_{2} \mathrm{Cl}_{2}, 0{ }^{\circ} \mathrm{C}, 1 \mathrm{~h}, 88 \%$.

The gem-dimethyl amino ester $\mathbf{1 0}$ was prepared by applying the above-described three-step sequence of $N$-allylation, esterification and Boc-deprotection to commercially available amino acid 20 (Scheme 3.6). Synthesis of gem-dimethyl amino carbonate 11 followed from bis- $\alpha$-methylation ${ }^{15}$ of the ester derived from amino acid 16.1 (Scheme 3.7). Mono-methylation could be identified by thin layer chromatography (TLC) since the $\mathrm{R}_{f}$ of bis- $\alpha$-methylated product was 0.53 and the mono- $\alpha-$ methylated product had an $\mathrm{R}_{f}$ of 0.50 . Incomplete $\alpha$-methylation could also be confirmed by the integration of the ${ }^{1} \mathrm{H}$ NMR spectrum (Figure 3.10). Subsequent chemoselective ester reduction ${ }^{29}$ and carbonate formation using the established method gave desired substrate $\mathbf{1 1 .}$ 


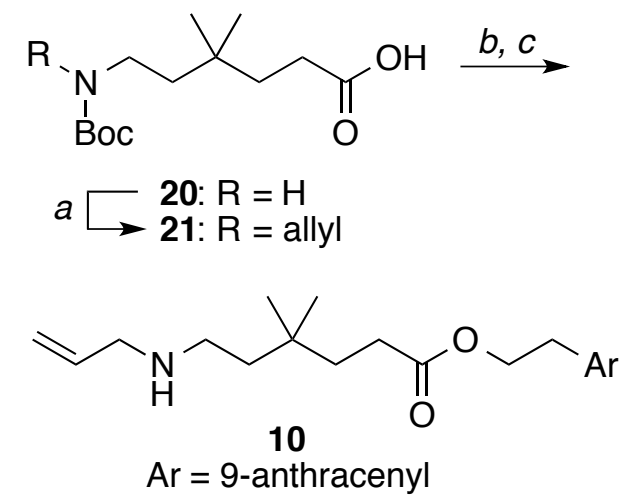

Scheme 3.6. Synthesis of gem-dimethylated ester 10. Conditions: $a$. allyl bromide, NaH, THF, $0{ }^{\circ} \mathrm{C}$ to $\mathrm{rt}, 68 \%$; $b$. 2-(9-anthracenyl)ethanol, DIC, cat. DMAP, $\mathrm{CH}_{2} \mathrm{Cl}_{2}, 12 \mathrm{~h}$, $87 \%$; c. TFA, $\mathrm{CH}_{2} \mathrm{Cl}_{2}, 0{ }^{\circ} \mathrm{C}, 1 \mathrm{~h}, 85 \%$.

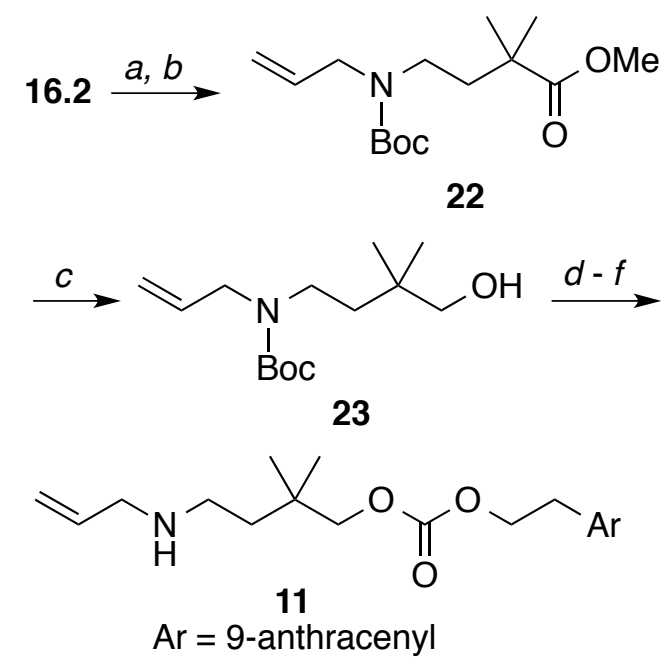

Scheme 3.7. Synthesis of gem-dimethylated carbonate 11. Conditions: a. MeOH, DIC, cat. DMAP, rt, 80\%; $b$. LiHMDS, MeI, THF, $-78{ }^{\circ} \mathrm{C}$ to rt, $67 \%$; c. $\mathrm{LiBH}_{4}, \mathrm{THF}, 0$ ${ }^{\circ} \mathrm{C}$ to $\mathrm{rt}, 73 \%$; $d$. (imid) ${ }_{2} \mathrm{C}=\mathrm{O},(i \text {-Pr })_{2} \mathrm{NEt}, \mathrm{CH}_{2} \mathrm{Cl}_{2}, 0{ }^{\circ} \mathrm{C}$ to rt, $92 \%$; e. 2-(9-anthracenyl)ethanol, $\mathrm{NaH}, \mathrm{THF},-5{ }^{\circ} \mathrm{C}$ to rt, $48 \% ; f$. TFA, $\mathrm{CH}_{2} \mathrm{Cl}_{2}, 0{ }^{\circ} \mathrm{C}, 1 \mathrm{~h}, 92 \%$. 


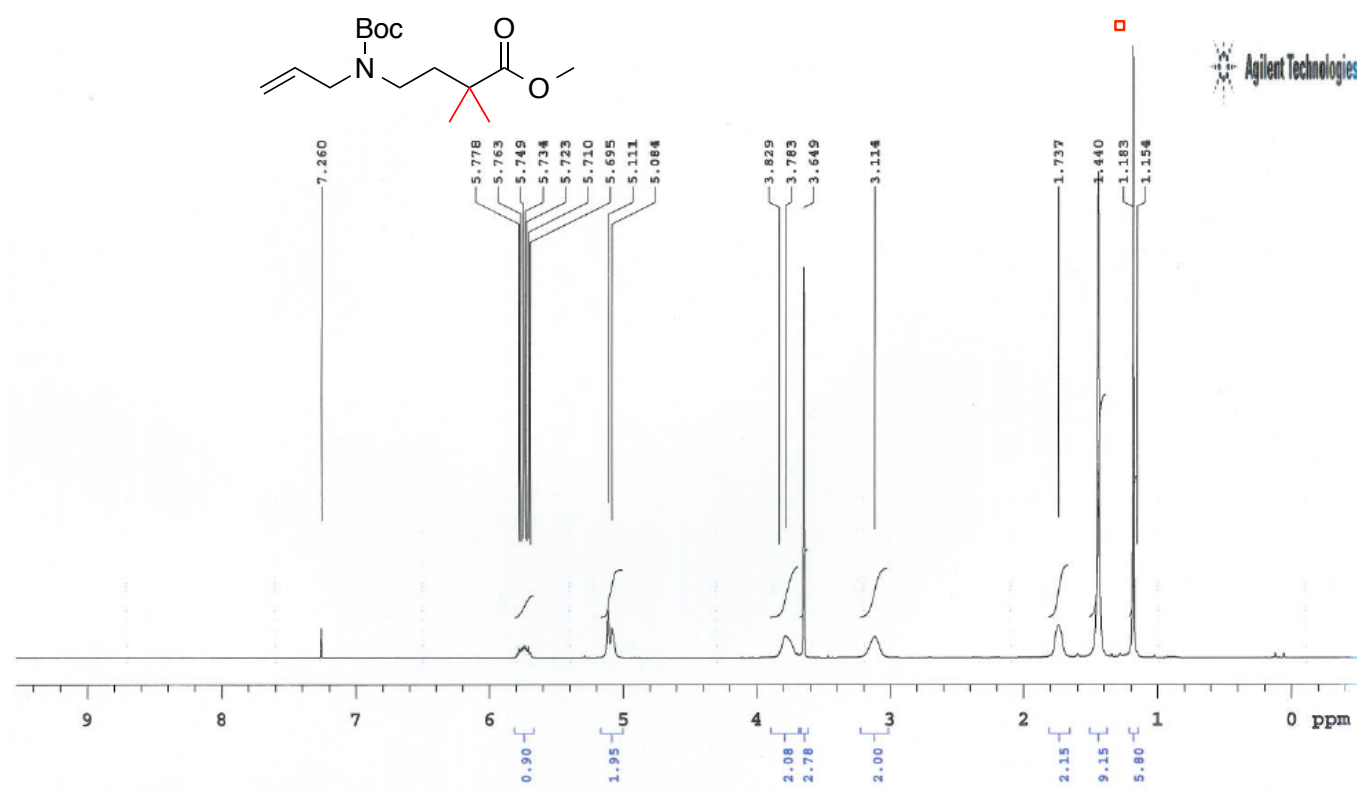

Figure 3.10. ${ }^{1} \mathrm{H}$ NMR spectrum $\left(\mathrm{CDCl}_{3}\right)$ of ester 22 after complete bis- $\alpha-$ methylation.

\subsection{CYCLIZATION STUDY}

To determine the release rates of the 9-substituted anthracenes (e.g., 25, Scheme 3.8) from the panel of amino-carbonyl substrates, dilute methanol solutions of each compound were heated at $55^{\circ} \mathrm{C}$. Aliquots taken at various times were analyzed by normal phase HPLC for the appearance of $\mathbf{2 5}$ or $\mathbf{2 6}$. Amino-carbamate $\mathbf{8}$ and aminoamide 9 were unreactive under the conditions and did not release detectable $\mathbf{2 5}$ or $\mathbf{2 6}$, respectively (Scheme 3.8). As could be expected, anthracene alcohol 25 was released from the amino-ester and amino-carbonate progenitors of the 5- and 6-membered lactams (24, $\left.\mathrm{Y}=\mathrm{CH}_{2}, \mathrm{n}=0,1\right)$ and oxazolidinones $(\mathbf{2 4}, \mathrm{Y}=\mathrm{O}, \mathrm{n}=0,1)$ at rates faster than from the progenitors of corresponding 7-membered rings (Figure 3.11 and Table 3.2). Control experiments indicated that ester or carbonate methanolysis did not occur under the conditions to yield 25. Whereas dilute conditions were used to reduce the incidence of 
intermolecular reactions, these cannot be ruled out as a possible source of $\mathbf{2 5}$ particularly with the substrates that lead to 7 -membered rings. In regards to potential heat-triggered release applications, the high thermal responsiveness of esters 6.1-2 and carbonates 7.1-2 make these constructs ideal for applications involving thermally sensitive substrates, such as in cell studies involving triggered release at or below $37^{\circ} \mathrm{C}$.

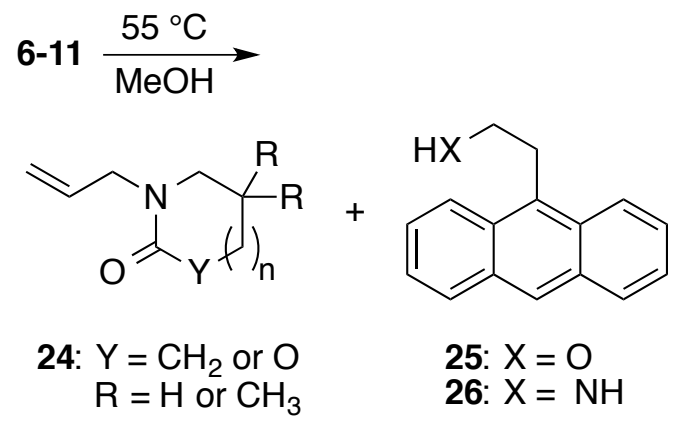

Scheme 3.8. Heat-induced release of anthracene probes. 


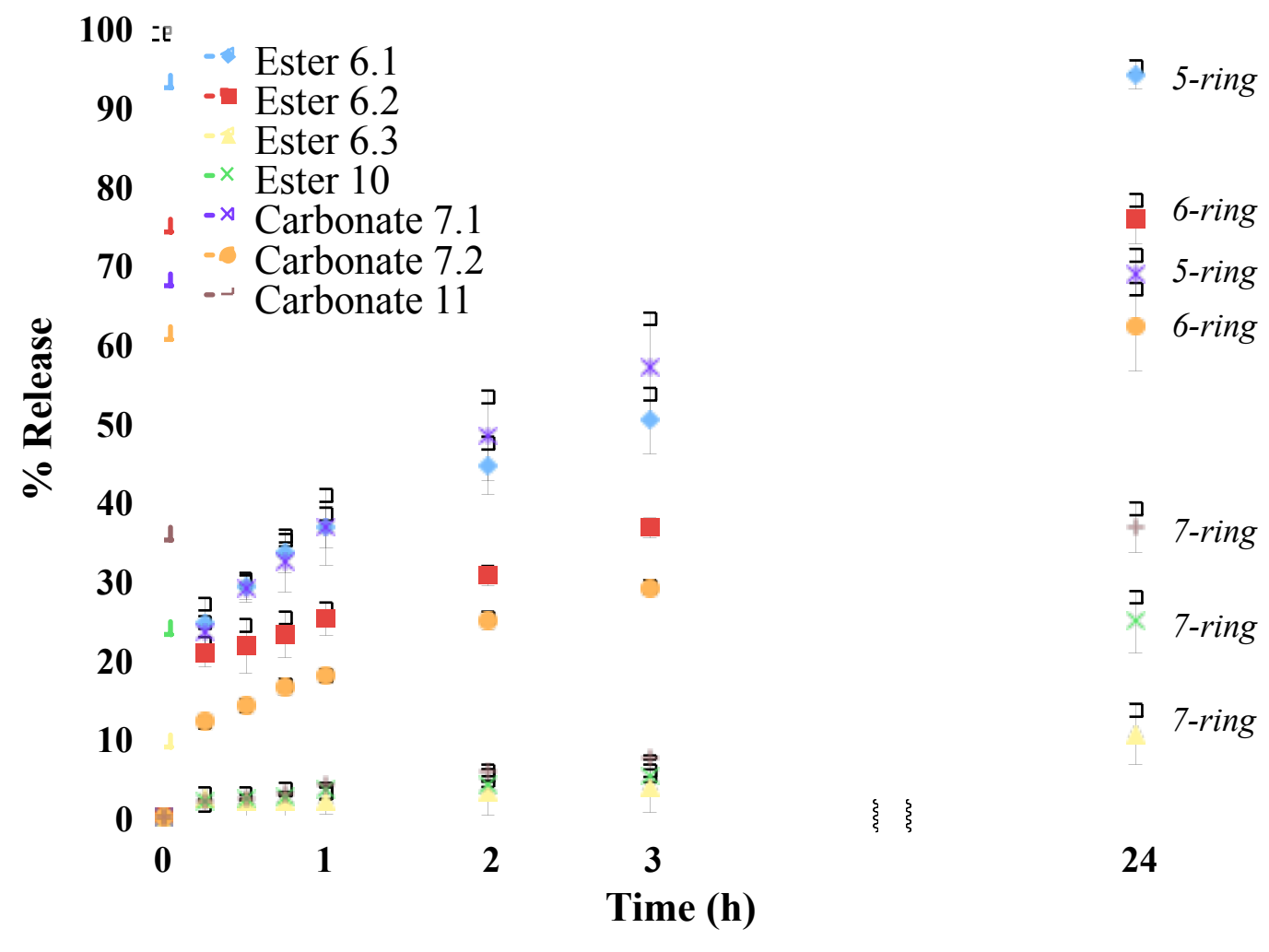

Figure 3.11. Percent release of $\mathbf{2 5}$ from indicated substrates in $\mathrm{MeOH}$ at $55^{\circ} \mathrm{C}$. Shown are the standard deviations from the mean $(n=3) .{ }^{30}$ 
Table 3.2. Heat-induced release of alcohol 25 (Scheme 3.8 and Figure 3.7). 30

\begin{tabular}{|c|c|c|c|c|c|c|c|c|c|c|}
\hline \multirow{2}{*}{ Entry } & $\begin{array}{c}\text { Substrate } \\
\text { (ring size) }\end{array}$ & \multicolumn{7}{|c|}{ \% release after heating $\left(\mathbf{5 5}{ }^{\circ} \mathbf{C}\right)$ at time (h) } & \multirow{2}{*}{ \% release } \\
at rt, 3.5h
\end{tabular}


In contrast, the resistance to cyclization of the 7-ring progenitor ester 6.3 is promising for higher temperature applications (Figure 3.11 and Table 3.2), although the overall release of 25 from 6.3 on heating at $55{ }^{\circ} \mathrm{C}$ was low. Comparison of amino-esters 6.3 and $\mathbf{1 0}$ shows that gem-dimethylation more than doubled the heat-induced release of anthracene 25 after heating $24 \mathrm{~h}$. The gem-dimethyl amino-carbonate $\mathbf{1 1}$ provided an even higher thermal response than gem-dimethyl ester $\mathbf{1 0}$ with a nearly $40 \%$ release of payload at $55{ }^{\circ} \mathrm{C}$ over $24 \mathrm{~h}$. Furthermore, the gem-dimethyl 7-ring carbonate motif resists cyclization at lower temperatures. Incubation of 11 at $37{ }^{\circ} \mathrm{C}$ for $24 \mathrm{~h}$ resulted in only $5.7 \% \pm 0.7 \%$ release of $\mathbf{2 5}$.

We also performed an NMR experiment to prove that the release $\mathbf{2 5}$ was due to intramolecular cyclization and not intermolecular polymerization. ${ }^{1} \mathrm{H}$ NMR spectra allowed us to not only see $\mathbf{2 5}$, but also the subsequent lactam or oxazolidinone. Samples of 6.1 and 7.1 were placed into NMR tubes with toluene- $\mathrm{d}_{8}$ and heated. The resulting ${ }^{1} \mathrm{H}$ NMR spectra (Figure 3.12) showed the formation of cyclized rings that were in agreement with literature while also showing the liberated alcohol $25^{31}$ No polymerization was observed in the spectra. 

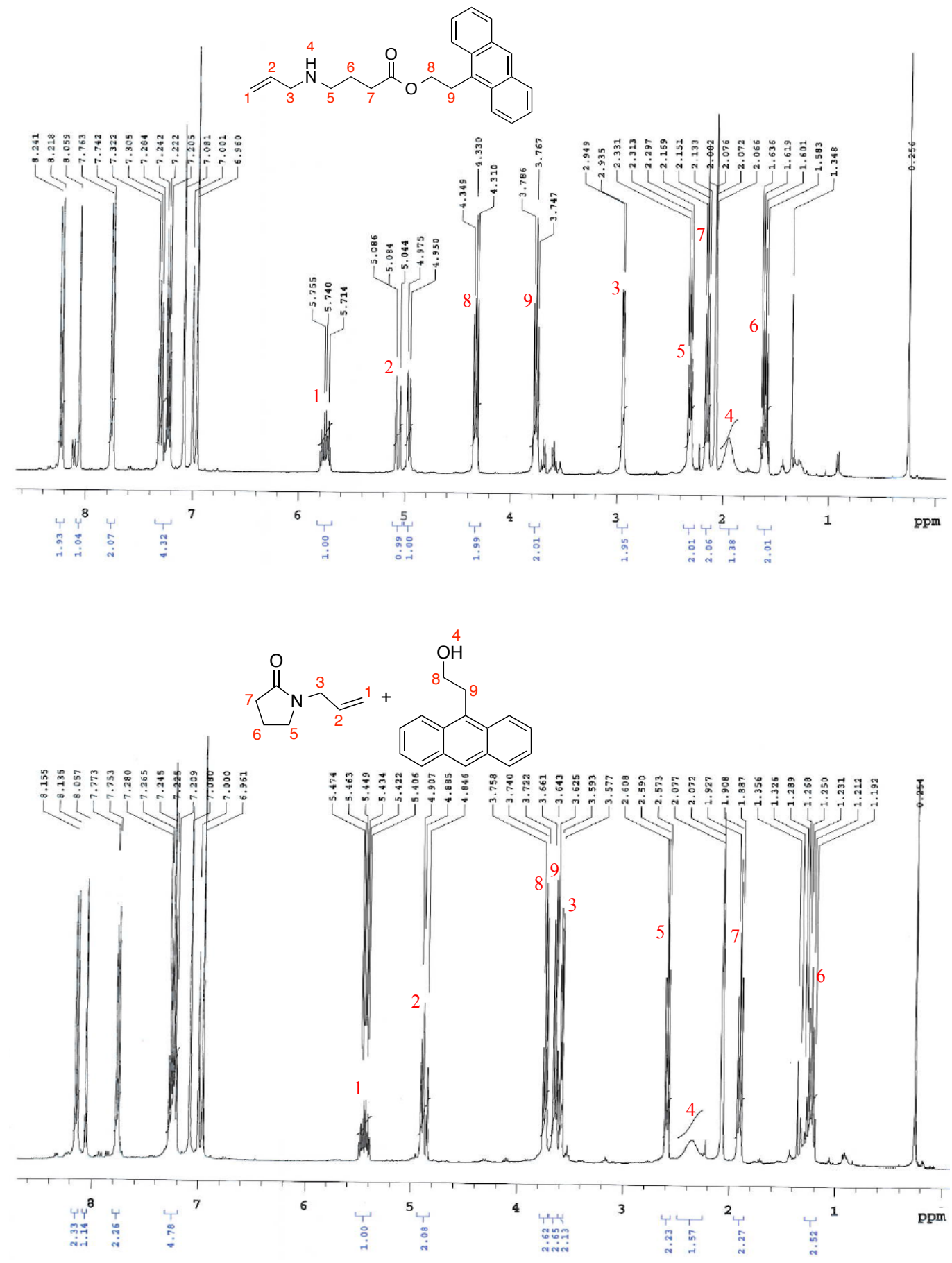

Figure 3.12. ${ }^{1} \mathrm{H}$ NMR (toluene- $\mathrm{d}_{6}$ ) spectra of ester $\mathbf{6 . 1}$ before heating (top spectrum) and after thermally-induced intramolecular cyclization (bottom spectrum). 


\subsection{MICROCHANNEL APPLICATION}

With these cyclization trends established, we set out to incorporate an aminooxy group into the linker chain. We felt that the addition of an aminooxy group would allow us to design a pre-concentrator for the chemoselective capture of carbonyl metabolites from an aqueous solution. After concentration of the aldehydes and ketones, the oxime ether adducts would be released from the linker with mild heating.

We chose to use a poly(dimethylsiloxane) (PDMS) microchannel (Figure 3.13) as a substrate from which we could covalently attach our linker and release its cargo upon heating. Microchannels fabricated using PDMS have shown great utility for molecular and cellular separations due to their extremely high surface to volume ratio. ${ }^{32}$

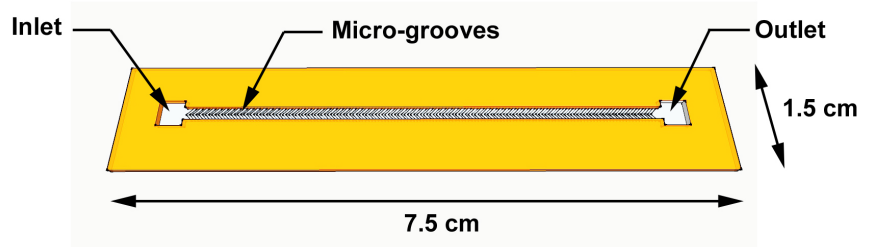

Figure 3.13. Schematic of poly(dimethylsiloxane) microchannel.

Further, small PDMS channel heights and incorporation of features, such as microfabricated grooves, enhance fluid mixing within the channel to increase interactions of functionalized surfaces with target molecules flowing through the channel. ${ }^{33}$

The $N$-allyl moiety of ammonium aminooxy ester 27 (Scheme 3.9), based on the ester motif of 6.2, was hydrosilylated ${ }^{24}$ to attach a terminal triethoxysilane group. The ammonium aminooxy substructure of ester 27 follows from our previously published 
work on ammonium aminooxy reagents for capture of aldehyde and ketone metabolites from aqueous cell extracts. ${ }^{34,35}$ Subsequent condensation ${ }^{36,37}$ with the PDMS microchannel (Figure 3.13) covalently attached 28 to afford an aminooxy-functionalized microchannel specific for reaction with aldehydes and ketones. Injection of an<smiles>[Z7]CCC(=O)OCC[N+](C)(C)CCON</smiles>

1. $(\mathrm{EtO})_{3} \mathrm{SiH}$, cat. $\mathrm{PtO}_{2}, 90^{\circ} \mathrm{C}$<smiles>CCO[SiH2]CCCN(CCCCC(=O)OCC[N+](C)(C)CCON)C(=O)OCc1ccccc1</smiles>

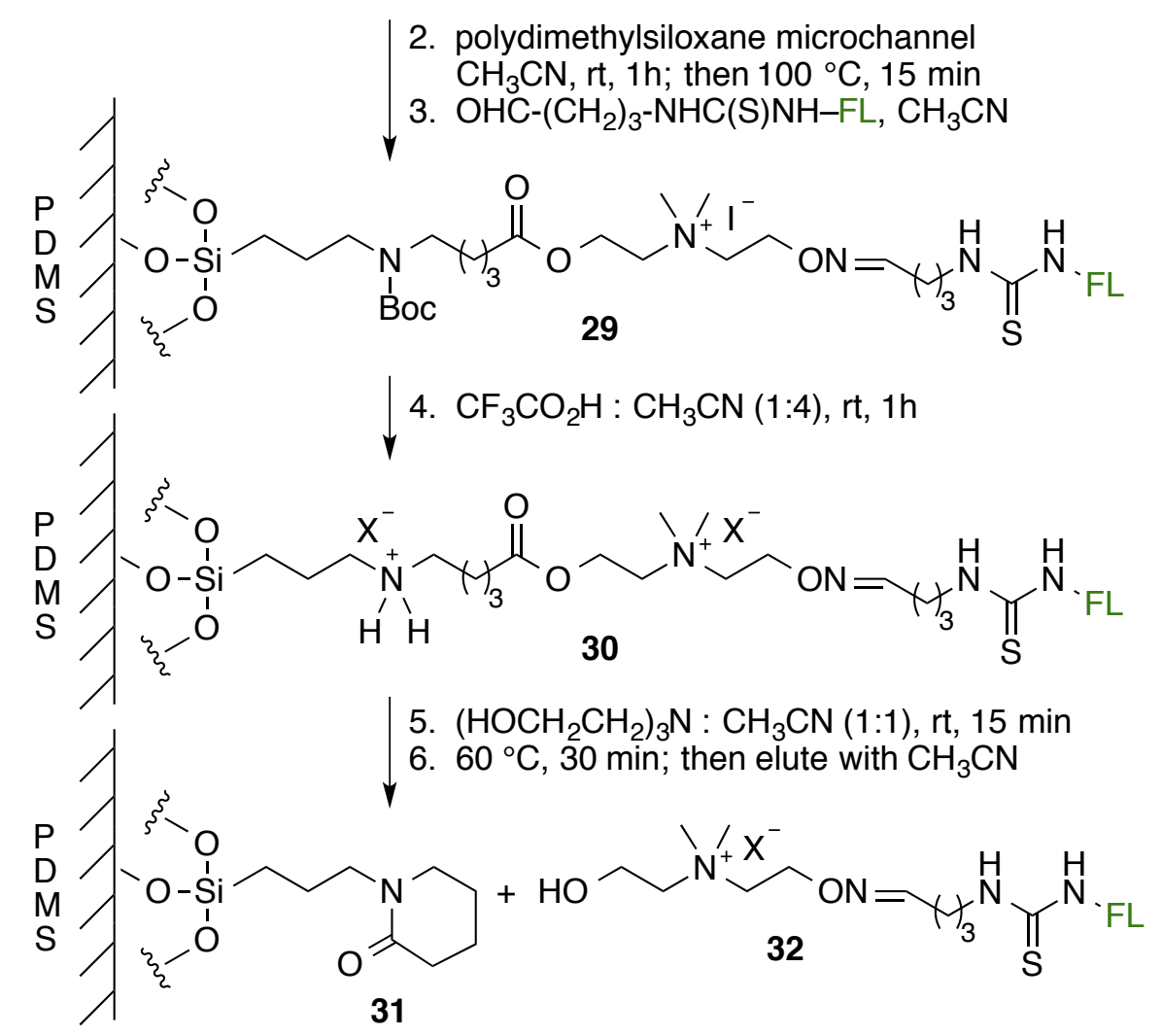

Scheme 3.9. Poly(dimethylsiloxane) microchannel functionalization and heat-induced release study $\left(\mathrm{FL}=\right.$ fluorescein, $\left.\mathrm{X}^{-}=\mathrm{CF}_{3} \mathrm{CO}_{2}^{-}\right)$. 
acetonitrile solution containing a fluorescent aldehyde probe, FITC (fluorescein isothiocyanate) reacted with a 4 -aminobutanal equivalent (Scheme 3.10), ${ }^{38}$ anchored the fluorophore to the surfaces of the microchannel via oxime ether formation. The
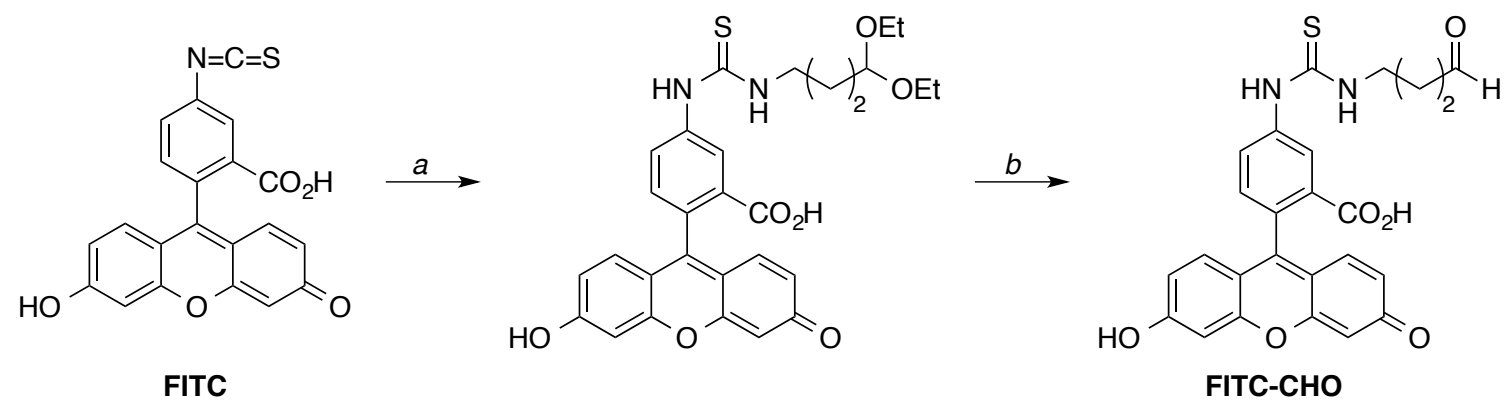

FITC-CHO

Scheme 3.10. Preparation of the aldehydic FITC moiety. Conditions: a. $\mathrm{H}_{2} \mathrm{~N}-\left(\mathrm{CH}_{2}\right)_{3}-\mathrm{CH}(\mathrm{OEt})_{2}, 94 \% ; b .30 \%$ aq. $\mathrm{AcOH}, 50 \%{ }^{38}$

quaternary ammonium salt of $\mathbf{2 8}$ ensures availability of the aminooxy moiety for reaction with aldehydes since charged polar groups are repelled by the hydrophobic PDMS matrix. The capture and covalent attachment of the FITC-derived fluorophore to the microchannel was verified using fluorescent microscopy (Figure 3.14a). Bocdeprotection using trifluoroacetic acid afforded trifluoroacetate salt 30. Concerned that loss of captured aldehyde probe may occur at this stage, we verified that no fluorescent probe was released prior to neutralization and thermal triggering. Indeed, no fluorescence was observed in the microchannel effluent after heating $\mathbf{3 0}$ at $60{ }^{\circ} \mathrm{C}$ for 30 min. Injection of a dilute solution of triethanolamine into the microchannel neutralized the linker. After subsequent rinsing $\left(\mathrm{CH}_{3} \mathrm{CN}\right)$ of the microchannel, we were gratified to observe that mild heating $\left(60^{\circ} \mathrm{C}, 30 \mathrm{~min}\right)$ promoted the intramolecular cyclization to release alcohol 32. As can be seen in Figure 3.14b, nearly complete release of the bound 
fluorophore was achieved. Further utilization of this release mechanism in microchannel studies of cell metabolites requiring hydroxide-free cleavage conditions are ongoing.

(a)

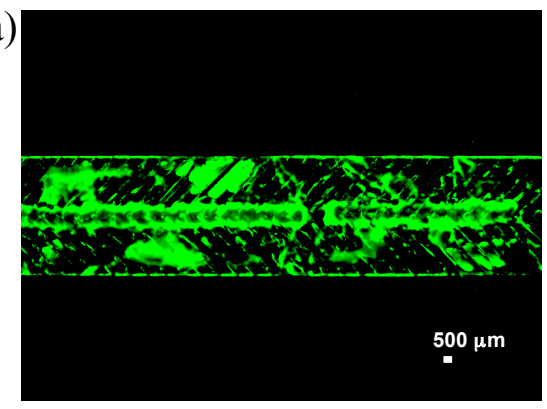

(b)

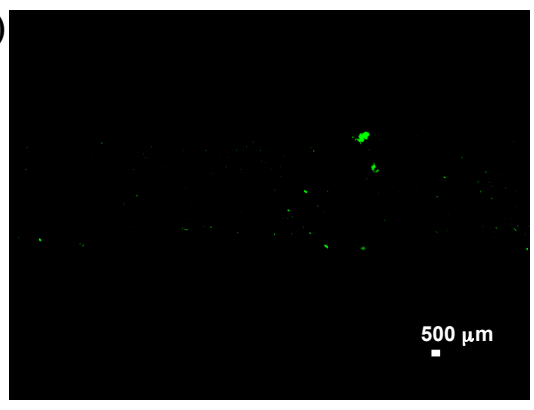

Figure 3.14. Fluorescence microscopy image of (a) FITCderived substrate 17 within the microchannel and (b) same field of view showing microchannel fluorescence after heating neutralized 18.

\subsection{CONCLUSION}

In summary, a brief study on the cyclization of amino esters and amino carbonates as a mode for substrate release revealed that both ring size and carbonyl functionality significantly influence the release rate. The process is facile at mild temperatures in cases where 5- and 6-membered rings are formed, even for non-phenolic alcohols as illustrated in a PDMS microchannel application. In contrast, an amino carbonate motif leading to a seven-membered ring appears well suited for applications requiring thermal 
stability at $37{ }^{\circ} \mathrm{C}$ while still releasing the alcohol substrate at slightly elevated temperatures. This particular secondary amine-carbonate structural combination may be a promising linker for drug delivery vehicles relying on the generation of local hyperthermia. 
CHAPTER 4

ALTERNATING MAGNETIC FIELD-INDUCED

SUBSTRATE RELEASE FROM IRON OXIDE NANOPARTICLES

\subsection{INTRODUCTION}

\subsection{RESULTS AND DISCUSSION}

4.2.1. Initial Loading

4.2.2. Formation of Covalent Bond to NP

4.2.3. Acidic Boc-deprotection on NPs

4.2.4. AMF vs. Non-AMF Induced Release: Hydrolysis

4.2.5. Suppression of Hydrolysis

4.2.6. Commencement of $\mathrm{SiO}_{2} @ \mathrm{Fe}_{3} \mathrm{O}_{4} \mathrm{NPs}$

4.2.7. Monodispersed $\mathrm{SiO}_{2} @ \mathrm{Fe}_{3} \mathrm{O}_{4}$ Nanoparticles

4.2.8. AMF-Induced Hydrolysis

4.3. CONCLUSION

4.4. FUTURE DIRECTIONS 


\subsection{INTRODUCTION}

An important attribute of a drug delivery system is regulated spatial and temporal drug release to minimize side effects as well as to improve the therapeutic efficacy of conventional pharmaceuticals. Iron oxide nanoparticles (NPs) possess many appropriate qualities that make them a viable choice for drug delivery formulations. Magnetite $\left(\mathrm{Fe}_{3} \mathrm{O}_{4}\right)$ NPs are biocompatible, ${ }^{1}$ have low cytotoxicity, ${ }^{2}$ and provide multiple means for surface modification. $\mathrm{Fe}_{3} \mathrm{O}_{4}$ NPs are set apart from other forms of iron oxide due to inherent paramagnetic or superparamagnetic properties. ${ }^{3}$ As a result of the magnetism, one highly-studied feature of $\mathrm{Fe}_{3} \mathrm{O}_{4} \mathrm{NPs}$ is their ability to heat a surrounding environment when exposed to an alternating magnetic field (AMF). Indeed, irradiation of $\mathrm{Fe}_{3} \mathrm{O}_{4} \mathrm{NPs}$ with an AMF to cause local hyperthermia (i.e., to raise target body tissue to $40-45^{\circ} \mathrm{C}$ ) is an emerging alternative treatment for several cancers, ${ }^{4}$ such as melanoma, ${ }^{5,6}$ glioblastoma, ${ }^{7,8}$ liver, ${ }^{9,10}$ or prostate ${ }^{11,12}$ cancer. Furthermore, when the $\mathrm{Fe}_{3} \mathrm{O}_{4}$ NPs are fitted with a drug, attached either through ionic interactions or via entrapment in a polymer gel coating, the drug may be guided to tumors using a magnet, a property first demonstrated by Meyers in $1963 .{ }^{13}$ Consequently, many $\mathrm{Fe}_{3} \mathrm{O}_{4}$ NP-based systems now are extensively functionalized for drug delivery applications. ${ }^{14,15}$

Some of the most common approaches for association of drug payloads with $\mathrm{Fe}_{3} \mathrm{O}_{4}$ NPs include encapsulation of $\mathrm{Fe}_{3} \mathrm{O}_{4}$ NPs within drug-loaded liposomal formulations, ${ }^{16,17}$ addition and subsequent functionalization of mesoporous silica shells ${ }^{18,19,20}$ and the use of polymer coatings to surround the $\mathrm{Fe}_{3} \mathrm{O}_{4}$ NPs (Figure 4.1). ${ }^{21,22}$ As the NPs reach 


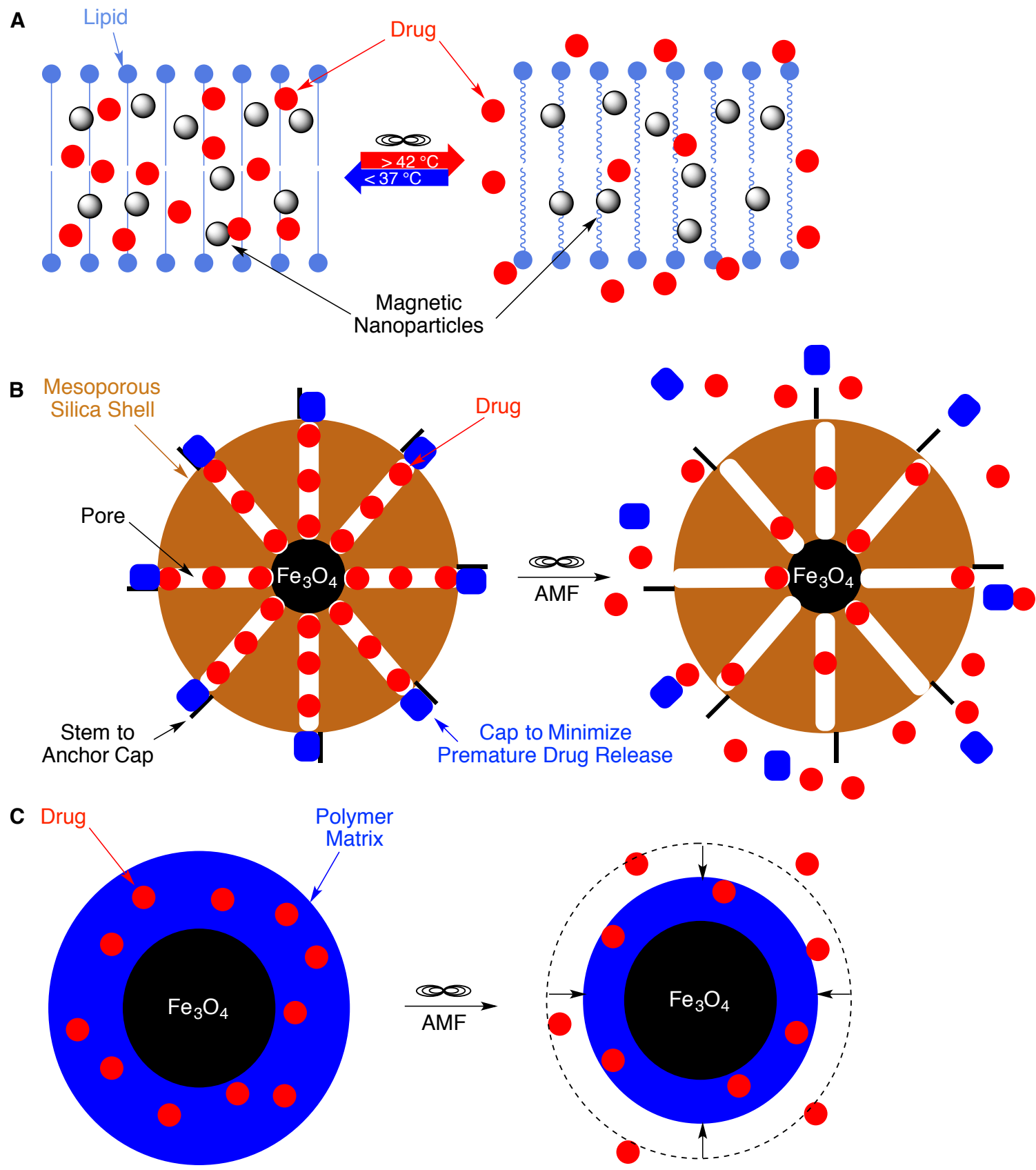

Figure 4.1. (a) Liposomal, (b) mesoporous silica and (c) polymeric iron oxide drug delivery platforms that use AMF-induced heat as a mechanism for controlled drug relesase.

a target (e.g., cancerous) tissue, a release mechanism is initiated so that the drug payloads are available only to the target tissue. The most popular release mechanisms 
include the use of $\mathrm{pH}$-sensitive — typically acid-labile - triggers (Figure 4.2a), ${ }^{23}$ photolabile linkers (Figure 4.2b) ${ }^{24}$ and AMF irradiation (Figure 4.1). ${ }^{25,26}$

A
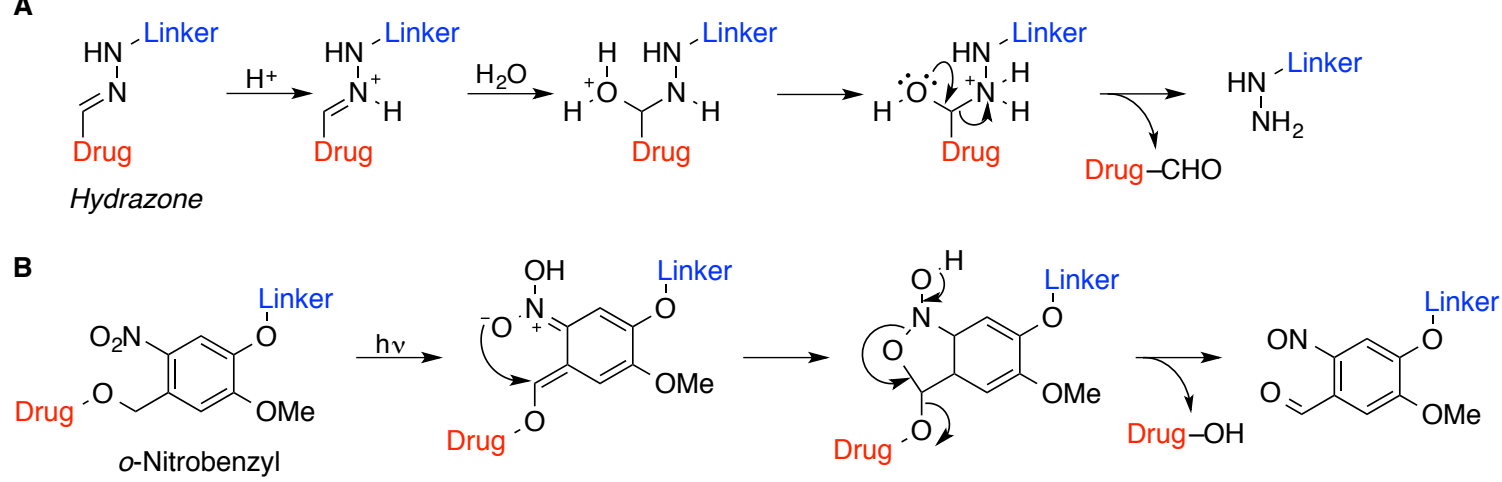

Figure 4.2. Drug release mechanism for (a) acid-labile hydrazone linkages and (b) photolabile $o$-nitrobenzyl linkages.

AMF-induced drug release has a distinct advantage over the $\mathrm{pH}$-mediated approaches in that the former exploits advantages associated with using a controllable, external stimulus whereas the later requires contact with an internal stimulus that cannot be easily controlled. AMF induction stimulates controlled release at a specific region and at a specific time without the need for a precise, and often unpredictable, internal environment. $^{27,28}$ Despite the advantage of controlled release using an AMF trigger, many such NP drug delivery systems still have a problem with premature drug release (i.e., payload leaching). In these instances, drugs are continuously released prior to application of the external stimulus. ${ }^{29,30}$ This problem is generally due to the inability of the payload to be covalently retained until the stimulus is applied. For example, Brule et al. ${ }^{31}$ have used AMF-induction to increase the porosity of drug-loaded, alginate microbeads containing $\mathrm{Fe}_{3} \mathrm{O}_{4}$ NPs. Whereas this elegant approach is quite functional for 
drug release, undesired diffusion of loaded drug from the microbeads is noted prior to AMF irradiation. Thus, the development of an AMF-responsive delivery system that covalently retains its payload until stimulated would constitute a desirable next-step and advance the evolution of nano-delivery systems.

\subsection{NANOPARTICLE FUNCTIONALIZATION AND RELEASE}

\subsubsection{Initial Loading}

Our initial concept was to covalently tether a drug to an $\mathrm{Fe}_{3} \mathrm{O}_{4} \mathrm{NP}$ via a thermally labile functionality using a linking chain that contains a secondary amine, such as shown by NP assembly 1 (Figure 4.3). With judicious choice in linker length, we expected that application of an AMF to raise the surrounding temperature would power an intramolecular cyclization via reaction of the amine with the carbonyl moiety, as in [2]. We aimed to achieve this goal using one of our thermally labile linkers (Figure 4.4) discussed in Chapter 3.

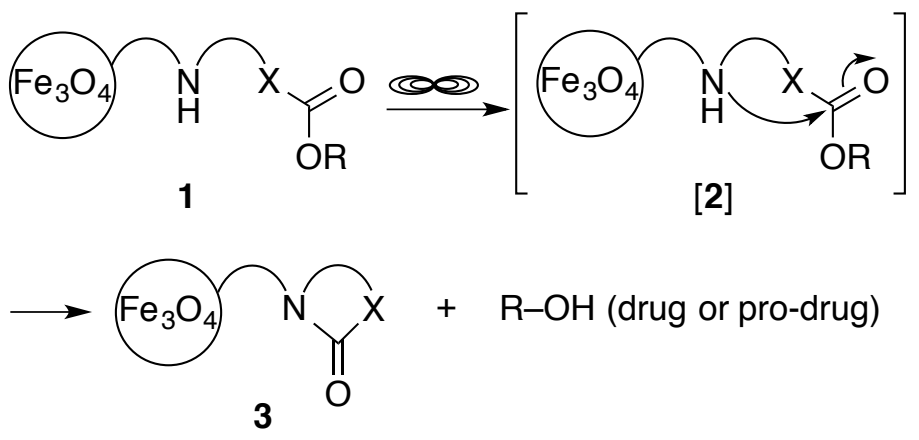

Figure 4.3. AMF-mediated intramolecular cyclization results in release of $\mathrm{ROH} . \mathrm{X}=\mathrm{CH}_{2}, \mathrm{O}, \mathrm{NH}$, or NR'. 
<smiles>C=CCNCCCCC(=O)OCC</smiles>

4.1: $\mathrm{n}=1$ (5-ring)

4.2: $\mathrm{n}=2$ (6-ring)

4.3: $\mathrm{n}=3$ (7-ring)<smiles>C=CCNCCNC(=O)OCC</smiles>

6: (5-ring)<smiles>C=CCNCC1CCCCC1OC(=O)OCC</smiles>

5.1: $\mathrm{n}=1$ (5-ring)

5.2: $\mathrm{n}=2$ (6-ring)<smiles>C=CCNCCCC(=O)NC</smiles>

7: (5-ring)<smiles>C=CCNCCC(C)(C)C[IH][Y](=O)OCC</smiles>

8: $\mathrm{Y}=\mathrm{CH}_{2}$ (7-ring)

9: $\mathrm{Y}=\mathrm{O} \quad$ (7-ring)

Figure 4.4. Cyclization precursors $(\mathrm{R}=2-(9-$ anthracenyl)ethyl). Ring size after intramolecular cyclization given in parentheses.

The first issue that we addressed was the formation of a covalent bond between $\mathrm{Fe}_{3} \mathrm{O}_{4}$ NPs and an organic linker. Despite the plethora of options for non-covalent bonding of $\mathrm{Fe}_{3} \mathrm{O}_{4} \mathrm{NPs}$, including but not limited to catechols, ${ }^{32}$ polysaccharides, ${ }^{33}$ liposomal,${ }^{34}$ polymers ${ }^{35}$ and cationic molecules,${ }^{36}$ there are limited options to effectively form a covalent bond to iron oxides. The three avenues to the formation of a covalent bond to $\mathrm{Fe}_{3} \mathrm{O}_{4}$ NPs (Figure 4.5) are 1) phosphonic acids/phosphonates ${ }^{37}$ 2) trichlorosilanes $^{38}$ and 3) alkoxysilanes. ${ }^{39,40}$ Out of these three, only the trichlorosilanes and the alkoxysilanes form a true covalent bond. It has been suggested that the phosphonic acids and phosphonates, similar to carboxylic acids, bind to the iron oxide surface through an interaction with a trivalent iron atom. ${ }^{41}$ The resulting interaction between the $\mathrm{Fe}_{3} \mathrm{O}_{4}$ and the carboxylic acid is relatively weak while the phosphonic acids and phosphonates form stronger interactions. ${ }^{42}$ The chlorosilanes, though quite effective 
at forming bonds to the $\mathrm{Fe}_{3} \mathrm{O}_{4} \mathrm{NP}$ surface, are quite hydrolytically unstable and are more difficult to handle when compared to the alkoxysilane. Due to the aforementioned reasons, the alkoxysilane is currently the primary method used to form a covalent bond to iron oxides. But formation of the $\mathrm{Fe}-\mathrm{O}-\mathrm{Si}$ bond is not as straightforward as the formation of the $\mathrm{Si}-\mathrm{O}-\mathrm{Si}$ bonds described in Chapter 3 between the linker and the PDMS microchannel. In Figure 4.6 Gelest, an industry leader in silanes and silicones, reports that the bonding effectiveness of an alkoxysilane with iron oxides is moderate. All Metal-O-Si bonds are inferior to the robust and hydrolytically stable $\mathrm{Si}-\mathrm{O}-\mathrm{Si}$ bonds. ${ }^{43}$

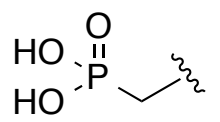

phosphonic acid/phosphonates

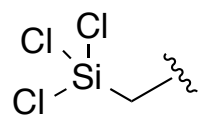

chlorosilanes

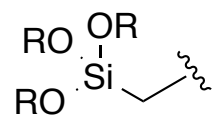

alkoxysilanes

Figure 4.5. Phosphonic acid/phosphonate, chlorosilane and alkoxysilane functionalities used to form moderate to strong bonds to $\mathrm{Fe}_{3} \mathrm{O}_{4}$ NPs. 


\begin{tabular}{|c|c|}
\hline \multicolumn{2}{|c|}{ Silane Bonding Effectiveness } \\
\hline & Substrate \\
\hline \multirow{6}{*}{$\begin{array}{c}\text { EXCELLENT } \\
\uparrow\end{array}$} & $\overline{\text { Silica }}$ \\
\hline & Quartz \\
\hline & Glass \\
\hline & Aluminum $(\mathrm{AlO}(\mathrm{OH}))$ \\
\hline & Alumino-silicates (e.g. clays) \\
\hline & Silicon \\
\hline \multirow{9}{*}{ GOOD } & Copper \\
\hline & Tin $(\mathrm{SnO})$ \\
\hline & Talc \\
\hline & Inorganic Oxides (e.g. $\mathrm{Fe}_{2} \mathrm{O}_{3}, \mathrm{TiO}_{2}, \mathrm{Cr}_{2} \mathrm{O}_{3}$ ) \\
\hline & Steel, Iron \\
\hline & Asbestos \\
\hline & Nickel \\
\hline & Zinc \\
\hline & Lead \\
\hline \multirow{2}{*}{ SLIGHT } & Marble, Chalk $\left(\mathrm{CaCO}_{3}\right)$ \\
\hline & Gypsum $\left(\mathrm{CaSO}_{-}\right)$ \\
\hline \multirow{3}{*}{ POOR } & Barytes $\left(\mathrm{BaSO}_{4}\right)$ \\
\hline & Graphite \\
\hline & Carbon Black \\
\hline
\end{tabular}

Figure 4.6. Effectiveness of silane bonding to various substrates. ${ }^{44}$

Using the same hydrosilylation protocol as described by Sabourault, ${ }^{75}$ we added a triethoxysilane to linker $\mathbf{4 . 3}$ to allow for loading onto $\mathrm{Fe}_{3} \mathrm{O}_{4} \mathrm{NPs}$. The NP loading was achieved following a procedure by Ma et $a l .{ }^{45}$ to afford the functionalized iron oxide NPs. There were two different means of loading alkoxysilylated molecules onto iron oxide NPs in literature. The two routes differ only after magnetically separating the NPs with the first route calling to dry the fuctionalized NPs under vacuum and the second route calling for the NPs to be dried by heating at $\sim 100{ }^{\circ} \mathrm{C}$ (Figure 4.7 , final bond formation/condensation step). The route described by Ma and coworkers called for the use of vacuum without heating. Abstaining from heating the NPs was attractive to us 
since our NPs were functionalized with a thermally labile linker. Since we wanted to induce the intramolecular cyclization using AMF, not conventional heat, using vacuum to induce the final condensation reaction was desirable.

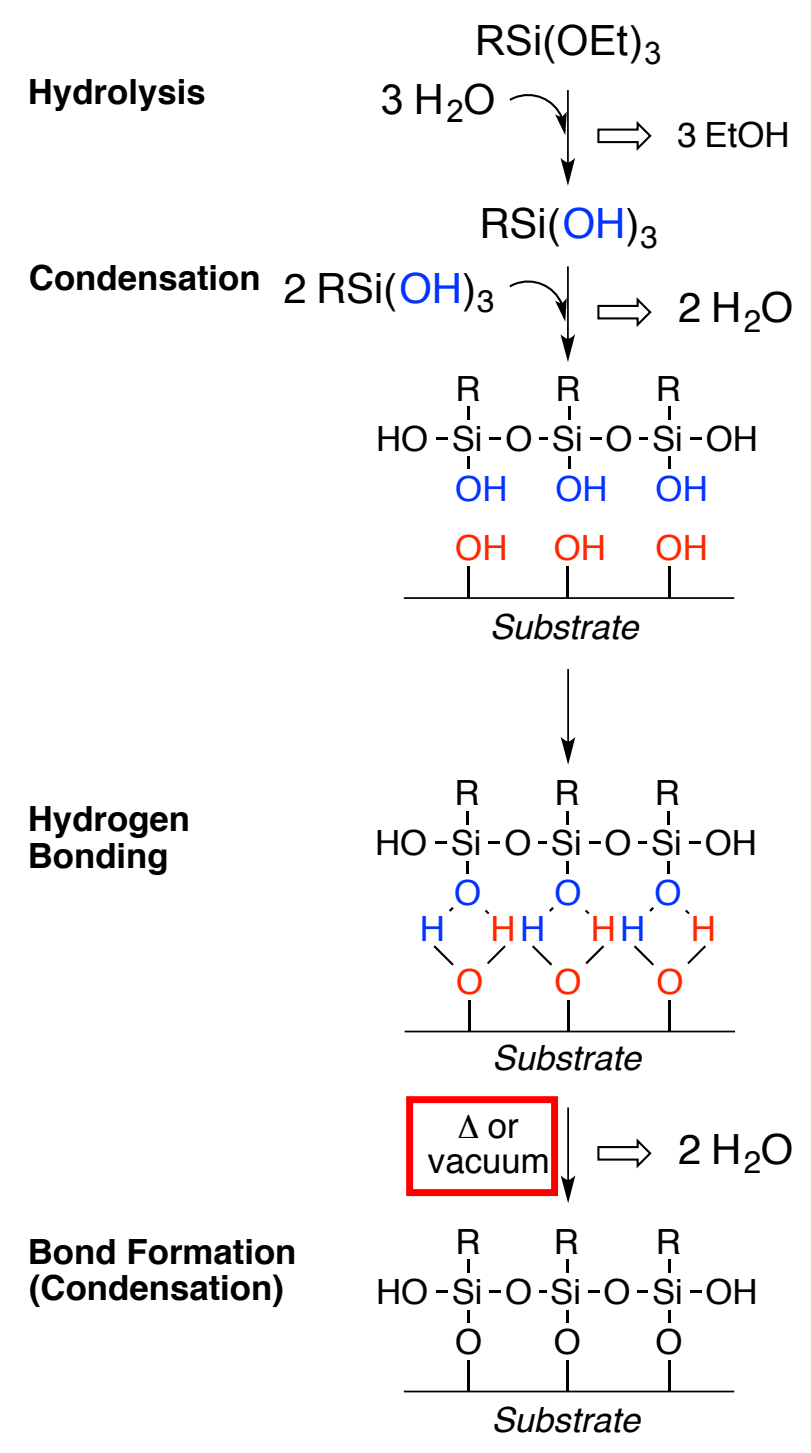

Figure 4.7. Hydrolytic deposition of silanes. ${ }^{46}$

The $\mathrm{Fe}_{3} \mathrm{O}_{4}$ NPs that were used for the loading were synthesized using the Massart method as described by Mikhaylova et al. ${ }^{47}$ and had an average diameter of $10 \mathrm{~nm}$. The 
NPs were synthesized by combining $\mathrm{FeCl}_{2} \bullet 4 \mathrm{H}_{2} \mathrm{O}$ and $\mathrm{FeCl}_{3} \bullet 6 \mathrm{H}_{2} \mathrm{O}$ in a 1:2 molar ratio in a $0.1 M$ solution of $\mathrm{NaOH}$ with rapid stirring. As the solution of the iron salts is added to the basic solution the black $\mathrm{Fe}_{3} \mathrm{O}_{4} \mathrm{NPs}$ form.

To functionalize the magnetic NPs, they were suspended by sonication in ethanol with a small amount of water added to aid in hydrolysis of the alkoxysilane to form the silanol. The alkoxysilane linker was added to the NP suspension and was mechanically stirred for $24 \mathrm{~h}$ at room temperature. The functionalized NPs were magnetically separated and washed with EtOH until there was no activity to ultraviolet (UV) light observed in the supernatant as determined using thin layer chromatography (TLC). Finally, the NPs were dried under vacuum for 12 hours to afford a tan powder. Using the weight difference between the starting, black $\mathrm{Fe}_{3} \mathrm{O}_{4} \mathrm{NPs}$ and the final, tan functionalized NPs, we had obtained a loading of $1.75 \mu \mathrm{mol} / \mathrm{mg}$. To verify that the linker was loaded onto the NPs and was still capable of undergoing an intramolecular cyclization to release, a portion of the NPs were suspended in toluene and heated to reflux for 22 hours. After magnetic separation, the supernatant was analyzed by TLC. TLC analysis (developed using UV and $p$-anisaldehyde stain) revealed the presence of 2-(9-anthracenyl)ethanol. This result confirmed that our linker was covalently bound to the $\mathrm{Fe}_{3} \mathrm{O}_{4} \mathrm{NP}$ and was still capable of undergoing an intramolecular cyclization for the release of an alcohol.

When we began to characterize the functionalized NPs by attenuated total reflectance (ATR) Fourier Transform (FT) infrared (IR) spectrometry we noticed that our signal intensity was high and that we had an intense signal at $1060 \mathrm{~cm}^{-1}$. This signal is attributed to $\mathrm{Si}-\mathrm{O}, \mathrm{Si}-\mathrm{O}-\mathrm{Si}$ and $\mathrm{Fe}-\mathrm{O}-\mathrm{Si}$ vibrations. ${ }^{48}$ In addition, Galeotti and coworkers report that the signal intensity of the $\mathrm{Fe}-\mathrm{O}$ bonds $\left(580-635 \mathrm{~cm}^{-1}\right)$ in a 
monolayer of silane should be stronger than the $\mathrm{Si}-\mathrm{O}, \mathrm{Si}-\mathrm{O}-\mathrm{Si}$ and $\mathrm{Fe}-\mathrm{O}-\mathrm{Si}$ vibration signal. As can be seen in Figure 4.8 our peak at $1060 \mathrm{~cm}^{-1}$ is very intense and the peak at $632 \mathrm{~cm}^{-1}$ is much weaker.

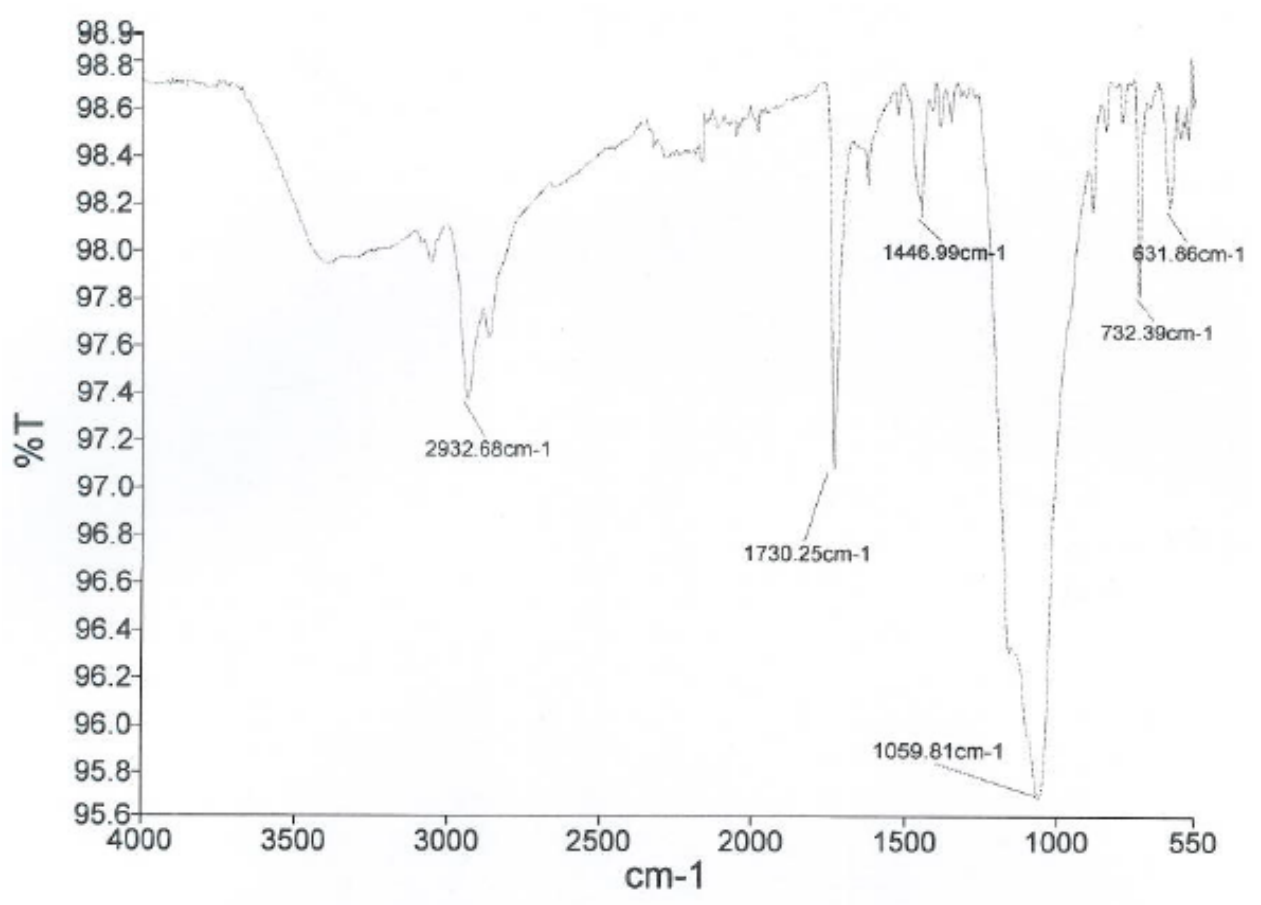

Figure 4.8. FT-IR spectrum of $\mathrm{Fe}_{3} \mathrm{O}_{4}$ NPs functionalized via an EtOH: $\mathrm{H}_{2} \mathrm{O}$ route with linker $\mathbf{4 . 3}$ obtained using ATR.

These results were indicative of a thick silica-linker shell around the iron oxide NP. These results were not desired despite the advantages provided by the silica shell including the robust $\mathrm{Si}-\mathrm{O}-\mathrm{Si}$ linkages between the linkers. We were targeting a silane monolayer for two main reasons, 1) to maximize the percent released when compared to the loading and 2) to increase the magnetism and thus the potential thermal response with the application of an AMF. Larumbe et $a l .{ }^{49}$ reported that as the thickness of the $\mathrm{SiO}_{2}$ layer increases there is a sharp reduction in the magnetic response of the $\mathrm{Fe}_{3} \mathrm{O}_{4}$ NPs when 
compared to analogous uncoated $\mathrm{Fe}_{3} \mathrm{O}_{4}$ NPs. After running the kinetic experiment, we knew that the 7-membered ring formation required temperatures above $55{ }^{\circ} \mathrm{C}$ and, therefore, were trying to maximize the AMF-induced release of thermal energy.

Before we could correct the thickness of the alkoxysilane coating we had to understand why the alkoxysilane was polymerizing instead of only binding to the surface of the NP. In order to form a $\mathrm{SiO}_{2}$ shell on an $\mathrm{Fe}_{3} \mathrm{O}_{4} \mathrm{NP}$ it is common to use the Stöber process, which is also performed in a solution of $\mathrm{EtOH}$ and water. ${ }^{50}$ But this process utilizes basic conditions to catalyze the hydrolysis of the alkoxysilane and the condensation of the silanols. Following the LaMer model, the nucleation is the fast process and the particle growth follows without further nucleation and can also be thought of as the addition of monomers. ${ }^{51}$ In our case, the base was likely from excess $\mathrm{NaOH}$ that had not been removed the synthesized $\mathrm{Fe}_{3} \mathrm{O}_{4}$ NPs. The presence of the base, in conjunction with the solvent conditions, had mimicked the Stöber process. Even if we were to eliminate the base from the prepared NPs, there was still worry that the water would eliminate our ability to positively obtain only a monolayer due to the inability to control the self-polymerization of the silanols in the presence of water as depicted in Figure 4.9. 
A

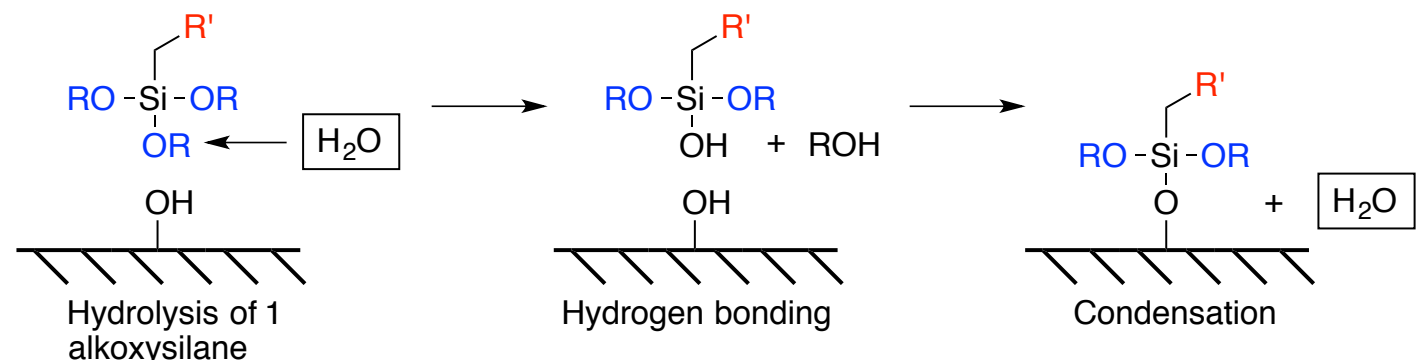

B

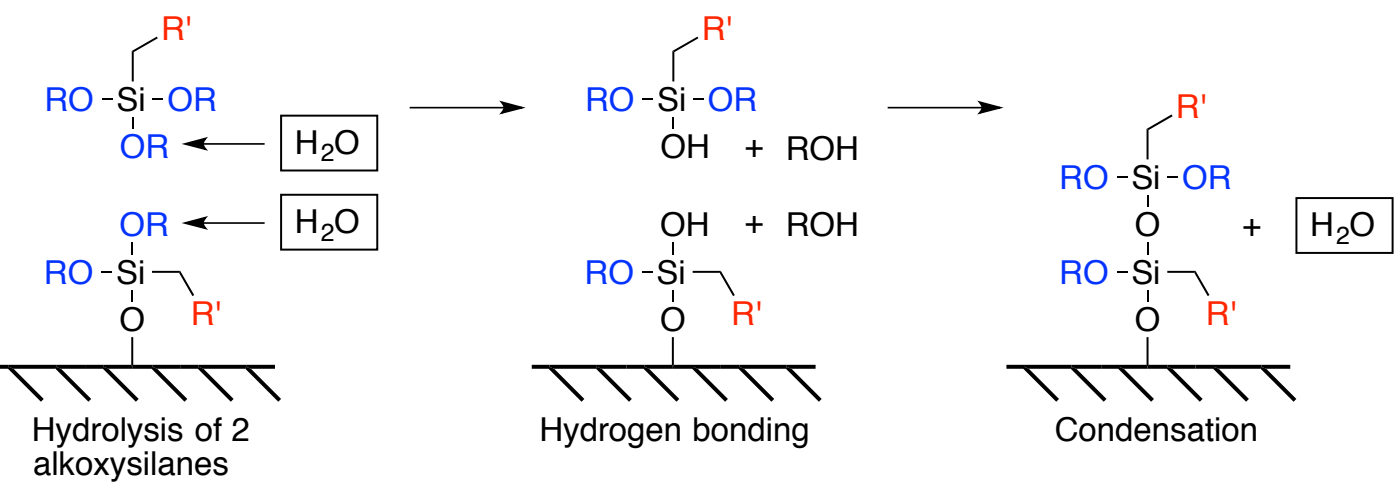

C

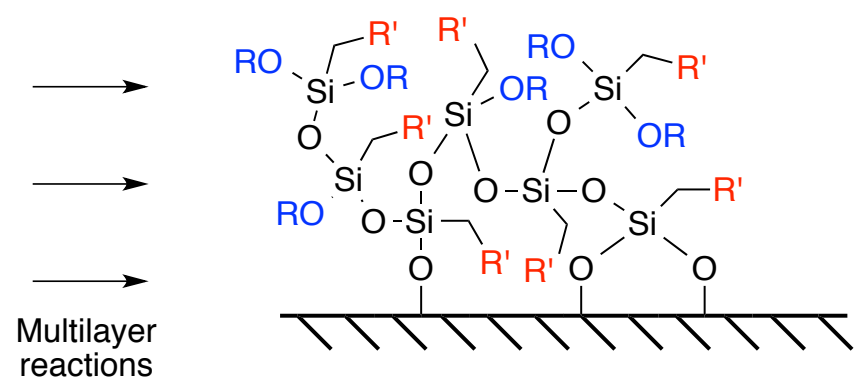

Figure 4.9. Examples of hydrolysis and condensation of alkoxysilanes with surface hydroxyl groups in the presence of water to afford A) a monolayer, B) a bilayer and C) an uncontrolled and disorderly polymeric shell.

For greater reliability, we switched from using in-house prepared $\mathrm{Fe}_{3} \mathrm{O}_{4} \mathrm{NPs}$ to commercial $\mathrm{Fe}_{3} \mathrm{O}_{4}$ NPs with a diameter of 20-30 nm (US Research Nanomaterials, $98+\%)$. This change reduced the risk of any problems arising from having used more basic NPs - commercial NPs are acid-washed. We also averted the unpredictability of aqueous loading by shifting to an anhydrous variation. Galeotti et $a l^{48}$ reported a route 
that would consistently provide a monolayer by performing the loading in chloroform instead of aqueous EtOH. The loading procedure involved suspending the $\mathrm{Fe}_{3} \mathrm{O}_{4} \mathrm{NPs}$ in chloroform via sonication, then adding a solution of the alkoxysilane linker in chloroform to the suspension with sonication. Upon completion of the addition, the reaction was moved to an overhead stirrer and heated to $65^{\circ} \mathrm{C}$ for at least 24 hours. Heat can be used instead of water to induce the hydrolysis of the alkoxysilane. ${ }^{44}$ The work-up was consistent with $\mathrm{Ma}$ et al. ${ }^{45}$ except the EtOH washes were replaced with chloroform washes. When the NPs functionalized via the procedure described by Galeotti and coworkers $^{48}$ were characterized by FT-IR, the spectra were similar to published data (Figure 4.10).

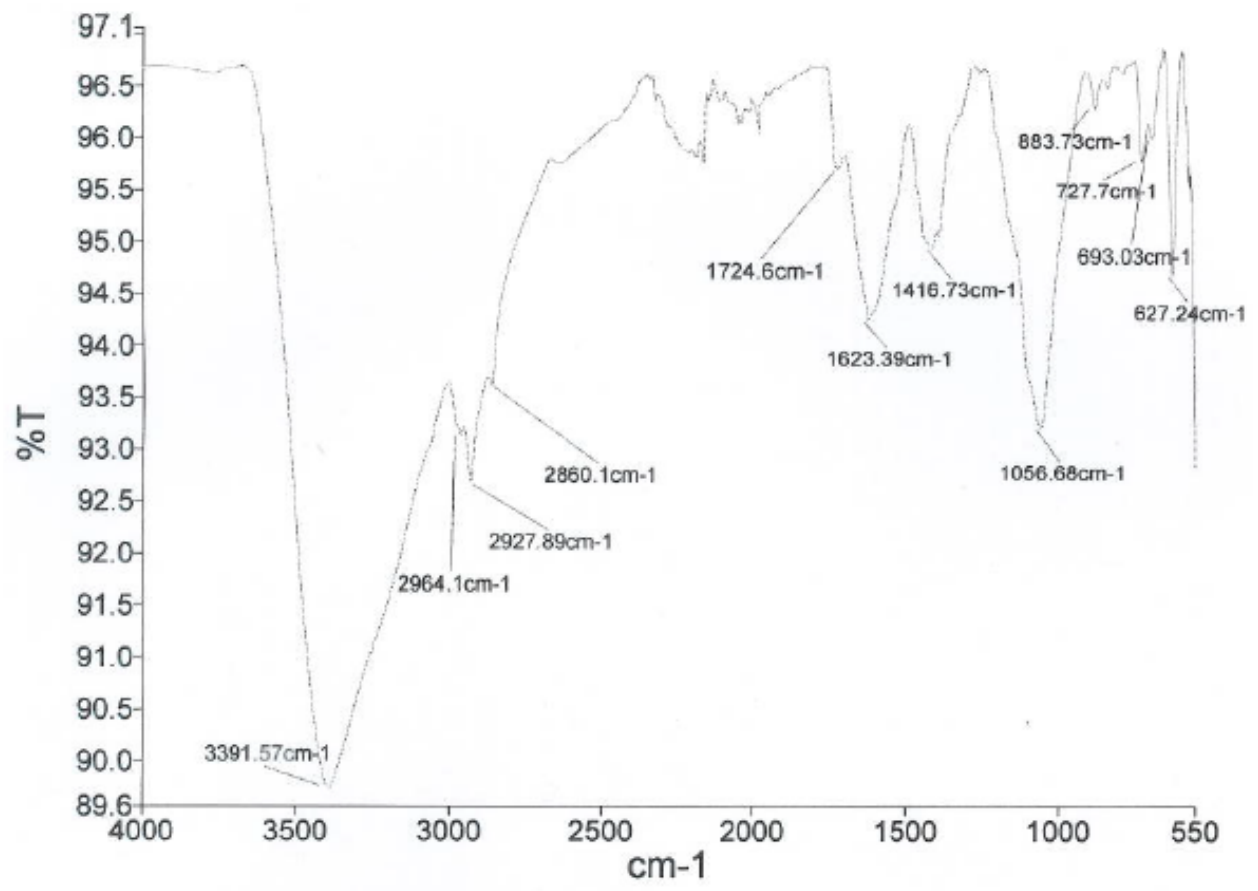

Figure 4.10. FT-IR spectrum of $\mathrm{Fe}_{3} \mathrm{O}_{4} \mathrm{NPs}$ functionalized via an anhydrous route with linker $\mathbf{4 . 3}$ obtained using ATR. 


\subsubsection{Formation of Covalent Bond to NP}

Now, with functionalized NPs similar to those published in the literature, we were able to begin experimenting with the exposure of the NPs to an AMF. We placed $\sim 4 \mathrm{mg}$ of the magnetic NPs functionalized with linker 4.3 in $0.75 \mathrm{~mL}$ of $2: 1$ phosphate buffer solution (PBS) : acetonitrile. The $\mathrm{pH}$ was adjusted to 7.4 with glacial acetic acid and the acetonitrile was used to keep the hydrophobic anthracene fluorophore in solution to

enable fluorescence measurements to be taken. As a control, one vial was allowed to sit on the bench at room temperature and a second vial was subjected to an AMF at 595 amps and $204 \mathrm{kHz}$ using a 5-turn coil. After separating the NPs by magnetic sedimentation, the supernatant could be conveniently analyzed. We were pleased to find that after 30 minutes of AMF exposure there was substantial fluorescence in the supernatant. However, much to our surprise, a similar result was obtained when analyzing the non-AMF exposed control (Figure 4.11). 


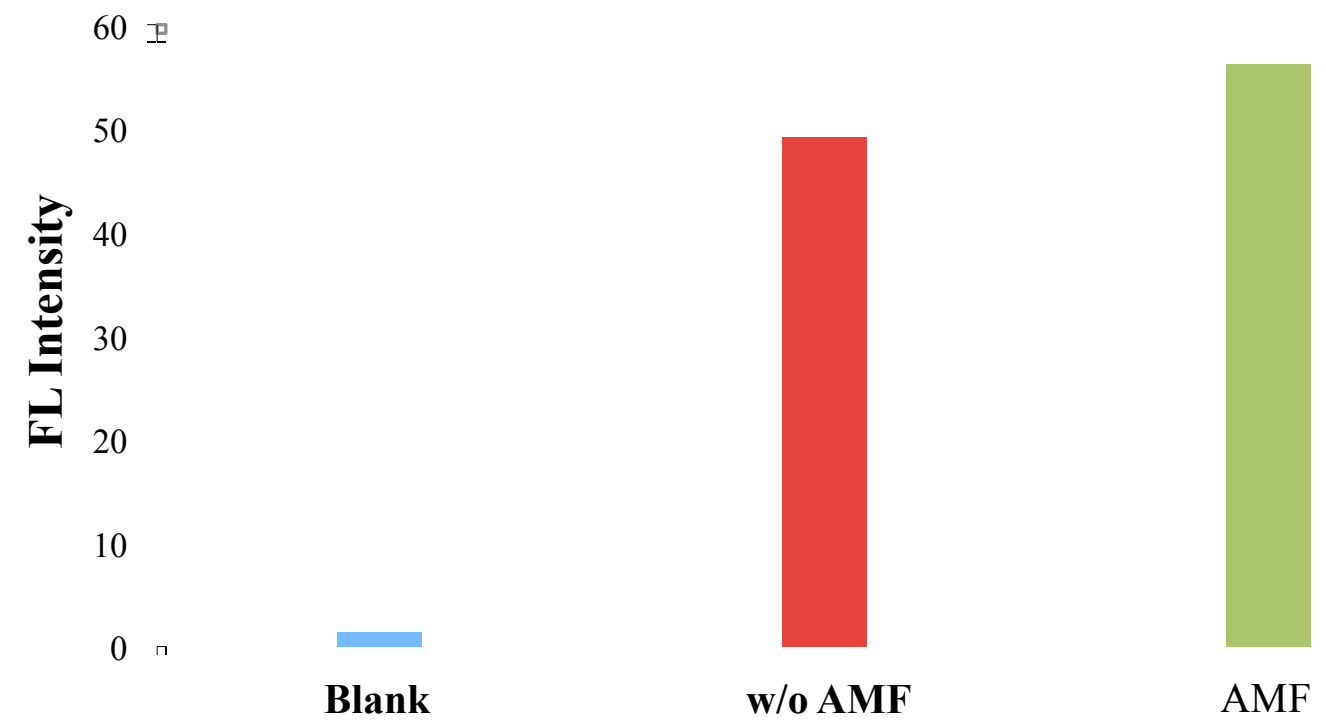

Figure 4.11. 595 Amp AMF and non-AMF induced release of fluorophore from $\sim 4 \mathrm{mg}$ of 20-30 $\mathrm{nm} \mathrm{Fe}_{3} \mathrm{O}_{4}$ NPs functionalized with linker 4.3 in 2:1 PBS:MeCN at $\mathrm{pH} 7.4$.

There were two possibilities to explain the minimal difference between non-AMF and AMF-induced release. The first possibility was that the intramolecular cyclization was occurring at a faster than expected rate when attached to the iron oxide NP. The second explanation was that we were not actually forming a covalent bond to the magnetic NPs. This would be the case if we had not successfully initiated the final condensation in the NP loading (Figure 4.12). 
A

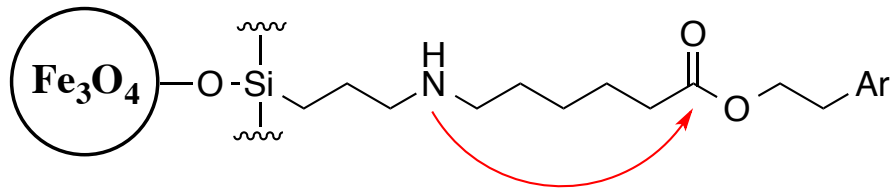

B

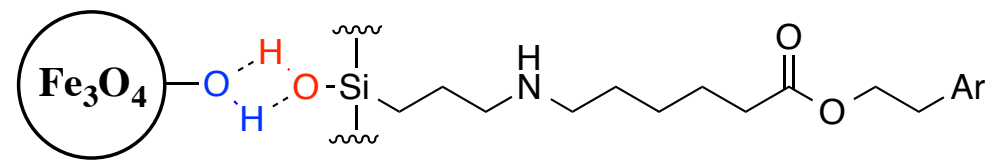

Figure 4.12. Possible explanations for non-AMF induced release of fluorophore. A) accelerated intramolecular cyclization or B) linker anchored to NPs through a noncovalent, hydrogen bond.

To discriminate these theories, I synthesized linker 10 (Figure 4.13) wherein the secondary amine involved in the intramolecular cyclization is replaced with a nonreactive methylene group. Also, the anthracenyl fluorophore was slightly modified to use the commercially available 9-anthracenemethanol instead of the 2-(9-anthracenyl)ethanol since no Boc-deprotection step is required for synthesis of 10. Having eliminated the possibility of an intramolecular cyclization for release of fluorophore, we expected it would be straightforward to differentiate this mechanism from the hypothesis that fluorophore release occurs via mere detachment of a hydrogen bound linker. 
A<smiles>C=CCNCCCCCC(=O)OCCBr</smiles>

4.3

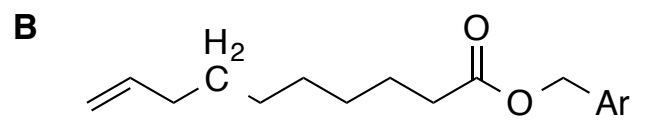

10

Figure 4.13. Linker 4.3, capable of intramolecular cyclization, and $\mathbf{1 0}$ with a methylene instead of a nucleophilic amine, rendering it incapable of intramolecular cyclization. Linker 10 was used to probe the origin of the observed non-AMF induced release.

Using the procedure described by Galeotti et al. ${ }^{48}$ the $\mathrm{Fe}_{3} \mathrm{O}_{4}$ NPs were functionalized with $\mathbf{1 0}$ in the same manner as the NPs were loaded with 4.3. The AMF experiment was repeated with $\sim 3 \mathrm{mg}$ of $\mathrm{Fe}_{3} \mathrm{O}_{4} \mathrm{NPs}$ coated with $\mathbf{1 0}$ and the addition of a 30 second sonication step to ensure that we would see fluorescence from any noncovalently bound fluorophore. The results of this experiment are shown in Figure 4.14. Though the non-AMF induced release without sonication has been reduced, sonication and AMF exposure resulted in substantial release of the fluorphore. These results led us to understand that we were not successfully initiating the final condensation step using vacuum in the absence of heat. 

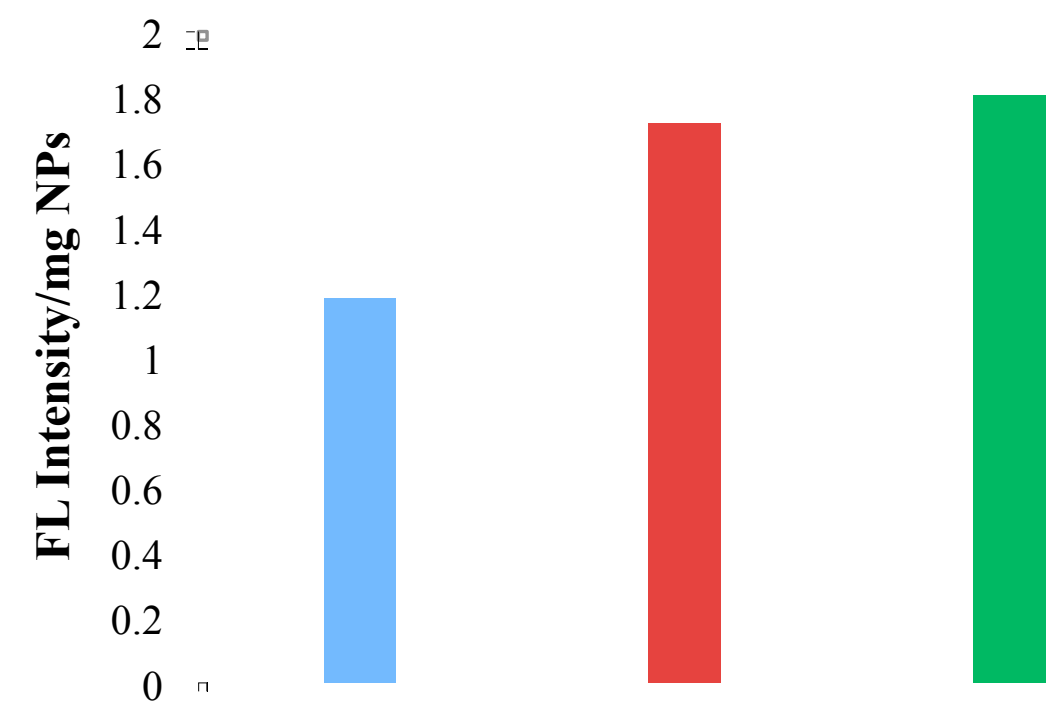

$30 \min 2: 1$

Sonicate

AMF

Blank PBS:MeCN

Figure 4.14. 595 Amp AMF and non-AMF induced release of fluorophore from $\sim 3 \mathrm{mg}$ of 20-30 $\mathrm{nm} \mathrm{Fe} \mathrm{O}_{4}$ NPs functionalized with linker 10 in 2:1 PBS:MeCN at $\mathrm{pH} 7.4$.

Dr. Jerry Larson, senior research fellow at Gelest Inc., Morrisville, PA, advised us that, despite what literature reports claim, $\mathrm{Fe}-\mathrm{O}-\mathrm{Si}$ bonds are difficult to form and that high temperatures $\left(\geq 100{ }^{\circ} \mathrm{C}\right)$ are required to induce the final condensation reaction for covalent attachment of the linker to the iron oxide NP. Armed with this knowledge, the NPs that were functionalized with 10 were heated to $110{ }^{\circ} \mathrm{C}$ for 24 hours at atmospheric pressure and the previous experiment was repeated, the results of which are combined with the results from Figure 4.14 and shown in Figure 4.15. When compared with the results obtained using conditions $1 \mathrm{a}-3 \mathrm{a}$, conditions $1 \mathrm{~b}-3 \mathrm{~b}$ showed almost no release of the fluorophore; a vast improvement in the minimization of undesired fluorophore release. These results also show that the use of heat to initiate the final condensation reaction and covalently bond the linker to the NP surface (Figure 4.7) is necessary and that vacuum 
alone cannot form the $\mathrm{Fe}-\mathrm{O}-\mathrm{Si}$ bond. Without the use of heat, the hydrolyzed alkoxysilane is only hydrogen bound to the $\mathrm{Fe}-\mathrm{OH}$ surface of the NP, thus explaining the high release rate when the functionalized NPs are subjected to sonication (condition 2a).
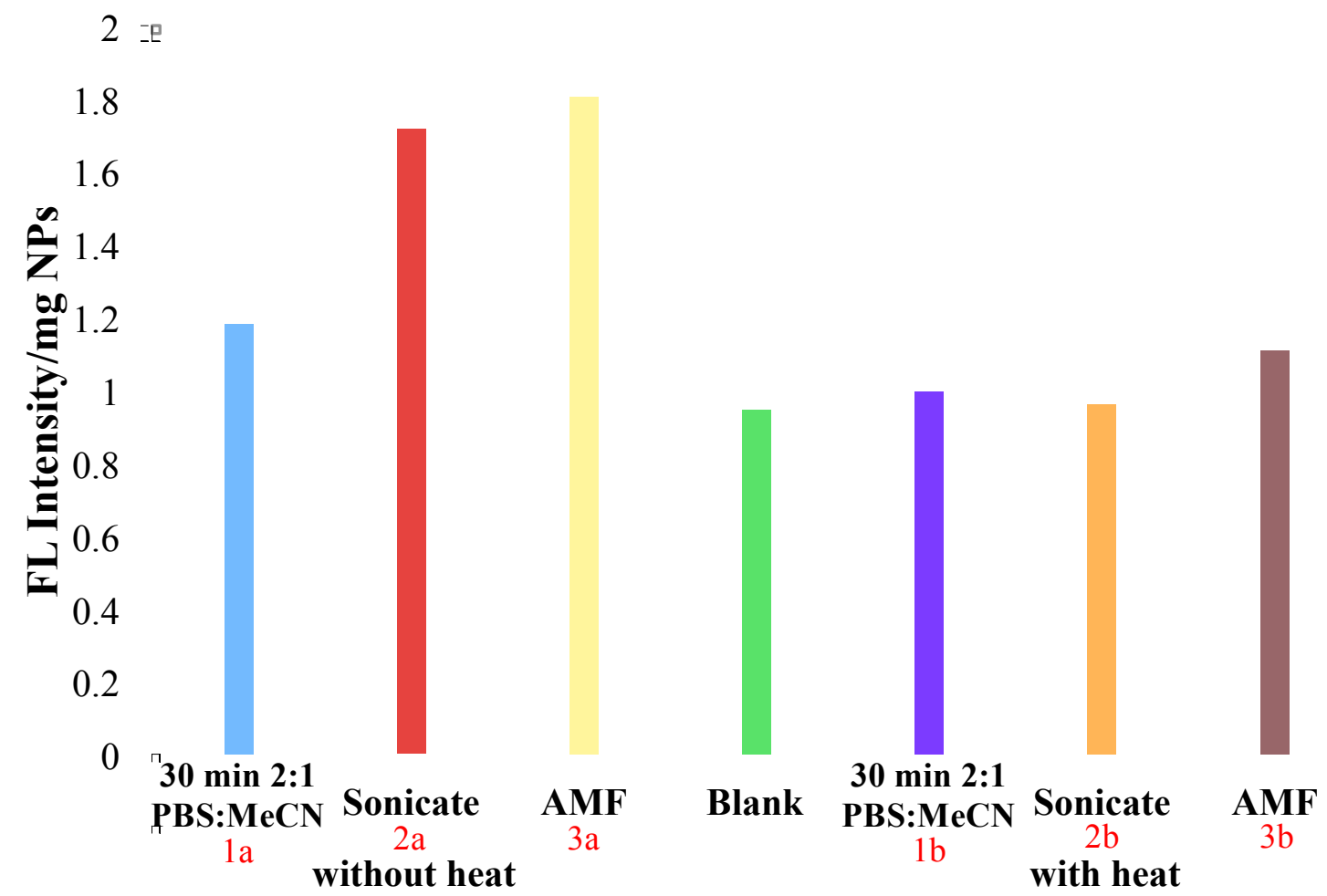

Figure 4.15. $595 \mathrm{Amp} A M F$ and non-AMF induced release of fluorophore from $\sim 3 \mathrm{mg}$ of $20-30 \mathrm{~nm} \mathrm{Fe}{ }_{3} \mathrm{O}_{4} \mathrm{NPs}$ functionalized with linker 10 in 2:1 PBS:MeCN at $\mathrm{pH}$ 7.4. With and without heat refers to the curing process of the functionalized NPs. Without heat NPs were cured under vacuum and with heat NPs were cured at $110^{\circ} \mathrm{C}$ for 24 hour at atmospheric pressure.

Though the results presented in Figure 4.15 were promising, it was necessary to confirm that the anthracene fluorophore was still attached to the $\mathrm{Fe}_{3} \mathrm{O}_{4}$ NPs. The NPs loaded with 10 and cured at $110{ }^{\circ} \mathrm{C}(\sim 3 \mathrm{mg})$ underwent saponification to liberate the esterified 9-anthracenemethanol. Briefly, bare $\mathrm{Fe}_{3} \mathrm{O}_{4}$ NPs (as a control) and NPs 
functionalized with $\mathbf{1 0}$ were suspended in a 2:1 solution of THF:MeOH. Then, a $0.03 \mathrm{M}$ aqueous solution of $\mathrm{NaOH}$ was added and the suspension was stirred overnight. After centrifuging the NPs out of solution the fluorescence of the supernatant was measured to find a fluorescent signal released only from the functionalized NPs.

\subsubsection{Acidic Boc-deprotection on NPs}

Since exposure of the NPs functionalized with the thermally labile linker to high temperatures were now required, we were no longer able to perform the acidic $-\mathrm{C}(\mathrm{O}) \mathrm{O} t$ $\mathrm{Bu}(\mathrm{Boc})$ deprotection prior to loading the linker onto the NPs. To test the acidic stability of the $\mathrm{Fe}_{3} \mathrm{O}_{4} \mathrm{NP}$ and the $\mathrm{Fe}-\mathrm{O}-\mathrm{Si}$ bonds we synthesized a small linker, 11, and attached it to the $\mathrm{Fe}_{3} \mathrm{O}_{4}$ NPs to form 12 (Scheme 4.1). We chose a secondary amine to best model our thermally responsive linkers.

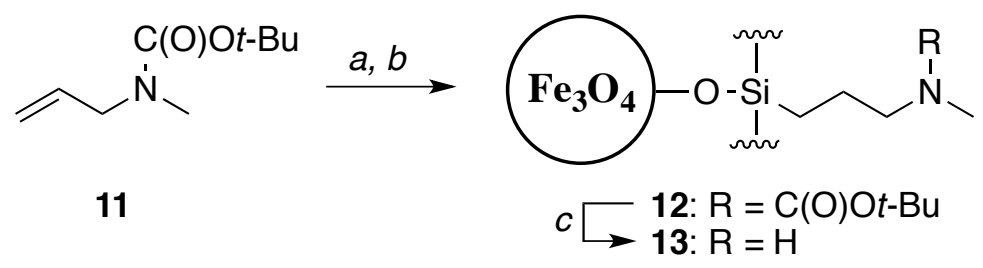

Scheme 4.1. Boc-deprotection of a $2^{\circ}$ amine bound to a 20 $30 \mathrm{~nm} \mathrm{Fe}_{3} \mathrm{O}_{4} \mathrm{NP}$ under acidic conditions. Conditions: $a$. (EtO) $)_{3} \mathrm{SiH}$, cat. $\mathrm{PtO}_{2}, 90{ }^{\circ} \mathrm{C} ; b . \mathrm{Fe}_{3} \mathrm{O}_{4}, \mathrm{CHCl}_{3}, 65^{\circ} \mathrm{C}$; $c$. i. $\mathrm{CF}_{3} \mathrm{CO}_{2} \mathrm{H}: \mathrm{CH}_{2} \mathrm{Cl}_{2}, 1: 1,0{ }^{\circ} \mathrm{C}, 1$ h; ii. $\mathrm{Et}_{3} \mathrm{~N}: \mathrm{CH}_{2} \mathrm{Cl}_{2}, 1: 1$, rt.

The Boc-deprotection of $\mathbf{1 2}$ was performed using 1:1 $\mathrm{CF}_{3} \mathrm{COOH}: \mathrm{CH}_{2} \mathrm{Cl}_{2}$ at $0{ }^{\circ} \mathrm{C}$ for 1 hour, the same as the Boc-deprotection performed to the linkers not bound to NPs. Upon completion the aminium functionalized NPs were magnetically separated, washed 
with $\mathrm{CH}_{2} \mathrm{Cl}_{2}$ and dried under vacuum. Thermogravimetric analysis (TGA) of $\mathbf{1 2}$ and $\mathbf{1 3 i}$ showed that $\sim 50 \%$ of the loading had been cleaved during the Boc-deprotection. As an affirmation of the successful deprotection and presence of nucleophilic 13, the NPs were neutralized with 1:1 $\mathrm{Et}_{3} \mathrm{~N}: \mathrm{CH}_{2} \mathrm{Cl}_{2}$ and fluorescein isothiocyanate (FITC) was added. 13, now functionalized with an FITC-thiourea fluorophore, was washed with methanol and ethyl ether, dried under vacuum and the fluorescent signal was observed using a Molecular Devices SpectraMax M5 fluorescent well plate reader. A well plate reader was necessary singe the solid NPs prohibit the standard fluorescent emission at $90^{\circ}$ to the excitation. The fluorescent signal confirmed the successful Boc-deprotection as can be seen in Figure 4.16, though costing $\sim 50 \%$ of the loading. Since we were still aiming for proof of concept this was deemed acceptable.

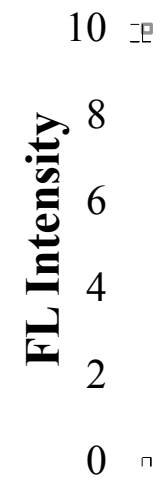

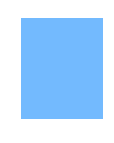

$\mathrm{MeCN}$

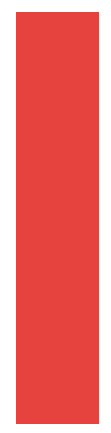

FITC-NPs

Figure 4.16. Fluorescent signal of $\mathbf{1 3}$ functionalized with FITC via a thiourea linkage in $\mathrm{MeCN}$.

We were now ready to load 14 (Scheme 4.2) onto the $\mathrm{Fe}_{3} \mathrm{O}_{4}$ NPs using the information we had learned. This included the information that anhydrous loading 
conditions decreased the uncontrolled polymerization and loading of the NPs, heat was necessary to initiate the final condensation reaction and acidic Boc-deprotection could effectively be performed on NP-bound linkers. The release experiment was set-up similar to earlier experiments with $16(\sim 7 \mathrm{mg})$ being suspended in 2:1 PBS:acetonitrile $(1.5 \mathrm{~mL})$ at $\mathrm{pH}$ 7.4. First, 16 was suspended and left on the bench for 30 minutes without exposure to sonication or an AMF. Using magnetic separation 16 was removed and the supernatant was collected and retained for fluorescent measurements. Then, the samples of 16 were again suspended in $1.5 \mathrm{~mL} 2: 1 \mathrm{PBS}$ :acetonitrile and were subjected to an AMF at 595 amps and $204 \mathrm{kHz}$ with a 5-turn coil for 30 minutes. The NPs were then magnetically separated and the supernatant was collected. Both samples of the supernatant were then analyzed using fluorescent spectrometry, the results of which are displayed in Figure 4.17. The only mystery that remained was an explanation for the continued release in the absence of an AMF.

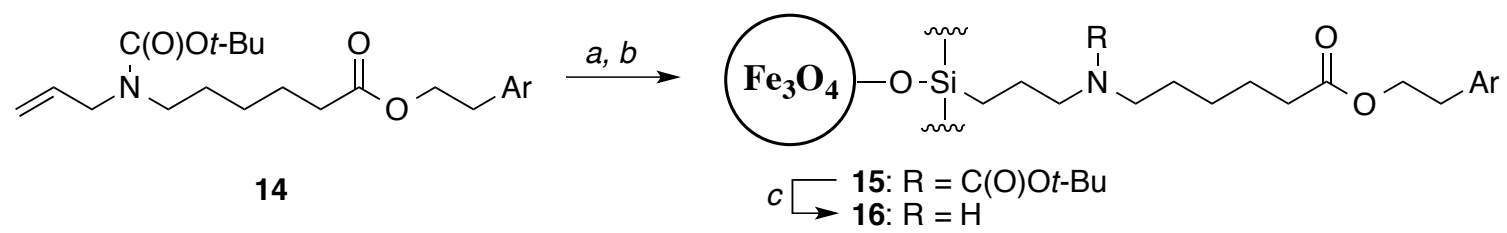

Scheme 4.2. Loading of 14 onto 20-30 $\mathrm{nm} \mathrm{Fe}{ }_{3} \mathrm{O}_{4} \mathrm{NPs}$ and Boc-deprotection of $\mathbf{1 5}$ under acidic conditions to afford a thermally labile linker. Conditions: $a .(\mathrm{EtO})_{3} \mathrm{SiH}$, cat. $\mathrm{PtO}_{2}$, $90{ }^{\circ} \mathrm{C} ;$ b. $\mathrm{Fe}_{3} \mathrm{O}_{4}, \mathrm{CHCl}_{3}, 65^{\circ} \mathrm{C}$; c. i. $\mathrm{CF}_{3} \mathrm{CO}_{2} \mathrm{H}: \mathrm{CH}_{2} \mathrm{Cl}_{2}, 1: 1$, $0{ }^{\circ} \mathrm{C}, 1$ h; ii. $\mathrm{Et}_{3} \mathrm{~N}: \mathrm{CH}_{2} \mathrm{Cl}_{2}, 1: 1$, rt. 


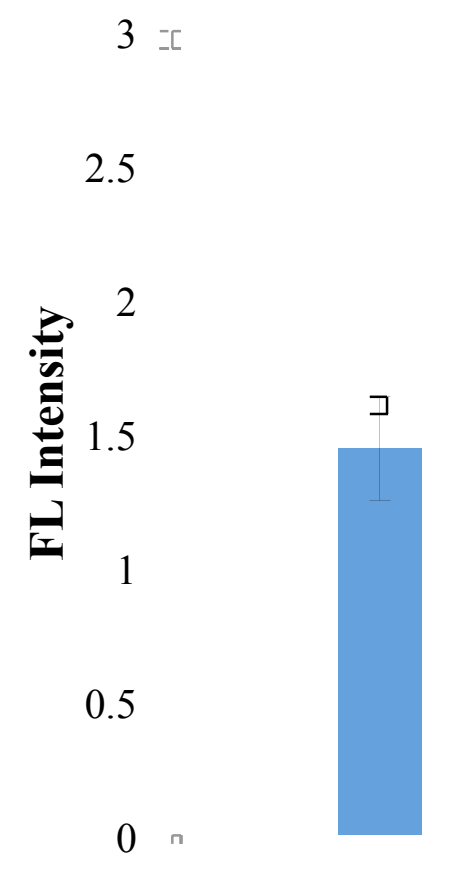

Blank

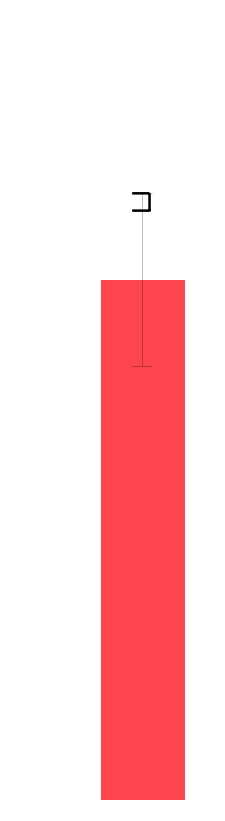

30 min, 2:1 PBS:MeCN
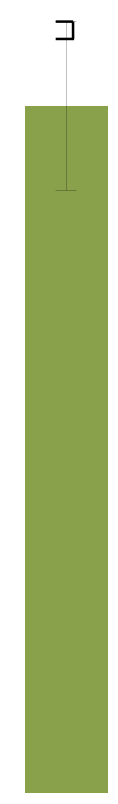

AMF

Figure 4.17. $595 \mathrm{Amp} A \mathrm{AMF}$ and non-AMF induced release of fluorophore from $\sim 7 \mathrm{mg}$ of 16 in $1.5 \mathrm{~mL}$ 2:1 PBS:MeCN at $\mathrm{pH}$ 7.4. The AMF-induced release is the result of what remained on the NPs following the incubation period of 30 minutes at room temperature in $1.5 \mathrm{~mL} \mathrm{2:1} \mathrm{PBS}: \mathrm{MeCN}$ at $\mathrm{pH}$ 7.4 .

\subsubsection{AMF vs. Non-AMF Induced Release: Hydrolysis}

We began to suspect that the hydrolytic susceptibility of the ester functionality might be the culprit responsible for non-triggered release of fluorophore. To test this theory we synthesized three short linkers, one with an ester functionality, one with a more hydrolytically stable carbonate functionality and one with an even more hydrolytically stable carbamate functionality (Scheme 4.3). The chemical structure of $\mathbf{1 7 . 1 - 3}$ was confirmed by ${ }^{13} \mathrm{C}$ NMR (Figure 4.18). Aside from the differences in the carbonyl carbon peak, the methylenes $\alpha$ to the carbonyl functionality also distinctly different chemical 
shifts. The ester methylenes have a chemical shift of $64.3 \mathrm{ppm}$ and $33.8 \mathrm{ppm}$ with the methylene $\alpha$ to the carbonyl being upfield of the methylene adjacent to the ester oxygen. The allyl methylene of the carbonate was at $67.4 \mathrm{ppm}$, upfield of the methylene next to the oxygen on the anthracene side of the molecule that was at $68.7 \mathrm{ppm}$. The methylene beside the carbamate nitrogen had an upfield chemical shift of $43.7 \mathrm{ppm}$ compared to the methylene near the oxygen, which had a chemical shift of $64.9 \mathrm{ppm}$. In addition to increasing the robustness of the carbonyl functionality, we also made several experimental adjustments: (1) the AMF power amperage was reduced from 595 amps to 200 amps (the frequency was kept at $204 \mathrm{kHz}$ since the coil diameter and number of turns dictate this frequency); (2) to begin modeling physiological conditions, we changed the control conditions from NPs incubated at room temperature to NPs incubated at $37^{\circ} \mathrm{C}$; and (3) we also changed the AMF application protocol from 30 minutes of continuous exposure to a pulsed application of AMF consisting of consecutive 5 minute 'on' and 5 minute 'off' cycles for a total of 30 minutes of AMF exposure. The pulsed modification in the experimental design allowed for collection of multiple data points needed to establish a release profile and provided a procedure that would be more agreeable with a biological system. Figure 4.19 shows the results of the AMF experiments. 


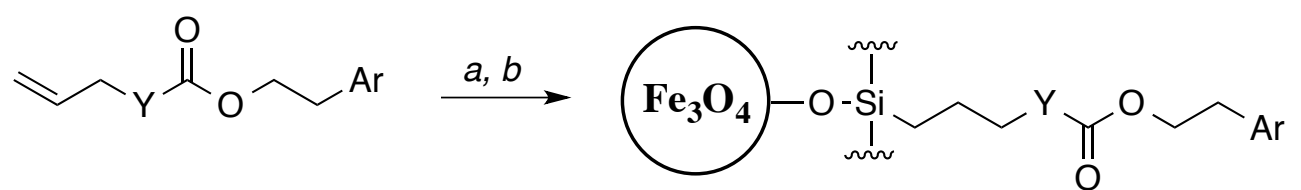

17.1: $Y=\mathrm{CH}_{2}$

17.2: $Y=O$

17.3: $Y=\mathrm{NH}$
18.1-3

Scheme 4.3. Short chain linkers bound to $\mathrm{Fe}_{3} \mathrm{O}_{4}$ NPs to test hydrolytic stability of ester, carbonate and carbamate functionalities. Conditions: $a$. (EtO) $)_{3} \mathrm{SiH}$, cat. $\mathrm{PtO}_{2}, 90^{\circ} \mathrm{C} ; b . \mathrm{Fe}_{3} \mathrm{O}_{4}, \mathrm{CHCl}_{3}, 65^{\circ} \mathrm{C}$. 

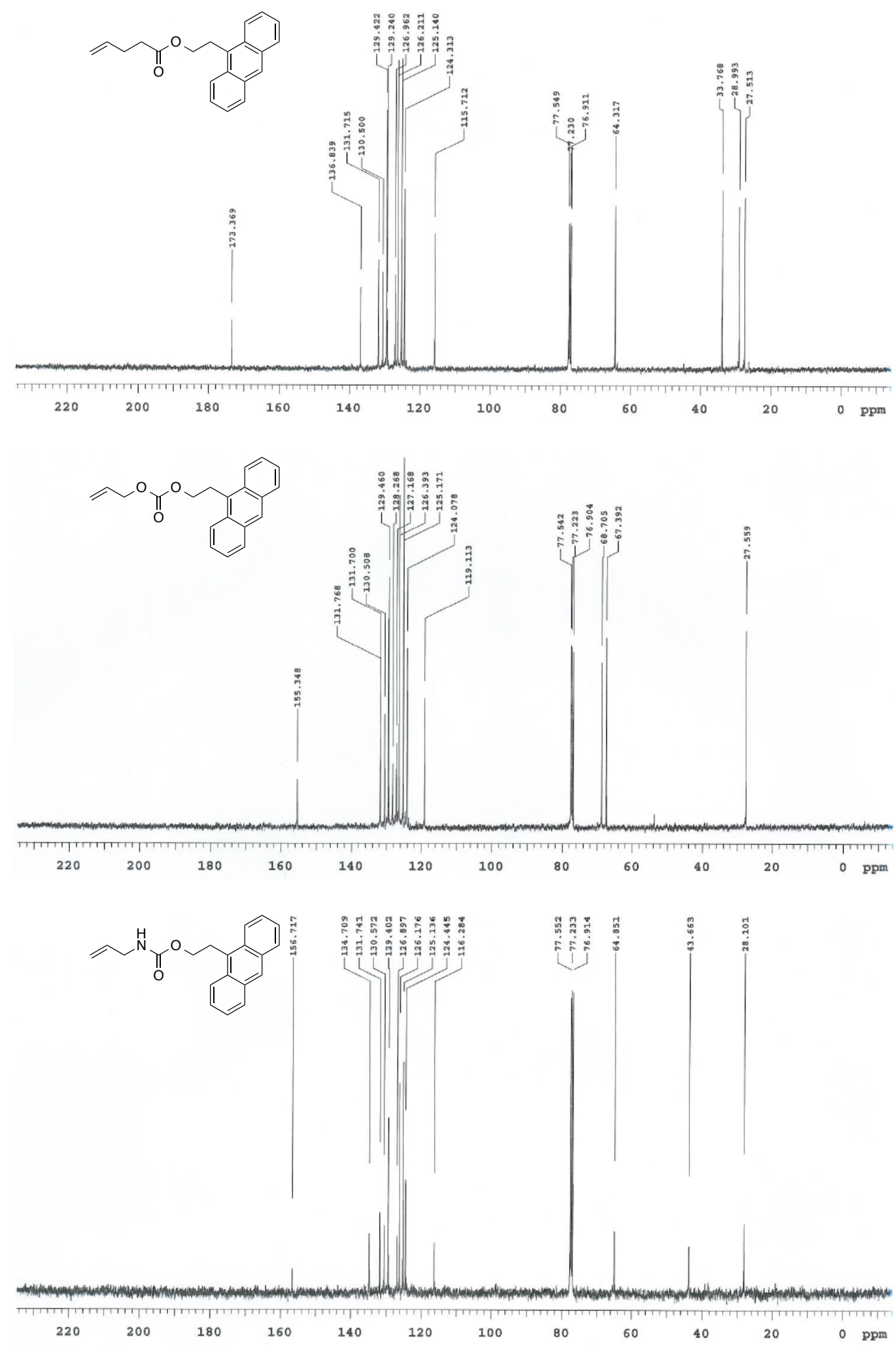

Figure 4.18. ${ }^{13} \mathrm{C}$ NMR spectra $\left(\mathrm{CDCl}_{3}\right)$ confirming the structures of 17.1-3. 


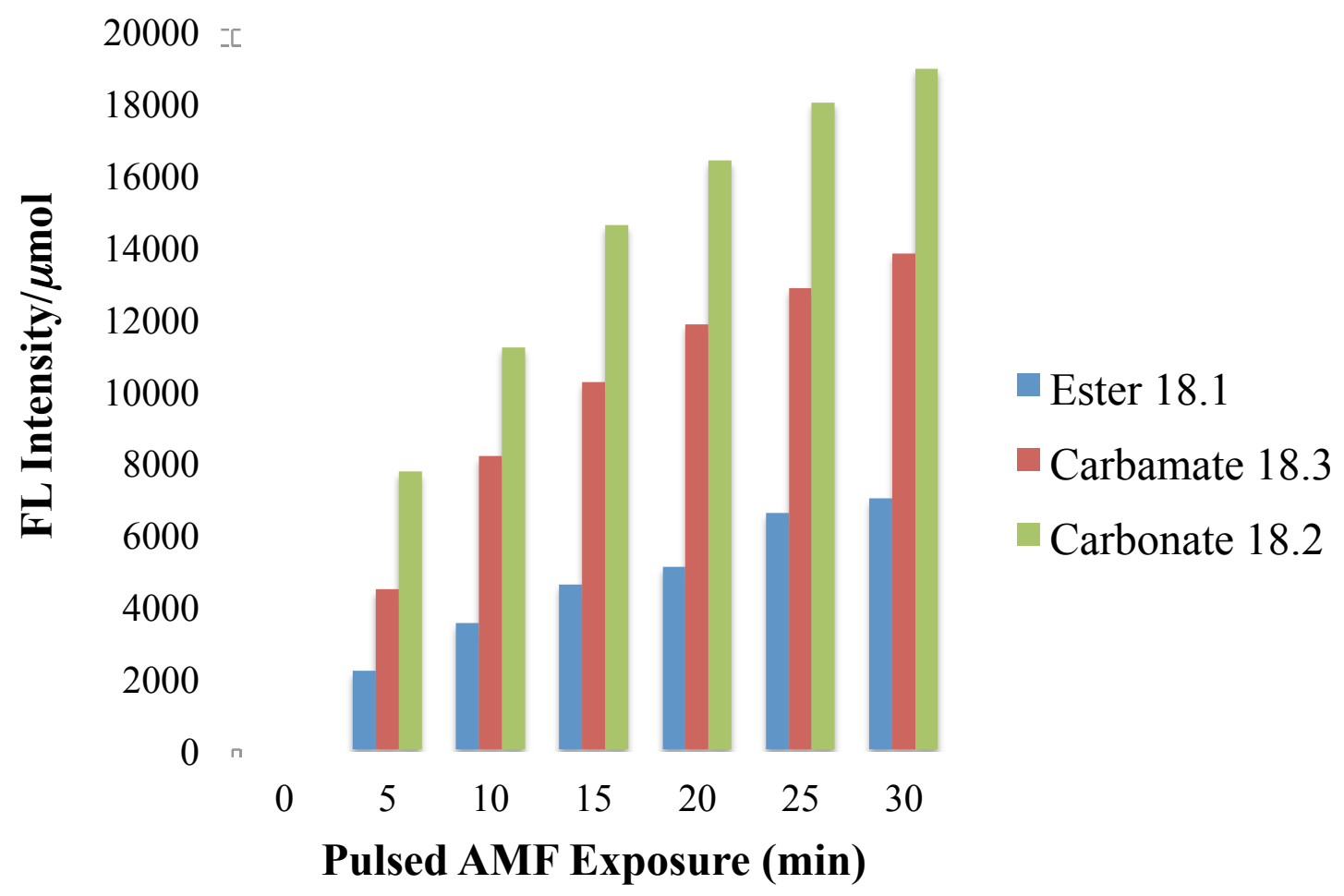

Figure 4.19. $200 \mathrm{amp}$ AMF induced release of fluorophore from $\sim 6$ mg of 18.1-3 in 0.75 mL 2:1 PBS:MeCN at $\mathrm{pH} 7.4$.

The release results from 18.1-3 were quite unexpected. The ester, the most easily hydrolyzed functionality of the panel, showed the lowest rate of release while the more robust linkages, carbonate and carbamate, showed higher release rates with the carbonate releasing the fastest. But the release patterns all appeared to be a stepwise release, indicating that it could be a result of AMF exposure. To test this theory we performed another experiment, but instead of exposing the NPs 18.1-3 to an AMF we incubated them at $37{ }^{\circ} \mathrm{C}$ in the 2:1 PBS:acetonitrile solution at $\mathrm{pH} 7.4$ for 20 hours. This experiment would allow us to see if the release was AMF-induced. Figure 4.20 depicts the incubation results. 


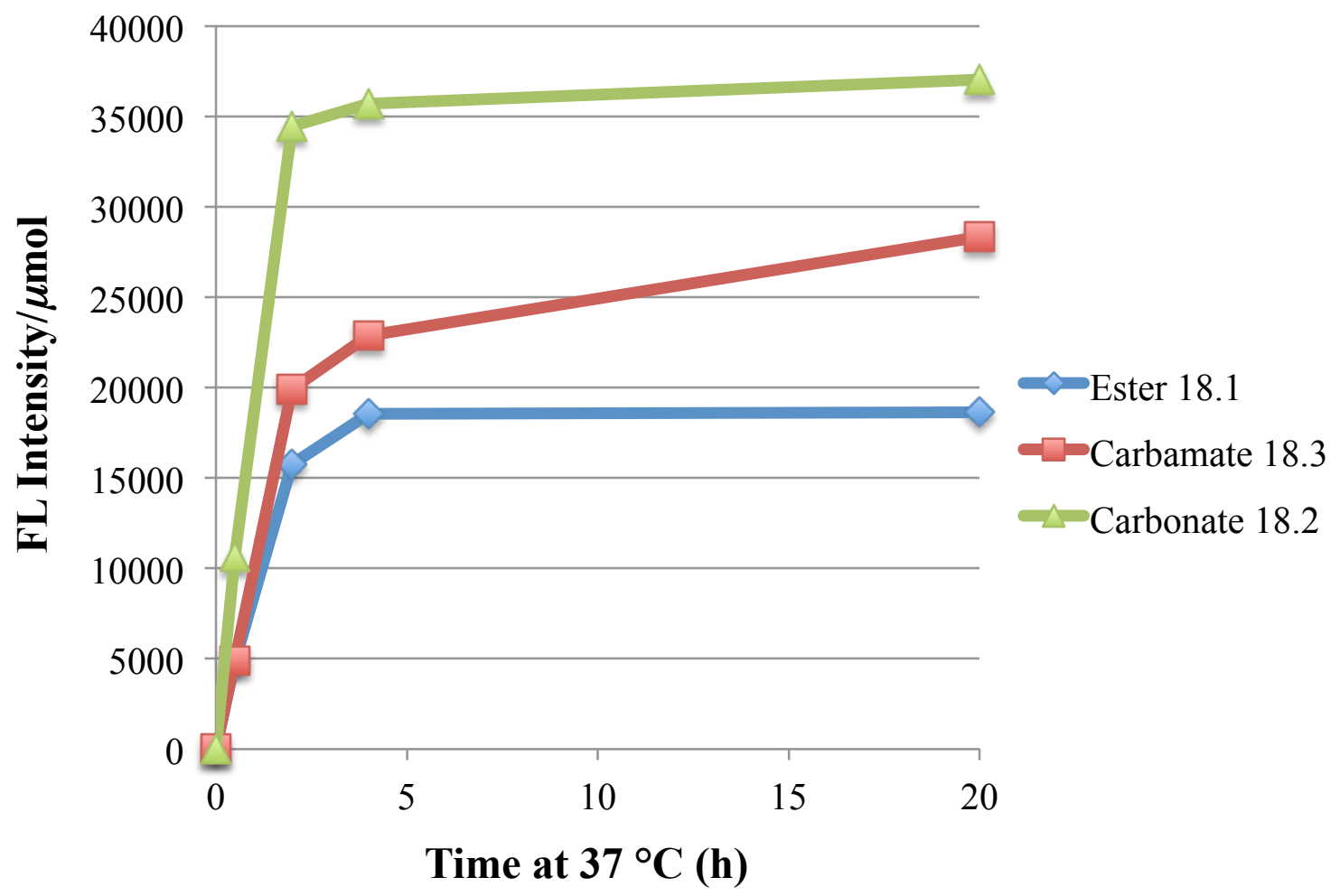

Figure 4.20. Release of fluorophore from $\sim 6 \mathrm{mg}$ of $\mathbf{1 8 . 1 - 3}$ in $0.75 \mathrm{~mL} \mathrm{2:1}$ PBS:MeCN at $\mathrm{pH} 7.4$ incubated at $37^{\circ} \mathrm{C}$ for $20 \mathrm{~h}$.

Incubation of 18.1-3 at $37{ }^{\circ} \mathrm{C}$ for 20 hours resulted in a release profile that was similar to the one obtained using an AMF. This result is remarkable - carbonate and carbamate functionalities undergoing hydrolysis at $\mathrm{pH} 7.4$ at $37{ }^{\circ} \mathrm{C}$. This import of this finding is put into perspective on comparison with current hydrolytic examples from the literature (Scheme 4.4) that show the forcing conditions needed to cleave carbonate and carbamate functionalities. It became obvious that the $\mathrm{Fe}_{3} \mathrm{O}_{4} \mathrm{NPs}$ must be exerting some effect to accelerate the hydrolyses, even in the absence of an AMF. 
A

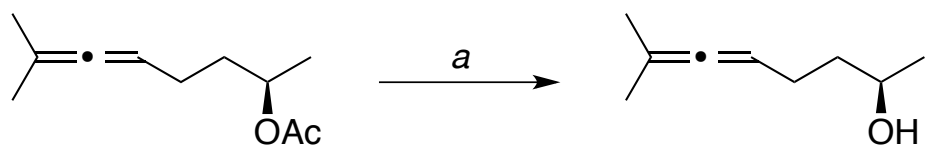

B

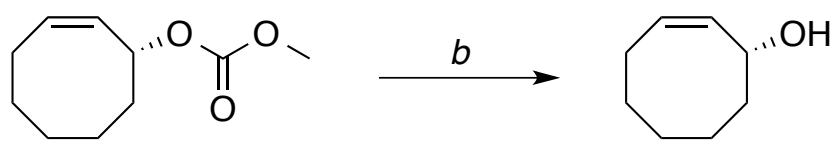

C

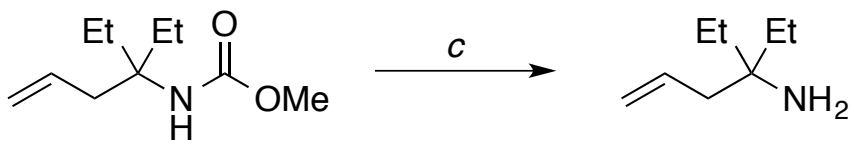

Scheme 4.4. Examples from current literature of the hydrolysis of A. an ester, ${ }^{52}$ B. a carbonate ${ }^{53}$ and C. a carbamate. ${ }^{54}$ Conditions: $a . \mathrm{H}_{2} \mathrm{O}$, DMSO, rt, $42 \mathrm{~h}, 58 \%$; $b$. $5 M \mathrm{NaOH}, \mathrm{MeOH}, \mathrm{rt}, 3 \mathrm{~d}, 90 \% ; c . \mathrm{KOH}, \mathrm{N}_{2} \mathrm{H}_{4}$, $\left(\mathrm{CH}_{2} \mathrm{OMe}\right)_{2}, 200{ }^{\circ} \mathrm{C}, 80 \%$.

To attempt to explain the iron oxide NP hydrolysis phenomenon we started to investigate the catalytic and physical attributes of iron. The $\mathrm{Fe}_{3} \mathrm{O}_{4} \mathrm{NPs}$ are comprised of a mixture of both $\mathrm{Fe}^{2+}$ and $\mathrm{Fe}^{3+}$ at a molar ratio of $1: 2$, respectively. ${ }^{47}$ Since $\mathrm{Fe}_{3} \mathrm{O}_{4}$ is readily oxidized to $\mathrm{Fe}_{2} \mathrm{O}_{3}\left(\mathrm{Fe}^{3+}\right)$ when exposed to air, changing the NP color from black to an orange/red color, it is not uncommon for the NPs to have an oxidized layer of $\mathrm{Fe}_{2} \mathrm{O}_{3}$ or $\mathrm{Fe}^{3+} \cdot 55$ With this in mind, we researched the properties of iron(III) and found that iron(III) oxides are a known Lewis acid, thus making them capable of selectively binding to Lewis bases (e.g., heteroatoms, particularly carbonyl oxygens). ${ }^{56}$ The Lewis acidLewis base concept could help explain why the carbonate and carbamate groups were hydrolyzed to a greater extent than the ester. The attribute of both the carbonate and carbamate that adds to their hydrolytic stability is the increased electron density supplied 
to the carbonyl, thus making it less susceptible to nucleophilic attack at the carbonyl carbon by water. As can be seen from Scheme 4.4, the hydrolysis of carbonates and carbamates require highly basic reaction conditions and, in the case of the carbamate, high temperatures. These conditions are all methods of enhancing the nucleophilicity of water/hydroxyl groups. But this hydrolytic acceleration can also be achieved with Lewis acids that, instead of increasing nucleophilicity, enhance the electrophilicity of the carbonyl and allow a weaker nucleophile to attack an otherwise stable functional group. This knowledge led us to postulate a possible mechanism for the $\mathrm{Fe}_{3} \mathrm{O}_{4} \mathrm{NP}$ accelerated hydrolysis that is shown in Figure 4.21.

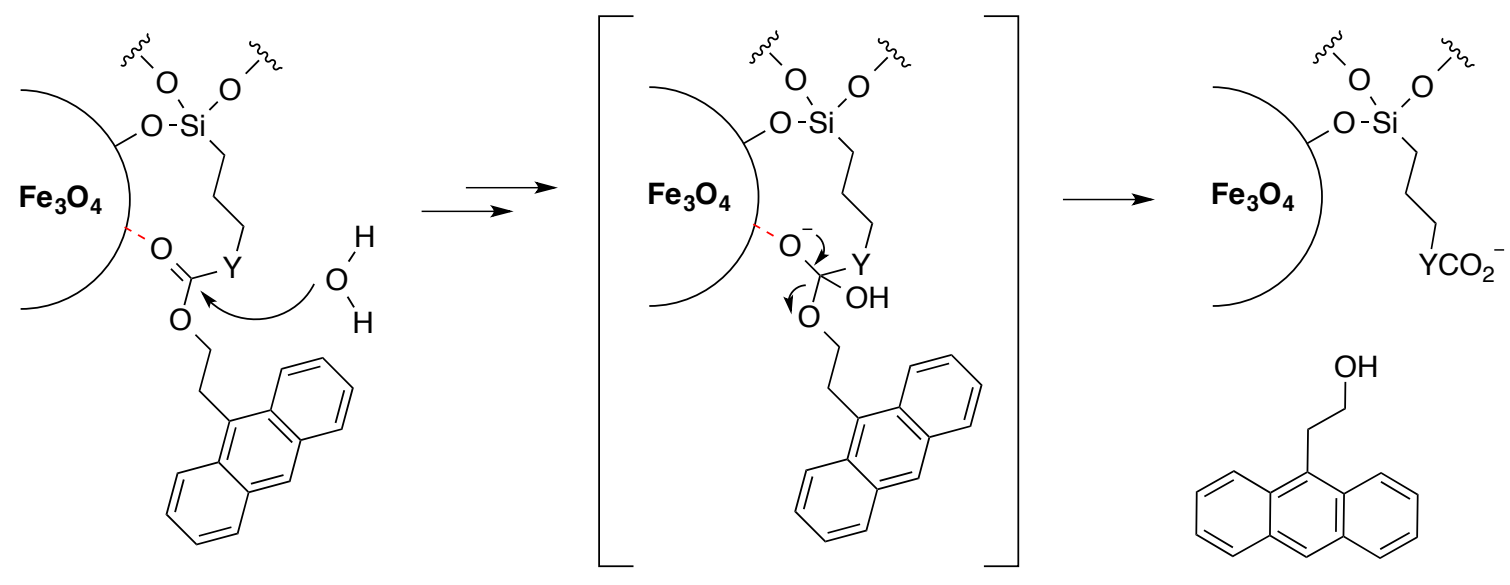

Figure 4.21. Proposed mechanism for $\mathrm{Fe}_{3} \mathrm{O}_{4} \mathrm{NP}$ accelerated hydrolysis of esters, carbonates and carbamates under mild conditions.

\subsubsection{Suppression of Hydrolysis}

Our attention was now turned to possibly masking the Lewis acidity at the surface of the magnetic NPs. The first approach we tried was to coat the functionalized NPs with either the disaccharide D-lactose or the polysaccharide dextran. The idea in using the these substrates was to effectively mask the unfunctionalized portions of magnetic NPs 
(Scheme 4.5a). The hydroxyl groups of the carbohydrate or polymer would hydrogen bond to the NP surface and prevent the Lewis basic carbonyl oxygen from coordinating with the NP surface. We postulated that application of an AMF would aid in the release of the hydrogen bound carbohydrate or polymer, allowing the NP accelerated hydrolysis to occur. The second method of preventing the rapid, non-triggered hydrolysis was to coat the unfunctionalized $\mathrm{Fe}_{3} \mathrm{O}_{4}$ NPs with a silica shell and then load the linkers onto the silica surface (Scheme 4.5b). Though silica can still serve as a Lewis acid, its acidity, in comparison to iron oxides, is far less since its Lewis acid centers are developed only from silicon atoms in a strained siloxane bridge. ${ }^{57}$ In this case the strained covalent bond causes the formation of a dipole, thus causing the silicon atom to become electron deficient and Lewis acidic. By using a silica shell around the iron oxide NP we would provide a permanent shield to prevent the linkers from interacting with the iron core. The linker-NP bond would also be strengthened and easier to form since we would be seeking to form $\mathrm{Si}-\mathrm{O}-\mathrm{Si}$ bonds instead of $\mathrm{Fe}-\mathrm{O}-\mathrm{Si}$ bonds during linker loading. As was previously stated, all Metal-O-Si bonds are inferior to the highly stable $\mathrm{Si}-\mathrm{O}-\mathrm{Si}$ bonds. ${ }^{43}$ 
A

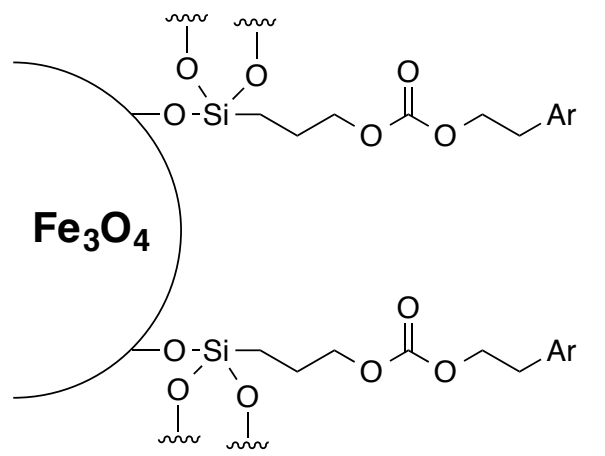

18.2

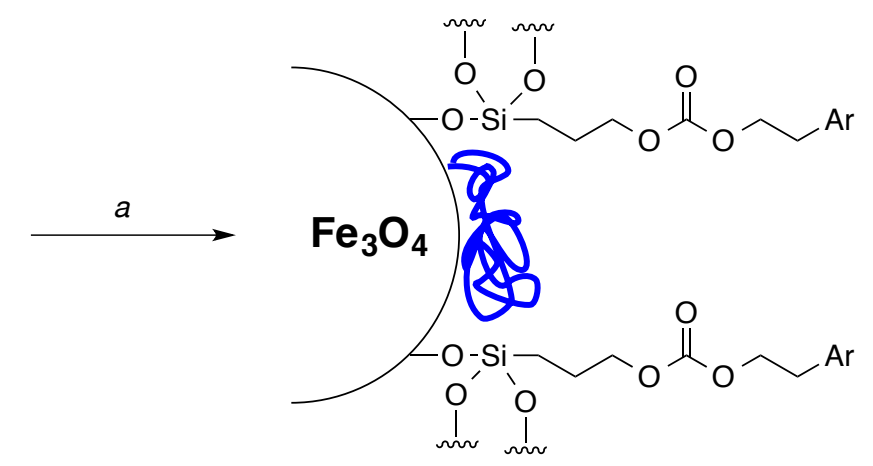

19.1: D-lactose

19.2: dextran

B
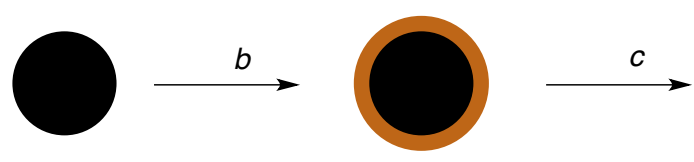

$\mathrm{Fe}_{3} \mathrm{O}_{4}$

$\mathrm{SiO}_{2} @ \mathrm{Fe}_{3} \mathrm{O}_{4}$

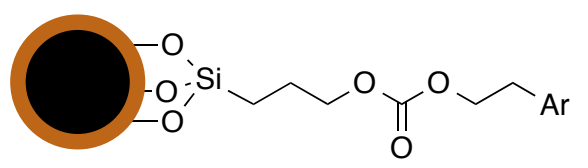

$\mathrm{Ar} @ \mathrm{SiO}_{2} @ \mathrm{Fe}_{3} \mathrm{O}_{4}$

20

Scheme 4.5. Attempts to mask the iron oxide NP surface. A: coating the functionalized NPs with polysaccharides; B: coating the iron oxide core with a silica shell. Conditions: $a$. D-lactose or dextran $(6 \mathrm{kd}) ; b$. tetraethyl orthosilicate, $\mathrm{NH}_{4} \mathrm{OH}, \mathrm{EtOH}, \mathrm{H}_{2} \mathrm{O}$; c. i. 17.2, $(\mathrm{EtO})_{3} \mathrm{SiH}$, cat. $\mathrm{PtO}_{2}, 90{ }^{\circ} \mathrm{C}$, ii. add alkoxysilane to NPs.

The $37{ }^{\circ} \mathrm{C}$ incubation experiment was repeated to compare the release rate of $\mathbf{1 8 . 2}$ with that of 19.1-2 and 20 and to see if we were able to effectively minimize non-AMF induced hydrolysis. As can be seen from Figure 4.22, the polysaccharides did slightly reduce the rate of hydrolysis with $\mathbf{1 9 . 2}$ being more effective. Since the vast majority of the non-AMF induced hydrolysis occurred soon after the incubation began, Figure 4.22 has focused only on the first 2 hours of incubation. This was especially true at the beginning of the experiment but the lack of a covalent bond resulted in a large amount of the mask dissociating from the NP surface. The silica shell of $\mathbf{2 0}$ greatly increased the 
hydrolytic stability of the linker. We continued all further experiments with $\mathrm{SiO}_{2} @ \mathrm{Fe}_{3} \mathrm{O}_{4}$ NPs.

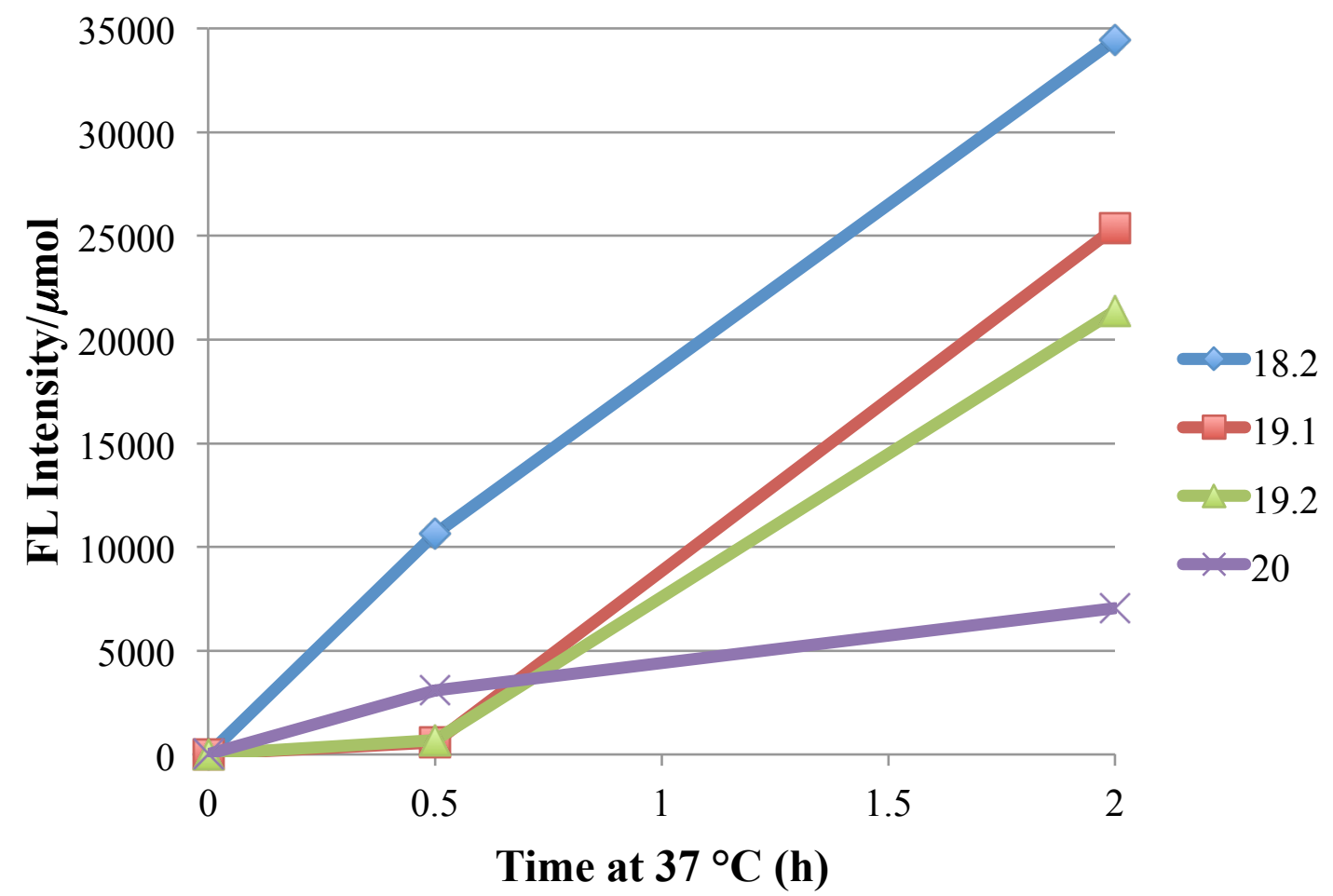

Figure 4.22. Non-triggered (i.e., no AMF application) hydrolysis of $\sim 7$ mg 18.2 compared with the suppressed hydrolysis of 19.1-2 and 20 at 37 ${ }^{\circ} \mathrm{C}$ in 2:1 PBS:MeCN pH 7.4.

\subsubsection{Commencement of $\mathrm{SiO}_{2} @ \mathrm{Fe}_{3} \mathrm{O}_{4} \mathrm{NPs}$}

With non-AMF induced hydrolysis sufficiently minimized, we returned to the investigation of AMF-induced intramolecular cyclization. Using the kinetic study discussed in Chapter 3 (Figure 3.11 and Table 3.2), we selected 9 as the optimal linker. It afforded thermal stability over short periods of time at $55{ }^{\circ} \mathrm{C}$ while remaining thermally responsive over extended intervals. Consequently, we set out to evaluate the feasibility 
of AMF-mediated oxazolidinone formation as a mechanism for drug release by covalently attaching 9 to $\mathrm{SiO}_{2} @ \mathrm{Fe}_{3} \mathrm{O}_{4}$ NPs (Scheme 4.6).

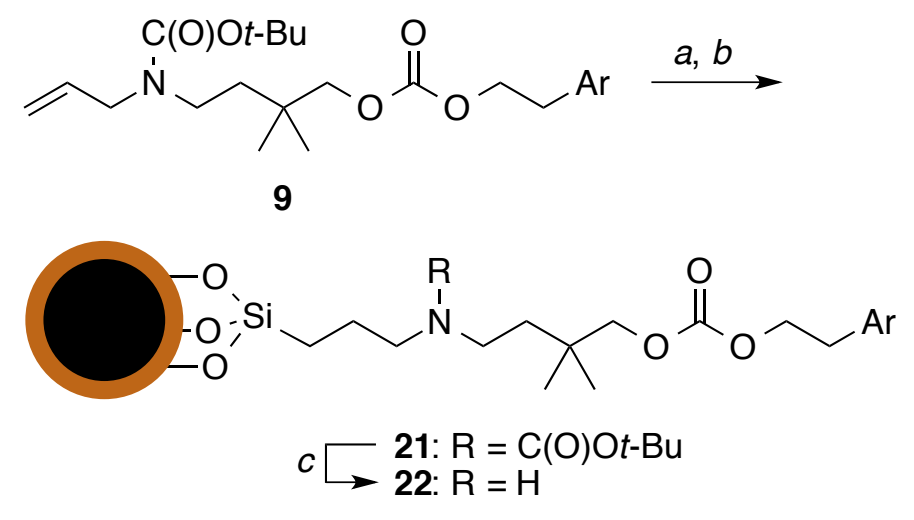

Scheme 4.6. Synthesis of NP-carbonate linker conjugates with anthracenyl fluorophore (Ar $=9$-anthracenyl). Conditions: $a$. (EtO) $)_{3} \mathrm{SiH}$, cat. $\mathrm{PtO}_{2}, 90{ }^{\circ} \mathrm{C} ; b . \mathrm{SiO}_{2} @ \mathrm{Fe}_{3} \mathrm{O}_{4}$, EtOH, $\mathrm{H}_{2} \mathrm{O} ;$ c. i. $\mathrm{CF}_{3} \mathrm{CO}_{2} \mathrm{H}: \mathrm{CH}_{2} \mathrm{Cl}_{2}, 1: 1,0{ }^{\circ} \mathrm{C}, 1 \mathrm{~h}$; ii. $\mathrm{Et}_{3} \mathrm{~N}: \mathrm{CH}_{2} \mathrm{Cl}_{2}, 1: 1, \mathrm{rt}$.

After adding the alkoxysilane to 9 through the $\mathrm{PtO}_{2}$-catalyzed hydrosilylation described by Sabourault et al. ${ }^{58}$ the linker was bound to $\mathrm{SiO}_{2} @ \mathrm{Fe}_{3} \mathrm{O}_{4}$ NPs. The Boc group was once again cleaved by treatment with trifluoroacetic acid followed by neutralization with triethylamine to afford $\mathbf{2 2}$ with the unmasked nucleophilic amine needed for the AMF-induced intramolecular cyclization. ${ }^{59}$ The success of the Bocdeprotection could be qualitatively observed by the formation of a white cloud when the 1:1 triethylamine: $\mathrm{CH}_{2} \mathrm{Cl}_{2}$ solution was added to the coated NPs. The basic solution was added after the removal of excess TFA with five washes of $\mathrm{CH}_{2} \mathrm{Cl}_{2}$.

A suspension of the free-amine NPs 22 in a 2:1 mixture of PBS:acetonitrile ( $\mathrm{pH}$ adjusted to 7.4) at room temperature was sequentially pulsed with an AMF for six 5- 
minute bursts with a delay time of 5 minutes between pulses. Magnetic-assisted sedimentation of the NPs allowed facile measurement of supernatant fluorescence at the end of each pulse (Figure 4.23). To confirm that we were releasing only the 2-(9anthracenyl)ethanol and not another fragment we analyzed the 2:1 PBS:acetonitrile supernatant that was used to suspend 22 during AMF exposure using matrix-assisted laser desorption ionization (MALDI) time of flight (TOF) mass spectrometry (MS) (Figure 4.24). The data show an AMF-promoted release of 2-(9-anthracenyl)ethanol (Figure 4.25) presumably as a consequence of the expected amine addition to the proximal carbonate moiety. 


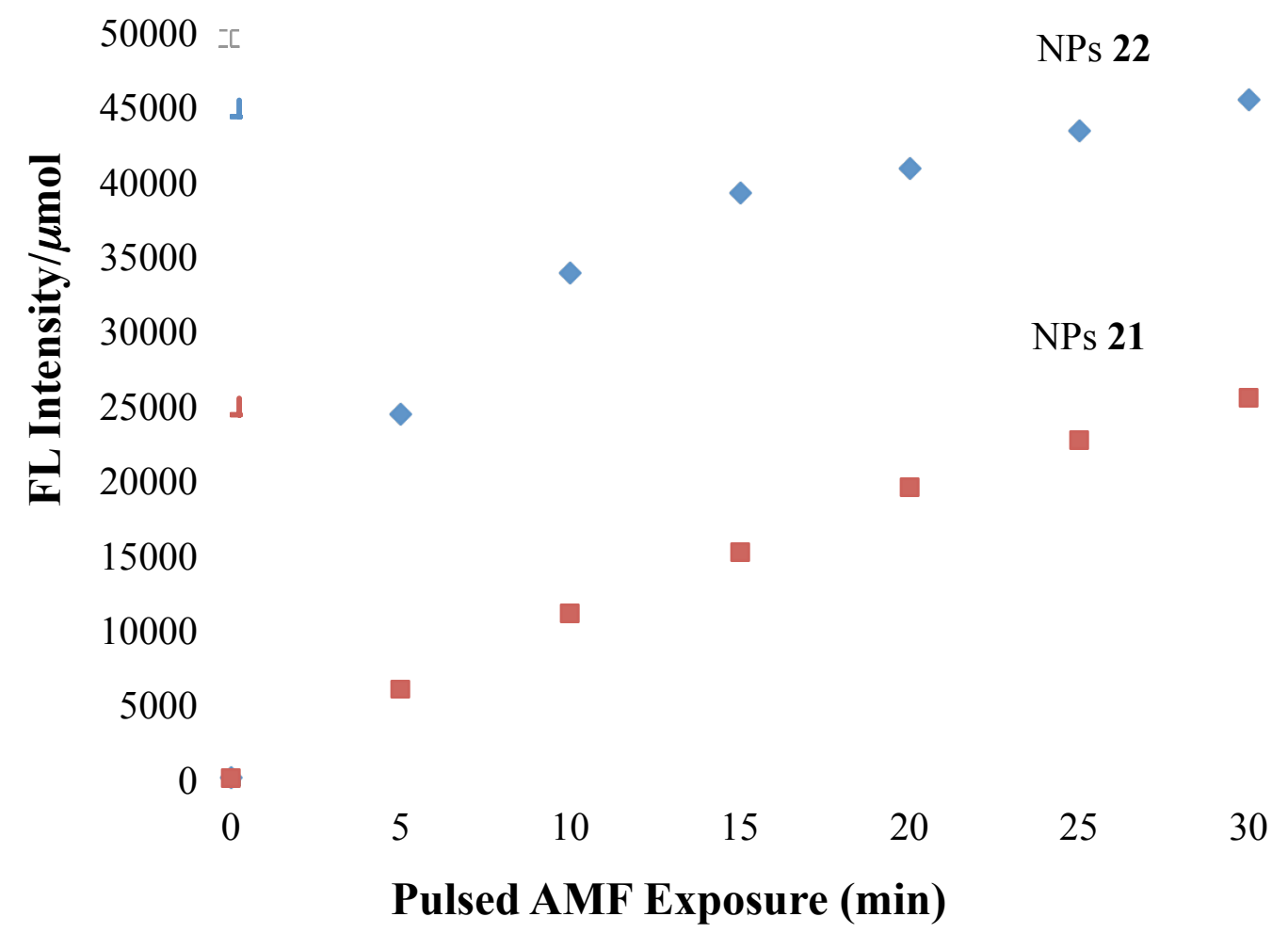

Figure 4.23. AMF-induced release of anthracene fluorophore from NPs 21 (red) and 22 (blue). NPs (6-8 mg) suspended in 2:1 PBS:acetonitrile $(0.75 \mathrm{~mL})$ at $\mathrm{pH} 7.4$ were irradiated with an AMF (501.6 amps, $204 \mathrm{kHz})$ for 5-minute bursts followed by 5-minute intervals. Vertical axis shows fluorescence intensity normalized to mmol of bound anthracene fluorophore as determined by thermogravimetric analysis. 


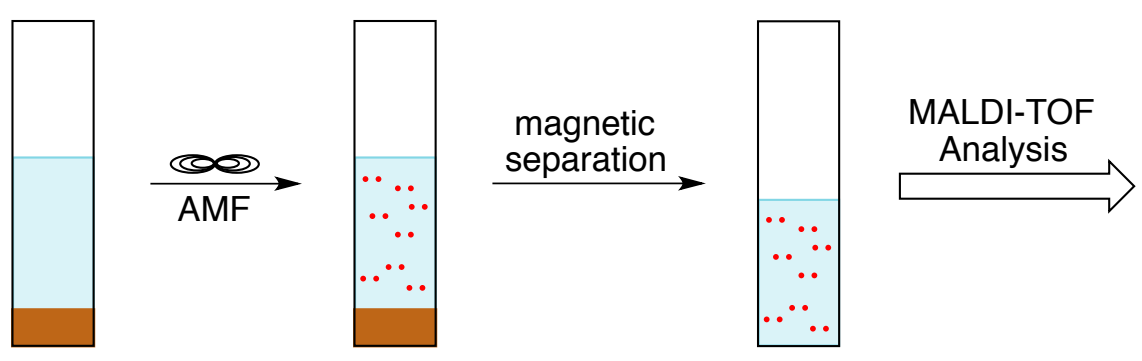

$\begin{aligned} & =\text { functionailzed } \mathrm{SiO}_{2} @ \mathrm{Fe}_{3} \mathrm{O}_{4} \mathrm{NPS} \\ \square & =2: 1 \text { PBS:acetonitrile solution } \\ \because \cdots & =\text { released fluorophore }\end{aligned}$

Figure 4.24. Procedure used to obtain sample for MALDI-TOF analysis and verification of released substrate.

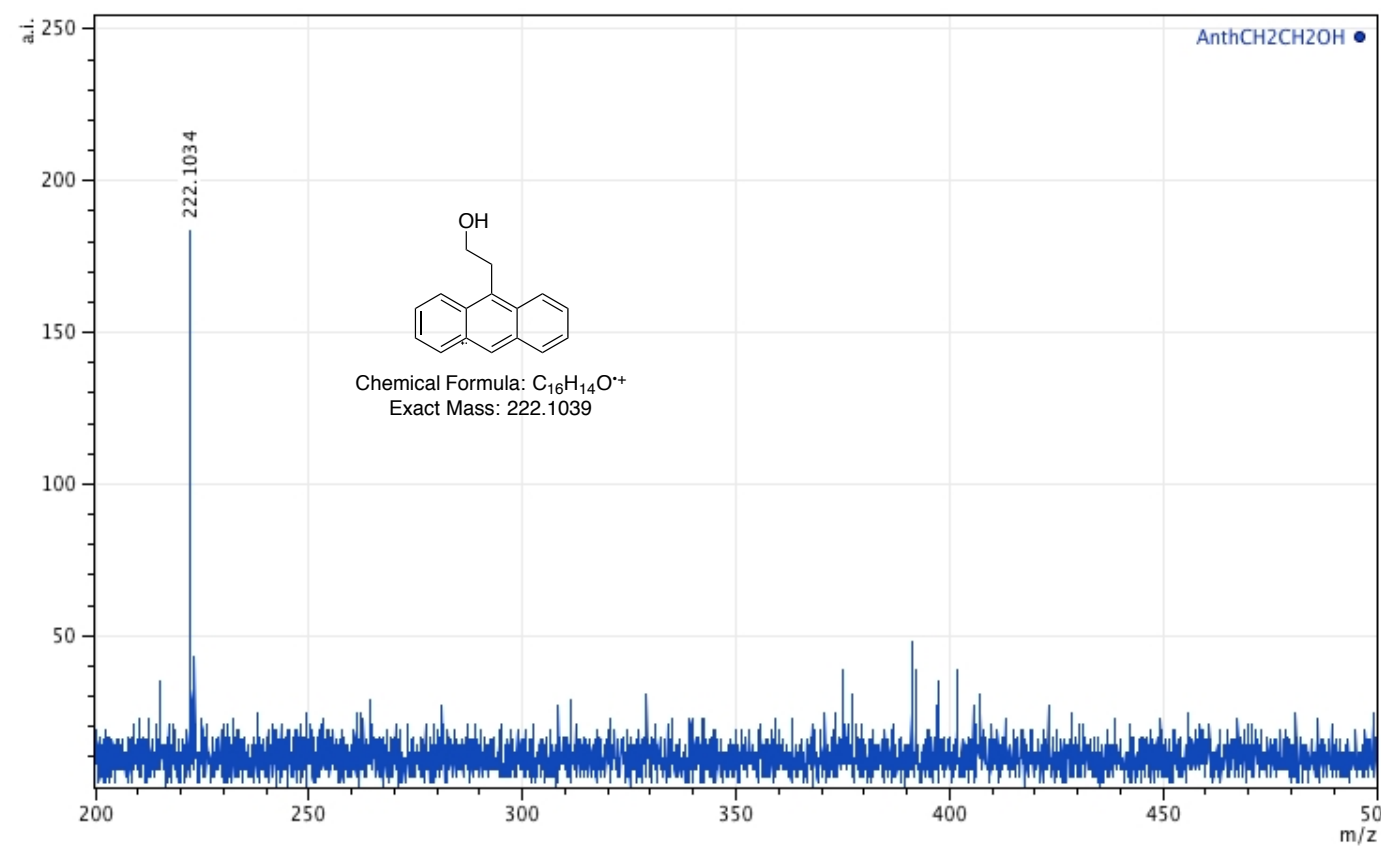

Figure 4.25. MALDI-TOF spectrum using a 2,5dihydroxybenzoic acid matrix of the radical carbocation ${ }^{60} 2-(9-$ anthracenyl)ethanol released from 22. The sample was acquired from the 2:1 PBS:acetonitrile supernatant used during the AMF treatment of $\mathbf{2 2}$. 
To test the proposed mechanism of substrate release, we examined the Bocprotected NPs 21, which would be incapable of undergoing the postulated intramolecular cyclization, under identical conditions. To our surprise, fluorophore release from $\mathbf{2 1}$ also occurred in response to AMF irradiation, albeit at a rate slower than the free amine formulation (Figure 4.23). This result pointed toward enhanced hydrolysis as a function of the energy deposited by AMF irradiation. Despite the reduced Lewis acidity of the $\mathrm{SiO}_{2} @ \mathrm{Fe}_{3} \mathrm{O}_{4} \mathrm{NPs}$, the significant, rapid heating within nanometers of an iron oxide NP surface could still explain this phenomenon. ${ }^{61}$ On examination of the stability of NPs $\mathbf{2 1}$ under the same conditions but without AMF irradiation, we noted the gradual release of 2-(anthracen-9-yl)ethan-1-ol over an hour at $37{ }^{\circ} \mathrm{C}$ to $\sim 50 \%$ of the cumulative level achieved by the six 5-minute bursts of AMF irradiation. This finding, while precluding the use of $\mathbf{2 1}$ (or 22) as a drug delivery system, is noteworthy because dialkylcarbonates are stable to such mild conditions and generally require highly basic conditions for their hydrolysis (e.g., $5 \mathrm{M} \mathrm{NaOH}, \mathrm{MeOH}, \mathrm{rt}, 3 \mathrm{~d}^{53}$ ). As a control, exposure of free carbonate 9 (Scheme 4.6) to $\mathrm{SiO}_{2} @ \mathrm{Fe}_{3} \mathrm{O}_{4} \mathrm{NPs}$ in 2:1 phosphate buffer solution (PBS):acetonitrile at $37^{\circ} \mathrm{C}$ resulted in no reaction, even after $24 \mathrm{~h}$ exposure. Consequently, we surmised that the close proximity and tethered relationship of the carbonate linkage in $\mathbf{2 1}$ to the iron oxide core and silica shell enhance, possibly through Lewis acid or hydrogen bonding interactions, respectively, the rate of hydrolysis of this linkage. This reasoning supports the previously postulated mechanism (Figure 4.21). 


\subsubsection{Monodispersed $\mathrm{SiO}_{2} @ \mathrm{Fe}_{3} \mathrm{O}_{4}$ Nanoparticles}

To gain a better understanding of 21, we had Dr. Jacek Jasinski (Conn Center for Renewable Energy, University of Louisville) obtain transmission electron microscopy (TEM) images. As can be seen from the High Angle Annular Dark Field (HAADF) scanning transmission electron microscopy (STEM) images in Figure 4.26, the iron oxide NPs are largely aggregated and the silica shell is visible (a somewhat cloudy surrounding) at the outer perimeter of each iron cluster. Iron oxide NPs are notorious for aggregating due to their anisotropic dipolar attraction. ${ }^{62}$ The most popular method of internalization of NPs into cells is through endocytosis. ${ }^{63}$ For clathrin-mediated endocytosis, the NPs can be up to $100 \mathrm{~nm}$ in diameter and for caveolae mediated endocytosis the diameter can be no greater than $60-80 \mathrm{~nm} .{ }^{64}$ As is evident, the mandates of endocytosis make NP aggregation an undesirable attribute for drug delivery. 


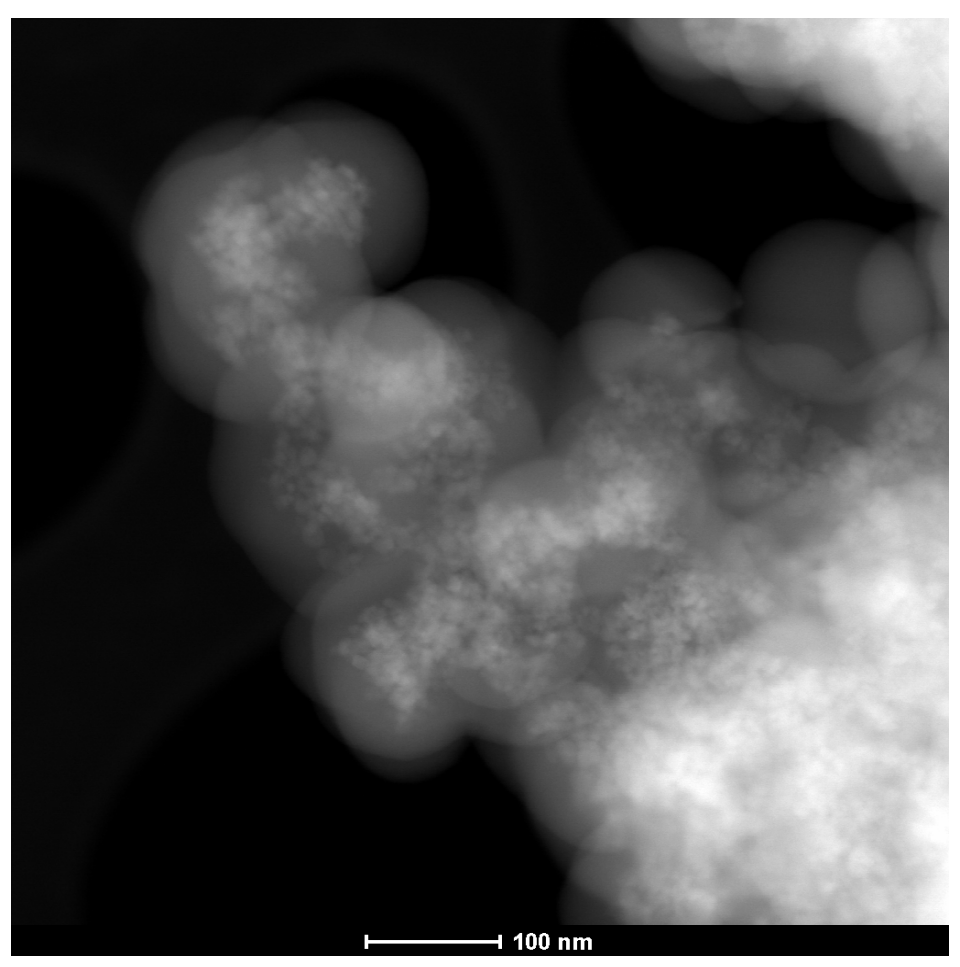

Figure 4.26. HAADF Z-contrast STEM image of aggregated $\mathrm{Fe}_{3} \mathrm{O}_{4}$ NPs with a thick silica shell surrounding them.

To prevent the aggregation the $\mathrm{Fe}_{3} \mathrm{O}_{4}$ NPs, a surfactant must immediately be applied to the surface of the NP. One of the most common surfactants used in the synthesis of mondispersed $\mathrm{Fe}_{3} \mathrm{O}_{4}$ NPs is oleic acid or its sodiated derivative. Following a two-step route described by Park et al. ${ }^{65}$ we synthesized oleate-coated $\mathrm{Fe}_{3} \mathrm{O}_{4} \mathrm{NPs}$ via high-temperature $\left(320^{\circ} \mathrm{C}\right)$ decomposition of the organic precursor that was generated in situ. $\mathrm{Fe}_{3} \mathrm{O}_{4}$-oleate NPs can also be prepared through a co-precipitation method as reported by Jiang et al. ${ }^{66}$ After obtaining the magnetic NPs we attempted to add the silica shell to the surface. Silica shells are usually added to iron oxide NPs using the Stöber process, ${ }^{50}$ but the presence of the oleate surfactant prevented the NPs from being dispersible in the standard ethanol/water mixture. To circumvent this issue using 
microemulsion, Ding and coworkers ${ }^{67}$ suspended the $\mathrm{Fe}_{3} \mathrm{O}_{4}$-oleate NPs in cyclohexane that was basified with $\mathrm{NH}_{4} \mathrm{OH}$. Using another surfactant, Igepal CO-520, the oleate surfactant is removed and replaced by the Igepal CO-520. This surfactant exchange allows the ammonia, in the form a droplet containing the hydrolyzed tetraethyl orthosilicate (TEOS), to draw near to the NP and deposit the silica onto the NP surface (Scheme 4.7). Though similar methods using Igepal CO-520 are frequently reported, ${ }^{68}$ we found the collection of the $\mathrm{SiO}_{2} @ \mathrm{Fe}_{3} \mathrm{O}_{4} \mathrm{NPs}$ to be very difficult. Magnetic separation cannot be used and centrifugation causes both the NPs and the surfactant to form a gelled pellet, resulting in an unusable paste. When the Stöber process was attempted with the $\mathrm{Fe}_{3} \mathrm{O}_{4}$-oleate NPs there was rampant aggregation resulting in particles with a hydrodynamic diameter of $2065.2 \pm 284.6 \mathrm{~nm}$. 

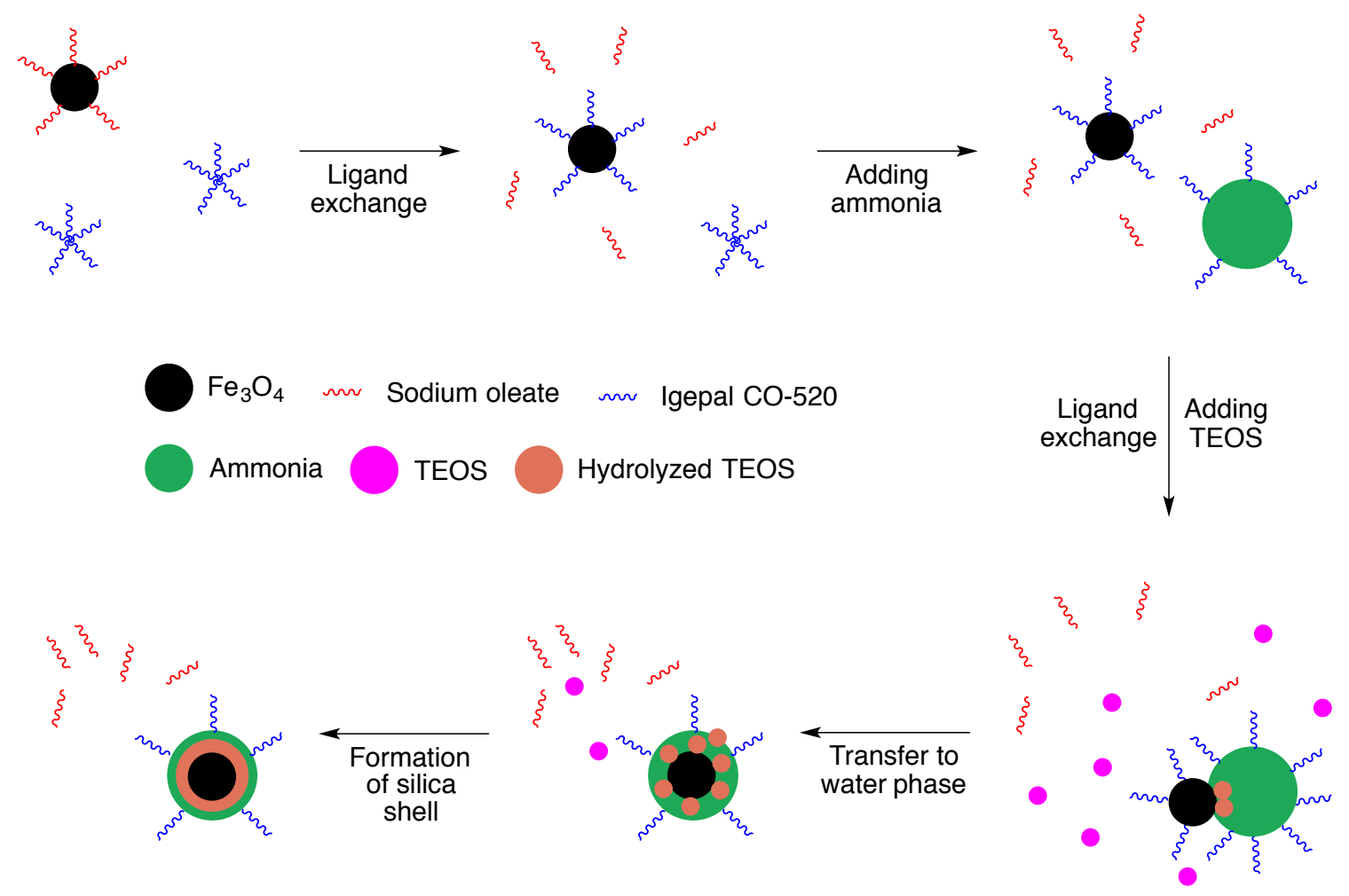

Scheme 4.7. Illustration of the microemulsion coating mechanism of $\mathrm{SiO}_{2}$ on the surface of $\mathrm{Fe}_{3} \mathrm{O}_{4}$-oleate NPs. ${ }^{67}$

We also investigated the use of hydrophilic surfactants. Jing et al. ${ }^{69}$ used iron(II) sulfate in an oxidative coprecipitation synthesis of $\mathrm{Fe}_{3} \mathrm{O}_{4} \mathrm{NPs}$. Briefly, $\mathrm{FeSO}_{4} \cdot 7 \mathrm{H}_{2} \mathrm{O}$ and sodium citrate were co-added to an aqueous solution of sodium nitrate and potassium hydroxide. The solution was heated to $90{ }^{\circ} \mathrm{C}$ for $5 \mathrm{~h}$ under an inert atmosphere before cooling to room temperature and precipitating the NPs from solution with acetone. The NPs could then be easily collected by magnetic separation. These NPs, though easy to suspend in water, still showed signs of aggregation with a hydrodynamic diameter of $294.8 \pm 11.7 \mathrm{~nm}$. When the silica shell was added to the $\mathrm{Fe}_{3} \mathrm{O}_{4}$-citrate NPs the hydrodynamic diameter increased to $453.8 \pm 8.8 \mathrm{~nm}$. 
We then looked into the use of commercial ferrofluid containing $\mathrm{Fe}_{3} \mathrm{O}_{4} \mathrm{NPs}$ (EMG 304, FerroTec). We chose a procedure reported by Lu and coworkers ${ }^{62}$ due to its rapid application (3 hours) of a thin silica shell around $\mathrm{Fe}_{3} \mathrm{O}_{4} \mathrm{NPs}$. As mentioned previously, as the thickness of the silica shell increases there is a sharp reduction in the magnetism of the NP. ${ }^{49}$ This reduction in magnetism decreases the AMF-induced energy and could potentially eliminate our ability to activate thermally-induced reactions. The dynamic light scattering (DLS) measurements showed a hydrodynamic diameter of $310 \pm 3.7 \mathrm{~nm}$ with a polydispersity of $0.23 \pm 0.01$ (Figure 4.27). The $\xi$-Potential of the $\mathrm{SiO}_{2} @ \mathrm{Fe}_{3} \mathrm{O}_{4}$ NPs was $-19.50 \pm 9.12 \mathrm{mV}$ with a mobility of $-0.39 \pm 0.18$. Though the DLS measurements still showed two distinct sizes of NPs with a larger than desired diameters we proceeded to obtain TEM images because Lu et al. ${ }^{62}$ had also used EMG 304 ferrofluid to create their $\mathrm{SiO}_{2} @ \mathrm{Fe}_{3} \mathrm{O}_{4}$ NPs. The TEM (Figure 4.28) show a very thin silica shell but there is large-scale aggregation as well.

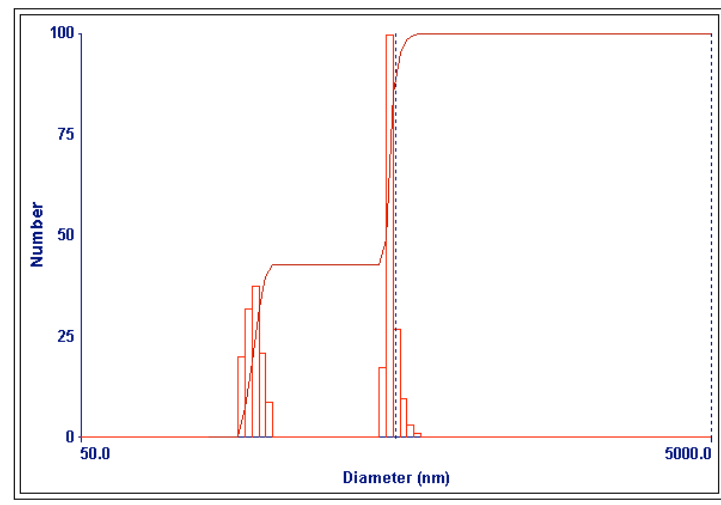

Figure 4.27. DLS of $\mathrm{SiO}_{2} @ \mathrm{Fe}_{3} \mathrm{O}_{4}$ NPs in EtOH showing the hydrodynamic diameter at $310 \mathrm{~nm}$ with a polydispersity of 0.23 . 


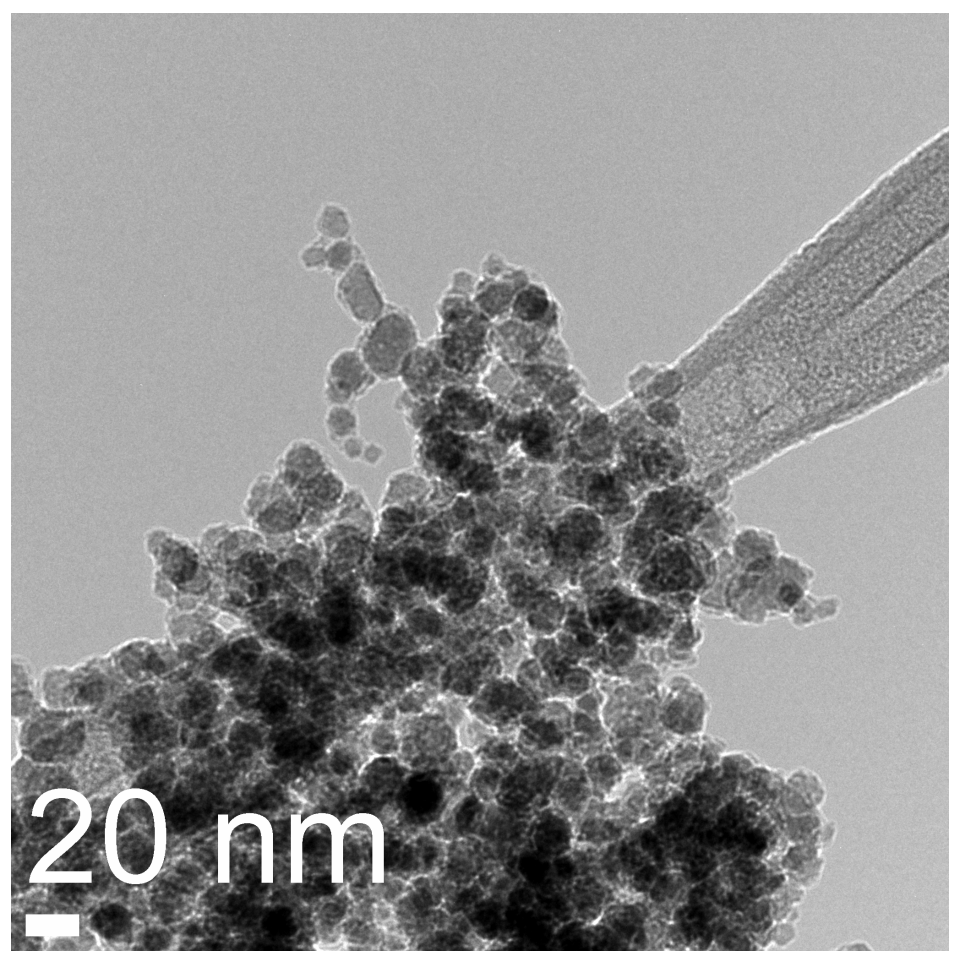

Figure 4.28. TEM image of $\mathrm{SiO}_{2} @ \mathrm{Fe}_{3} \mathrm{O}_{4} \mathrm{NPs}$ synthesized from EMG 304 showing excessive aggregation.

Because we were unable to reproduce the results of $\mathrm{Lu}$ et al., ${ }^{62}$ we moved to a modified version of the Stöber process as reported by Pinho et al. ${ }^{70}$ This procedure was especially attractive as it included an equation, that allowed for the thickness of the silica shell to be adjusted by varying the amount of TEOS. Using the volume of TEOS needed to afford a $4 \mathrm{~nm}$ thick silica shell, $\mathrm{Fe}_{3} \mathrm{O}_{4}$ NPs in the EMG 304 ferrofluid with a reported average diameter of $10 \mathrm{~nm}$ were silylated to form monodispersed superparamagnetic (Figure 4.29) $\mathrm{SiO}_{2} @ \mathrm{Fe}_{3} \mathrm{O}_{4} \mathrm{NPs}$ with a particle size distribution of $24 \pm 6 \mathrm{~nm}$ and a silica shell thickness of 6-7 nm, as determined by TEM (Figure 4.30). The monodispersed $\mathrm{SiO}_{2} @ \mathrm{Fe}_{3} \mathrm{O}_{4} \mathrm{NPs}$ had a $\zeta-$ Potential of $-46.58 \pm 0.47 \mathrm{mV}$ and a mobility of $-3.64 \pm 0.04$ in Millipore water. The DLS measurements (Figure 4.31) showed a hydrodynamic diameter of $103 \mathrm{~nm}$ and a polydispersity of 0.17 . 


$$
V_{\text {TEOS }}=N_{\text {part }}\left[\frac{\rho_{\mathrm{SiO}_{2}} M_{\text {TEOS }}}{M_{\mathrm{SiO}_{2}} \rho_{\text {TEOS }}}\right]\left[\frac{4}{3} \pi\left(\left(r+e_{\text {shell }}\right)^{3}-r^{3}\right)\right]
$$

Equation 4.1. Equation used to calculate the volume of TEOS required to obtain a silica shell of a desired thickness, where $V_{T E O S}$ is the volume of TEOS required, $N_{\text {part }}$ is the number of $\mathrm{Fe}_{3} \mathrm{O}_{4} \mathrm{NPs}$ to be coated, $\rho_{\mathrm{SiO}_{2}}$ is the density of silica, $M_{\mathrm{TEOS}}$ is the molecular weight of TEOS, $\mathrm{MSiO}_{2}$ is the molecular weight of silica, $\rho_{\mathrm{TEOS}}$ is the density of TEOS, $r$ is the average radius of the $\mathrm{Fe}_{3} \mathrm{O}_{4} \mathrm{NPs}$, and $e_{\text {shell }}$ is the desired thickness of the silica shell.

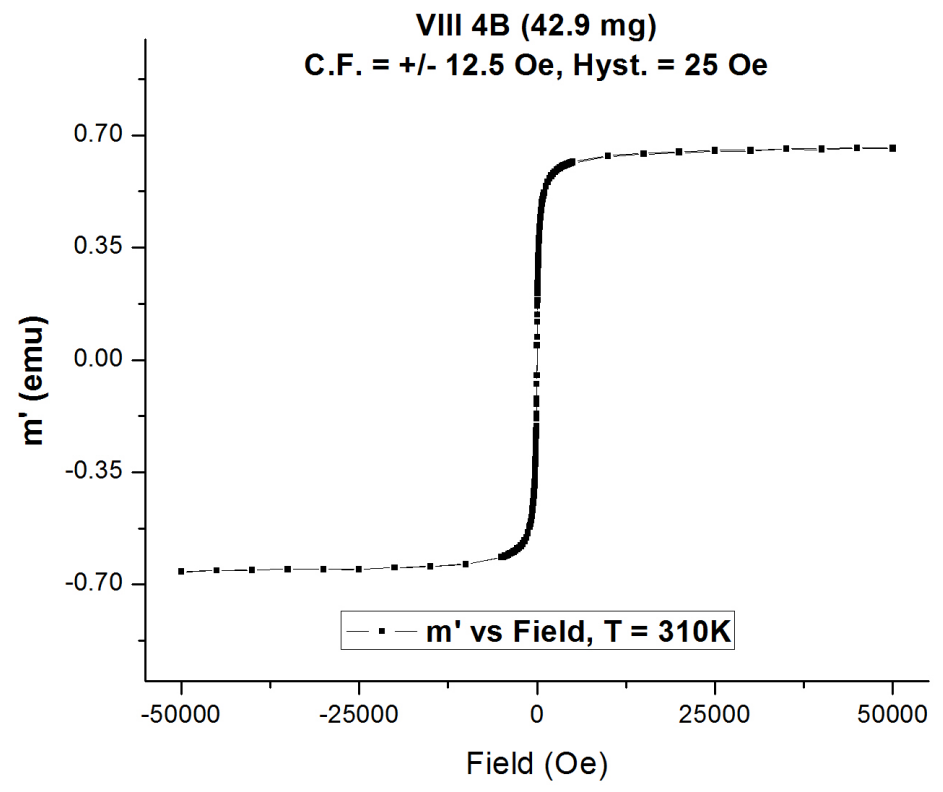

Figure 4.29. Superconducting quantum interference device (SQUID) measurement of $\mathrm{SiO}_{2} @ \mathrm{Fe}_{3} \mathrm{O}_{4}$ NPs prepared from EMG 304. Measurements were taken at temperatures from 2 to $400 \mathrm{~K}$ and magnetization data was taken by cycling from $-5 \mathrm{~T}$ to $5 \mathrm{~T}$. SQUID measurements were taken by Professor Lance DeLong, University of Kentucky. 

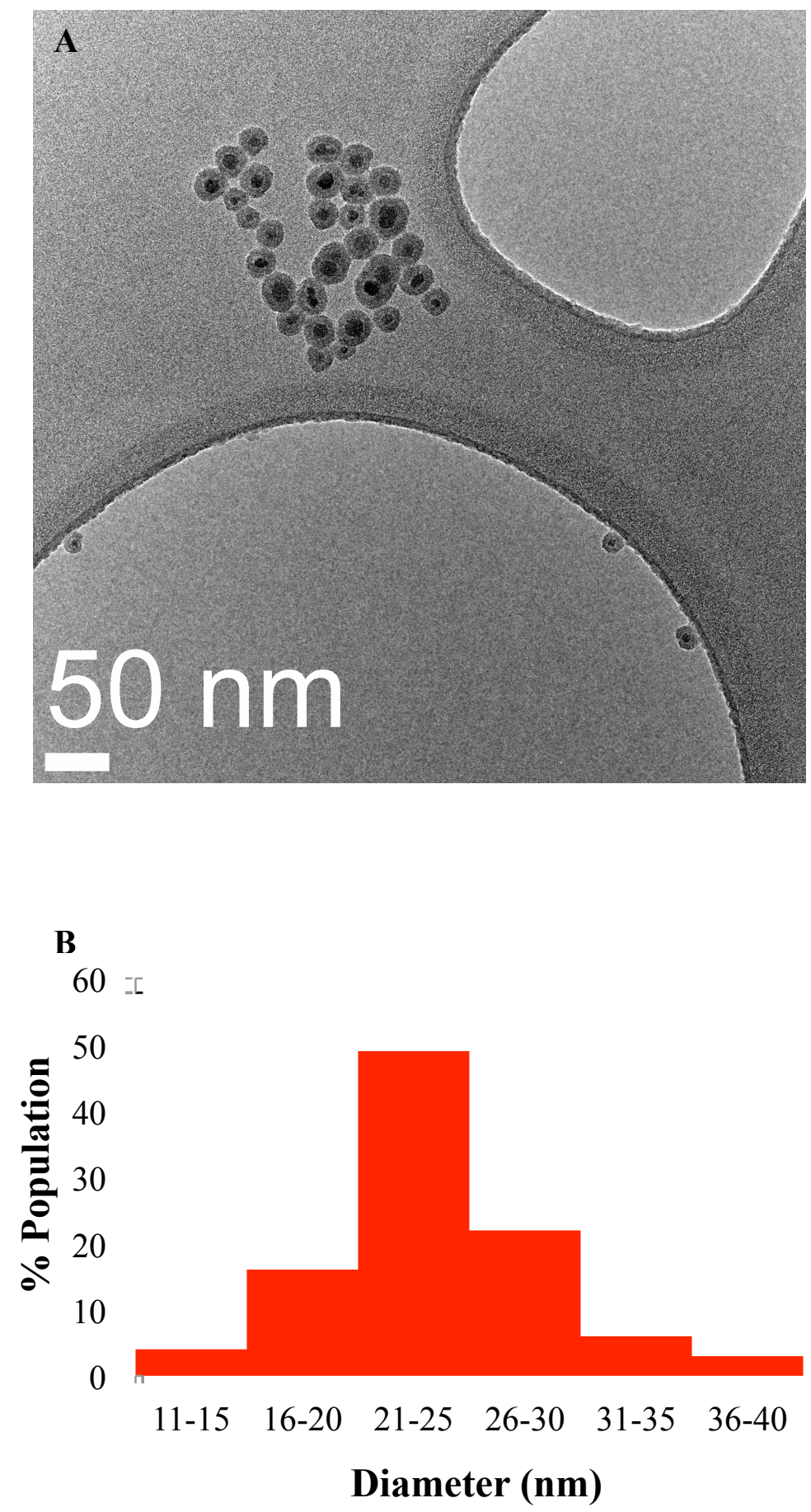

Figure 4.30. A. TEM image of $\mathrm{SiO}_{2} @ \mathrm{Fe}_{3} \mathrm{O}_{4} \quad \mathrm{NPs}$ synthesized from EMG 304 with a B. particle size distribution of $24 \pm 6 \mathrm{~nm}$ and a silica shell thickness of 6-7 nm. 

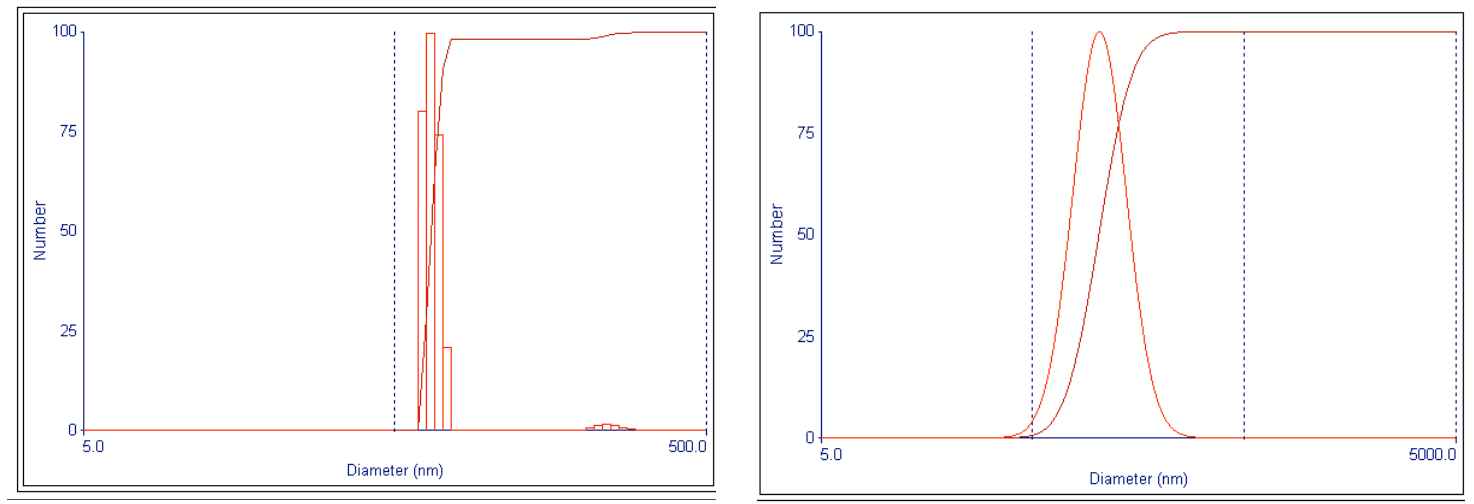

Figure 4.31. DLS of $\mathrm{SiO}_{2} @ \mathrm{Fe}_{3} \mathrm{O}_{4} \mathrm{NPs}$ in Millipore water showing the hydrodynamic diameter at $103 \mathrm{~nm}$ with a polydispersity of 0.17 .

When the monodispersed $\mathrm{SiO}_{2} @ \mathrm{Fe}_{3} \mathrm{O}_{4}$ NPs with a $10 \mathrm{~nm} \mathrm{Fe} \mathrm{O}_{4}$ core and a 6-7 nm thick silica shell were subjected to pulsed AMF irradiation at a concentration of $7 \mathrm{mg}$ of NPs in 2:1 PBS:acetonitrile $(0.75 \mathrm{~mL})$, the temperature of the bulk suspension, as confirmed by measurements taken using a fiber optic temperature sensor, increased +16.4 $\pm 0.3{ }^{\circ} \mathrm{C}$ from the start of the AMF pulse to the end of the AMF pulse and increased +4 ${ }^{\circ} \mathrm{C}$ from start of the first pulse to the start of the sixth pulse (Figure 4.32). 


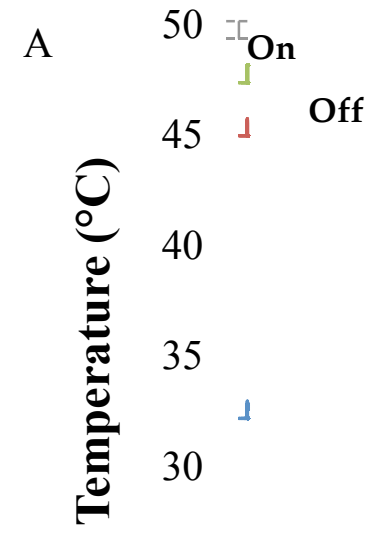

25

$\begin{array}{rr}20 & \text { n } \\ & 0\end{array}$

20

40

Time (min)
$60 \quad 80$

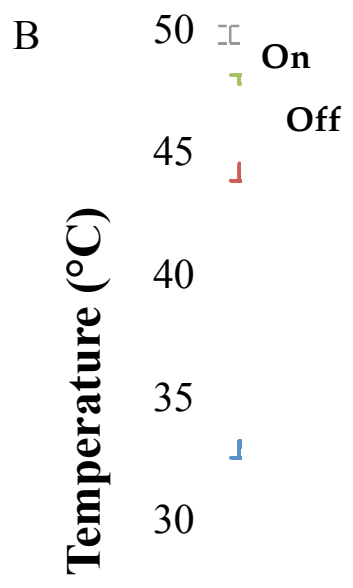

25

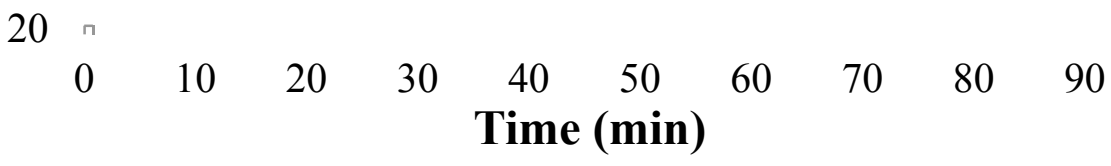

Figure 4.32. AMF-induced heating/cooling of $2: 1$ PBS:acetonitrile (0.75 mL) (blue), $\mathrm{SiO}_{2} @ \mathrm{Fe}_{3} \mathrm{O}_{4} \mathrm{NPs}(7 \mathrm{mg})$ in 2:1 PBS:acetonitrile $(0.75 \mathrm{~mL})$ (red), AMF $5 \mathrm{~min}$ on/off sequence (green). A. 5 minute pulse duration and B. 2 minute pulse duration. AMF conditions: $500 \mathrm{amps}, 204 \mathrm{kHz}$. 


\subsubsection{AMF-Induced Hydrolysis}

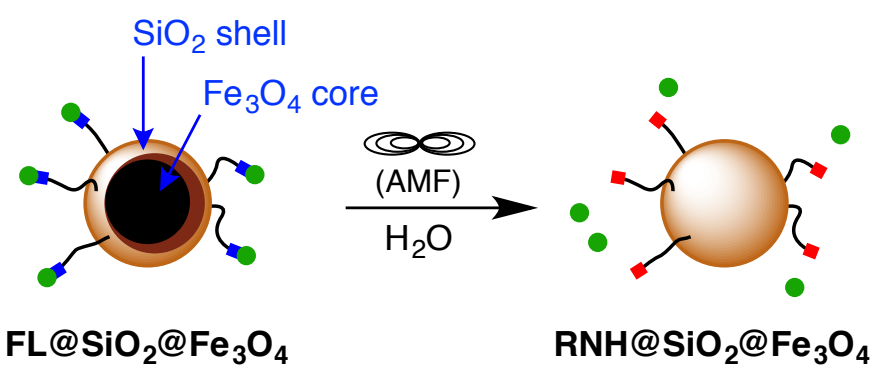

Figure 4.33. AMF-triggered hydrolysis of pendant functionality $(\mathrm{FL}=$ anthracene fluorophore, •; carbamate moiety, n; terminal amine residue, $n$ ).

Since we were still observing significant AMF-induced hydrolysis (Figure 4.23, 21) and continuing to lose $\sim 50 \%$ of the NP loading during Boc-deprotection, we decided to embrace the observed AMF-induced hydrolysis. As depicted graphically in Figure 4.33, we engineered a new linker by first removing the nucleophilic element and, second, we replaced the carbonate moiety with an $N$-methyl carbamate linkage in order to effectively increase the hydrolytic stability of the carbonyl (Scheme 4.8). Even unsubstituted carbamates are extremely resistant to hydrolysis and typically require treatment with base under forcing conditions for cleavage (e.g., $\mathrm{KOH}$, diglyme, $\left.200{ }^{\circ} \mathrm{C}\right) .{ }^{54}$ We also adopted a strategy for convenient attachment of substrate to the simplified carbamate-linked NPs, namely oximation (Schemes 4.8 and 4.9). $\mathrm{We}^{71}$ and others ${ }^{72}$ have used the click chemistry reaction $^{73}$ of NP-bound aminooxy groups with aldehydes to form oxime ethers as a convenient means for surface functionalization. Any newly formed oxime ether linkage would be sufficiently resistant to hydrolysis and thereby decrease the risk of a non-AMF induced release. ${ }^{74}$ 

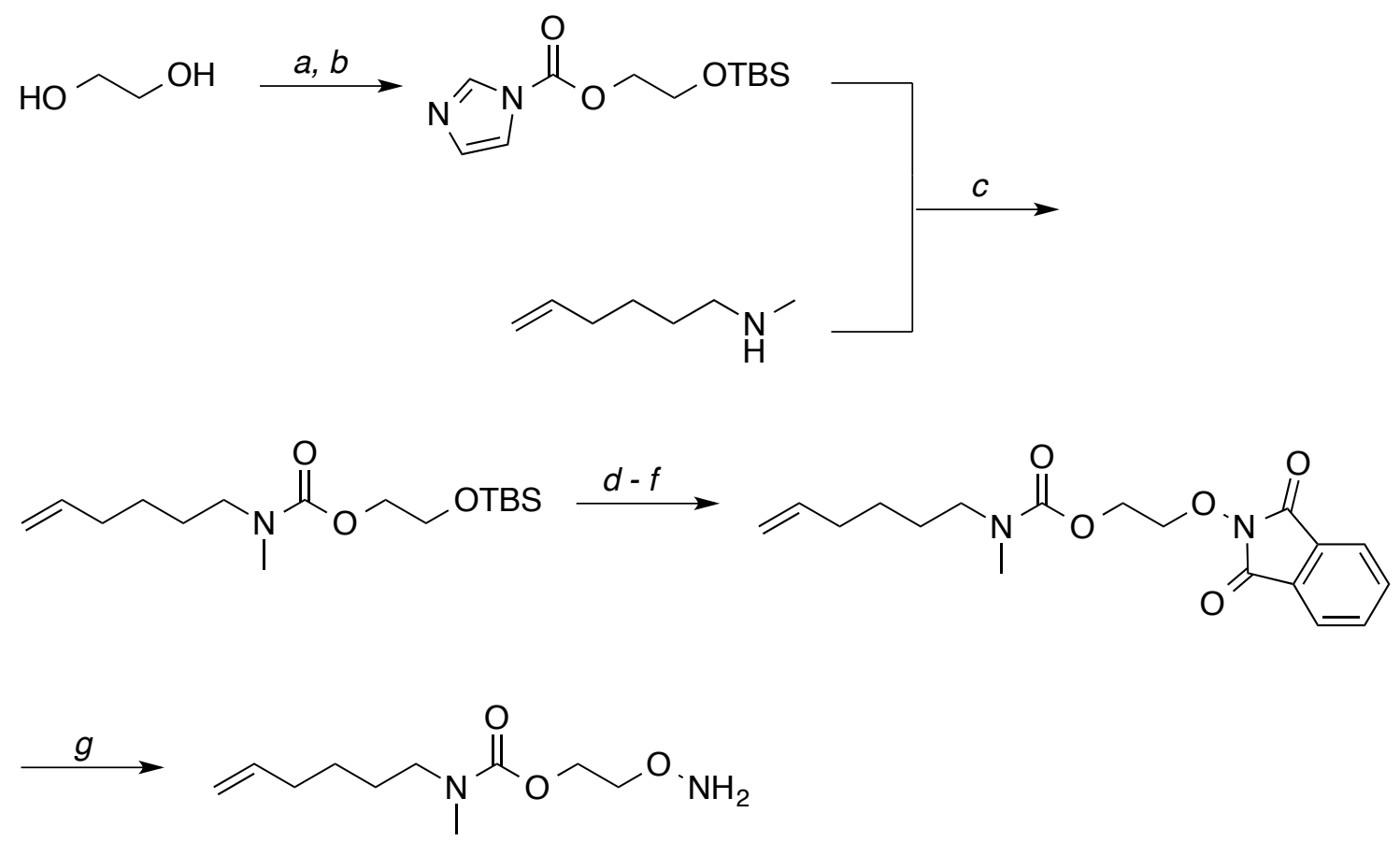

23

Scheme 4.8. Synthesis of linkers for substrate release via AMF-induced hydrolysis. Conditions: a. TBSCl, $\mathrm{Et}_{3} \mathrm{~N}, \mathrm{CH}_{2} \mathrm{Cl}_{2}, 0{ }^{\circ} \mathrm{C} ;$ b. 1,1'carbonyldiimidazole, $(i-\mathrm{Pr})_{2} \mathrm{NEt}, \mathrm{CH}_{2} \mathrm{Cl}_{2}, 0{ }^{\circ} \mathrm{C} ; c$. DBU, $\mathrm{MeCN}, \mathrm{rt}, 79 \%$ over 3 steps; $d$. TBAF, THF, $0{ }^{\circ} \mathrm{C}$; e. $\mathrm{MsCl}, \mathrm{Et}_{3} \mathrm{~N}, \mathrm{CH}_{2} \mathrm{Cl}_{2}, 0{ }^{\circ} \mathrm{C} ; f . N$ hydroxyphthalimide, $\mathrm{K}_{2} \mathrm{CO}_{3}$, DMSO, $75{ }^{\circ} \mathrm{C}, 71 \%$ over 3 steps; $g$. $\mathrm{N}_{2} \mathrm{H}_{4} \cdot \mathrm{H}_{2} \mathrm{O}, \mathrm{CH}_{2} \mathrm{Cl}_{2}, 0{ }^{\circ} \mathrm{C}, 96 \%$.

After preparing the $\mathrm{SiO}_{2} @ \mathrm{Fe}_{3} \mathrm{O}_{4} \mathrm{NPs}$, the residual ammonia from the Stöber process was removed prior to NP functionalization by placing the ethanol/water suspension of $\mathrm{SiO}_{2} @ \mathrm{Fe}_{3} \mathrm{O}_{4} \mathrm{NPs}$ under mild vacuum and then heating at $60{ }^{\circ} \mathrm{C}$ for $8 \mathrm{~h}$. Removal of the ammonia in this way decreased the $\mathrm{pH}$ of the suspension from 11.7 to 8.8. The resultant $\mathrm{SiO}_{2} @ \mathrm{Fe}_{3} \mathrm{O}_{4} \mathrm{NPs}$ then were functionalized by adding an ethanolic 
solution of the triethoxysilane-derived linker obtained from $\mathrm{PtO}_{2}$-catalyzed ${ }^{75}$ hydrosilylation of alkene $\mathbf{2 3}$ with (EtO) $)_{3} \mathrm{SiH}$ to obtain $\mathbf{2 4}$ (Scheme 4.9).

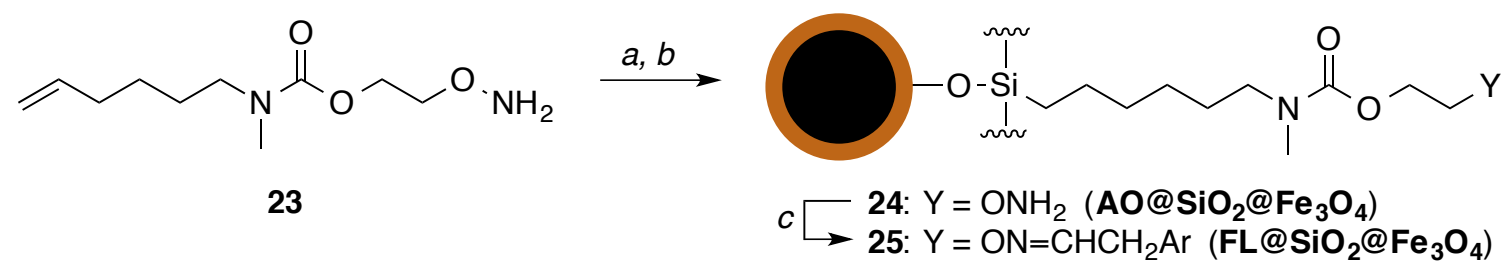

Scheme 4.9. Linkers designed for release via AMF-induced hydrolysis bound to $\mathrm{SiO}_{2} @ \mathrm{Fe}_{3} \mathrm{O}_{4} \mathrm{NPs}$. Conditions: a. (EtO) $)_{3} \mathrm{SiH}$, cat. $\mathrm{PtO}_{2}, 90{ }^{\circ} \mathrm{C}$; b. $\mathrm{SiO}_{2} @ \mathrm{Fe}_{3} \mathrm{O}_{4}, \mathrm{EtOH}, \mathrm{H}_{2} \mathrm{O}, \mathrm{rt}, 12$ h, c. 2-(9-anthryl)acetaldehyde, ${ }^{76}$ (26), EtOH, $\mathrm{H}_{2} \mathrm{O}, \mathrm{rt}, 8 \mathrm{~h}$.

The functionalization of the $\mathrm{SiO}_{2} @ \mathrm{Fe}_{3} \mathrm{O}_{4} \mathrm{NPs}$ with hydrosilylated 23 increased the shell thickness by 3 nm to afford $\mathrm{AO} @ \mathrm{SiO}_{2} @ \mathrm{Fe}_{3} \mathrm{O}_{4} \mathrm{NPs}$ with a diameter of $30 \pm 8 \mathrm{~nm}$ as determined by TEM (Figure 4.34). The hydrodynamic diameter of aqueous $\mathrm{AO} @ \mathrm{SiO}_{2} @ \mathrm{Fe}_{3} \mathrm{O}_{4} \mathrm{NPs}$ was measured at $96 \mathrm{~nm}$ with a polydispersity of 0.16 , roughly similar to the hydrodynamic diameter of the starting $\mathrm{SiO}_{2} @ \mathrm{Fe}_{3} \mathrm{O}_{4} \mathrm{NPs}$ at $103 \mathrm{~nm}$ with a polydispersity of 0.17 (Figure 4.35). We also observed little change in $\zeta$-potential, where both the $\mathrm{SiO}_{2} @ \mathrm{Fe}_{3} \mathrm{O}_{4}$ and $\mathrm{AO} @ \mathrm{SiO}_{2} @ \mathrm{Fe}_{3} \mathrm{O}_{4} \mathrm{NPs}$ registered ca. $-45 \mathrm{mV}$ as aqueous suspensions. Simple mixing of $\mathrm{AO} @ \mathrm{SiO}_{2} @ \mathrm{Fe}_{3} \mathrm{O}_{4}$ with anthracene aldehyde 26 (Scheme 4.9) in aqueous ethanol gave NPs $25\left(\mathrm{FL} @ \mathrm{SiO}_{2} @ \mathrm{Fe}_{3} \mathrm{O}_{4}\right)$. In an alternate approach, we conducted the oximation step first by reacting anthracene aldehyde $\mathbf{2 6}$ with $\mathbf{2 3}$ (Scheme 4.9) followed by hydrosilylation of the oxime ether adduct and then loading onto $\mathrm{SiO}_{2} @ \mathrm{Fe}_{3} \mathrm{O}_{4} \mathrm{NPs}$ to obtain 25. 

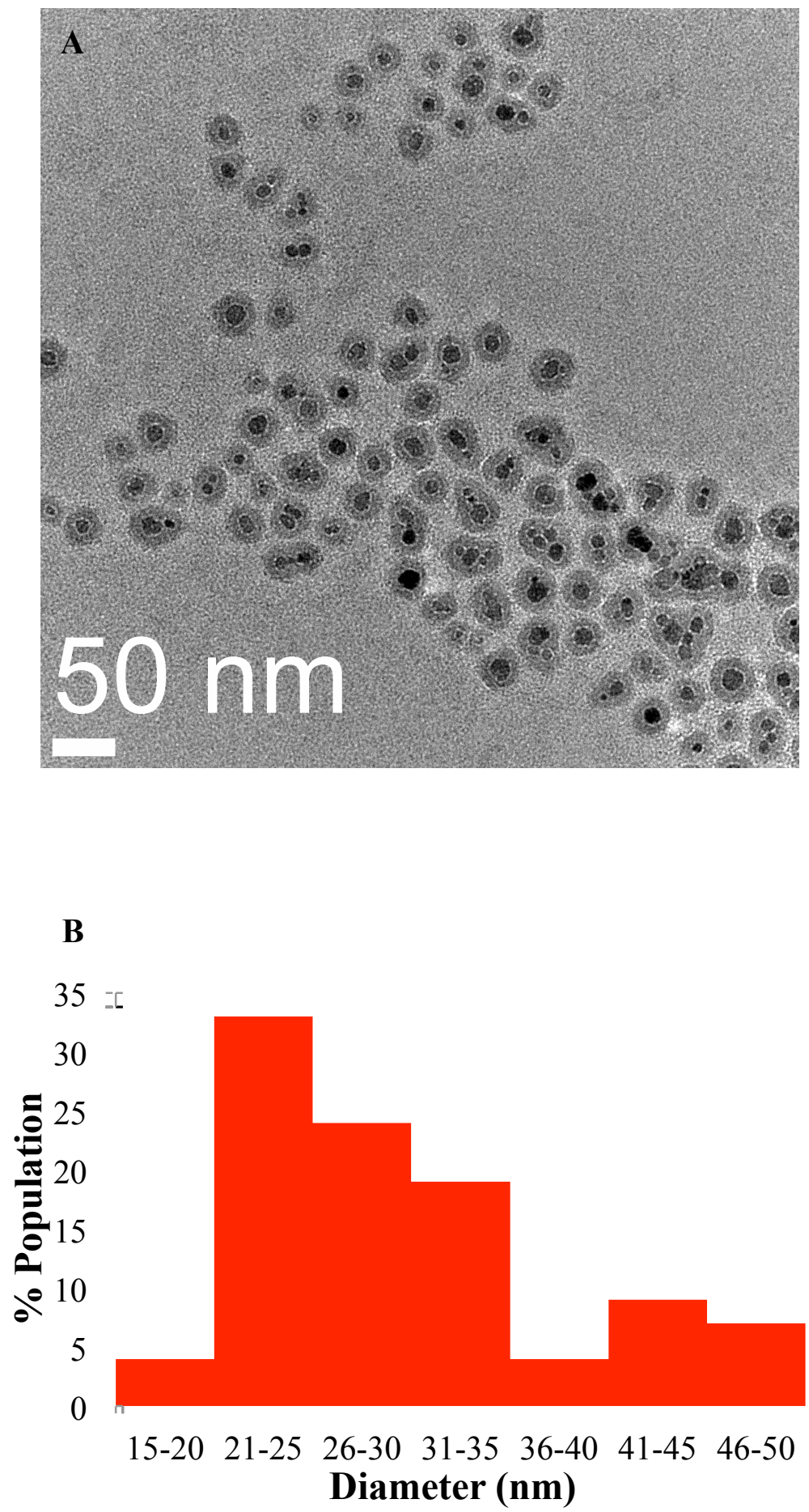

Figure 4.34. A. TEM image of $\mathrm{AO} @ \mathrm{SiO}_{2} @ \mathrm{Fe}_{3} \mathrm{O}_{4} \mathrm{NPs}$ with a B. particle size distribution $(30.4 \pm 0.8 \mathrm{~nm})$ resulting in $\mathrm{a} \sim 3 \mathrm{~nm}$ increase in the NP radius. 

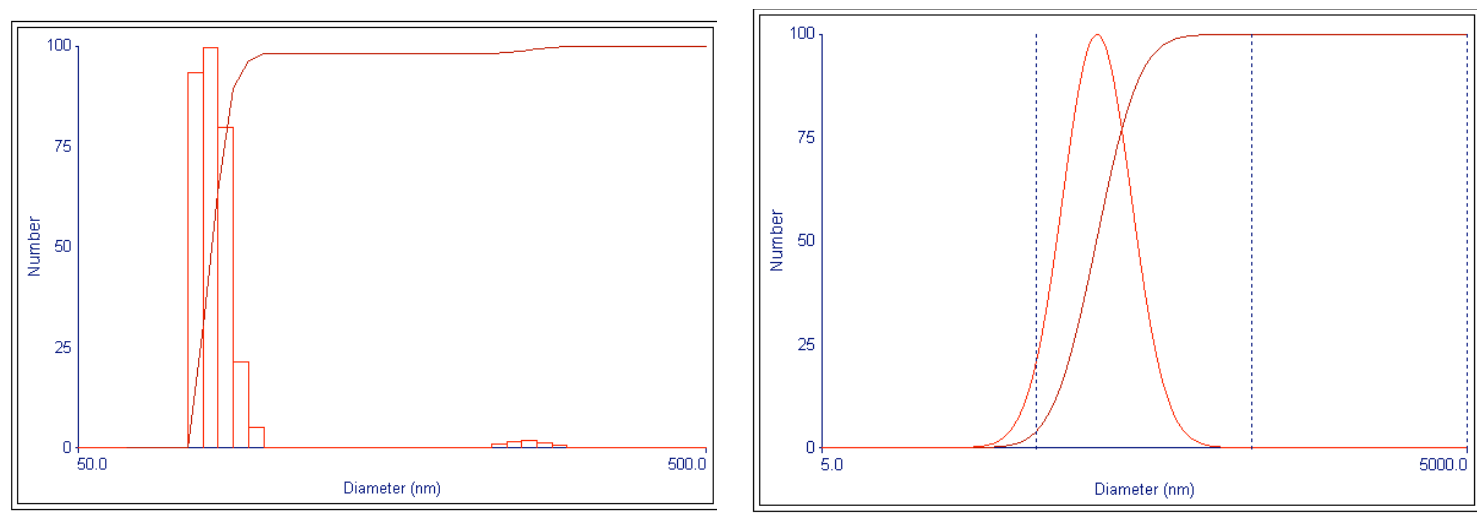

Figure 4.35. DLS of $\mathrm{AO} @ \mathrm{SiO}_{2} @ \mathrm{Fe}_{3} \mathrm{O}_{4} \mathrm{NPs}$ in Millipore water showing the hydrodynamic diameter at $96 \mathrm{~nm}$ with a polydispersity of 0.16 .

With the FL@SiO $@ \mathrm{Fe}_{3} \mathrm{O}_{4}$ NPs in hand, we studied the AMF-induced hydrolysis of the carbamate linkage by measuring the release of anthracene oxime ether fragment 27 (Scheme 4.10). The AMF experiments were conducted as described above $(2: 1$ PBS:acetonitrile solution, $\mathrm{pH}$ 7.4). As can be seen in Figure 4.36, AMF irradiation stimulated a rapid release of substrate from $\mathrm{FL} @ \mathrm{SiO}_{2} @ \mathrm{Fe}_{3} \mathrm{O}_{4}$. MALDI-TOF analysis confirmed that the oxime ether-anthracene fragment $\mathbf{2 7}$ was liberated from the NP formulation on AMF irradiation (Figure 4.37). Based on fluorescent measurements, 40 minutes of AMF exposure resulted in $67 \pm 3 \%$ of payload release; longer exposure times did not release additional 27. We also tested different currents by subjecting the NPs to 100 amps and 300 amps and compared the release with the results from the previous experiment (Figure 4.38). In addition, we also varied the duration of each AMF pulse from 5-minute on/off pulses to 2-minute on/off pulse with a total AMF exposure time of 40 minutes for both samples. As can be seen in Figure 4.39, the release of 27 is controlled by total AMF exposure time and not by the duration of the pulsing sequence. We were also gratified to find that the switch from carbonate to carbamate dramatically 
reduced non-AMF release of substrate - less than $10 \%$ of the payload was released at 37 ${ }^{\circ} \mathrm{C}$ after 40 minutes. The rapid release of the fluorophore fragment on AMF application is quite remarkable in that $N, N$-dialkyl carbamates are not expected to cleave under these mild conditions (e.g., a previous kinetic study estimates a typical half-life of $>550$ years at $25^{\circ} \mathrm{C}$ and $\left.\mathrm{pH} 7\right) .^{77}$

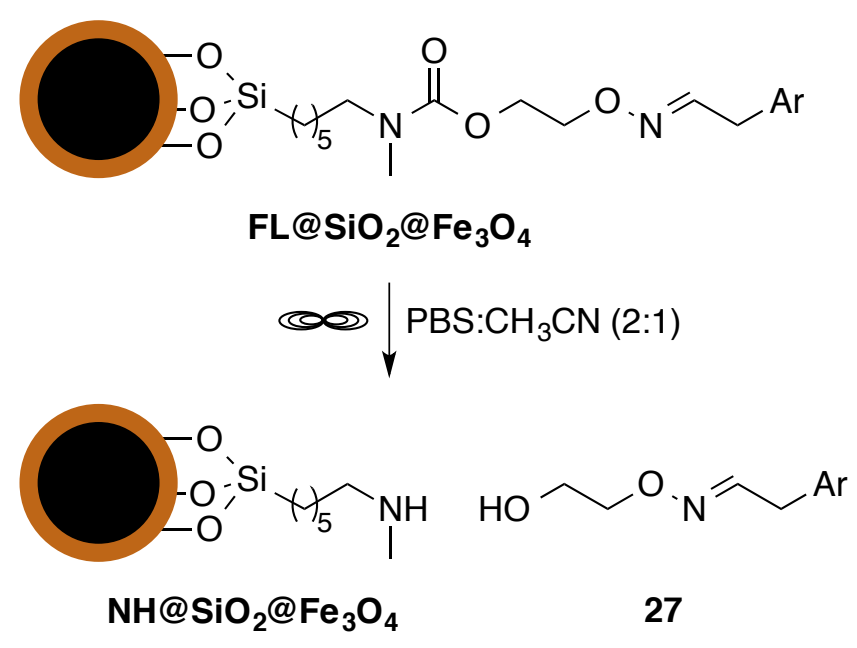

Scheme 4.10. AMF-induced carbamate hydrolysis. 


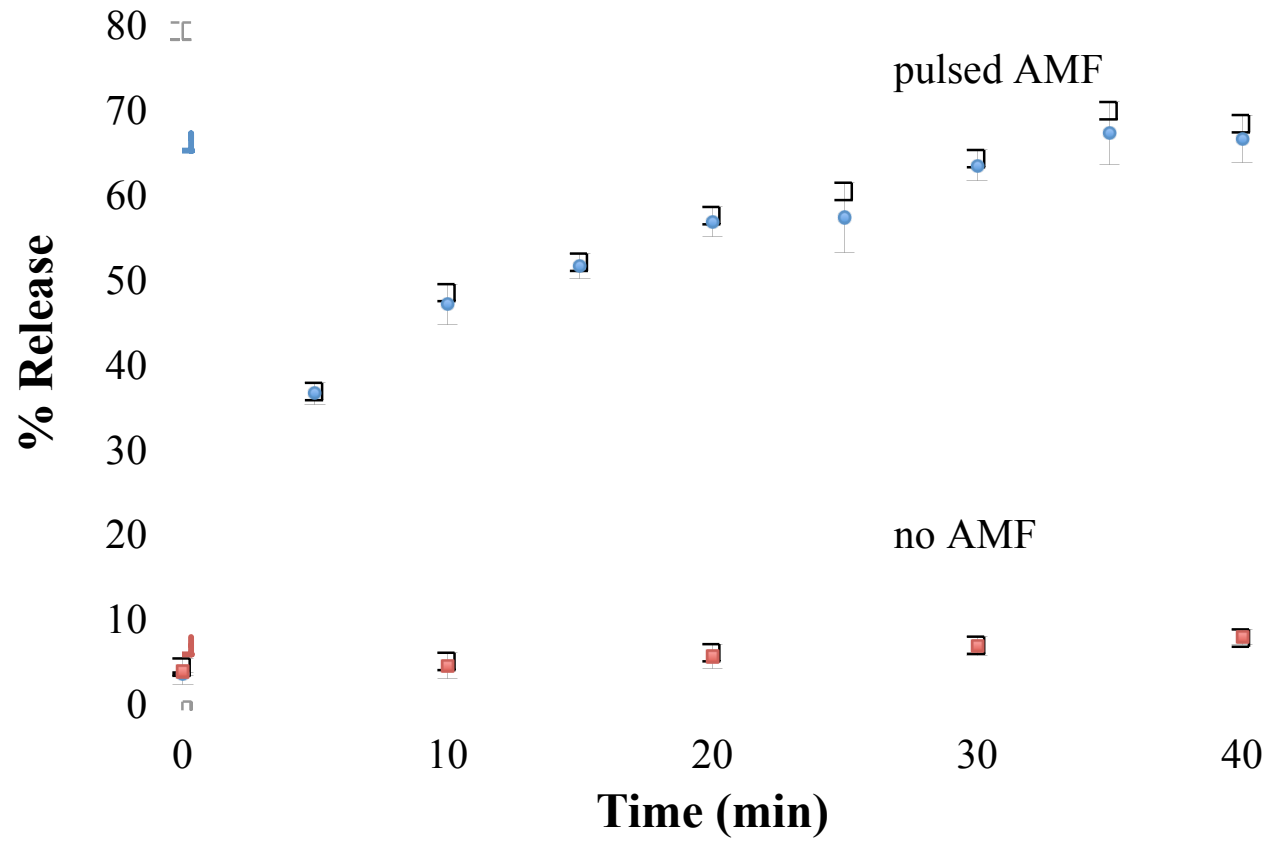

Figure 4.36. AMF-induced release of 27 from FL@ $\mathrm{SiO}_{2} @ \mathrm{Fe}_{3} \mathrm{O}_{4}$ (blue): NPs in PBS:acetonitrile were irradiated with an AMF (501.6 amps, $204 \mathrm{kHz}$ ) for 5minute bursts followed by 5-minute intervals; release of $\mathbf{2 7}$ from FL@ $\mathrm{SiO}_{2} @ \mathrm{Fe}_{3} \mathrm{O}_{4}$ (red): NPs in PBS:acetonitrile were incubated at $37{ }^{\circ} \mathrm{C}$ for the indicated time. Vertical axis shows the percent of 27 released as determined by fluorescent measurements; $\mathrm{n}=3$. 


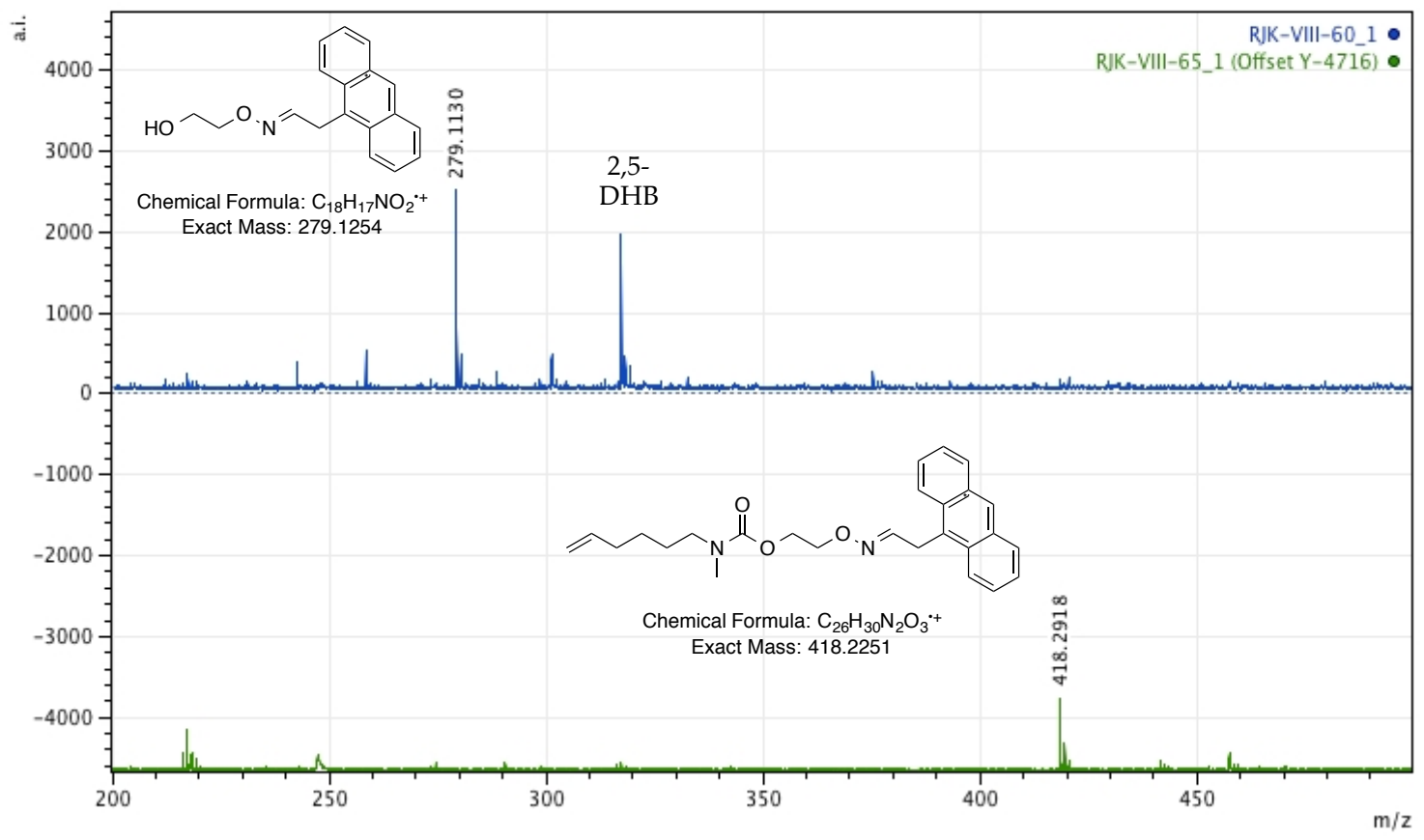

Figure 4.37. MALDI-TOF spectra of the radical carbocation ${ }^{60}$ of the anthracynyl oxime ether of $\mathbf{2 3}$ (bottom, green) and 27 (top, blue) after AMFinduced hydrolysis and release from FL@ $\mathrm{SiO}_{2} @ \mathrm{Fe}_{3} \mathrm{O}_{4} \mathrm{NPs}$ (25). The MALDI-TOF analysis of the anthracenyl oxime ether of $\mathbf{2 3}$ shows that the appearance of $\mathbf{2 7}$ is not a result of fragmentation during MS analysis. 


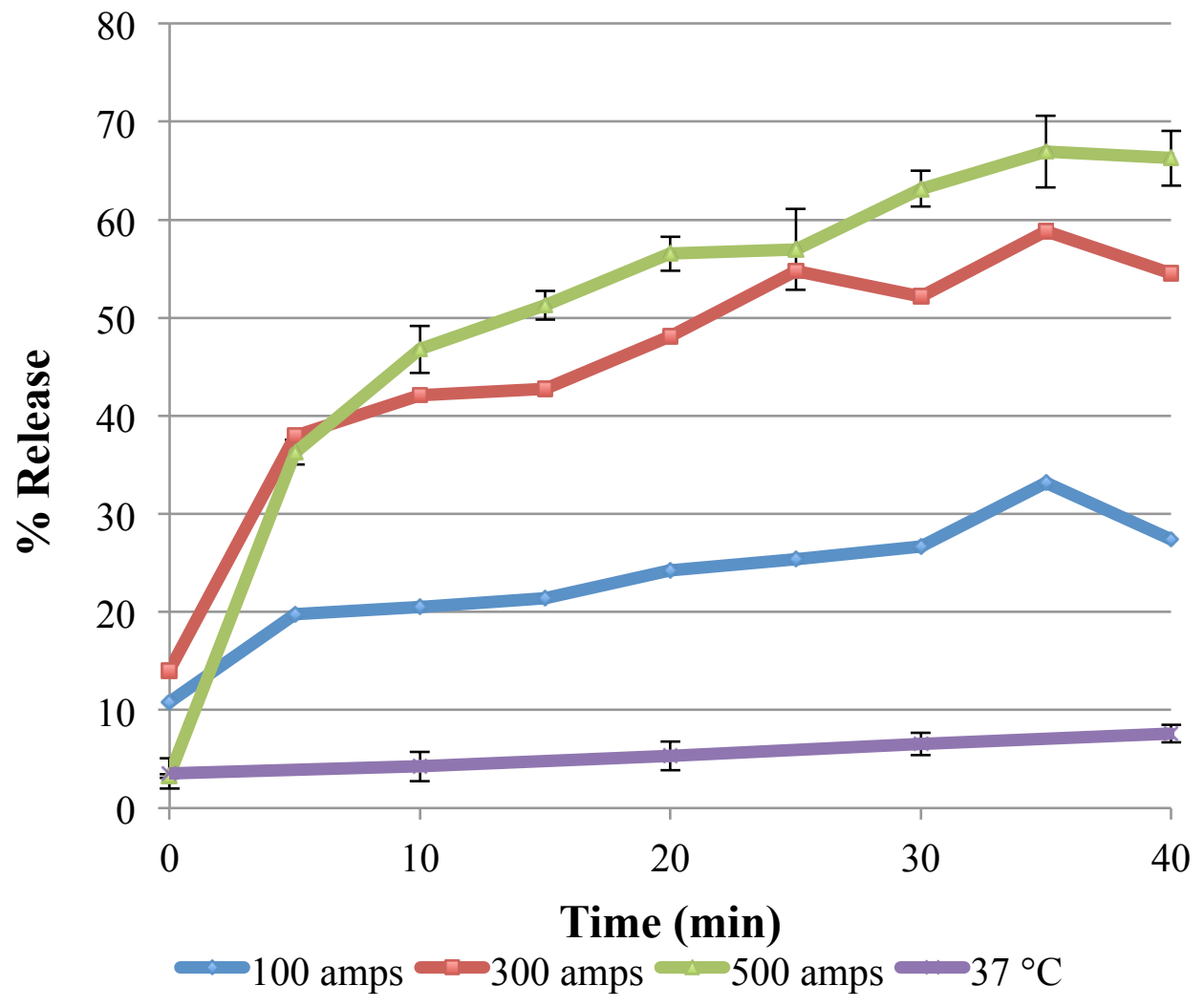

Figure 4.38. AMF-induced release, as determined by fluorescence measurements, of 27 from $\mathrm{FL} @ \mathrm{SiO}_{2} @ \mathrm{Fe}_{3} \mathrm{O}_{4}$ NPs in 2:1 PBS:acetonitrile at AMF amperages of 100.7 $(n=1), 300.2(n=1)$ or $501.6(n=3)$. The control, incubated at $37^{\circ} \mathrm{C}$ for the indicated time, received no AMF pulses. 


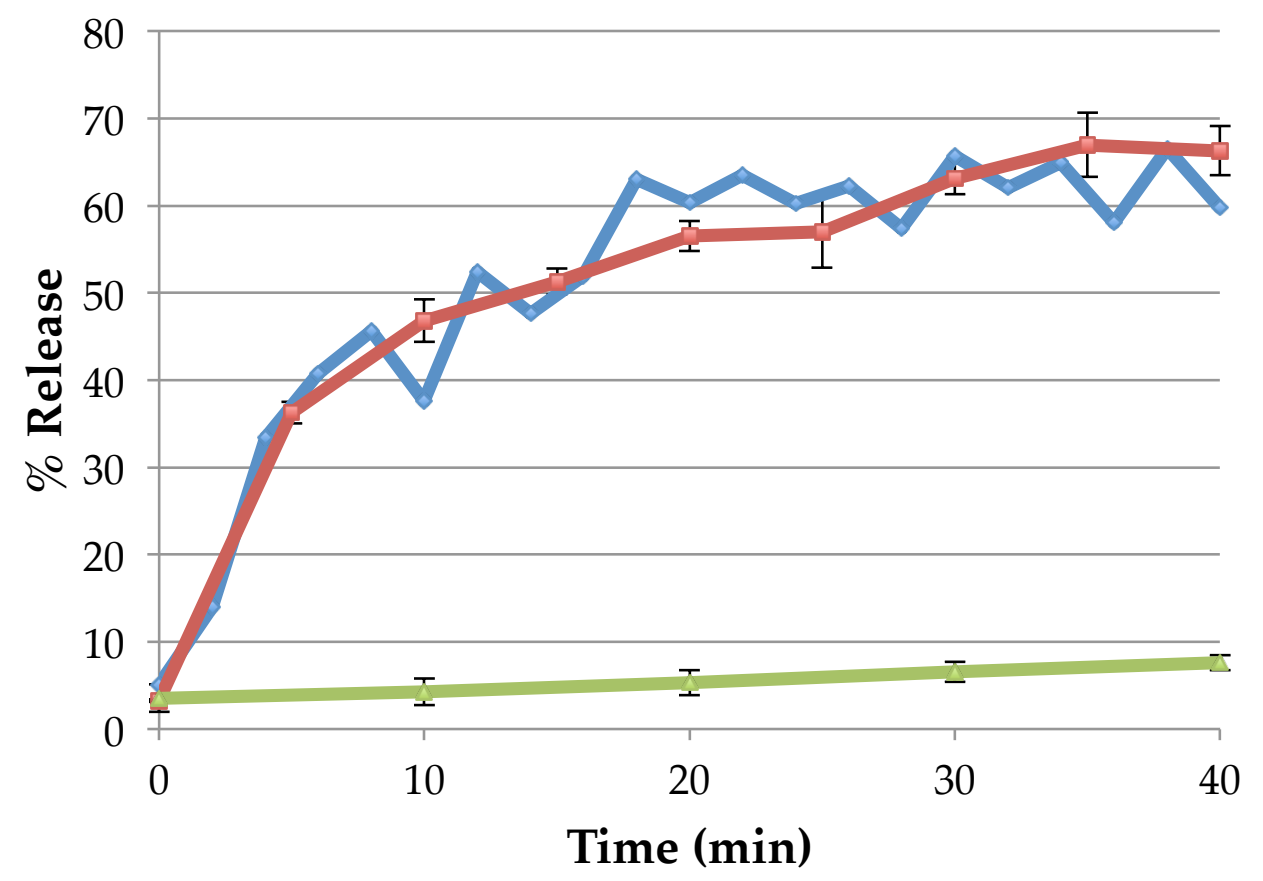

$\longrightarrow 2$ min pulses@500 A

-5 min pulses@ $500 \mathrm{~A}$

$-37^{\circ} \mathrm{C} \mathrm{w} / \mathrm{o} \mathrm{AMF}$

Figure 4.39. AMF-induced release of 27 from $\mathrm{FL} @ \mathrm{SiO}_{2} @ \mathrm{Fe}_{3} \mathrm{O}_{4} \mathrm{NPs}$ during 2 minute (n=1) or 5 minute $(\mathrm{n}=3)$ AMF pulses at 501.6 amps versus incubation at $37^{\circ} \mathrm{C}$.

\subsection{CONCLUSION.}

The use of a thermally susceptible bond, such as in the azo system recently disclosed by Riedinger et al., ${ }^{61}$ enables several possibilities for controlled drug delivery from iron oxide NPs when using an AMF as an external stimulus. In the present study, we have observed the AMF-stimulated release of fluorescent substrates from short-chain carbonate- and carbamate-based linkers covalently attached to magnetic iron oxide-silica, core-shell NPs. Although the mode of catalysis for the cleavage of the carbonate and carbamate functional groups is unclear at this time, AMF-induction, likely through 
accompanying local hyperthermia, dramatically enhances hydrolyses of these otherwise highly stable chemical linkages. This first example of an AMF-induced hydrolysis of a carbamate linkage may find utility in the development of externally triggerable delivery systems. In particular, the ease of carbamate synthesis, especially in the context of conjugating substrates to NPs, and the lower hydrolytic susceptibility of the $N, N$-dialkyl carbamate linkage in the absence of AMF exposure combine to make AMF-mediated functional group hydrolysis a promising strategy for regulated nanoparticle delivery of substrates.

\subsection{FUTURE DIRECTIONS.}

It is still unclear why the release of the fluorophore always stops at approximately $65 \%$. As was stated by Riedinger et al., ${ }^{61}$ the energy produced by the iron oxide NPs in the presence of an AMF dissipates rapidly as the distance from the NP core is increased. We suspect that the remaining $\sim 35 \%$ of the loading that does not release is too far away from the iron oxide core to induce the accelerated hydrolysis. This hypothesis is quite reasonable in that the alkoxysilane can undergo self-condensation, or polymerization, prior to being attached to the NP surface. This theory could be tested by incorporating poly(ethyleneglycol) (PEG) chains of varying length into the linker between the NP and the carbonyl functionality. Modification of the linker with PEG would increase the distance of the carbonyl from the NP and help to determine at what distance the hydrolytic phenomenon ceases. Elucidation of the reaction mechanism of the enhanced hydrolysis would also supply much needed information to further explain this surprising reaction. 
To advance this research to the next level, i.e., in vivo studies, we have partnered with Dr. Ralf Schirrmacher, a research scientist in Oncology at the University of Alberta Medical Isotope \& Cyclotron facility. Together with his post doctoral student Dr. Jun Zhu, they obtained gold coated $\mathrm{Fe}_{3} \mathrm{O}_{4}$ NPs using a procedure outlined in Scheme 4.11.

Figure 4.40 shows the TEM image of the $\mathrm{Au} @ \mathrm{Fe}_{3} \mathrm{O}_{4}$ NPs and Figure 4.41 are the SQUID measurements showing superparamagnetism.

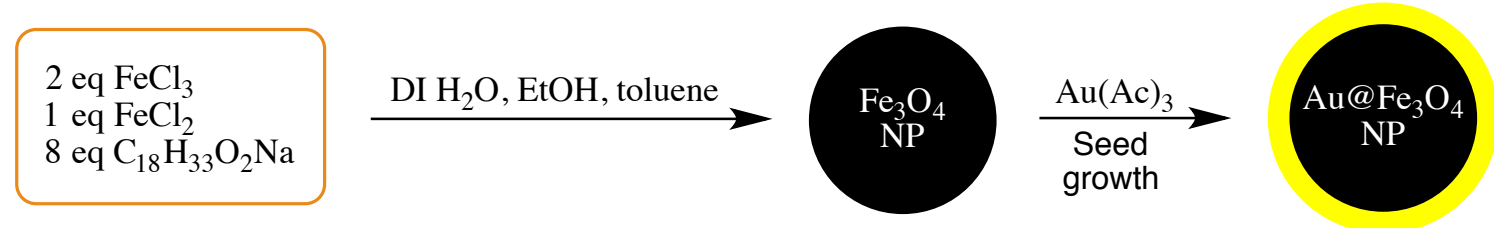

Scheme 4.11. Synthesis of $\mathrm{Au} @ \mathrm{Fe}_{3} \mathrm{O}_{4} \mathrm{NPs}$.

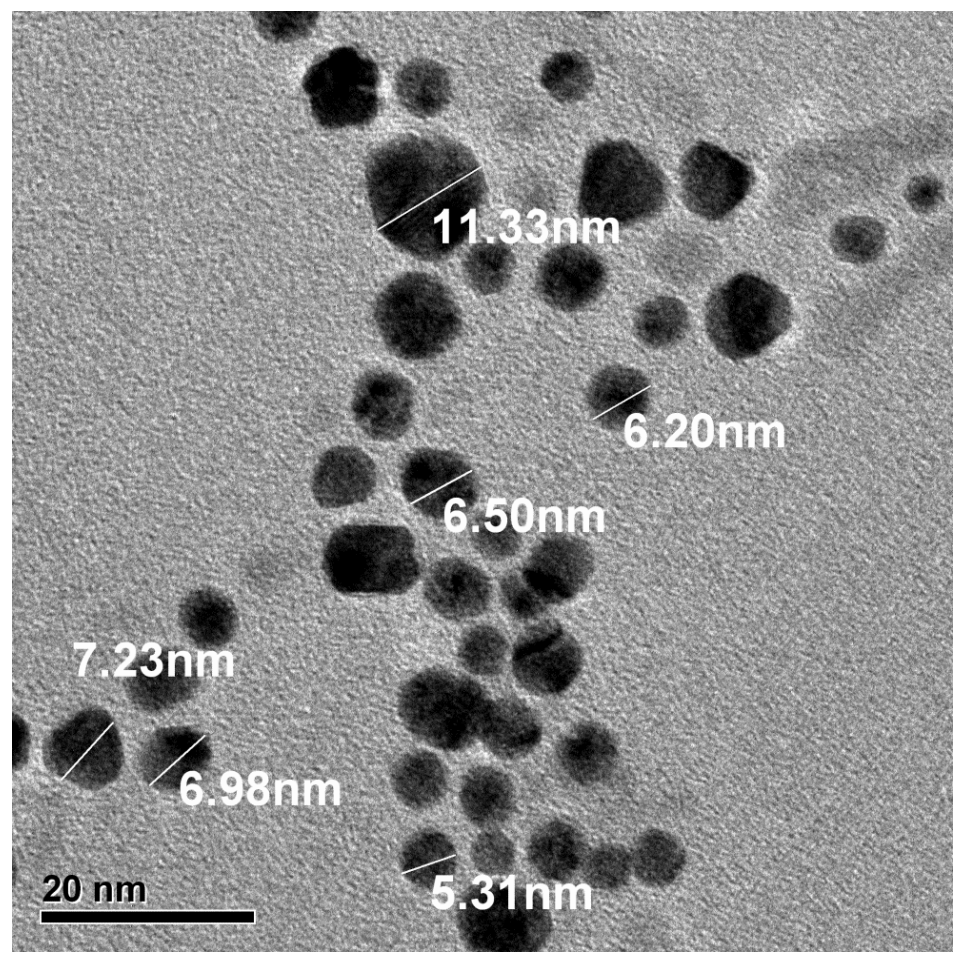

Figure 4.40. TEM image of $\mathrm{Au} @ \mathrm{Fe}_{3} \mathrm{O}_{4} \mathrm{NPs}$. 


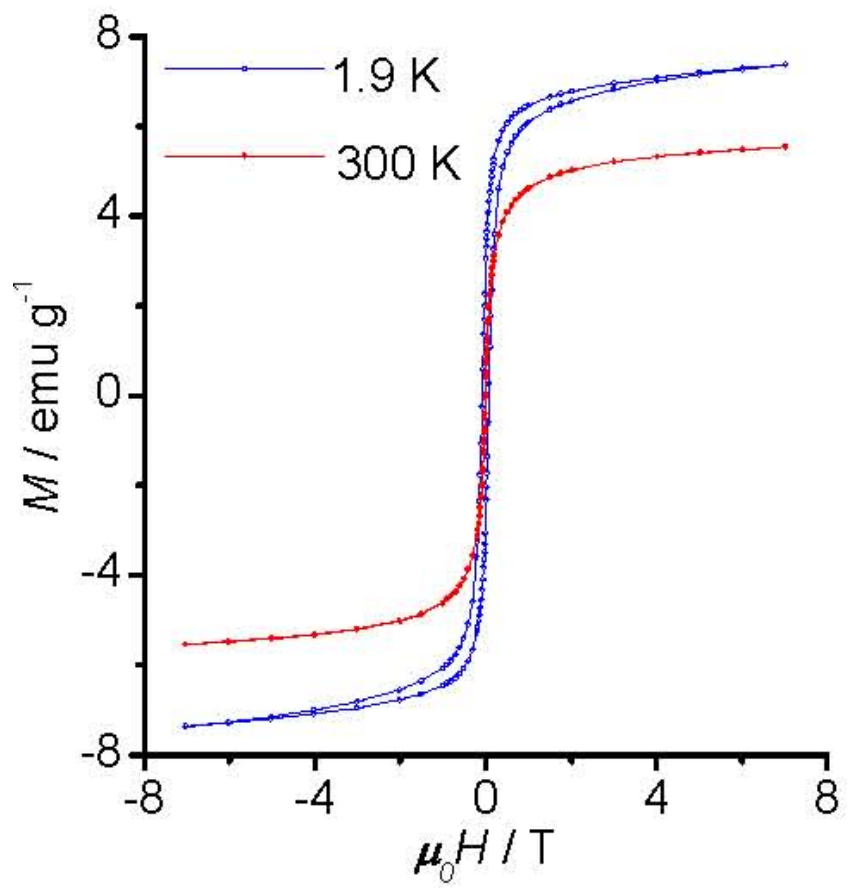

Figure 4.41. SQUID measuments of $\mathrm{Au} @ \mathrm{Fe}_{3} \mathrm{O}_{4}$ NPs showing superparamagnetism.

To allow for covalent binding of our linkers to the surface of the $\mathrm{Au} @ \mathrm{Fe}_{3} \mathrm{O}_{4} \mathrm{NPs}$ we replaced the unsaturation with a protected thiol (Scheme 4.12). Gold is a very attractive surface because it allows for easy covalent attachment of the linker to the NP through the $\mathrm{Au}-\mathrm{S}$ bond and is known to have very good biocompatibility. ${ }^{78}$ 


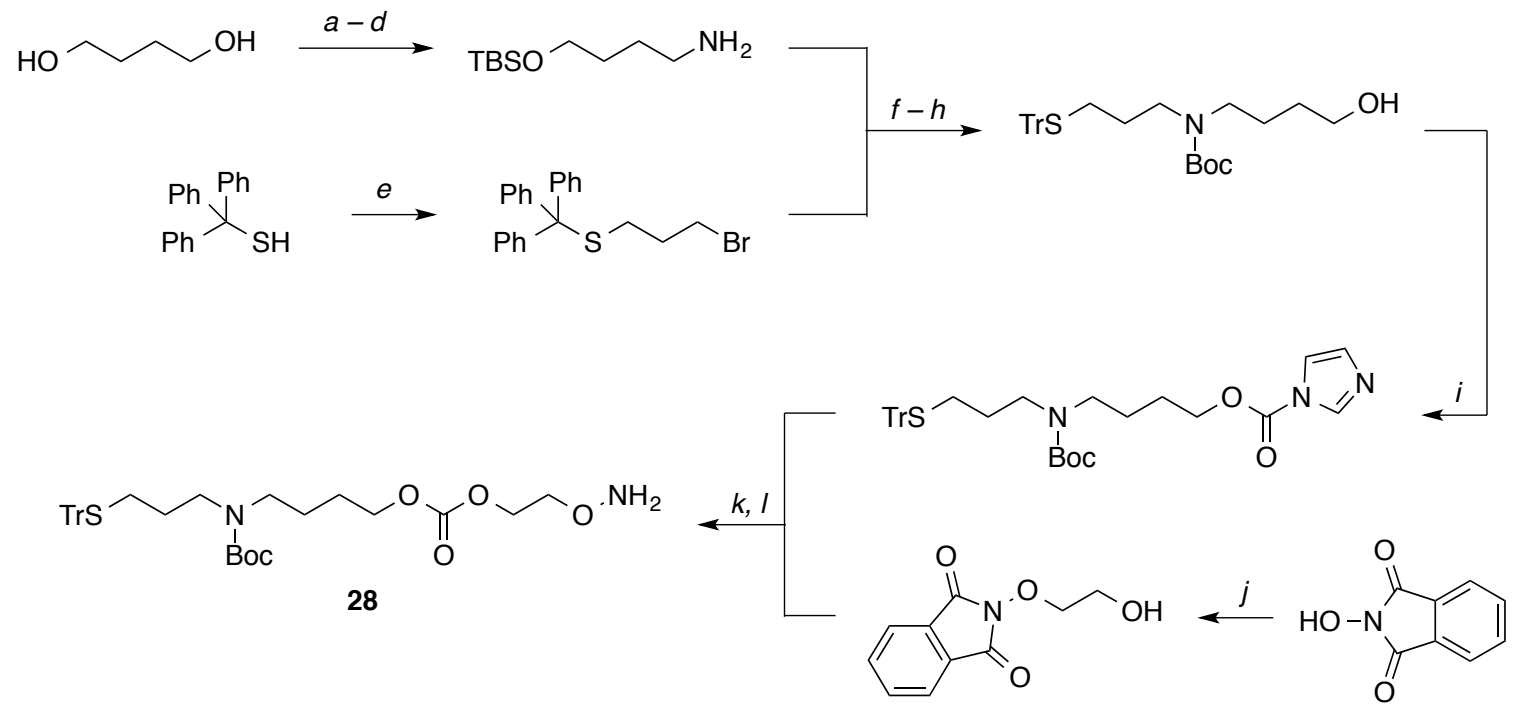

Scheme 4.12. Synthesis of carbonate linker 28 for attachment to $\mathrm{Au} @ \mathrm{Fe}_{3} \mathrm{O}_{4}$ NPs. Conditions: $a$. TBSCl, $\mathrm{Et}_{3} \mathrm{~N}, \mathrm{CH}_{2} \mathrm{Cl}_{2}, 0{ }^{\circ} \mathrm{C}, 17 \mathrm{~h} ; b$. $\mathrm{MsCl}, \mathrm{Et}_{3} \mathrm{~N}, \mathrm{CH}_{2} \mathrm{Cl}_{2}, 0{ }^{\circ} \mathrm{C}, 1.5 \mathrm{~h}$; c. phthalimide, $\mathrm{K}_{2} \mathrm{CO}_{3}, \mathrm{DMSO}, 7{ }^{\circ} \mathrm{C}$, $18 \mathrm{~h} ;$ d. $\mathrm{N}_{2} \mathrm{H}_{4} \cdot \mathrm{H}_{2} \mathrm{O}, 1: 2 \mathrm{EtOH}: \mathrm{CH}_{2} \mathrm{Cl}_{2}, 20 \mathrm{~h} ;$ e. 1,2-dibromoethane, $\mathrm{K}_{2} \mathrm{CO}_{3}$, THF, reflux, $24 \mathrm{~h}, 98 \%$; $f$. MeCN, $55{ }^{\circ} \mathrm{C}, 24 \mathrm{~h}, 82 \%$; $g . \mathrm{Boc}_{2} \mathrm{O}$, $\mathrm{Et}_{3} \mathrm{~N}, \mathrm{CH}_{2} \mathrm{Cl}_{2}, 0{ }^{\circ} \mathrm{C}, 2 \mathrm{~h} ; h .1 \mathrm{M}$ TBAF in THF, THF, $0{ }^{\circ} \mathrm{C}, 20 \mathrm{~h}, 62 \% ; i$. 1,1-carbonyldiimidazole, ( $i$-Pr)NEt, $\mathrm{CH}_{2} \mathrm{Cl}_{2}, 0{ }^{\circ} \mathrm{C}, j$. 2-bromoethanol, AcONa, DMSO, $70{ }^{\circ} \mathrm{C}, 67 \%$; $k$. DBU, MeCN, 18 h, 55\%; l. $\mathrm{N}_{2} \mathrm{H}_{4} \cdot \mathrm{H}_{2} \mathrm{O}$, $\mathrm{CH}_{2} \mathrm{Cl}_{2}, 0{ }^{\circ} \mathrm{C}, 92 \%$.

By transitioning from $\mathrm{SiO}_{2} @ \mathrm{Fe}_{3} \mathrm{O}_{4}$ NPs to $\mathrm{Au} @ \mathrm{Fe}_{3} \mathrm{O}_{4}$ NPs we suspect that hydrolysis will no longer occur. This hypothesis is based on the elimination of the Lewis acidic iron oxide surface. In the absence of hydrolysis we have resorted back to intramolecular cyclization as a release mechanism. Another problem that we had previously faced was the loss of $\sim 50 \%$ of the NP loading during the acidic Bocdeprotection when performed on the functionalized NPs. By using a thiol attachment and careful choice of protecting groups we have circumvented this issue. Standard Bocdeprotection usually involves a reaction with trifluoroacetic acid. These acidic conditions 
will also cleave the trityl thiol-protecting group. After performing the dual acidic deprotection, the $\mathrm{Au} @ \mathrm{Fe}_{3} \mathrm{O}_{4}$ NPs can be loaded without the risk of premature intramolecular cyclization. Leaving the linker in the form of an aminium salt prevents nucleophilic attack of the carbonyl by the amine. After the $\mathrm{Au} @ \mathrm{Fe}_{3} \mathrm{O}_{4}$ NPs are functionalized, a base wash restores the nucleophilic amine, thus enabling intramolecular cyclization.

My laboratory co-worker, Ms. Sara Biladeau, is currently attaching the aldehyde functionalized fluorophore 4,4-difluoro-4-bora-3a,4a-diaza-s-indacene (BODIPY) ${ }^{79}$ through the oximation reaction and loading the linkers onto the $\mathrm{Au} @ \mathrm{Fe}_{3} \mathrm{O}_{4} \mathrm{NPs}$. 
CHAPTER 5

EXPERIMENTAL PROCEDURES

\subsection{GENERAL STATEMENT AND INDEX OF EXPERIMENTAL PROCEDURES}

\subsection{EXPERIMENTAL PROCEDURES OF CHAPTER 2}

5.2.1. Open- and Closed-Chip Microreactor Fabrication

5.2.2. Open-Chip Sample Collection

5.2.3. Determination of AMAH Capture Efficiency

5.2.4. FT-ICR-MS Instrumentation

5.2.5. GC-MS Instrumentation

5.2.6. Synthesis of AMAH

5.2.7. Procedure for Neutralization of AMAH-adducts

5.2.8. Synthesis of ADMH

5.2.9. Synthesis of AAn

\subsection{EXPERIMENTAL PROCEDURES OF CHAPTER 3}

\subsubsection{First Generation}

5.3.2. Synthesis of 2-(9-Anthracenyl)ethanol 25

5.3.3. Synthesis of Amino Esters and Amide

5.3.4. Synthesis of Amino Carbonates 
5.3.5. Synthesis of Carbamate

5.3.6. Synthesis of gem-Dimethyl Ester

5.3.7. Synthesis of gem-Dimethyl Carbonate

5.3.8. Experimental Procedures for Determination of Release Rate of 25 and 26

5.3.9. Experimental Procedure for ${ }^{1} \mathrm{H}$ NMR Verification of Cyclization

5.3.10. Synthesis of Ester 27

5.3.11. Experimental Procedures for Manufacture of PDMS Microchannel 5.3.12.

Procedure for Loading 27 onto PDMS Microchannel

5.3.13. Experimental Procedures for PDMS Microchannel Reaction and Release

\subsection{EXPERIMENTAL PROCEDURES OF CHAPTER 4}

5.4.1. Synthesis of Non-Nucleophilic Ester Linker

5.4.2. Synthesis of 11

5.4.3. Anhydrous NP Loading Procedure (with heat)

5.4.4. Acidic Boc-Deprotection of Functionalized NPs to Afford $2^{\circ}$ Amine

5.4.5. Synthesis of Non-Nucleophilic Olefinic Ester

5.4.6. Synthesis of Non-Nucleophilic Olefinic Carbonate

5.4.7. Synthesis of Non-Nucleophilic Olefinic Carbamate

5.4.8. Procedure for the Addition of Polysaccharides to Functionalized NPs

5.4.9. Synthesis of Monodispersed $\mathrm{SiO}_{2} @ \mathrm{Fe}_{3} \mathrm{O}_{4} \mathrm{NPs}$

5.4.10. Synthesis of Carbamate Linker for AMF-Induced Hydrolytic Release

5.4.11. SQUID Measurements 
5.4.12. Synthesis of $\mathrm{AO} @ \mathrm{SiO}_{2} @ \mathrm{Fe}_{3} \mathrm{O}_{4}$ and $\mathrm{FL} @ \mathrm{SiO}_{2} @ \mathrm{Fe}_{3} \mathrm{O}_{4} \mathrm{NPs}$

5.4.13. AMF-Induced Heating of Bulk Solution

5.4.14. Assay of Percent 27 Released from NPs

5.4.15. Synthesis of Thiol Linker for Loading onto $\mathrm{Au} @ \mathrm{Fe}_{3} \mathrm{O}_{4} \mathrm{NPs}$ 


\subsection{GENERAL STATEMENT}

All solvents and reagents used in this thesis were reagent grade and were used as received unless otherwise indicated. Dry THF and $\mathrm{CH}_{2} \mathrm{Cl}_{2}$ were obtained from SPBT-101 Bench Top Solvent Purification System (LC Technology Solutions, Inc., USA). Anhydrous DMF was purchased from Sigma-Aldrich. Dry acetonitrile was obtained by distillation from $\mathrm{CaH}_{2}$. Unless otherwise noted, all reagents were purchased from commercial suppliers and were used without further purification. The progress of reactions was monitored by thin-layer chromatography (TLC) using pre-coated silica plates (EMD Silica Gel 60 F254). Visualization was accomplished by staining the plates with iodine, PMA (3\% phosphomolybdic acid/ethanol solution), PAA stain $(2.5 \%$ p-anisaldehyde acid/ethanol solution) or ninhydrin stain $(10 \mathrm{~g}$ ninhydrin in $100 \mathrm{~mL}$ of $n$-butanol and add 3 $\mathrm{mL} \mathrm{AcOH})$. UV active compounds were visualized by UV light $(254 \mathrm{~nm})$. Silica gel 60 (230-400 mesh) was used for flash column chromatography. ${ }^{1}$ H NMR spectra were recorded at 400,500 or $700 \mathrm{MHz}$, and ${ }^{13} \mathrm{C}$ spectra were recorded at 100,125 or 175 $\mathrm{MHz}$, respectively, in the indicated solvents. The chemical shifts are reported in ppm values relative to the solvent residual peak $\mathrm{CDCl}_{3}\left(7.26 \mathrm{ppm}\right.$ for ${ }^{1} \mathrm{H}$ NMR and $77.23 \mathrm{ppm}$ for ${ }^{13} \mathrm{C}$ NMR), DMSO- $d_{6}$ (2.50 ppm for ${ }^{1} \mathrm{H}$ NMR and $39.52 \mathrm{ppm}$ for ${ }^{13} \mathrm{C}$ NMR), $\mathrm{CD}_{3} \mathrm{OD}$ (3.31 ppm for ${ }^{1} \mathrm{H}$ NMR and $49.00 \mathrm{ppm}$ for ${ }^{13} \mathrm{C}$ NMR) or toluene- $d_{8}\left(2.08\right.$ ppm for ${ }^{1} \mathrm{H}$ NMR and 20.43 ppm for ${ }^{13} \mathrm{C}$ NMR). Coupling constants are reported in hertz (Hz). High resolution ESI-MS were obtained using a FT-ICR-MS system (LTQ FT, Thermo Electron Corp.) at the Center for Regulatory and Environmental Analytical Metabolomics (CREAM) Mass Spectrometry Facility, University of Louisville, Kentucky. Fluorescent measurements were taken on a Molecular Devices SpectraMax M5 in fluorescent mode at 
an excitation of $366 \mathrm{~nm}$ and emission wavelength of $408 \mathrm{~nm}$. The fluorescent measurements were taken using a quartz semi-micro VWR Spectrosil spectrophotometer cell with a $10 \mathrm{~mm}$ light path. Transmission electron microscopy (TEM) was used to analyze size distribution and morphology of fabricated nanoparticles, including uniformity and thickness of silica coating. For this, ethanol-based nanoparticle dispersions were prepared and drop-casted on commercially available 300 mesh TEM support $\mathrm{Cu}$ grids coated with ultra thin carbon films. After ethanol evaporation, samples were transferred to and analyzed using a field emission gun FEI Tecnaci F20 transmission electron microscope operating at the accelerating voltage of $200 \mathrm{kV}$. TGA measurements were made on a TA Insruments Hi-Res TGA 2950 Thermogravi-metric Analyzer using a Pt basket and maintaining a flow of $\mathrm{N}_{2}$ gas through the oven. Each TGA experiment was run from $35^{\circ} \mathrm{C}$ to $800{ }^{\circ} \mathrm{C}$ at a ramp rate of $20^{\circ} \mathrm{C} / \mathrm{min}$. DLS and $\zeta$ potential measurements were taken using a Brookhaven Instruments 90Plus Particle Size Analyzer. All measurements were taken using aqueous colloids of the nanoparticles in Millipore water. The alternating magnetic field (AMF) was generated with an Ambrell EasyHeat L1 set at 501.6 amps and $204 \mathrm{kHz}$ using a 5-turn coil. MALDI-TOF analysis was done on a Voyager DE-Pro MALDI-TOF instrument (PE Biosystems). Spectra were acquired in positive reflectron mode and calibration was achieved by using known peaks from the 2,5-dihydroxybenzoic acid (2,5-DHB) matrix. Data was analyzed with mMASS data analysis software. 


\subsection{EXPERIMENTAL PROCEDURES FROM CHAPTER 2}

\subsubsection{Open- and Closed-Chip Microreactor Fabrication}

Dr. Xiao-An Fu's research group designed and fabricated the open-chip microreactor in a way that mirrored the microelectromechanical system procedures described previously. ${ }^{1,2,3}$ Deep reactive ion etching (DRIE) was used to create microfluidic channels and micropillars with a height of roughly $250 \mu \mathrm{m}$ on a silicon wafer. The microreactor channels and micropillars were thermally oxidized to form a 50 $\mathrm{nm} \mathrm{SiO} 2$ thin film in an $\mathrm{O}_{2}$ and $\mathrm{H}_{2} \mathrm{O}$ atmosphere. The silicon microreactors have a 7 x 5 $\mathrm{mm}$ microfluidic channel consisting of over 2500 micropillars with a surface area of about $130 \mathrm{~mm}^{2}$.

The open-chip microreactors were completed by directly dicing the silicon wafer after the thermal oxidation process. The microreactors were functionalized with AMAH by placing the chip into a $20 \mathrm{~mL}$ clean-air sampling vial. Then, a solution of AMAH $\left(140 \mu \mathrm{g}, 7.67 \times 10^{-7} \mathrm{~mol}\right)$ dissolved in methanol $(20 \mu \mathrm{L})$ was added into the microfluidic channel of the chip. The vial was put into an oven and dried for 5 minutes at $85{ }^{\circ} \mathrm{C}$.

Closed-chip microreactors were prepared by bonding the wafer-supported microreactors with a Pyrex glass wafer using an anodic wafer bonding process. Each wafer was subsequently diced, and the connection ports were opened for connecting fused silica tubes to the microreactors. The surface of the micropillars in the closed-chip was functionalized with AMAH by infusing a solution of AMAH (67 $\mu \mathrm{g}, 3.65 \times 10^{-7}$ mol) in methanol $(15 \mu \mathrm{L})$ into the microreactors followed by evaporation of the solvent under vacuum. Finally, fused silica tubes with $190 \mu \mathrm{m}$ o.d. and $100 \mu \mathrm{m}$ i.d. were 
connected to the inlet and outlet ports of the microreactor, respectfully, with a silicabased bonding agent.

\subsubsection{Open-Chip Sample Collection}

To test the open chip for capture of trace carbonyl compounds, exhaled breath samples were collected. After approval by the Internal Review Board of the institution and after having obtained written informed consent, exhaled breath samples were collected from healthy never-smokers, current smokers and lung cancer patients. To collect a breath sample, the subject used a straw to blow into the sampling vial containing the open-chip for 15 minutes. During this process, the carbonyl compounds in the exhaled breath react with the AMAH coating and are retained in the microreactor while the rest of the breath sample moves freely away and out of the sampling vial. After the subject had finished blowing, methanol $(10 \mu \mathrm{L})$ was added into the microfluidic channel to dissolve the analytes. The methanol solution was then removed from the microreactor using a pipette. This process was repeated four more times to collect an approximate total of $50 \mu \mathrm{L}$ of a methanol-analyte mixture for analysis.

\subsubsection{Determination of AMAH Capture Efficiency}

To test the microreactor for capturing trace levels of carbonyl compounds, a solution of acetaldehyde, acetone or 2-butanone in methanol $\left(7.67 \times 10^{-7}\right.$ to $7.67 \times 10^{-10}$ mol) was injected to a 1 liter air Tedlar bag. The vapor-filled Tedlar bag then was connected to the preconcentration setup as shown in Figure 2.2 before a vacuum pump was used to pull the gaseous vapor from the Tedlar bag through the closed microreactor 
at a flow rate of $3.5 \mathrm{~mL} / \mathrm{min}$. The microreactor then was disconnected from the system after the air sample in the bag had been completely evacuated.

The reacted AMAH adduct and unreacted AMAH were eluted from the closed microreactor by flowing $100 \mu \mathrm{L}$ methanol from one slightly pressurized vial through the microreactor, and into an empty collecting sample vial. An internal reference for FTICR-MS analysis was established by adding a solution of $1.53 \times 10^{-8}$ mol of AMAHacetone- $d_{6}$ adduct in methanol to each sample of eluent. The resulting solutions were directly injected into the FT-ICR-MS for analysis without any further process.

\subsubsection{FT-ICR-MS Instrumentation}

The methanol-eluted mixtures of AMAH-VOC adducts were analyzed on a hybrid linear ion trap- FT-ICR-MS instrument (Finnigan LTQ-FT, Thermo Electron, Bremen, Germany) equipped with a TriVersa NanoMate ion source (Advion BioSciences, Ithaca, NY) with an electrospray chip (nozzle inner diameter $5.5 \mu \mathrm{m}$ ). The TriVersa NanoMate was operated in positive ion mode by applying $2.0 \mathrm{kV}$ with no head pressure. Initially, low-resolution MS scans were acquired for 1 min to ensure the stability of ionization, after which high mass accuracy data were collected using the FT-ICR analyzer where MS scans were acquired for $8.5 \mathrm{~min}$ and at the target mass resolution of 100,000 at $800 \mathrm{~m} / \mathrm{z}$. AMAH and AMAH-adduct species were assigned on the basis of their accurate mass by

first applying a small (typically $<0.0005$ ) linear correction based on the observed mass of the internal standard. 


\subsubsection{GC-MS Instrumentation}

A Thermo Scientific GC/MS instrument equipped with an AI 1310 automatic sampler, a TRACE 1310 GC with a split/splitless injector and an ITQ 1100 series iron trap MS was used for analysis. The GC was fitted with an Agilent J\&W DB-17ms column $(30 \mathrm{~m} \times 0.25 \mathrm{~mm} \times 0.25 \mu \mathrm{m}$ film thickness $)$. The carrier helium flow rate was set to $1.5 \mathrm{~mL} / \mathrm{min}$. The injection port temperature was set to $280{ }^{\circ} \mathrm{C}$ and the initial column temperature was $50{ }^{\circ} \mathrm{C}$ for 1 minute, with a $12{ }^{\circ} \mathrm{C} / \mathrm{min}$ ramp up to $290{ }^{\circ} \mathrm{C}$ and was held at $290{ }^{\circ} \mathrm{C}$ for 5 minutes. The total running time was 26 minutes. The samples were split injected with split flow $15 \mathrm{ml} / \mathrm{min}$ and split ratio of 10 .

Carbonyl adduct standards for retention time determination were prepared by individually reacting excess AMA $\left(5.1 \times 10^{-7} \mathrm{~mol}\right)$ with commercially available aldehydes or ketones $\left(3.5 \times 10^{-7} \mathrm{~mol}\right)$ in a 1:1 mixture of acetonitrile:MTBSTFA $(\mathrm{v} / \mathrm{v})(100 \mu \mathrm{L})$. MTBSTFA reacted with the AMA-3-hydroxy-2-butanone and AMAhydroxyacetaldehyde adducts to form AMA-3-(tert-butyldimethylsilyloxy)-2-butanone (AMA-3-TBSO-2-butanone) and AMA-(tert-butyldimethyl-silyloxy)acetaldehyde (AMA-TBSO-acetaldehyde), respectively for GC-MS analysis.

\subsubsection{Synthesis of AMAH}<smiles>O=C1c2ccccc2C(=O)N1OCCN1CCOCC1</smiles>

2-(2-Morpholinoethoxy)isoindoline-1,3-dione. By analogy to our published method ${ }^{13}$ for synthesis of quaternary ammonium aminooxy compounds as well as to a literature procedure, ${ }^{14}$ we prepared AMAH as follows: diisopropyl azodicarboxylate (DIAD) (3.60 
$\mathrm{mL}, 18.3 \mathrm{mmol}$ ) was added dropwise to a stirred solution of 2-(4-morpholinyl) ethanol (2.00 g, $15.3 \mathrm{mmol}), N$-hydroxyphthalimide (2.98 g, $18.3 \mathrm{mmol})$, and triphenylphosphine (4.80 g, $18.3 \mathrm{mmol})$ in dry THF $(95 \mathrm{~mL})$ at $0{ }^{\circ} \mathrm{C}$ under nitrogen. The resulting solution was allowed to warm to rt and then stirred. After $16 \mathrm{~h}$, the THF was removed in vacuo and the resulting oil was dissolved in EtOAc $(150 \mathrm{~mL})$ and washed with sat. $\mathrm{NaHCO}_{3}(3 \mathrm{x}$ $50 \mathrm{~mL}$ ) and brine $(50 \mathrm{~mL})$. The organic phase was reduced to $50 \mathrm{~mL}$ in vacuo followed by the addition of cold $5 \% \mathrm{HCl}$ to $\mathrm{pH} 3$. The aqueous phase was separated, washed with $\mathrm{Et}_{2} \mathrm{O}(3 \times 20 \mathrm{~mL})$, and then basified with sat. $\mathrm{NaHCO}_{3}$ to $\mathrm{pH}$ 7.5. The aqueous phase then was extracted with $\mathrm{CHCl}_{3}(3 \times 50 \mathrm{~mL})$. The combined organic extract was dried $\left(\mathrm{Na}_{2} \mathrm{SO}_{4}\right)$, filtered, and the solvent was removed in vacuo to afford 2-(2morpholinoethoxy)isoindoline-1,3-dione as a light yellow solid (3.67 g, 87\%); mp, 78-79 ${ }^{\circ} \mathrm{C}$; TLC, $R_{f} 0.37$ (EtOAc); ${ }^{1} \mathrm{H}$ NMR $\left(\mathrm{CDCl}_{3}\right) \delta 2.55(\mathrm{~s}, 4 \mathrm{H}), 2.84(\mathrm{t}, J=4.8 \mathrm{~Hz}, 2 \mathrm{H})$, $3.60(\mathrm{t}, J=3.8 \mathrm{HZ}, 4 \mathrm{H}), 4.36(\mathrm{t}, J=5 \mathrm{~Hz}, 2 \mathrm{H}), 7.74-7.76(\mathrm{~m}, 2 \mathrm{H}), 7.82-7.84(\mathrm{~m}, 2 \mathrm{H})$; ${ }^{13} \mathrm{C} \mathrm{NMR}\left(\mathrm{CDCl}_{3}\right) \delta 53.8,57.2,66.9,74.2,123.7,129.2,134.7,163.7$.

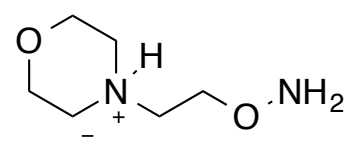

$\mathrm{Cl}^{-}$

AMAH

4-(2-Aminooxyethyl)-morpholin-4-ium chloride (AMAH). Hydrazine monohydrate (527 $\mu \mathrm{L}, \quad 10.9 \mathrm{mmol})$ was added to a stirred solution of 2-(2morpholinoethoxy)isoindoline-1,3-dione (735 $\mathrm{mg}, 2.66 \mathrm{mmol})$ in $\mathrm{CH}_{2} \mathrm{Cl}_{2}(11 \mathrm{~mL})$ at $\mathrm{rt}$. After stirring for $20 \mathrm{~h}$, the suspension was filtered through a fritted glass funnel and the cake was washed with ample $\mathrm{CH}_{2} \mathrm{Cl}_{2}$. The filtrate and combined $\mathrm{CH}_{2} \mathrm{Cl}_{2}$ washes were concentrated in vacuo and the residue was distilled using a Kugelrohr apparatus (10 Torr, 
$\left.150{ }^{\circ} \mathrm{C}\right)$. The distillate was dissolved in $\mathrm{Et}_{2} \mathrm{O}(11 \mathrm{~mL})$ and gaseous $\mathrm{HCl}$ was bubbled into the solution. The acidic solution was stirred at $\mathrm{rt}$ for $1 \mathrm{~h}$, and then dried in vacuo to provide 4-(2-aminooxyethyl)-morpholin-4-ium chloride (AMAH, $486 \mathrm{mg}, 100 \%$ yield) as a white solid; $\mathrm{mp}, 148-151{ }^{\circ} \mathrm{C}$; IR $v\left(\mathrm{~cm}^{-1}\right) 902,1030,1444,2022,2640,3418 ;{ }^{1} \mathrm{H}$ NMR (DMSO-d f $_{\text {) }} 3.29$ (s, 4 H), 3.47 (t, $J=4.4 \mathrm{~Hz}, 2 \mathrm{H}$ ), 3.89 (t, $\left.J=4.4 \mathrm{~Hz}, 4 \mathrm{H}\right), 4.47$ (t, $J=4.6 \mathrm{~Hz}, 2 \mathrm{H}), 11.18(\mathrm{br} \mathrm{s}, 1 \mathrm{H}) ;{ }^{13} \mathrm{C}$ NMR $\left(\mathrm{DMSO}_{6}\right)$ ) $51.2,53.5,63.1,68.2$.

\subsubsection{Procedure for Neutralization of AMAH-adducts}<smiles>[R]C([18OH])=NOCCN1CCOCC1</smiles>

A $35 \mu \mathrm{L}$ aliquot of the methanol AMAH-adduct mixture was transferred to a $200 \mu \mathrm{L}$ insert containing $2 \mathrm{mg}$ of poly(4-vinylpyridine) (PVP) for the neutralization of AMAHadducts to AMA-adducts. After shaking the vial for 30 seconds, the vial was centrifuged at $1000 \mathrm{rpm}$ for 5 minutes to settle the PVP. A $2 \mu \mathrm{L}$ aliquot was taken from the supernatant and directly injected into the GC-MS.

\subsubsection{Synthesis of ADMH}<smiles>CN(C)CCOC1NC(=O)c2ccccc2C1=O</smiles>

2-(2-(Dimethylamino)ethoxy)-2,3-dihydro-1H-isoindole-1,3-dione was synthesized following a procedure described by Biswas et al. ${ }^{4}$ 


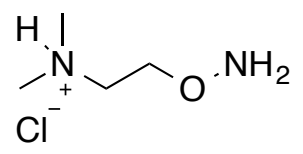

ADMH

\section{$O$-[2-(Dimethylamino)ethyl]hydroxylamine hydrochloride (ADMH). Methyl}

hydrazine ( $247 \mu \mathrm{L}, 4.70 \mathrm{mmol}$ ) was added to a solution of 2-[2-(dimethylamino)ethoxy]2,3-dihydro- $1 \mathrm{H}$-isoindole-1,3-dione $(1.02 \mathrm{~g}, 4.35 \mathrm{mmol})$ in dry $\mathrm{CH}_{2} \mathrm{Cl}_{2}(14 \mathrm{~mL})$ at $0{ }^{\circ} \mathrm{C}$ and was stirred for $4.5 \mathrm{~h}$. At completion, the solvent was removed by distillation and the residue was Kugelrohr distilled at atmospheric pressure. A was a small amount of oxime present, so the distilled product was dissolved in $6 \mathrm{M}$ aq. $\mathrm{HCl}(5 \mathrm{~mL})$ and refluxed for 22 h. The majority of the water was removed by distillation and the product was finally azeo-dried with hexanes. Isopropanol was added to the remaining residue and the solution was stirred until white crystal formation ceased. The crystals were collected by filtration, washed once with $i$-PrOH and dried under reduced pressure to yield $O$-[2(dimethylamino)ethyl]hydroxylamine hydrochloride (ADMH, $224 \mathrm{mg}, 37 \%$ ) as a white

crystal. ${ }^{1} \mathrm{H}$ NMR (DMSO- $\left.d_{6}, 700 \mathrm{MHz}\right) \delta 2.79$ (s, 6H), 3.43 (t, $J=4.6 \mathrm{~Hz}, 2 \mathrm{H}$ ), $4.43(\mathrm{t}, J$ $=4.9 \mathrm{~Hz}, 2 \mathrm{H}), 11.14,(\mathrm{br} \mathrm{s}, 3 \mathrm{H}) ;{ }^{13} \mathrm{C}$ NMR $\left(\mathrm{DMSO}-d_{6}, 176 \mathrm{MHz}\right) \delta 42.3,53.7,68.4$; FTICR-MS calcd for $\mathrm{C}_{4} \mathrm{H}_{13} \mathrm{~N}_{2} \mathrm{O}^{+}[\mathrm{M}+\mathrm{H}]^{+} m / z$ 105.1022, found.

\subsubsection{Synthesis of AAn}<smiles>OCc1c2ccccc2cc2ccccc12</smiles>

9-Anthracenemethanol. Sodium borohydride $(275 \mathrm{mg}, 7.27 \mathrm{mmol})$ was added to a solution of 9-anthracenecarboxaldehyde $(1.02 \mathrm{~g}, 4.93 \mathrm{mmol})$ in $\mathrm{EtOH}(30 \mathrm{~mL})$ at $0{ }^{\circ} \mathrm{C}$. 
The reaction was then heated to $55{ }^{\circ} \mathrm{C}$ for $3.5 \mathrm{~h}$. After cooling to rt, the reaction was quenched with sat $\mathrm{NH}_{4} \mathrm{Cl}(50 \mathrm{~mL})$ and extracted three times with $\mathrm{CH}_{2} \mathrm{Cl}_{2}$. The combined organic phases were dried over $\mathrm{Na}_{2} \mathrm{SO}_{4}$, filtered and concentrated in vacuo to give a crude material of 9-anthracenemethanol (yellow crystal, $1.07 \mathrm{~g}$ ) that was used in the next step without further purification. $R_{f} 0.49\left(2: 23, \mathrm{EtOAc}: \mathrm{CH}_{2} \mathrm{Cl}_{2}\right)$.

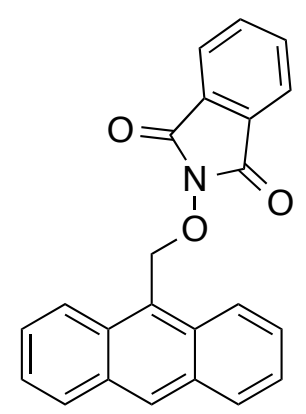

2-(Anthracen-9-ylmethoxy)-2,3-dihydro-1H-isoindole-1,3-dione. DIAD (1.26 mL, $6.40 \mathrm{mmol})$ was added dropwise to a solution of crude 9-anthracenemethanol (1.03 g, $4.92 \mathrm{mmol}), N$-hydroxyphthalimide (942 $\mathrm{mg}, 6.40 \mathrm{mmol})$, and $\mathrm{PPh}_{3}(1.68 \mathrm{~g}, 6.40 \mathrm{mmol})$ in dry THF $(25 \mathrm{~mL})$ at $0{ }^{\circ} \mathrm{C}$ and the reaction was stirred overnight. The solvent was then removed and the residue was diluted with $\mathrm{CH}_{2} \mathrm{Cl}_{2}$. The solution was then washed twice with sat. $\mathrm{NaHCO}_{3}$ and the aqueous phase was extracted twice with $\mathrm{CH}_{2} \mathrm{Cl}_{2}$. The combined organic phases were washed with brine, dried over $\mathrm{Na}_{2} \mathrm{SO}_{4}$, filtered and concentrated in vacuo. The crude material was purified by column chromatography $\left(\mathrm{SiO}_{2}, \mathrm{CH}_{2} \mathrm{Cl}_{2}\right)$ to give 2-(anthracen-9-ylmethoxy)-2,3-dihydro-1 $H$-isoindole-1,3-dione as a yellow solid (1.68 g, 96\%). $\quad R_{f} 0.73\left(1: 19, \mathrm{EtOAc}: \mathrm{CH}_{2} \mathrm{Cl}_{2}\right) ;{ }^{1} \mathrm{H}$ NMR $(400 \mathrm{MHz}$, DMSO-d $\left._{6}\right) \delta 6.18(\mathrm{~s}, 2 \mathrm{H}), 7.59(\mathrm{t}, J=7.6 \mathrm{~Hz}, 2 \mathrm{H}), 7.71(\mathrm{t}, J=7.6 \mathrm{~Hz}, 2 \mathrm{H}), 7.92-7.94(\mathrm{~m}$, 4H), $8.17(\mathrm{~d}, J=8.4 \mathrm{~Hz}, 2 \mathrm{H}), 8.66(\mathrm{~d}, J=8.8 \mathrm{~Hz}, 2 \mathrm{H}), 8.78(\mathrm{~s}, 1 \mathrm{H}) ;{ }^{13} \mathrm{C} \mathrm{NMR}(100 \mathrm{MHz}$, 
DMSO-d $\left._{6}\right) \delta 71.3,123.3,124.0,124.2,125.4,127.1,128.8,128.9,129.8,130.8,131.2$, $134.9,163.4$.

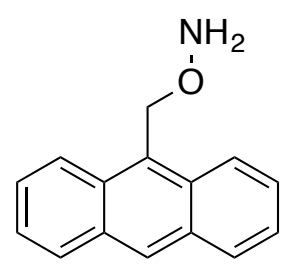

AAn

$O$-(Anthracen-9-ylmethyl)hydroxylamine (AAn). Hydrazine monohydrate (343 $\mu \mathrm{L}$, $7.08 \mathrm{mmol}$ ) was added to a suspension of 2-(anthracen-9-ylmethoxy)-2,3-dihydro- $1 \mathrm{H}$ isoindole-1,3-dione $(531 \mathrm{mg}, 1.50 \mathrm{mmol})$ in $\mathrm{EtOH}(36 \mathrm{~mL})$ at $0{ }^{\circ} \mathrm{C}$. After the addition was complete, the ice bath was removed and 2-(anthracen-9-ylmethoxy)-2,3-dihydro- $1 H$ isoindole-1,3-dione slowly went into solution before a white precipitate began to drop from the solution. After $5 \mathrm{~h}$ the white solids were filtered, washed with $\mathrm{CH}_{2} \mathrm{Cl}_{2}$, and the filtrate was concentrated in vacuo. Dilute $\mathrm{HCl}(30 \mathrm{~mL}, 10 \%)$ was added to the residue and stirred for 10 minutes. The solution was washed once with EtOAc before being basified with sat. $\mathrm{NaHCO}_{3}$. The basic solution was extracted three times with $\mathrm{CHCl}_{3}$, the combined organic phase was dried over $\mathrm{Na}_{2} \mathrm{SO}_{4}$, filtered and concentrated in vacuo to give $O$-(anthracen-9-ylmethyl)hydroxylamine (AAn, $200 \mathrm{mg}, 60 \%$ ) as yellow crystals. $R_{f}$ 0.35 (1:19, EtOAc: $\left.\mathrm{CH}_{2} \mathrm{Cl}_{2}\right) ;{ }^{1} \mathrm{H}$ NMR (400 MHz, $\left.\mathrm{CDCl}_{3}\right) \delta 5.73(\mathrm{~s}, 2 \mathrm{H}), 7.48(\mathrm{dd}, J=7.2$ $\mathrm{Hz}, 2 \mathrm{H}), 7.57(\mathrm{dd}, J=6.4,8.8 \mathrm{~Hz}, 2 \mathrm{H}), 8.02(\mathrm{~d}, J=8.0 \mathrm{~Hz}, 2 \mathrm{H}), 8.44-8.47(\mathrm{~m}, 3 \mathrm{H}) ;{ }^{13} \mathrm{C}$ NMR (100 MHz, DMSO-d $\left.{ }_{6}\right) \delta$ 70.0, 124.6, 125.2, 126.4, 127.9, 128.8, 129.2, 131.4, 131.6 . 


\subsection{EXPERIMENTAL PROCEDURES FROM CHAPTER 3}

\subsubsection{First Generation}

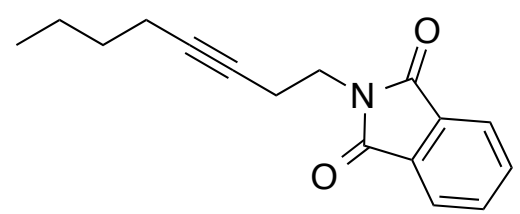

2-(Oct-3-yn-1-yl)-2,3-dihydro-1 $\boldsymbol{H}$-isoindole-1,3-dione. DIAD (1.84 mL, 9.34 mmol) was added to a solution of oct-3-yn-1-ol (907 mg, $7.18 \mathrm{mmol})$, phthalimide $(1.37 \mathrm{~g}, 9.34$ mmol) and triphenylphosphine $(2.45 \mathrm{~g}, 9.34 \mathrm{mmol})$ in dry $\mathrm{THF}(36 \mathrm{~mL})$ at $0{ }^{\circ} \mathrm{C}$ and stirred overnight. The solution was then concentrated in vacuo and diluted with EtOAc $(50 \mathrm{~mL})$. The solution was washed with $\mathrm{NaHCO}_{3}(3 \times 40 \mathrm{~mL})$ and the combined aqueous layers were extracted with EtOAc $(20 \mathrm{~mL})$. The combined organics were washed with brine $(45 \mathrm{~mL})$, dried over $\mathrm{Na}_{2} \mathrm{SO}_{4}$, filtered and concentrated in vacuo. The crude material was purified by column chromatography $\left(\mathrm{SiO}_{2}, 1: 19\right.$ to $1: 4$, EtOAc:hexanes gradient) to give 2-(oct-3-yn-1-yl)-2,3-dihydro-1H-isoindole-1,3-dione (1.77 g, 97\%) as a white solid. $R_{f} 0.78$ (1:1, EtOAc:hexanes); ${ }^{1} \mathrm{H}$ NMR $\left(500 \mathrm{MHz}, \mathrm{CDCl}_{3}\right) \delta 0.76(\mathrm{t}, J=7.3 \mathrm{~Hz}, 3 \mathrm{H})$, $1.20-1.25(\mathrm{~m}, 2 \mathrm{H}), 1.29-1.32(\mathrm{~m}, 2 \mathrm{H}), 2.00-2.03(\mathrm{~m}, 2 \mathrm{H}), 2.50-2.53(\mathrm{~m}, 2 \mathrm{H}), 3.78(\mathrm{t}, J=$ $7.3 \mathrm{~Hz}, 2 \mathrm{H})$, 7.66-7.68 (m, 2H), 7.78-7.80 (m, 2H); $\left.{ }^{13} \mathrm{C} \mathrm{NMR} \mathrm{(100} \mathrm{MHz,} \mathrm{CDCl}_{3}\right) \delta$ 13.5, $18.2,18.6,21.8,30.8,37.1,75.8,82.3,123.1,132.0,133.9,167.9$.

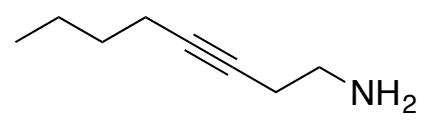

Oct-3-yn-1-amine. Hydrazine monohydrate $(1.68 \mathrm{~mL}, 34.6 \mathrm{mmol})$ was added to a solution of 2-(oct-3-yn-1-yl)-2,3-dihydro-1H-isoindole-1,3-dione (1.77 g, $6.93 \mathrm{mmol})$ in 1:1 $\mathrm{CH}_{2} \mathrm{Cl}_{2}: \mathrm{EtOH}(5 \mathrm{~mL})$ at $0{ }^{\circ} \mathrm{C}$ with stirring. The reaction was allowed to reach room 
temperature and stir overnight. The white precipitate was removed through filtration and the filter cake was washed with ample $\mathrm{CH}_{2} \mathrm{Cl}_{2}$ and the filtrate was concentrated in vacuo. The crude material was purified by column chromatography $\left(\mathrm{SiO}_{2}, 1: 1: 98\right.$ to $10: 1: 89$ $\mathrm{MeOH}: \mathrm{NH}_{4} \mathrm{OH}: \mathrm{CH}_{2} \mathrm{Cl}_{2}$ gradient) to give oct-3-yn-1-amine (555 $\mathrm{mg}, 64 \%$ ) as a yellow oil. $R_{f} 0.46\left(10: 1: 89, \mathrm{MeOH}: \mathrm{NH}_{4} \mathrm{OH}: \mathrm{CH}_{2} \mathrm{Cl}_{2}\right) ;{ }^{1} \mathrm{H} \mathrm{NMR}\left(500 \mathrm{MHz}, \mathrm{CDCl}_{3}\right) \delta 0.82(\mathrm{t}, J=$ $7.3 \mathrm{~Hz}, 3 \mathrm{H}), 1.29-1.34$ (m, 2H), 1.36-1.39 (m, 2H), 1.72 (br s, 2H), 2.06-2.09 (m, 2H), 2.20-2.23 (m, 2H), $2.71(\mathrm{t}, J=6.3 \mathrm{~Hz}, 2 \mathrm{H}) ;{ }^{13} \mathrm{C} \mathrm{NMR}\left(100 \mathrm{MHz}, \mathrm{CDCl}_{3}\right) \delta$ 13.6, 18.4, $22.0,23.9,31.2,41.5,77.8,81.9$.

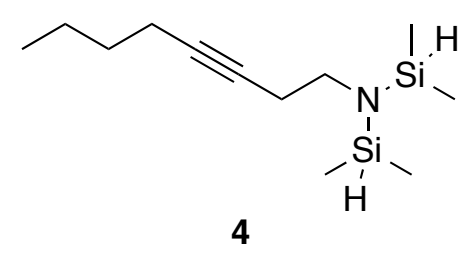

Bis(dimethylsilyl)(oct-3-yn-1-yl)amine (4). $n$-BuLi (3.15 mL $1.6 M$ in hexanes, 5.04 mmol) was added dropwise to a solution of oct-3-yn-1-amine (517 mg, $4.13 \mathrm{mmol})$ in dry THF $(21 \mathrm{~mL})$ at $-78{ }^{\circ} \mathrm{C}$ and stirred for $0.5 \mathrm{~h}$, then warmed to $-40{ }^{\circ} \mathrm{C}$ and stirred for another $0.5 \mathrm{~h}$. After cooling the solution back to $-78{ }^{\circ} \mathrm{C}$, chlorodimethylsilane $(504 \mu \mathrm{L}$, $4.54 \mathrm{mmol}$ ) was added and the solution was warmed to $-40{ }^{\circ} \mathrm{C}$ and stirred for $1 \mathrm{~h}$. The solution was cooled back to $-78{ }^{\circ} \mathrm{C}$ and a second portion of $n$-BuLi $(3.44 \mathrm{~mL} 1.6 \mathrm{M}$ in hexanes, $5.50 \mathrm{mmol}$ ) was added dropwise and stirred for $1 \mathrm{~h}$ after returning to $-40{ }^{\circ} \mathrm{C}$. After cooling back to $-78^{\circ} \mathrm{C}$ the second portion of chlorodimethylsilane (550 $\mu \mathrm{L}, 4.95$ mmol) was added and the solution was stirred overnight. Hexanes $(33 \mathrm{~mL})$ was added to the reaction to precipitate $\mathrm{LiCl}$, filtered and concentrated in vacuo. The crude was purified by Kugelrohr distillation to provide 4 (731 mg, 73\%) as a colorless oil. ${ }^{1} \mathrm{H}$ NMR 
(400 MHz, $\left.\mathrm{CDCl}_{3}\right) \delta 0.16(\mathrm{~s}, 12 \mathrm{H}), 0.90(\mathrm{t}, J=7.2 \mathrm{~Hz}, 3 \mathrm{H}), 1.38-1.46(\mathrm{~m}, 4 \mathrm{H}), 2.13(\mathrm{t}, J$ $=6.8 \mathrm{~Hz}, 2 \mathrm{H}), 2.19(\mathrm{t}, J=8 \mathrm{~Hz}, 2 \mathrm{H}), 2.95(\mathrm{t}, J=7.8 \mathrm{~Hz}, 2 \mathrm{H}), 4.43(\mathrm{t}, J=3.4 \mathrm{~Hz}, 2 \mathrm{H})$;

${ }^{13} \mathrm{C}$ NMR $\left(100 \mathrm{MHz}, \mathrm{CDCl}_{3}\right) \delta-0.3,13.8,18.7,22.2,24.1,31.4,46.1,78.1,81.6$.

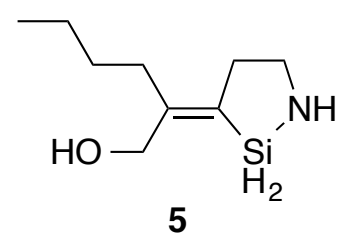

2-[(3E)-2,2-Dimethyl-1,2-azasilolidin-3-ylidene]hexan-1-ol (5). Bis(dimethylsilyl)(oct3-yn-1-yl)amine (142 $\mathrm{mg}, 0.59 \mathrm{mmol})$ in toluene $(10 \mathrm{~mL})$ was added to a solution of cat. $\mathrm{Rh}_{4}(\mathrm{CO})_{12}(2 \mathrm{mg}, 0.0025 \mathrm{mmol})$ in toluene $(5 \mathrm{~mL})$. The autoclave was then sealed and purged with $\mathrm{CO}$ before adjusting the pressure to $10 \mathrm{~atm}$ with $\mathrm{CO}$ gas. The reactor was then heated to $60{ }^{\circ} \mathrm{C}$ for $14 \mathrm{~h}$, at which point the pressure was relieved and the reaction solution was poured into a solution of $\mathrm{NaBH}_{4}(189 \mathrm{mg}, 5.0 \mathrm{mmol})$ in $\mathrm{MeOH}(5 \mathrm{~mL})$ at 0 ${ }^{\circ} \mathrm{C}$ and stirred for $10 \mathrm{~min}$. The reaction was quenched with $\mathrm{NH}_{4} \mathrm{Cl}(20 \mathrm{~mL})$ and extracted with $\mathrm{Et}_{2} \mathrm{O}(3 \times 10 \mathrm{~mL})$. The combined organics were dried over $\mathrm{K}_{2} \mathrm{CO}_{3}$, filtered and concentrated in vacuo. The crude material was purified by column chromatography $\left(\mathrm{SiO}_{2}, 1: 1: 98\right.$ to 5:1:94 MeOH: $\mathrm{NH}_{4} \mathrm{OH}: \mathrm{CH}_{2} \mathrm{Cl}_{2}$ gradient) to give 5 ( $\left.83 \mathrm{mg}, 66 \%\right) . R_{f} 0.57$ (10:1:89, MeOH:NH$\left.{ }_{4} \mathrm{OH}: \mathrm{CH}_{2} \mathrm{Cl}_{2}\right) ;{ }^{1} \mathrm{H}$ NMR $\left(400 \mathrm{MHz}, \mathrm{CDCl}_{3}\right) \delta 0.23(\mathrm{~s}, 6 \mathrm{H}), 0.90(\mathrm{t}, J$ $=6.6 \mathrm{~Hz}, 3 \mathrm{H}), 1.25-1.36(\mathrm{~m}, 4 \mathrm{H}), 1.56(\mathrm{br} \mathrm{s}, 1 \mathrm{H}), 2.14(\mathrm{t}, J=7.2 \mathrm{~Hz}, 2 \mathrm{H}), 2.34(\mathrm{t}, J=6.6$ $\mathrm{Hz}, 2 \mathrm{H}), 2.76$ (br s, 2H), 4.48 (s, 2H); ${ }^{13} \mathrm{C}$ NMR (100 MHz, $\left.\mathrm{CDCl}_{3}\right) \delta$ 1.4, 14.1, 23.0, $27.9,30.9,31.9,42.3,74.1,130.3,155.0$. 


\subsubsection{Synthesis of 2-(9-Anthracenyl)ethanol ${ }^{5}$ (25)}<smiles>CO/C=C\c1c2ccccc2cc2ccccc12</smiles>

(E/Z)-9-(2-Methoxyvinyl)anthracene. (Methoxymethyl)triphenylphosphonium chloride (13.5 g, $39.5 \mathrm{mmol})$ was suspended in dry THF $(105 \mathrm{~mL})$ under nitrogen with stirring to which KHMDS (68.5 mL of $0.5 \mathrm{M}$ solution in toluene, $34.2 \mathrm{mmol}$ ) was added dropwise via syringe at room temperature and stirred for 1 hour. The addition of KHMDS turned the white suspension to a dark red solution. The solution was then cooled to $-78{ }^{\circ} \mathrm{C}$ and a solution of 9-anthraldehyde $(5.43 \mathrm{~g}, 26.3 \mathrm{mmol})$ in dry THF $(20 \mathrm{~mL})$ was added dropwise. The reaction was allowed to warm to room temperature and was stirred overnight. The reaction solution was then poured into water $(75 \mathrm{~mL})$ and $\mathrm{EtO}_{2}(50 \mathrm{~mL})$ was added. After separation, the aqueous phase was extracted with $\mathrm{EtO}_{2}(75 \mathrm{~mL})$ and $\mathrm{CH}_{2} \mathrm{Cl}_{2}(75 \mathrm{~mL})$. The combined organics were then dried over $\mathrm{Na}_{2} \mathrm{SO}_{4}$, filtered, and concentrated in vacuo. The crude material was purified by column chromatography $\left(\mathrm{SiO}_{2}, \quad 1: 1 \quad \mathrm{CH}_{2} \mathrm{Cl}_{2}:\right.$ hexanes $)$ to give a dark orange solid (E/Z)-9-(2methoxyvinyl)anthracene (6.05 g, E/Z 1:1,98\%). $R_{f} 0.6\left(1: 1, \mathrm{CH}_{2} \mathrm{Cl}_{2}\right.$ :hexanes); ${ }^{1} \mathrm{H} \mathrm{NMR}$ $\left(400 \mathrm{MHz}, \mathrm{CDCl}_{3}\right) E$ isomer $\delta 3.94(\mathrm{~s}, 3 \mathrm{H}), 6.43(\mathrm{~d}, J=13.2 \mathrm{~Hz}, 1 \mathrm{H}), 6.77(\mathrm{~d}, J=13.2$ $\mathrm{Hz}), 7.45-7.52(\mathrm{~m}, 4 \mathrm{H}), 7.99-8.00(\mathrm{~m}, 2 \mathrm{H}), 8.25(\mathrm{~d}, J=9.2 \mathrm{~Hz}), 8.37-8.41(\mathrm{~m}, 2 \mathrm{H}) ;{ }^{13} \mathrm{C}$ NMR (100 MHz, $\left.\mathrm{CDCl}_{3}\right) E$ isomer $\delta 56.7,99.1,125.3,125.9,126.5,126.9,128.7,129.9$, 130.8, 131.8, 153.1. 
<smiles>O=CCc1c2ccccc2cc2ccccc12</smiles>

2-(Anthracen-9-yl)acetaldehyde. (E/Z)-9-(2-methoxyvinyl)anthracene (6.05 g, 25.8 mmol) was dissolved in THF $(129 \mathrm{~mL})$ with stirring. Aqueous $2 \mathrm{M} \mathrm{HCl}$ solution $(51.6$ $\mathrm{mL}, 103.2 \mathrm{mmol}$ ) was added and the reaction solution was heated to $60{ }^{\circ} \mathrm{C}$ and stirred overnight. The reaction was then quenched with aqueous $\mathrm{NaHCO}_{3}(125 \mathrm{~mL})$, extracted with $\mathrm{EtO}_{2}(3 \times 80 \mathrm{~mL})$, and washed with brine $(100 \mathrm{~mL})$. The resulting solution was dried over $\mathrm{Na}_{2} \mathrm{SO}_{4}$, filtered and concentrated in vacuo to give a crude material 2(anthracen-9-yl)acetaldehyde as orange crystals that were used in the next step without further purification. $R_{f} 0.34\left(1: 1 \mathrm{CH}_{2} \mathrm{Cl}_{2}\right.$ :hexanes); ${ }^{1} \mathrm{H}$ NMR $\left(700 \mathrm{MHz}, \mathrm{CDCl}_{3}\right) \delta 4.70$ $(\mathrm{d}, J=2.8 \mathrm{~Hz}, 2 \mathrm{H}), 7.50-7.52(\mathrm{~m}, 2 \mathrm{H}), 7.56-7.58(\mathrm{~m}, 2 \mathrm{H}), 8.05(\mathrm{~d}, J=8.4 \mathrm{~Hz}, 2 \mathrm{H}), 8.17$ $(\mathrm{d}, J=8.4 \mathrm{~Hz}, 2 \mathrm{H}), 8.48(\mathrm{~s}, 1 \mathrm{H}) ;{ }^{13} \mathrm{C} \mathrm{NMR}\left(176 \mathrm{MHz}, \mathrm{CDCl}_{3}\right) \delta$ 44.5, 122.0, 122.4, $123.8,125.2,126.4,127.9,129.2,130.0,195.1$.

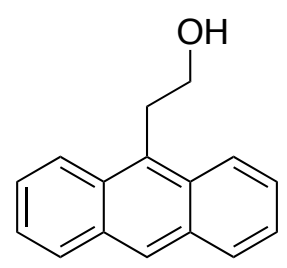

25

2-(Anthracen-9-yl)ethanol (25). Crude 2-(anthracen-9-yl)acetaldehyde (5.68 g, 25.8 mmol) was dissolved in 5:1 THF:MeOH (129 mL) with stirring at room temperature. $\mathrm{NaBH}_{4}(1.17 \mathrm{~g}, 31.0 \mathrm{mmol})$ was added portion-wise to the reaction solution and allowed to stir for $12 \mathrm{~h}$. The reaction was quenched with aqueous $\mathrm{NH}_{4} \mathrm{Cl}(70 \mathrm{~mL})$ and extracted 
with $\mathrm{EtO}_{2}(3 \times 50 \mathrm{~mL})$. The combined organics were washed with brine $(75 \mathrm{~mL})$, dried over $\mathrm{Na}_{2} \mathrm{SO}_{4}$, filtered and concentrated in vacuo. The crude material was purified by column chromatography ( $\mathrm{SiO}_{2}, 0: 100$ to $6: 94$ EtOAc: $\mathrm{CH}_{2} \mathrm{Cl}_{2}$ gradient) to give a yellow solid 25 (4.61 g, 80\% over two steps). $R_{f} 0.45$ (5:95, EtOAc: $\left.\mathrm{CH}_{2} \mathrm{Cl}_{2}\right) ;{ }^{1} \mathrm{H}$ NMR (400 $\left.\mathrm{MHz}, \mathrm{CDCl}_{3}\right) \delta 1.56(\mathrm{~s}, 1 \mathrm{H}), 3.94(\mathrm{t}, J=7.0 \mathrm{~Hz}, 2 \mathrm{H}), 4.09(\mathrm{t}, J=7.0 \mathrm{~Hz}, 2 \mathrm{H}), 7.45-7.55$ $(\mathrm{m}, 4 \mathrm{H}), 8.01(\mathrm{~d}, J=8.4 \mathrm{~Hz}, 2 \mathrm{H}), 8.33(\mathrm{~d}, J=8.8 \mathrm{~Hz}, 2 \mathrm{H}), 8.39(\mathrm{~s}, 1 \mathrm{H}) ;{ }^{13} \mathrm{C}$ NMR $(100$ $\left.\mathrm{MHz}, \mathrm{CDCl}_{3}\right) \delta 31.3,63.6,124.5,125.2,126.0,126.7,129.4,130.4,130.6,131.8$.

\subsubsection{Synthesis of Amino Esters and Amide (Scheme 3.3)}

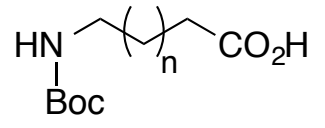

12.1-3

5-((tert-Butoxycarbonyl)amino)pentanoic acid (12.2). 5-aminopentanoic acid (1.99 g, $17.0 \mathrm{mmol})$ was dissolved in a 2:1 mixture of 1,4-dioxane: $\mathrm{H}_{2} \mathrm{O}(51 \mathrm{~mL})$ and cooled to 0 ${ }^{\circ} \mathrm{C}$. A $1 M$ solution of $\mathrm{NaOH}(0.68 \mathrm{~g}, 17.1 \mathrm{mmol})$ was added, followed by the addition of di-tert-butyl dicarbonate $(4.10 \mathrm{~g}, 18.8 \mathrm{mmol})$. After $18 \mathrm{~h}$, the dioxane was removed in vacuo and the remaining aqueous layer was washed with EtOAc $(19 \mathrm{~mL})$. The aqueous phase was then acidified to $\mathrm{pH} \sim 3$ with $1 \mathrm{M} \mathrm{HCl}$ and extracted with EtOAc $(3 \times 19 \mathrm{~mL})$. The combined organics were dried over $\mathrm{MgSO}_{4}$, filtered, and concentrated in vacuo to give a crude material 12.2 (white crystals) which were used in the next step without further purification. ${ }^{1} \mathrm{H} \mathrm{NMR}\left(400 \mathrm{MHz}, \mathrm{CDCl}_{3}\right) \delta: 1.43(\mathrm{~s}, 9 \mathrm{H}), 1.52(\mathrm{dt}, J=7.2 \mathrm{~Hz}$, 2H), 1.65 (dt, $J=7.4 \mathrm{~Hz}, 2 \mathrm{H}), 2.36(\mathrm{t}, J=7.2 \mathrm{~Hz}, 2 \mathrm{H}), 4.61$ (br s, $1 \mathrm{H}) .{ }^{13} \mathrm{C} \mathrm{NMR}(100$

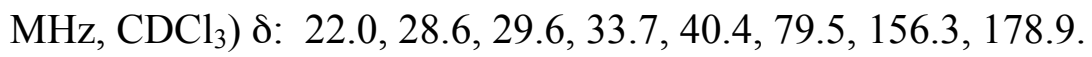


6-((tert-Butoxycarbonyl)amino)hexanoic acid (12.1). ${ }^{1} \mathrm{H} \mathrm{NMR}\left(400 \mathrm{MHz}, \mathrm{CDCl}_{3}\right) \delta$ : 1.31-1.52 (m, 13H), $1.63(\mathrm{dt}, J=7.6 \mathrm{~Hz}, 2 \mathrm{H}), 2.33(\mathrm{t}, J=7.4 \mathrm{~Hz}, 2 \mathrm{H}), 3.08(\mathrm{br} \mathrm{s}, 2 \mathrm{H})$,

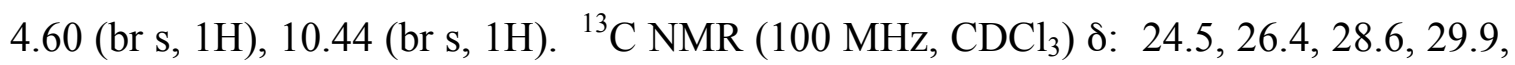
$34.1,40.6,79.6,156.1,179.2$.

4-((tert-Butoxycarbonyl)amino)butanoic acid (12.3). ${ }^{1} \mathrm{H}$ NMR $\left(400 \mathrm{MHz}, \mathrm{CDCl}_{3}\right) \delta$ : $1.43(\mathrm{~s}, 9 \mathrm{H}), 1.80(\mathrm{dt}, J=7.2 \mathrm{~Hz}, 2 \mathrm{H}), 2.38(\mathrm{t}, J=7.0 \mathrm{~Hz}, 2 \mathrm{H}), 3.16$ (br s, 2H), 4.72 (br s, 1H), 9.34 (br s, $1 \mathrm{H}) .{ }^{13} \mathrm{C} \mathrm{NMR}\left(100 \mathrm{MHz}, \mathrm{CDCl}_{3}\right) \delta:$ 25.3, 28.6, 31.5, 40.0, 79.8, 156.4, 178.5 .

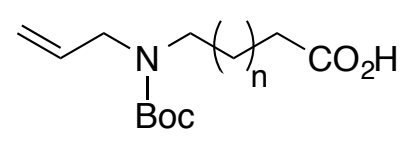

13.1: $\mathrm{n}=1$

13.2: $\mathrm{n}=2$

13.3: $n=3$

5-(Allyl(tert-butoxycarbonyl)amino)pentanoic acid (13.2). Boc-protected amine 12.2 (4.65 g, $21.4 \mathrm{mmol})$ was added to a slurry of $60 \% \mathrm{NaH}(4.28 \mathrm{~g}, 107 \mathrm{mmol})$ in dry THF $(140 \mathrm{~mL})$ at $0{ }^{\circ} \mathrm{C}$. After one-hour of stirring, allyl bromide $(5.56 \mathrm{~mL}, 64.2 \mathrm{mmol})$ was added dropwise. After $24 \mathrm{~h}$, the reaction mixture was cooled to $0{ }^{\circ} \mathrm{C}$ and quenched with water until the reaction became transparent. The reaction was acidified to $\mathrm{pH} \sim 3$ by addition of $1 \mathrm{M} \mathrm{HCl}$ and the layers were separated. The aqueous phase was extracted with EtOAc $(2 \times 30 \mathrm{~mL})$ and the combined organic phase was washed with brine (50 $\mathrm{mL}$ ), dried over $\mathrm{Na}_{2} \mathrm{SO}_{4}$, filtered and concentrated in vacuo. The crude material was purified by column chromatography $\left(\mathrm{SiO}_{2}, 1: 1\right.$ EtOAc:hexanes) to give $\mathbf{1 3 . 2}$ as an oil (4.77 g, 87\%); $R_{f} 0.36$ (1:1 EtOAc:hexanes); ${ }^{1} \mathrm{H}$ NMR (400 MHz, $\left.\mathrm{CDCl}_{3}\right) \delta 1.43$ (s, 9H), 1.51-1.64 (m, 4H), 2.35 (t, $J=7.0 \mathrm{~Hz}, 2 \mathrm{H}), 3.17$ (br s, 2H), 3.78 (br s, 2H), 5.09 (d, $J=$ 
11.6 Hz, 2H), 5.70-5.78 (m, 1H); ${ }^{13} \mathrm{C}$ NMR (100 MHz, $\left.\mathrm{CDCl}_{3}\right)$ \& 22.1, 27.8, 28.6, 33.9, 46.2, 49.7, 79.9, 116.5, 134.4, 155.8, 179.6; FT-ICR-MS calcd for $\mathrm{C}_{13} \mathrm{H}_{22} \mathrm{NO}_{4}^{-}[\mathrm{M}-\mathrm{H}]^{-}$ $m / z 256.1554$, found 256.1555 .

4-(Allyl(tert-butoxycarbonyl)amino)butanoic acid (13.1). ${ }^{1} \mathrm{H}$ NMR (400 MHz, $\left.\mathrm{CDCl}_{3}\right)$

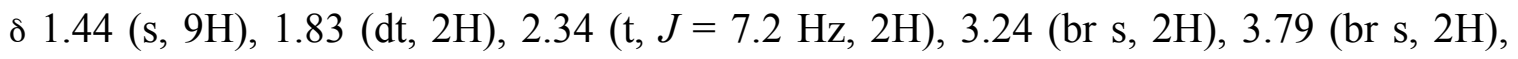
5.08-5.12 (m, 2H), 5.71-5.80 (m, 1H); ${ }^{13} \mathrm{C}$ NMR $\left(100 \mathrm{MHz}, \mathrm{CDCl}_{3}\right) \delta$ 23.5, 28.6, 31.4, 45.8, 49.7, 80.1, 116.7, 134.1, 155.9, 178.9; FT-ICR-MS calcd for $\mathrm{C}_{12} \mathrm{H}_{20} \mathrm{NO}_{4}^{-}[\mathrm{M}-\mathrm{H}]^{-}$ $(m / z) 242.1398$, found 242.1398 .

6-(Allyl(tert-butoxycarbonyl)amino)hexanoic acid (13.3). $\quad{ }^{1} \mathrm{H} \quad \mathrm{NMR}(400 \mathrm{MHz}$, $\left.\mathrm{CDCl}_{3}\right) \delta 1.30(\mathrm{dt}, J=7.6 \mathrm{~Hz}, 2 \mathrm{H}), 1.43(\mathrm{~s}, 9 \mathrm{H}), 1.83(\mathrm{dt}, J=7.6 \mathrm{~Hz}, 2 \mathrm{H}), 1.63$ (dt, $J=$ $7.6 \mathrm{~Hz}, 2 \mathrm{H}), 2.33$ (t, $J=7.4 \mathrm{~Hz}, 2 \mathrm{H}), 3.15(\mathrm{t}, J=7.0 \mathrm{~Hz}, 2 \mathrm{H}), 3.77$ (br s, $2 \mathrm{H}), 5.07-5.11$ (m, 2H), 5.70-5.80 (m, 1H), 10.02 (br s, $1 \mathrm{H}) ;{ }^{13} \mathrm{C} \mathrm{NMR}\left(100 \mathrm{MHz}, \mathrm{CDCl}_{3}\right)$ \& 24.6, 26.4, 28.1, 28.6, 34.2, 46.6, 49.7, 79.7, 116.3, 134.5, 155.8, 179.7; FT-ICR-MS calcd for $\mathrm{C}_{14} \mathrm{H}_{24} \mathrm{NO}_{4}^{-}[\mathrm{M}-\mathrm{H}]^{-}(\mathrm{m} / \mathrm{z}) 2$ 270.1711, found 270.1710.

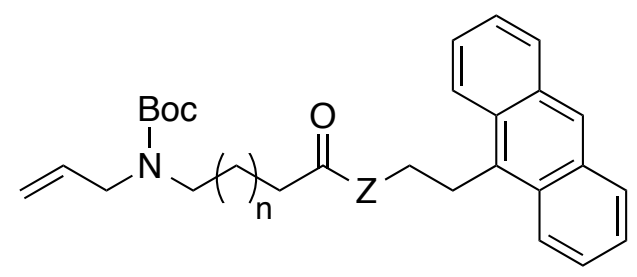

14.1-3: $Z=O$

14.4: $\mathrm{Z}=\mathrm{NH}$

2-(Anthracen-9-yl)ethyl 5-(allyl(tert-butoxycarbonyl)amino)pentanoate (14.2). To amino acid $13.2(255 \mathrm{mg}, 0.99 \mathrm{mmol})$ and alcohol $25(197 \mathrm{mg}, 0.88 \mathrm{mmol})$ in dry $\mathrm{CH}_{2} \mathrm{Cl}_{2}$ $(8 \mathrm{~mL})$ was added DIC $(211 \mu \mathrm{L}, 1.35 \mathrm{mmol})$ and 4 -( $N, N$-dimethylamino)pyridine (DMAP, pinch). After $3 \mathrm{~h}$, the white solids were filtered and the filter cake was washed 
with $\mathrm{CH}_{2} \mathrm{Cl}_{2}$. The combined filtrate was condensed in vacuo and the crude material was purified by column chromatography $\left(\mathrm{SiO}_{2}, 0: 100\right.$ to 1:19 EtOAc: $\mathrm{CH}_{2} \mathrm{Cl}_{2}$ gradient) to give 14.2 (242 mg, 59\%) as an oil; $R_{f} 0.46\left(1: 19\right.$ EtOAc: $\left.\mathrm{CH}_{2} \mathrm{Cl}_{2}\right)$; FT-IR 3058, 2981, 1729, $1685 \mathrm{~cm}^{-1} ;{ }^{1} \mathrm{H}$ NMR $\left(400 \mathrm{MHz}, \mathrm{CDCl}_{3}\right) \delta 1.46(\mathrm{~s}, 9 \mathrm{H}), 1.50-1.61(\mathrm{~m}, 4 \mathrm{H}), 2.33(\mathrm{t}, J=7.4$ Hz, 2H), 3.16 (br s, 2H), 3.79 (br s, 2H), 3.97 (t, $J=7.8 \mathrm{~Hz}, 2 \mathrm{H}), 4.48$ (t, $J=7.8 \mathrm{~Hz}, 2 \mathrm{H})$, $5.11(\mathrm{~d}, J=11.6 \mathrm{~Hz}, 2 \mathrm{H}), 5.72-5.82(\mathrm{~m}, 1 \mathrm{H}), 7.45-7.49(\mathrm{~m}, 2 \mathrm{H}), 7.51-7.59(\mathrm{~m}, 2 \mathrm{H}), 8.01$ $(\mathrm{d}, J=8.4 \mathrm{~Hz}, 2 \mathrm{H}), 8.34(\mathrm{~d}, J=9.2 \mathrm{~Hz}, 2 \mathrm{H}), 8.39(\mathrm{~s}, 1 \mathrm{H}) ;{ }^{13} \mathrm{C} \mathrm{NMR}\left(100 \mathrm{MHz}, \mathrm{CDCl}_{3}\right) \delta$ $22.3,27.5,27.9,28.6,34.2,46.3,49.9,64.3,79.6,116.2,124.3,125.1,126.2,127.0$, 129.2, 129.4, 130.5, 131.7, 134.5, 155.7, 173.8; FT-ICR-MS calcd for $\mathrm{C}_{29} \mathrm{H}_{35} \mathrm{NNaO}_{4}{ }^{+}[\mathrm{M}$ $+\mathrm{Na}]^{+} m / z$ 484.2458, found 484.2459 .

2-(Anthracen-9-yl)ethyl 4-(allyl(tert-butoxycarbonyl)amino)butanoate (14.1). FT-IR: 3058, 2981, 1730, $1685 \mathrm{~cm}^{-1}$; ${ }^{1} \mathrm{H}$ NMR (400 MHz, $\mathrm{CDCl}_{3}$ ) $\delta 1.45$ (s, 9H), 1.81 (br s, 2H), 2.31 (br s, 2H), 3.17 (br s, 2H), 3.78 (br s, 2H), 3.98 (t, $J=7.8 \mathrm{~Hz}, 2 \mathrm{H}), 4.48$ (t, $J=7.8$ $\mathrm{Hz}, 2 \mathrm{H}), 5.07-5.12(\mathrm{~m}, 2 \mathrm{H}), 5.71-5.80(\mathrm{~m}, 1 \mathrm{H})$, 7.45-7.49 (m, 2H), 7.53-7.56 (m, 2H), $8.00(\mathrm{~d}, J=8.4 \mathrm{~Hz}, 2 \mathrm{H}), 8.34$ (d, $J=8.8 \mathrm{~Hz}, 2 \mathrm{H}), 8.38(\mathrm{~s}, 1 \mathrm{H}) ;{ }^{13} \mathrm{C}$ NMR $(100 \mathrm{MHz}$, $\left.\mathrm{CDCl}_{3}\right) \delta$ 23.7, 27.5, 28.6, 31.7, 45.8, 49.9, 64.4, 79.8, 116.7, 124.3, 125.1, 126.2, 127.0, 129.2, 129.4, 130.5, 131.7, 134.3, 155.7, 173.5; FT-ICR-MS calcd for $\mathrm{C}_{28} \mathrm{H}_{33} \mathrm{NNaO}_{4}{ }^{+}[\mathrm{M}$ $+\mathrm{Na}]^{+}(m / z) 470.2302$, found 470.2303 .

2-(Anthracen-9-yl)ethyl 6-(allyl(tert-butoxycarbonyl)amino)hexanoate (14.3). FT-IR: 3058, 2936, 1730, $1684 \mathrm{~cm}^{-1}$; ${ }^{1} \mathrm{H}$ NMR (400 MHz, $\left.\mathrm{CDCl}_{3}\right) \delta 1.26(\mathrm{dt}, J=7.8 \mathrm{~Hz}, 2 \mathrm{H})$, $1.42-1.54(\mathrm{~m}, 11 \mathrm{H}), 1.58-1.64(\mathrm{dt}, J=7.6 \mathrm{~Hz}, 2 \mathrm{H}), 2.31(\mathrm{t}, J=7.6 \mathrm{~Hz}, 2 \mathrm{H}), 3.15$ (br s, 2H), 3.80 (br s, 2H), 3.97 (t, $J=7.8 \mathrm{~Hz}, 2 \mathrm{H}), 4.47$ (t, $J=8.0 \mathrm{~Hz}, 2 \mathrm{H}), 5.11$ (d, $J=12.0$ $\mathrm{Hz}, 2 \mathrm{H}), 5.73-5.83(\mathrm{~m}, 1 \mathrm{H}), 7.45-7.48(\mathrm{~m}, 2 \mathrm{H}), 7.52-7.56(\mathrm{~m}, 2 \mathrm{H}), 8.00(\mathrm{~d}, J=8.4 \mathrm{~Hz}$, 
$2 \mathrm{H}), 8.34(\mathrm{~d}, J=8.8 \mathrm{~Hz}, 2 \mathrm{H}), 8.38(\mathrm{~s}, 1 \mathrm{H}) ;{ }^{13} \mathrm{C} \mathrm{NMR}\left(100 \mathrm{MHz}, \mathrm{CDCl}_{3}\right) \delta 24.8,26.5$, $27.5,28.2,28.6,34.4,46.6,49.6,64.2,79.5,116.2,124.3,125.1,126.2,126.8,126.9$, 129.2, 129.4, 130.5, 131.7, 134.6, 155.7, 173.9; FT-ICR-MS calcd for $\mathrm{C}_{30} \mathrm{H}_{37} \mathrm{NNaO}_{4}{ }^{+}[\mathrm{M}$ $+\mathrm{Na}^{+}(\mathrm{m} / \mathrm{z})$ 498.2615, found 498.2615.

tert-Butyl allyl(4-((2-(anthracen-9-yl)ethyl)amino)-4-oxobutyl)carbamate (14.4). $R_{f}$ 0.59 (EtOAc); FT-IR: 3445, 3058, 2970, 1679, $1520 \mathrm{~cm}^{-1} ;{ }^{1} \mathrm{H}$ NMR (400 MHz, $\left.\mathrm{CDCl}_{3}\right) \delta$ 1.43 (s, 9H), 1.80 (quin, $J=6.8 \mathrm{~Hz}, 2 \mathrm{H}), 2.11$ (br s, 2H), 3.19 (br s, 2H), 3.65-3.70 (m, 2H), $3.76(\mathrm{br} \mathrm{s}, 2 \mathrm{H}), 3.87(\mathrm{t}, J=7.6 \mathrm{~Hz}, 2 \mathrm{H}), 5.08-5.11(\mathrm{~m}, 2 \mathrm{H}), 2 \mathrm{H}), 5.70-5.79(\mathrm{~m}, 1 \mathrm{H})$, 6.74 (br s, 1H), 7.43-7.46 (m, 2H), 7.49-7.53 (m, 2H), 7.98 (d, $J=8.4 \mathrm{~Hz}, 2 \mathrm{H}), 8.34$ (br s, $2 \mathrm{H}) ;{ }^{13} \mathrm{C}$ NMR (100 MHz, $\mathrm{CDCl}_{3}$ ) \& 24.4, 27.9, 28.6, 33.7, 40.6, 45.7, 49.8, 79.9, 116.5, $124.5,125.1,126.0,126.5,129.3,130.3,131.3,131.7,134.2,156.3,173.1 ;$ FT-ICR-MS calcd for $\mathrm{C}_{28} \mathrm{H}_{34} \mathrm{~N}_{2} \mathrm{NaO}_{3}{ }^{+}[\mathrm{M}+\mathrm{Na}]^{+}(m / z)$ 469.2462, found 469.2464.

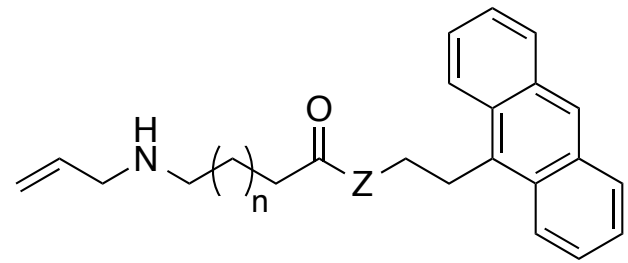

$\begin{aligned} \text { 6.1: } & n=1, Z=O \\ \text { 6.2: } & n=2, Z=O \\ \text { 6.3: } & n=3, Z=O \\ \text { 9: } & n=1, Z=N H\end{aligned}$

2-(Anthracen-9-yl)ethyl 5-(allylamino)pentanoate (6.2). Trifluoroacetic acid (0.74 $\mathrm{mL}, 9.60 \mathrm{mmol})$ was added to a solution of $\mathbf{1 4 . 2}(68 \mathrm{mg}, 0.15 \mathrm{mmol})$ in dry $\mathrm{CH}_{2} \mathrm{Cl}_{2}(0.74$ $\mathrm{mL}$ ) at $0{ }^{\circ} \mathrm{C}$. After stirring $1 \mathrm{~h}$, the volatiles were removed in vacuo and the remaining residue was diluted with $\mathrm{Et}_{2} \mathrm{O}(10 \mathrm{~mL})$ and washed with $\mathrm{NaHCO}_{3}(3 \times 5 \mathrm{~mL})$. The organic phase was washed with brine $(5 \mathrm{~mL})$, dried over $\mathrm{Na}_{2} \mathrm{SO}_{4}$, filtered and concentrated in vacuo to give $\mathbf{6 . 2}\left(53 \mathrm{mg}, 100 \%\right.$ yield) as an oil; $R_{f} 0.20$ (1:9 MeOH: $\left.\mathrm{CH}_{2} \mathrm{Cl}_{2}\right) ;{ }^{1} \mathrm{H} \mathrm{NMR}\left(400 \mathrm{MHz}, \mathrm{CDCl}_{3}\right) \delta 1.49(\mathrm{dt}, J=7.4 \mathrm{~Hz}, 2 \mathrm{H}), 1.64(\mathrm{dt}, J=$ 
$7.6 \mathrm{~Hz}, 2 \mathrm{H}), 1.84$ (br s, 1H), 2.32 (t, $J=7.4 \mathrm{~Hz}, 2 \mathrm{H}), 2.59$ (t, $J=7.2 \mathrm{~Hz}, 2 \mathrm{H}), 3.24$ (d, $J=$ $5.6 \mathrm{~Hz}, 2 \mathrm{H}), 3.97(\mathrm{t}, J=7.8 \mathrm{~Hz}, 2 \mathrm{H}), 4.48(\mathrm{t}, J=7.8 \mathrm{~Hz}, 2 \mathrm{H}), 5.11(\mathrm{~d}, J=10.4 \mathrm{~Hz}, 1 \mathrm{H})$, $5.18(\mathrm{~d}, J=17.2 \mathrm{~Hz}, 1 \mathrm{H}), 5.86-5.96(\mathrm{~m}, 1 \mathrm{H}), 7.47(\mathrm{t}, J=7.4 \mathrm{~Hz}, 2 \mathrm{H}), 7.55(\mathrm{t}, J=7.6 \mathrm{~Hz}$, $2 \mathrm{H}), 8.01(\mathrm{~d}, J=8.4 \mathrm{~Hz}, 2 \mathrm{H}), 8.34(\mathrm{~d}, J=9.6 \mathrm{~Hz}, 2 \mathrm{H}), 8.38(\mathrm{~s}, 1 \mathrm{H}) ;{ }^{13} \mathrm{C} \mathrm{NMR}(100 \mathrm{MHz}$, $\left.\mathrm{CDCl}_{3}\right)$ \& 22.8, 27.5, 29.5, 34.3, 48.9, 52.5, 64.3, 116.4, 124.3, 124.6, 125.1, 126.2, 126.9, 129.4, 130.5, 131.7, 136.7, 173.9; FT-ICR-MS calcd for $\mathrm{C}_{24} \mathrm{H}_{28} \mathrm{NO}_{2}{ }^{+}[\mathrm{M}+\mathrm{H}]^{+} \mathrm{m} / \mathrm{z}$ 362.2115 , found 362.2141 .

2-(Anthracen-9-yl)ethyl 4-(allylamino)butanoate (6.1). ${ }^{1} \mathrm{H} \mathrm{NMR}\left(400 \mathrm{MHz}, \mathrm{CDCl}_{3}\right) \delta$ 1.64 (br s, 1H), 1.79 (dt, $J=7.2 \mathrm{~Hz}, 2 \mathrm{H}), 2.37$ (t, $J=7.2 \mathrm{~Hz}, 2 \mathrm{H}), 2.59$ (t, $J=7.2 \mathrm{~Hz}$, 2H), $3.21(\mathrm{~d}, J=6.4 \mathrm{~Hz}, 2 \mathrm{H}), 3.97$ (t, $J=7.8 \mathrm{~Hz}, 2 \mathrm{H}), 4.48$ (t, $J=7.8 \mathrm{~Hz}, 2 \mathrm{H}), 5.09$ (d, $J$ $=10.0 \mathrm{~Hz}, 1 \mathrm{H}), 5.17(\mathrm{dd}, J=17.2,1.4 \mathrm{~Hz}, 1 \mathrm{H}), 5.83-5.93(\mathrm{~m}, 1 \mathrm{H}), 7.45-7.49(\mathrm{~m}, 2 \mathrm{H})$, 7.52-7.55 (m, 2H), $8.01(\mathrm{~d}, J=8.4 \mathrm{~Hz}, 2 \mathrm{H}), 8.34(\mathrm{~d}, J=8.8 \mathrm{~Hz}, 2 \mathrm{H}), 8.38(\mathrm{~s}, 1 \mathrm{H}) ;{ }^{13} \mathrm{C}$ NMR (100 MHz, $\left.\mathrm{CDCl}_{3}\right)$ \& 25.3, 27.5, 32.3, 48.6, 52.3, 64.3, 116.3, 124.4, 124.9, 125.7, $126.8,129.3,129.5,130.5,131.7,136.7,173.8$; FT-ICR-MS calcd for $\mathrm{C}_{23} \mathrm{H}_{26} \mathrm{NO}_{2}{ }^{+}[\mathrm{M}+$ $\mathrm{H}]^{+} m / z 348.1958$, found 348.1964 .

2-(Anthracen-9-yl)ethyl 6-(allylamino)hexanoate (6.3). ${ }^{1} \mathrm{H} \mathrm{NMR}\left(400 \mathrm{MHz}, \mathrm{CDCl}_{3}\right) \delta$ $1.32(\mathrm{dt}, J=7.8 \mathrm{~Hz}, 2 \mathrm{H}), 1.49$ (dt, $J=7.4 \mathrm{~Hz}, 2 \mathrm{H}), 1.62$ (quin, $J=7.6 \mathrm{~Hz}, 2 \mathrm{H}), 1.77$ (br s, 1H), $2.31(\mathrm{t}, J=7.6 \mathrm{~Hz}, 2 \mathrm{H}), 2.60(\mathrm{t}, J=7.0 \mathrm{~Hz}, 2 \mathrm{H}), 3.26(\mathrm{~d}, J=5.6 \mathrm{~Hz}, 2 \mathrm{H}), 3.98(\mathrm{t}$, $J=7.8 \mathrm{~Hz}, 2 \mathrm{H}), 4.48(\mathrm{t}, J=8.0 \mathrm{~Hz}, 2 \mathrm{H}), 5.10(\mathrm{~d}, J=10.4 \mathrm{~Hz}, 1 \mathrm{H}), 5.19(\mathrm{~d}, J=17.6 \mathrm{~Hz}$, 1H), 5.87-5.97 (m, 1H), 7.45-7.49 (m, 2H), 7.53-7.57 (m, 2H), $8.01(\mathrm{~d}, J=8.4 \mathrm{~Hz}, 2 \mathrm{H})$, $8.34(\mathrm{~d}, J=8.8 \mathrm{~Hz}, 2 \mathrm{H}), 8.38$ (s, $1 \mathrm{H}) ;{ }^{13} \mathrm{C} \mathrm{NMR}\left(100 \mathrm{MHz}, \mathrm{CDCl}_{3}\right) \delta$ 24.9, 27.0, 27.5, $29.8,34.4,49.2,52.6,64.2,116.3,124.3,125.1,126.2,126.9,129.3,129.4,130.5,131.7$, 
136.8, 174.0; FT-ICR-MS calcd for $\mathrm{C}_{25} \mathrm{H}_{30} \mathrm{NO}_{2}{ }^{+}[\mathrm{M}+\mathrm{H}]^{+}(m / z)$ 376.2271, found 376.2278 .

N-(2-(Anthracen-9-yl)ethyl)-4-(allylamino)butanamide (9, TFA salt). Trifluoroacetic acid $(0.50 \mathrm{~mL}, 6.53 \mathrm{mmol})$ was added to a solution of $14.4(20 \mathrm{mg}, 0.045 \mathrm{mmol})$ in dry $\mathrm{CH}_{2} \mathrm{Cl}_{2}(0.50 \mathrm{~mL})$ at $0{ }^{\circ} \mathrm{C}$. After stirring $1 \mathrm{~h}$, the volatiles were removed in vacuo to afford the TFA salt of 9 (15 mg, 72\%) as an oil; ${ }^{1} \mathrm{H}$ NMR (400 MHz, $\left.\mathrm{CDCl}_{3}\right) \delta 1.97(\mathrm{t}, J$ $=6.4 \mathrm{~Hz}, 2 \mathrm{H}), 2.29(\mathrm{t}, J=6.4 \mathrm{~Hz}, 2 \mathrm{H}), 2.97(\mathrm{br} \mathrm{s}, 2 \mathrm{H}), 3.54(\mathrm{br} \mathrm{s}, 2 \mathrm{H}), 3.60(\mathrm{q}, J=7.2$ $\mathrm{Hz}, 2 \mathrm{H}), 3.81(\mathrm{t}, J=7.8 \mathrm{~Hz}, 2 \mathrm{H}), 5.40-5.46(\mathrm{~m}, 2 \mathrm{H}), 5.84-5.93(\mathrm{~m}, 1 \mathrm{H}), 6.81(\mathrm{~s}, 1 \mathrm{H})$, 7.42-7.52 (m, 4H), $7.97(\mathrm{~d}, J=8.0 \mathrm{~Hz}, 2 \mathrm{H}), 8.28(\mathrm{~d}, J=8.8 \mathrm{~Hz}, 2 \mathrm{H}), 8.34(\mathrm{~s}, 1 \mathrm{H}), 9.81$ (br s, $2 \mathrm{H}) ;{ }^{13} \mathrm{C} \mathrm{NMR}\left(100 \mathrm{MHz}, \mathrm{CDCl}_{3}\right) \delta 21.9,27.7,33.7,40.7,46.7,50.0,124.0,124.3$, $125.2,126.2,126.7,127.9,129.4,130.3,130.8,131.7,173.0$; FT-ICR-MS calcd for $\mathrm{C}_{23} \mathrm{H}_{27} \mathrm{~N}_{2} \mathrm{O}^{+}[\mathrm{M}+\mathrm{H}]^{+}(m / z) 347.2118$, found 347.2143.

\subsubsection{Synthesis of Amino Carbonates (Scheme 3.4)}<smiles>CC(O)C(C)CCNC(=O)O</smiles>

15.1-2

tert-Butyl (2-hydroxyethyl)carbamate (15.1). $\mathrm{Et}_{3} \mathrm{~N}(2.51 \mathrm{~mL}, 18.0 \mathrm{mmol})$ was added to a solution of 2-ethanolamine $(1.02 \mathrm{~g}, 16.7 \mathrm{mmol})$ in dry $\mathrm{CH}_{2} \mathrm{Cl}_{2}(33 \mathrm{~mL})$ with stirring at $0{ }^{\circ} \mathrm{C}$. Boc $_{2} \mathrm{O}(3.93 \mathrm{~g}, 18.0 \mathrm{mmol})$ was then added to the reaction, turning the solution to an opaque white color that slowly cleared as the reaction proceeded. The reaction was stirred for $19 \mathrm{~h}$ and then was quenched with sat. $\mathrm{NH}_{4} \mathrm{Cl}(30 \mathrm{~mL})$ and the aqueous phase was extracted with $\mathrm{CH}_{2} \mathrm{Cl}_{2}(2 \times 20 \mathrm{~mL})$. The combined organics were washed with brine (30 mL), dried over $\mathrm{Na}_{2} \mathrm{SO}_{4}$, filtered and concentrated in vacuo to give crude $\mathbf{1 5 . 1}$ as a 
light yellow oil which was used in the next step without further purification. $R_{f} 0.38$ (1:19, $\left.\mathrm{MeOH}: \mathrm{CH}_{2} \mathrm{Cl}_{2}\right) ;{ }^{1} \mathrm{H}$ NMR (400 MHz, $\mathrm{CDCl}_{3}$ ) $\delta 1.40$ (s, 9H), 2.86 (br s, $1 \mathrm{H}$ ), 3.23 (br s, 2H), 3.63 (br s, 2H), 5.03 (br s, $1 \mathrm{H}) ;{ }^{13} \mathrm{C} \mathrm{NMR}\left(100 \mathrm{MHz}, \mathrm{CDCl}_{3}\right) \delta$ 28.6, 43.3, $62.6,79.8,157.0$.

tert-Butyl (3-hydroxypropyl)carbamate (15.2). ${ }^{1} \mathrm{H} \mathrm{NMR}\left(400 \mathrm{MHz}, \mathrm{CDCl}_{3}\right) \delta 1.42$ (s, 9H), $1.64(\mathrm{dt}, J=5.8 \mathrm{~Hz}, 2 \mathrm{H}), 3.18(\mathrm{br} \mathrm{s}, 1 \mathrm{H}), 3.25(\mathrm{dt}, J=6.4,5.6 \mathrm{~Hz}, 2 \mathrm{H}), 3.63$ (br s, 2H), 4.87 (br s, 1H); ${ }^{13} \mathrm{C}$ NMR (100 MHz, $\left.\mathrm{CDCl}_{3}\right) \delta$ 28.6, 33.0, 37.1, 59.4, 79.8, 157.3.

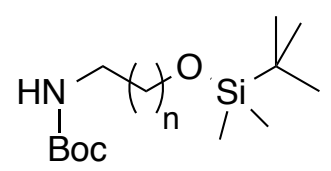

tert-Butyl (2-((tert-butyldimethylsilyl)oxy)ethyl)carbamate. TBSCl (3.06 g, 20.3 mmol) was added to a solution of $\mathbf{1 . 1}(2.70 \mathrm{~g}, 16.7 \mathrm{mmol}), \mathrm{Et}_{3} \mathrm{~N}(2.85 \mathrm{~mL}, 20.3 \mathrm{mmol})$ and imidazole $(3.14 \mathrm{~g}, 46.1 \mathrm{mmol})$ in dry $\mathrm{CH}_{2} \mathrm{Cl}_{2}(40 \mathrm{~mL})$ with stirring at $0{ }^{\circ} \mathrm{C}$. After 23 $\mathrm{h}$, the reaction was quenched with water $(25 \mathrm{~mL})$ and the aqueous phase was extracted with $\mathrm{CH}_{2} \mathrm{Cl}_{2}(2 \times 10 \mathrm{~mL})$. The combined organics were washed with sat. $\mathrm{NH}_{4} \mathrm{Cl}$, dried over $\mathrm{Na}_{2} \mathrm{SO}_{4}$, filtered and condensed in vacuo. The crude material was purified by column chromatography $\left(\mathrm{SiO}_{2}, 3: 7\right.$ EtOAc:hexanes) to give tert-butyl (2-((tertbutyldimethylsilyl)oxy)ethyl)carbamate $(4.52 \mathrm{~g}, 98 \%)$ as a colorless oil. $R_{f} 0.67(1: 1$, EtOAc:hexanes); ${ }^{1} \mathrm{H}$ NMR (400 MHz, $\left.\mathrm{CDCl}_{3}\right) \delta 0.06$ (s, 6H), 0.89 (s, 9H), 1.44 (s, 9H), 3.23 (br s, 2H), 3.65 (br s, 2H), 4.84 (br s, $\left.1 \mathrm{H}) ;{ }^{13} \mathrm{C} \mathrm{NMR} \mathrm{(100} \mathrm{MHz,} \mathrm{CDCl}_{3}\right) \delta-5.1,18.5$, $26.1,28.6,43.1,62.5,79.4,156.2$.

tert-Butyl (3-((tert-butyldimethylsilyl)oxy)propyl)carbamate. ${ }^{1} \mathrm{H}$ NMR $(400 \mathrm{MHz}$, $\left.\mathrm{CDCl}_{3}\right) \delta 0.05(\mathrm{~s}, 6 \mathrm{H}), 0.89(\mathrm{~s}, 9 \mathrm{H}), 1.42(\mathrm{~s}, 9 \mathrm{H}), 1.68(\mathrm{dt}, J=6.0 \mathrm{~Hz}, 2 \mathrm{H}), 3.23(\mathrm{br} \mathrm{s}$, 
2H), $3.70(\mathrm{t}, J=5.6 \mathrm{~Hz}, 2 \mathrm{H}), 5.10(\mathrm{br} \mathrm{s}, 1 \mathrm{H}) ;{ }^{13} \mathrm{C} \mathrm{NMR}\left(100 \mathrm{MHz}, \mathrm{CDCl}_{3}\right) \delta$-3.4, 18.4, $26.1,28.6,32.2,39.4,62.4,79.0,156.2$.

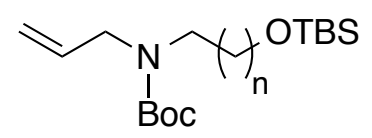

tert-Butyl allyl(2-((tert-butyldimethylsilyl)oxy)ethyl)carbamate. tert-Butyl (2-((tertbutyldimethylsilyl)oxy)ethyl)carbamate $(4.52 \mathrm{~g}, 16.4 \mathrm{mmol})$ was added to a slurry of $\mathrm{NaH}(1.08 \mathrm{~g}$ of $60 \%$ in mineral oil, $26.9 \mathrm{mmol})$ in dry THF $(36 \mathrm{~mL})$ at $0{ }^{\circ} \mathrm{C}$ and stirred for $1 \mathrm{~h}$, and then allyl bromide $(3.88 \mathrm{~mL}, 44.8 \mathrm{mmol})$ was added dropwise. The slurry was stirred for two days before cooling it to $0{ }^{\circ} \mathrm{C}$ and quenching it with water $(20 \mathrm{~mL})$. The aqueous phase was extracted with EtOAc $(2 \times 15 \mathrm{~mL})$. The combined organics were washed with brine $(20 \mathrm{~mL})$, dried over $\mathrm{Na}_{2} \mathrm{SO}_{4}$, filtered and concentrated in vacuo. The crude material was purified by column chromatography $\left(\mathrm{SiO}_{2}, 1: 19 \mathrm{Et}_{2} \mathrm{O}\right.$ :hexanes) to give tert-butyl allyl(2-((tert-butyldimethylsilyl)oxy)ethyl)carbamate (4.36 g, 84\%) as a light yellow oil. $R_{f} 0.25$ (1:19, $\mathrm{Et}_{2} \mathrm{O}$ :hexanes); ${ }^{1} \mathrm{H}$ NMR (400 MHz, $\left.\mathrm{CDCl}_{3}\right) \delta 0.04$ (s, 6H), 0.88 (s, 9H), 1.44 (s, 9H), 3.29 (br s, 2H), 3.71 (br s, 2H), 3.89 (br s, 2H), 5.10 (br s, 2H), 5.76 (br s, $1 \mathrm{H}) ;{ }^{13} \mathrm{C} \mathrm{NMR}\left(100 \mathrm{MHz}, \mathrm{CDCl}_{3}\right) \delta-5.2,18.5,26.1,28.7,49.0,51.6$, $62.0,79.6,116.5,134.6,155.6$.

tert-Butyl allyl(3-((tert-butyldimethylsilyl)oxy)propyl)carbamate. ${ }^{1} \mathrm{H}$ NMR (400 $\left.\mathrm{MHz}, \mathrm{CDCl}_{3}\right) \delta 0.04$ (s, 6H), 0.88 (s, 9H), 1.44 (s, 9H), $1.72(\mathrm{dt}, J=6.0 \mathrm{~Hz}, 2 \mathrm{H}), 3.23$ (br $\mathrm{s}, 2 \mathrm{H}), 3.61(\mathrm{t}, J=6.0 \mathrm{~Hz}, 2 \mathrm{H}), 3.82(\mathrm{br} \mathrm{s}, 2 \mathrm{H}), 5.10(\mathrm{~d}, J=11.2 \mathrm{~Hz}, 2 \mathrm{H}), 5.72-5.80(\mathrm{~m}$, $1 \mathrm{H}) ;{ }^{13} \mathrm{C}$ NMR $\left(100 \mathrm{MHz}, \mathrm{CDCl}_{3}\right) \delta-5.1,26.1,28.7,32.0,44.0,49.2,61.0,79.5,116.5$, $134.6,155.7$. 


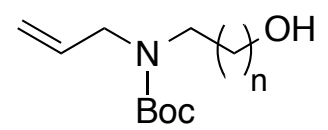

16.1-2

tert-Butyl allyl(2-hydroxyethyl)carbamate (16.1). TBAF $(18.0 \mathrm{~mL}$ of a $1 \mathrm{M}$ solution, $18.0 \mathrm{mmol}$ ) was added dropwise to a solution of the corresponding $\mathrm{N}$-allyl silyl ether of $10.1(4.36 \mathrm{~g}, 13.8 \mathrm{mmol})$ in dry THF $(28 \mathrm{~mL})$ with stirring at $0{ }^{\circ} \mathrm{C}$. After $20 \mathrm{~h}$, the reaction solution was diluted with $\mathrm{Et}_{2} \mathrm{O}(50 \mathrm{~mL})$ and washed with sat. $\mathrm{NaHCO}_{3}(3 \mathrm{x} 40$ $\mathrm{mL})$. The reaction solution was then washed with brine $(40 \mathrm{~mL})$, dried over $\mathrm{Na}_{2} \mathrm{SO}_{4}$, filtered and concentrated in vacuo. The crude material was purified by column chromatography $\left(\mathrm{SiO}_{2}, 1: 1 \mathrm{EtOAc}\right.$ :hexanes) to give $\mathbf{1 6 . 1}(2.65 \mathrm{~g}, 95 \%)$ as a light yellow oil. $R_{f} 0.33$ (1:1 EtOAc:hexanes); ${ }^{1} \mathrm{H}$ NMR (400 MHz, $\left.\mathrm{CDCl}_{3}\right) \delta 1.45$ (s, 9H), 3.07 (br s, 1H), 3.37 (br s, 2H), 3.72 (br s, 2H), 3.84 (br s, 2H), 5.10-5.14 (m, 2H), 5.78 (br s, 1H); ${ }^{13} \mathrm{C} \mathrm{NMR}\left(100 \mathrm{MHz}, \mathrm{CDCl}_{3}\right) \delta$ 28.5, 50.0, 51.4, 62.5, 80.4, 116.6, 134.2, 157.4; FT-ICRMS calcd for $\mathrm{C}_{10} \mathrm{H}_{20} \mathrm{NO}_{3}{ }^{+}[\mathrm{M}+\mathrm{H}]^{+}(\mathrm{m} / z)$ 202.1438, found 202.1439.

tert-Butyl allyl(3-hydroxypropyl)carbamate (16.2). ${ }^{1} \mathrm{H}$ NMR $\left(400 \mathrm{MHz}, \mathrm{CDCl}_{3}\right) \delta$ 1.45 (s, 9H), 1.66 (br s, 2H), 3.37 (br s, 2H), 3.55 (br s, 2H), 3.75 (br s, 2H), 5.12 (d, J= 11.2 Hz, 2H), 5.71-5.81 (m, 1H); ${ }^{13} \mathrm{C}$ NMR (100 MHz, $\left.\mathrm{CDCl}_{3}\right) \delta$ 28.5, 30.7, 42.6, 50.0, $58.5,80.4,116.7,134.1,157.1$.

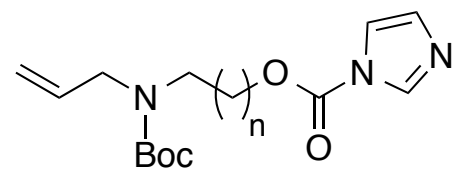

17.1-2

3-(Allyl(tert-butoxycarbonyl)amino)propyl $1 \mathrm{H}$-imidazole-1-carboxylate (17.2). $\mathrm{N}, \mathrm{N}$ Diiso-propylethylamine $(566 \mu \mathrm{L}, 3.25 \mathrm{mmol})$ was added to a solution of alcohol $\mathbf{1 6 . 2}$ 
(399 mg, $1.85 \mathrm{mmol})$ in dry $\mathrm{CH}_{2} \mathrm{Cl}_{2}(46 \mathrm{~mL})$ at $0{ }^{\circ} \mathrm{C} .1,1^{\prime}$-Carbonyldiimidazole $(527 \mathrm{mg}$, $3.25 \mathrm{mmol}$ ) was then added to the cooled solution and the cooling bath was removed to allow the reaction to slowly warm to room temperature. After $24 \mathrm{~h}$, the reaction was washed with water $(2 \times 20 \mathrm{~mL})$, brine $(20 \mathrm{~mL})$, and then dried $\left(\mathrm{Na}_{2} \mathrm{SO}_{4}\right)$. After filtration and concentration in vacuo, the residue was purified by column chromatography $\left(\mathrm{SiO}_{2}\right.$, EtOAc) to give 17.2 (543 mg, 95\%) as a colorless oil; $R_{f} 0.50$ (EtOAc); ${ }^{1} \mathrm{H}$ NMR (400 $\left.\mathrm{MHz}, \mathrm{CDCl}_{3}\right) \delta 1.43(\mathrm{~s}, 9 \mathrm{H}), 2.01(\mathrm{dt}, J=6.6 \mathrm{~Hz}, 2 \mathrm{H}), 3.34$ (br s, 2H), 3.82 (br s, 2H), $4.43(\mathrm{t}, J=6.4 \mathrm{~Hz}, 2 \mathrm{H}), 5.09-5.14(\mathrm{~m}, 2 \mathrm{H}), 5.74-5.81(\mathrm{~m}, 1 \mathrm{H}), 7.06(\mathrm{~s}, 1 \mathrm{H}), 7.41(\mathrm{~s}, 1 \mathrm{H})$,

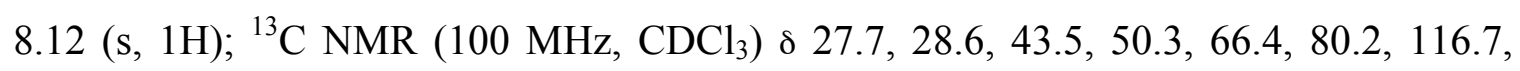
117.3, 130.9, 134.2, 137.3, 148.8, 155.6; FT-ICR-MS calcd for $\mathrm{C}_{15} \mathrm{H}_{24} \mathrm{~N}_{3} \mathrm{O}_{4}{ }^{+}[\mathrm{M}+\mathrm{H}]^{+}$ $m / z 310.1761$, found 310.1765 .

2-(Allyl(tert-butoxycarbonyl)amino)ethyl $1 \mathrm{H}$-imidazole-1-carboxylate (17.1). $R_{f} 0.50$ (EtOAc); ${ }^{1} \mathrm{H}$ NMR (400 MHz, $\mathrm{CDCl}_{3}$ ) $\delta 1.41$ (s, 9H), 3.59 (br s, 2H), 3.87 (br s, 2H), $4.48(\mathrm{t}, J=5.2 \mathrm{~Hz}, 2 \mathrm{H}), 5.11(\mathrm{~d}, J=10.8 \mathrm{~Hz}, 2 \mathrm{H}), 5.76(\mathrm{br} \mathrm{s}, 1 \mathrm{H}), 7.05(\mathrm{~s}, 1 \mathrm{H}), 7.41$ (s, 1H), $8.12(\mathrm{~s}, 1 \mathrm{H}) ;{ }^{13} \mathrm{C} \mathrm{NMR}\left(100 \mathrm{MHz}, \mathrm{CDCl}_{3}\right) \delta$ 28.5, 45.4, 51.0, 66.0, 80.5, 116.7, $117.3,130.9,133.9,137.3,148.8,155.7$; FT-ICR-MS calcd for $\mathrm{C}_{14} \mathrm{H}_{21} \mathrm{~N}_{3} \mathrm{NaO}_{4}{ }^{+}[\mathrm{M}+$ $\mathrm{Na}]^{+}(m / z) 318.1424$, found 318.1428. 


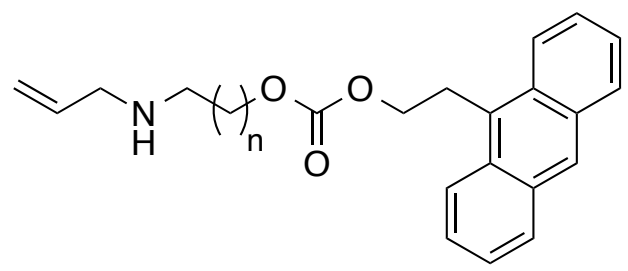

7.1: $\mathrm{n}=1$

7.2: $n=2$

2-(Anthracen-9-yl)ethyl 3-((allyl)amino)propyl carbonate (7.2, TFA salt). Alcohol $25(131 \mathrm{mg}, 0.59 \mathrm{mmol})$ was added to a mixture of $\mathbf{1 7 . 2}(210 \mathrm{mg}, 0.65 \mathrm{mmol})$ and $\mathrm{KOH}$ (s, 1 pellet) in dry toluene $(3 \mathrm{~mL})$ at $60{ }^{\circ} \mathrm{C}$. After $5 \mathrm{~h}$ the reaction was concentrated in vacuo and the residue was diluted with $\mathrm{CH}_{2} \mathrm{Cl}_{2}(5 \mathrm{~mL})$. The solution was washed with water $(3 \times 5 \mathrm{~mL})$, dried over $\mathrm{Na}_{2} \mathrm{SO}_{4}$, filtered and concentrated in vacuo. The crude material was purified by column chromatography $\left(\mathrm{SiO}_{2}, 1: 19\right.$ EtOAc: $\left.\mathrm{CH}_{2} \mathrm{Cl}_{2}\right)$ to give Boc-protected 7.2 as an orange oil (93 mg, 34\%). $R_{f} 0.63$ (1:19 EtOAc:hexanes); FT-IR: 3017, 2971, 1739, $1229 \mathrm{~cm}^{-1} ;{ }^{1} \mathrm{H}$ NMR (400 MHz, $\mathrm{CDCl}_{3}$ ) $\delta 1.47$ (s, 9H), 1.92 (br s, 2H), 3.30 (br s, 2H), 3.83 (br s, 2H), 4.05 (t, $J=8.0 \mathrm{~Hz}, 2 \mathrm{H}), 4.19$ (t, $J=6.2 \mathrm{~Hz}, 2 \mathrm{H}), 4.49$ (t, $J$ $=8.2 \mathrm{~Hz}, 2 \mathrm{H}), 5.13(\mathrm{~d}, J=11.2 \mathrm{~Hz}, 2 \mathrm{H}), 5.74-5.84(\mathrm{~m}, 1 \mathrm{H}), 7.46-7.50(\mathrm{~m}, 2 \mathrm{H}), 7.54-7.58$ $(\mathrm{m}, 2 \mathrm{H}), 8.02(\mathrm{~d}, J=8.0 \mathrm{~Hz}, 2 \mathrm{H}), 8.34(\mathrm{~d}, J=8.8 \mathrm{~Hz}, 2 \mathrm{H}), 8.40(\mathrm{~s}, 1 \mathrm{H}) ;{ }^{13} \mathrm{C}$ NMR $(100$ $\left.\mathrm{MHz}, \mathrm{CDCl}_{3}\right) \delta$ 27.6, 28.0, 28.6, 43.9, 49.8, 66.0, 67.3, 79.9, 116.8, 124.1, 125.2, 126.4, $127.2,128.3,129.5,130.5,131.7,134.3,155.5,155.6$; FT-ICR-MS calcd for $\mathrm{C}_{28} \mathrm{H}_{33} \mathrm{NNaO}_{5}^{+}[\mathrm{M}+\mathrm{Na}]^{+} m / z$ 486.2251, found 486.2251.

Trifluoroacetic acid $(0.50 \mathrm{~mL}, 6.53 \mathrm{mmol})$ was added to a solution of Boc-protected $7.2(12 \mathrm{mg}, 0.026 \mathrm{mmol})$ in dry $\mathrm{CH}_{2} \mathrm{Cl}_{2}(0.50 \mathrm{~mL})$ at $0{ }^{\circ} \mathrm{C}$. After stirring $1 \mathrm{~h}$, the volatiles were removed in vacuo to afford the TFA salt of $7.2(12 \mathrm{mg}, 96 \%)$ as an oil; ${ }^{1} \mathrm{H}$ NMR (400 MHz, $\left.\mathrm{CDCl}_{3}\right) \delta 2.10$ (t, $\left.J=6.8 \mathrm{~Hz}, 2 \mathrm{H}\right), 3.00(\mathrm{br} \mathrm{s}, 2 \mathrm{H}), 3.53(\mathrm{br} \mathrm{s}, 2 \mathrm{H}), 4.02$ (t, $J=$ $8.4 \mathrm{~Hz}, 2 \mathrm{H}), 4.23$ (t, $J=6.0 \mathrm{~Hz}, 2 \mathrm{H}), 4.50$ (t, $J=8.4 \mathrm{~Hz}, 2 \mathrm{H}), 5.12-5.48$ (m, 2H), 5.83- 
$5.96(\mathrm{~m}, 1 \mathrm{H}), 7.45-7.56(\mathrm{~m}, 4 \mathrm{H}), 8.00(\mathrm{~d}, J=8.4 \mathrm{~Hz}, 2 \mathrm{H}), 8.30(\mathrm{~d}, J=8.8 \mathrm{~Hz}, 2 \mathrm{H}), 8.39$ (s, 1H), 9.64 (br s, 2H); ${ }^{13} \mathrm{C}$ NMR (100 MHz, $\left.\mathrm{CDCl}_{3}\right)$ \& 25.7, 27.4, 43.9, 50.1, 64.6, 67.7, 124.1, 124.3, 125.2, 126.4, 127.2, 127.6, 128.3, 129.5, 130.5, 131.7, 155.3; FT-ICR-MS calcd for $\mathrm{C}_{23} \mathrm{H}_{26} \mathrm{NO}_{3}{ }^{+}[\mathrm{M}+\mathrm{H}]^{+} \mathrm{m} / \mathrm{z} 364.1907$, found 364.1911.

tert-Butyl allyl(2-(((2-(anthracen-9-yl)ethoxy)carbonyl)oxy)ethyl)carbamate (7.1,

Boc-protected). $R_{f} 0.64$ (1:19 EtOAc:hexanes); FT-IR: 3017, 2971, 1740, $1230 \mathrm{~cm}^{-1} ;{ }^{1} \mathrm{H}$ NMR (400 MHz, $\left.\mathrm{CDCl}_{3}\right) \delta 1.47$ (s, 9H), 3.46 (br s, 2H), 3.95 (br s, 2H), 4.04 (t, $J=8.0$ Hz, 2H), 4.27 (br s, 2H), 4.50 (t, $J=8.2 \mathrm{~Hz}, 2 \mathrm{H}$ ), 5.13 (br s, 2H), 5.77 (br s, 1H), 7.44$7.50(\mathrm{~m}, 2 \mathrm{H}), 7.51-7.58(\mathrm{~m}, 2 \mathrm{H}), 8.02(\mathrm{~d}, J=8.0 \mathrm{~Hz}, 2 \mathrm{H}), 8.34(\mathrm{~d}, J=8.8 \mathrm{~Hz}, 2 \mathrm{H}), 8.40$ (s, 1H); ${ }^{13} \mathrm{C}$ NMR $\left(100 \mathrm{MHz}, \mathrm{CDCl}_{3}\right) \delta 27.5,28.6,45.5,50.4,66.2,67.5,79.4,117.1$, 124.1, 125.2, 126.4, 127.2, 128.3, 129.5, 130.5, 131.7, 134.1, 155.0, 155.4; FT-ICR-MS calcd for $\mathrm{C}_{27} \mathrm{H}_{31} \mathrm{NNaO}_{5}^{+}[\mathrm{M}+\mathrm{Na}]^{+}(\mathrm{m} / z)$ 472.2094, found 472.2096.

\section{2-(Anthracen-9-yl)ethyl 3-((allyl)amino)ethyl carbonate (7.1, TFA salt).} Trifluoroacetic acid $(0.50 \mathrm{~mL}, 6.53 \mathrm{mmol})$ was added to a solution of Boc-protected 7.1 (13 mg, $0.030 \mathrm{mmol})$ in dry $\mathrm{CH}_{2} \mathrm{Cl}_{2}(0.50 \mathrm{~mL})$ at $0{ }^{\circ} \mathrm{C}$. After stirring $1 \mathrm{~h}$, the volatiles were removed in vacuo to afford the TFA salt of $2.1(14 \mathrm{mg}, 100 \%)$ as an oil; ${ }^{1} \mathrm{H}$ NMR (400 MHz, $\left.\mathrm{CDCl}_{3}\right) \delta 3.24$ (t, $\left.J=4.8 \mathrm{~Hz}, 2 \mathrm{H}\right), 3.62-3.64(\mathrm{~m}, 2 \mathrm{H}), 4.00(\mathrm{t}, J=8.0 \mathrm{~Hz}, 2 \mathrm{H})$, 4.44-4.48 (m, 4H), 5.42-5.46 (m, 2H), 5.85-5.96 (m, 1H), 7.43-7.54 (m, 4H), $7.99(\mathrm{~d} J=$ $8.0 \mathrm{~Hz}, 2 \mathrm{H}), 8.26(\mathrm{~d}, J=8.8 \mathrm{~Hz}, 2 \mathrm{H}), 8.37(\mathrm{~s}, 1 \mathrm{H}) ;{ }^{13} \mathrm{C} \mathrm{NMR}\left(100 \mathrm{MHz}, \mathrm{CDCl}_{3}\right) \delta 27.2$, $45.3,50.2,63.0,68.0,124.0,124.6,125.2,126.4,127.2,127.6,129.5,130.5,131.7$, 134.3, 154.9; FT-ICR-MS calcd for $\mathrm{C}_{22} \mathrm{H}_{24} \mathrm{NO}_{3}{ }^{+}[\mathrm{M}+\mathrm{H}]^{+}(\mathrm{m} / \mathrm{z})$ 350.1751, found 350.1758. 


\subsubsection{Synthesis of Carbamate (Scheme 3.5)}<smiles>CC(C)(C)OC(=O)NCCN</smiles>

18

tert-Butyl (2-aminoethyl)carbamate (18). Ethylene diamine $(9.2 \mathrm{~mL}, 138 \mathrm{mmol})$ was added dropwise via syringe to dry $\mathrm{CH}_{2} \mathrm{Cl}_{2}(82 \mathrm{~mL})$ at $0{ }^{\circ} \mathrm{C}$ with stirring. A solution of $\mathrm{Boc}_{2} \mathrm{O}(5.60 \mathrm{~g}, 25.7 \mathrm{mmol})$ in dry $\mathrm{CH}_{2} \mathrm{Cl}_{2}(76 \mathrm{~mL})$ was added dropwise over $8 \mathrm{~h}$ with continued chilling. The reaction was allowed to stir overnight to form a colorless solution with clumps of white solids. The solids were filtered off and the filter cake was washed with $\mathrm{CH}_{2} \mathrm{Cl}_{2}$. The filtrate was condensed in vacuo, and then poured into $\mathrm{NaHCO}_{3}$ solution $(75 \mathrm{~mL})$, which produced an exotherm, and shaken. The aqueous solution was extracted with $\mathrm{CH}_{2} \mathrm{Cl}_{2}(3 \times 50 \mathrm{~mL})$, and then the combined organics were dried over $\mathrm{Na}_{2} \mathrm{SO}_{4}$, filtered and condensed in vacuo to give crude $\mathbf{1 8}$ (turbid oil, $4.50 \mathrm{~g}$ ) which was used in the next step without further purification. $R_{f} 0.24$ (10:2:88, MeOH:NH $4{ }_{4} \mathrm{OH}: \mathrm{CH}_{2} \mathrm{Cl}_{2} ;{ }^{1} \mathrm{H}$ NMR (400 MHz, $\mathrm{CDCl}_{3}$ ) $\delta 1.40$ (s, 9H), 2.76 (br s, 2H), 3.14 (br s, 2H), 5.05 (br s, 1H); ${ }^{13} \mathrm{C}$ NMR (100 MHz, $\left.\mathrm{CDCl}_{3}\right) \delta 28.5,41.9,43.4,79.3,156.4$.

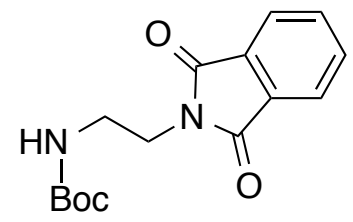

tert-Butyl $N$-(2-(1,3-dioxo-2,3-dihydro-1H-isoindol-2-yl)ethyl)carbamate. Phthalic anhydride (4.18 g, $28.2 \mathrm{mmol})$ was added to a solution of crude $18(4.11 \mathrm{~g}, 25.7 \mathrm{mmol})$ in toluene $(86 \mathrm{~mL})$ with stirring and the reaction flask was fitted with a Dean-Stark apparatus. The mixture was heated to reflux for $6.5 \mathrm{~h}$ with the phthalic anhydride slowly 
going into solution. After the heat was removed, the solution was allowed to stir overnight where a precipitate dropped out of solution. The mixture was washed with water $(3 \times 50 \mathrm{~mL})$ and the combined aqueous phases were extracted with EtOAc $(2 \times 30$ $\mathrm{mL})$. The combined organics were washed with brine $(50 \mathrm{~mL})$, dried over $\mathrm{Na}_{2} \mathrm{SO}_{4}$, filtered and concentrated in vacuo. The crude material was purified by column chromatography $\left(\mathrm{SiO}_{2}, 1: 19\right.$ to 1:1, EtOAc: $\mathrm{CH}_{2} \mathrm{Cl}_{2}$ gradient) to give tert-butyl $N$-(2-(1,3dioxo-2,3-dihydro- $1 H$-isoindol-2-yl)ethyl)carbamate $(5.76 \mathrm{~g}, 77 \%)$ as a white solid. $R_{f}$ 0.27 (1:19, EtOAc:hexanes); ${ }^{1} \mathrm{H}$ NMR (400 MHz, $\left.\mathrm{CDCl}_{3}\right) \delta 1.33$ (s, 9H), 3.42 (br s, 2H), $3.82(\mathrm{t}, J=5.6 \mathrm{~Hz}, 2 \mathrm{H}), 4.83(\mathrm{br} \mathrm{s}, 1 \mathrm{H}), 7.70-7.72(\mathrm{~m}, 2 \mathrm{H}), 7.82-7.86(\mathrm{~m}, 2 \mathrm{H}) ;{ }^{13} \mathrm{C} \mathrm{NMR}$ $\left(100 \mathrm{MHz}, \mathrm{CDCl}_{3}\right) \delta 28.4,38.3,39.8,79.7,123.5,132.3,134.2,156.2,168.7$.

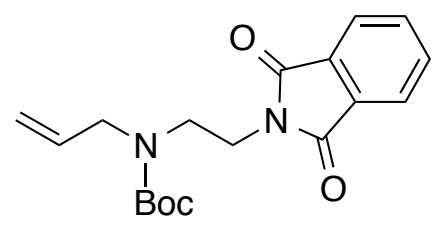

19

tert-Butyl allyl(2-(1,3-dioxoisoindolin-2-yl)ethyl)carbamate (19). tert-Butyl $N$-(2(1,3-dioxo-2,3-dihydro-1 $H$-isoindol-2-yl)ethyl)carbamate phthalimide $\left(\begin{array}{llll}5.76 & \mathrm{~g}, & 19.8\end{array}\right.$ mmol) was added to a slurry of $\mathrm{NaH}(1.59 \mathrm{~g}$ of $60 \%$ in mineral oil, $39.7 \mathrm{mmol})$ in dry THF $(83 \mathrm{~mL})$ at $0{ }^{\circ} \mathrm{C}$. After stirring $1 \mathrm{~h}$, allyl bromide $(2.23 \mathrm{~mL}, 25.8 \mathrm{mmol})$ was added dropwise. The slurry was stirred for three days and then quenched by addition into water $(50 \mathrm{~mL})$. The layers were separated and the aqueous phase was extracted with $\mathrm{Et}_{2} \mathrm{O}(2 \mathrm{x}$ $35 \mathrm{~mL})$. The combined organic phase was washed with brine $(50 \mathrm{~mL})$, dried over $\mathrm{Na}_{2} \mathrm{SO}_{4}$, filtered and concentrated in vacuo. The crude material was purified by column chromatography $\left(\mathrm{SiO}_{2}, 1: 19 \mathrm{EtOAc}: \mathrm{CH}_{2} \mathrm{Cl}_{2}\right)$ to give 19 (3.77 g, 58\%) as a white solid; $R_{f}$ 
0.55 (1:19 EtOAc:hexanes); $\mathrm{mp}=73-76{ }^{\circ} \mathrm{C} ;{ }^{1} \mathrm{H}$ NMR (400 MHz, $\left.\mathrm{CDCl}_{3}\right) \delta 1.27$ (s, 9H), 3.46 (br s, 2H), 3.81 (br s, 2H), 3.87 (br s, 2H), 5.01-5.13 (m, 2H), 5.71-5.77 (m, 1H), 7.70 (br s, 2H), 7.82 (br s, 2H); ${ }^{13} \mathrm{C}$ NMR (100 MHz, $\mathrm{CDCl}_{3}$ ) \& 28.2, 44.5, 49.3, 50.2, $80.1,117.2,123.4,132.3,133.9,134.2,155.3,168.3$.

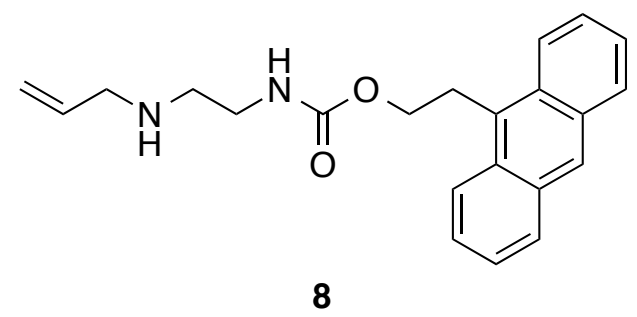

2-(Anthracen-9-yl)ethyl $N$-(2-(allylamino)ethyl)carbamate (8, TFA salt). Hydrazine monohydrate (147 $\mu \mathrm{L}, 3.03 \mathrm{mmol})$ was added to a solution of $19(217 \mathrm{mg}, 0.66 \mathrm{mmol})$ in 2:1 $\mathrm{CH}_{2} \mathrm{Cl}_{2}: \mathrm{EtOH}(6 \mathrm{~mL})$ with stirring at $0{ }^{\circ} \mathrm{C}$. The reaction was stirred for $18 \mathrm{~h}$, allowing it to warm slowly to room temperature. The white precipitate was then filtered and the filter cake was washed with $\mathrm{CH}_{2} \mathrm{Cl}_{2}$ and concentrated in vacuo. The concentrate was diluted with $\mathrm{CH}_{2} \mathrm{Cl}_{2}$ and the precipitate was filtered, the cake washed with $\mathrm{CH}_{2} \mathrm{Cl}_{2}$ and concentrated in vacuo again to give the crude amine (light yellow oil, $122 \mathrm{mg}, 92 \%$ ) which was used in the next step without further purification. $R_{f} 0.47$ (10:2:88 MeOH: $\mathrm{NH}_{4} \mathrm{OH}: \mathrm{CH}_{2} \mathrm{Cl}_{2}$ ); ${ }^{1} \mathrm{H}$ NMR (500 MHz, $\mathrm{CDCl}_{3}$ ) \& 1.40 (s, 2H), 1.43 (s, 9H), 2.79 (t, $J=5.0 \mathrm{~Hz}, 2 \mathrm{H}), 3.22($ br s, 2H), 3.81 (br s, 2H), 5.09-5.12 (m, 2H), 5.74-5.79 (m, 1H); ${ }^{13} \mathrm{C}$ NMR $\left(100 \mathrm{MHz}, \mathrm{CDCl}_{3}\right) \delta$ 28.6, 40.8, 50.1, 79.8, 116.4, 134.3, 156.0; FT-ICR-MS calcd for $\mathrm{C}_{10} \mathrm{H}_{21} \mathrm{~N}_{2} \mathrm{O}_{2}{ }^{+}[\mathrm{M}+\mathrm{H}]^{+}(m / z)$ 201.1598, found 201.1599.

The amine (188 $\mathrm{mg}, 0.94 \mathrm{mmol})$ was added dropwise with stirring to a solution of $\mathrm{ClC}(\mathrm{O}) \mathrm{OCH}_{2} \mathrm{CH}_{2}\left(9\right.$-anthracenyl) $(303 \mathrm{mg}, 1.06 \mathrm{mmol})$ in dry $\mathrm{CH}_{2} \mathrm{Cl}_{2}(3.5 \mathrm{~mL})$ at $0{ }^{\circ} \mathrm{C}$. After 10 minutes, $\mathrm{Et}_{3} \mathrm{~N}(148 \mu \mathrm{L}, 1.06 \mathrm{mmol})$ was added dropwise to the reaction, causing 
the solution to darken. After stirring $17 \mathrm{~h}$, the reaction mixture was quenched by addition of sat. $\mathrm{NH}_{4} \mathrm{Cl}(5 \mathrm{~mL})$ and the aqueous phase was extracted with $\mathrm{CH}_{2} \mathrm{Cl}_{2}(5 \mathrm{~mL})$. Combined organic phase was dried over $\mathrm{Na}_{2} \mathrm{SO}_{4}$, filtered and concentrated in vacuo. The crude material was purified by column chromatography $\left(\mathrm{SiO}_{2}, \quad 5: 1: 4\right.$ $\mathrm{CH}_{2} \mathrm{Cl}_{2}$ :hexanes:EtOAc) to give Boc-protected $3(171 \mathrm{mg}, 41 \%)$ as a yellow gum; $R_{f}$ 0.33 (1:19 EtOAc: $\mathrm{CH}_{2} \mathrm{Cl}_{2}$ ); FT-IR: 3449, 3058, 2971, 1724, $1514 \mathrm{~cm}^{-1} ;{ }^{1} \mathrm{H}$ NMR (400 $\left.\mathrm{MHz}, \mathrm{CDCl}_{3}\right) \delta 1.46$ (s, 9H), 3.13 (br s, 2H), 3.36 (br s, 2H), 3.83 (br s, 2H), 3.97 (t, $J=$ $8.0 \mathrm{~Hz}, 2 \mathrm{H}), 4.43$ (br s, 2H), 5.10-5.15 (m, 2H), 5.75-5.81 (m, 1H), 7.44-7.48 (m, 2H), 7.52-7.56 (m, 2H), 8.00 (d, $J=8.4 \mathrm{~Hz}, 2 \mathrm{H}), 8.35-8.37$ (m, 3H); ${ }^{13} \mathrm{C}$ NMR (100 MHz, $\left.\mathrm{CDCl}_{3}\right) \delta 28.1,28.5,40.5,46.2,50.6,64.6,80.3,116.6,124.4,125.1,126.1,126.8,129.3$, 130.5, 131.7, 134.0, 155.3, 157.0; FT-ICR-MS calcd for $\mathrm{C}_{27} \mathrm{H}_{32} \mathrm{~N}_{2} \mathrm{NaO}_{4}{ }^{+}[\mathrm{M}+\mathrm{Na}]^{+} m / z$ 471.2254, found 471.2256.

Trifluoroacetic acid $(0.50 \mathrm{~mL}, 6.53 \mathrm{mmol})$ was added to a solution of Boc-protected $8(20 \mathrm{mg}, 0.045 \mathrm{mmol})$ in dry $\mathrm{CH}_{2} \mathrm{Cl}_{2}(0.50 \mathrm{~mL})$ at $0{ }^{\circ} \mathrm{C}$. After stirring $1 \mathrm{~h}$, the volatiles were removed in vacuo to afford the TFA salt of $\mathbf{3}(18 \mathrm{mg}, 88 \%)$ as an oil; ${ }^{1} \mathrm{H}$ NMR (400 $\left.\mathrm{MHz}, \mathrm{CDCl}_{3}\right) \delta 3.09$ (br s, 2H), 3.48-3.56 (m, 4H), 3.90 (t, $\left.J=8.0 \mathrm{~Hz}, 2 \mathrm{H}\right), 4.36(\mathrm{t}, J=$ $8.0 \mathrm{~Hz}, 2 \mathrm{H}), 5.35-5.47(\mathrm{~m}, 2 \mathrm{H}), 5.81-5.88(\mathrm{~m}, 1 \mathrm{H}), 7.41-7.51(\mathrm{~m}, 4 \mathrm{H}), 7.97(\mathrm{~d}, J=8.4$ $\mathrm{Hz}, 2 \mathrm{H}), 8.27$ (d, $J=8.8 \mathrm{~Hz}, 2 \mathrm{H}), 8.34$ (s, 1H), 9.47 (br s, $2 \mathrm{H}) ;{ }^{13} \mathrm{C}$ NMR $(100 \mathrm{MHz}$, $\left.\mathrm{CDCl}_{3}\right) \delta 27.8,37.8,47.2,50.2,65.1,124.3,124.6,125.2,126.2,126.9,127.5,129.4$, 130.5, 131.7, 134.3, 157.5; FT-ICR-MS calcd for $\mathrm{C}_{22} \mathrm{H}_{25} \mathrm{~N}_{2} \mathrm{O}_{2}{ }^{+}[\mathrm{M}+\mathrm{H}]^{+} m / z$ 349.1910, found 349.1913 


\subsubsection{Synthesis of gem-Dimethylester (Scheme 3.6)}<smiles>CC1(C)CCC(=O)CC1</smiles>

4,4-Dimethylcyclohexanone. $10 \%$ Palladium on carbon $(10 \mathrm{mg}, 0.0094 \mathrm{mmol})$ was added to a solution of 4,4-dimethyl-2-cyclohexen-1-one (1.52 g, $12.2 \mathrm{mmol})$ in hexanes (15 mL). The atmosphere was purged with $\mathrm{H}_{2}$ and sealed with a $\mathrm{H}_{2}$ balloon attached. After $48 \mathrm{~h}$ the reaction was filtered through Celite and the filter cake was rinsed with hexanes. The filtrate was concentrated in vacuo to give 4,4-dimethylcyclohexanone (1.29 $\mathrm{g}$ white crystals, $83 \%$ ) that was used in the next step without further purification. $R_{f} 0.46$ (1:3, EtOAc:Hexanes); ${ }^{1} \mathrm{H}$ NMR (400 MHz, $\left.\mathrm{CDCl}_{3}\right) \delta 1.09(\mathrm{~s}, 6 \mathrm{H}), 1.66(\mathrm{t}, J=6.0 \mathrm{~Hz}$, 4H), $2.33(\mathrm{t}, J=6.0 \mathrm{~Hz}, 4 \mathrm{H}) ;{ }^{13} \mathrm{C} \mathrm{NMR}\left(100 \mathrm{MHz}, \mathrm{CDCl}_{3}\right) \delta 27.7,30.1,38.2,39.3$, 212.8.

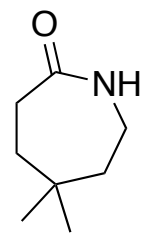

5,5-Dimethylazepan-2-one. A solution of 4,4-dimethylcyclohexanone (1.29 g, 10.2 mmol) in formic acid $(10 \mathrm{~mL})$ was added dropwise to a solution of hydroxylamine- $O$ sulfonic acid $(1.73 \mathrm{~g}, 15.3 \mathrm{mmol})$ in formic acid $(7 \mathrm{~mL})$ and allowed to stir at $\mathrm{rt}$ for 15 min. The reaction flask was then heated to reflux. After $24 \mathrm{~h}$, the reaction solution was cooled to rt and quenched with $10 \mathrm{~N} \mathrm{NaOH}(40 \mathrm{~mL})$. The aqueous mixture was extracted with chloroform $(4 \times 20 \mathrm{~mL})$ and the combined organics were washed with water $(2 \times 10$ 
$\mathrm{mL})$ and brine $(10 \mathrm{~mL})$. The solution was dried over $\mathrm{MgSO}_{4}$, filtered and concentrated in vacuo to give 5,5-dimethylazepan-2-one (1.06 g brown crystals, $73 \%$ ) that was used in the next step without further purification. $R_{f} 0.13$ (EtOAc); ${ }^{1} \mathrm{H}$ NMR $\left(400 \mathrm{MHz}, \mathrm{CDCl}_{3}\right.$ ) $\delta 0.97(\mathrm{~s}, 6 \mathrm{H}), 1.42-1.44(\mathrm{~m}, 2 \mathrm{H}), 1.48-1.50(\mathrm{~m}, 2 \mathrm{H}), 2.39-2.42(\mathrm{~m}, 2 \mathrm{H}), 3.15(\mathrm{q}, J=5.2$ $\mathrm{Hz}, 2 \mathrm{H}), 6.50$ (br s, $1 \mathrm{H}) ;{ }^{13} \mathrm{C} \mathrm{NMR}\left(100 \mathrm{MHz}, \mathrm{CDCl}_{3}\right) \delta 29.0,32.0,33.0,36.0,38.3$, $42.4,179.1$

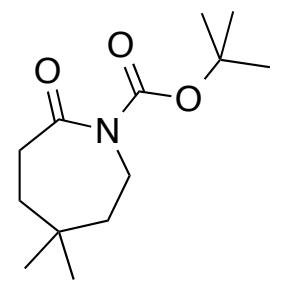

tert-Butyl 5,5-dimethyl-2-oxoazepane-1-carboxylate. Di-tert-butyl dicarbonate (1.54 g, $7.01 \mathrm{mmol})$ and DMAP $(856 \mathrm{mg}, 7.01 \mathrm{mmol})$ were added to a solution of 5,5dimethylazepan-2-one $(899 \mathrm{mg}, 6.37 \mathrm{mmol})$ in dry THF $(16 \mathrm{~mL})$ at rt. After purging the head-space with $\mathrm{N}_{2}$, the reaction was heated to reflux. After $2.5 \mathrm{~h}$ the reaction was cooled to rt and the volatiles were removed in vacuo and the crude material was purified by column chromatography $\left(\mathrm{SiO}_{2}, 1: 9\right.$ to $3: 7$, EtOAc:hexanes gradient) to give tert-butyl 5,5-dimethyl-2-oxoazepane-1-carboxylate as yellow crystals $(1.05 \mathrm{~g}, 68 \%) . \quad R_{f} 0.68$ (EtOAc); ${ }^{1} \mathrm{H}$ NMR $\left(400 \mathrm{MHz}, \mathrm{CDCl}_{3}\right) \delta 0.94$ (s, 6H), 1.46-1.49 (m, 11H), 1.52-1.55 (m,

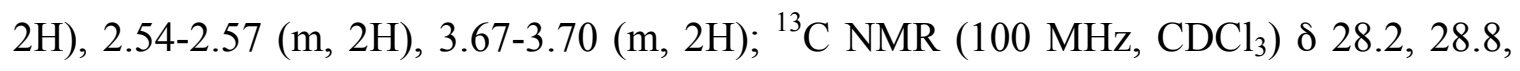
32.2, 35.2, 36.5, 41.8, 41.9, 83.0, 153.0, 175.8 . 


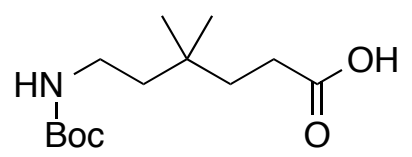

20

6-((tert-Butoxycarbonyl)amino)-4,4-dimethylhexanoic acid (20). Lithium hydroxide monohydrate (340 $\mathrm{mg}, 8.10 \mathrm{mmol})$ was added to a solution of tert-butyl 5,5-dimethyl-2oxoazepane-1-carboxylate $(888 \mathrm{mg}, 3.68 \mathrm{mmol})$ in $2: 1 \mathrm{THF}: \mathrm{H}_{2} \mathrm{O}(18 \mathrm{~mL})$ and the reaction was heated to $60{ }^{\circ} \mathrm{C}$. After $4 \mathrm{~h}$ the reaction was cooled to $\mathrm{rt}$ and partitioned between $\mathrm{Et}_{2} \mathrm{O}$ and $\mathrm{H}_{2} \mathrm{O}$ and the organic layer separated. The aqueous phase was acidified to $\mathrm{pH} \sim 4$ with $10 \% \mathrm{HCl}$ and extracted with EtOAc $(3 \times 15 \mathrm{~mL})$. The combined organics were washed with brine $(15 \mathrm{~mL})$, dried over $\mathrm{Na}_{2} \mathrm{SO}_{4}$, filtered and concentrated in vacuo to give 20 (912 mg yellow crystals, 96\%) that was used in the next step without further purification. $R_{f} 0.29$ (1:1, EtOAc:Hexanes with $\left.0.5 \% \mathrm{AcOH}\right) ;{ }^{1} \mathrm{H}$ NMR $(400 \mathrm{MHz}$, $\left.\mathrm{CDCl}_{3}\right) \delta 1.37-1.43(\mathrm{~m}, 11 \mathrm{H}), 1.58(\mathrm{t}, J=8.4 \mathrm{~Hz}, 2 \mathrm{H}), 2.30(\mathrm{t}, J=8.4 \mathrm{~Hz}, 2 \mathrm{H}), 3.11($ br s, 2H), 4.49 (br s, $1 \mathrm{H}) ;{ }^{13} \mathrm{C}$ NMR (100 MHz, $\left.\mathrm{CDCl}_{3}\right) \delta$ 26.9, 28.6, 29.5, 32.1, 36.6, 36.9, $41.6,79.5,156.2,179.7$.

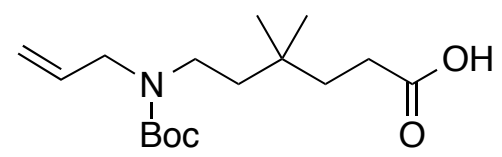

21

6-(Allyl(tert-butoxycarbonyl)amino)-4,4-dimethylhexanoic acid (21). Amino acid 20 (912 mg, $3.52 \mathrm{mmol})$ was added to a slurry of $60 \% \mathrm{NaH}(703 \mathrm{mg}, 17.6 \mathrm{mmol})$ in dry THF $(18 \mathrm{~mL})$ at $0{ }^{\circ} \mathrm{C}$. After stirring $1 \mathrm{~h}$, allyl bromide $(913 \mu \mathrm{L}, 10.5 \mathrm{mmol})$ was added dropwise. After $24 \mathrm{~h}$, the reaction mixture was cooled to $0{ }^{\circ} \mathrm{C}$ and quenched with water until the reaction became transparent. The reaction was acidified to $\mathrm{pH} \sim 3$ by addition of 
$1 M \mathrm{HCl}$ and the layers were separated. The aqueous phase was extracted with EtOAc (3 $\mathrm{x} 10 \mathrm{~mL})$ and the combined organic phase was washed with brine $(15 \mathrm{~mL})$, dried over $\mathrm{Na}_{2} \mathrm{SO}_{4}$, filtered and concentrated in vacuo. The crude material was purified by column chromatography $\left(\mathrm{SiO}_{2}, 1: 1\right.$ EtOAc:hexanes with $\left.0.5 \% \mathrm{AcOH}\right)$ to give 21 (712 mg, 68\%) as a light yellow oil; $R_{f} 0.43$ (1:1 EtOAc:hexanes with $\left.0.5 \% \mathrm{AcOH}\right) ;{ }^{1} \mathrm{H}$ NMR $(400 \mathrm{MHz}$, $\left.\mathrm{CDCl}_{3}\right) \delta 0.88(\mathrm{~s}, 6 \mathrm{H}), 1.39-1.44(\mathrm{~m}, 11 \mathrm{H}), 1.56(\mathrm{t}, J=8.0 \mathrm{~Hz}, 2 \mathrm{H}), 2.31(\mathrm{t}, J=8.2 \mathrm{~Hz}$, $2 \mathrm{H}), 3.14$ (br s, $2 \mathrm{H}), 3.80$ (br s, $2 \mathrm{H}), 5.11(\mathrm{~d}, J=11.6 \mathrm{~Hz}, 2 \mathrm{H}), 5.71-5.81(\mathrm{~m}, 1 \mathrm{H}) ;{ }^{13} \mathrm{C}$

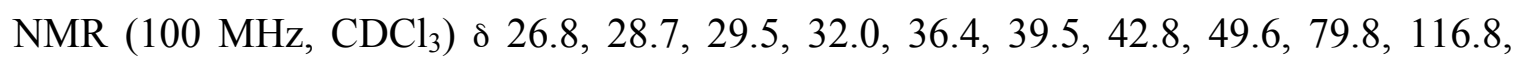
134.6, 155.6, 180.2; FT-ICR-MS calcd for $\mathrm{C}_{16} \mathrm{H}_{28} \mathrm{NO}_{4}^{-}[\mathrm{M}-\mathrm{H}]^{-} \mathrm{m} / \mathrm{z} 298.2024$, found 298.2024 .

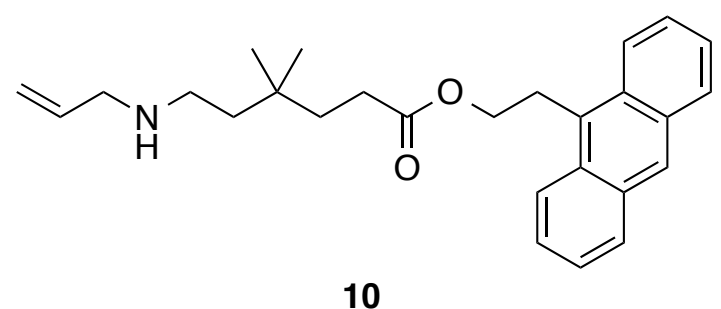

2-(Anthracen-9-yl)ethyl 4,4-dimethyl-6-(allylamino)-hexanoate (10, TFA salt). To a mixture of carboxylic acid 21 (370 mg, $1.24 \mathrm{mmol})$ and 25 (249 mg, $1.12 \mathrm{mmol})$ in dry $\mathrm{CH}_{2} \mathrm{Cl}_{2}(10 \mathrm{~mL})$ at $0{ }^{\circ} \mathrm{C}$ was added DIC (264 $\left.\mu \mathrm{L}, 1.69 \mathrm{mmol}\right)$ and DMAP (pinch). After $16 \mathrm{~h}$, the precipitated white solids were filtered and the filter cake was washed with $\mathrm{CH}_{2} \mathrm{Cl}_{2}$. The combined filtrate was condensed in vacuo and the crude material was purified by column chromatography $\left(\mathrm{SiO}_{2}, 0: 100\right.$ to 1:19 EtOAc: $\mathrm{CH}_{2} \mathrm{Cl}_{2}$ gradient) to give Boc-protected gem-dimethyl ester $\mathbf{1 0}$ as a yellow oil (494 mg, 87\%); $R_{f} 0.58(1: 19$ EtOAc: $\mathrm{CH}_{2} \mathrm{Cl}_{2}$ ); FT-IR 3058, 2963, 1728, $1684 \mathrm{~cm}^{-1} ;{ }^{1} \mathrm{H}$ NMR (400 MHz, $\left.\mathrm{CDCl}_{3}\right) \delta 0.87$ (s, 6H), 1.39 (br s, 2H), 1.46 (s, 9H), 1.51 (t, $J=8.4 \mathrm{~Hz}, 2 \mathrm{H}), 2.28$ (t, $J=8.2 \mathrm{~Hz}, 2 \mathrm{H})$, 
3.12 (br s, 2H), 3.81 (br s, 2H), 3.98 (t, $J=7.8 \mathrm{~Hz}, 2 \mathrm{H}), 4.48$ (t, $J=7.8 \mathrm{~Hz}, 2 \mathrm{H}), 5.12$ (d, $J=11.6 \mathrm{~Hz}, 2 \mathrm{H}), 5.73-5.82(\mathrm{~m}, 1 \mathrm{H}), 7.46-7.49(\mathrm{~m}, 2 \mathrm{H}), 7.53-7.57(\mathrm{~m}, 2 \mathrm{H}), 8.02(\mathrm{~d}, J=$ $8.4 \mathrm{~Hz}, 2 \mathrm{H}), 8.34(\mathrm{~d}, J=9.2 \mathrm{~Hz}, 2 \mathrm{H}), 8.39(\mathrm{~s}, 1 \mathrm{H}) ;{ }^{13} \mathrm{C} \mathrm{NMR}\left(100 \mathrm{MHz}, \mathrm{CDCl}_{3}\right) \delta 26.8$, $27.5,28.7,29.8,32.0,36.6,39.2,42.8,49.6,53.6,64.3,79.6,116.6,124.3,125.2,126.2$, 127.0, 129.5, 130.5, 131.7, 134.7, 155.5, 174.5; FT-ICR-MS calcd for $\mathrm{C}_{32} \mathrm{H}_{41} \mathrm{NNaO}_{4}{ }^{+}[\mathrm{M}$ $+\mathrm{Na}]^{+} m / z$ 526.2928, found 526.2927.

Trifluoroacetic acid $(0.50 \mathrm{~mL}, 6.53 \mathrm{mmol})$ was added to a solution of Boc-protected $10(11 \mathrm{mg}, 0.022 \mathrm{mmol})$ in dry $\mathrm{CH}_{2} \mathrm{Cl}_{2}(0.50 \mathrm{~mL})$ at $0{ }^{\circ} \mathrm{C}$. After stirring $1 \mathrm{~h}$, the volatiles were removed in vacuo to afford the TFA salt of $\mathbf{1 0}(10 \mathrm{mg}, 85 \%)$ as an oil; ${ }^{1} \mathrm{H}$ NMR (400 MHz, $\left.\mathrm{CDCl}_{3}\right) \delta 0.86(\mathrm{~s}, 6 \mathrm{H}), 1.47(\mathrm{t}, J=8.4 \mathrm{~Hz}, 2 \mathrm{H}), 1.58(\mathrm{t}, J=8.6 \mathrm{~Hz}, 2 \mathrm{H}), 2.24$ (t, $J=8.4 \mathrm{~Hz}, 2 \mathrm{H}), 2.88($ br s, 2H), $3.54($ br s, 2H), $3.96(\mathrm{t}, J=7.6 \mathrm{~Hz}, 2 \mathrm{H}), 4.46$ (t, $J=$ $8.0 \mathrm{~Hz}, 2 \mathrm{H}), 5.40-5.47(\mathrm{~m}, 2 \mathrm{H}), 5.84-5.94(\mathrm{~m}, 1 \mathrm{H}), 7.44-7.56(\mathrm{~m}, 4 \mathrm{H}), 8.00(\mathrm{~d}, J=8.8$ $\mathrm{Hz}, 2 \mathrm{H}), 8.32$ (d, $J=9.2 \mathrm{~Hz}, 2 \mathrm{H}), 8.38$ (s, 1H), 9.47 (br s, 2H); ${ }^{13} \mathrm{C}$ NMR (100 MHz, $\left.\mathrm{CDCl}_{3}\right)$ $\delta$ 26.5, 27.4, 29.4, 32.1, 36.1, 37.1, 43.2, 49.9, 64.4, 124.1, 124.3, 125.2, 126.2, $127.0,127.9,129.2,129.5,130.5,131.7,174.1$; FT-ICR-MS calcd for $\mathrm{C}_{27} \mathrm{H}_{34} \mathrm{NO}_{2}{ }^{+}[\mathrm{M}+$ $\mathrm{H}]^{+} m / z 404.2584$, found 404.2588 .

\subsubsection{Synthesis of gem-Dimethylcarbonate (Scheme 3.7)}

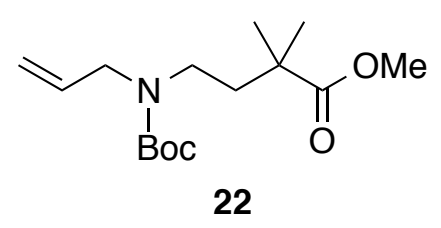

Methyl 4-(allyl(tert-butoxycarbonyl)amino)-2,2-dimethylbutanoate (22). DIC (837

$\mu \mathrm{L}, 5.34 \mathrm{mmol}$ ) and DMAP (pinch) were added to a mixture of amino acid $\mathbf{1 6 . 2}(631 \mathrm{mg}$, 
$2.59 \mathrm{mmol})$ and dry $\mathrm{MeOH}(173 \mu \mathrm{L}, 4.28 \mathrm{mmol})$ in $\mathrm{CH}_{2} \mathrm{Cl}_{2}(32 \mathrm{~mL})$ at rt. After $12 \mathrm{~h}$ the precipitated solids were filtered and the filter cake was washed with $\mathrm{CH}_{2} \mathrm{Cl}_{2}$. The combined filtrate was concentrated in vacuo and the resulting residue was purified by column chromatography $\left(\mathrm{SiO}_{2}, 1: 1: 8\right.$, EtOAc:THF:hexanes) to give methyl 4-(allyl(tertbutoxycarbonyl)amino)butanoate as a pale yellow oil (532 $\mathrm{mg}, 80 \%) ; R_{f} 0.65(1: 1: 8$, EtOAc:THF:hexanes); ${ }^{1} \mathrm{H}$ NMR (400 MHz, $\mathrm{CDCl}_{3}$ ) $\delta 1.44$ (s, 9H), 1.82 (dt, $J=7.0 \mathrm{~Hz}$, 2H), $2.30(\mathrm{t}, J=7.4 \mathrm{~Hz}, 2 \mathrm{H}), 3.20(\mathrm{br} \mathrm{s}, 2 \mathrm{H}), 3.65(\mathrm{~s}, 3 \mathrm{H}), 3.79(\mathrm{br} \mathrm{s}, 2 \mathrm{H}), 5.10(\mathrm{~d}, J=$ $12.8 \mathrm{~Hz}, 2 \mathrm{H}), 5.72-5.80(\mathrm{~m}, 1 \mathrm{H}) ;{ }^{13} \mathrm{C} \mathrm{NMR}\left(100 \mathrm{MHz}, \mathrm{CDCl}_{3}\right)$ \& 23.7, 28.6, 31.4, 45.9, $49.7,51.8,79.8,116.7,134.3,155.7,173.8$.

The methyl ester (532 mg, $2.06 \mathrm{mmol})$ was dissolved in dry THF $(10 \mathrm{~mL})$ and cooled to $-78^{\circ} \mathrm{C}$ with stirring. A solution of lithium bis(trimethylsilyl)amide (LiHMDS) in THF $(6.20 \mathrm{~mL}$ of $1 \mathrm{M}$ solution, $6.20 \mathrm{mmol})$ was added dropwise to the reaction solution. After stirring $1 \mathrm{~h}$, methyl iodide $(772 \mu \mathrm{L}, 12.4 \mathrm{mmol})$ was added dropwise and the reaction was stirred overnight while slowly warming to rt. After $22 \mathrm{~h}$, the reaction was cooled to $0{ }^{\circ} \mathrm{C}$ and quenched with water $(5 \mathrm{~mL})$, followed by $1 M \mathrm{HCl}(5 \mathrm{~mL})$. The phases were separated and the aqueous phase was extracted with $\mathrm{Et}_{2} \mathrm{O}(3 \times 15 \mathrm{~mL})$. The combined organic phase was washed with $\mathrm{NaHCO}_{3}(10 \mathrm{~mL})$ and brine $(10 \mathrm{~mL})$ and then dried over $\mathrm{Na}_{2} \mathrm{SO}_{4}$, filtered and concentrated in vacuo. The crude material was purified by column chromatography $\left(\mathrm{SiO}_{2}, 1: 4\right.$ EtOAc:hexanes) to give $22(397 \mathrm{mg}, 67 \%)$ as a yellow oil; $R_{f} 0.48$ (1:4, EtOAc:hexanes); ${ }^{1} \mathrm{H}$ NMR (400 MHz, $\left.\mathrm{CDCl}_{3}\right) \delta 1.18(\mathrm{~s}, 6 \mathrm{H})$, 1.44 (s, 9H), 1.74 (br s, 2H), 3.11 (br s, 2H), 3.65 (s, 3H), 3.79 (br s, 2H), 5.10 (d, $J=$ $10.8 \mathrm{~Hz}, 2 \mathrm{H}), 5.70-5.79(\mathrm{~m}, 1 \mathrm{H}) ;{ }^{13} \mathrm{C} \mathrm{NMR}\left(100 \mathrm{MHz}, \mathrm{CDCl}_{3}\right) \delta$ 25.3, 28.6, 38.1, 41.1, 
43.1, 49.4, 51.9, 79.7, 116.1, 134.2, 155.5, 178.0; FT-ICR-MS calcd for $\mathrm{C}_{15} \mathrm{H}_{27} \mathrm{NNaO}_{4}{ }^{+}$ $[\mathrm{M}+\mathrm{Na}]^{+} \mathrm{m} / z$ 308.1832, found 308.1836 .

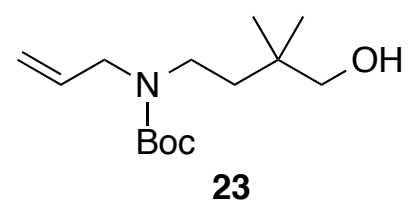

tert-Butyl allyl(4-hydroxy-3,3-dimethylbutyl)carbamate (23). $\mathrm{LiBH}_{4}(45 \mathrm{mg}, 2.08$ mmol) was added to a solution of $22(265 \mathrm{mg}, 0.93 \mathrm{mmol})$ in dry THF $(21 \mathrm{~mL})$ at $0{ }^{\circ} \mathrm{C}$. After 5 minutes of stirring the reaction was warmed to rt and stirred overnight. The reaction was then carefully quenched with $\mathrm{NH}_{4} \mathrm{Cl}(25 \mathrm{~mL})$ and extracted with $\mathrm{Et}_{2} \mathrm{O}(3 \mathrm{x}$ $10 \mathrm{~mL})$. The combined organic phase was washed with brine $(15 \mathrm{~mL})$, dried over $\mathrm{Na}_{2} \mathrm{SO}_{4}$, filtered and concentrated in vacuo. The crude material was purified by column chromatography $\left(\mathrm{SiO}_{2}, 1: 1\right.$ EtOAc:hexanes) to give $\mathbf{2 3}$ (175 mg, 73\%) as a colorless oil; $R_{f} 0.47$ (1:1, EtOAc:hexanes); ${ }^{1} \mathrm{H}$ NMR (400 MHz, $\left.\mathrm{CDCl}_{3}\right) \delta 0.86(\mathrm{~s}, 6 \mathrm{H}), 1.43-1.49(\mathrm{~m}$, 11H), 2.89 (br s, 1H), 3.13-3.17 (m, 2H), 3.32 (s, 2H), 3.78 (br s, 2H), 5.09-5.13 (m, 2H), 5.71-5.81 (m, 1H); ${ }^{13} \mathrm{C}$ NMR (100 MHz, $\left.\mathrm{CDCl}_{3}\right) \delta 24.4,28.6,34.7,36.5,42.9,50.4$, 70.8, 79.8, 116.4, 134.6, 155.8; FT-ICR-MS calcd for $\mathrm{C}_{14} \mathrm{H}_{27} \mathrm{NNaO}_{3}{ }^{+}[\mathrm{M}+\mathrm{Na}]^{+} \mathrm{m} / \mathrm{z}$ 280.1883 , found 280.1886 . 


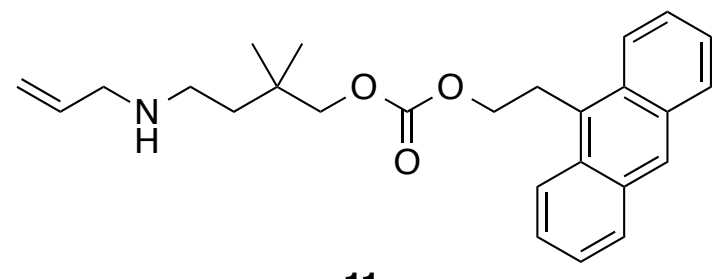

11

2-(Anthracen-9-yl)ethyl 2,2-dimethyl-4-(allylamino)butyl carbonate (11, TFA salt).

$N, N$-Diisopropylethylamine (304 $\mu \mathrm{L}, 1.74 \mathrm{mmol})$ was added to a solution of alcohol 23 (251 mg, $0.98 \mathrm{mmol})$ in dry $\mathrm{CH}_{2} \mathrm{Cl}_{2}(25 \mathrm{~mL})$ at $0{ }^{\circ} \mathrm{C} .1,1$ '-Carbonyldiimidazole $(283 \mathrm{mg}$, $1.74 \mathrm{mmol}$ ) was then added to the solution followed by warming to rt. After $24 \mathrm{~h}$, the reaction was washed with water $(2 \times 10 \mathrm{~mL})$, brine $(10 \mathrm{~mL})$ and then dried over $\mathrm{Na}_{2} \mathrm{SO}_{4}$. After filtration and concentration in vacuo, the crude residue was purified by column chromatography $\left(\mathrm{SiO}_{2}\right.$, EtOAc) to give the imidazole carbamate as a colorless oil (316 mg, 92\%); $R_{f} 0.61$ (EtOAc); ${ }^{1} \mathrm{H}$ NMR (400 MHz, $\left.\mathrm{CDCl}_{3}\right) \delta 1.01(\mathrm{~s}, 6 \mathrm{H}), 1.42$ (s, 9H), $1.56(\mathrm{t}, J=7.8 \mathrm{~Hz}, 2 \mathrm{H}), 3.21(\mathrm{br} \mathrm{s}, 2 \mathrm{H}), 3.76(\mathrm{br} \mathrm{s}, 2 \mathrm{H}), 4.12(\mathrm{~s}, 2 \mathrm{H}), 5.07-5.09(\mathrm{~m}, 2 \mathrm{H})$, 5.70-5.79 (m, 1H), $7.07(\mathrm{~s}, 1 \mathrm{H}), 7.42(\mathrm{~s}, 1 \mathrm{H}), 8.13(\mathrm{~s}, 1 \mathrm{H}) ;{ }^{13} \mathrm{C} \mathrm{NMR}\left(100 \mathrm{MHz}, \mathrm{CDCl}_{3}\right)$ б $24.2,28.6,33.7,36.7,42.5,50.0,75.9,79.8,116.4,117.2,130.9,134.5,137.2,148.9$, 155.4 .

A solution of alcohol 25 (191 mg, $0.86 \mathrm{mmol})$ in dry THF (1 mL) was added dropwise to a slurry of $\mathrm{NaH}(103 \mathrm{mg}$ of $60 \%$ in mineral oil, $2.57 \mathrm{mmol})$ in dry THF (5 $\mathrm{mL}$ ) at $-5^{\circ} \mathrm{C}$ and stirred for 30 minutes. A solution of the above imidazole carbamate (316 $\mathrm{mg}, 0.90 \mathrm{mmol})$ in dry THF $(1 \mathrm{~mL})$ was then added dropwise to the reaction mixture. The reaction was stirred overnight, then the mixture was filtered through Celite and the filter cake was washed with $\mathrm{Et}_{2} \mathrm{O}$. The filtrate was washed with water $(2 \mathrm{x} 10$ $\mathrm{mL})$ and the combined aqueous layers were extracted with $\mathrm{Et}_{2} \mathrm{O}(3 \times 10 \mathrm{~mL})$. The combined organic phase was washed with brine, dried over $\mathrm{Na}_{2} \mathrm{SO}_{4}$, filtered and 
concentrated in vacuo. The crude material was purified by column chromatography $\left(\mathrm{SiO}_{2}, 1: 19 \mathrm{EtOAc}: \mathrm{CH}_{2} \mathrm{Cl}_{2}\right)$ to give Boc-protected $11(207 \mathrm{mg}, 48 \%)$ as a yellow oil; $R_{f}$

0.64 (1:19, EtOAc: $\mathrm{CH}_{2} \mathrm{Cl}_{2}$ ); FT-IR 3008, 2974, 1743, 1685, $1256 \mathrm{~cm}^{-1} ;{ }^{1} \mathrm{H}$ NMR (400 $\left.\mathrm{MHz}, \mathrm{CDCl}_{3}\right) \delta 0.98$ (s, 6H), 1.46 (s, 9H), 1.53 (t, $\left.J=7.8 \mathrm{~Hz}, 2 \mathrm{H}\right), 3.19$ (br s, 2H), 3.77$3.82(\mathrm{~m}, 2 \mathrm{H}), 3.90(\mathrm{~s}, 2 \mathrm{H}), 4.05(\mathrm{t}, J=8.0 \mathrm{~Hz}, 2 \mathrm{H}), 4.50(\mathrm{t}, J=8.4 \mathrm{~Hz}, 2 \mathrm{H}), 5.10-5.13$ (m, 2H), 5.74-5.80 (m, 1H), 7.45-7.49 (m, 2H), 7.53-7.57 (m, 2H), $8.01(\mathrm{~d}, J=8.4 \mathrm{~Hz}$, 2H), $8.34(\mathrm{~d}, J=9.2 \mathrm{~Hz}, 2 \mathrm{H}), 8.40(\mathrm{~s}, 1 \mathrm{H}) ;{ }^{13} \mathrm{C} \mathrm{NMR}\left(100 \mathrm{MHz}, \mathrm{CDCl}_{3}\right) \delta$ 24.1, 27.6, $28.7,33.5,37.1,42.5,49.7,67.3,76.2,79.7,116.2,124.1,125.2,126.4,127.2,128.3$, 129.5, 130.5, 131.7, 134.5, 155.5, 155.7; FT-ICR-MS calcd for $\mathrm{C}_{26} \mathrm{H}_{32} \mathrm{NO}_{3}{ }^{+}[\mathrm{M}+\mathrm{Na}]^{+}$ $m / z 528.2928$, found 528.2720 .

Trifluoroacetic acid $(0.50 \mathrm{~mL}, 6.53 \mathrm{mmol})$ was added to a solution of Boc-protected $11(12 \mathrm{mg}, 0.024 \mathrm{mmol})$ in dry $\mathrm{CH}_{2} \mathrm{Cl}_{2}(0.50 \mathrm{~mL})$ at $0{ }^{\circ} \mathrm{C}$. After stirring $1 \mathrm{~h}$, the volatiles were removed in vacuo to afford the TFA salt of $\mathbf{1 1}(11 \mathrm{mg}, 92 \%)$ as an oil; ${ }^{1} \mathrm{H}$ NMR (400 MHz, $\left.\mathrm{CDCl}_{3}\right) \delta 0.96(\mathrm{~s}, 6 \mathrm{H}), 1.70(\mathrm{t}, J=8.4 \mathrm{~Hz}, 2 \mathrm{H}), 2.99$ (br s, 2H), 3.86 (br s, 2H), $4.03(\mathrm{t}, J=8.0 \mathrm{~Hz}, 2 \mathrm{H}), 4.49(\mathrm{t}, J=8.0 \mathrm{~Hz}, 2 \mathrm{H}), 5.40-5.48(\mathrm{~m}, 2 \mathrm{H}), 5.81-5.91(\mathrm{~m}$, 1H), 7.45-7.60 (m, 4H), 8.00 (d, $J=8.4 \mathrm{~Hz}, 2 \mathrm{H}), 8.32$ (d, $J=8.4 \mathrm{~Hz}, 2 \mathrm{H}), 8.39$ (s, 1H), 9.06 (br s, 2H); ${ }^{13} \mathrm{C}$ NMR (100 MHz, $\left.\mathrm{CDCl}_{3}\right) \delta$ 24.0, 27.4, 33.6, 34.8, 43.3, 50.0, 67.5, $75.3,124.1,124.3,125.2,126.4,127.2,127.5,128.3,129.5,130.5,131.7,155.5$; FTICR-MS calcd for $\mathrm{C}_{26} \mathrm{H}_{32} \mathrm{NO}_{3}{ }^{+}[\mathrm{M}+\mathrm{H}]^{+} m / z$ 406.2377, found 406.2379.

\subsubsection{Experimental Procedures for Determination of Release Rate of 25 and 26.}

The Boc protecting group was removed from each linker as described for $\mathbf{6 . 2}$ and immediately placed into a $0.01 \mathrm{M}$ solution in $\mathrm{MeOH}$ and heated at $55^{\circ} \mathrm{C}$ for $24 \mathrm{~h} .200$ 
$\mu \mathrm{L}$ aliquots were pulled at $\mathrm{t}=0,0.25,0.5,0.75,1,2,3$ and $24 \mathrm{~h}$ and were stored at -20 ${ }^{\circ} \mathrm{C}$ to prevent further release. The solvent was removed under vacuum for $3.5 \mathrm{~h}$ at $\mathrm{rt}$ and was then returned to $-20{ }^{\circ} \mathrm{C}$ until analyzed by HPLC. The remaining residue was diluted to $500 \mu \mathrm{L}$ with HPLC grade $\mathrm{CH}_{2} \mathrm{Cl}_{2}$ and a $50 \mu \mathrm{L}$ aliquot was injected into the HPLC fitted with a Waters Nova-Pak HR Silica $6 \mu \mathrm{m} 60$ Å 3.9x300 mm Prep Column using 1\% MeOH: $\mathrm{CH}_{2} \mathrm{Cl}_{2}$ with $0.1 \%$ triethylamine as the eluent. The percent release was determined by the ratio of the starting linker to the release of $\mathbf{2 5}$ or $\mathbf{2 6}$.

To test thermal stability of $\mathbf{1 1}$ at $37^{\circ} \mathrm{C}$, three samples were incubated at $37^{\circ} \mathrm{C}$ for $24 \mathrm{~h}$. The solvent was then evaporated and analyzed as described above.

\subsubsection{Experimental Procedure for ${ }^{1}$ H NMR Verification of Cyclization}

Samples of 6.1 and 7.1 (10 mg) were placed into separate screw-cap NMR tubes. Each sample was diluted with toluene- $\mathrm{d}_{8}(0.5 \mathrm{~mL})$ and a ${ }^{1} \mathrm{H}$ NMR was taken. This NMR measurement was considered $\mathrm{t}=0 \mathrm{~h}$. The samples were then heated to $55^{\circ} \mathrm{C}$ and held for $3 \mathrm{~h}(\mathbf{6 . 1})$ and $5 \mathrm{~h}(\mathbf{7 . 1})$, at which time another ${ }^{1} \mathrm{H}$ NMR spectrum of each sample was obtained. The samples were then heated to $\geq 90{ }^{\circ} \mathrm{C}$ and held at that temperature overnight to complete the cyclization. ${ }^{1} \mathrm{H}$ NMR spectra were once again obtained. To verify the formation of the respective lactam and oxazolidinone, the toluene- $\mathrm{d}_{8}$ was removed in vacuo and the samples were diluted with $\mathrm{CDCl}_{3}$ and the spectral results were found to be in agreement with published literature. ${ }^{6}$ 


\subsubsection{Synthesis of Ester 27 (Scheme 3.9)}

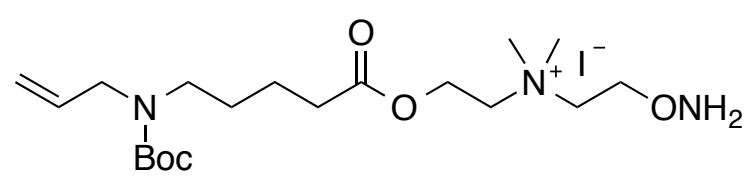

27

\section{2-((5-(Allyl(tert-butoxycarbonyl)amino)pentanoyl)oxy)- $N-(2-((1,3-$ dioxoisoindolin-2-}

yl)oxy)ethyl)- $N, N$-dimethylethanaminium iodide (27). Amino acid $\mathbf{1 3 . 2}$ (98 mg, 0.38

mmol) and the 2-(2-((2-hydroxyethyl)(methyl)amino)ethoxy)-2,3-dihydro- $1 H$-isoindole1,3-dione mono- $O$-phthalimide of $\mathrm{N}$-methyl-diethanolamine ${ }^{4}$ were dissolved in dry $\mathrm{CH}_{2} \mathrm{Cl}_{2}(1.7 \mathrm{~mL})$ with stirring. DIC $(82 \mu \mathrm{L}, 0.52 \mathrm{mmol})$ was added to the reaction solution followed by cat. DMAP. After $2 \mathrm{~h}$, the white solids were filtered out and the filter cake was washed with $\mathrm{CH}_{2} \mathrm{Cl}_{2}$. The filtrate was condensed in vacuo and the crude material was purified by column chromatography $\left(\mathrm{SiO}_{2}, 3: 1\right.$ to $1: 0$, EtOAc:hexanes gradient) to give the corresponding ester as a light yellow oil (96 mg, 55\%). $R_{f} 0.55$ (EtOAc); ${ }^{1} \mathrm{H}$ NMR (400 MHz, $\left.\mathrm{CDCl}_{3}\right) \delta 1.43(\mathrm{~s}, 9 \mathrm{H}), 1.49-1.62(\mathrm{~m}, 4 \mathrm{H}), 2.33(\mathrm{t}, J=7.4$ $\mathrm{Hz}, 2 \mathrm{H}), 2.39(\mathrm{~s}, 3 \mathrm{H}), 2.77(\mathrm{t}, J=6.0 \mathrm{~Hz}, 2 \mathrm{H}), 2.91(\mathrm{t}, J=5.6 \mathrm{~Hz}, 2 \mathrm{H}), 3.16$ (br s, 2H), $3.78($ br s, 2H), $4.16(\mathrm{t}, J=5.8 \mathrm{~Hz}, 2 \mathrm{H}), 4.30(\mathrm{t}, J=5.4 \mathrm{~Hz}, 2 \mathrm{H}), 5.09(\mathrm{~d}, J=11.2 \mathrm{~Hz}$, 2H), 5.72-5.78 (m, 1H), 7.72-7.75 (m, 2H), 7.76-7.84 (m, 2H); ${ }^{13} \mathrm{C}$ NMR (100 MHz, $\left.\mathrm{CDCl}_{3}\right) \delta 22.4,28.0,28.6,34.1,42.9,46.3,55.9,60.6,62.3,76.1,79.6,116.5,123.7$, $129.2,134.6,155.7,163.6,173.6$.

To the ester $(96 \mathrm{mg}, 0.19 \mathrm{mmol})$ in dry $\mathrm{CH}_{2} \mathrm{Cl}_{2}(1 \mathrm{~mL})$ in a pressure tube was added iodomethane $(24 \mu \mathrm{L}, 0.38 \mathrm{mmol})$. The tube was sealed and heated to $60{ }^{\circ} \mathrm{C}$ for $18 \mathrm{~h}$. The solution was then concentrated in vacuo to give a crude ammonium salt (yellow gum, $122 \mathrm{mg}, 100 \%$ ) that was used in the next step without further purification. $R_{f} 0.20$ 
(1:9, $\left.\mathrm{MeOH}: \mathrm{CH}_{2} \mathrm{Cl}_{2}\right) ;{ }^{1} \mathrm{H}$ NMR (400 MHz, $\mathrm{CDCl}_{3}$ ) $\delta 1.39$ (s, 9H), 1.50-1.56 (m, 4H), $2.40(\mathrm{t}, J=6.6 \mathrm{~Hz}, 2 \mathrm{H}), 3.12(\mathrm{t}, J=7.0 \mathrm{~Hz}, 2 \mathrm{H}), 3.66(\mathrm{~s}, 6 \mathrm{H}), 3.73(\mathrm{br} \mathrm{s}, 2 \mathrm{H}), 4.26(\mathrm{br} \mathrm{s}$, 2H), 4.39 (br s, 2H), 4.64 (br s, 2H), 4.77 (br s, 2H), 5.06 (d, $J=12.0 \mathrm{~Hz}, 2 \mathrm{H}), 5.68-5.74$ (m, 1H), 7.76-7.81 (m, 4H); ${ }^{13} \mathrm{C} \mathrm{NMR}\left(100 \mathrm{MHz}, \mathrm{CDCl}_{3}\right)$ o 21.9, 27.5, 28.5, 46.2, 49.9, $53.2,57.8,63.2,64.9,72.4,79.5,116.2,124.1,128.6,134.3,135.2,155.6,163.2,172.5$.

Methyl hydrazine (24 $\mu \mathrm{L}, 0.46 \mathrm{mmol}$ ) was added to a stirred solution of the crude ammonium salt $(49 \mathrm{mg}, 0.076 \mathrm{mmol})$ in $1: 1 \mathrm{CH}_{2} \mathrm{Cl}_{2}: \mathrm{EtOH}(2 \mathrm{~mL})$ at $-40{ }^{\circ} \mathrm{C}$. After $1.5 \mathrm{~h}$, the solution was concentrated in vacuo and diluted with $\mathrm{CH}_{2} \mathrm{Cl}_{2}$, causing a white precipitate to form. The solid was filtered and the filter cake was washed with $\mathrm{CH}_{2} \mathrm{Cl}_{2}$. The filtrate was concentrated in vacuo to give 27 (yellow gum, $39 \mathrm{mg}, 100 \%$ ) that was used in the next step without further purification; $R_{f} 0.14\left(1: 9, \mathrm{MeOH}: \mathrm{CH}_{2} \mathrm{Cl}_{2}\right) ;{ }^{1} \mathrm{H} \mathrm{NMR}$ (400 MHz, $\left.\mathrm{CDCl}_{3}\right) \delta 1.41(\mathrm{~s}, 9 \mathrm{H}), 1.51-1.59(\mathrm{~m}, 4 \mathrm{H}), 2.39(\mathrm{t}, J=7.0 \mathrm{~Hz}, 2 \mathrm{H}), 3.14(\mathrm{t}, J=$ $6.4 \mathrm{~Hz}, 2 \mathrm{H}), 3.48$ (s, 6H), 3.74 (br s, 2H), 4.02 (br s, 2H), 4.08 (br s, 2H), 4.19 (br s, 2H), 4.57 (br s, 2H), 5.07 (d, $J=15.6 \mathrm{~Hz}, 2 \mathrm{H}), 5.70-5.74(\mathrm{~m}, 1 \mathrm{H}) ;{ }^{13} \mathrm{C}$ NMR $(100 \mathrm{MHz}$, $\left.\mathrm{CDCl}_{3}\right) \delta 22.0,27.6,28.6,33.8,46.2,49.9,53.1,57.8,63.9,64.2,69.2,79.7,116.3$, 134.3, 155.7, 172.6; FT-ICR-MS calcd for $\mathrm{C}_{19} \mathrm{H}_{38} \mathrm{~N}_{3} \mathrm{O}_{5}^{+}[\mathrm{M}]^{+} \mathrm{m} / z$ 388.2806, found 388.2807 .

\subsubsection{Experimental Procedures for Manufacture of PDMS Microchannel}

The device design and detailed fabrication protocol was based on a previous device by

Sethu et al. ${ }^{7}$ Briefly, the device was fabricated using standard soft lithographic techniques. A silicon wafer was first treated with oxygen plasma in an asher (March Instruments, Concord, MA) and then spin coated with SU-8, a negative photoresist (SU-8 
50, MicroChem, Newton, MA). Standard photolithography using a transparency mask (CAD ART Services Inc., Poway, CA) was generated using AutoCAD layout software (Autodesk, Inc., San Rafael, CA), and used to create negative replicas of the channel structures. A silicone elastomer, polydimethylsiloxane (PDMS) (Dow Corning, Midland, MI) was mixed 10:1 with a cross linking agent and poured on top of the silicon wafer, and cured at $60{ }^{\circ} \mathrm{C}$ for 12 hours in a petri dish. The cured elastomer with the replicated channel structure was released and access holes to the channels were punched using a 22gauge syringe needle. The PDMS piece with the channels was bonded irreversibly to a glass slide after treatment with oxygen plasma in the asher. Access tubing (Tygon, Small Parts Inc. Miami Lakes, FL) with a slightly larger diameter than the holes was subsequently press fitted into the punched holes.

\subsubsection{Procedure for Loading 27 onto PDMS Microchannel}

Ester $27(39 \mathrm{mg}, 0.076 \mathrm{mmol})$ was placed into a pressure tube with a stir-bar and the headspace was purged with nitrogen. Catalytic $\mathrm{PtO}_{2}$ was then added, followed by triethoxysilane ( $14 \mu \mathrm{L}, 0.076 \mathrm{mmol})$. The headspace was purged with nitrogen and the

pressure tube was sealed and heated to $80^{\circ} \mathrm{C}$. After two days, the reaction was cooled to room temperature and the solution was filtered under nitrogen through Celite and the filter cake was washed with dry $\mathrm{CH}_{2} \mathrm{Cl}_{2}$. The filtrate was concentrated in vacuo to give a moisture-sensitive residue, triethoxylsilane intermediate 28, which was immediately loaded onto the PDMS microchannel without further purification by first dissolving in $\mathrm{CH}_{3} \mathrm{CN}(0.8 M)$ and then injecting the solution $(10 \mu \mathrm{L}, 80 \mu \mathrm{mol})$ into the microchannel. After $1 \mathrm{~h}$, the microchannel was washed with $\mathrm{CH}_{3} \mathrm{CN}(5 \times 10 \mu \mathrm{L})$ and placed in $110{ }^{\circ} \mathrm{C}$ 
oven for 15 minutes. On cooling, the loaded microchannel was stored at room temperature in a sealed bag until needed.

\subsubsection{Experimental Procedures for PDMS Microchannel Reaction and Release FITC-CHO Attachment}

A solution of FITC-CHO (10 $\mu \mathrm{L}$ of $0.02 M, 0.2 \mu \mathrm{mol})$ was injected into the aminooxyfunctionalized microchannel and allowed to react for $1 \mathrm{~h}$. The remaining FITC-CHO solution was then removed and the microchannel was washed with $\mathrm{MeCN}(5 \times 10 \mu \mathrm{L})$. The microchannel was then observed with a fluorescence microscope (excitation/emission, $495 \mathrm{~nm} / 521 \mathrm{~nm}$ ) and the microchannel was fluorescent.

\section{Boc Deprotection}

The linker was activated by removing the Boc protecting group by injecting a 1:4 solution of TFA:MeCN $(10 \mu \mathrm{L})$ into the microchannel and allowing it to react for $1 \mathrm{~h}$. The microchannel was then evacuated and washed with $\mathrm{MeCN}(3 \times 10 \mu \mathrm{L})$. The basification of the ammonium salt was achieved by injecting a 1:1 solution of TEOA:MeCN into the microchannel $(2 \times 10 \mu \mathrm{L})$ and allowing each aliquot to sit for 5 minutes. The microchannel was then washed with $\operatorname{MeCN}(5 \times 10 \mu \mathrm{L})$.

\section{Thermally induced cyclization/substrate release}

The microchannel with the activated linker was placed on a hot plate at $\sim 60{ }^{\circ} \mathrm{C}$ for 30 minutes to induce an intramolecular cyclization and release of the FITC-CHO substrate. After cooling the microchannel to $\mathrm{rt}$, it was washed with $\mathrm{MeCN}(5 \times 10 \mu \mathrm{L})$ and the washings were collected. The microchannel was then observed with a fluorescence microscope (excitation/emission, $495 \mathrm{~nm} / 521 \mathrm{~nm}$ ) and it was no longer fluorescent. To 
ensure that release of the FITC-CHO moiety occurred upon heating, a fluorescent measurement was taken of the collected washings and fluorescence was observed.

\subsection{EXPERIMENTAL PROCEDURES OF CHAPTER 4}

\subsubsection{Synthesis of Non-Nucleophilic Ester Linker}

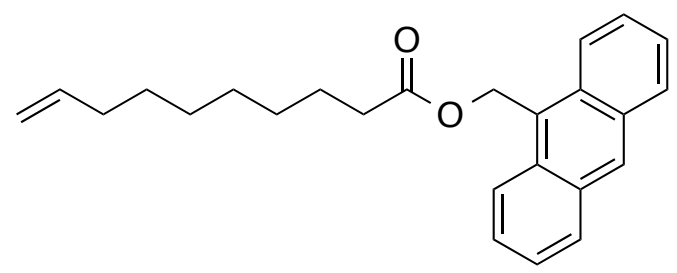

10

Anthracen-9-ylmethyl dec-9-enoate (10). 9-Decenoic acid (343 mg, $2.02 \mathrm{mmol})$ and 9anthracenemethanol (361 $\mathrm{mg}, 1.73 \mathrm{mmol})$ were dissolved in dry $\mathrm{CH}_{2} \mathrm{Cl}_{2}(15 \mathrm{~mL})$ with stirring. DIC (395 $\mu \mathrm{L}, 2.52 \mathrm{mmol})$ was added to the reaction solution followed by cat. DMAP. After $2 \mathrm{~h}$, the white solids were filtered out and the filter cake was washed with $\mathrm{CH}_{2} \mathrm{Cl}_{2}$. The filtrate was condensed in vacuo and the crude material was purified by column chromatography $\left(\mathrm{SiO}_{2}, 0: 100\right.$ to 1:9 EtOAc: $\mathrm{CH}_{2} \mathrm{Cl}_{2}$ gradient) to give 10 (506 mg, 81\%) as a yellow oil. $R_{f} 0.67\left(\mathrm{CH}_{2} \mathrm{Cl}_{2}\right) ;{ }^{1} \mathrm{H}$ NMR $\left(400 \mathrm{MHz}, \mathrm{CDCl}_{3}\right) \delta$ 1.24-1.33 (m, $8 \mathrm{H}), 1.62$ (quin, $J=5.6 \mathrm{~Hz}, 2 \mathrm{H}), 2.00(\mathrm{q}, J=5.6 \mathrm{~Hz}, 2 \mathrm{H}), 2.33(\mathrm{t}, J=5.8 \mathrm{~Hz}, 2 \mathrm{H}), 4.96$ (dd, $J=8.0$ and $13.6 \mathrm{~Hz}, 2 \mathrm{H}), 5.76-5.82(\mathrm{~m}, 1 \mathrm{H}), 6.16(\mathrm{~s}, 2 \mathrm{H}), 7.48-7.51(\mathrm{~m}, 2 \mathrm{H}), 7.56-$ $7.59(\mathrm{~m}, 2 \mathrm{H}), 8.03(\mathrm{~d}, J=6.8 \mathrm{~Hz}, 2 \mathrm{H}), 8.34(\mathrm{~d}, J=7.2 \mathrm{~Hz}, 2 \mathrm{H}), 8.50(\mathrm{~s}, 1 \mathrm{H}) ;{ }^{13} \mathrm{C} \mathrm{NMR}$ $\left(100 \mathrm{MHz}, \mathrm{CDCl}_{3}\right) \delta$ 25.2, 29.0, 29.1, 29.2, 33.9, 34.5, 58.8, 114.3, 124.1, 125.3, 126.6, $126.8,129.3,131.2,131.6,139.3,174.3$. 


\subsubsection{Synthesis of 11}<smiles>C=CCNC(=O)OC(C)(C)C</smiles>

tert-Butyl allylcarbamate. Allyl amine (997 mg, $17.5 \mathrm{mmol})$ was dissolved in dry $\mathrm{CH}_{2} \mathrm{Cl}_{2}(35 \mathrm{~mL})$ and cooled to $0{ }^{\circ} \mathrm{C}$ with stirring. Boc $2 \mathrm{O}$ was added to the reaction solution and stirred for $18 \mathrm{~h}$, allowing the reaction to slowly warm to room temperature. Upon completion, the solution was washed with $\mathrm{HCl}(2 \times 13 \mathrm{~mL}$ of $1 M$ solution $)$, dried over $\mathrm{Na}_{2} \mathrm{SO}_{4}$, filtered and concentrated in vacuo to give tert-butyl allylcarbamate $(2.55 \mathrm{~g}$, 93\%) as white crystals which were used in the next step without further purification. $R_{f}$ 0.65 (1:1, EtOAc:hexanes); ${ }^{1} \mathrm{H}$ NMR (400 MHz, $\left.\mathrm{CDCl}_{3}\right) \delta 1.44$ (s, 9H), 3.73 (br s, 2H), $4.60($ br s, $1 \mathrm{H}), 5.08-5.19(\mathrm{~m}, 2 \mathrm{H}), 5.78-5.88(\mathrm{~m}, 1 \mathrm{H}) ;{ }^{13} \mathrm{C} \mathrm{NMR}\left(100 \mathrm{MHz}, \mathrm{CDCl}_{3}\right) \delta$ 28.6, 43.3, 79.6, 115.9, 135.1, 156.0.

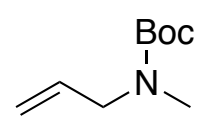

11

tert-Butyl allyl(methyl)carbamate (11). A suspension of $\mathrm{NaH}(579 \mathrm{mg}, 24.1 \mathrm{mmol})$ in dry THF (100 mL) was cooled to $0{ }^{\circ} \mathrm{C}$ with stirring. A solution of tert-butyl allylcarbamate $(2.53 \mathrm{~g}, 16.1 \mathrm{mmol})$ in dry THF $(10 \mathrm{~mL})$ was added to the reaction flask dropwise and stirred for $20 \mathrm{~min}$ at $0{ }^{\circ} \mathrm{C}$. Methyl iodide $(1.5 \mathrm{~mL}, 24.1 \mathrm{mmol})$ was added and the reaction was stirred for $16 \mathrm{~h}$ allowing it to slowly come to room temperature. The reaction was quenched with sat. $\mathrm{NH}_{4} \mathrm{Cl}(150 \mathrm{~mL})$ and the aqueous phase was extracted with $\mathrm{EtO}_{2}(3 \times 90 \mathrm{~mL})$ and wash with brine $(100 \mathrm{~mL})$. The combined organics 
were dried over $\mathrm{Na}_{2} \mathrm{SO}_{4}$, filtered and concentrated in vacuo. The crude material was purified by column chromatography $\left(\mathrm{SiO}_{2}, 1: 3\right.$, EtOAc:hexanes) to give $11(1.03 \mathrm{~g}, 38 \%)$ as a light yellow oil. $R_{f} 0.53$ (1:3, EtOAc:hexanes); ${ }^{1} \mathrm{H} \mathrm{NMR}\left(400 \mathrm{MHz}, \mathrm{CDCl}_{3}\right) \delta 1.45$ $(\mathrm{s}, 9 \mathrm{H}), 2.81(\mathrm{~s}, 3 \mathrm{H}), 3.80(\mathrm{br} \mathrm{s}, 2 \mathrm{H}), 5.08-5.13(\mathrm{~m}, 2 \mathrm{H}), 5.70-5.80(\mathrm{~m}, 1 \mathrm{H}) ;{ }^{13} \mathrm{C} \mathrm{NMR}$ $\left(100 \mathrm{MHz}, \mathrm{CDCl}_{3}\right) \delta 28.6,33.9,51.5,79.6,116.5,133.9,155.9$.

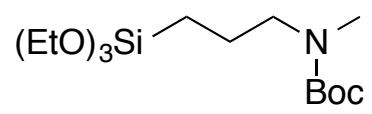

tert-Butyl methyl(3-(triethoxysilyl)propyl)carbamate. $11(518 \mathrm{mg}, 3.02 \mathrm{mmol})$ and cat. $\mathrm{PtO}_{2}$ were placed into a pressure vial with a stir bar and the vial was purged with nitrogen. Triethoxysilane ( $600 \mu \mathrm{L}, 3.25 \mathrm{mmol}$ ) was then added and the vial was purged with nitrogen and then sealed. The reaction vial was heated to $85-95{ }^{\circ} \mathrm{C}$ and stirred for one week. Upon completion, the reaction mixture was filtered through Celite and the filter cake was washed with dry $\mathrm{CH}_{2} \mathrm{Cl}_{2}$. The solution was then concentrated in vacuo to give crude tert-butyl methyl(3-(triethoxysilyl)propyl)carbamate as a brown oil that was used in the next step without further purification. ${ }^{1} \mathrm{H} \mathrm{NMR}\left(400 \mathrm{MHz}, \mathrm{CDCl}_{3}\right) \delta 0.55(\mathrm{t}$, $J=8.4 \mathrm{~Hz}, 2 \mathrm{H}), 1.20-1.25(\mathrm{~m}, 9 \mathrm{H}), 1.44(\mathrm{~s}, 9 \mathrm{H}), 1.58-1.61(\mathrm{~m}, 2 \mathrm{H}), 2.82(\mathrm{~s}, 3 \mathrm{H}), 3.16$ (br $\mathrm{s}, 2 \mathrm{H}$ ), 3.78-3.87 (quin, $J=6.8 \mathrm{~Hz}, 6 \mathrm{H}) ;{ }^{13} \mathrm{C} \mathrm{NMR} \mathrm{(100} \mathrm{MHz,} \mathrm{CDCl}_{3}$ ) $\mathrm{N} 7.6,18.5,21.5$, 28.7, 34.4, 58.6, 59.3, 79.2, 156.0 . 


\subsubsection{Anhydrous NP Loading Procedure (with heat)}

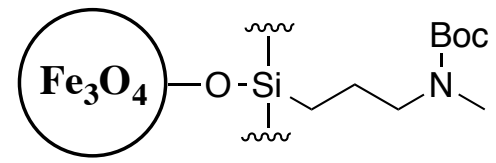

12

The functionalization of the iron oxide NPs was done following a modified procedure as described by Galeotti et al. ${ }^{8}$ Briefly, $\mathrm{Fe}_{3} \mathrm{O}_{4} \mathrm{NPs}(150 \mathrm{mg})$ were placed in a round bottom flask and purged with nitrogen. $\mathrm{CHCl}_{3}(20 \mathrm{~mL})$ was then added via syringe and the NPs were suspended with sonication ( $3 \times 10$ min with 5 min pause between sonication). While maintaining sonication, a solution of the alkoxysilane linker (819 mg, $2.44 \mathrm{mmol})$ in $\mathrm{CHCl}_{3}(5 \mathrm{~mL})$ was added dropwise via a syringe. Sonication was continued for $10 \mathrm{~min}$ after completion of the addition of the silylated linker and was then mechanically stirred for $2.5 \mathrm{~h}$ at room temperature. Then, the reaction flask was heated to $60-65{ }^{\circ} \mathrm{C}$ and stirred for $48 \mathrm{~h}$. After cooling, the coated NPs were magnetically separated and the supernatant was removed followed by washing with $\mathrm{CHCl}_{3}(5 \times 10 \mathrm{~mL})$. The coated NPs were then dried with an oil vacuum pump for $30 \mathrm{~min}$. The vial was then purged with nitrogen and heated to $100-110^{\circ} \mathrm{C}$ for $24 \mathrm{~h}$ to yield $12\left(197 \mathrm{mg}, 10.8\right.$ molecules $\left./ \mathrm{nm}^{2}\right)$.

\subsubsection{Acidic Boc-Deprotection of Functionalized NPs to Afford $2^{\circ}$ Amine}

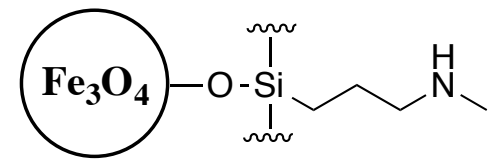

13

A suspension of $12(13.2 \mathrm{mg})$ in $\mathrm{CH}_{2} \mathrm{Cl}_{2}$ was sonicated for 15 seconds to break any aggregation and was then cooled to $0{ }^{\circ} \mathrm{C}$. Trifluoroacetic acid was then added and the vial was allowed to stand for $1 \mathrm{~h}$. Upon completion, the NPs were magnetically 
separated, washed with $\mathrm{CH}_{2} \mathrm{Cl}_{2}(5 \times 5 \mathrm{~mL})$ and dried with an oil vacuum pump for $2 \mathrm{~h}$ to afford 13 (5.5 molecules $\left./ \mathrm{nm}^{2}\right)$.

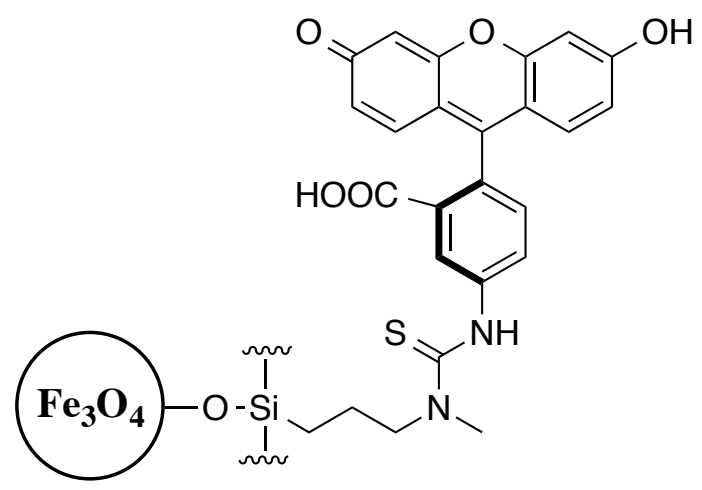

Excess fluorescein isothiocyanate was added to $\mathbf{1 3}(12 \mathrm{mg})$ suspended in dry THF ( $2 \mathrm{~mL}$ ) and sonicated for 30 seconds. The suspension was allowed to stand for $4.5 \mathrm{~h}$ before the NPs were magnetically separated and the supernatant was removed. The NPs were washed with sat. $\mathrm{NaHCO}_{3}(3 \times 5 \mathrm{~mL}), \mathrm{MeOH}(5 \times 5 \mathrm{~mL})$, and $\mathrm{EtO}_{2}(2 \times 5 \mathrm{~mL})$. The washed NPs were dried with an oil vacuum pump to yield the fluorescent NPs.

\subsubsection{Synthesis of Non-Nucleophilic Olefinic Ester}<smiles>C=CCCC(=O)OCCc1c2ccccc2cc2ccccc12</smiles>

17.1

2-(Anthracen-9-yl)ethyl pent-4-enoate (17.1). 4-Pentenoic acid (159 $\mu \mathrm{L}, 1.56 \mathrm{mmol})$ and 2-(anthracen-9-yl)ethanol (281 $\mathrm{mg}, 1.26 \mathrm{mmol})$ were dissolved in dry $\mathrm{CH}_{2} \mathrm{Cl}_{2}(15$ $\mathrm{mL})$ with stirring. DIC (305 $\mu \mathrm{L}, 1.95 \mathrm{mmol})$ was added to the reaction solution followed by cat. DMAP. After $2 \mathrm{~h}$, the white solids were filtered out and the filter cake was 
washed with $\mathrm{CH}_{2} \mathrm{Cl}_{2}$. The filtrate was condensed in vacuo and the crude material was purified by column chromatography $\left(\mathrm{SiO}_{2}, 1: 1\right.$ hexanes: $\left.\mathrm{CH}_{2} \mathrm{Cl}_{2}\right)$ to give $\mathbf{1 7 . 1}$ (359 $\mathrm{mg}$, 93\%) as a yellow oil. $R_{f} 0.36\left(1: 1\right.$ hexanes: $\left.\mathrm{CH}_{2} \mathrm{Cl}_{2}\right) ;{ }^{1} \mathrm{H}$ NMR $\left(400 \mathrm{MHz}, \mathrm{CDCl}_{3}\right) \delta 2.35$ $2.46(\mathrm{~m}, 4 \mathrm{H}), 3.99(\mathrm{t}, J=8.0 \mathrm{~Hz}, 2 \mathrm{H}), 4.50(\mathrm{t}, J=7.8 \mathrm{~Hz}, 2 \mathrm{H}), 5.00-5.08(\mathrm{~m}, 2 \mathrm{H}), 5.79-$ $5.88(\mathrm{~m}, 1 \mathrm{H}), 7.46-7.50(\mathrm{~m}, 2 \mathrm{H}), 7.54-7.58(\mathrm{~m}, 2 \mathrm{H}), 8.02(\mathrm{~d}, J=8.4 \mathrm{~Hz}, 2 \mathrm{H}), 8.38(\mathrm{~d}, J=$ $8.8 \mathrm{~Hz}, 2 \mathrm{H}), 8.40(\mathrm{~s}, 1 \mathrm{H}) ;{ }^{13} \mathrm{C} \mathrm{NMR}\left(100 \mathrm{MHz}, \mathrm{CDCl}_{3}\right) \delta 27.5,29.0,33.8,64.3,115.7$, $124.3,125.1,126.2,127.0,129.2,129.4,130.5,131.7,136.8,173.4$.

\subsubsection{Synthesis of Non-Nucleophilic Olefinic Carbonate}

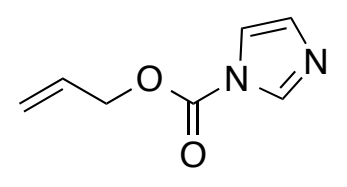

Allyl $\mathbf{1 H}$-imidazole-1-carboxylate. $N, N$-diisopropylethylamine $(5.37 \mathrm{~mL}, 30.8 \mathrm{mmol})$ was added to a solution of allyl alcohol $(1.21 \mathrm{~g}, 20.8 \mathrm{mmol})$ in dry $\mathrm{CH}_{2} \mathrm{Cl}_{2}(69 \mathrm{~mL})$. The solution was cooled to $0{ }^{\circ} \mathrm{C}$ and $1,1^{\prime}$-carbonyldiimidazole $(5.07 \mathrm{~g}, 31.2 \mathrm{mmol})$ was added and the reaction was stirred overnight. The reaction was washed with water $(2 \mathrm{x} 40 \mathrm{~mL})$, brine $(40 \mathrm{~mL})$ and dried over $\mathrm{Na}_{2} \mathrm{SO}_{4}$, filtered and concentrated in vacuo. The crude material was purified by column chromatography $\left(\mathrm{SiO}_{2}\right.$, EtOAc) to give allyl $1 H$ imidazole-1-carboxylate $(2.24 \mathrm{~g}, 71 \%)$ as a light yellow oil. $R_{f} 0.51$ (EtOAc); ${ }^{1} \mathrm{H}$ NMR $\left(400 \mathrm{MHz}, \mathrm{CDCl}_{3}\right) \delta 4.87(\mathrm{~d}, J=5.6 \mathrm{~Hz}, 2 \mathrm{H}), 5.37(\mathrm{~d}, J=10.0 \mathrm{~Hz}, 1 \mathrm{H}), 5.44(\mathrm{~d}, J=16.8$ $\mathrm{Hz}, 1 \mathrm{H})$, 5.95-6.03 (m, 1H), $7.05(\mathrm{~s}, 1 \mathrm{H}), 7.42(\mathrm{~s}, 1 \mathrm{H}), 8.13(\mathrm{~s}, 1 \mathrm{H}) ;{ }^{13} \mathrm{C} \mathrm{NMR}(100 \mathrm{MHz}$ $\left.\mathrm{CDCl}_{3}\right) \delta 68.8,117.3,120.6,130.6,130.9,137.3,148.6$. 


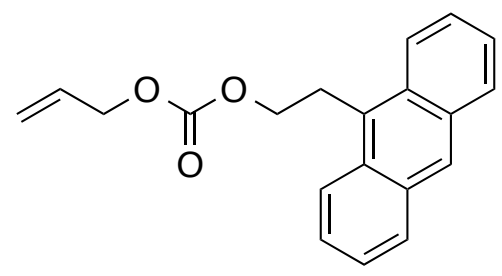

17.2

Allyl (2-(anthracen-9-yl)ethyl) carbonate (17.2). 1,8-Diazabicyclo[5.4.0]undec-7-ene (1.68 mL, $11.2 \mathrm{mmol})$ was added to a solution of allyl $1 H$-imidazole-1-carboxylate $(1.71$ $\mathrm{g}, 11.2 \mathrm{mmol})$ in dry $\mathrm{CH}_{3} \mathrm{CN}(56 \mathrm{~mL})$. The reaction solution was stirred for 10 minutes before adding 2-(anthracen-9-yl)ethanol (2.50 g, $11.2 \mathrm{mmol})$. The reaction was stirred overnight before quenching with sat. $\mathrm{NH}_{4} \mathrm{Cl}(40 \mathrm{~mL})$. The aqueous phase was extracted with EtOAc (2 x $40 \mathrm{~mL})$ and the combined organic phases were washed with brine, dried over $\mathrm{Na}_{2} \mathrm{SO}_{4}$, filtered and concentrated in vacuo. The crude material was purified by column chromatography $\left(\mathrm{SiO}_{2}, 3: 1, \mathrm{CH}_{2} \mathrm{Cl}_{2}\right.$ :hexanes $)$ to give $17.2(2.33 \mathrm{~g}, 68 \%)$ as yellow crystals. $R_{f} 0.63\left(3: 1, \mathrm{CH}_{2} \mathrm{Cl}_{2}\right.$ :hexanes); ${ }^{1} \mathrm{H}$ NMR $\left(400 \mathrm{MHz}, \mathrm{CDCl}_{3}\right) \delta 4.06(\mathrm{t}, J$ $=8.0 \mathrm{~Hz}, 2 \mathrm{H}), 4.52(\mathrm{t}, J=8.4 \mathrm{~Hz}, 2 \mathrm{H}), 4.69(\mathrm{~d}, J=5.6 \mathrm{~Hz}, 2 \mathrm{H}), 5.31(\mathrm{dd}, J=10.8,1.8$ $\mathrm{Hz}, 1 \mathrm{H}), 5.40(\mathrm{dd}, J=17.6,1.2 \mathrm{~Hz}, 1 \mathrm{H}), 5.93-6.03(\mathrm{~m}, 1 \mathrm{H}), 7.47-7.50(\mathrm{~m}, 2 \mathrm{H}), 7.55-7.59$ $(\mathrm{m}, 2 \mathrm{H}), 8.02(\mathrm{~d} J=8.4 \mathrm{~Hz}, 2 \mathrm{H}), 8.35(\mathrm{~d}, J=8.8 \mathrm{~Hz}, 2 \mathrm{H}), 8.40(\mathrm{~s}, 1 \mathrm{H}) ;{ }^{13} \mathrm{C}$ NMR $(100$ $\left.\mathrm{MHz}, \mathrm{CDCl}_{3}\right) \delta 27.6,67.4,68.7,119.1,124.1,125.2,126.4,127.2,128.3,129.5,130.5$, $131.7,131.8,155.3$. 


\subsubsection{Synthesis of Non-Nucleophilic Olefinic Carbamate}<smiles>O=C(OCCc1c2ccccc2cc2ccccc12)n1ccnc1</smiles>

2-(Anthracen-9-yl)ethyl $\mathbf{1 H}$-imidazole-1-carboxylate. $\quad N, N$-Diisopropylethylamine (408 $\mu \mathrm{L}, 2.34 \mathrm{mmol})$ was added to a solution of 2-(anthracen-9-yl)ethanol (346 mg, 1.55 mmol $)$ in dry $\mathrm{CH}_{2} \mathrm{Cl}_{2}(8 \mathrm{~mL})$, the solution was cooled to $0{ }^{\circ} \mathrm{C}$ and 1,1 'carbonyldiimidazole (380 mg, $2.34 \mathrm{mmol}$ ) was added. After stirring overnight, the reaction solution was washed with water $(2 \times 5 \mathrm{~mL})$, brine, dried over $\mathrm{Na}_{2} \mathrm{SO}_{4}$, filtered and concentrated in vacuo. The crude material was purified by column chromatography $\left(\mathrm{SiO}_{2}, \mathrm{CH}_{2} \mathrm{Cl}_{2}\right.$ to $3: 2$, EtOAc: $\mathrm{CH}_{2} \mathrm{Cl}_{2}$ gradient) to give 2-(anthracen-9-yl)ethyl $1 \mathrm{H}$ imidazole-1-carboxylate (436 $\mathrm{mg}, 89 \%)$ as a pale yellow solid. $R_{f} 0.23$ (1:19, EtOAc: $\left.\mathrm{CH}_{2} \mathrm{Cl}_{2}\right) ;{ }^{1} \mathrm{H}$ NMR $\left(400 \mathrm{MHz}, \mathrm{CDCl}_{3}\right) \delta 4.16(\mathrm{t}, J=7.4 \mathrm{~Hz}, 2 \mathrm{H}), 4.80(\mathrm{t}, J=7.6$ $\mathrm{Hz}, 2 \mathrm{H}), 7.02(\mathrm{~s}, 1 \mathrm{H}), 7.31(\mathrm{~s}, 1 \mathrm{H}), 7.50(\mathrm{t}, J=7.6 \mathrm{~Hz}, 2 \mathrm{H}), 7.58(\mathrm{t}, J=7.4 \mathrm{~Hz}, 2 \mathrm{H}), 8.00$ $(\mathrm{s}, 1 \mathrm{H}), 8.04(\mathrm{~d}, J=8.0 \mathrm{~Hz}, 2 \mathrm{H}), 8.33(\mathrm{~d}, J=8.8 \mathrm{~Hz}, 2 \mathrm{H}), 8.44(\mathrm{~s}, 1 \mathrm{H}) ;{ }^{13} \mathrm{C} \mathrm{NMR}(100$ $\left.\mathrm{MHz}, \mathrm{CDCl}_{3}\right) \delta 27.0,68.0,117.3,123.8,125.3,126.6,127.5,127.9,129.7,130.5,130.8$, $131.7,137.3,149.0$. 


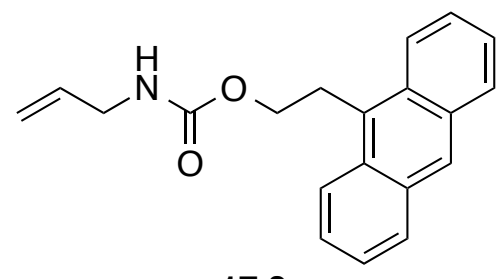

17.3

2-(Anthracen-9-yl)ethyl allylcarbamate (17.3). 1,8-Diazabicyclo[5.4.0]undec-7-ene (206 $\mu \mathrm{L}, 1.38 \mathrm{mmol})$ was added to a solution of 2-(anthracen-9-yl)ethyl $1 H$-imidazole-1carboxylate $(436 \mathrm{mg}, 1.38 \mathrm{mmol})$ in dry $\mathrm{CH}_{3} \mathrm{CN}(7 \mathrm{~mL})$. The reaction solution was stirred for 10 minutes before adding allylamine $(114 \mu \mathrm{L}, 1.51 \mathrm{mmol})$. The reaction was stirred overnight before quenching with sat. $\mathrm{NH}_{4} \mathrm{Cl}(40 \mathrm{~mL})$. The aqueous phase was extracted with EtOAc $(2 \times 5 \mathrm{~mL})$ and the combined organic phases were washed with brine, dried over $\mathrm{Na}_{2} \mathrm{SO}_{4}$, filtered and concentrated in vacuo. The crude material was purified by column chromatography $\left(\mathrm{SiO}_{2}, \mathrm{CH}_{2} \mathrm{Cl}_{2}\right.$ to $2: 3, \mathrm{EtOAc:} \mathrm{CH}_{2} \mathrm{Cl}_{2}$ gradient) to give $17.3(356 \mathrm{mg}, 85 \%)$ as pale yellow crystals. $R_{f} 0.72\left(1: 19\right.$, EtOAc: $\left.\mathrm{CH}_{2} \mathrm{Cl}_{2}\right) ;{ }^{1} \mathrm{H}$ NMR (400 MHz, $\left.\mathrm{CDCl}_{3}\right) \delta 3.85$ (br s, 2H), 4.00 (t, $\left.J=7.8 \mathrm{~Hz}, 2 \mathrm{H}\right), 4.47$ (t, $J=7.6 \mathrm{~Hz}$, 2H), 4.74 (br s, $1 \mathrm{H}), 5.13-5.22(\mathrm{~m}, 2 \mathrm{H}), 5.84-5.90(\mathrm{~m}, 1 \mathrm{H}), 7.45-7.49(\mathrm{~m}, 2 \mathrm{H}), 7.53-7.56$ $(\mathrm{m}, 2 \mathrm{H}), 8.01(\mathrm{~d}, J=8.4 \mathrm{~Hz}, 2 \mathrm{H}), 8.36(\mathrm{~d}, J=8.8 \mathrm{~Hz}, 2 \mathrm{H}), 8.39(\mathrm{~s}, 1 \mathrm{H}) ;{ }^{13} \mathrm{C} \mathrm{NMR}(100$ $\left.\mathrm{MHz}, \mathrm{CDCl}_{3}\right) \delta 28.1,43.7,64.9,116.3,124.4,125.1,126.2,126.9,129.4,130.6,131.7$ $134.7,156.7$.

\subsubsection{Procedure for the Addition of Polysaccharides to Functionalized NPs}

$\mathrm{Fe}_{3} \mathrm{O}_{4}$ NPs functionalized with linker $17.2(11 \mathrm{mg})$ were suspended in DMSO $(1 \mathrm{~mL})$. Then, excess polysaccharide (D-lactose or $6 \mathrm{kDa}$ dextran) was added to the suspension and the mixture was stirred overnight. The coated NPs were magnetically separated and 
the supernatant was decanted. The resulting NPs were washed once with DMSO and twice with $\mathrm{MeOH}$ before being dried using high vacuum to afford polysaccharide coated NPs (12 mg).

\subsubsection{Synthesis of Monodispersed $\mathrm{SiO}_{2} @ \mathrm{Fe}_{3} \mathrm{O}_{4} \mathrm{NPs}$}

The $\mathrm{Fe}_{3} \mathrm{O}_{4}$ NPs were coated with a thin silica shell using the modified procedure of Pinho et al. ${ }^{9}$ Briefly, the EMG 304 ferrofluid (1 mL, $233 \mathrm{mg} \mathrm{NPs}, 8.55 \times 10^{16} \mathrm{NPs}$ ) was added to Millipore water $(98 \mathrm{~mL})$ and was then added to a solution of EtOH $(312 \mathrm{~mL})$ and $\mathrm{NH}_{4} \mathrm{OH}(6.2 \mathrm{~mL}, 28-30 \%)$ with rapid mechanical stirring. Tetraethyl orthosilicate (TEOS) $(2.13 \mathrm{~mL})$ was then added to the colloidal suspension and was stirred for $12 \mathrm{~h}$. An aliquot was removed to allow for characterization. The NPs were magnetically separated and the remaining colloidal supernatant was centrifuged at 13,200 RPM for 20 min. The NPs were then washed $5 \mathrm{x}$ with $\mathrm{EtOH}$ with magnetic separation and centrifugation after each wash. The excess EtOH was then removed by rotary evaporation and the NPs were dried under vacuum for $3 \mathrm{~h}$. The resulting $\mathrm{SiO}_{2} @ \mathrm{Fe}_{3} \mathrm{O}_{4}$ NPs were characterized by IR, TGA, DLS, $\zeta$-potential, SQUID and TEM.

\subsubsection{Synthesis of Carbamate Linker for AMF-Induced Hydrolytic Release} $\mathrm{HO} \frown$ OTBS

2-((tert-Butyldimethylsilyl)oxy)ethan-1-ol. A solution of $\mathrm{TBSCl}(2.34 \mathrm{~g}, 15.5 \mathrm{mmol})$ in dry $\mathrm{CH}_{2} \mathrm{Cl}_{2}(20 \mathrm{~mL})$ was added dropwise over $1 \mathrm{~h}$ to a solution of ethylene glycol $(8 \mathrm{~mL}$, $143 \mathrm{mmol})$ and $\mathrm{Et}_{3} \mathrm{~N}(2.80 \mathrm{~mL}, 19.9 \mathrm{mmol})$ in dry $\mathrm{CH}_{2} \mathrm{Cl}_{2}(25 \mathrm{~mL})$ at $0{ }^{\circ} \mathrm{C}$ and was stirred overnight. The solvent was removed in vacuo and the remaining oil was extracted 
with hexanes $(4 \mathrm{x})$ and the combined extractions were washed twice with sat. $\mathrm{NH}_{4} \mathrm{Cl}$, once with brine and was dried over $\mathrm{Na}_{2} \mathrm{SO}_{4}$, filtered and concentrated in vacuo to afford the mono-TBS protected diol 2-((tert-butyldimethylsilyl)oxy)ethan-1-ol (2.52 g, 92\%) as a colorless oil and was used without further purification. $R_{f} 0.59$ (1:3, EtOAc:hexanes).

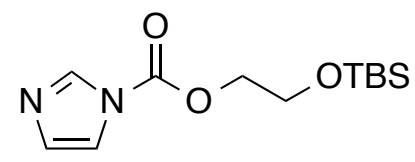

\section{2-((tert-Butyldimethylsilyl)oxy)ethyl $\quad \mathbf{1} \boldsymbol{H}$-imidazole-1-carboxylate. $\quad 1,1^{\prime}-$} Carbonyldiimidazole $(3.47 \mathrm{~g}, 21.4 \mathrm{mmol})$ was added to a solution of the mono-TBS protected diol (2.52 g, $14.3 \mathrm{mmol})$ and $N, N$-diisopropylethylamine $(3.70 \mathrm{~mL}, 21.4 \mathrm{mmol})$ in dry $\mathrm{CH}_{2} \mathrm{Cl}_{2}(48 \mathrm{~mL})$ at $0{ }^{\circ} \mathrm{C}$ and was stirred for $6 \mathrm{~h}$. The reaction was washed twice with water and the combined aqueous phases were extracted once with $\mathrm{CH}_{2} \mathrm{Cl}_{2}$. The combined organic layers were then washed twice with sat. $\mathrm{NH}_{4} \mathrm{Cl}$, once with brine and were dried over $\mathrm{Na}_{2} \mathrm{SO}_{4}$, filtered and concentrated in vacuo to afford crude 2-((tertbutyldimethylsilyl)oxy)ethyl $1 H$-imidazole-1-carboxylate as a colorless oil and was used without further purification. $R_{f} 0.36$ (1:3, EtOAc:hexanes); ${ }^{1} \mathrm{H}$ NMR $\left(400 \mathrm{MHz}, \mathrm{CDCl}_{3}\right)$ $\delta 0.06(\mathrm{~s}, 6 \mathrm{H}), 0.88,(\mathrm{~s}, 9 \mathrm{H}), 3.93(\mathrm{t}, J=4.8 \mathrm{~Hz}, 2 \mathrm{H}), 4.71(\mathrm{t}, J=4.8 \mathrm{~Hz}, 2 \mathrm{H}), 7.06$ (s, 1H), $7.42(\mathrm{~s}, 1 \mathrm{H}), 8.13(\mathrm{~s}, 1 \mathrm{H}) ;{ }^{13} \mathrm{C} \mathrm{NMR}\left(100 \mathrm{MHz}, \mathrm{CDCl}_{3}\right) \delta$-5.2, 18.4, 25.9, 61.0, $69.4,117.3,130.9,137.3,149.0$. 
<smiles>C=CCCCCNC(=O)OC</smiles>

Methyl $\boldsymbol{N}$-(hex-5-en-1-yl)carbamate. Triethylamine $(12.8 \mathrm{~mL}, 91.0 \mathrm{mmol})$ was slowly added to a solution of hex-5-en-1-amine hydrochloride (4.11 g, $30.334 \mathrm{mmol})$ in dry $\mathrm{CH}_{2} \mathrm{Cl}_{2}(300 \mathrm{~mL})$ at $0{ }^{\circ} \mathrm{C}$, then methyl chloroformate $(3.52 \mathrm{~mL}, 45.5 \mathrm{mmol})$ was added dropwise and the reaction was stirred overnight. The remaining white solids were removed by filtration and the filter cake was washed with $\mathrm{CH}_{2} \mathrm{Cl}_{2}$. The filtrate was then washed with $1 \mathrm{M} \mathrm{HCl}(2 \times 100 \mathrm{~mL})$ and the combined aqueous phases were extracted once with $\mathrm{CH}_{2} \mathrm{Cl}_{2}$. The combined organic phases were washed with brine, dried over $\mathrm{Na}_{2} \mathrm{SO}_{4}$, filtered and concentrated in vacuo to afford the crude methyl $N$-(hex-5-en-1yl)carbamate as a yellow oil which was used without further purification. $R_{f} 0.42$ (1:3, EtOAc:hexanes); ${ }^{1} \mathrm{H}$ NMR (400 MHz, $\mathrm{CDCl}_{3}$ ) $\delta 1.40$ (quin, $J=7.2 \mathrm{~Hz}, 2 \mathrm{H}$ ), 1.50 (quin, $J$ $=7.2 \mathrm{~Hz}, 2 \mathrm{H}), 2.05(\mathrm{q}, J=7.2 \mathrm{~Hz}, 2 \mathrm{H}), 3.15-3.16(\mathrm{~m}, 2 \mathrm{H}), 3.64(\mathrm{~s}, 3 \mathrm{H}), 4.68(\mathrm{br} \mathrm{s}, 1 \mathrm{H})$, 4.92-5.01 (m, 2H), 5.72-5.82 (m, 1H); ${ }^{13} \mathrm{C}$ NMR (100 MHz, $\left.\mathrm{CDCl}_{3}\right) \delta 26.2,29.7,33.5$, $41.2,52.1,114.9,138.6,157.3$.<smiles>C=CCCCCNC</smiles>

$N$-methyl-1-aminohex-5-ene. Lithium aluminum hydride (2.30 g, $60.7 \mathrm{mmol})$ was slowly added to a solution of crude $N$-(hex-5-en-1-yl)carbamate $(30.334 \mathrm{mmol})$ in dry $\mathrm{Et}_{2} \mathrm{O}(75 \mathrm{~mL})$ at $0{ }^{\circ} \mathrm{C}$, then the cooling bath was removed and the reaction was heated at reflux overnight. The reaction was then cooled to $0{ }^{\circ} \mathrm{C}$ and carfully quenched with aq. Rochelle's Salt $(50 \%, 40 \mathrm{~mL})$ and stirred for $0.5 \mathrm{~h}$. The layers were separated and the aqueous phase was extracted twice with $\mathrm{Et}_{2} \mathrm{O}$. The combined organic phases were 
washed with brine, dried over $\mathrm{Na}_{2} \mathrm{SO}_{4}$, filtered and the solvent was removed by distillation followed by distillation of the product to afford $N$-methyl-1-aminohex-5-ene (3.26 g ,95\%) as a colorless oil. $R_{f} 0.38\left(1: 9, \mathrm{MeOH}: \mathrm{CH}_{2} \mathrm{Cl}_{2}\right.$ with $\left.1 \% \mathrm{NH}_{4} \mathrm{OH}\right) ;{ }^{1} \mathrm{H}$ NMR (400 MHz, $\left.\mathrm{CDCl}_{3}\right) \delta 1.39-1.55(\mathrm{~m}, 4 \mathrm{H}), 2.05$ (q, $\left.J=6.8 \mathrm{~Hz}, 2 \mathrm{H}\right), 2.41(\mathrm{~s}, 3 \mathrm{H}), 2.56$ $(\mathrm{t}, J=6.6 \mathrm{~Hz}, 2 \mathrm{H}), 4.91-5.01(\mathrm{~m}, 2 \mathrm{H}), 5.72-5.84(\mathrm{~m}, 1 \mathrm{H}) ;{ }^{13} \mathrm{C} \mathrm{NMR}\left(100 \mathrm{MHz}, \mathrm{CDCl}_{3}\right) \delta$ $26.9,29.6,33.8,36.7,52.2,114.6,139.0$.<smiles>C=CCCCCN(C)C(=O)OCC[Se-]</smiles>

2-((tert-Butyldimethylsilyl)oxy)ethyl $\quad N$-(hex-5-en-1-yl)-N-methylcarbamate. The carbamate was synthesized as described by Heller, et.al. ${ }^{10}$ DBU $(2.13 \mathrm{~mL}, 14.3 \mathrm{mmol})$ was added to a solution of crude 2-((tert-butyldimethylsilyl)oxy)ethyl $1 H$-imidazole-1carboxylate $(14.3 \mathrm{mmol})$ in dry $\mathrm{MeCN}(70 \mathrm{~mL})$ and was stirred for $10 \mathrm{~min}$, then $\mathrm{N}$ methyl-1-aminohex-5-ene $(1.60 \mathrm{~g}, 14.3 \mathrm{mmol})$ was added and the reaction was stirred overnight. The reaction was then washed twice with sat. $\mathrm{NH}_{4} \mathrm{Cl}$ and the combined aqueous layers were extracted three times with EtOAc. The combined organic phases were washed with brine, dried over $\mathrm{Na}_{2} \mathrm{SO}_{4}$, filtered and concentrated in vacuo. The crude material was purified by column chromatography $\left(\mathrm{SiO}_{2}, 1: 3\right.$, EtOAc:hexanes) to give a colorless oil 2-((tert-Butyldimethylsilyl)oxy)ethyl $N$-(hex-5-en-1-yl)- $N$ methylcarbamate (3.56 g, 79\%). $\quad R_{f} 0.40$ (1:3, EtOAc:hexanes); ${ }^{1} \mathrm{H}$ NMR (400 MHz, $\left.\mathrm{CDCl}_{3}\right) \delta 0.06(\mathrm{~s}, 6 \mathrm{H}), 0.89(\mathrm{~s}, 9 \mathrm{H}), 1.38$ (quin, $J=7.4 \mathrm{~Hz}, 2 \mathrm{H}$ ), 1.52, (quin, $J=7.6 \mathrm{~Hz}$, 2H), 2.07 (q, $J=7.2 \mathrm{~Hz}, 2 \mathrm{H}), 2.88(\mathrm{~s}, 3 \mathrm{H}), 3.25$ (t, $J=7.2 \mathrm{~Hz}, 2 \mathrm{H}), 3.80$ (t, $J=5.0 \mathrm{~Hz}$, $2 \mathrm{H}), 4.13(\mathrm{t}, J=5.0 \mathrm{~Hz}, 2 \mathrm{H}), 4.93-5.02(\mathrm{~m}, 2 \mathrm{H}), 5.73-5.84(\mathrm{~m}, 1 \mathrm{H}) ;{ }^{13} \mathrm{C} \mathrm{NMR}(100 \mathrm{MHz}$, 
$\left.\mathrm{CDCl}_{3}\right) \delta$-5.1, 18.5, 26.1, 27.6, 33.6, 34.1, 49.0, 62.0, 66.8, 114.9, 138.7, 156.7; FT-ICRMS calcd for $\mathrm{C}_{16} \mathrm{H}_{34} \mathrm{NO}_{3} \mathrm{Si}^{+}[\mathrm{M}+\mathrm{H}]^{+} m / z$ 316.2302, found 316.2307.<smiles>C=CCCCCN(C)C(=O)OCCO</smiles>

2-Hydroxyethyl $N$-(hex-5-en-1-yl)- $N$-methylcarbamate. TBAF ( $1 M$ in THF, $10.6 \mathrm{~mL}$, $10.6 \mathrm{mmol})$ was added dropwise to a solution of 2-((tert-Butyldimethylsilyl)oxy)ethyl $\mathrm{N}$ (hex-5-en-1-yl)- $N$-methylcarbamate $(2.58 \mathrm{~g}, 8.17 \mathrm{mmol})$ in dry THF $(16 \mathrm{~mL})$ at $0{ }^{\circ} \mathrm{C}$ and was stirred overnight. The reaction solution was washed twice with sat. $\mathrm{NaHCO}_{3}$ and the combined aqueous layers were extracted three times with $\mathrm{Et}_{2} \mathrm{O}$. The combined organic phases were washed with brine, dried over $\mathrm{Na}_{2} \mathrm{SO}_{4}$, filtered and concentrated in vacuo to afford crude 2-hydroxyethyl $N$-(hex-5-en-1-yl)- $N$-methylcarbamate as a yellow oil and was used without further purification. $R_{f} 0.12$ (1:3, EtOAc:hexanes); ${ }^{1} \mathrm{H}$ NMR (400 $\mathrm{MHz}, \mathrm{CDCl}_{3}$ ) $\delta 1.40$ (quin, $J=7.8 \mathrm{~Hz}, 2 \mathrm{H}$ ), 1.55 (quin, $J=7.6 \mathrm{~Hz}, 2 \mathrm{H}$ ), 2.08 (q, $J=6.8$ Hz, 2H), 2.90 (s, 3H), 3.26 (t, $J=7.2 \mathrm{~Hz}, 2 \mathrm{H}), 3.78-3.82$ (q, $J=4.4 \mathrm{~Hz}, 2 \mathrm{H}), 4.23$ (t, $J=$ 4.4 Hz, 2H), 4.94-5.02 (m, 2H), 5.74-5.84 (m, 1H); $\left.{ }^{13} \mathrm{C} \mathrm{NMR} \mathrm{(100} \mathrm{MHz,} \mathrm{CDCl}_{3}\right) \delta$ 25.9, $27.4,33.6,34.7,48.9,62.6,67.7,115.0,138.6,157.4$.<smiles>C=CCCCCN(C)C(=O)OCCOC</smiles>

2-(Methanesulfonyloxy)ethyl $N$-(hex-5-en-yl)- $N$-methylcarbamate. Methanesulfonyl chloride (728 $\mathrm{mL}, 9.40 \mathrm{mmol}$ ) was added dropwise to a solution of the crude 2hydroxyethyl $N$-(hex-5-en-1-yl)- $N$-methylcarbamate $(8.17 \mathrm{mmol})$ and triethylamine $(1.72$ 
$\mathrm{mL}, 12.3 \mathrm{mmol})$ in $\mathrm{CH}_{2} \mathrm{Cl}_{2}(27 \mathrm{~mL})$ at $0{ }^{\circ} \mathrm{C}$ and stirred overnight. The reaction was then washed twice with sat. $\mathrm{NH}_{4} \mathrm{Cl}$ and the combined aqueous layers were extracted twice with $\mathrm{CH}_{2} \mathrm{Cl}_{2}$. The combined organic phases were washed with brine, dried over $\mathrm{Na}_{2} \mathrm{SO}_{4}$, filtered and concentrated in vacuo to give crude 2-(methanesulfonyloxy)ethyl $\mathrm{N}$-(hex-5en-yl)- $N$-methylcarbamate as a light yellow oil $(2.15 \mathrm{~g}) . \quad R_{f} 0.36$ (major product) \& 0.68 (1:1, EtOAc:hexanes); ${ }^{1} \mathrm{H}$ NMR (400 MHz, $\left.\mathrm{CDCl}_{3}, R_{f} 0.36\right) \delta 1.37$ (quin, $J=7.6 \mathrm{~Hz}$, 2H), 1.54 (quin, $J=7.6 \mathrm{~Hz}, 2 \mathrm{H}), 2.07$ (q, $J=7.2 \mathrm{~Hz}, 2 \mathrm{H}), 2.89(\mathrm{~s}, 3 \mathrm{H}), 3.01(\mathrm{~s}, 3 \mathrm{H}), 3.26$ $(\mathrm{t}, J=7.2 \mathrm{~Hz}, 2 \mathrm{H}), 4.32-4.34(\mathrm{~m}, 2 \mathrm{H}), 4.39-4.41(\mathrm{~m}, 2 \mathrm{H}), 4.93-5.02(\mathrm{~m}, 2 \mathrm{H}), 5.74-5.81$ $(\mathrm{m}, 1 \mathrm{H}) ;{ }^{13} \mathrm{C} \mathrm{NMR}\left(100 \mathrm{MHz}, \mathrm{CDCl}_{3}, R_{f} 0.36\right) \delta$ 26.1, 27.0, 33.5, 34.1, 37.9, 49.2, 62.8, $68.1,114.9,138.6,155.9$.<smiles>C=CCCCCN(C)C(=O)OCCON1C(=O)c2ccccc2C1=O</smiles>

2-((1,3-Dioxo-2,3-dihydro-1H-isoindol-2-yl)oxy)ethyl $N-($ hex-5-en-1-yl)- $N-$ methylcarbamate. $\mathrm{K}_{2} \mathrm{CO}_{3}(1.17 \mathrm{~g}, 8.47 \mathrm{mmol})$ was added to a solution of the combined 2-(methanesulfonyloxy)ethyl $N$-(hex-5-en-yl)- $N$-methylcarbamate products $\left(R_{f} 0.36 \&\right.$ 0.68) (2.15 g, $7.70 \mathrm{mmol})$ in DMSO $(26 \mathrm{~mL})$, followed by the addition of $N$ hydroxyphthalimide $(2.39 \mathrm{~g}, 14.6 \mathrm{mmol})$ and the dark red solution was heated at $75{ }^{\circ} \mathrm{C}$ overnight. The yellow solution was cooled to $\mathrm{rt}$ and slowly quenched with water and extracted four times with EtOAc. The combined organic phase was washed three times with water, once with brine, dried over $\mathrm{Na}_{2} \mathrm{SO}_{4}$, filtered and concentrated in vacuo. The crude material was purified by column chromatography $\left(\mathrm{SiO}_{2}, 1: 1\right.$, EtOAc:hexanes) to give 2-((1,3-Dioxo-2,3-dihydro-1H-isoindol-2-yl)oxy)ethyl $\quad N$-(hex-5-en-1-yl)- $N$ - 
methylcarbamate $(2.02 \mathrm{~g}, 71 \%$ over 3 steps $)$ as a yellow oil. $R_{f} 0.55$ (1:1, EtOAc:hexanes); ${ }^{1} \mathrm{H} \mathrm{NMR}\left(400 \mathrm{MHz}, \mathrm{CDCl}_{3}\right.$ ) $\delta 1.35$ (quin, $J=7.6 \mathrm{~Hz}, 2 \mathrm{H}$ ), 1.52 (quin, $J$ $=7.6 \mathrm{~Hz}, 2 \mathrm{H}), 2.05(\mathrm{q}, J=7.2 \mathrm{~Hz}, 2 \mathrm{H}), 2.85(\mathrm{br} \mathrm{s}, 3 \mathrm{H}), 3.24(\mathrm{t}, J=7.2 \mathrm{~Hz}, 2 \mathrm{H}), 4.41(\mathrm{~s}$, $4 \mathrm{H}), 4.91-5.00(\mathrm{~m}, 2 \mathrm{H}), 5.72-5.82(\mathrm{~m}, 1 \mathrm{H}), 7.72-7.75(\mathrm{~m}, 2 \mathrm{H}), 7.80-7.82(\mathrm{~m}, 2 \mathrm{H}) ;{ }^{13} \mathrm{C}$ NMR (100 MHz, $\left.\mathrm{CDCl}_{3}\right) \delta$ 26.1, 27.2, 33.5, 34.6, 49.1, 62.8, 76.7, 114.8, 123.7, 129.2, 134.7, 138.7, 156.1, 163.5; FT-ICR-MS calcd for $\mathrm{C}_{18} \mathrm{H}_{23} \mathrm{~N}_{2} \mathrm{O}_{5}^{+}[\mathrm{M}+\mathrm{H}]^{+} m / z$ 347.1601, found 347.1606 .

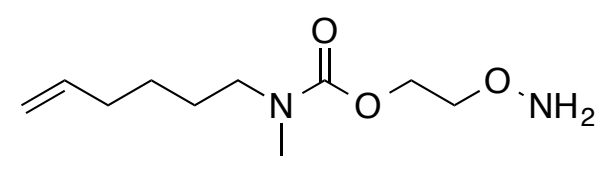

23

2-(Aminooxy)ethyl $\quad N$-(hex-5-en-1-yl)- $N$-methylcarbamate (23). $\quad$ Hydrazine monohydrate (34 $\mathrm{mL}, 0.70 \mathrm{mmol})$ was added to a solution of 2-((1,3-Dioxo-2,3-dihydro$1 H$-isoindol-2-yl)oxy)ethyl $N$-(hex-5-en-1-yl)- $N$-methylcarbamate (258 mg, $0.74 \mathrm{mmol}$ ) in $\mathrm{CH}_{2} \mathrm{Cl}_{2}(4 \mathrm{~mL})$ at $0{ }^{\circ} \mathrm{C}$ and was stirred for $1 \mathrm{~h}$. The solids were then filtered off and the filter cake was washed with ample $\mathrm{CH}_{2} \mathrm{Cl}_{2}$. The filtrate was then concentrated in vacuo to afford $23(154 \mathrm{mg}, 96 \%)$ as a colorless oil without further purification. $R_{f} 0.24$ (1:1, EtOAc:hexanes); ${ }^{1} \mathrm{H}$ NMR (400 MHz, $\mathrm{CDCl}_{3}$ ) $\delta 1.36$ (quin, $J=7.6 \mathrm{~Hz}, 2 \mathrm{H}$ ), 1.52 (quin, $J=7.6 \mathrm{~Hz}, 2 \mathrm{H}), 2.05$ (q, $J=7.2 \mathrm{~Hz}, 2 \mathrm{H}), 2.86(\mathrm{~s}, 3 \mathrm{H}), 3.24(\mathrm{t}, J=7.2 \mathrm{~Hz}, 2 \mathrm{H}$ ), $3.80(\mathrm{t}, J=3.6 \mathrm{~Hz}, 2 \mathrm{H}), 4.26(\mathrm{t}, J=4.4 \mathrm{~Hz}, 2 \mathrm{H}), 4.91-5.00(\mathrm{~m}, 2 \mathrm{H}), 5.42(\mathrm{br} \mathrm{s}, 2 \mathrm{H}), 5.71-$ $5.82(\mathrm{~m}, 1 \mathrm{H}) ;{ }^{13} \mathrm{C} \mathrm{NMR}\left(100 \mathrm{MHz}, \mathrm{CDCl}_{3}\right) \delta 26.0,27.3,33.5,34.6,48.8,62.9,74.3$, 114.8, 138.7, 156.7; FT-ICR-MS calcd for $\mathrm{C}_{10} \mathrm{H}_{21} \mathrm{~N}_{2} \mathrm{O}_{3}{ }^{+}[\mathrm{M}+\mathrm{H}]^{+} \mathrm{m} / \mathrm{z}$ 217.1547, found 217.1548. 


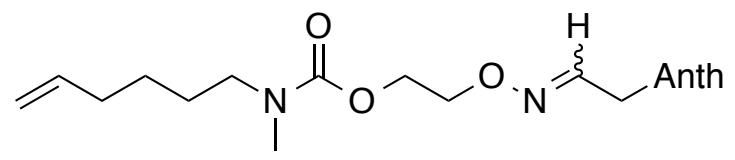

26

2-(((E/Z)-(2-(Anthracen-9-yl)ethylidene)amino)oxy)ethyl

$N-($ hex-5-en-1-yl)-Nmethylcarbamate (26). 2-(Anthracen-9-yl)acetaldehyde ${ }^{11}(175 \mathrm{mg}, 0.79 \mathrm{mmol})$ was added to a solution of $23(156 \mathrm{mg}, 0.72 \mathrm{mmol})$ in $\mathrm{CH}_{2} \mathrm{Cl}_{2}(4 \mathrm{~mL})$ and stirred for $1 \mathrm{~h}$. The reaction solution was concentrated in vacuo. The crude material was purified by column chromatography $\left(\mathrm{SiO}_{2}\right.$, 0:1 to 3:17, EtOAc: $\mathrm{CH}_{2} \mathrm{Cl}_{2}$ gradient) to give $\mathbf{2 6}(291 \mathrm{mg}, 96 \%)$ as a yellow oil. $R_{f} 0.55$ (1:19, EtOAc: $\left.\mathrm{CH}_{2} \mathrm{Cl}_{2}\right) ;{ }^{1} \mathrm{H}$ NMR $\left(400 \mathrm{MHz}, \mathrm{CDCl}_{3}\right) \delta 0.78$ (br s, $0.25 \mathrm{H}), 0.87$ (br s, 0.25H), 1.26 (br s, 1.44H), 1.35-1.41 (m, 2H), 1.55-1.63 (m, 2H), 1.92-2.07 (m, 2H), 2.78 (br s, 0.68H), 2.95 (s, 1.91H), 3.14 (br s, 0.46H), 3.22 (br s, $0.46 \mathrm{H}), 3.30-3.34(\mathrm{~m}, 1.33 \mathrm{H}), 4.26(\mathrm{dd}, J=16.8,5.4 \mathrm{~Hz}, 1.78 \mathrm{H}), 4.49-4.52(\mathrm{~m}, 3.48 \mathrm{H})$, $4.68(\mathrm{~d}, J=4.4 \mathrm{~Hz}, 1.31 \mathrm{H}), 4.88-5.02(\mathrm{~m}, 2 \mathrm{H}), 5.69-5.79(\mathrm{~m}, 1 \mathrm{H}), 6.74(\mathrm{t}, J=5.0 \mathrm{~Hz}$, 0.60H), 7.47-7.57 (m, 4.48H), 8.01-8.05 (m, 2H), 8.19 (d, $J=8.4 \mathrm{~Hz}, 1 \mathrm{H}), 8.30$ (d, $J=$ $8.8 \mathrm{~Hz}, 1 \mathrm{H}), 8.43(\mathrm{~s}, 1 \mathrm{H}) ;{ }^{13} \mathrm{C} \mathrm{NMR}\left(100 \mathrm{MHz}, \mathrm{CDCl}_{3}\right) \delta 25.3,26.6,28.7,33.6,34.8$, $48.8,64.1,72.9,114.9,124.2,125.3,126.3,127.1,128.7,129.4,130.3,131.7,138.7$, 150.3, 156.6; FT-ICR-MS calcd for $\mathrm{C}_{26} \mathrm{H}_{31} \mathrm{~N}_{2} \mathrm{O}_{3}{ }^{+}[\mathrm{M}+\mathrm{H}]^{+} \mathrm{m} / z$ 419.2329, found 419.2333.<smiles>[Y]CCOC(=O)N(C)CCCCCC[SiH2]CC</smiles>

$\mathrm{Y}=\mathrm{ONH}_{2}$ or $\mathrm{ON}=\mathrm{CHCH}_{2} \mathrm{Ar}$

2-(Aminooxy)ethyl $N$-methyl- $N$-(6-(triethoxysilyl)hexyl)carbamate or 2-(((E/Z)-(2(anthracen-9-yl)ethylidene)amino)oxy)ethyl $\quad N$-methyl- $N$-(6-(triethoxysilyl)hexyl) carbamate. Following a procedure outlined by Sabourault et al., ${ }^{12}$ catalytic $\mathrm{PtO}_{2}$ and 
triethoxysilane (131 $\mathrm{mL}, 0.71 \mathrm{mmol})$ was added to 23 or $26(153 \mathrm{mg}, 0.71 \mathrm{mmol})$ in a pressure tube. The tube was flushed with $\mathrm{N}_{2}$, sealed and heated to $90{ }^{\circ} \mathrm{C}$ for $48 \mathrm{~h}$. The reaction solution was cooled to rt and filtered through Celite into a flask flushed with $\mathrm{N}_{2}$ and the filter cake was washed with ample dry $\mathrm{CH}_{2} \mathrm{Cl}_{2}$. The filtrate was concentrated in vacuo to afford the crude material as a light brown oil which was used without further characterization or purification due to its sensitivity to moisture.

\subsubsection{SQUID Measurements}

The magnetic properties of the magnetic NPs were measured using a Quantum Design MPMS-5S superconducting quantum interference device (SQUID) magnetometer (in the temperature range from 2 to $400 \mathrm{~K}$ ) courtesy of Professor Lance DeLong (University of Kentucky). The samples were loaded in a gel capsule and secured inside a standard drinking straw with small holes punched in both the straw and capsule for equalization of pressure and temperature. $\mathrm{M}(\mathrm{H})$ data was taken by starting the sample at saturation, 5 $\mathrm{T}$, and then cycling to $-5 \mathrm{~T}$ and back to $5 \mathrm{~T}$ in different step ranges to see the details of the hysteresis. The data was taken using DC magnetization to disturb the magnetic NPs as little as possible during the measurement.

\subsubsection{Synthesis of $\mathrm{AO} @ \mathrm{SiO}_{2} @ \mathrm{Fe}_{3} \mathrm{O}_{4}$ and $\mathrm{FL} @ \mathrm{SiO}_{2} @ \mathrm{Fe}_{3} \mathrm{O}_{4} \mathrm{NPs}$}

The suspension of $\mathrm{SiO}_{2} @ \mathrm{Fe}_{3} \mathrm{O}_{4} \mathrm{NPs}$ was placed under mild vacuum and heated to $60{ }^{\circ} \mathrm{C}$ for $8 \mathrm{~h}$. This process was used to remove the catalytic ammonia thus increasing the chances of obtaining a thin organic monolayer on the surface of the NPs. The removal of the ammonia decreased the $\mathrm{pH}$ of the solution from 11.7 to 8.8. Then, $\mathrm{EtOH}(5 \mathrm{~mL})$ was 
added to 2-(aminooxy)ethyl $N$-methyl- $N$-(6-(triethoxysilyl)hexyl)carbamate or 2-(((E/Z)(2-(anthracen-9-yl)ethylidene)amino)oxy)ethyl $\quad N$-methyl- $N$-(6-(triethoxysilyl)hexyl) carbamate $(1.454 \mathrm{mmol})$ and the solution was added to the NP suspension with rapid mechanical stirring. The mixture was stirred for $20 \mathrm{~h}$. The majority of the $\mathrm{AO} @ \mathrm{SiO}_{2} @ \mathrm{Fe}_{3} \mathrm{O}_{4}$ or $\mathrm{FL} @ \mathrm{SiO}_{2} @ \mathrm{Fe}_{3} \mathrm{O}_{4} \mathrm{NPs}$ were then magnetically separated and the remaining colloidal supernatant was centrifuged at 13,200 RPM for 20 min. The NPs were then washed $5 \mathrm{x}$ with EtOH with magnetic separation and centrifugation after each wash. The excess EtOH was then removed by rotary evaporation and the NPs were dried under vacuum for $3 \mathrm{~h}$. The resulting NPs were characterized by IR, TGA, DLS, $\zeta$ potential and TEM.

\subsubsection{AMF-Induced Heating of Bulk Solution}

Temperature measurements were taken with a Neoptix Nomad fitted with a fiber optic temperature sensor. All data was recorded using Neoptix NeoLink software. AMF conditions were set to 501.6 amps and a frequency of $204 \mathrm{kHz}$. Each AMF pulse lasted for 2 or $5 \mathrm{~min}$ followed by 2 or $5 \mathrm{~min}$ of without an AMF for a combined total of $40 \mathrm{~min}$

of AMF exposure. All samples were started at room temperature and were allowed to cool at room temperature; all heating was induced using only an AMF. The control was $0.75 \mathrm{~mL}$ of 2:1 PBS:acetonitrile without $\mathrm{SiO}_{2} @ \mathrm{Fe}_{3} \mathrm{O}_{4} \mathrm{NPs}$, which gave a $\Delta \mathrm{T}$ of $4.8 \pm 0.1$ ${ }^{\circ} \mathrm{C}$ with a 5 min AMF pulse. The $\Delta \mathrm{T}$ of a 5 min AMF pulse with $7.0 \mathrm{mg}$ of $\mathrm{SiO}_{2} @ \mathrm{Fe}_{3} \mathrm{O}_{4}$ NPs in $0.75 \mathrm{~mL} 2: 1$ PBS:acetonitrile was $+16.4 \pm 0.3{ }^{\circ} \mathrm{C}$. The $\Delta \mathrm{T}$ from the start of the first AMF pulse to the start of the sixth AMF pulse was $+4{ }^{\circ} \mathrm{C}$. 


\subsubsection{Assay of Percent 27 Released from NPs}

To determine the percent of 27 released from FL@ $\mathrm{SiO}_{2} @ \mathrm{Fe}_{3} \mathrm{O}_{4} \mathrm{NPs}$, it is necessary to determine the residual amount of 2-(((E/Z)-(2-(anthracen-9yl)ethylidene)amino)oxy)ethyl $N$-methyl- $N$-(6-(triethoxysilyl)hexyl)carbamate remaining on the FL@SiO $\mathrm{SiO}_{2} @ \mathrm{Fe}_{3} \mathrm{O}_{4}$ NPs post AMF exposure. After recording the fluorescence intensity of the last (eighth) AMF pulse, the NPs were magnetically separated and the AMF supernatant was removed. The isolated NPs then were washed $5 \mathrm{x}$ with $\mathrm{MeCN}$ followed by magnetic separation to remove any hydrogen bound 27. To the washed NPs was added $1 \mathrm{~mL}$ of $5 \% \mathrm{HF}$ in EtOH. The suspension was stirred until all NPs had dissolved resulting in a light yellow solution. The acidic solution was added to a separatory funnel and then basified using sat. aq. $\mathrm{NaHCO}_{3}$. The basic solution was extracted $3 \mathrm{x}$ with $\mathrm{Et}_{2} \mathrm{O}$ and the combined extracts were concentrated by rotary evaporation and dried under vacuum. The resulting residue was dissolved in a 2:1 mixture of PBS:MeCN $(0.75 \mathrm{~mL})$ at $\mathrm{pH} 7.4$ and the fluorescence was measured. This measured fluorescence intensity was then added to the total fluorescence intensity measured in the supernatant after the final AMF pulse, and the combined intensity was set to $100 \%$. The results from all three experiments were normalized by dividing the fluorescent intensity by the milligrams of $\mathrm{FL} @ \mathrm{SiO}_{2} @ \mathrm{Fe}_{3} \mathrm{O}_{4}$ NPs used for the experiment. 


\subsubsection{Synthesis of Thiol Linker for Loading onto $\mathrm{Au} @ \mathrm{Fe}_{3} \mathrm{O}_{4} \mathrm{NPs}$}

$\mathrm{HO} \curvearrowright$ OTBS

4-((tert-Butyldimethylsilyl)oxy)butan-1-ol. A solution of $\mathrm{TBSCl}(5.20 \mathrm{~g}, 34.5 \mathrm{mmol})$ in dry $\mathrm{CH}_{2} \mathrm{Cl}_{2}(60 \mathrm{~mL})$ was added dropwise over $1 \mathrm{~h}$ to a solution of 1,4-butanediol (14.7 $\mathrm{mL}, 166 \mathrm{mmol})$ and $\mathrm{Et}_{3} \mathrm{~N}(6.99 \mathrm{~mL}, 49.8 \mathrm{mmol})$ in dry $\mathrm{CH}_{2} \mathrm{Cl}_{2}(50 \mathrm{~mL})$ at $0{ }^{\circ} \mathrm{C}$ and was stirred overnight. The solvent was removed in vacuo and the remaining oil was extracted with hexanes $(4 \mathrm{x})$ and the combined extractions were washed twice with sat. $\mathrm{NH}_{4} \mathrm{Cl}$, once with brine and was dried over $\mathrm{Na}_{2} \mathrm{SO}_{4}$, filtered and concentrated in vacuo to afford crude 4-((tert-butyldimethylsilyl)oxy)butan-1-ol (6.58 g, 93\%) as a colorless oil and was used without further purification. $R_{f} 0.44$ (1:3, EtOAc:hexanes).<smiles>COCCCCO[Sb]</smiles>

4-((tert-Butyldimethylsilyl)oxy)butyl methanesulfonate. Methanesulfonyl chloride (2.87 mL, $37.0 \mathrm{mmol})$ was added to a solution of crude 4-((tertbutyldimethylsilyl)oxy)butan-1-ol (6.58 g, $32.2 \mathrm{mmol})$ and $\mathrm{Et}_{3} \mathrm{~N}(6.79 \mathrm{~mL}, 48.3 \mathrm{mmol})$ in dry $\mathrm{CH}_{2} \mathrm{Cl}_{2}(107 \mathrm{~mL})$ at $0{ }^{\circ} \mathrm{C}$ and was stirred for $2 \mathrm{~h}$. The reaction solution was washed twice with sat. $\mathrm{NH}_{4} \mathrm{Cl}$ and the combined aqueous layers were extracted twice with $\mathrm{CH}_{2} \mathrm{Cl}_{2}$. The combined organic phases were washed once with brine and was dried over $\mathrm{Na}_{2} \mathrm{SO}_{4}$, filtered and concentrated in vacuo to afford 4-((tert-butyldimethylsilyl)oxy)butyl methanesulfonate $(9.03 \mathrm{~g}, 99 \%)$ as an orange oil and was used without further purification. $R_{f} 0.52$ (1:3, EtOAc:hexanes); ${ }^{1} \mathrm{H}$ NMR $\left(400 \mathrm{MHz}, \mathrm{CDCl}_{3}\right) \delta 0.04(\mathrm{~s}, 6 \mathrm{H})$, $0.88(\mathrm{~s}, 9 \mathrm{H}), 1.62$ (quin, $J=6.0 \mathrm{~Hz}, 2 \mathrm{H}), 1.83$ (quin, $J=7.2 \mathrm{~Hz}, 2 \mathrm{H}$ ), $2.99(\mathrm{~s}, 3 \mathrm{H}), 3.64$ 
$(\mathrm{t}, J=6.0 \mathrm{~Hz}, 2 \mathrm{H}), 4.26(\mathrm{t}, J=6.6 \mathrm{~Hz}, 2 \mathrm{H}) ;{ }^{13} \mathrm{C} \mathrm{NMR}\left(100 \mathrm{MHz}, \mathrm{CDCl}_{3}\right) \delta-5.2,18.5$, $26.1,28.8,37.6,62.4,70.3$.

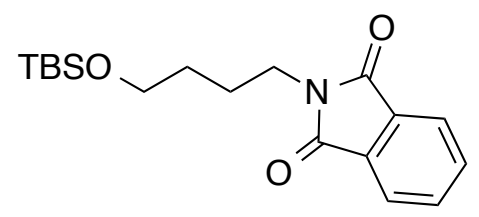

\section{2-(4-((tert-Butyldimethylsilyl)oxy)butyl)-2,3-dihydro-1H-isoindole-1,3-dione.}

Phthalimide (8.55 $\mathrm{g}, \quad 58.1 \mathrm{mmol})$ was added to a solution of 4-((tertbutyldimethylsilyl)oxy)butyl methanesulfonate $(8.64 \mathrm{~g}, 30.6 \mathrm{mmol})$ and $\mathrm{K}_{2} \mathrm{CO}_{3}(5.07 \mathrm{~g}$, $36.7 \mathrm{mmol})$ in DMSO $(180 \mathrm{~mL})$ and the solution was heated to $75^{\circ} \mathrm{C}$ for $17 \mathrm{~h}$. The reaction was then cooled to rt and was quenched with water. The aqueous solution was extracted with EtOAc $(4 \mathrm{x})$ and the combined organic phases were washed with water (3x). The organic phase was then washed with brine, dried over $\mathrm{Na}_{2} \mathrm{SO}_{4}$, filtered and concentrated in vacuo to afford 2-(4-((tert-butyldimethylsilyl)oxy)butyl)-2,3-dihydro- $1 \mathrm{H}$ isoindole-1,3-dione $(9.27 \mathrm{~g}, 91 \%)$ as white crystals and was used without further purification. $R_{f} 0.59$ (1:3, EtOAc:hexanes); ${ }^{1} \mathrm{H}$ NMR $\left(400 \mathrm{MHz}, \mathrm{CDCl}_{3}\right) \delta 0.03(\mathrm{~s}, 6 \mathrm{H})$, 0.87 (s, 9H), 1.55 (quin, $J=6.4 \mathrm{~Hz}, 2 \mathrm{H}$ ), 1.74 (quin, $J=7.4 \mathrm{~Hz}, 2 \mathrm{H}$ ), 3.63 (t, $J=6.4 \mathrm{~Hz}$, 2H), $3.70(\mathrm{t}, J=7.2 \mathrm{~Hz}, 2 \mathrm{H}), 7.65(\mathrm{dd}, J=2.4,2.8 \mathrm{~Hz}, 2 \mathrm{H}), 7.83(\mathrm{dd}, J=1.6,3.4 \mathrm{~Hz}$, $2 \mathrm{H}) ;{ }^{13} \mathrm{C} \mathrm{NMR}\left(100 \mathrm{MHz}, \mathrm{CDCl}_{3}\right) \delta-5.1,18.5,25.3,26.1,30.2,38.1,62.7,123.4,132.4$, 134.0, 168.6. 
TBSO $\mathrm{NH}_{2}$

(4-Aminobutoxy)(tert-butyl)dimethylsilane. Hydrazine monohydrate $(6.74 \mathrm{~mL}, 139$ mmol) was added to a solution of crude 2-(4-((tert-butyldimethylsilyl)oxy)butyl)-2,3dihydro- $1 \mathrm{H}$-isoindole-1,3-dione $(9.27 \mathrm{~g}, 27.8 \mathrm{mmol})$ in $\mathrm{CH}_{2} \mathrm{Cl}_{2}(140 \mathrm{~mL})$ at $0{ }^{\circ} \mathrm{C}$ and was stirred overnight, allowing the reaction to come to rt. The white precipitate was filtered and the filter cake was washed with ample $\mathrm{CH}_{2} \mathrm{Cl}_{2}$. The crude solution was concentrated in vacuo to afford (4-aminobutoxy)(tert-butyl)dimethylsilane (3.99 g, 71\%) as a light yellow oil and was used without further purification. $R_{f} 0.26\left(1: 9, \mathrm{MeOH}: \mathrm{CH}_{2} \mathrm{Cl}_{2}\right.$ with $\left.1 \% \mathrm{NH}_{4} \mathrm{OH}\right) ;{ }^{1} \mathrm{H}$ NMR (400 MHz, $\left.\mathrm{CDCl}_{3}\right) \delta 0.03(\mathrm{~s}, 6 \mathrm{H}), 0.87$ (s, 9H), 1.44-1.55 (m, 4H), $1.61($ br s, $2 \mathrm{H}), 2.68(\mathrm{t}, J=6.8 \mathrm{~Hz}, 2 \mathrm{H}), 3.60(\mathrm{t}, J=6.2 \mathrm{~Hz}) ;{ }^{13} \mathrm{C} \mathrm{NMR}(100 \mathrm{MHz}$, $\left.\mathrm{CDCl}_{3}\right) \delta-5.1,18.5,26.1,30.4,30.5,42.3,63.3$.<smiles>BrCCCSC(c1ccccc1)(c1ccccc1)c1ccccc1</smiles>

(((3-Bromopropyl)sulfanyl)diphenylmethyl)benzene. $\mathrm{K}_{2} \mathrm{CO}_{3}(2.28 \mathrm{~g}, 16.5 \mathrm{mmol})$ followed by 1,3-dibromopropane $(7.61 \mathrm{~mL}, 75 \mathrm{mmol})$ was added to a solution of triphenylmethanethiol $(4.22 \mathrm{~g}, 15.3 \mathrm{mmol})$ in dry THF $(75 \mathrm{~mL})$ under $\mathrm{N}_{2}$. The reaction was refluxed for $24 \mathrm{~h}$ before cooling to rt. The reaction solution was washed twice with water, extracted twice with $\mathrm{Et}_{2} \mathrm{O}$, washed with brine, dried over $\mathrm{Na}_{2} \mathrm{SO}_{4}$, filtered and concentrated in vacuo. The excess 1,3-dibromopropane was distilled off to afford (((3bromopropyl)sulfanyl)diphenylmethyl)benzene $(5.94 \mathrm{~g}, 98 \%)$ as white crystals. $R_{f} 0.54$ (1:3, $\mathrm{CH}_{2} \mathrm{Cl}_{2}$ :hexanes); ${ }^{1} \mathrm{H} \mathrm{NMR}\left(400 \mathrm{MHz}, \mathrm{CDCl}_{3}\right) \delta 1.81$ (quin, $J=6.8 \mathrm{~Hz}, 2 \mathrm{H}$ ), 2.32 
(t, $J=6.8 \mathrm{~Hz}, 2 \mathrm{H}), 3.32(\mathrm{t}, J=6.8 \mathrm{~Hz}, 2 \mathrm{H}), 7.19-7.30(\mathrm{~m}, 9 \mathrm{H}), 7.40-7.44(\mathrm{~m}, 6 \mathrm{H}) ;{ }^{13} \mathrm{C}$ NMR (100 MHz, $\left.\mathrm{CDCl}_{3}\right) \delta$ 30.5, 31.8, 32.5, 66.9, 126.9, 128.1, 129.8, 144.9.<smiles>[18OH]CCCCNCCC[AsH3]</smiles>

\section{2,12,13,13-Tetramethyl-1,1,1-triphenyl-11-oxa-2-thia-6-aza-12-silatetradecane.} (((3-bromopropyl)sulfanyl)diphenylmethyl)benzene $(800 \mathrm{mg}, 2.01 \mathrm{mmol})$ was added to a solution of (4-aminobutoxy)(tert-butyl)dimethylsilane (1.02 g, $5.03 \mathrm{mmol})$ in $\mathrm{MeCN}$ (20 $\mathrm{mL}$ ) and the reaction was heated to $55^{\circ} \mathrm{C}$ for $24 \mathrm{~h}$. After cooling to rt, the reaction was quenched with sat. $\mathrm{NaHCO}_{3}$ and extracted three times with EtOAc. The combined organic phases were washed with brine, dried over $\mathrm{Na}_{2} \mathrm{SO}_{4}$, filtered and concentrated in vacuo. The crude material was purified by column chromatography $\left(\mathrm{SiO}_{2}, 0: 1\right.$ to $1: 4$, $\mathrm{MeOH}: \mathrm{CH}_{2} \mathrm{Cl}_{2}$ with $1 \% \mathrm{NH}_{4} \mathrm{OH}$ gradient) to give 12,12,13,13-tetramethyl-1,1,1triphenyl-11-oxa-2-thia-6-aza-12-silatetradecane $(852 \mathrm{mg}, 82 \%)$ as an orange oil. $R_{f} 0.33$ (1:4, MeOH: $\mathrm{CH}_{2} \mathrm{Cl}_{2}$ with $\left.1 \% \mathrm{NH}_{4} \mathrm{OH}\right) ;{ }^{1} \mathrm{H}$ NMR $\left(400 \mathrm{MHz}, \mathrm{CDCl}_{3}\right) \delta 0.04(\mathrm{~s}, 6 \mathrm{H}), 0.88$ (s, 9H), 1.51-1.52 (m, 4H), 1.60 (quin, $J=7.4 \mathrm{~Hz}, 2 \mathrm{H}), 2.17$ (t, $J=7.0 \mathrm{~Hz}, 2 \mathrm{H}$ ), 2.57 (t, $J$ $=7.2 \mathrm{~Hz}, 4 \mathrm{H}), 3.59(\mathrm{t}, J=6.0 \mathrm{~Hz}, 2 \mathrm{H}), 7.17-7.28(\mathrm{~m}, 9 \mathrm{H}), 7.39-7.41(\mathrm{~m}, 6 \mathrm{H}) ;{ }^{13} \mathrm{C} \mathrm{NMR}$ $\left(100 \mathrm{MHz}, \mathrm{CDCl}_{3}\right) \delta$-5.1, 18.6, 25.9, 26.2, 28.5, 29.9, 30.7, 48.6, 49.5, 63.2, 66.8, 126.8, $128.1,129.8,145.1$.

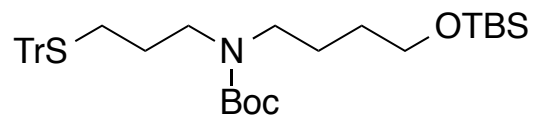

tert-Butyl $\quad N$-(4((tert-butyldimethylsiyl)oxy)butyl)- $N$-(3-((triphenylmethyl)sufanyl) propyl)carbamate. $\mathrm{Boc}_{2} \mathrm{O}(348 \mathrm{mg}, 1.60 \mathrm{mmol})$ was added to a solution of $12,12,13,13$ - 
tetramethyl-1,1,1-triphenyl-11-oxa-2-thia-6-aza-12-silatetradecane (754 mg, $1.45 \mathrm{mmol}$ ) and $\mathrm{Et}_{3} \mathrm{~N}(224 \mu \mathrm{L}, 1.60 \mathrm{mmol})$ in $\mathrm{CH}_{2} \mathrm{Cl}_{2}(5 \mathrm{~mL})$ at $0{ }^{\circ} \mathrm{C}$. After $3 \mathrm{~h}$, the reaction solution was washed twice with sat. $\mathrm{NH}_{4} \mathrm{Cl}$ and the aqueous phase was extracted twice with $\mathrm{CH}_{2} \mathrm{Cl}_{2}$. The combined organic phases were washed with brine, dried over $\mathrm{Na}_{2} \mathrm{SO}_{4}$, filtered and concentrated in vacuo to afford crude tert-butyl $N-(4(($ tertbutyldimethylsiyl)oxy)butyl)- $N$-(3-((triphenylmethyl)sufanyl)propyl)carbamate as an orange oil and was used without further purification. $R_{f} 0.81$ (1:19, EtOAc: $\left.\mathrm{CH}_{2} \mathrm{Cl}_{2}\right) ;{ }^{1} \mathrm{H}$ NMR (400 MHz, $\left.\mathrm{CDCl}_{3}\right) \delta 0.03$ (s, 6H), 0.88 (s, 9H), 1.39 (br s, 4H), 1.45 (br s, 2H), $1.52(\mathrm{~s}, 9 \mathrm{H}), 2.13(\mathrm{t}, J=7.4 \mathrm{~Hz}, 2 \mathrm{H}), 3.05(\mathrm{br} \mathrm{s}, 4 \mathrm{H}), 3.58(\mathrm{t}, J=6.0 \mathrm{~Hz}, 2 \mathrm{H}), 7.18-7.29$ (m, 9H), 7.39-7.41 (m, 6H); ${ }^{13} \mathrm{C}$ NMR $\left(100 \mathrm{MHz}, \mathrm{CDCl}_{3}\right) \delta-5.1,18.5,25.9,26.2$, 27.6, 28.6, 29.6, 30.3, 46.5, 47.1, 63.1, 66.8, 79.3, 126.8, 128.1, 129.8, 145.1, 155.6.

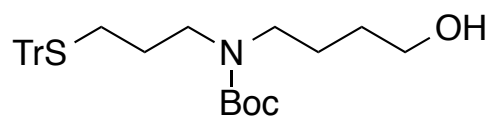

tert-Butyl $\quad N$-(4-hydroxybutyl)- $N$-(3-((triphenylmethyl)sulfanyl)propyl)carbamate. TBAF (1.89 $\mathrm{mL}$ of $1 M$ solution in THF, $1.89 \mathrm{mmol}$ ) was added to a solution of crude tert-butyl $\quad N$-(4((tert-butyldimethylsiyl)oxy)butyl)-N-(3-((triphenylmethyl)sufanyl) propyl)carbamate $(1.45 \mathrm{mmol})$ in dry $\mathrm{THF}(3 \mathrm{~mL})$ at $0{ }^{\circ} \mathrm{C}$ and the reaction was stirred overnight, allowing the reaction to warm to rt. Upon completion, the reaction was washed twice with sat. $\mathrm{NaHCO}_{3}$ and the combined aqueous phases were extracted three times with $\mathrm{Et}_{2} \mathrm{O}$. The combined organic layers were washed with brine, dried over $\mathrm{Na}_{2} \mathrm{SO}_{4}$, filtered and concentrated in vacuo. The crude material was purified by column chromatography $\left(\mathrm{SiO}_{2}, 3: 7, \mathrm{EtOAc}: \mathrm{CH}_{2} \mathrm{Cl}_{2}\right)$ to give tert-butyl $N$-(4-hydroxybutyl)- $N$-(3- 
((triphenylmethyl)sulfanyl)propyl)carbamate $(453 \mathrm{mg}, 62 \%$ over 2 steps) as a light yellow oil. $R_{f} 0.52$ (3:7, EtOAc: $\left.\mathrm{CH}_{2} \mathrm{Cl}_{2}\right) ;{ }^{1} \mathrm{H}$ NMR $\left(400 \mathrm{MHz}, \mathrm{CDCl}_{3}\right) \delta 1.40(\mathrm{~s}, 9 \mathrm{H})$, 1.47-1.51 (m, 4H), 1.58 (quin, $J=7.6 \mathrm{~Hz}, 2 \mathrm{H}), 3.08(\mathrm{br} \mathrm{s}, 4 \mathrm{H}), 3.63(\mathrm{t}, J=6.0 \mathrm{~Hz}, 2 \mathrm{H}$ ), 7.19-7.30 (m, 9H), 7.41-7.43 (m, 6H); ${ }^{13} \mathrm{C}$ NMR (100 MHz, $\left.\mathrm{CDCl}_{3}\right) \delta$ 24.8, 28.2, 28.6, 29.5, 29.7, 46.6, 46.9, 62.6, 66.8, 79.5, 126.8, 128.0, 129.7, 145.0, 155.7.

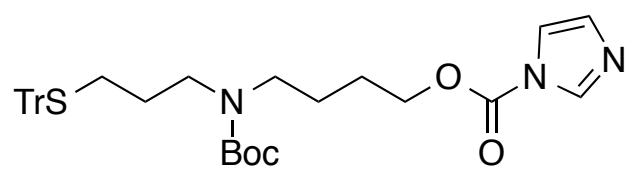

4-(((tert-Butoxy)carbonyl)((3-((triphenylmethyl)sulfanyl)propyl))amino) butyl $1 \mathrm{H}$ imidazole-1-carboxylate. 1,1'-Carbonyldiimidazole (214 $\mathrm{mg}, 1.32 \mathrm{mmol})$ was added to a solution of tert-butyl $N$-(4-hydroxybutyl)- $N$-(3-((triphenylmethyl)sulfanyl)propyl) carbamate (444 mg, $0.88 \mathrm{mmol})$ and $(i-\operatorname{Pr})_{2} \mathrm{NEt}(228 \mu \mathrm{L}, 1.32 \mathrm{mmol})$ in dry $\mathrm{CH}_{2} \mathrm{Cl}_{2}(3$ $\mathrm{mL}$ ) at $0{ }^{\circ} \mathrm{C}$ and was stirred overnight. The reaction solution was washed twice with water and extracted once with $\mathrm{CH}_{2} \mathrm{Cl}_{2}$. The combined organic layers were washed twice with sat. $\mathrm{NH}_{4} \mathrm{Cl}$, once with brine, were dried over $\mathrm{Na}_{2} \mathrm{SO}_{4}$, filtered and concentrated in vacuo to afford crude 4-(((tert-butoxy)carbonyl)((3-((triphenylmethyl)sulfanyl)propyl)) amino) butyl $1 H$-imidazole-1-carboxylate $(517 \mathrm{mg}, 98 \%)$ as a light yellow oil and was used without further purification. $R_{f} 0.50\left(3: 7, \mathrm{EtOAc}: \mathrm{CH}_{2} \mathrm{Cl}_{2}\right) ;{ }^{1} \mathrm{H} \mathrm{NMR}(400 \mathrm{MHz}$, $\left.\mathrm{CDCl}_{3}\right) \delta 1.42$ (s, 9H), 1.59 (br s, 4H), 1.76 (quin, $J=6.8 \mathrm{~Hz}, 2 \mathrm{H}$ ), 2.18 (t, $J=7.4 \mathrm{~Hz}$, 2H), 3.11 (br s, 4H), 4.42 (t, $J=6.6 \mathrm{~Hz}, 2 \mathrm{H}), 7.08(\mathrm{~s}, 1 \mathrm{H}), 7.20-7.31$ (m, 9H), 7.42-7.44 (m, 7H), $8.15(\mathrm{~s}, 1 \mathrm{H}) ;{ }^{13} \mathrm{C} \mathrm{NMR}\left(100 \mathrm{MHz}, \mathrm{CDCl}_{3}\right) \delta$ 24.6, 25.9, 28.2, 28.5, 29.4, $46.5(2$ C's), 66.8, 68.1, 79.6, 117.2, 126.7, 128.0, 129.7, 130.8, 137.2, 144.9, 148.8, 155.5. 
<smiles>O=C(OCCCCN(CCC[As])C(=O)OCc1ccccc1)OCCOC(=O)OCc1ccccc1</smiles>

\section{2-(2-(((4-(((tert-Butoxy)carbonyl)((3-((triphenylmethyl)sulfanyl)propyl))amino)}

butoxy)carbonyl)oxy)ethoxy)-2,3-dihydro-1 $\boldsymbol{H}$-isoindole-1,3-dione. $\quad$ DBU (129 $\mu \mathrm{L}$,

$0.86 \mathrm{mmol})$ was added to a solution of crude tert-butyl $N$-(4-hydroxybutyl)- $N$-(3((triphenylmethyl)sulfanyl)propyl)carbamate $(517 \mathrm{mg}, 0.86 \mathrm{mmol})$ in dry $\mathrm{MeCN}(4 \mathrm{~mL})$. After stirring for $10 \mathrm{~min}, 2$-(2-hydroxyethoxy)-2,3-dihydro- $1 H$-isoindole-1,3-dione (178 mg, $0.86 \mathrm{mmol}$ ) was added and the reaction was stirred overnight. Upon completion, the reaction was washed twice with sat. $\mathrm{NH}_{4} \mathrm{Cl}$ and the combined aqueous layers were extracted three times with EtOAc. The combined organic layers were washed with brine, dried over $\mathrm{Na}_{2} \mathrm{SO}_{4}$, filtered and concentrated in vacuo. The crude material was purified by column chromatography $\left(\mathrm{SiO}_{2}, 3: 17\right.$, EtOAc: $\left.\mathrm{CH}_{2} \mathrm{Cl}_{2}\right)$ to give 2-(2-(((4-)((tertbutoxy)carbonyl)((3-((triphenylmethyl)sulfanyl) propyl))amino)butoxy)carbonyl)oxy) ethoxy)-2,3-dihydro-1H-isoindole-1,3-dione (350 mg, 55\% over 2 steps) as a colorless oil. $R_{f} 0.75$ (3:17, EtOAc: $\left.\mathrm{CH}_{2} \mathrm{Cl}_{2}\right) ;{ }^{1} \mathrm{H} \mathrm{NMR}\left(400 \mathrm{MHz}, \mathrm{CDCl}_{3}\right) \delta 1.40(\mathrm{~s}, 9 \mathrm{H}), 1.46-1.64$ $(\mathrm{m}, 6 \mathrm{H}), 2.15(\mathrm{t}, J=7.4 \mathrm{~Hz}, 2 \mathrm{H}), 3.06(\mathrm{br} \mathrm{s}, 4 \mathrm{H}), 4.14(\mathrm{t}, J=6.4 \mathrm{~Hz}, 2 \mathrm{H}), 4.43-4.48(\mathrm{~m}$, 4H), 7.18-7.29 (m, 9H), 7.39-7.42 (m, 6H), 7.73-7.76 (m, 2H), 7.81-7.85 (m, 2H); ${ }^{13} \mathrm{C}$ NMR (100 MHz, $\left.\mathrm{CDCl}_{3}\right) \delta$ 24.6, 26.1, 28.2, 28.6, 29.5, 46.5, 46.6, 65.2, 68.1, 75.6, 79.5, $123.8,126.8,128.0,129.0,129.7,134.7,145.0,155.1,155.5,163.5$. 


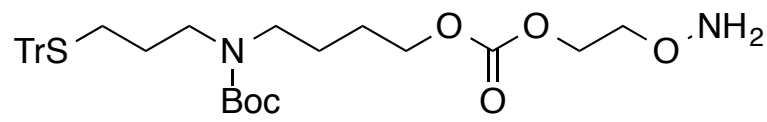

28

$N$-(4-(((2-(aminooxy)ethoxy)carbonyl)oxy)butyl)- $N$-((tert-butoxy)carbonyl)-3-

((triphenylmethyl)sulfanyl)propan-1-amine. Hydrazine monohydrate $(51 \mu \mathrm{L}, 1.04$

mmol) was added to a solution of 2-(2-(((4-(((tert-butoxy)carbonyl)((3((triphenylmethyl)sulfanyl)propyl))amino)butoxy)carbonyl) oxy)ethoxy)-2,3-dihydro$1 \mathrm{H}$-isoindole-1,3-dione $(154 \mathrm{mg}, 0.21 \mathrm{mmol})$ in $\mathrm{CH}_{2} \mathrm{Cl}_{2}$ at $0{ }^{\circ} \mathrm{C}$ and stirred for $2 \mathrm{~h}$. When complete, the white precipitate was removed by filtration and the filter cake was washed with ample $\mathrm{CH}_{2} \mathrm{Cl}_{2}$. The filtrate was concentrated in vacuo to afford 28 (127 mg, $100 \%)$ as a colorless oil and did not require further purification. $R_{f} 0.52(1: 3$, EtOAc: $\left.\mathrm{CH}_{2} \mathrm{Cl}_{2}\right) ;{ }^{1} \mathrm{H} \mathrm{NMR}\left(400 \mathrm{MHz}, \mathrm{CDCl}_{3}\right) \delta 1.38(\mathrm{~s}, 9 \mathrm{H}), 1.48-1.62(\mathrm{~m}, 6 \mathrm{H}), 2.14(\mathrm{t}, J$ $=7.4 \mathrm{~Hz}, 2 \mathrm{H}), 3.05(\mathrm{br} \mathrm{s}, 4 \mathrm{H}), 3.85(\mathrm{t}, J=4.6 \mathrm{~Hz}, 2 \mathrm{H}), 4.12(\mathrm{t}, J=6.4 \mathrm{~Hz}, 2 \mathrm{H}), 4.33(\mathrm{t}, J$ $=4.4 \mathrm{~Hz}, 2 \mathrm{H}), 5.51($ br s, $2 \mathrm{H}), 7.18-7.21(\mathrm{~m}, 3 \mathrm{H}), 7.25-7.29(\mathrm{~m}, 6 \mathrm{H}), 7.39-7.41(\mathrm{~m}, 6 \mathrm{H})$;

${ }^{13} \mathrm{C}$ NMR (100 MHz, $\left.\mathrm{CDCl}_{3}\right) \delta$ 24.7, 26.2, 27.5, 28.6, 29.5, 46.7 (2 C's), 65.5, 66.8, 68.0, $73.4,79.5,126.8,128.0,129.7,145.0,155.5,155.6$. 


\section{REFERENCES}

\section{R.1 CHAPTER 1 REFERENCES}

R.2. CHAPTER 2 REFERENCES

R.3. CHAPTER 3 REFERENCES

R.4. CHAPTER 4 REFERENCES

R.5. CHAPTER 5 REFERENCES 


\section{R.1. CHAPTER 1 REFERENCES}

1. Kolb, H. C.; Finn, M. G.; Sharpless, K. B., Angew. Chem. Int. Ed. 2001, 40, 20042021.

2. Grekov, A. P.; Veselov, V. Y. Russ. Chem. Rev. 1978, 47, 631-648.

3. Edwards, J. O.; Pearson, R. G. J. Am. Chem. Soc. 1962, 84, 16-24.

4. Khomutov, A. R.; Vepsäläinen, J. J.; Shvetsov, A. S.; Hyvönen, T.; Keinänen, T. A.; Pustobaev, V. N.; Eloranta, T. O.; Khomutov, R. M. Tetrahedron 1996, 52, 13751-13766.

5. Kalia, J.; Raines, R. T. Angew. Chem. Int. Ed. 2008, 47, 7523-7526.

6. Wiberg, K. B.; Glaser, R. J. Am. Chem. Soc. 1992, 114, 841-850.

7. Pauling, L. The Nature of the Chemical Bond, 1st ed., Cornell University Press, Ithaca, 1939, p. 60.

8. Crisalli, P.; Kool, E. T. J. Org. Chem. 2013, 78, 1184-1189.

9. (a) Dirksen, A.; Hackeng, T. M.; Dawson, P. E. Angew. Chem. Int. Ed. 2006, 45, 7581-7584; (b) Dirksen, A.; Dawson, P. E. Bioconjugate Chem. 2008, 19, 25432548; (c) Dirksen, A.; Dirken, S.; Hackeng, T. M.; Dawson, P. E. J. Am. Chem. Soc. 2006, 128, 15602-15603.

10. Thygesen, M. B.; Munch, H.; Sauer, J.; Cló, E.; Jørgensen, M. R.; Hindsgaul, O.; Jensen, K. J. J. Org. Chem. 2010, 75, 1752-1755.

11. Borch, R.; Bernstein, M.; Durst, H. J. Am. Chem. Soc. 1971, 93, 2897-2904.

12. Rashidian, M.; Mahmoodi, M. M.; Shah, R.; Dozier, J. K.; Wagner, C. R.; Distefano, M. D. Bioconjugate Chem. 2013, 24, 333-342.

13. Nauman, D.; Bertozzi, C. Biochim. Biophys. Acta, Gen. Subj. 2001, 1568, 147-154.

14. Hang, H. C.; Bertozzi, C. R. Acc. Chem. Res. 2001, 34, 727-736.

15. Boturyn, D.; Coll, J.-L.; Garanger, E.; Favrot, M.-C.; Dumy, P. J. Am. Chem. Soc. 2004, 126, 5730-5739.

16. Stromblad, S.; Cheresh, D. A. Chem. Biol. 1996, 3, 881-885.

17. (a) Desgrosellier, J. S.; Cheresh, D. A. Nat. Rev. Cancer 2010, 10, 9-22; (b) Jin, H.; J. Varner, J. Br. J. Cancer 2004, 90, 561-565. 
18. Wenk, C. H. F.; Ponce, F.; Guillerment, S.; Tenaud, C.; Boturyn, D.; Dumy, P.; Watrelot-Virieux, D.; Carozzo, C.; Josserand, V.; Coll, J.-L. Cancer Lett. 2013, 334, 188-195.

19. Garanger, E.; Boturyn, D.; Coll, J.-L.; Favrot, M.-C.; Dumy, P. Org. Biomol. Chem. 2006, 4, 1958-1965.

20. Zeng, W.; Azzopardi, K.; Hocking, D.; Wong, C. Y.; Robevska, G.; Tauschek, M.; Robins-Browne, R. M.; Jackson, D. C. Vaccine 2012, 30, 4800-4806.

21. Lempens, E. H.; Helms, B. A.; Merkx, M.; Meijer, E. W. ChemBioChem 2009, 10, 658-662.

22. Chan, E. W. L.; Lee, D.-C.; Ng, M.-K.; Wu, G.; Lee, K. Y. C.; Yu, L. J. Am. Chem. Soc. 2002, 124, 12238-12243.

23. (a) Friend, R. H.; Gymer, R. W.; Holmes, A. B.; Burroughes, J. H.; Marks, R. N.; Taliani, C.; Bradley, D. D. C.; Dos Santos, D. A.; Bredas, J. L.; Logdlund, M.; Salaneck, W. R. Nature 1999, 397, 121-128; (b) Hubbell, J. A. Nat. Biotechnol. 1995, 13, 565-576.

24. Thygesen, M. B.; Sørensen, K. K.; Cló, E.; Jensen, K. J. Chem. Comm. 2009, 63676369.

25. (a) Biswas, S.; Gordon, L. E.; Clark, G. J.; Nantz, M. H. Biomaterials 2011, 32, 2683-2688; (b) Biswas, S.; Knipp, R. J.; Gordon, L. E.; Nandula, S. R.; Gorr, S. U.; Clark, G. J.; Nantz M. H. ChemMedChem 2011, 6, 2063-2069.

26. Zhang, Y.; Yu, M.; Zhang, C.; Ma, W.; Zhang, Y.; Wang, C.; Lu, H. Anal. Chem. 2014, 86, 7920-7924.

27. Torres-Lugo, M.; Rinaldi, C. Nanomedicine 2013, 8, 1689-1707.

28. Shokrollahi, H. Mater. Sci. Eng., C. 2013 33, 4485-4497.

29. Huang, J.; Zhong, X.; Wang, L.; Yang, L.; Mao, H.; Theranostics 2012, 2, 86-102.

30. Yang, L.; Peng, X.-H.; Wang, Y. A.; Wang, X.; Cao, Z.; Ni, C.; Karna, P.; Zhang, X.; Wood, W. C.; Gao, X.; Nie, S.; Mao, H. Clin. Cancer Res. 2009, 15, 47224732 .

31. Massoud, T. F.; Gambhir S. S. Genes Dev. 2003, 17, 545-580.

32. Moore, A.; Marecos, E.; Bogdanov, A. Jr.; Weissleder, R. Radiology 2000, 214, 568-574. 
33. Gao, X.; Cui, Y. Levenson, R. M.; Chung, L. W. K.; Nie, S. Nat. Biotechnol. 2004, 22, 969-976.

34. Teja, A. S.; Koh, P.-Y. Prog. Cryst. Growth Charact. Mater. 2009, 55, 22-45.

35. Cornell, R. M.; Schwertmann, U. The Iron Oxides: Structure, Properties, Reactions, Occurrences, and Uses. 2nd Ed. Wiley-VCH, Weinheim, 2003.

36. Jun, Y.-W.; Seo, J.-W.; Cheon, J. Acc. Chem. Res. 2008, 41, 179-189.

37. Cheng, D.; Li, X.; Zhang, G.; Shi, H. Nanoscale Res. Lett. 2014, 9, 195.

38. Sapsford, K. E.; Algar, W. R.; Berti, L.; Gemmill, K. B.; Casey, B. J.; Oh, E.; Stewart, M. H.; Medintz, I. L. Chem. Rev. 2013, 113, 1904-2074.

39. Thiesen, B.; Jordan, A. Int. J. Hyperthermia 2008, 24, 467-474.

40. Mody, V. V.; Singh, A.; Wesley, B. Eur. J. Nanomed. 2013, 5, 11-21.

41. American Cancer Society, Types of Treatment, Available from: http://www.cancer.org/docroot.ETO/ETO_1.asp.

42. (a) Wust, P.; Kildebrandt, B.; Sreenivasa, G.; Rau, B.; Gellermann, J.; Riess, H.; Felix, R.; Schlag, P. M. Lancet Oncol. 2002, 3, 487-497; (b) Moroz, P, Jones, S. K.; Gray, B. N. Int. J. Hyperthermia 2002, 18, 267-284.

43. Aoki, H.; Kakinuma, K.; Morita, K.; Kato, M.; Uzuka, T.; Igor, G.; Takahashi, H.; Tanaka, R. Int. J. Hyperthermia 2004, 20, 595-605.

44. Purushotham, S.; Ramanujan, R. V. J. Appl. Phys. 2010, 107, 114701/1-114701/9.

45. Alexiou, C.; Arnold, W.; Klein, R. J.; Parak, F. G.; Hulin, P.; Bergemann, C.; Erhardt, W.; Wagenpfeil, S.; Lübbe, A. S. Cancer Res. 2000, 60, 6641-6648. 


\section{R.2. CHAPTER 2 REFERENCES}

1. U.S. National Institutes of Health. National Cancer Institute. SEER Cancer Statistics Review, 1975-2011.

2. Luque de Castro, M. D.; Fernández-Peralbo, M. A. TRAC-Trend. Anal. Chem. 2012, 38, 13-20.

3. Johnson, F. L.; Turic, B.; Kemp, R.; Palcic, B.; Sussman, R.; Voelker, K. G.; Robinette, E. Chest 2004, 125, 157S-158S.

4. Varella-Garcia, M.; Kittelson, J.; Schulte, A. P.; Vu, K. O.; Wolf, H. J.; Zeng, C.; Hirsch, F. R.; Byers, T.; Kennedy, T.; Miller, Y. E., Keith, R. L.; Franklin, W. A. Cancer Detect. Prev. 2004, 28, 244-251.

5. Helmig, S.; Schneider, J. Expert Rev. Mol. Diagn. 2007, 7, 555-568.

6. Yildiz, P. B.; Shyr, Y.; Rahman, J. S.; Wardwell, N. R.; Zimmerman, L. J.; Shakhtour, B.; Gray, W. H.; Chen, S.; Li, M.; Roder, H.; Liebler, D. C.; Bigbee, W. L.; Siegfried, J. M.; Weissfeld, J. L.; Gonzalez, A. L.; Ninan, M.; Johnson, D. H.; Carbone, D. P.; capriole, R. M.; Massion, P. P. J. Thorac. Oncol. 2007, 2, 893901.

7. Maciel, C. M.; Junqueira, M.; Paschoal, M. E.; Kawamura, M. T.; Duarte, R. L.; Carvalho, M. G.; Domont, G. B. J. Exp. Ther. Oncol. 2005, 5, 31-38.

8. Kikuchi, T.; Carbone, D. P. Respirology 2007, 12, 22-28.

9. Diederich, S. Cancer Imaging 2007, 8, S24-S26.

10. Aisner, J. Clin. Cancer Res. 2007, 13, 4951-4953.

11. Lichy, M. P.; Aschoff, P.; Plathow, C.; Stemmer, A.; Horger, W.; Mueller-Horvat, C.; Steidle, G.; Horger, M.; Schafer, J.; Eshmann, S. M.; Kiefer, B.; Claussen, C. D.; Pfannenberg, C.; Schlemmer, H.-P. Invest. Radiol. 2007, 42, 605-613.

12. Fossella, F. V.; Komaki, R.; Putnam, J. B. Lung Cancer, Springer-verlag, New York, USA, 2003.

13. Kennedy, T. C.; Hirsch, F. R. Lung Cancer 2004, 45, S21-S27.

14. Hilbe, W.; Dirnhofer, S.; Greil, R.; Woll, E. J. Cancer Prev. 2004, 13, 425-436.

15. Dammas, S.; Patz, E. F.; Goodman, P. C. Lung Cancer 2001, 33, 11-16. 
16. Pauling, L.; Robinson, A. B.; Teranishi, R.; Cary, P. Proc. Nat. Acad. Sci. USA. 1971, 68, 2374-2376.

17. O’Neill, H. J.; Gordon, S. M.; O’Neill, M. H.; Gibbons, R. D.; Szidon, J. P. Clin. Chem. 1988, 34, 1613-1618.

18. Gordon, S. M.; Szidon, J. P.; Krotoszynski, B. K.; Gibbons, R. D.; O’Neill, H. J. Clin. Chem. 1985, 31, 1278-1282.

19. Preti, G.; Labows, J. N.; Kostelc, J. G.; Aldinger, S.; Daniele, R. J. Chromatogr. 1988, 432, 1-11.

20. Khyshiktyev, B. S.; Khyshiktueva, N. A.; Ivanov, V. N.; Darenskaia, S. D.; Novikov, S. V. Vopr. Onkol. 1994, 247, 161-164.

21. Phillips, M.; Gleeson, K.; Hughes, J. M. B.; Greenberg, J.; Cataneo, R. N.; Baker, L.; McVay, W. P. Lancet 1999, 353, 1930-1933.

22. Williams, H.; Pembroke, A. Lancet 1989, 1, 734.

23. Walker, D. B.; Cavnar, P.; Taylor, J.; et.al., Paper, Meeting Assoc. Chemorecept. Sci., April 2003, Sarasota, FL, USA.

24. Malerba, M.; Montuschi, P. Curr. Med. Chem. 2012, 19, 187-196.

25. Di Natale, C.; Paolesse, R.; Martinelli, E.; Capuano, R. Anal. Chim. Acta 2014, $824,1-17$.

26. Alagirisamy, N.; Hardas, S.; Jayaraman, S. Anal. Chim. Acta 2010, 661, 97-102.

27. Liu, C.; Hayashi, K.; Toko, K. Sensors Actuat. B-Chem. 2002, 161, 504-509.

28. Mitsubayashi, K.; Minamide, T.; Otsuka, K.; Kudo, H.; Saito, H. Anal. Chim. Acta 2006, 573, 75-80.

29. Righettoni, M.; Tricoli, A.; Gass, S.; Schmid, A.; Amann, A.; Pratsinis, S. Anal. Chim. Acta 2012, 738, 69-75.

30. Nasutionet, T.; Nainggolan, I.; Hutagalung, S.; Ahmad, K.; Ahmad, Z. Sensors Actuat. B-Chem. 2013, 177, 522-528.

31. Neri, G.; Bonavita, A.; Milcali, G.; Donato, N. IEEE Sens. J. 2010, 10, 131-136.

32. Choi, S.; Less, I.; Jang, B.; Ryu, W.; Park, C.; Kim, I. Anal. Chem. 2012, 85, 17921796. 
33. Wang, C.; Mbi, A.; Sheperd, M. IEEE Sens. J. 2010, 10, 54-63.

34. Shuster, G.; Gallimadi, Z.; Reiss, A.; Dovgolevsky, E.; Billan, S.; Abdah-Bortynak, R.; Kuten, A.; Engel, A.; Shiban, A.; Tisch, U.; Haick, H. Breast Cancer Res. Tr. 2011, 126, 791-796.

35. Wiedemair, J.; Van Dorp, H.; Olthuis, W.; Van der Berg, A. Electrophoresis 2012, 33, 3181-3196.

36. Komkova, M.; Karyakina, E.; Marken, F.; Karyakin, A. Anal. Chem. 2013, 85, 2574-2577.

37. Mondal, S. P.; Dutta, P.; Hunter, G.; Ward, B.; Laskowsi, D. Sensors Actuat. BChem. 2011, 158, 292-298.

38. Pantalei, S.; Zampetti, E.; Bearzotti, A.; De Cesare, F.; Macagnano, A. Sensors Actuat. B-Chem. 2013, 179, 87-94.

39. Namjou, K.; Roller, C.; Reich, T.; Jeffers, J.; McMillen, G.; McCann, P.; Camp, M. Appl. Phys. B 2006, 85, 427-435.

40. McCurdy, M.; Bakhirkin, Y.; Tittel, F. Appl. Phys. B 2006, 85, 445-452.

41. Kuzmych, O.; Allen, B.; Star, A. Nanotechnology 2007, 18, 37502.

42. Liu, H.; Huang, W.; Chen, Y.; Lu, C.; Huang, J. IEEE Sens. J. 2013, 13, 2737-274.

43. Toda, K.; Li, J.; Dasgupta, P. Anal. Chem. 2006, 78, 7284-7291.

44. Gouma, P.; Kalyanasundaram, K.; Yun, X. IEEE Sens. J. 2010, 10, 49-53.

45. DuBois, S.; Eng, S.; Bhattacharya, R.; Rulyak, S.; Hubbard, T.; Putnam, D.; Kearney, D. Digest. Dis. Sci. 2005, 50, 1780-1784.

46. Fernandez-Sanchez, J.; Cannas, R.; Spichiger, S.; Steiger, R.; Spichiger-Keller, U. Sensors Actuat. B-Chem. 2007, 128, 145-153.

47. Mills, A.; Lepre, A.; Wild, L. Sensors Actuat. B-Chem. 1997, 38, 419-425.

48. Shen, F.; Wang, J.; Xu, Z.; Wu, Y.; Chen, Q.; Li, X.; Jie, X.; Li, L.; Yao, M.; Guo, X.; Zhu, T. Nano Lett. 2012, 12, 3722-3730.

49. Driskell, J.; Jones, C.; Tompkins, M.; Tripp, R. Analyst 2011, 136, 3083. 
50. Mitsubayashi, K.; Matsunaga, H.; Nishio, G.; Toda, S.; Nakanishi, Y.; Saito, H.; Ogawa, M.; Otsuka, K. Sensors Actuat. B-Chem. 2005, 108, 660-664.

51. Biswas, S. Functionalized Nanoparticles for AMF-Induced Gene and Drug Delivery. Dissertation, University of Louisville, 2011.

52. (a) Fu, X.-A.; Li, M.; Knipp, R. J.; Nantz, M. H.; Bousamra, M. Cancer Med. 2014, 3, 174-181; (b) Bousamra, M.; Schumer, E.; Li, M.; Knipp, R. J.; Nantz, M. H.; van Berkel, V. H.; Fu, X.-A. J. Thorac. Cardiovasc. Surg. 2014, 148, 1074-1081.

53. Li, M.; Biswas, S.; Nantz, M. H.; Higashi, R. M.; Fu, X.-A. Sens. Actuators B Chem. 2013, 180.

54. Fu, X.-A.; Li, M.; Biswas, S.; Nantz, M. H.; and Higashi, R. M. Analyst 2011, 136, 4662-4666.

55. Li, M.; Biswas, S.; Nantz, M. H.; Higashi, R. M.; Fu, X.-A. Anal. Chem. 2012, 84, 1288-1293.

56. Hakim, M., Broza, Y. Y., Barash, O., Peled, N., Phillips, M., Amann, A., Haick, H. Chem. Rev. 2012, 112, 5949-5966.

57. Bajtarevic, A., Ager, C., Pienz, M., Klieber, M., Schwarz, K., Ligor, M., Ligor, T., Filipiak, W., Denz, H., Fiegl, M., Hilbe, W., Weiss, W., Lukas, P., Jamnig, H., Hackl, M., Haidenberger, A., Buszewski, B., Miekisch, W., Schubert, J., Amann, A. BMC Cancer 2009, 9, 348-363.

58. Fuchs, P., Loeseken, C., Schubert, J. K., Miekisch, W. Int. J. Cancer 2010, 126, 2663-2670.

59. Poli, D., Goldoni, M., Corradi, M., Acampa, O., Carbognani, P., Internullo, E., Casalini, A., Mutti, A. J. Chromatography B 2010, 878, 2643-2651.

60. Phillips, M., Altorki, N., Austin, J. H. M., Cameron, R. B., Cataneo, R. N., Kloss, R., Maxfield, R. A., Munawar, M. I., Pass, H. I., Rashid, A., Rom, W. N., Schmitt, P., Wai, J. Clin. Chim. Acta 2008, 393, 76-84.

61. Fujioka, K., Shibamoto, T. Environ Toxicol. 2006, 21, 47-54.

62. Mattingly, S. J.; Xu, T.; Nantz, M. H.; Higashi, R. M.; Fan, T. W.-M. Metabolomics 2012, 8, 989-996.

63. Crampton, M. R.; Robotham, I. A. J. Chem. Res. (S) 1997, 22-23. 
64. Deng, C.; Zhang, J.; Yu, X.; Zhang, W.; Zhang, X. J. Chromatogr., B: Anal. Technol. Biomed. Life Sci. 2004, 810, 269-275.

65. Ueta, I.; Saito, Y.; Hosoe, M.; Okamoto, M.; Ohkita, H.; Shirai, S.; Tamura, H.; Jinno, K. J. Chromatogr., B: Anal. Technol. Biomed. Life Sci. 2009, 877, 25512556.

66. Dirksen, A.; Dawson, P. E. Bioconjugate Chem. 2008, 19, 2543-2548.

67. Goto, J.; Saisho, Y.; Nambara, T. Anal. Sci. 1989, 5, 399-402. 


\section{R.3. CHAPTER 3 REFERENCES}

1. Parvatkar, P. T.; Kadam, H. K.; Tilve, S. G. Tetrahedron 2014, 70, 2857-2888.

2. Kondoh, A.; Aoki, T.; Terada, M. Org. Lett. 2014, 16, 3528-3531.

3. Sakulsombat, M.; Angelin, M.; Ramström, O. Tet. Lett. 2010, 51, 75-78.

4. Zhdanko, A.; Schmauder, A.; Ma, C. I.; Sibley, L. D.; Sept, D.; Sasse, F.; Maier, M. E. Chem. Eur. J. 2011, 17, 13349-13357.

5. Tsui, G. C.; Ninnemann, N. M.; Hosotani, A.; Lautens, M. Org. Lett. 2013, 15, 1064-1067.

6. Wong, A. D.; DeWit, M. A.; Gillies, E. R. Adv. Drug Delivery Rev. 2012, 64, 1031-1045.

7. Shan, D.; Nicolaou, M. G.; Borchardt, R. T.; Wang, B. J. Pharm. Sci. 1997, 86, 765-767.

8. $\quad$ Sykes, B. M.; Atwell, G. J.; Hogg, A.; Wison, W. R.; O'Connor, C. J.; Denny, W. A. J. Med. Chem. 1999, 42, 346-355.

9. Saari, W. S.; Schwering, J. E.; Lyle, P. A.; Smith, S. J.; Engelhardt, E. L. J. Med. Chem. 1990, 33, 97-101.

10. Greenwald, R. B.; Choe, Y. H.; Conover, C. D.; Shum, K.; Wu, D.; Royzen, M. J. Med. Chem. 2000, 43, 475-487.

11. Chen, E. K. Y.; McBride, R. A.; Gillies, E. R. Macromolecules 2012, 45, 7364-7374.

12. Wakselman, M. Nouv. J. Chim. 1983, 7, 439-447.

13. Carl, P. L.; Chakravarty, P. K.; Katzenellenbogen, J. A. J. Med. Chem. 1981, 24, 479-480.

14. Senter, P. D.; Pearce, W. E.; Greenfield, R. S. J. Org. Chem. 1990, 55, 2975-2978.

15. DeWit, M. A.; Gillies, E. R. Org. Biomol. Chem. 2011, 9, 1846-1854.

16. Crimmins, M. T.; Carroll, C. A.; Wells, A. J. Tetrahedron Lett. 1998, 39, 70057008 . 
17. Zhu, J.; Munn, R. J.; Nantz, M. H. J. Am. Chem. Soc. 2000, 122, 2645-2646.

18. Saari, W. S.; Schwering, J. E.; Lyle, P. A.; Smith, S. J.; Engelhardt, E. L. J. Med. Chem. 1990, 33, 2590-2595.

19. Vinšová, J.; Imramovský, A. Chem. Listy 2005, 99, 21-29.

20. Wei, Y.; Pei, D. Biorg. Med. Chem. Lett. 2000, 10, 1073-1076.

21. Ojima, I.; Vidal, E. S., Organometallics 1999, 18, 5103-5107.

22. Arkles, B. "Silanes and Surfaces: Hydrophobicity, Hydrophilicity, and Coupling Agents." Gelest Silicon Compounds: Silanes \& Silicones 2013, 3, 160-198.

23. Arkles, B. CHEMTECH 1977, 7, 766.

24. Sabaurault, N.; Mignani, G.; Wagner, A.; Mioskowski, C. Org. Lett. 2002, 4, $2117-$ 2119.

25. Beesley, R. M.; Ingold, C. K.; J.F. Thorpe, J. F. J. Chem. Soc. Trans. 1915, 107, 1080-1106.

26. Russo, O.; Berthouze, M.; Giner, M.; Soulier, J.-L.; Rivail, L.; Sicsic, S.; Lezoualc'h, F.; Jockers, R.; Berque-Bestel, I. J. Med. Chem. 2007, 50, 4482-4492.

27. Revell, J. D.; Dörner, B.; White, P. D.; Ganesan, A. Org. Lett. 2005, 7, 831-833.

28. Gardener, R. A.; Delcros, J.-G.; Konate, F.; Breitbeil III, F.; Martin, B.; Sigman, M.; Huang, M.; Phanstiel, O. IV J. Med. Chem. 2004, 47, 6055-6069.

29. Lemen, G. S.; Wolfe, J. P. Org. Lett. 2010, 12, 2322-2325.

30. (a) Knipp, R. J.; Nantz, M. H. (University of Louisville, USA). US Patent Application US2014015413, February 7, 2014; Priority: US Provisional Application 61/762,832 February 8, 2013; (b) Knipp, R. J.; Estrada, R.; Sethu, P.; Nantz, M. H. Tetrahedron 2014, 70, 3422-3429.

31. Ohmura, N.; Nakamura, A.; Hamasaki, A.; Tokunaga, M. Eur. J. Org. Chem. 2008, $5042-5045$.

32. Livak-Dahl, E.; Sinn, I.; Burns, M. Annu. Rev. Chem. Biomol. Eng. 2011, 2, 325353. 
33. Sethu, P.; Moldawer, L. L.; Mindrinos, M. N.; Scumpia, P. O.; Tannahill, C. L.; Wilhelmy, J.; Efron, P. A.; Brownstein, B. H.; Tompkins, R.G.; Toner, M. Anal. Chem. 2006, 78, 5453-5461.

34. Mattingly, S. J.; Xu, T.; Nantz, M. H.; Higashi, R. M.; Fan, T. W.-M. Metabolomics 2012, 8, 989-996.

35. Biswas, S.; Huang, X.; Badger, W. R.; Nantz, M. H. Tetrahedron Lett. 2010, 51, 1727-1729.

36. Murthy, S. K.; Sin, A.; Tompkins, R. G.; Toner, M. Langmuir 2004, 20, 1164911655.

37. Lee, J. N.; Park, C.; Whitesides, G. M. Anal. Chem. 2003, 75, 6544-6554.

38. Trévisiol, E.; Defrancq, E.; Lhomme, J.; Laayoun, A.; Cros, P. Eur. J. Org. Chem. 2000, 211-217. 


\section{R.4. CHAPTER 4 REFERENCES}

1. Kievit, F. M.; Zhang, M. Acc. Chem. Res. 2011, 44, 853-862.

2. Bulte, J. W. M.; Kraitchman, D. L. NMR Biomed. 2004, 17, 484-499.

3. Yang, C.; Wu, J.; Hou, Y. Chem. Commun. 2011, 47, 5130-5141.

4. Mody, V. V.; Singh, A.; Wesley, B. Eur. J. Nanomed. 2013, 5, 11-21.

5. Tanaka, K.; Ito, A.; Kobayashi, T.; Kawamura, T.; Shimada, S.; Matsumoto, K.; Saida, T.; Honda, H. Int. J. Cancer 2005, 116, 624-633.

6. Sato, M.; Yamashita, T.; Ohkura, M.; Osai, Y.; Sato, A.; Takada, T.; Matsusaka, H.; Ono, I.; Tamura, Y.; Sato, N.; Sasaki, Y. Ito, A.; Honda, H.; Wakamatsu, K.; Ito, S.; Jimbow, K. J. Invest. Dermatol. 2009, 129, 2233-2241.

7. Shinkai, M.; Yanase, M.; Suzuki, M.; Honda, H.; Wakabayashi, T.; Yoshida, J.; Kobayashi, T. J. Magn. Magn. Mater. 1999, 194, 176-184.

8. Le, B.; Shinkai, M.; Kitade, T.; Honda, H.; Yoshida, J.; Wakabayashi, T.; Kobayashi, T. J. Chem. Eng. Jpn. 2001, 34, 66-72.

9. Moroz, P.; Jones, S. K.; Winter, J.; Gray, B. N. J. Surg. Oncol. 2001, 78, $22-29$.

10. Moroz, P.; Jones, S. K.; Metcalf, C.; Gray, B. N. Int. J. Hyperthermia 2003, 19, 23-34.

11. Johannsen, M.; Gneveckow, U.; Eckelt, L.; Feussner, A.; Waldöfner, N.; Scholz, R.; Deger, S.; Wust, P.; Loening, S. A.; Jordan, A. Int. J. Hyperthermia 2005, 21, 637-647.

12. Kawai, N.; Ito, A.; Nakahara, Y.; Futakuchi, M.; Shirai, T.; Honda, H.; Kobayashi, T.; Kohri, K. Prostate 2005, 64, 373-381.

13. Meyers, P.H.; Cronic, F.; Nice, C. M. Jr. Am. J. Roentgenol. Radium Ther. Nucl. Med. 1963, 90, 1068-1077.

14. Kim, J.; Lee, J. E.; Lee, S. H.; Yu, J. H.; Lee, J. H.; Park, T. G.; Hyeon, T. $A d v$. Mater. 2008, 20, 478-483.

15. Zhang, Z.; Lee, S. H.; Feng, S.-S. Biomaterials 2007, 28, 1889-1899. 
16. Amstad, E.; Kohlbrecher, J.; Müller, E.; Schweizer, T.; Textor, M.; Reimhult, E. Nano Lett. 2011, 11, 1664-1670.

17. Mengesha, A. E.; Wydra, R. J.; Hilt, J. Z.; Bummer, P. M. Pharm. Res. 2013, 30, 3214-3224.

18. Meng, H.; Liong, M.; Xia, T.; Zongxi, J. Z.; Ji, Z.; Zink, J. I.; New, A. E. ACS Nano 2010, 4, 4539-4550.

19. Bringas, E.; Köysüren, Ö.; Quach, D. V.; Mahmoudi, M.; Aznar, E.; Roehling, J. D.; Marcos, M. D.; Martínez-Máñez, R.; Stroeve, P. Chem. Commun. 2012, 48, 5647-5649.

20. Lin, M. M.; Kim, H.-H.; Kim, H.; Muhammed, M.; Kim, D. K. Nano Rev. 2010, 1 , 4883.

21. Yu, M. K.; Jeong, Y. Y.; Park, J.; Park, S.; Kim, J. W.; Min, J. J.; Kim, K.; Jon, S. Angew. Chem. Int. Ed. 2008, 47, 5362-5365.

22. Rahimi, M.; Wadajkar, A.; Subramanian, K.; Yousef, M.; Cui, W.; Hsieh, J.-T.; Nguyen, K. T. Nanomedicine 2010, 6, 672-680.

23. Aryal, S.; Grailer, J. J.; Pilla, S.; Steeber, D. A.; Gong, S. J. Mater. Chem. 2009, 19, 7879-7884.

24. Choi, S. K.; Verma, M.; Silpe, J.; Moody, R. E.; Tang, K.; Hanson, J. J.; Baker, J. R. Jr. Bioorg. Med. Chem. 2012, 20, 1281-1290.

25. Liu, T.-Y.; Hu, S.-H.; Liu, K.-H.; Shaiu, R.-S.; Liu, D.-M.; Chen, S.-Y. Langmuir 2008, 24, 13306-13311.

26. Liu, J.; Zhang, Y.; Wang, C.; Xu, R.; Chen, Z.; Gu, N. J. Phys. Chem. C 2010, 114, 7673-7679.

27. Chiang, W.-L.; Ke, C.-J.; Liao, Z.-X.; Chen, S.-Y.; Chen, F.-R.; Tsai, C.-Y.; Xia, Y.; Sung, H.-W. Small 2012, 8, 3584-3588.

28. Thomas, C. R.; Ferris, D. P.; Lee, J.-H.; Choi, E.; Cho, M. H.; Kim, E. S.; J. Fraser Stoddart, J. F.; Shin, J.-S.; Cheon, J.; Zink, J. I. J. Am. Chem. Soc. 2010, 132, 10623-10625.

29. Hawkins, A. M.; Bottom, C. E.; Liang, Z.; Puleo, D. A.; Hilt, J. Z. Adv. Healthcare Mater. 2012, 1, 96-100.

30. Zhou, L.; He, B.; Zhang, F. ACS Appl. Mater. Interfaces 2012, 4, 192-199. 
31. Brulé, S.; Levy, M.; Wilhelm, C.; Letourneur, D.; Gazeau, F.; Ménager, C.; Le Visage, C. Adv. Materials 2011, 23, 787-790.

32. Rachakatla, R. S.; Balivada, S.; Seo, G.-M.; Myers, C. B.; Wang, H.; Samarakoon, T. N.; Dani, R.; Pyle, M.; Kroh, F. O.; Walker, B.; Leaym, X.; Koper, O. B.; Chikan, V.; Bossmann, S. H.; Tamura, M.; Troyer, D. L. ACS Nano 2010, 4, 70937104.

33. Silva, V. A. J.; Andrade, P. L.; Silva, M. P. C.; Bustamante, D. A.; Valladares, L. D. L. S.; Aguiar, J. A. J Magn. Magn. Mater. 2013, 343, 138-143.

34. Peiris, P. M.; Bauer, L.; Toy, R.; Tran, E.; Pansky, J.; Doolittle, E.; Schmidt, E.; Hayden, E.; Mayer, A.; Keri, R. A.; Griswold, M. A.; Karathanasis, E. ACS Nano 2012, 6, 4157-4168.

35. Sundaresan, V.; Menon, J. U.; Rahimi, M.; Nguyen, K. T.; Wadajkar, A. S. Int. J. Pharm. 2014, 466, 1-7.

36. Nantz, M. H.; Biswas, S. (University of Louisville, USA). US Patent 20120302516, November 29, 2012.

37. (a) Matsuno, R.; Yamamoto, K.; Otsuka, H.; Takahara. A. Chem. Mater. 2003, 15, 3-5; (b) Matsuno, R.; Yamamoto, K.; Otsuka, H.; Takahara, A. Macromolecules 2004, 37, 2203-2209; (c) Kobayashi, M.; Matsuno, R.; Otsuka, H.; Takahara, A. Sci. Technol. Adv. Mater. 2006, 7, 617-628; (d) Babu, K.; Dhamodharan, R. Nanoscale Res. Lett. 2008, 3, 109-117.

38. (a) García, I.; Zafeiropoulos, N. E.; Janke, A.; Tercjak, A.; Eceiza, A.; Stamm, M.; Mondragon, I. J. Polym. Sci., Part A: Polym. Chem. 2007, 45, 925-932; (b) García, I.; Tercjak, A.; Zafeiropoulos, N. E.; Stamm, M.; Mondragon, I. J. Polym. Sci., Part A: Polym. Chem. 2007, 45, 4744-4750; (c) García, I.; Tercjak, A.; Zafeiropoulos, N. E.; Stamm, M.; Mondragon, I. Macromol. Rapid Commun. 2007, 28, 2361-2365; (d) Wuang, S. C.; Neoh, K. G.; Kang, E.-T.; Pack, D. W.; Leckband, D. E. Adv. Funct. Mater. 2006, 16, 1723-1730; (e) Marutani, E.; Yamamoto, S.; Ninjbadgar, T.; Tsujii, Y.; Fukuda, T.; Takano, M. Polymer 2004, 45, 2231-2235; (f) Hu, F. X.; Neoh, K. G.; Cen, L.; Kang, E.-T. Biomacromolecules 2006, 7, 809-816.

39. Barbey, R.; Lavanant, L.; Paripovic, D.; Schüwer, N.; Sugnaux, C.; Tugulu, S.; Klok, H.-A. Chem. Rev. 2009, 109, 5437-5527.

40. (a) Zhou, Y.; Wang, S. X.; Ding, B. J.; Yang, Z. M. Chem. Eng. J. 2008, 138, 578585; (b) Sun, Y. B.; Ding, X. B.; Zheng, Z. H.; Cheng, X.; Hu, X. H.; Peng, Y. X. Eur. Polym. J. 2007, 43, 762-772. 
41. Lattuada, M.; Hatton, T. A. Langmuir 2007, 23, 2158-2168.

42. Maliakal, A.; Katz, H.; Cotts, P. M.; Subramoney, S.; Mirau, P. J. Am. Chem. Soc. 2005, 127, 14655-14662.

43. Belyavskii, S. G.; Mingalev, P. G.; Lisichkin, G. V. Colloid J. 2004, 66, 128-136.

44. Arkles, B. "Silanes and Surfaces: Hydrophobicity, Hydrophilicity, and Coupling Agents." Gelest Silicon Compounds: Silanes \& Silicones 2013, 3, 160-198.

45. Ma, M.; Zhang, Y.; Yu, W.; Shen, H.-Y.; Zhang, H.-Q.; Gu, N. Colloids Surf., A 2003, 212, 219-226.

46. Arkles, B. CHEMTECH 1977, 7, 766.

47. Mikhaylova, M.; Kim, D. K.; Bobrysheva, N.; Osmolowsky, M.; Semenov, V.; Tsakalakos, T.; Muhammed, M. Langmuir 2004, 20, 2472-2477.

48. Galeotti, F.; Bertini, F.; Scavia, G.; Bolognesi, A. J. Colloid Interface Sci. 2011, $360,540-547$.

49. Larumbe, S.; Gomez-Polo, C.; Perez-Landazabal, J.; Pastor, J. M. J. J. Phys.: Condens. Matter 2012, 24, 266007/1-266007/6.

50. Stöber, W.; Fink, A.; Bohn, E. J. Colloid Interface Sci. 1968, 26, 62-69.

51. (a) Matsoukas, T.; Gulari, E. J. Colloid Interface Sci. 1988, 124, 252-261; (b) Matsoukas, T.; Gulari, E. J. Colloid Interface Sci. 1989, 132, 13-21; (c) Matsoukas, T.; Gulari, E. J. Colloid Interface Sci. 1991, 145, 557-562.

52. Wang, Z. J.; Clary, K. N.; Bergman, R. G.; Raymond, K. N.; Toste, F. D. Nat. Chem. 2013, 5, 100-103.

53. Zhang, J.; Matta, M. E.; Martinez, H.; Hillmyer, M. A. Macromolecules 2013, 46, 2535-2543.

54. Melamed, J. Y.; Zartman, A. E.; Kett, N. R.; Gotter, A. L.; Uebele, V. N.; Reiss, D. R.; Condra, C. L.; Fandozzi, C.; Lubbers, L. S.; Rowe, B. A.; McGaughey, G. B.; Henault, M.; Stocco, R.; Renger, J. J.; Hartman, G. D.; Bilodeau, M. T.; Trotter, B. W. Bioorg. Med. Chem. Lett. 2010, 20, 4700-4703.

55. Ong, B. H.; Devaraj, N. K.; Matsumoto, M.; Abdullah, M. H. Mater. Res. Soc. Symp. Proc. 2008, Paper \#: 1118-K03-09. 
56. Sarkar, S.; Chatterjee, P. K.; Cumbal, L. H.; SenGupta, A. K. Chem. Eng. J. 2011, $166,923-931$.

57. Morrow, B. A.; Cody, I. A. J. Phys. Chem. 1976, 80, 1995-1998.

58. Sabourault, N.; Mignani, G.; Wagner, A.; Mioskowski, C. Org. Lett. 2002, 4, 2117 2119.

59. Knipp, R. J.; Estrada, R.; Sethu, P.; Nantz, M. H. Tetrahedron 2014, 70, 34223429.

60. Macha, S. F.; Limbach, P. A.; Savickas, P. J. J. Am. Soc. Mass Spectrom. 2000, 11, 731-737.

61. Riedinger, A.; Guardia, P.; Curcio, A.; Garcia, M. A.; Cingolani, R.; Manna, L.; Pellegrino, T. Nano Lett. 2013, 13, 2399-2406.

62. Lu, Y.; Yin, Y.; Mayers, B. T.; Xia, Y. Nano Lett. 2002, 2, 183-186.

63. Kou, L.; Sun, J.; Zhai, Y.; He, Z. Asian J. Pharm. Sci. 2013, 8, 1-10.

64. Benmerah, A.; Lamaze, C. Traffic 2007, 8, 970-982.

65. Park, J.; An, K.; Hwang, Y.; Park, J.-G.; Noh, H.-J.; Kim, J.-Y.; Park, J.-H.; Hwang, N.-M.; Hyeon, T. Nat. Mat. 2004, 3, 891-895.

66. Jiang, W.; Lai, K.-L.; Hu, H.; Zeng, X.-B.; Lan, F.; Liu, K.-X.; Wu, Y.; Gu, Z.-W. J. Nanopart. Res. 2011, 13, 5135-5145.

67. Ding, H. L.; Zhang, Y. X.; Wang, S.; Xu, J. M.; Xu, S. C.; Li, G. H. Chem. Mater. 2012, 24, 4572-4580.

68. (a) Yi, D.; Lee, S.; Papaefthymiou, G.; Ying, J. Chem. Mater. 2006, 18, 614-619; (b) Kang, K.; Choi, J.; Nam, J. H.; Lee, S. C.; Kim, K. J.; Lee, S.-W. W.; Chang, J. H. J. Phys. Chem. B 2009, 113, 536-543; (c) Malvindi, M. A.; Matteis, V. De; Galeone, A.; Brunetti, V.; Anyfantis, G. C.; Athanassiou, A.; Cingolani, R.; Pompa, P. P. PloS One 2014, 9, e85835; (d) Yi, D. K.; Selvan, S. T.; Lee, S. S.; Papaefthymiou, G. C.; Kundaliya, D.; Ying, J. Y. J. Am. Chem. Soc. 2005, 127 , 4990-4991; (e) Laurent, S.; Forge, D.; Port, M.; Roch, A.; Robic, C.; Vander Elst, L.; Muller, R. N. Chem. Rev. 2008, 108, 2064-2110.

69. Jing, J.; Zhang, Y.; Liang, J.; Zhang, Q.; Bryant, E.; Avendano, C.; Colvin, V.; Wang, Y.; Li, W.; Yu, W. J. Nanopart. Res. 2012, 14, 827/1-827/8. 
70. Pinho, S.; Pereira, G.; Voisin, P.; Kassem, J.; Bouchaud, V.; Etienne, L.; Peters, J.; Carlos, L.; Mornet, S.; Geraldes, C.; Rocha, J.; Delville, M.-H. ACS Nano 2010, 4, 5339-5349.

71. Biswas, S.; Gordon, L. E.; Clark, G. J.; Nantz, M. H. Biomaterials 2011, 32, 26832688.

72. Beaudette, T. T.; Cohen, J. A.; Bachelder, E. M.; Broaders, K. E.; Cohen, J. L.; Engleman, E. G.; Fréchet, J. M. J. J. Am. Chem. Soc. 2009, 131, 10360-10361.

73. Kolb, H. C.; Finn, M. G.; Sharpless, K. B., Angew. Chem. Int. Ed. 2001, 40, 20042021.

74. Kalia, J.; Raines, R. Angew. Chem. Int. Ed. 2008, 47, 7523-7526.

75. Sabourault, N.; Mignani, G.; Wagner, A.; Mioskowski, C. Org. Lett. 2002, 4, 2117 2119.

76. Jiang, H.; Rodrígues-Escrich, C.; Johansen, T. K.; Davis, R. L.; Jørgensen, K. A. Angew. Chem. Int. Ed. 2012, 51, 10271-10274.

77. Dittert, L. W.; Higuchi, T. J. Pharm. Sci. 1963, 52, 852-857.

78. Chen, C.-C.; Wu, C.-J.; Yeh, M.-K. Int. J. Nanotechnol. 2013, 10, 840-849.

79. Waldo, J. P.; Larock, R. C. Org. Lett. 2005, 7, 5203-5205. 


\section{R.5. CHAPTER 5 REFERENCES}

1. Alfeeli, B.; Cho, D.; Ashraf-Khorassani, M.; Taylor, L. T.; Agah, M. Sens. Actuators 2008, 133, 24-32.

2. Alfeeli, B.; Agah, M. IEEE Sens. J. 2009, 9, 1068-1075.

3. Voiculescu, I.; Zaghloul, M.; Harasimhan, N. Trends Anal. Chem. 2008, 27, 327-343.

4. Biswas, S.; Huang, X.; Badger, W. R.; Nantz, M. H. Tet. Lett. 2010, 51, 17271729.

5. Revell, J. D.; Dörner, B.; White, P. D.; Ganesan, A. Org. Lett. 2005, 7, 831-833.

6. Ohmura, N.; Nakamura, A.; Hamasaki, A.; Tokunaga, M. Eur. J. Org. Chem. 2008, 5042-5045.

7. Sethu, P.; Moldawer, L. L.; Mindrinos, M. N.; Scumpia, P. O.; Tannahill, C. L.; Wilhelmy, J.; Efron, P. A.; Brownstein, B. H.; Tompkins, R.G.; Toner, M. Anal. Chem. 2006, 78, 5453-5461.

8. Galeotti, F.; Bertini, F.; Scavia, G.; Bolognesi, A. J. Colloid Interface Sci. 2011, $360,540-547$.

9. Pinho, S.; Pereira, G.; Voisin, P.; Kassem, J.; Bouchaud, V.; Etienne, L.; Peters, J.; Carlos, L.; Mornet, S.; Geraldes, C.; Rocha, J.; Delville, M.-H. ACS Nano 2010, 4, 5339-5349.

10. Heller, S. T.; Schultz, E. E.; Sarpong, R. Angew. Chem., Int. Ed. 2012, 51, 83048308 .

11. Jiang, H.; Rodrígues-Escrich, C.; Johansen, T. K.; Davis, R. L.; Jørgensen, K. A. Angew. Chem. Int. Ed. 2012, 51, 10271-10274.

12. Sabourault, N.; Mignani, G.; Wagner, A.; Mioskowski, C. Org. Lett. 2002, 4, 2117-2119. 
APPENDIX A

SPECTRA

A.1. Index of NMR Spectra and Other Spectroscopic Data

A.2. Selected NMR Spectra From Chapter 2

A.3. Selected NMR Spectra From Chapter 3

A.4. Selected NMR and FT-IR Spectra From Chapter 4 


\section{A.1. Index Of NMR Spectra}

A.2. ${ }^{1} \mathrm{H}$ NMR Spectrum of ATM 248

${ }^{13} \mathrm{C}$ NMR Spectrum of ATM 249

${ }^{1} \mathrm{H}$ NMR Spectrum of AMAH 250

${ }^{13} \mathrm{C}$ NMR Spectrum of AMAH 251

${ }^{1} \mathrm{H}$ NMR Spectrum of ADMH 252

${ }^{13} \mathrm{C}$ NMR Spectrum of ADMH 253

${ }^{1} \mathrm{H}$ NMR Spectrum of AAn $\quad 254$

${ }^{13}$ C NMR Spectrum of AAn 255

A.3. ${ }^{1}$ H NMR Spectrum of 5

${ }^{13} \mathrm{C}$ NMR Spectrum of 5

${ }^{1}$ H NMR Spectrum of $\mathbf{1 4 . 1} 258$

${ }^{13}$ C NMR Spectrum of $\mathbf{1 4 . 1} 259$

${ }^{1} \mathrm{H}$ NMR Spectrum of $\mathbf{1 4 . 2} \quad 260$

${ }^{13} \mathrm{C}$ NMR Spectrum of $\mathbf{1 4 . 2} 261$

${ }^{1} \mathrm{H}$ NMR Spectrum of $\mathbf{1 4 . 3} 262$

${ }^{13} \mathrm{C}$ NMR Spectrum of $\mathbf{1 4 . 3} 263$

${ }^{1} \mathrm{H}$ NMR Spectrum of $\mathbf{1 4 . 4}$

${ }^{13}$ C NMR Spectrum of $\mathbf{1 4 . 4}$

${ }^{1}$ H NMR Spectrum of Boc-Protected 7.1 266

${ }^{13}$ C NMR Spectrum of Boc-Protected 7.1 267

${ }^{1}$ H NMR Spectrum of Boc-Protected 7.2 268

${ }^{13}$ C NMR Spectrum of Boc-Protected 7.2 269

${ }^{1}$ H NMR Spectrum of Boc-Protected 8

${ }^{13}$ C NMR Spectrum of Boc-Protected 8

${ }^{1}$ H NMR Spectrum of Boc-Protected $\mathbf{1 0} 272$

${ }^{13} \mathrm{C}$ NMR Spectrum of Boc-Protected 10

${ }^{1}$ H NMR Spectrum of Boc-Protected 11

${ }^{13}$ C NMR Spectrum of Boc-Protected 11

${ }^{1}$ H NMR Spectrum of Boc-Protected 27

${ }^{13}$ C NMR Spectrum of Boc-Protected 27 
$\begin{array}{lll}\text { A.4. } & { }^{1} \mathrm{H} \text { NMR Spectrum of } \mathbf{1 7 . 1} & 278\end{array}$

${ }^{13}$ C NMR Spectrum of $\mathbf{1 7 . 1} \quad 279$

${ }^{1} \mathrm{H}$ NMR Spectrum of $\mathbf{1 7 . 2} \quad 280$

${ }^{13}$ C NMR Spectrum of $\mathbf{1 7 . 2}$

${ }^{1} \mathrm{H}$ NMR Spectrum of $\mathbf{1 7 . 3} \quad 282$

${ }^{13}$ C NMR Spectrum of $\mathbf{1 7 . 3} \quad 283$

${ }^{1} \mathrm{H}$ NMR Spectrum of $\mathbf{2 3} \quad 284$

${ }^{13} \mathrm{C}$ NMR Spectrum of $\mathbf{2 3} \quad 285$

${ }^{1}$ H NMR Spectrum of 23 After Oximation With 2-(9-anthryl) 286 acetaldehyde

${ }^{13}$ C NMR Spectrum of 23 After Oximation With 2-(9-anthryl) 287 acetaldehyde

FT-IR Spectrum of FL@SiO $\mathbf{S i}_{\mathbf{2}} \mathbf{F e}_{3} \mathbf{O}_{4}$

${ }^{1} \mathrm{H}$ NMR Spectrum of $\mathbf{2 8} \quad 289$

${ }^{13}$ C NMR Spectrum of $\mathbf{2 8} 290$ 


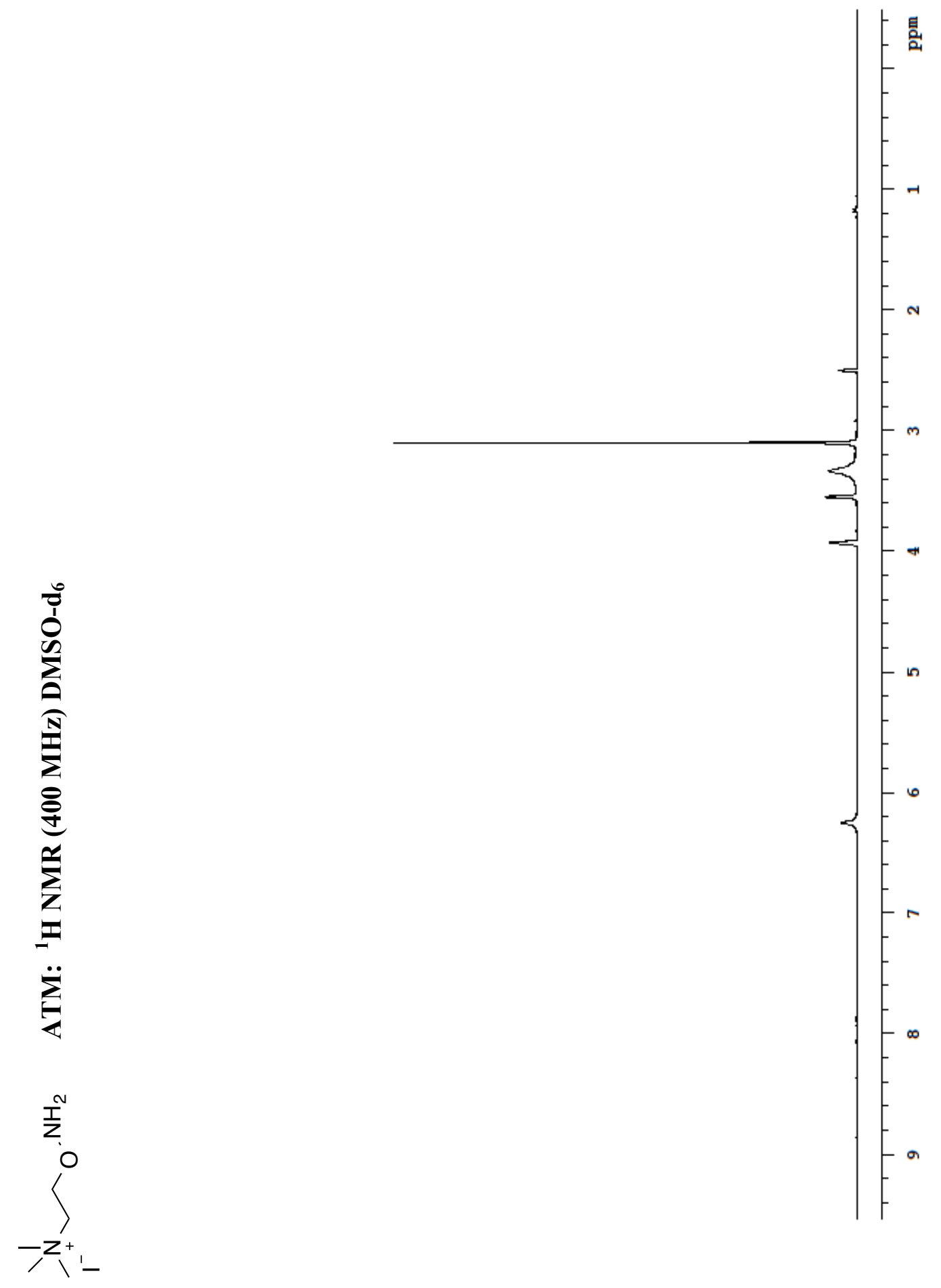




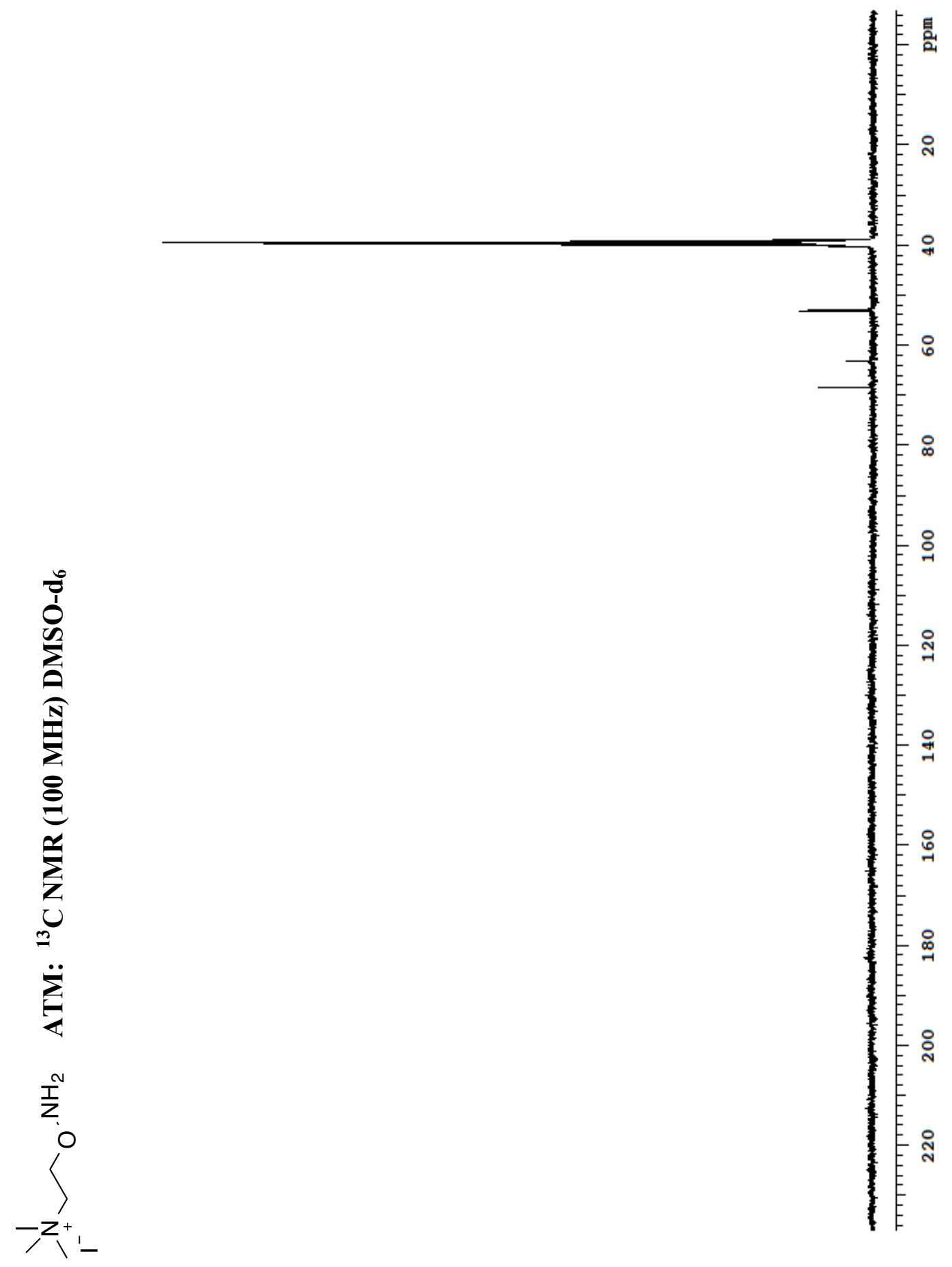




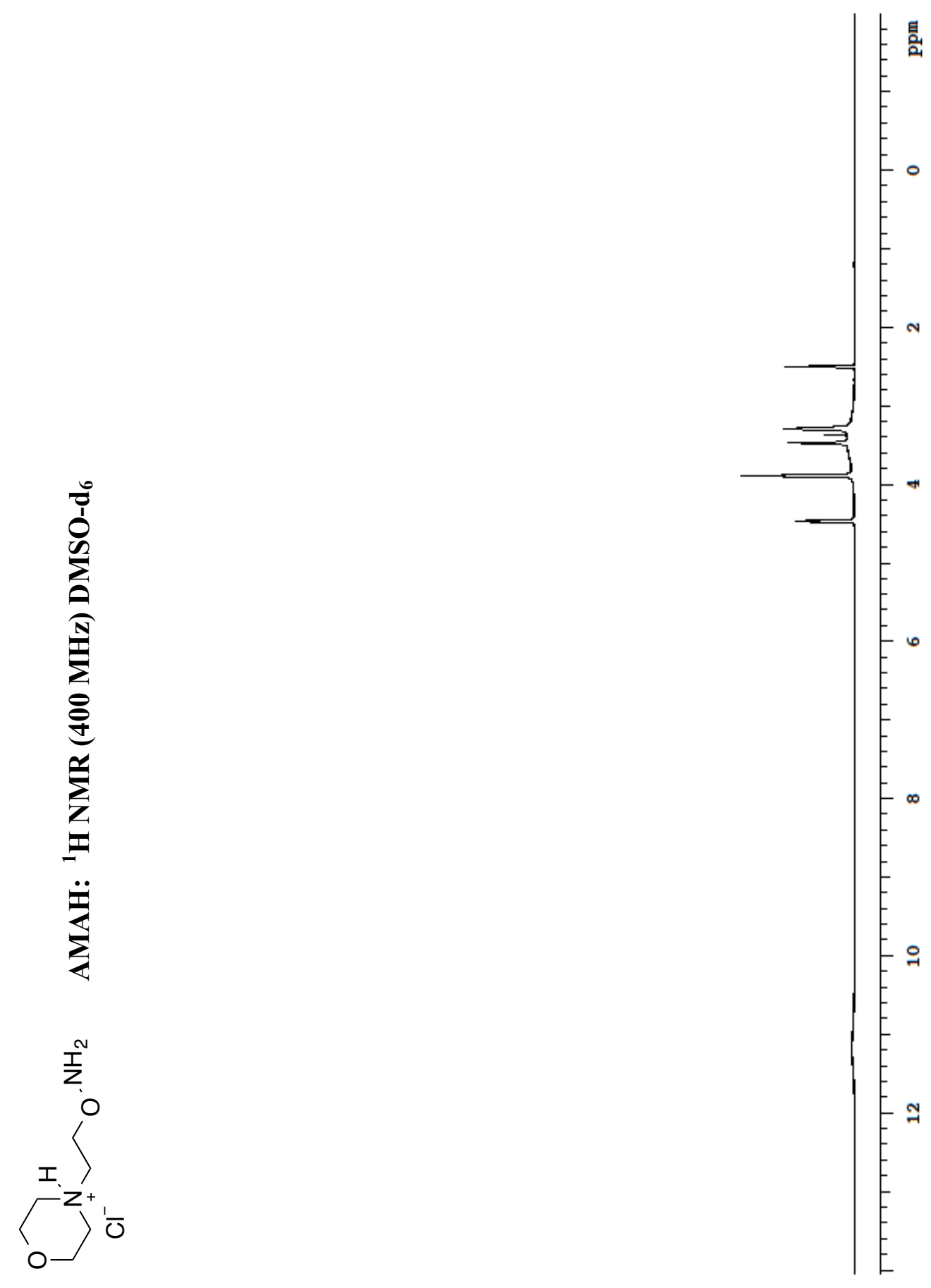




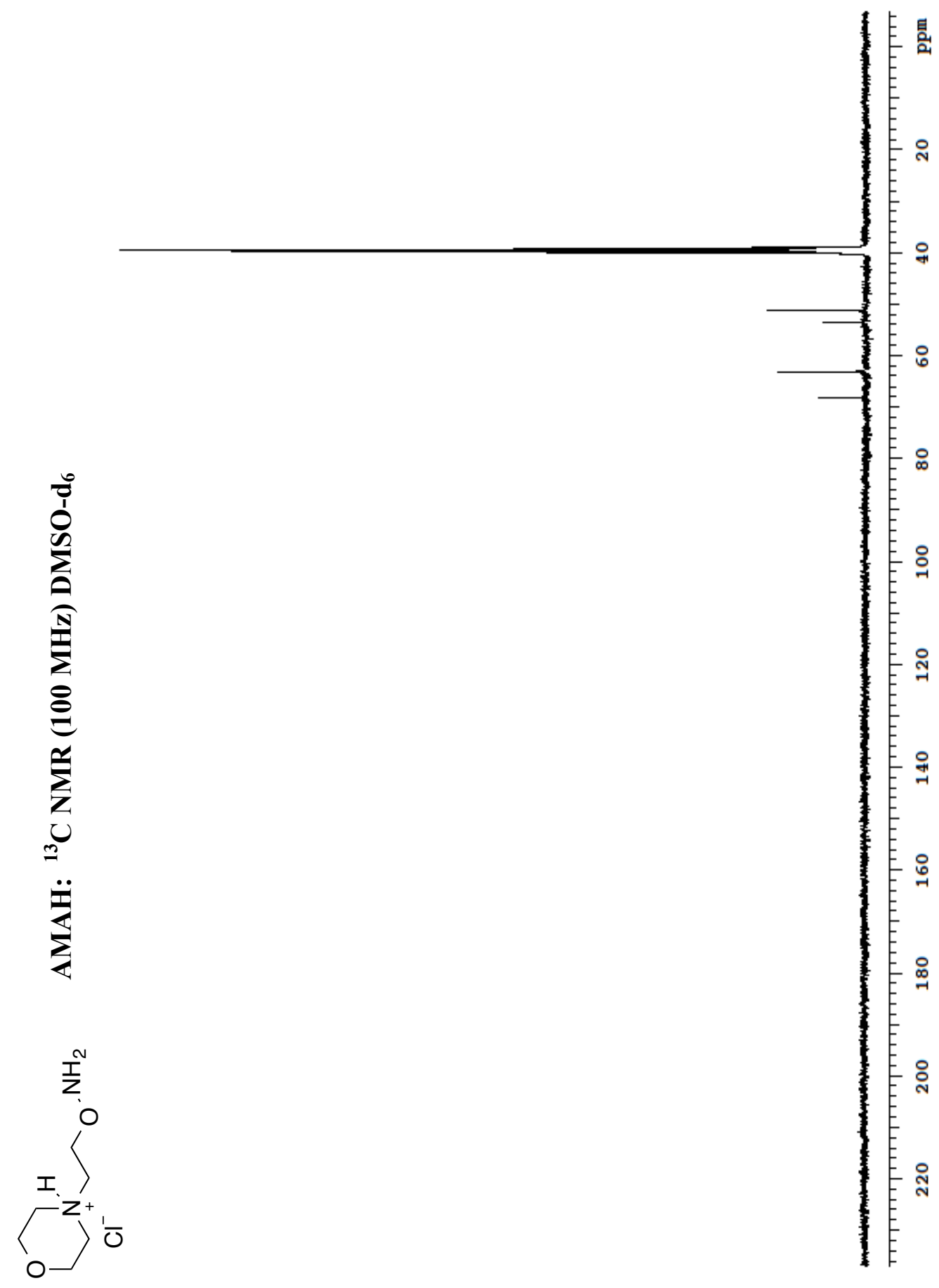




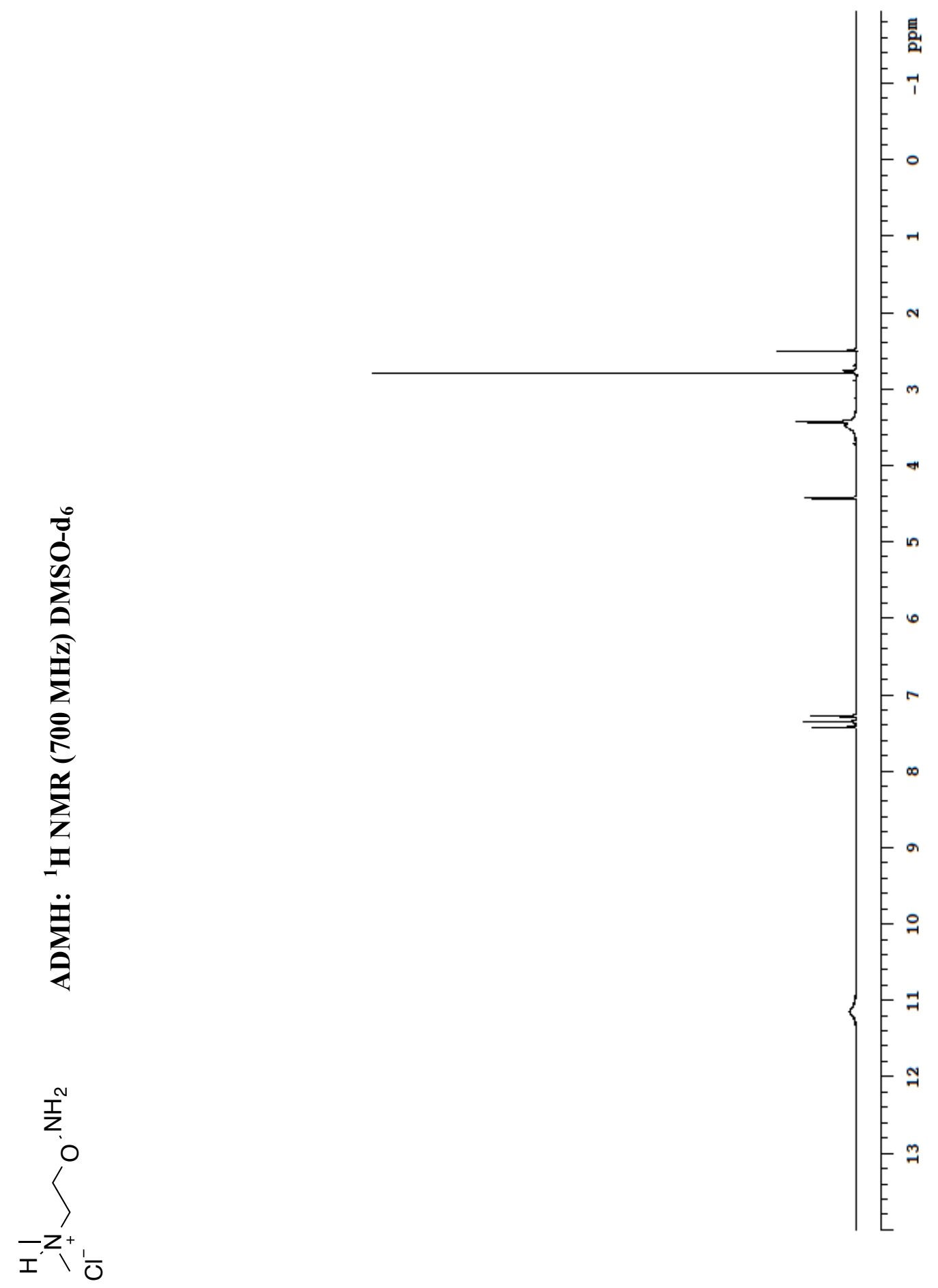




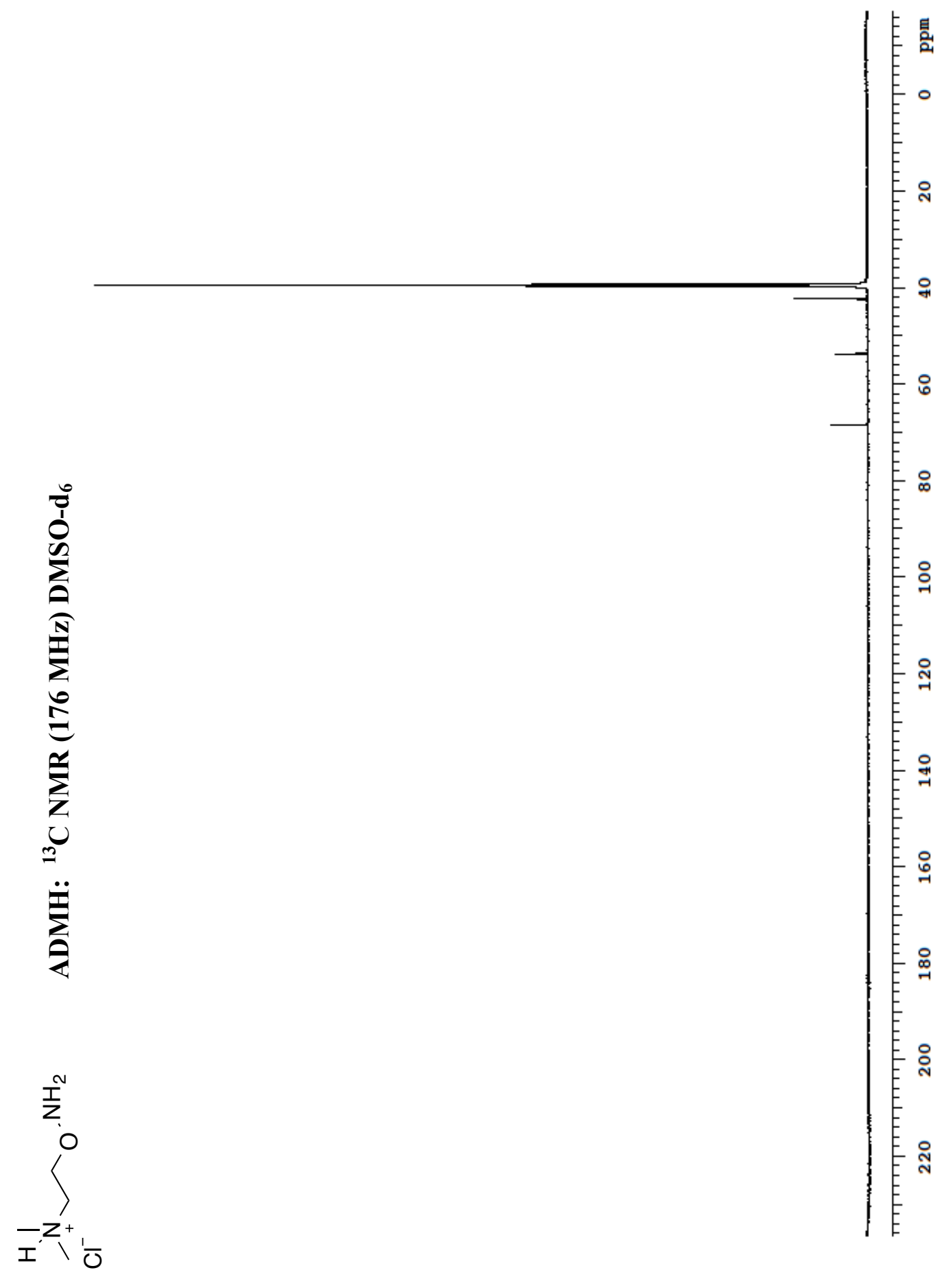




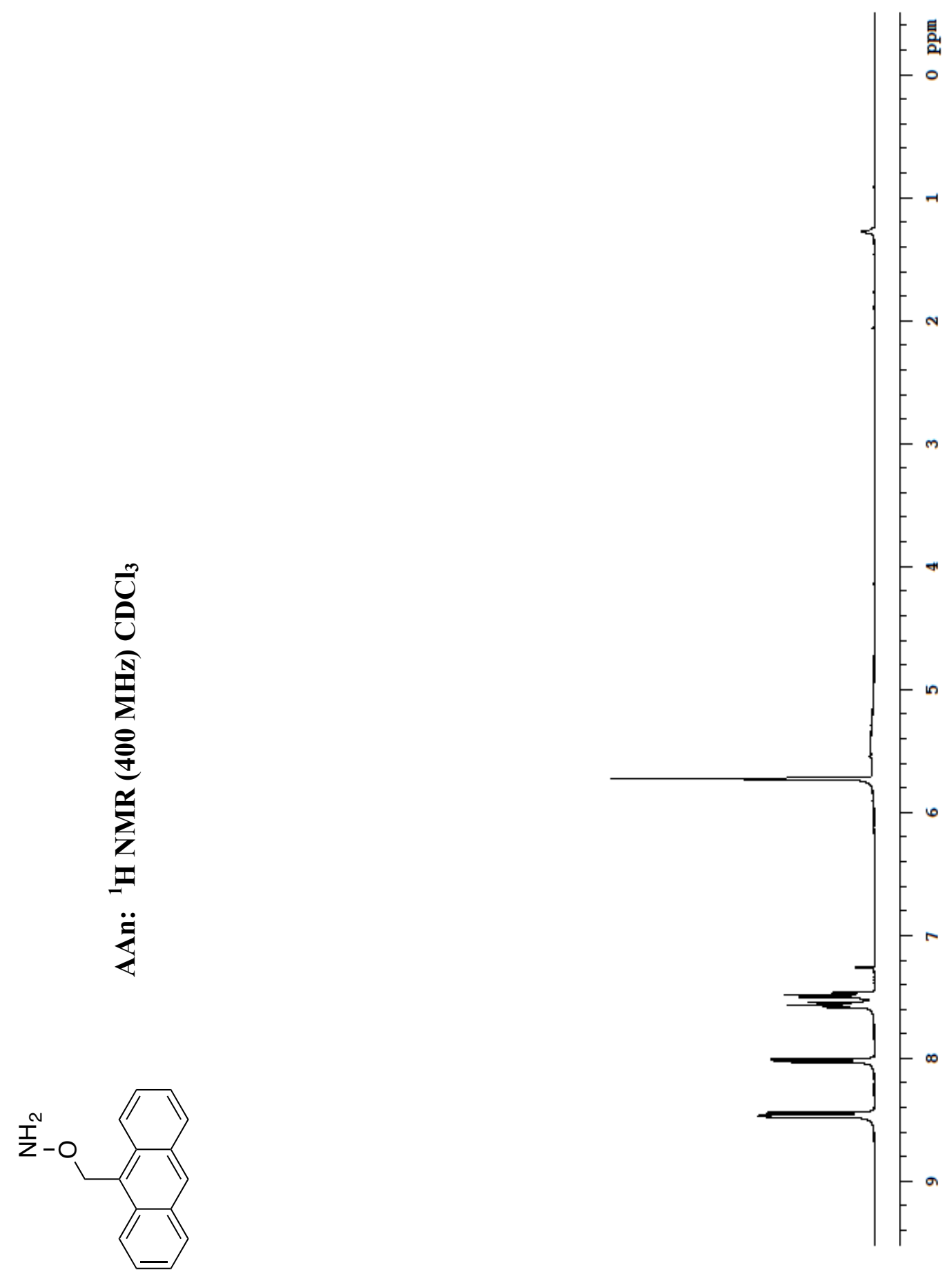




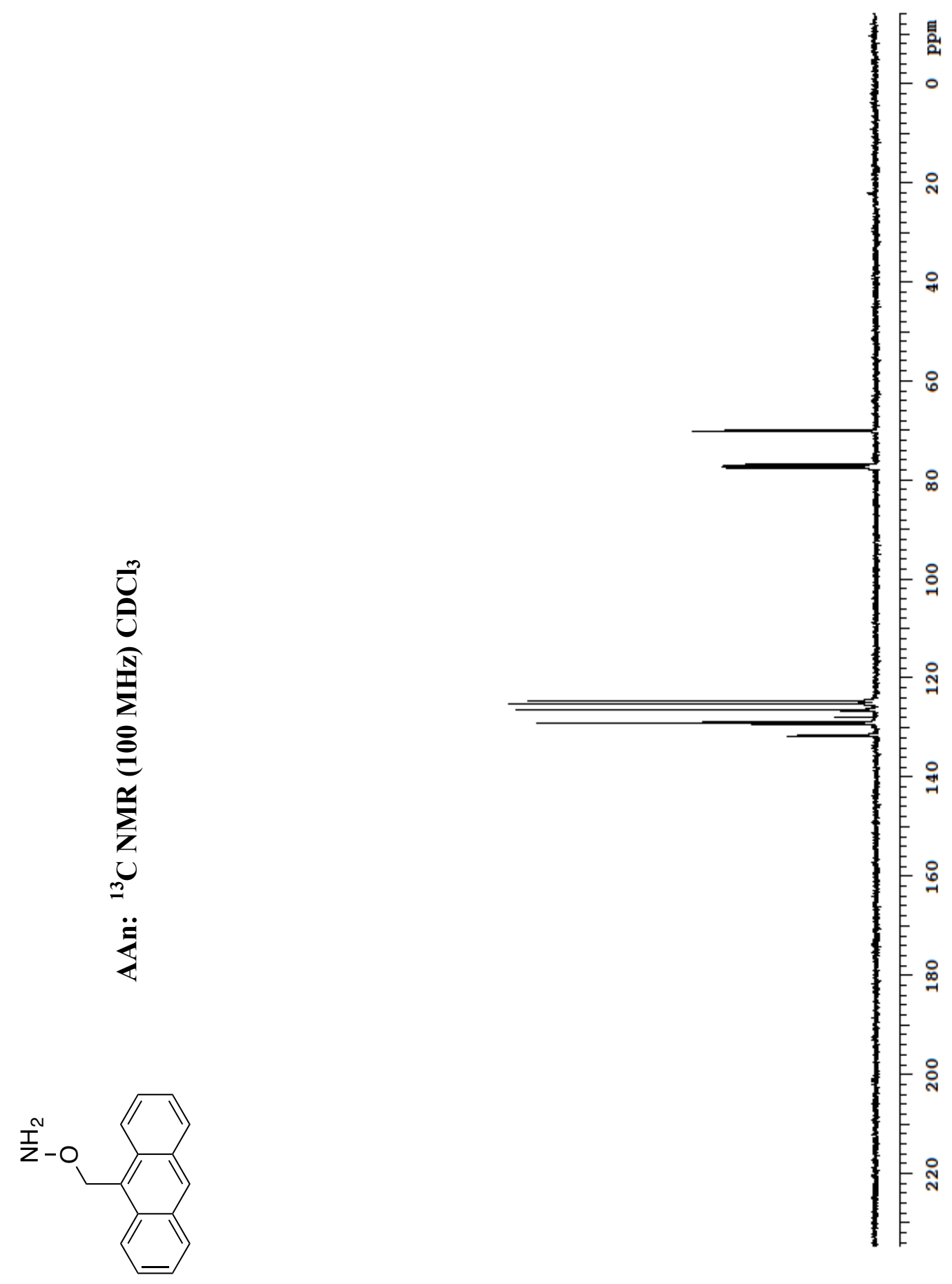




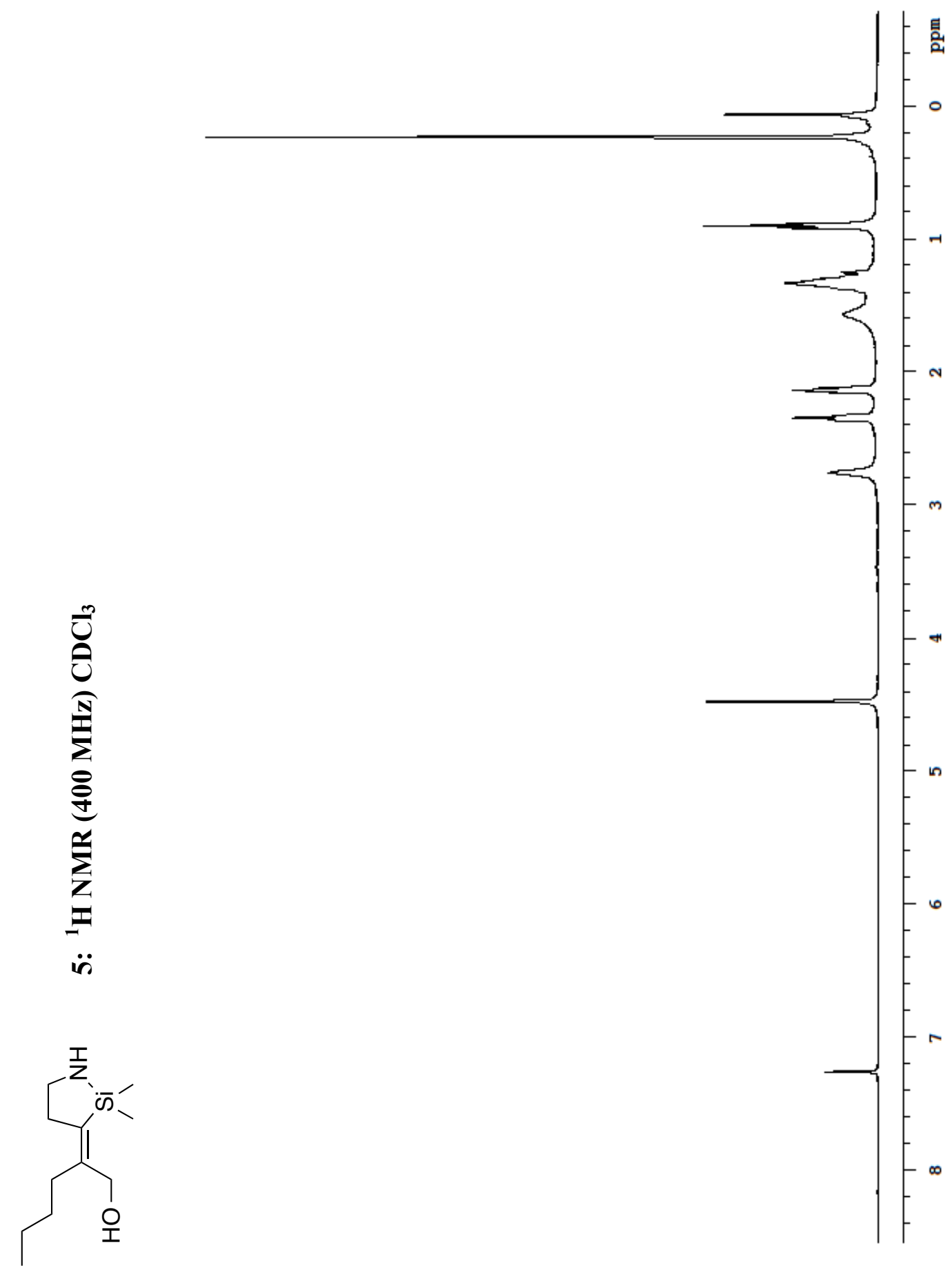



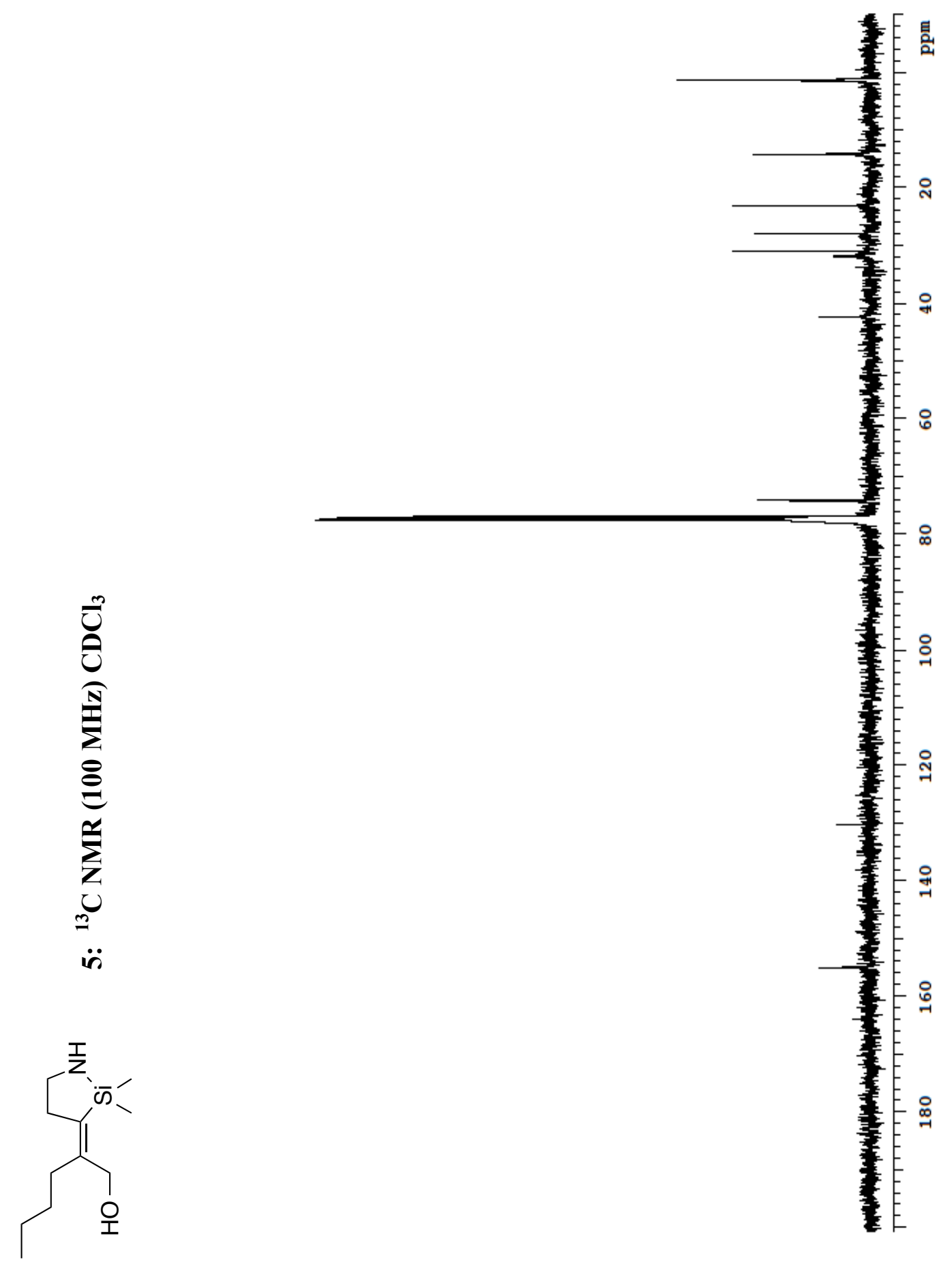


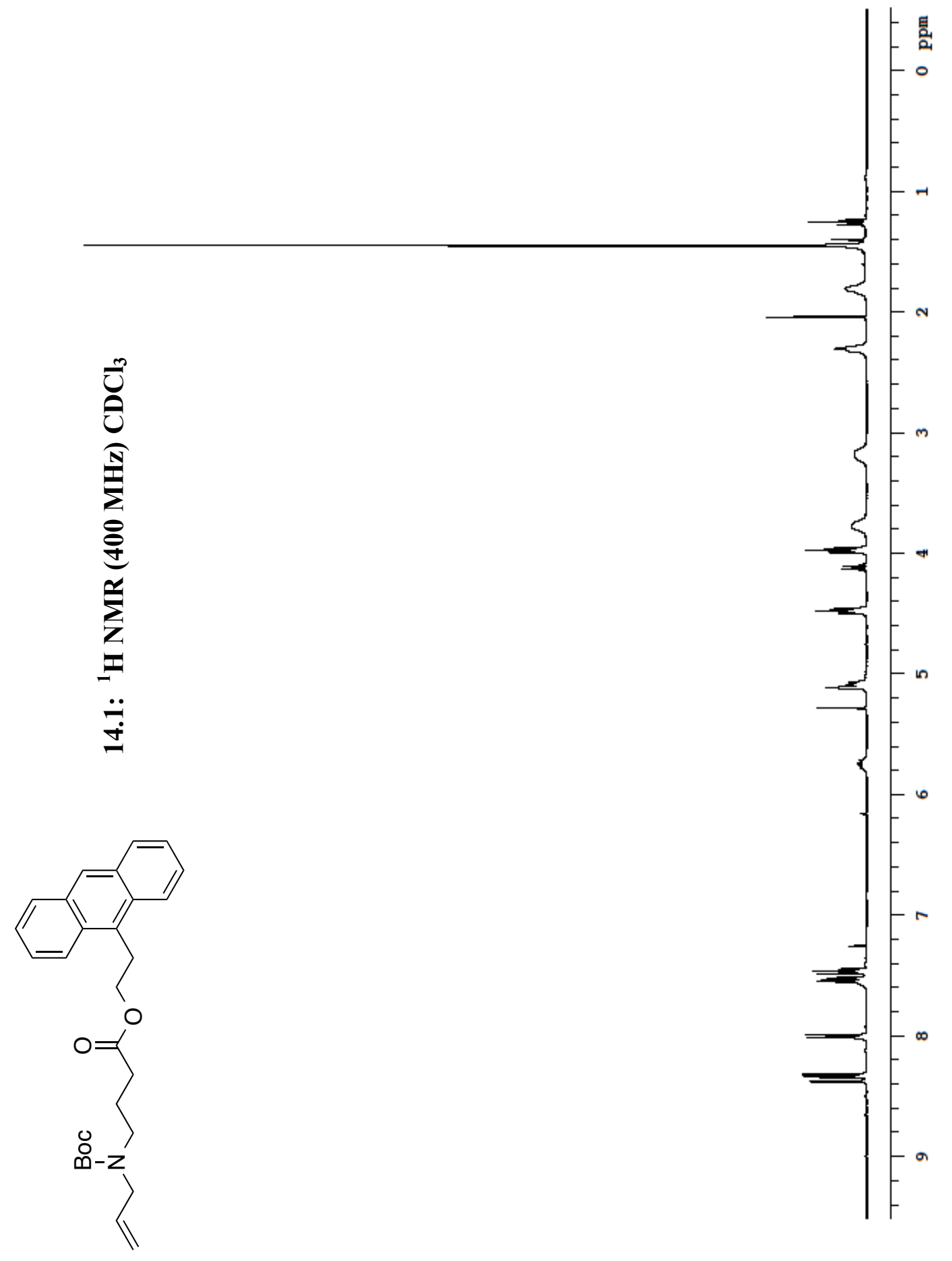




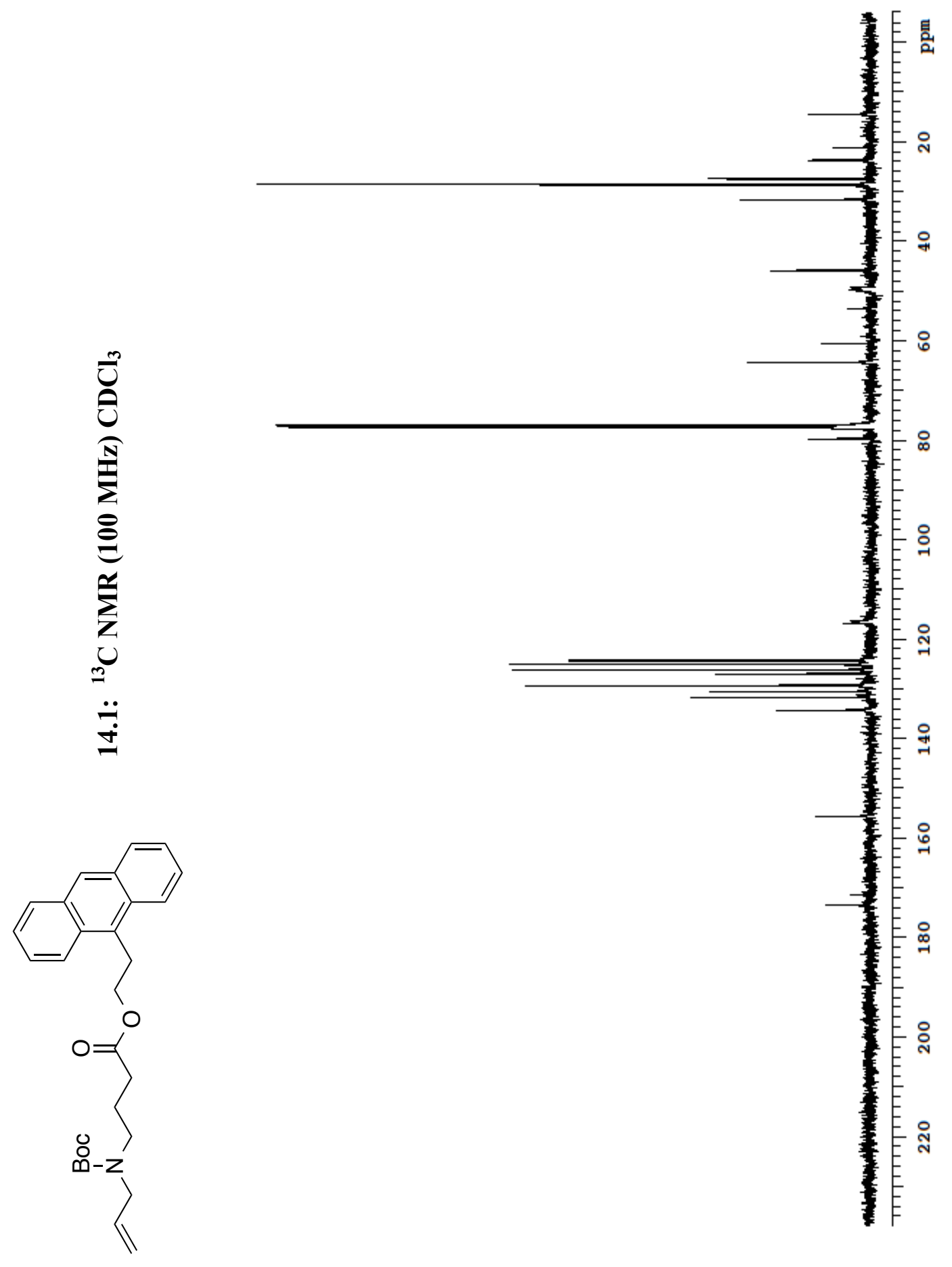




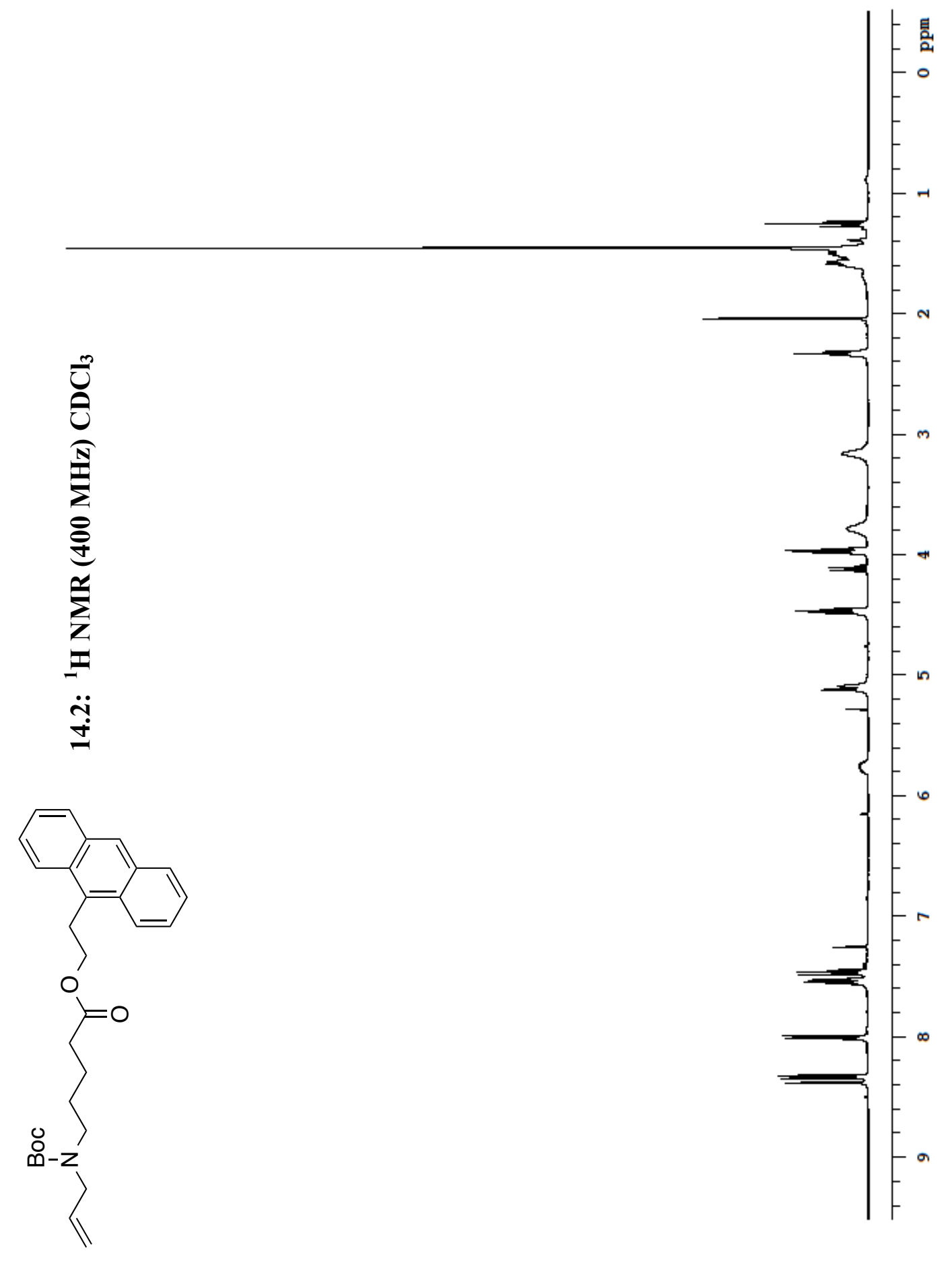



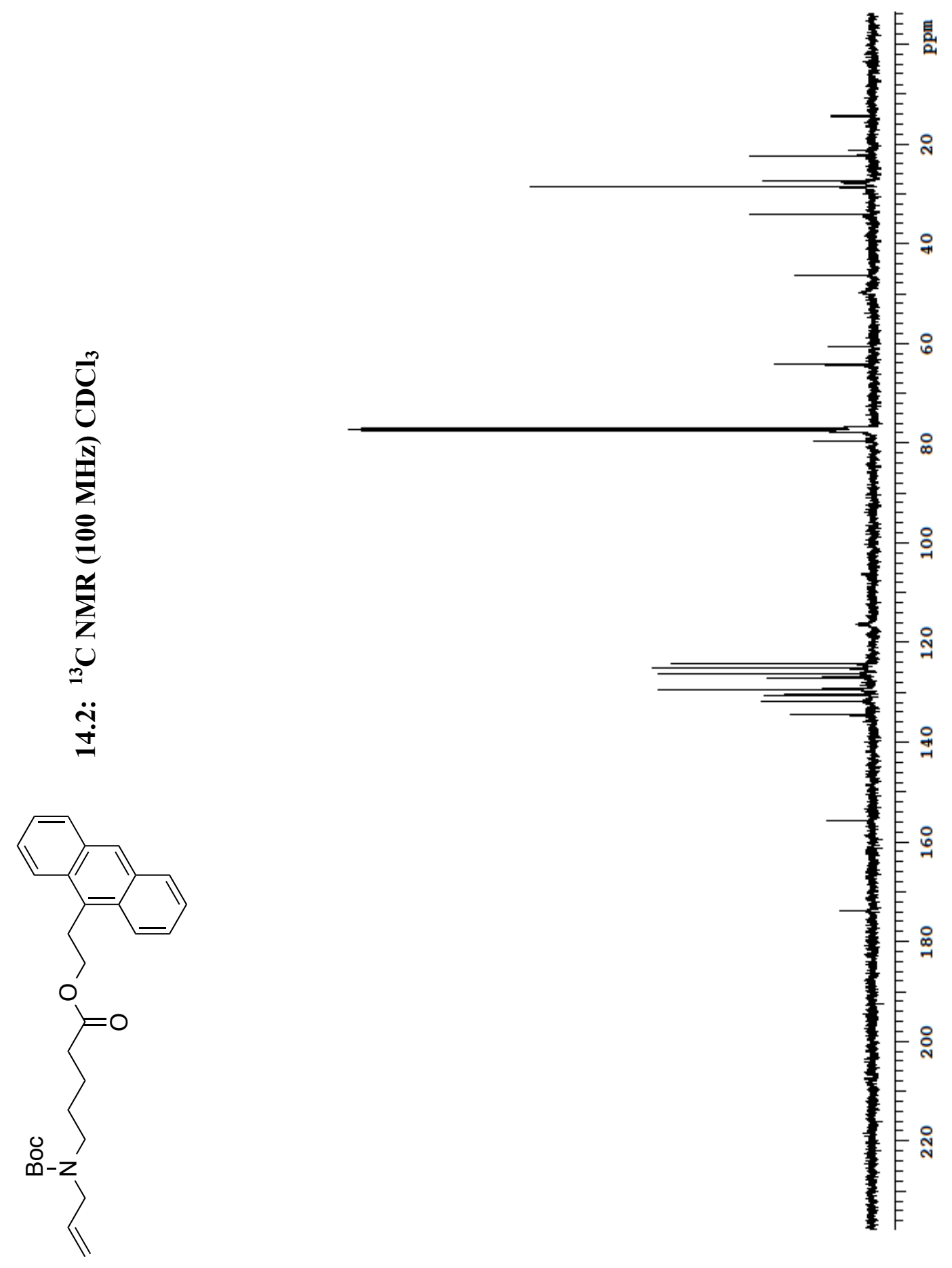


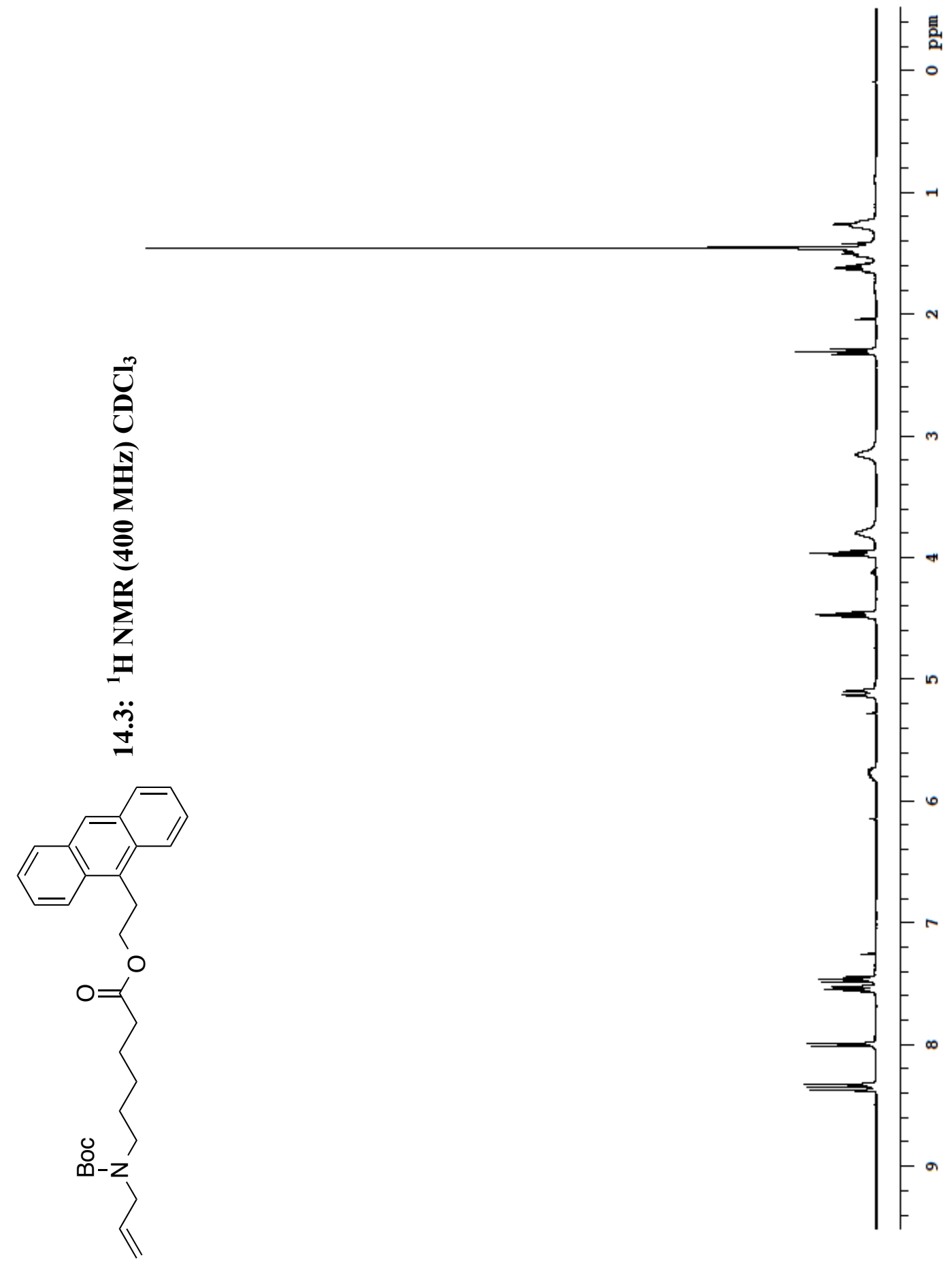




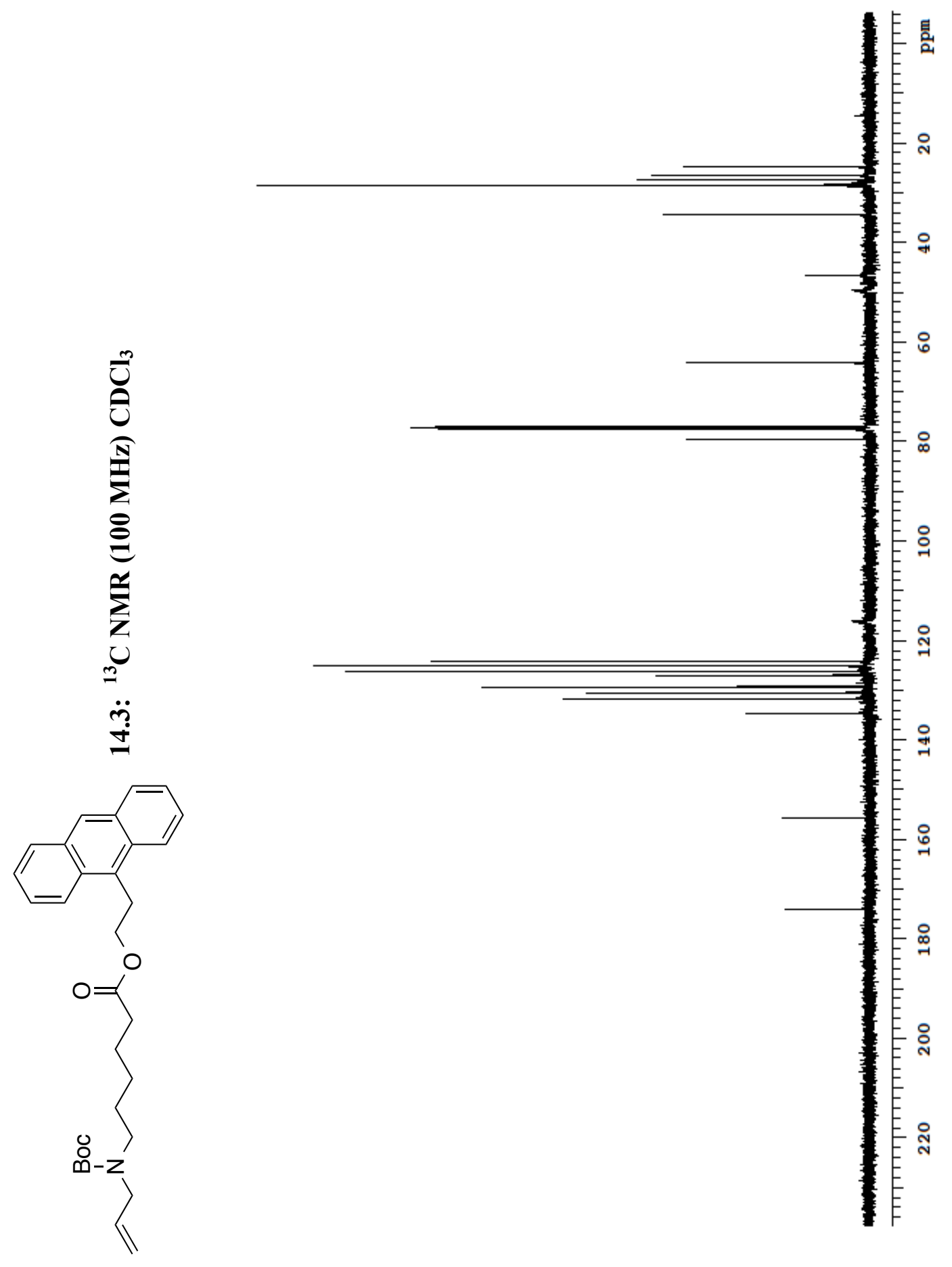




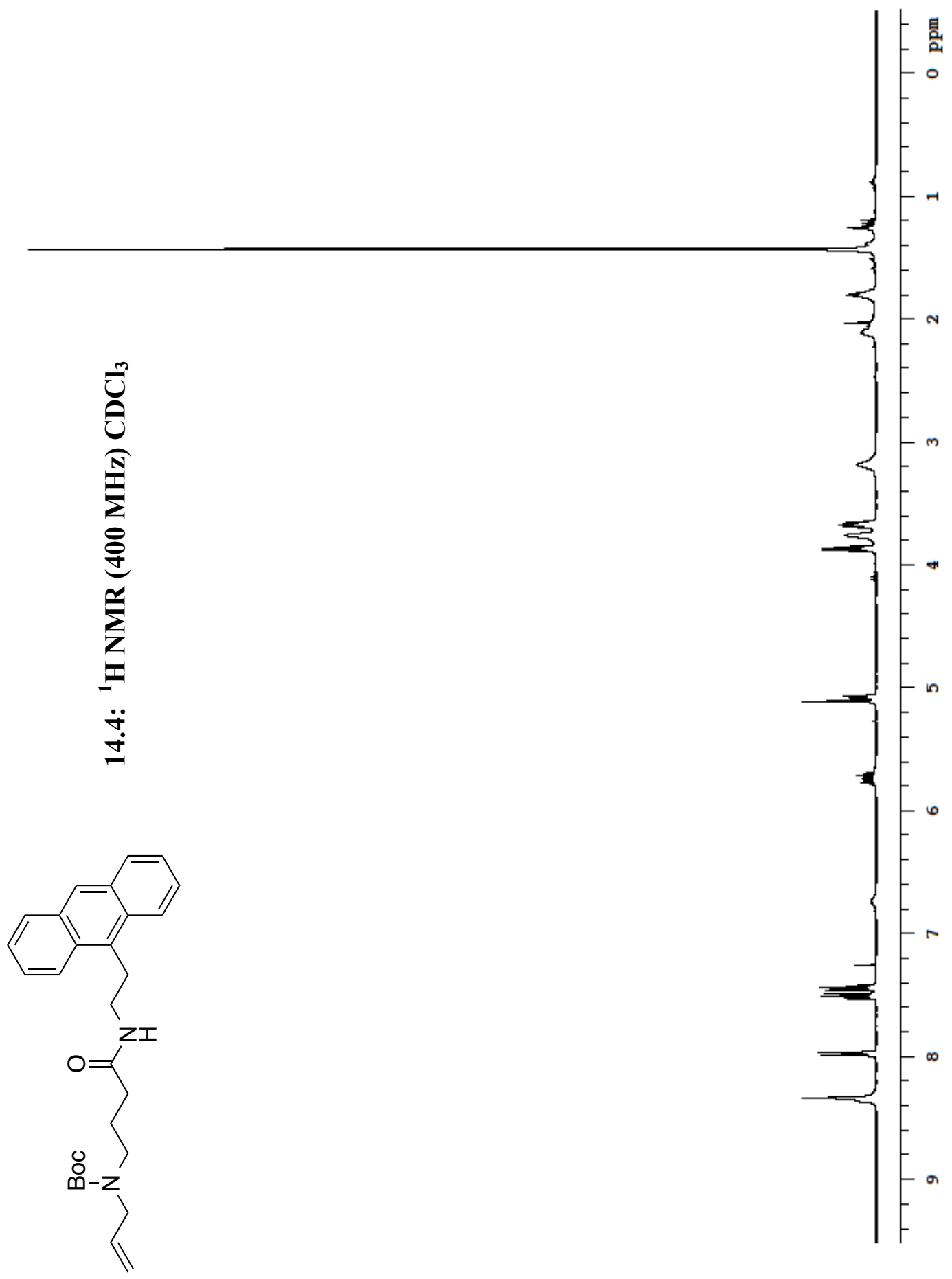




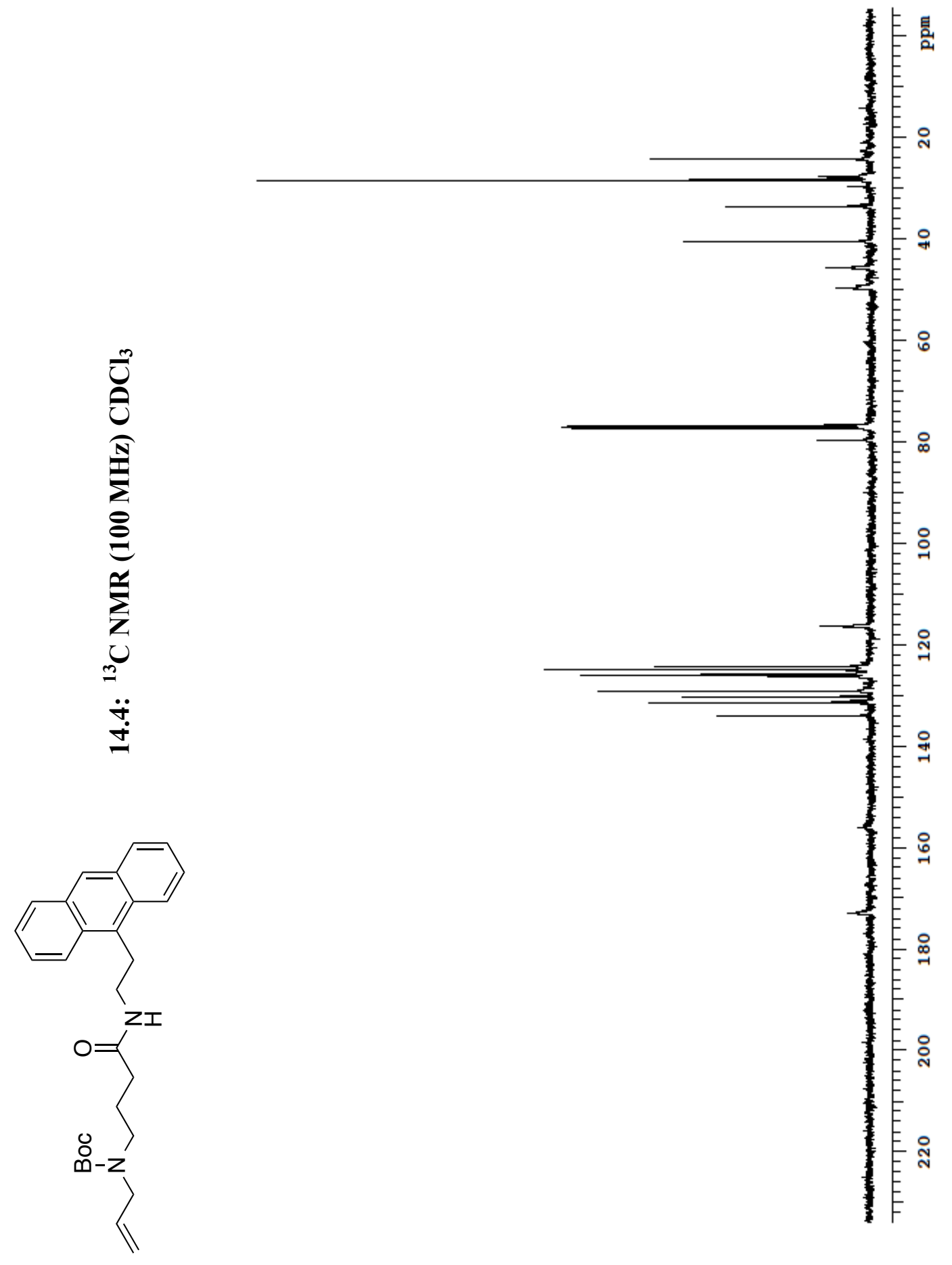




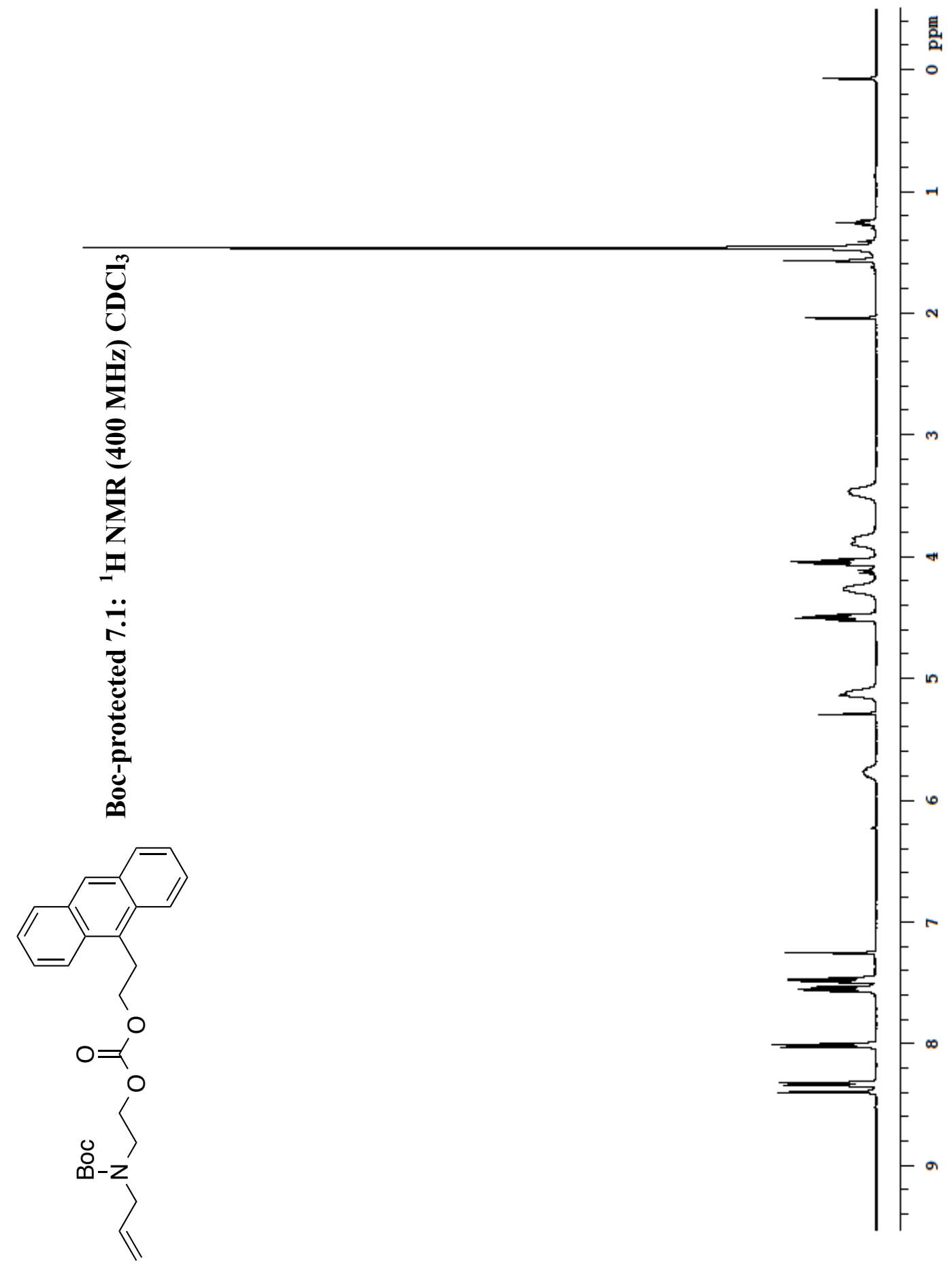




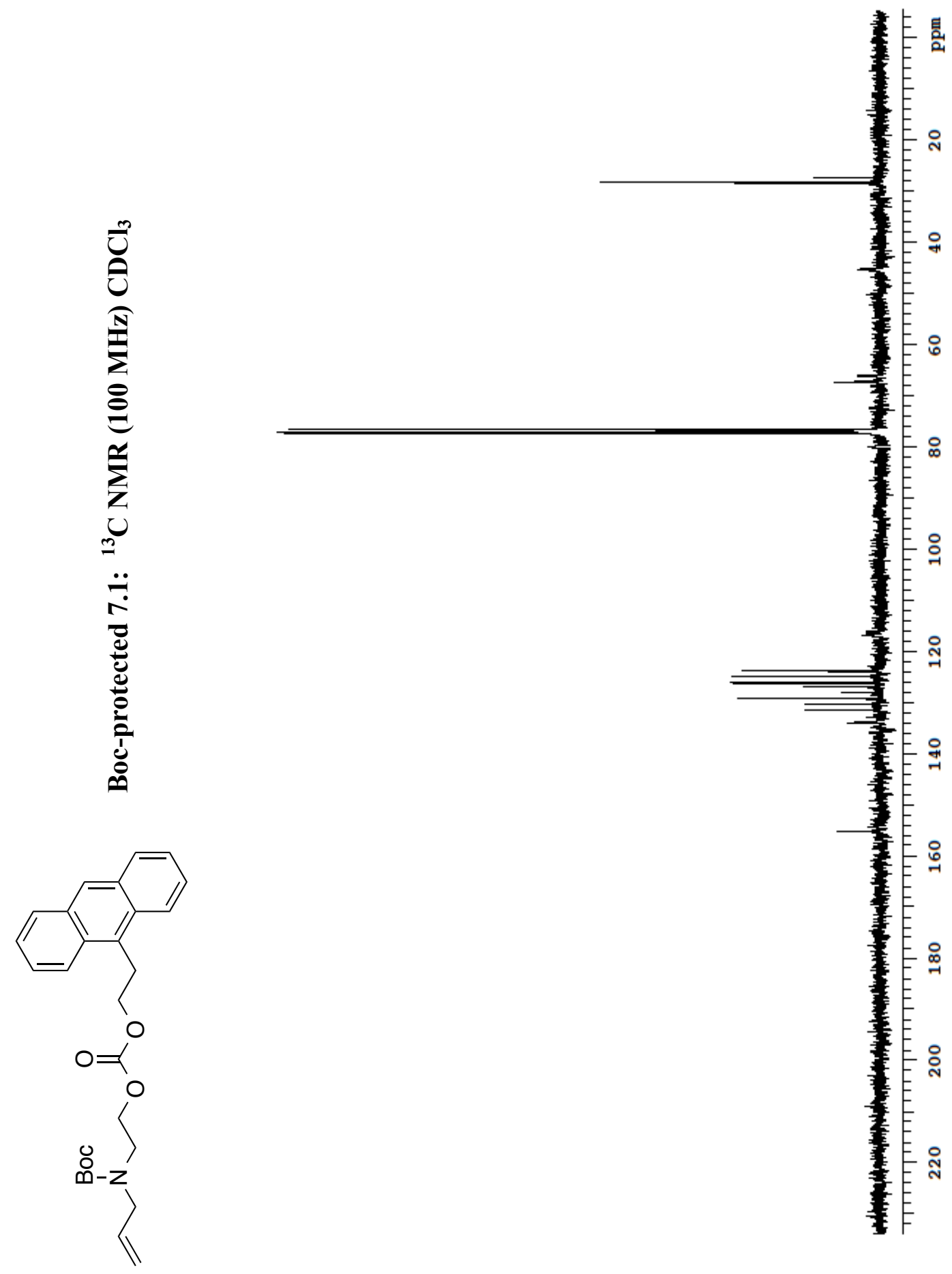




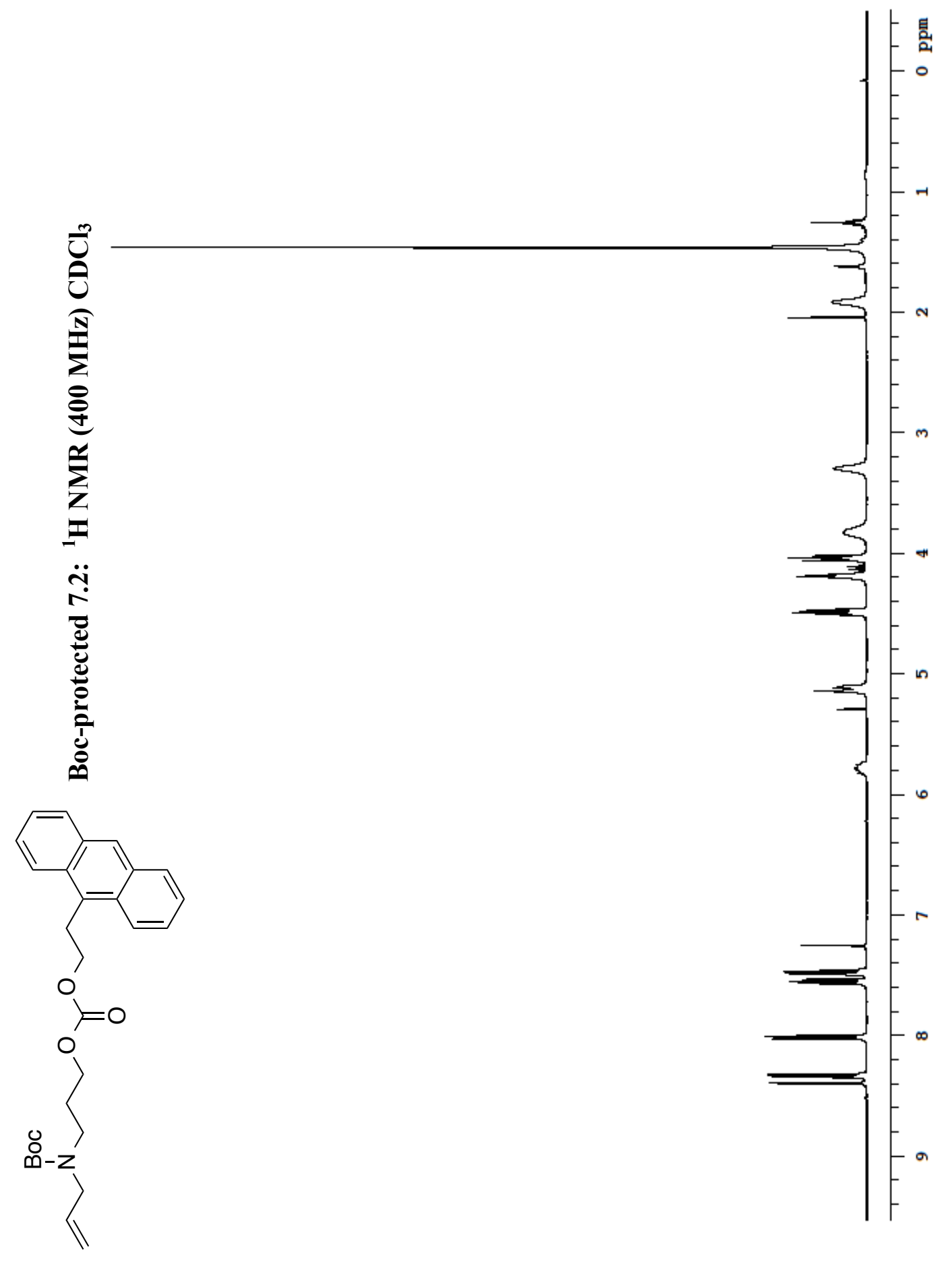




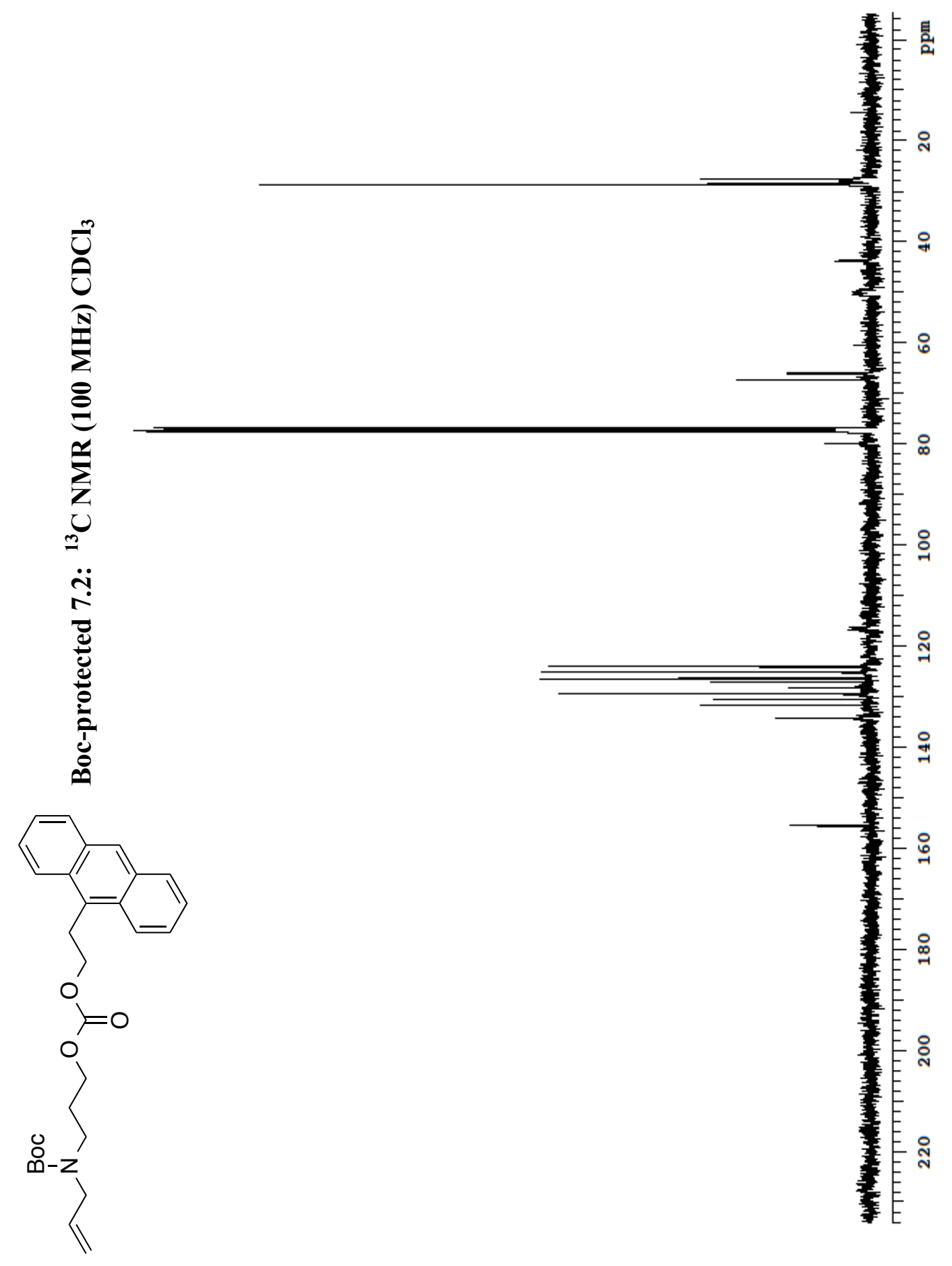




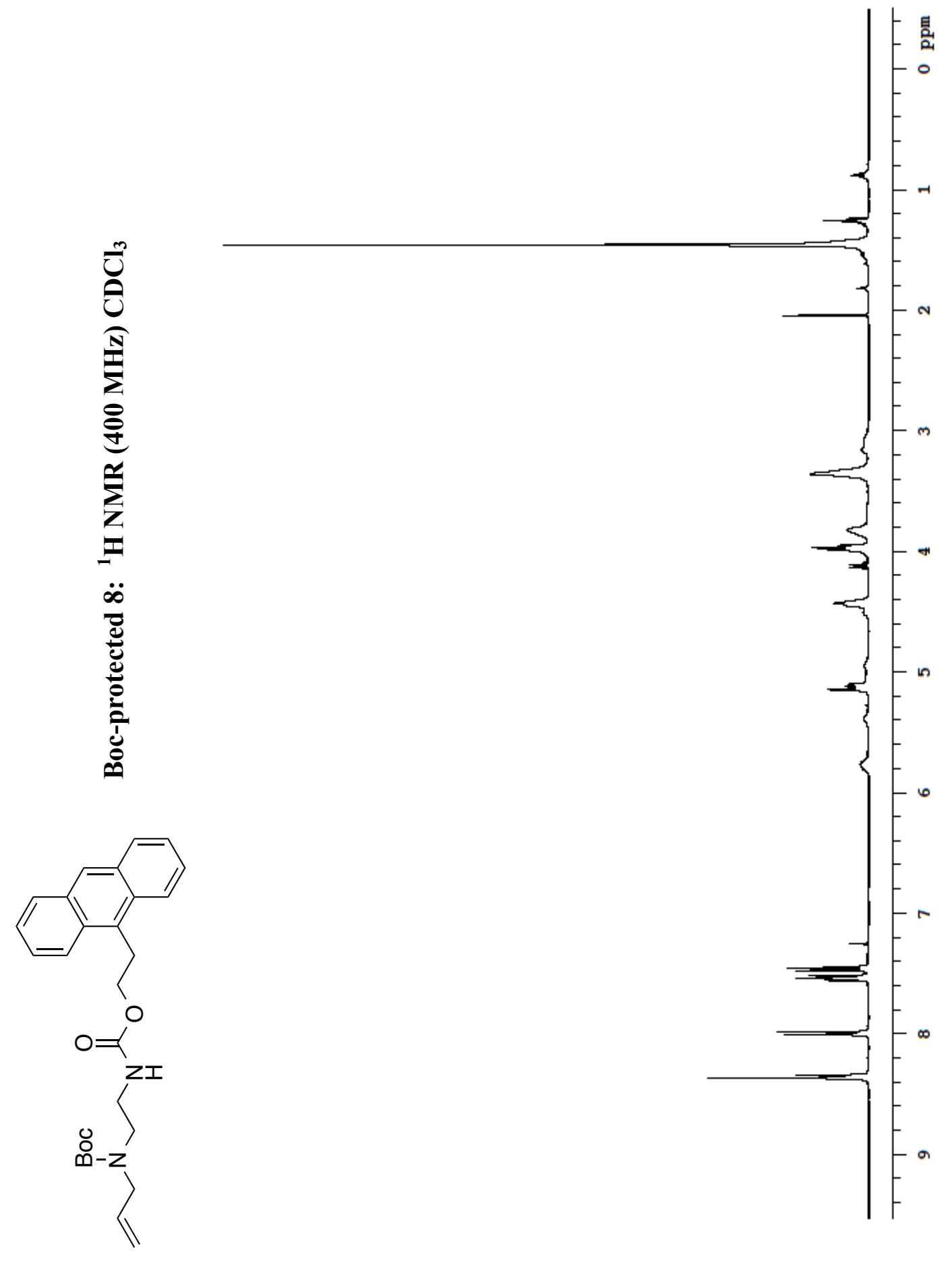




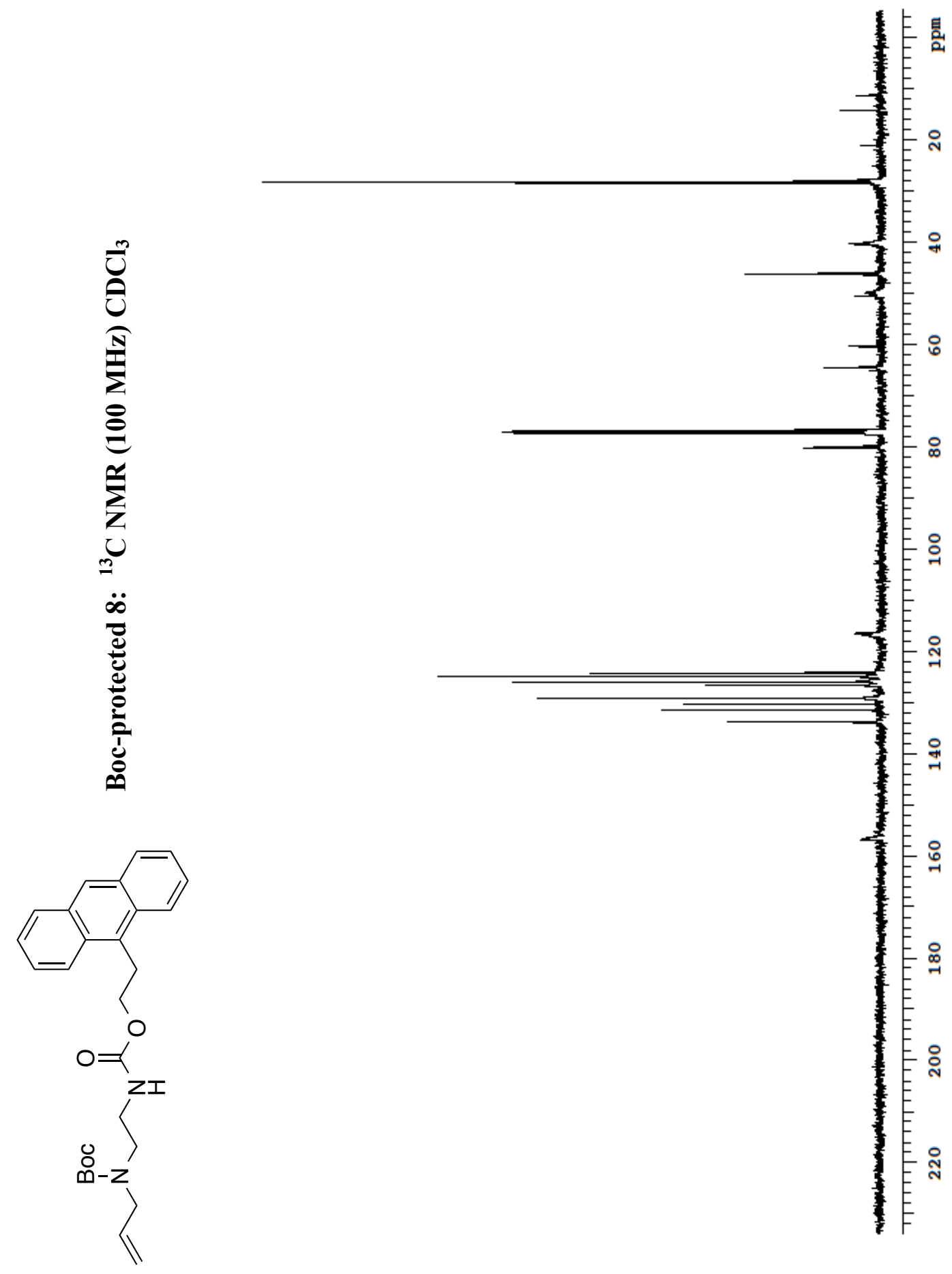




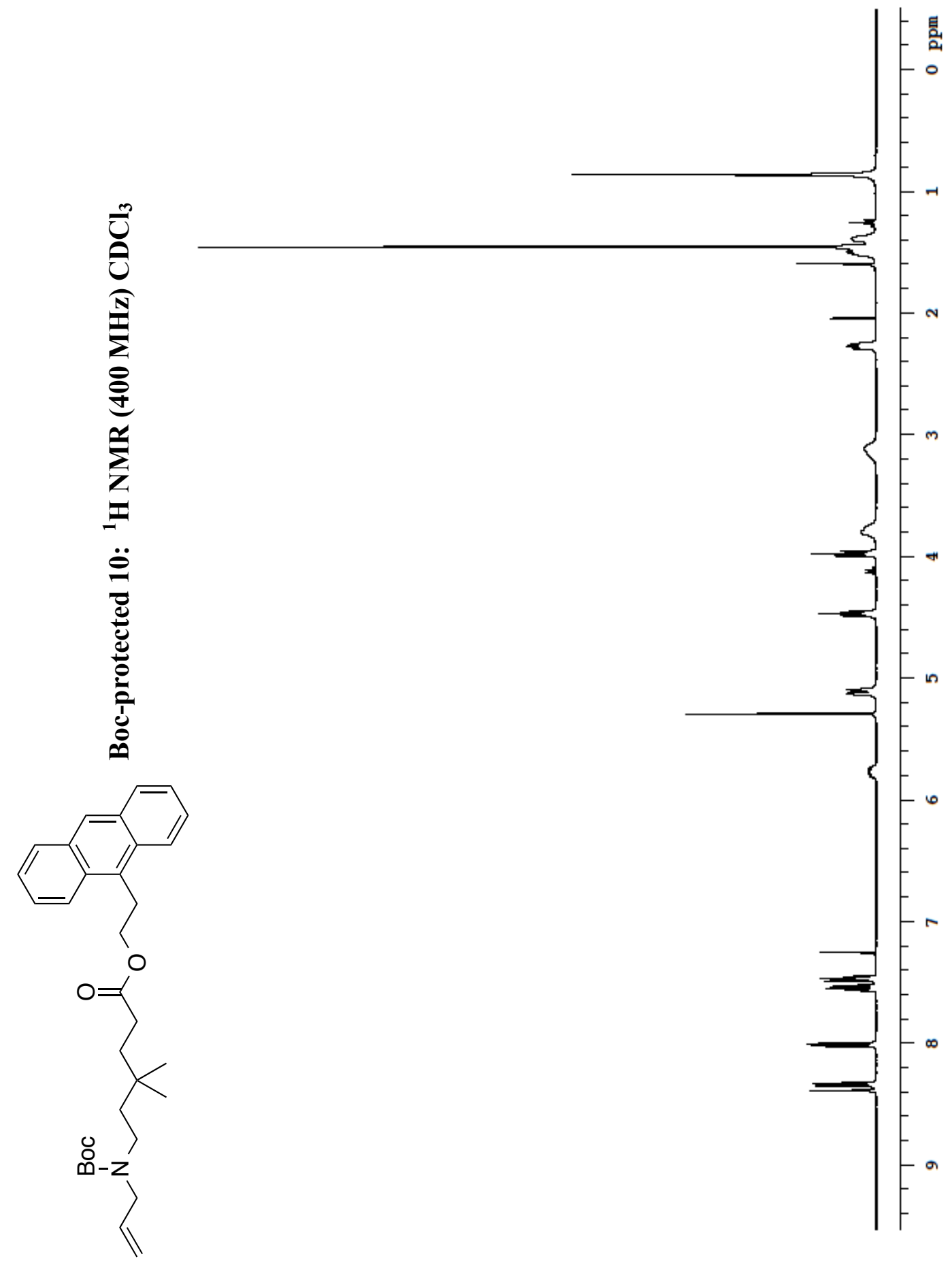




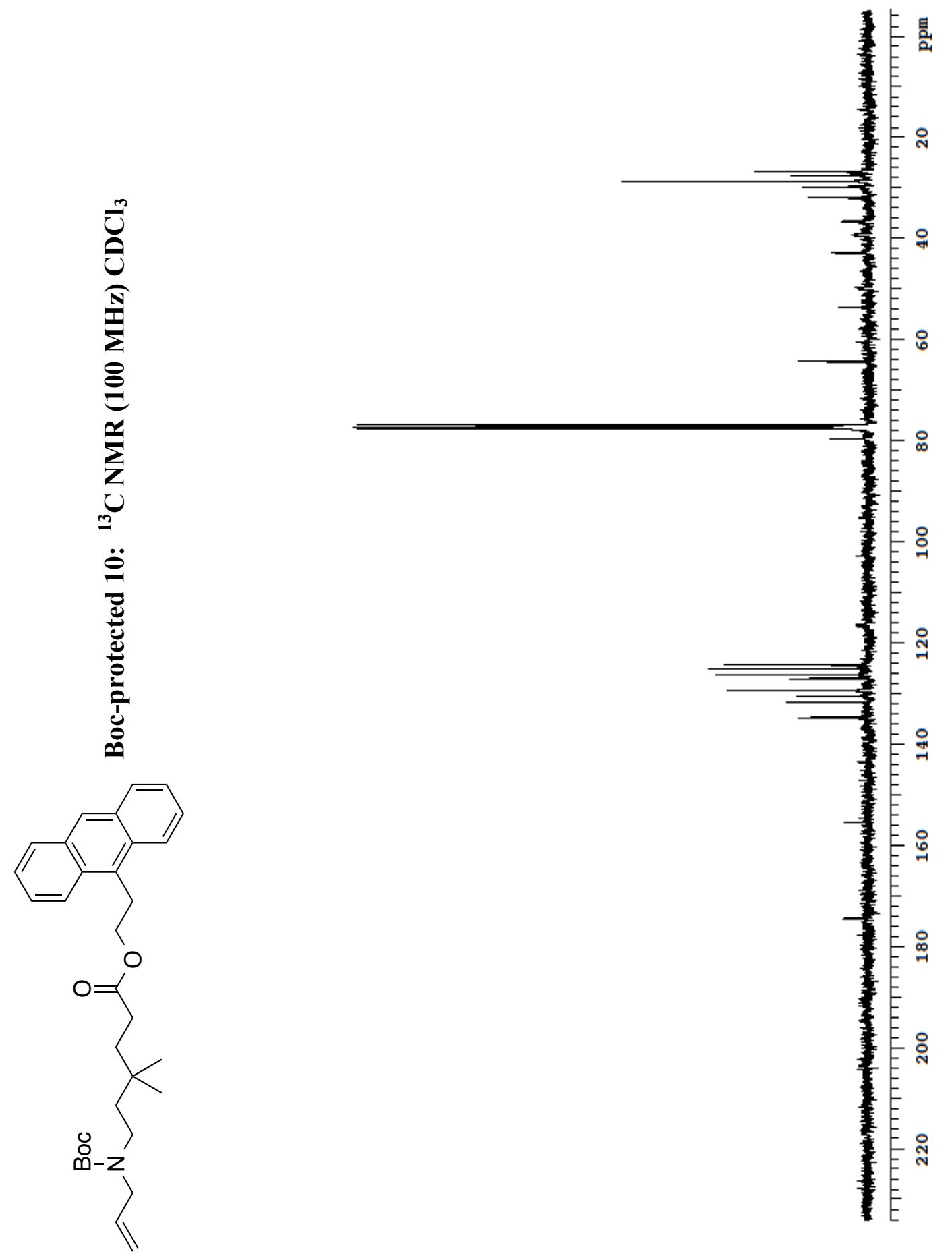




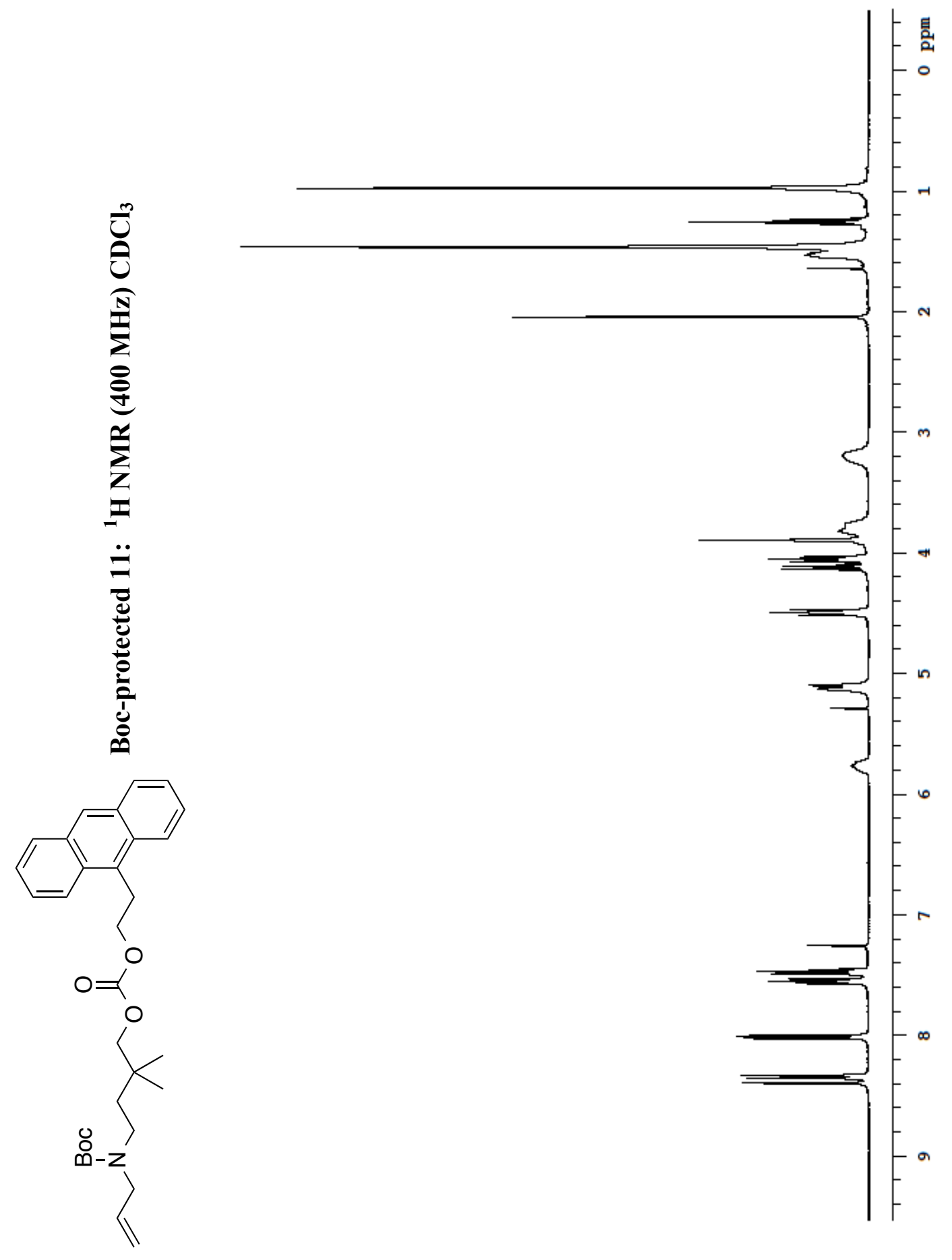



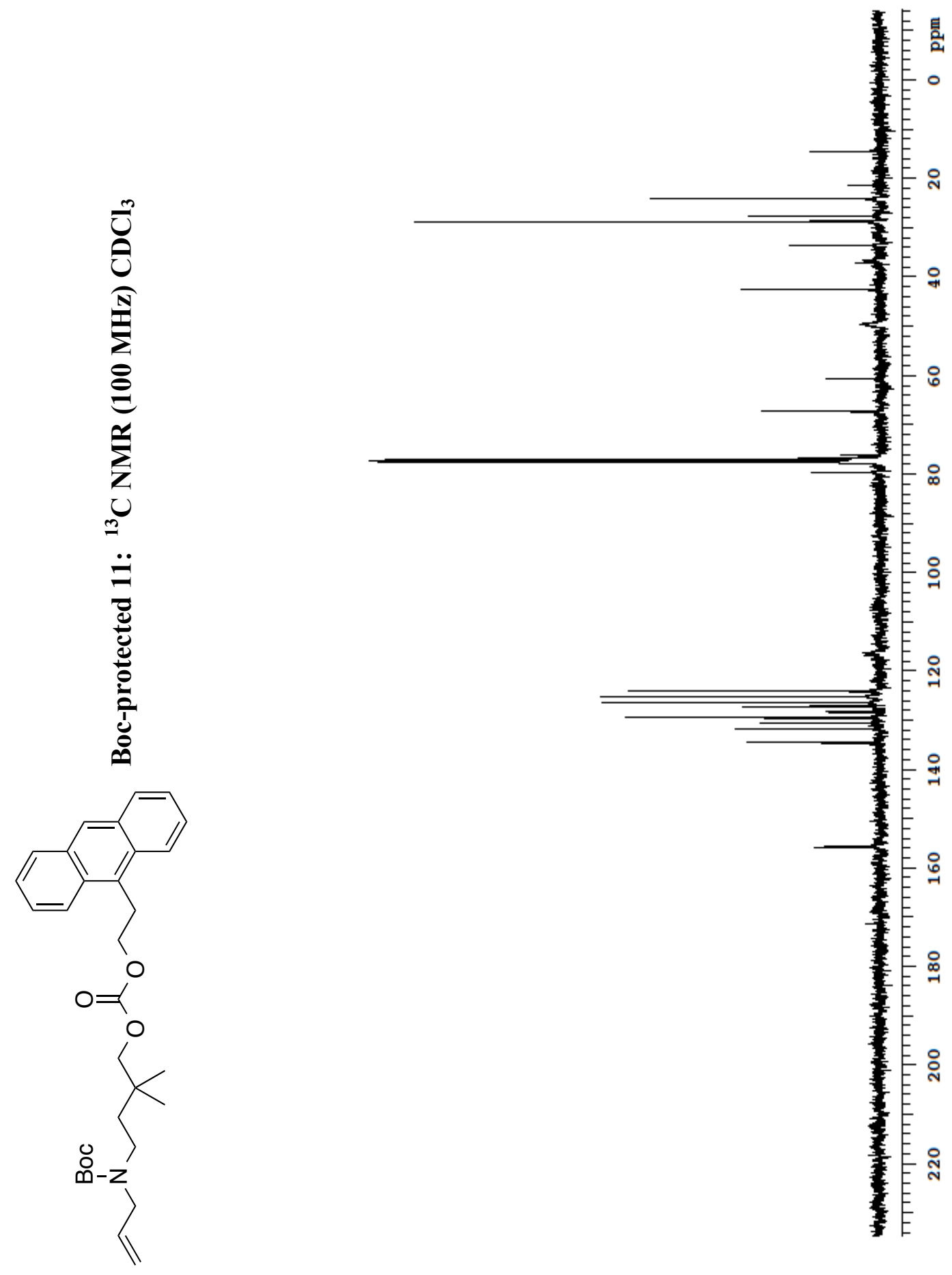

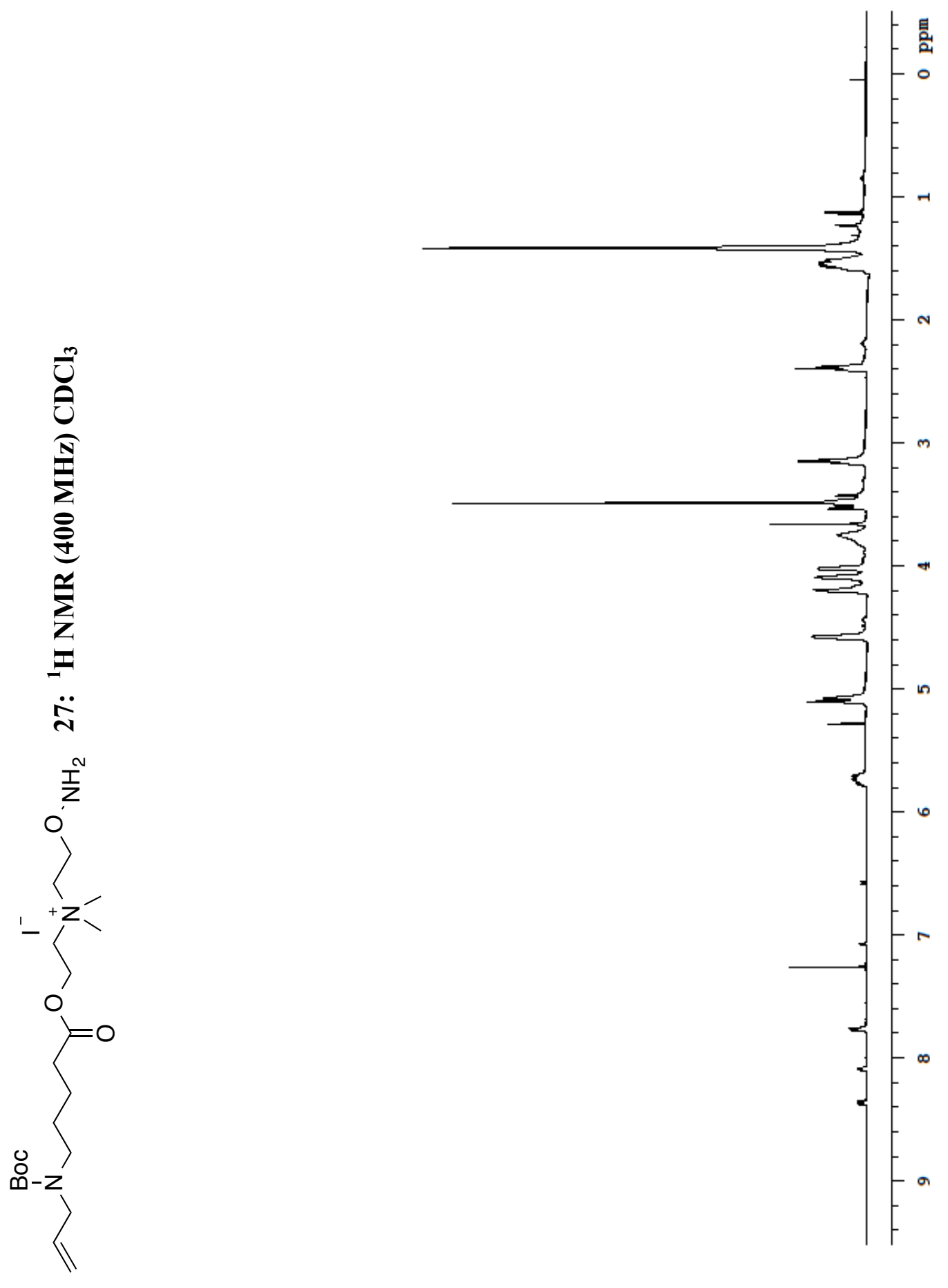


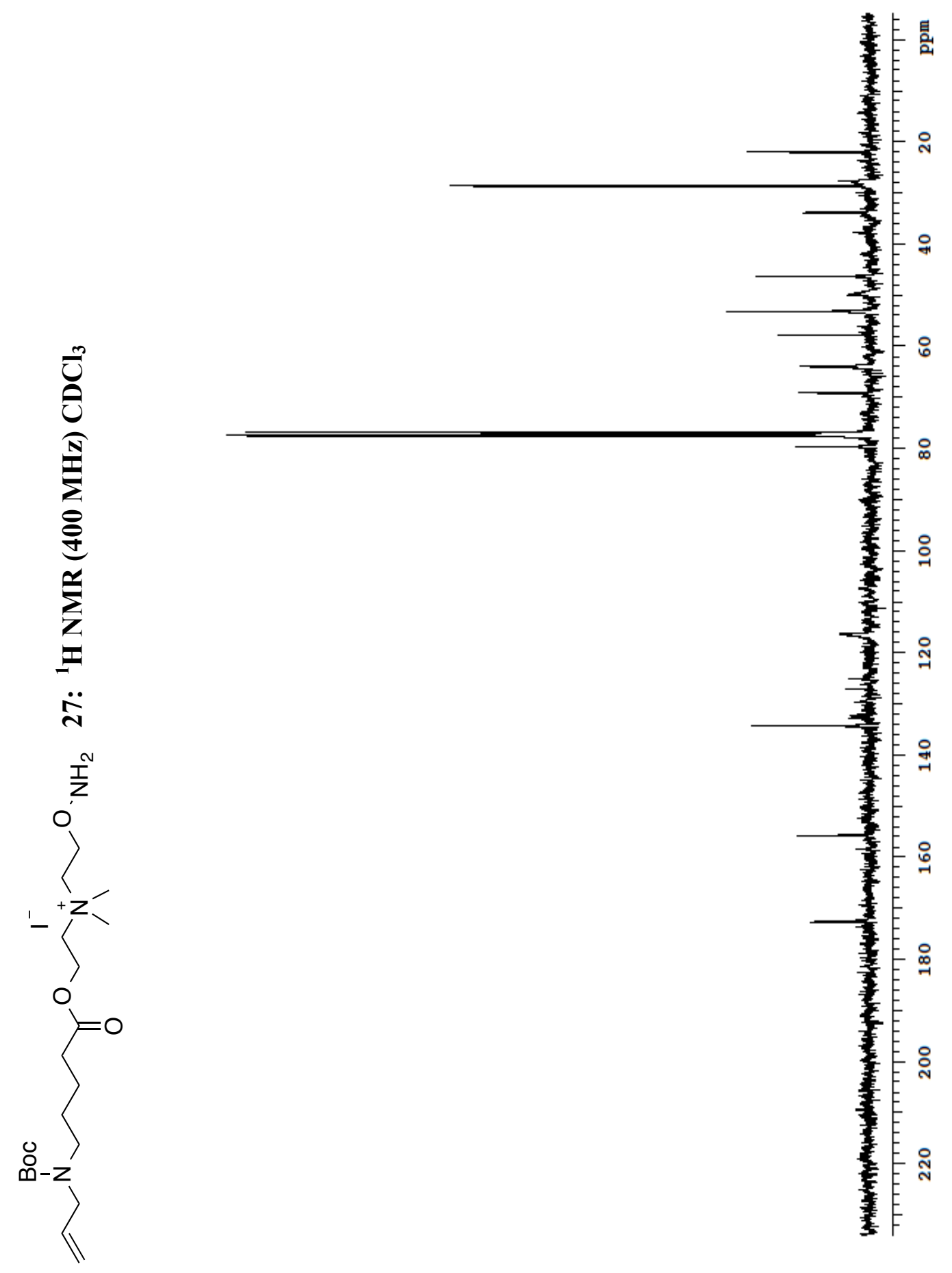



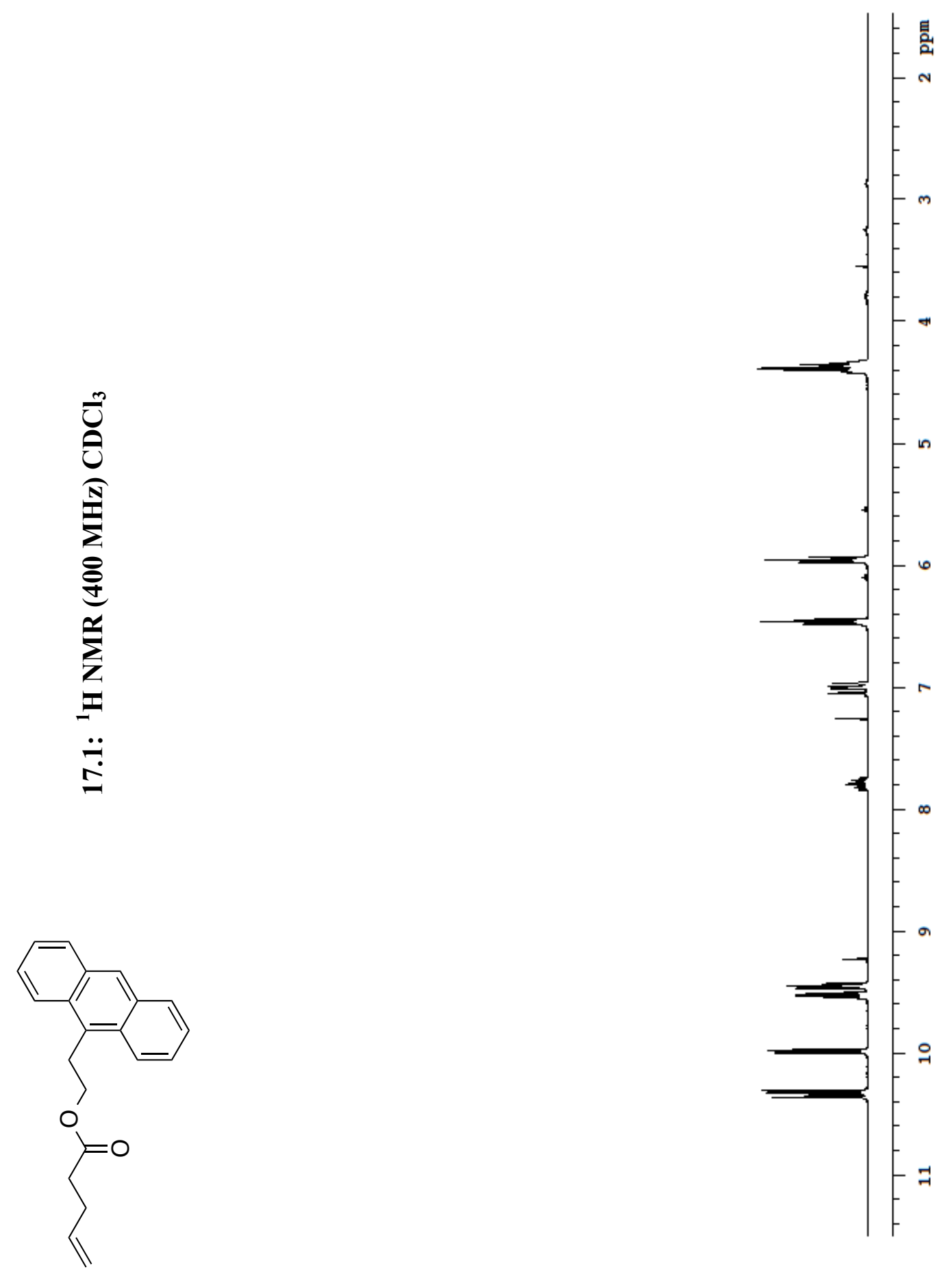


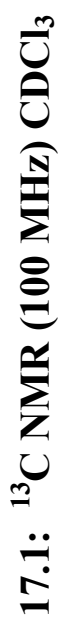
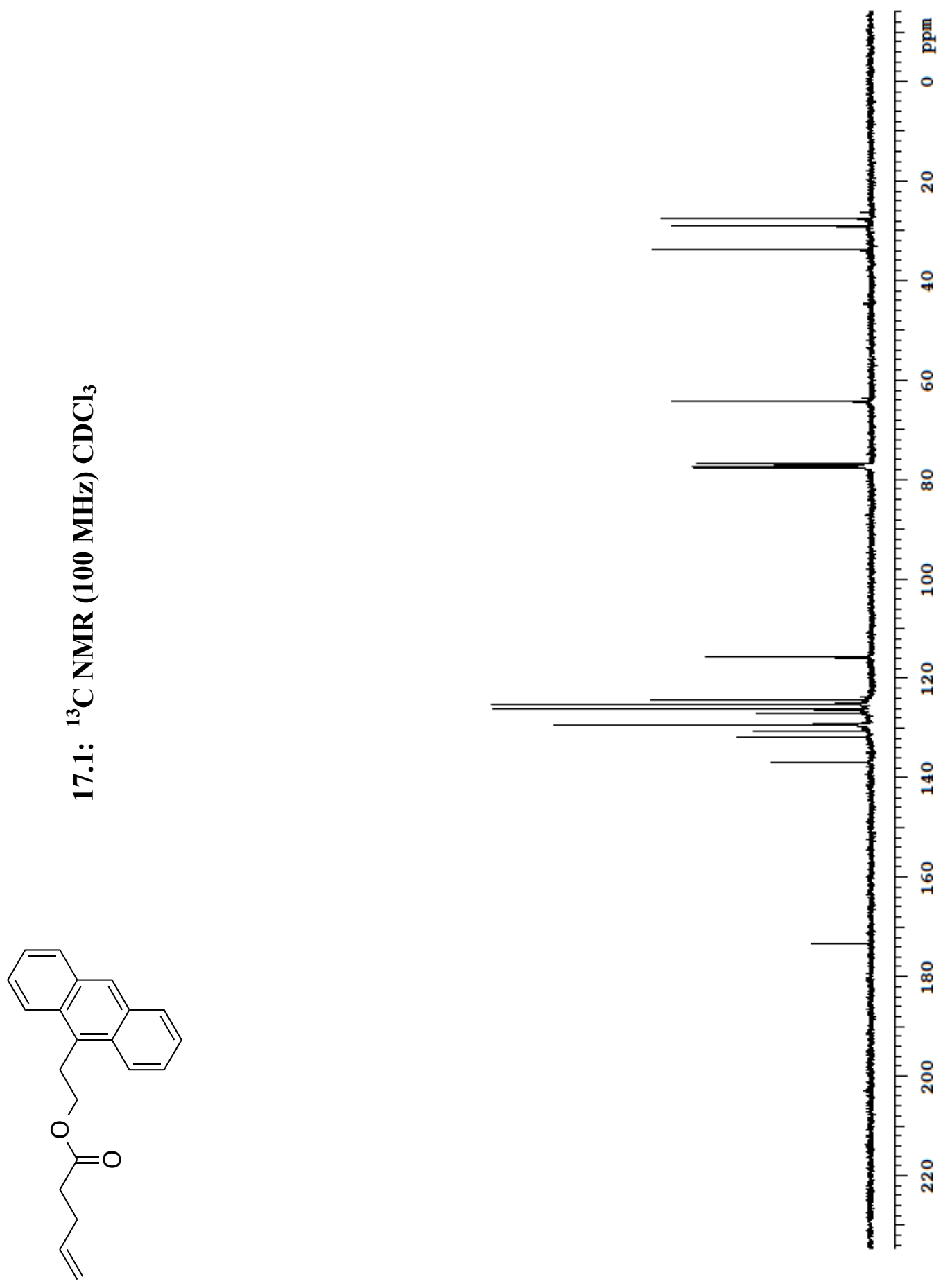

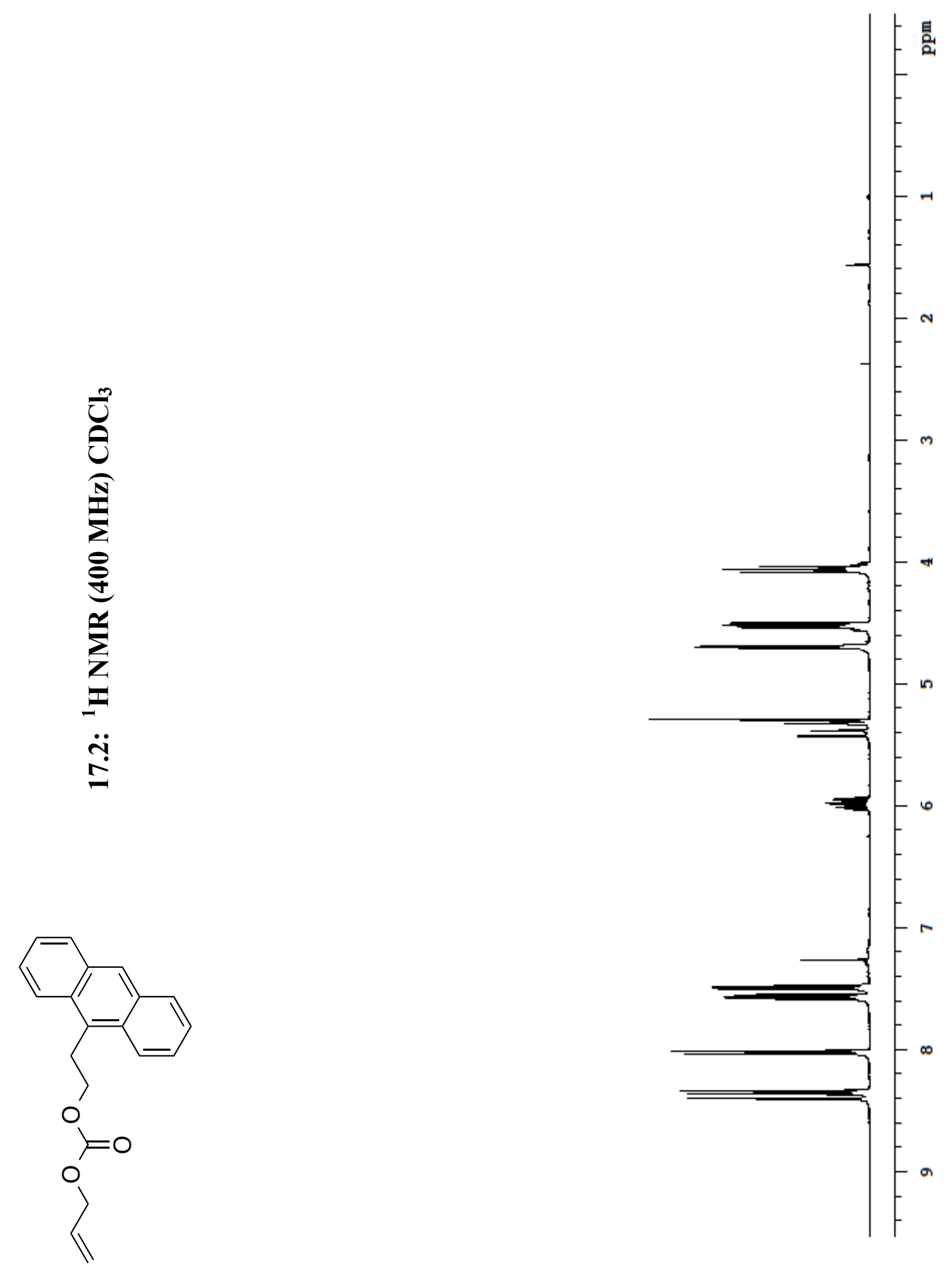

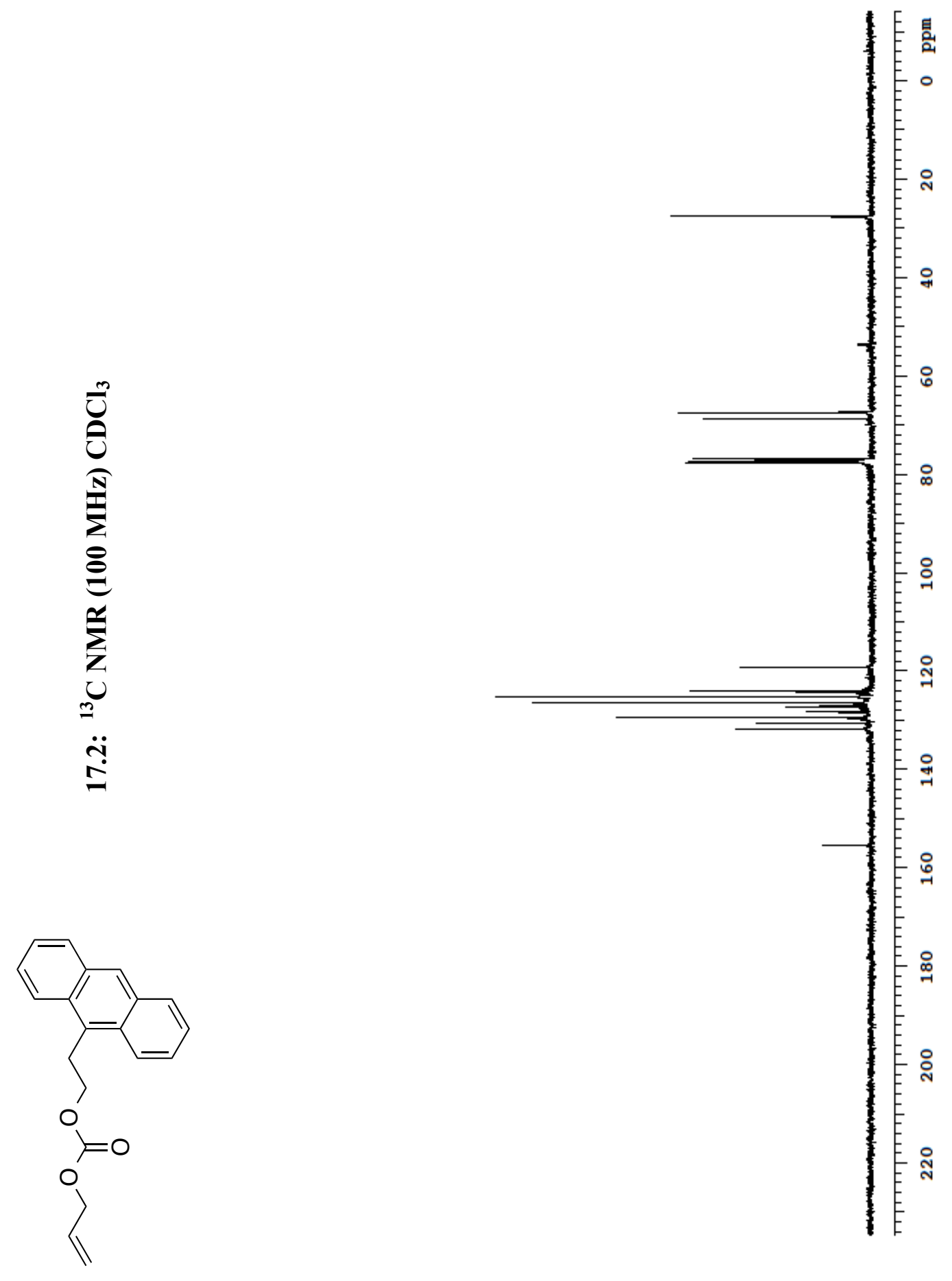
Uิ
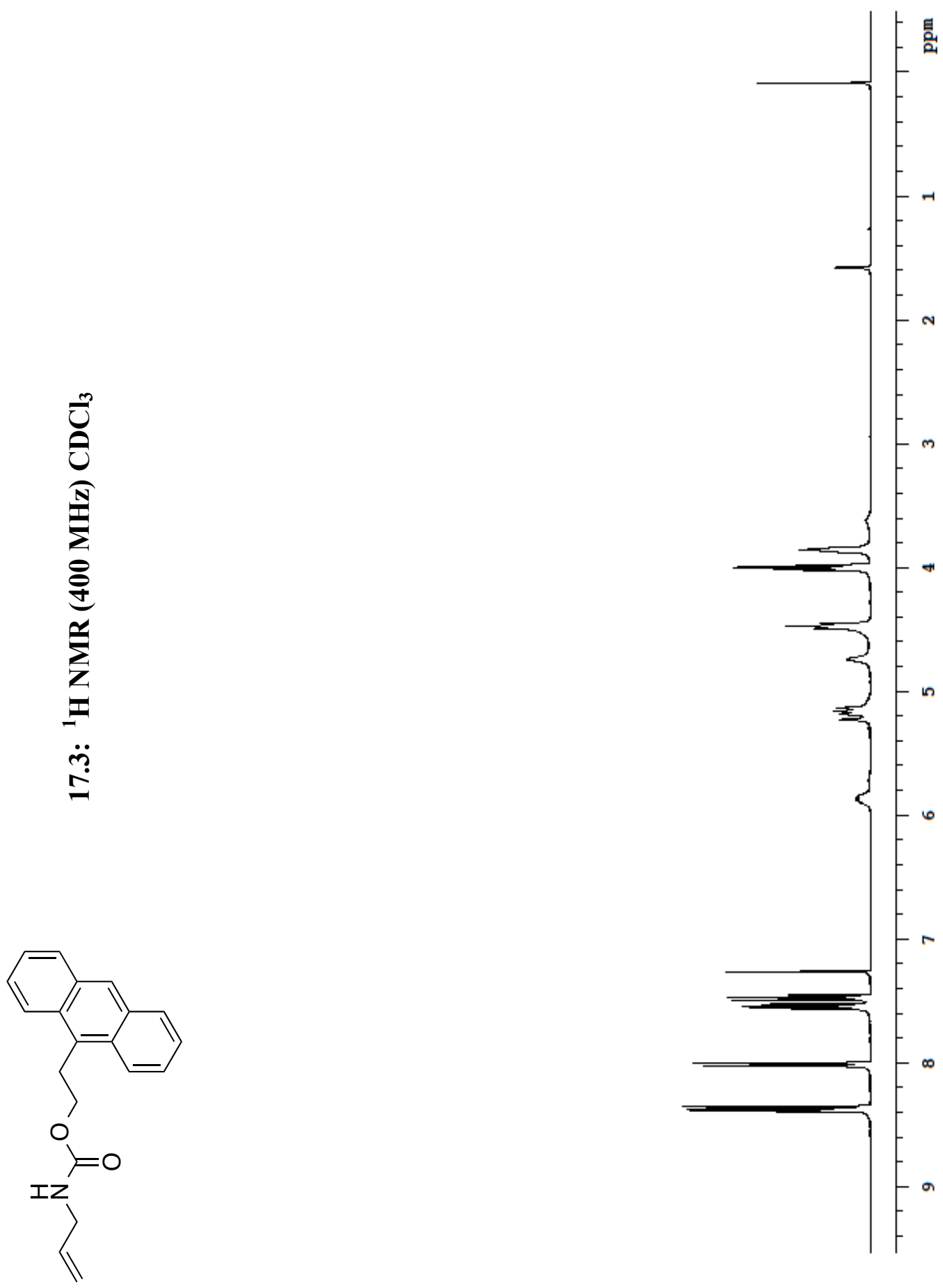

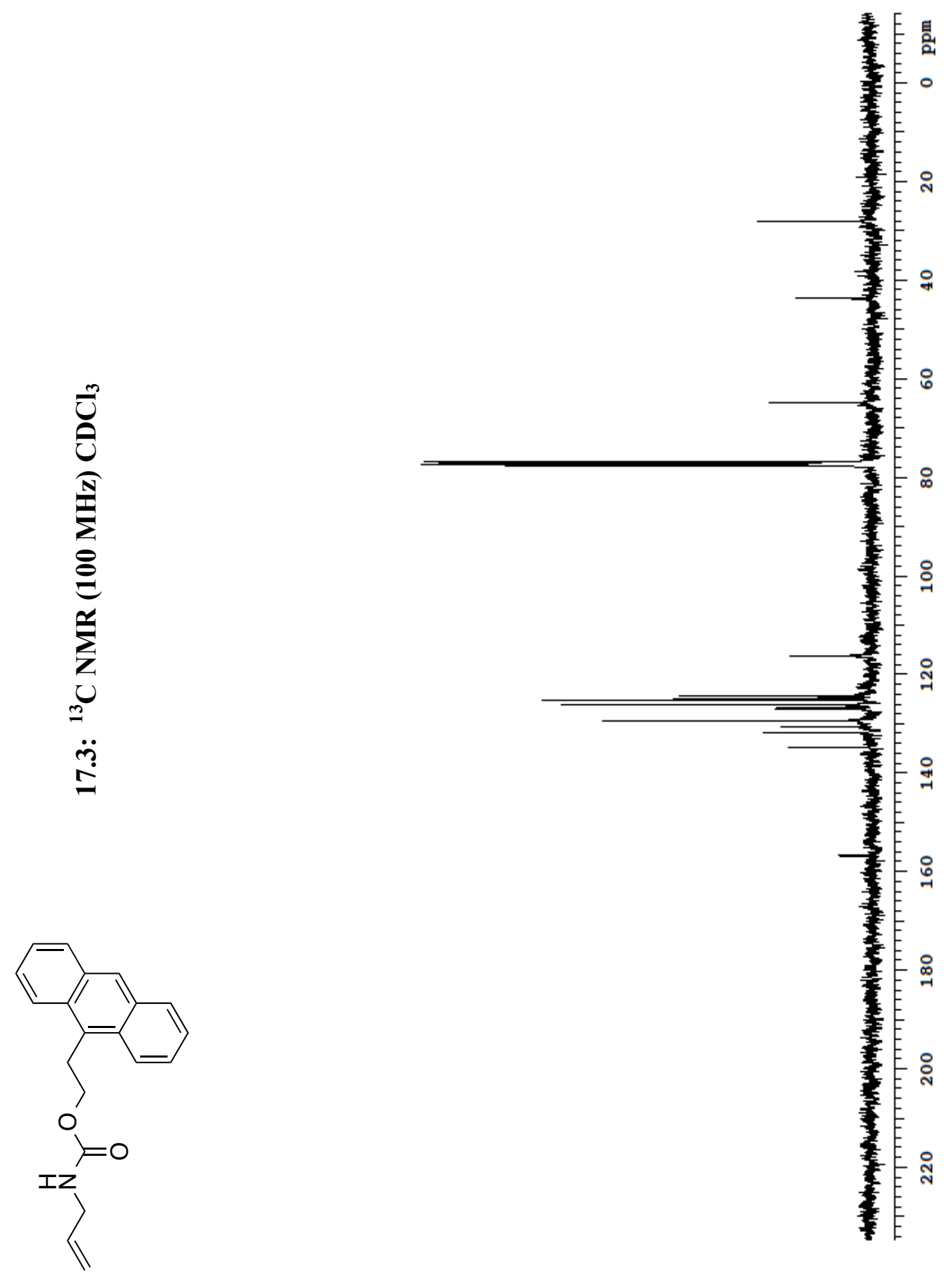


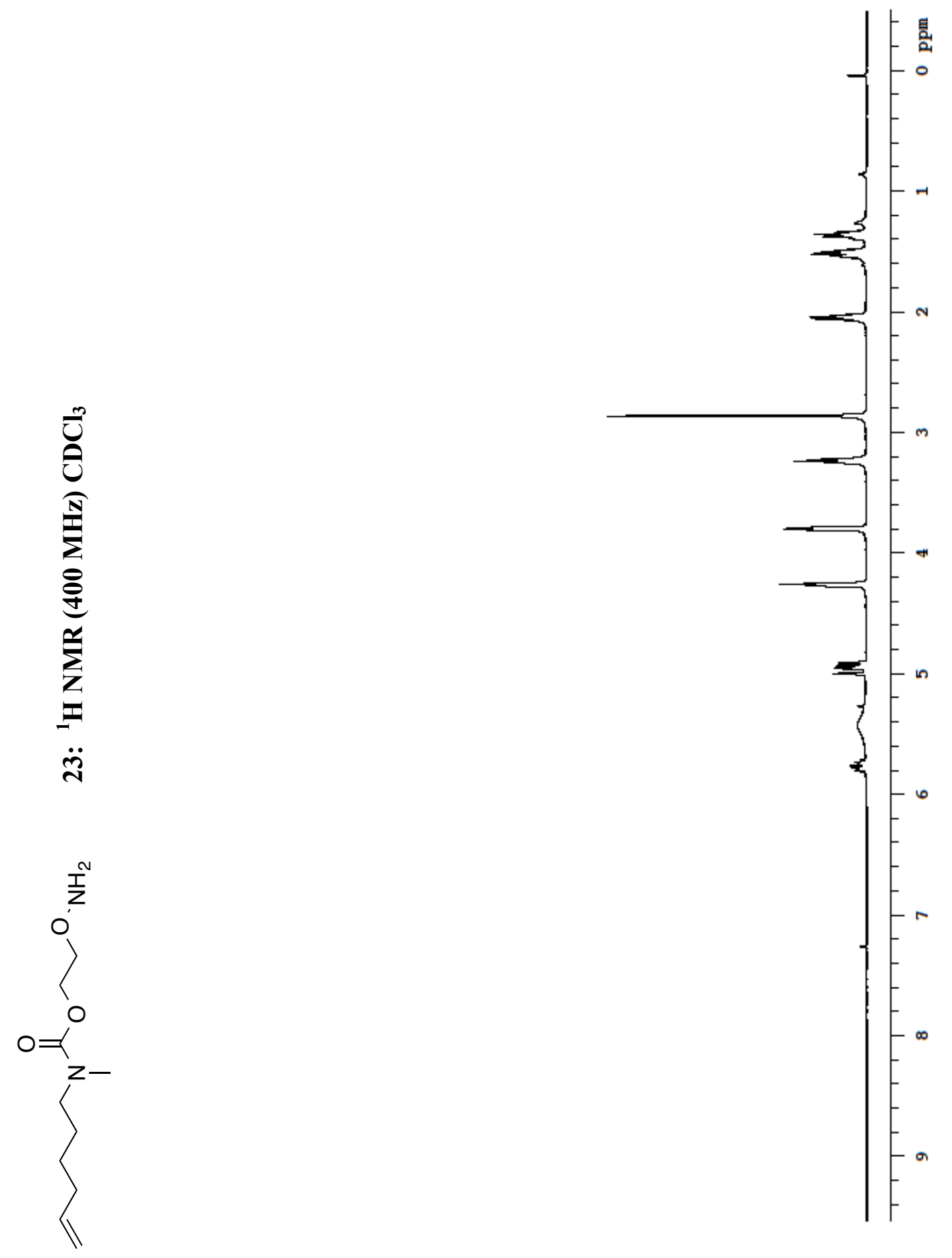




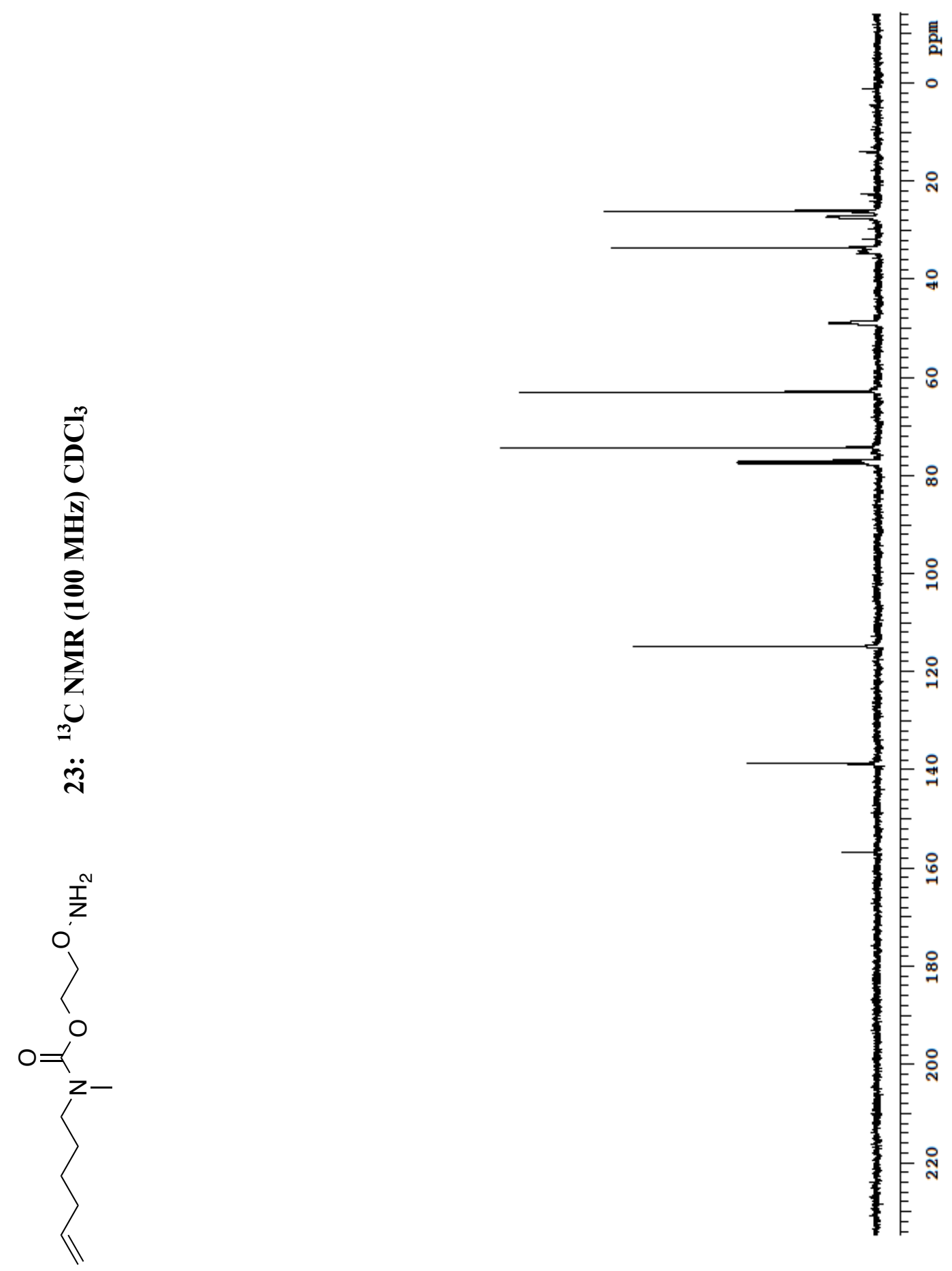



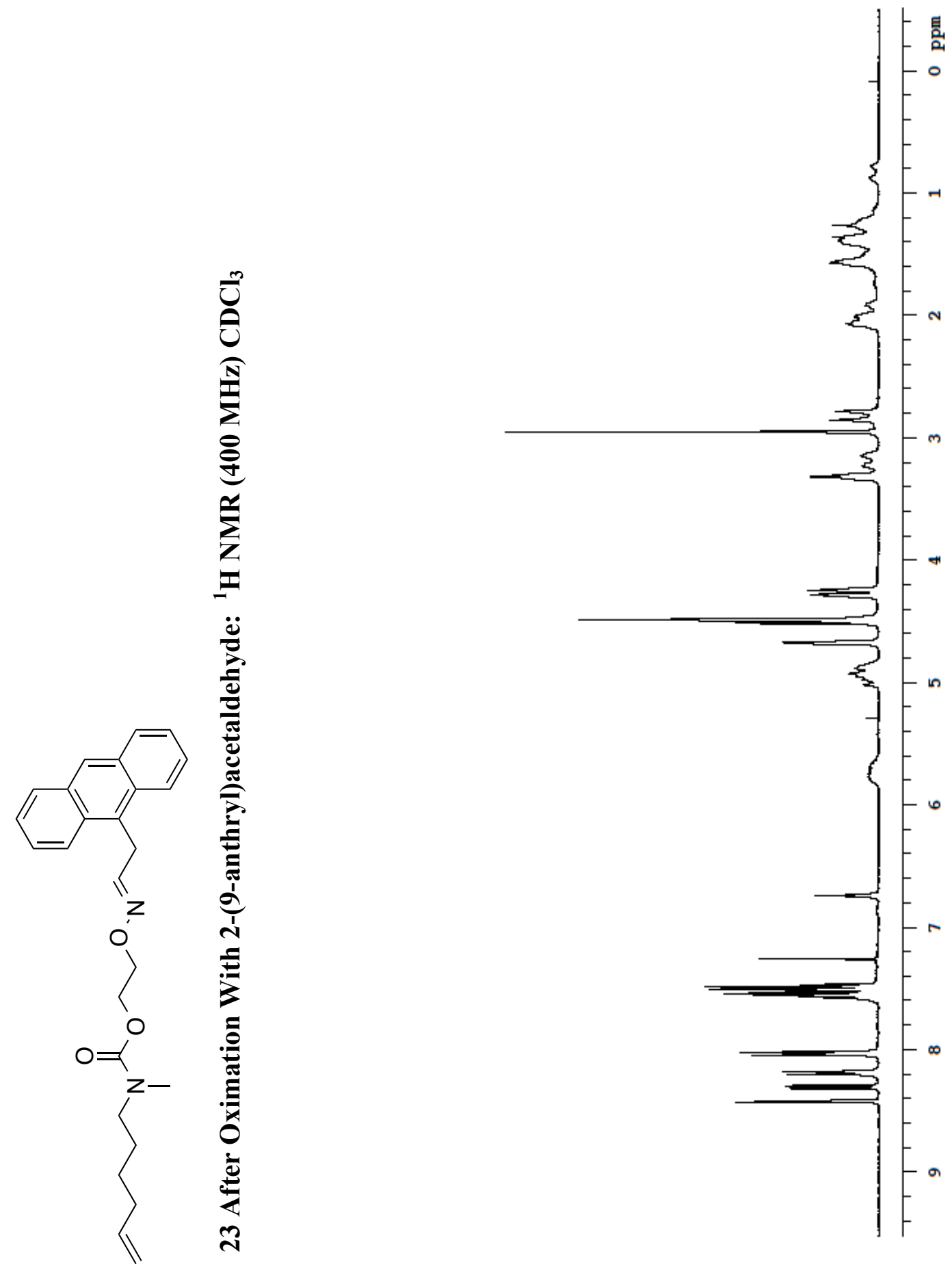


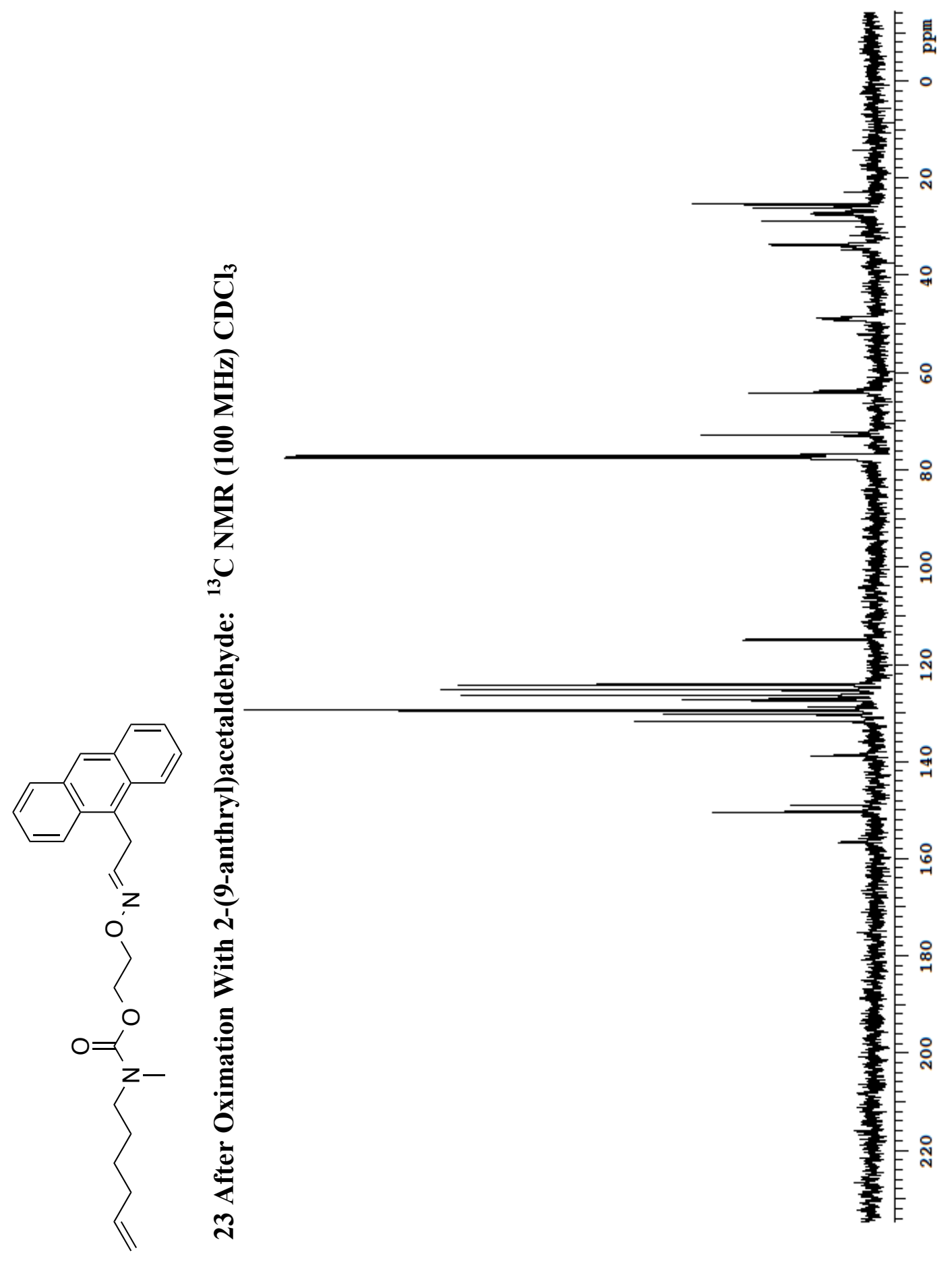




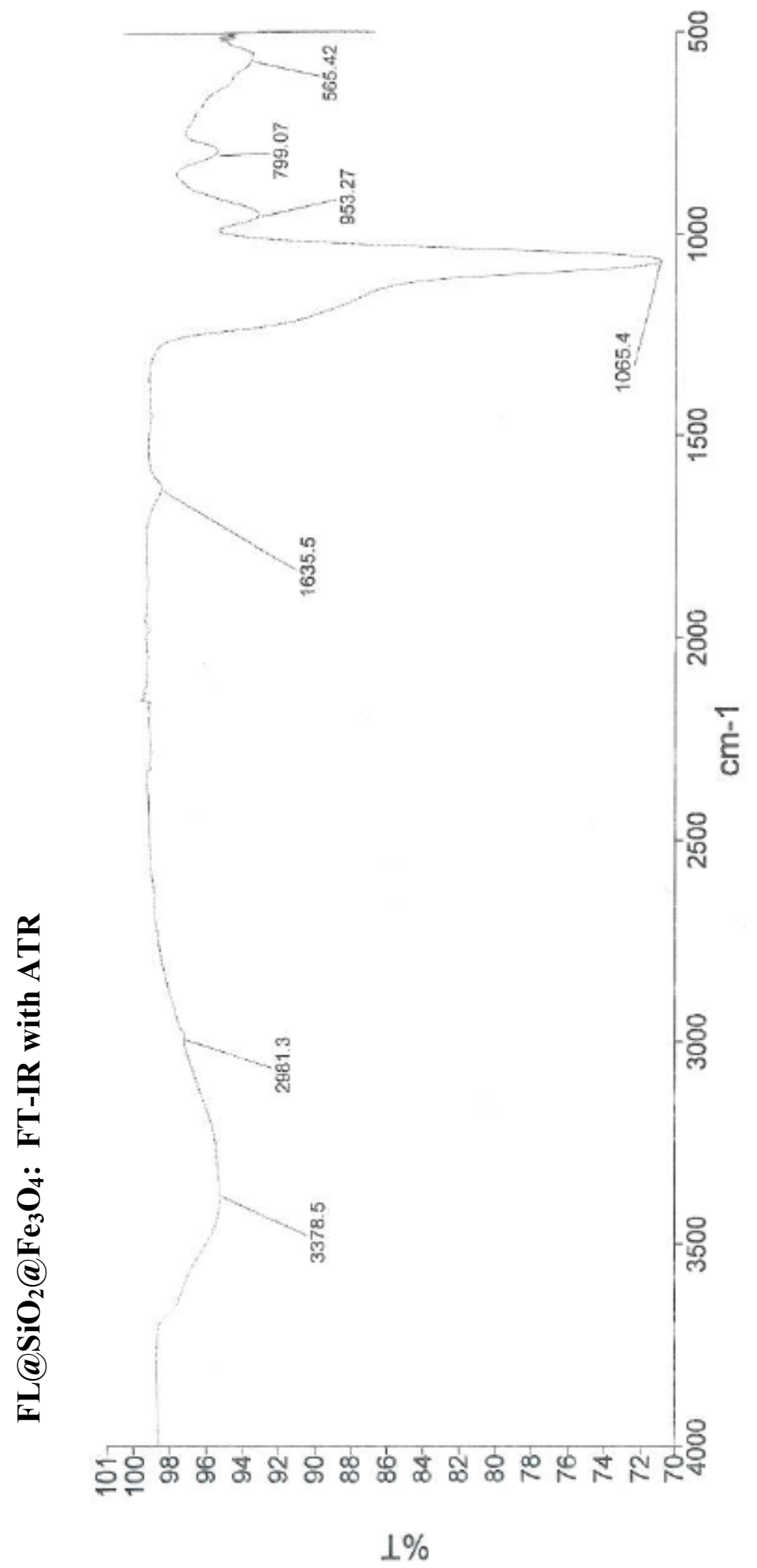



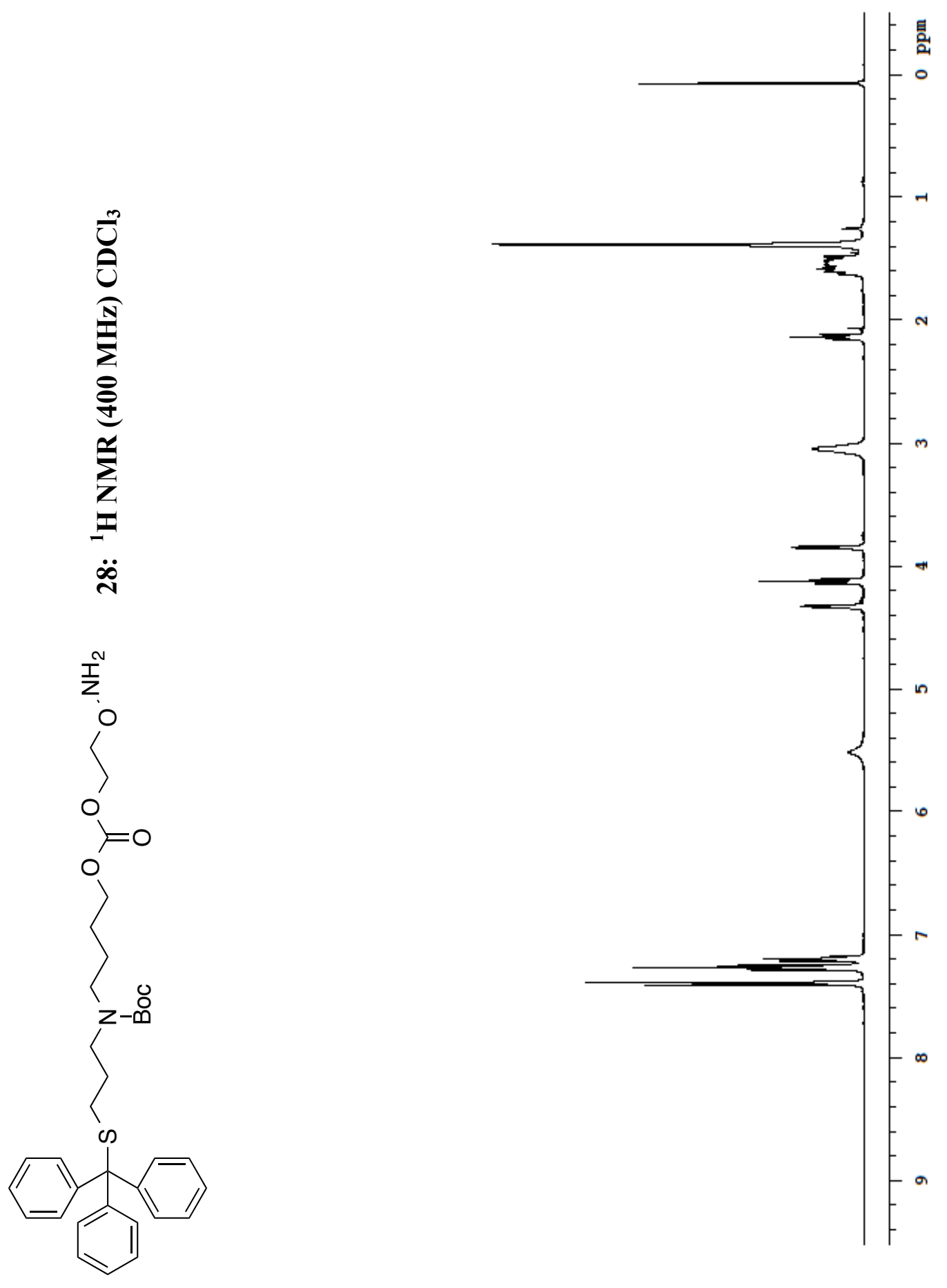

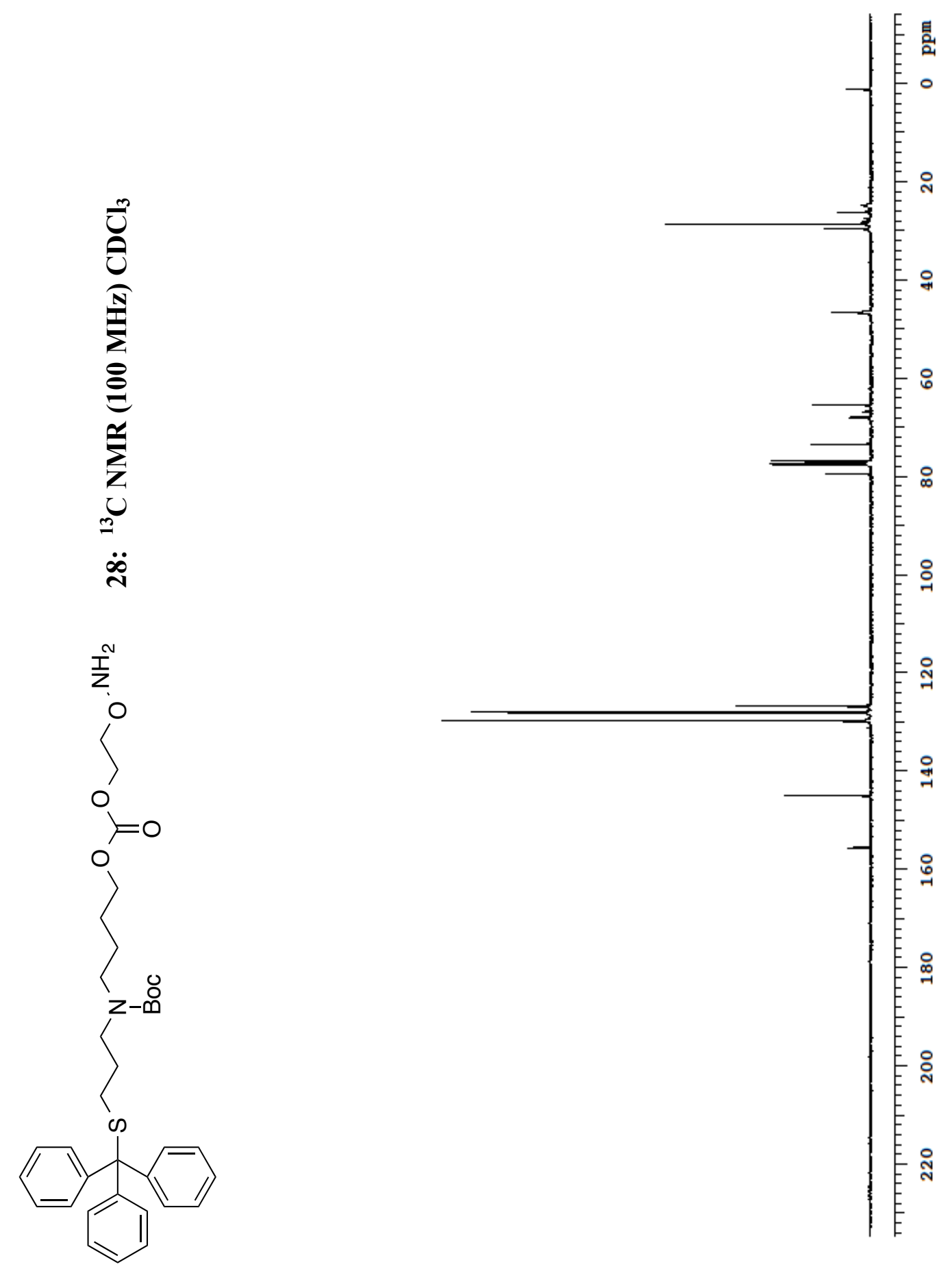
APPENDIX B

LIST OF PUBLICATIONS

B.1. List of Publications

B.2. Copyright Permissions

B.3. Manuscript Title Pages 


\section{B.1. List of Publications}

The work presented in this dissertation was published in various peer-reviewed journals and/or patented. The following list summarizes the publications and patents that arose from my research, and the chapters in which the work is described.

\section{Publications:}

1. Biswas, S.; Knipp, R. J.; Gordon, L. E.; Nandula, S. R.; Gorr, S. U.; Clark, G. J.; Nantz M. H. Hydrophobic Oxime Ethers: A Versitile Class of pDNA and siRNA Transfection Lipids. ChemMedChem 2011, 6, 2063-2069. [Chapter 1]

2. Fu, X.-A.; Li, M.; Knipp, R. J.; Nantz, M. H.; Bousamra, M. Noninvasive Detection of Lung Cancer Using Exhaled Breath. Cancer Medicine 2014, 3, 174181. [Chapter 2]

3. Bousamra, M.; Schumer, E.; Li, M.; Knipp, R. J.; Nantz, M. H.; van Berkel, V. H.; Fu, X.-A. Quantitative Analysis of Exhaled Carbonyl Compounds Distinguishes Benign From Malignant Pulmonary Disease. Journal of Thoracic and Cardiovascular Surgery 2014, 148, 1074-1081. [Chapter 2]

4. Knipp, R. J.; Li, M.; Fu, X.-A.; Nantz, M. H. A Versatile Probe for Chemoselective Capture and Analysis of Carbonyl Compounds in Exhaled Breath. Submitted to Analytical Chemistry [Chapter 2]

5. Li, M.; Knipp, R. J.; Nantz, M. H.; Bousamra, M.; Fu, X.-A. Analysis of Exhaled Breath for Identification of Lung Cancer Biomarkers. Submitted to Clinica Chimica Acta. [Chapter 2]

6. Knipp, R. J.; Estrada, R.; Sethu, P.; Nantz, M. H. Thermally-Induced Substrate Release Via Intramolecular Cyclizations of Amino Esters and Amino Carbonates. Tetrahedron 2014, 70, 3422-3429. [Chapter 3 and Chapter 4]

7. Knipp, R. J.; Nantz, M. H. Nanoparticles for Drug Delivery; US Patent Application US2014015413; filed February 7, 2014; Priority: US Provisional Application 61/762,832; Filed February 8, 2013. [Chapter 3 and Chapter 4]

8. Nantz, M. H.; Knipp, R. J. Nanoparticles for Drug Delivery; US Provisional Application 62/034,059; filed Ausust 6, 2014. [Chapter 4]

9. Knipp, R. J.; Mattingly, S. J.; Nantz, M. H. Magnetic-Field Induced Hydrolysis as a Mechanism for Rapid Release of Nanoparticle-Bound Substrates. Submitted to Advanced Materials Interfaces. [Chapter 4] 


\section{B.2. Copyright Permissions}

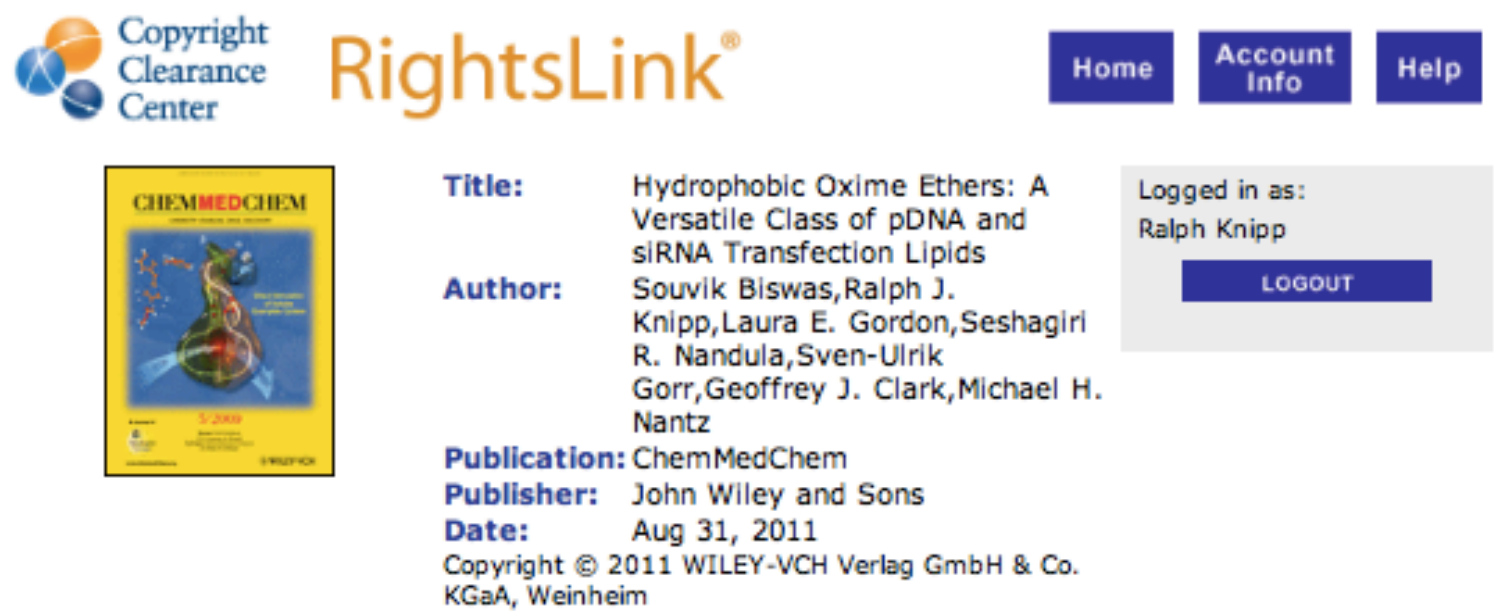

\section{Order Completed}

Thank you very much for your order.

This is a License Agreement between Ralph J Knipp ("You") and John Wiley and Sons ("John Wiley and Sons"). The license consists of your order details, the terms and conditions provided by John Wiley and Sons, and the payment terms and conditions.

\section{Get the printable license.}

\begin{tabular}{|c|c|}
\hline License Number & 3493830633502 \\
\hline License date & Oct 21,2014 \\
\hline $\begin{array}{l}\text { Licensed content } \\
\text { publisher }\end{array}$ & John Wiley and Sons \\
\hline $\begin{array}{l}\text { Licensed content } \\
\text { publication }\end{array}$ & ChemMedChem \\
\hline Licensed content title & Hydrophobic Oxime Ethers: A Versatile Class of pDNA and siRNA Transfection Lipids \\
\hline Licensed copyright line & Copyright @2011 WILEY-VCH Verlag GmbH \& Co. KGaA, Weinheim \\
\hline $\begin{array}{l}\text { Licensed content } \\
\text { author }\end{array}$ & $\begin{array}{l}\text { Souvik Biswas, Ralph J. Knipp,Laura E. Gordon,Seshagiri R. Nandula,Sven-Ulrik Gorr,Geoffrey } \\
\text { J. Clark,Michael H. Nantz }\end{array}$ \\
\hline Licensed content date & Aug 31, 2011 \\
\hline Start page & 2063 \\
\hline End page & 2069 \\
\hline Type of use & Dissertation/Thesis \\
\hline Requestor type & Author of this Wiley article \\
\hline Format & Electronic \\
\hline Portion & Full article \\
\hline $\begin{array}{l}\text { Will you be } \\
\text { translating? }\end{array}$ & No \\
\hline $\begin{array}{l}\text { Title of your thesis / } \\
\text { dissertation }\end{array}$ & $\begin{array}{l}\text { Triggered Release of Small Molecules from Solid Supports Using Heat or an Applied } \\
\text { Magnetic Field }\end{array}$ \\
\hline $\begin{array}{l}\text { Expected completion } \\
\text { date }\end{array}$ & Dec 2014 \\
\hline $\begin{array}{l}\text { Expected size } \\
\text { (number of pages) }\end{array}$ & 300 \\
\hline Total & 0.00 USD \\
\hline
\end{tabular}


Copyright Clearance Center

\section{WI LEY}

Title:

Author:
Noninvasive detection of lung cancer using exhaled breath

Xiao-An Fu,Mingxiao Li,Ralph J. Knipp,Michael H. Nantz,Michael Bousamra

Publication: Cancer Medicine

Publisher: John Wiley and Sons

Date: Nov 20, 2013

(c) 2013 The Authors. Cancer Medicine published by John Wiley \& Sons Ltd.
Logged in as:

Ralph Knipp

LOGOUT

\section{Welcome to RightsLink}

This article is available under the terms of the Creative Commons Attribution License (CC BY) (which may be updated from time to time) and permits use, distribution and reproduction in any medium, provided that the Contribution is properly cited.

For an understanding of what is meant by the terms of the Creative Commons License, please refer to Wiley's Open Access Terms and Conditions.

Permission is not required for this type of reuse.

Wiley offers a professional reprint service for high quality reproduction of articles from over 1400 scientific and medical journals. Wiley's reprint service offers:

- Peer reviewed research or reviews

- Tailored collections of articles

- A professional high quality finish

- Glossy journal style color covers

- Company or brand customisation

- Language translations

- Prompt turnaround times and delivery directly to your office, warehouse or congress.

Please contact our Reprints department for a quotation. Email corporatesaleseurope@wiley.com or corporatesalesusa@wiley.com or corporatesalesDE@wiley.com. 


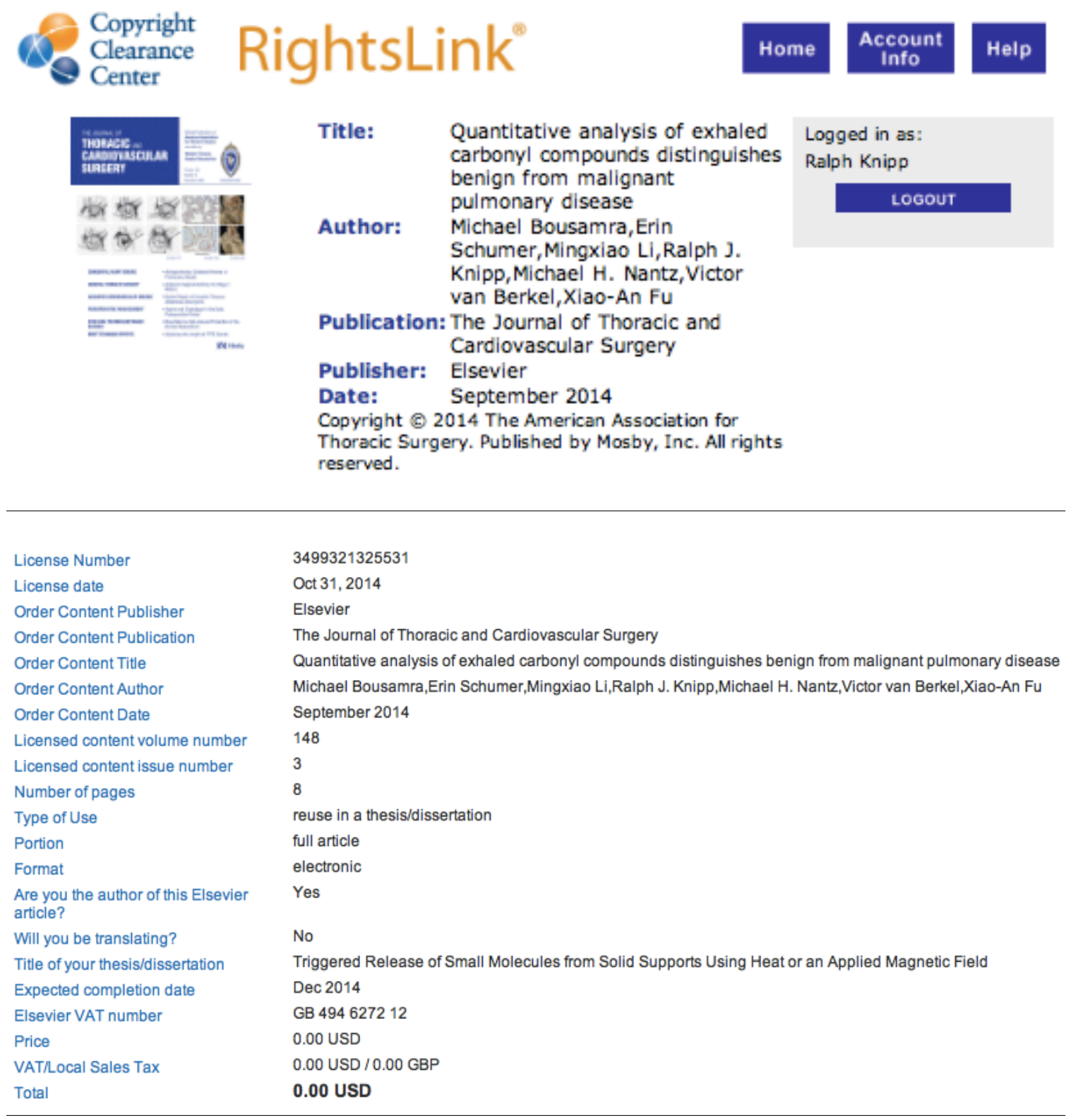


Copyright Clearance Center

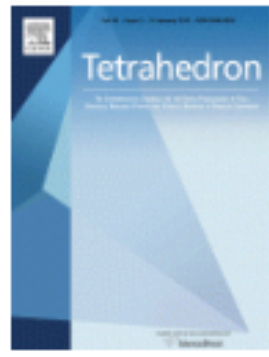

Title: Thermally induced substrate release via intramolecular cyclizations of Amino esters and Amino carbonates

Logged in as:

Ralph Knipp

$\begin{array}{ll}\text { Author: } & \text { Ralph J. Knipp,Rosendo } \\ & \text { Estrada,Palaniappan }\end{array}$

Sethu, Michael H. Nantz

Publication: Tetrahedron

Publisher: Elsevier

Date: 27 May 2014

Copyright (6) 2014 Elsevier Ltd. All rights reserved.

\section{Order Completed}

Thank you very much for your order.

This is a License Agreement between Ralph J Knipp ("You") and Elsevier ("Elsevier"). The license consists of your order details, the terms and conditions provided by Elsevier, and the payment terms and conditions.

\section{Get the printable license.}

\begin{tabular}{|c|c|}
\hline License Number & 3493830967445 \\
\hline License date & Oct 21,2014 \\
\hline Licensed content publisher & Elsevier \\
\hline \multicolumn{2}{|c|}{ Licensed content publication Tetrahedron } \\
\hline Licensed content title & $\begin{array}{l}\text { Thermally induced substrate release via intramolecular cyclizations of Amino esters } \\
\text { and Amino carbonates }\end{array}$ \\
\hline Licensed content author & Ralph J. Knipp,Rosendo Estrada,Palaniappan Sethu,Michael H. Nantz \\
\hline Licensed content date & 27 May 2014 \\
\hline $\begin{array}{l}\text { Licensed content volume } \\
\text { number }\end{array}$ & 70 \\
\hline $\begin{array}{l}\text { Licensed content issue } \\
\text { number }\end{array}$ & 21 \\
\hline Number of pages & 8 \\
\hline Type of Use & reuse in a thesis/dissertation \\
\hline Portion & full article \\
\hline Format & electronic \\
\hline $\begin{array}{l}\text { Are you the author of this } \\
\text { Elsevier article? }\end{array}$ & Yes \\
\hline Will you be translating? & No \\
\hline $\begin{array}{l}\text { Title of your } \\
\text { thesis/dissertation }\end{array}$ & $\begin{array}{l}\text { Triggered Release of Small Molecules from Solid Supports Using Heat or an Applied } \\
\text { Magnetic Field }\end{array}$ \\
\hline Expected completion date & Dec 2014 \\
\hline $\begin{array}{l}\text { Estimated size (number of } \\
\text { pages) }\end{array}$ & 300 \\
\hline Elsevier VAT number & GB 494627212 \\
\hline Permissions price & 0.00 USD \\
\hline VAT/Local Sales Tax & 0.00 USD / 0.00 GBP \\
\hline Total & 0.00 USD \\
\hline
\end{tabular}




\title{
B.3. Manuscript Title Pages
}

FULL PAPERS

\author{
DO: $10.1002 / \mathrm{cmd}$ c.201100259
}

\section{Hydrophobic Oxime Ethers: A Versatile Class of pDNA and siRNA Transfection Lipids}

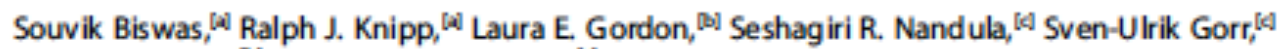
Geoffrey J. Clark, ${ }^{\text {Did }}$ and Michael H. Nantz $z^{[\text {[a] }}$

The manipulation of the carionic Ipid structures to inamse polynudeotide binding and delvery propertes, while also min imising assodated cytotouldty, has been a prindpal strategy for developing nestgenention transfection agents. The polar (DNA bindling) and hydrophobic domains of transfectibn Ipids have been extencivdy studied; however, the linking domain comprising the substructure used to tether the polar and hy. drophobic domains has attneted considershly less attentbn as an optimmtibn arable. Hare, we examine the use of an oume ether as the linking domain. Hydrophobic ouime ethers ware readly assembled via click chemistry by ouimation of hy. drophobic aldehydes uing $\mathbf{m}$ aminooxy salt $\mathbf{A}$ fadle lignton rextibn delivered the desired compounds with hydrophobic domain asymmetry. Uaing the MCF-7 breast cancer, H1792 lung cancer and PAR CIO salvary epithelal cell lines, our find ings show that Ipoplexes detwed fom oume ather lipids transfect in the presence of serum at higher levels than com monly used Iposome formulatibna, baed on both ludfarase and grean fuorescent protein (GFP) ascays. Given the biolog; cal compatbilty of arime ethers and their ease of formation this functional group should find signficant application as a linking doman in future designs of transfection vectors.

\section{Introduction}

Catbric Ipids and their derived Iposomes have become the most wellstudied and widely used syntheti, nomiral gene de Ivery wehides since felgner etal[t frst dsdosed Dorma. medated gene transfer in 1987. ${ }^{D, A l}$ Due to the major Imta tons of vial vectors, such as assochted immune responses. Imitad polynucleotde camyling capacty and high cost, ${ }^{p, S \mid}$ cat ionic lipids remain an attractive alternative. The advantages of low mmunoganidity the bilty to transfect RNA or DNA of nearly unimithed sine,,$M /$ and the rehtive exe of cationic lipidplasmid DNA (pDNA) or RNA complex flpoplexd formuhtbr? continue to stract interest aimed at deseloping safer and more effident cationic Ipids for use as transfection agents ${ }^{\text {[* }}$

Catonic Ipid molecules, such as the prototypical dual dhain Inids DOTMA ${ }^{10}$ and DOThpoa (Aigure 1l, contain a polr, posi. twely changed (DNA binding) head group connected to a hy. drophobic domain via a Inling functionsilty. These three prin. dps structunal components of catbric glycerditype lipids

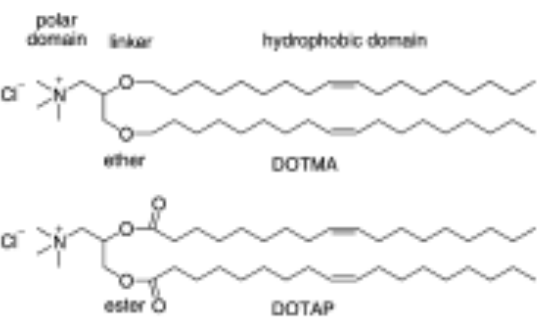

Fige 1. Structural doma ins of common tramifucton lpida. have been artanshely studied in efforts to improve lipid-med, ated intraceluhr ddivery of polynuckotides to mammalim celk. ${ }^{[1]}$ Many structure-activity relatonships have been deter.

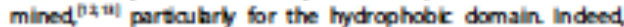
the hydrophobic domain's structural vartables of chain langth degree of uncatuntion, and domain asymmetry are among the strongest contributors to transfection efficaxy ${ }^{[0 \mid}$ Fewer direct structural comprisons of changes in the carionic lipid back bone, or Inling domain, have been reported, with the most wellknown compartson being that of the diether DOTMA versus the diester DOTAP. ${ }^{04}$ The linking functionality, and to a lesser extent the cationic head group, seem to be the principal determinants of toxdaty ${ }^{[\mathrm{ti}-1 \mid}$ The Inler determines conforms tional fexbilty, degree of stablity, and blodegradabilty. Among the most studied chamical functionalties compitaing the Inising domain of tansfection lipids are the ether, ester.

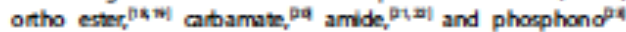
moleter. 
ORIGINAL RESEARCH

\title{
Noninvasive detection of lung cancer using exhaled breath
}

\author{
Xiao-An Fu', Mingxiao $L^{1}$, Ralph J. Knipp ${ }^{2}$, Michael H. Nant $z^{2} \&$ Michael Bousamra ${ }^{3,4}$
}

'Doparment of Chamical Enginearing Univerity of Lousulb, Louksub, Kentudy 4008

"Dopartment of Chamistry, Universty at Loukvib, Loukvile, Kentudky $40 x 08$

'Deparmant of Surgry, University of Lousulb, Louivile, Kentudy 4008

hamex Gaham Brown Cancer Conar, Universty of Loukdle, Loukvile, Kentudky 4030

Keymords

Bianaders, broath andyek, early dagnosis. of cancer, lung cancor, volatb ongric compounds

\section{Comespondence}

Xla An Fu, Depantmert of Chamical

Enginoemg, Unwerity of Louisile, 230 South Thind Streot, Lais/lk, $\mathrm{KY} 40208$.

Td: 01.502-852 E49, Fax 01.502.8526355

E-mat xacan tualousulb abs

Funding Information

The authors adknowledge funding from the Nationd Sclence Foundation (CBET:119829)

and the intial finandil support from the

Unverainy of Louisile (Cinical and

Tranational Solence Abt Grant Frogram).

Recaived: 18 buly 2013 Rovisd: 27 September 2013, Accopted: 11 Octobor 2013

Cancer Medidine 2014; 3(1): 174-181

\begin{abstract}
Early detection of lung cancer is a key factor for increasing the survival rates of lung cancer patient. The andysis of exhaled breath is promising as a nonimvasive diagnostic tool for dingnosis of lung cancer. We demonstrate the quantitative analysis of carbonyl volatile organic compounds (VOCs) and identification of lung cancer VOC markers in exhaled breath using unique silicon microreactor technology. The microrextor consists of thousands of micropillars coated with an ammonium aminooxy salt for capture of carbomyl VOCs in exhaled breath by means of oximation reactions. Captured aminooxy-VOC adducts are analyzed by nanoelectrospray Fourier trans form-ion cydotron resonance (FT-ICR) mass spectrometry (MS). The concentrations of 2 -butanone, 2-hydroxyacetzldehyde, 3-hydroxy-2-butanone, and 4-hydroxyhexenal (4-HHE) in the exhaled breath of lung cancer patients $(n-97)$ were significantly higher than in the achaled breath of healthy smoker and nonsmoker controls $(n-88)$ and patients with benign pulmonary nodules $(n-32)$. The concentration of 2-butanone in exhaled breath of patients $(n-51)$ with stages II though IV non-small cell lung ancer (NSCLC) was significantly higher than in exhaled brexth of patients with stage I $(n-34)$. The carbonyl VOC profile in exhaled brexth determined using this new silicon microreactor technology provides for the nonimasive detection of lung cancer.
\end{abstract}

dot $10.1002 x a m 4.162$

\section{Introduction}

The US Nutional Lung Screening Trial recently found that periodic computed tomography (CT) screening of heavy smokers could reduce mortalities of lung cancer patients by a much a $20 \% 6[1,2]$. Currently, CT and bronchoscopy are the principal techniques used for lung cancer detection $[3,4]$. In recent years, the analysis of ahaled breath has become an international research frontier because of its applicability for nonimvasive diagnosis of diseases [5-17]. Several approaches have been developed to analyze exhaled breath induding the use of sensor arrays $[7-9,15-17]$, proton-transfer reaction mass spectrometry (PTR-MS) [18-20], selected ion flow tube mass spectrometry (SIFT-MS) [21, 22], and gas chromatogr aphy- mass spectrometry (GC-MS) [13, 18, 23-26). Real-time analysis of volatile organic compounds (VOCs) in exhaled breath by PTR-MS has been established to monitor variation in $\mathrm{VOCs}$ with time $[27-31]$. Although some VOCs in achaled breath have bean reported as lung cancer markers, there has been no clinical adoption of breath andysis methods for diagnosis because of the large number of VOCs in ahaled breath and the lad of cancer-specific VOC markers for reliably predicting lung cancer [5-17].

Here, we describe the quantituive analysis of carbonyl VOCs in exhaled bresth and the identification of specific carbomyl VOCs relited to lung cancer stages and histology using silion microreactors for the capture of carbonyl VOCs. Our approach only requires a patient to fill a 1-L 


\title{
Quantitative analysis of exhaled carbonyl compounds distinguishes benign from malignant pulmonary disease
}

\author{
Michael Bousamra II, MD, ${ }^{\text {a }}$ Erin Schumer, MS, MD, ${ }^{\text {a }}$ Mingxiao Li, PhD, ${ }^{b}$ Ralph J. Knipp, MS, \\ Michael H. Nantz, PhD, ${ }^{2}$ Victor van Berkel, MD, PhD, and Xiao-An Fu, $\mathrm{PhD}^{5}$
}

Objectives: The anslysis of exhaled treath is a promising noninvasive tool for the diagnosis of lung cancer, bu its clinical relevance has yet to be established. We report the analysis of exhaled volatile carbonyl compounds for the identification of specific carbonyl cancer markers to differentiate benign pulmonary disease from early-stage lung cancer and to compare its diagnostic accuracy with positron emision tomography (PET) scans.

Methods: Aminooxy-costed silicon microchips were used for the selective capture of exhaledcarbonyls by an oximation resction. B reath samples were collected then directed through the silicon chips by applying avacuum. Carbony1 adklucts were analyzed by Fourier transform mass spectrometry. Eighty-eight control subjects, 107 patients with lung cancer ( 64 stage $0, \mathrm{~L}$, or II), 40 patients with benign pulmonary disease, and 7 patients with a solitary pulmonary metastasis participated. Anslysis of cancer markers was performed blinded to the pathologic results.

Results: Four carbonyls were defined as cancer markers with significantly higher concentrations in patients with lung cancer. The number of increased cancer markers distinguished benign disease from both early and stage III and IV lung cancer. For early-stage disease, defining greater than 2 increased markers as diagnostic of lung cancer resulted in $83 \%$ sensitivity and $74 \%$ specificity. PET scans for this same cohort resulted in $90 \%$ sensitivity but only $39 \%$ specificity. Markers normalized for 3 of the 4 markers after resection of the lung cancer.

Conclusions: Anslysis of specific exhaled carbonyls can differentiate early lung cancer from benign pulmonary disease. Breath analysis was more specific than PET for a lung cancer diagnosis. Judicious use of these data may expecite the care of patients with lung cancer. (J Thorac Carcliovase Surg 2014;148:1074-81)

With the advert and increasing acceptance of computed to. mography (CT) screening for lung cancer, the importance of distinguishing berign from malignant intrathoracic dis ease is increasing. The reported $20 \%$ reduction in lung can cer mortality from the National Lung Cancer Screening Trial is partially offset by the morbidity, cost and occasional mortality incurred by pursuing nonmalignant pulmonary noduks and adencpathy.

Given a resectable pulmonary nodule, and depending on its degree of saspicion, there are multiple options for disg nostic workup. ${ }^{2}$ Serial $C T$ scanning to ohserve growth, res olution, or stability is commonly used for subcentimeter lesions. Positron emission tomography (PET) scintigraphy

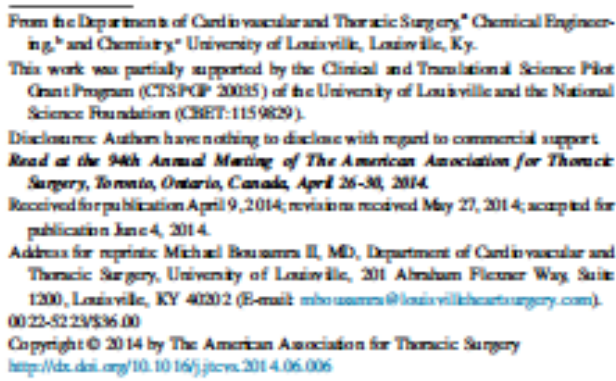

of the ksion may be used to assess the prohability of malig. nancy in nodules larger than $8 \mathrm{~mm}$. Bronchoscopy and percutaneous biopsy are options for tissue acquisition but the associated costs and risks are significant. In addition. diagnostic yiekds are highly conditionsl with respect to thmor size, kcation, and operator skill. Proceeding directly to surgical resection is appropriate when the probability of malignancy is high and the sargical risk is low. Conversely, surgical resection of nonmalignant disease is a clinical failure because, in most such instances, the natural course of the benign process would never have harmed the patient. Furthermore, PET scans are frequently falsely positive both in cases of solitary pulmonary nodules and in cases with hilar and mediestinal adencpathy. All may lead to increased clinical suspicion of lung cancer and an obliga. tion to ruk out malignancy by surgical intervention. Thus, it is important to develop reliable methods that minimize the diagnostic burden to patients who have no significant disease while expediting treatment in patients who have lung cancer. $^{2-4}$

Breath analysis of patients with suspected lung cancer is a developing modality with the potential to fulfill this gosl." Linus Pauling firs studied the volatile constituents of treath in individuals maintained on an elemental diet in $1971 .{ }^{\circ}$ In recent years, the analysis of exhaled breath has become a brosd research fromtier. ${ }^{373}$ Several approsches have been developed, including sensor arrays, ${ }^{21}$ prowon transfer 


\title{
A Versatile Probe for Chemoselective Capture and Analysis of Carbonyl Compounds in Exhaled Breath
}

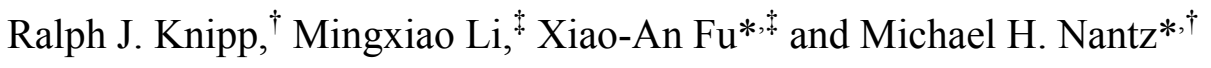 \\ ${ }^{\dagger}$ Department of Chemistry, ${ }^{\star}$ Department of Chemical Engineering, University of Louisville, \\ Louisville, Kentucky 40292, United States
}

\begin{abstract}
We describe an aminooxy reagent for the capture of trace aldehyde and ketone volatile organic compounds (VOCs) in exhaled breath. The reagent, 4-(2aminooxyethyl)-morpholin-4-ium chloride (AMAH), when coated onto micropillars within a silicon microreactor, chemoselectively and covalently retains carbonyl VOCs from exhaled breath. The AMAH-carbonyl adducts are then rinsed from the microreactor with methanol and directly analyzed by Fourier transform-ion cyclotron resonance (FTICR) mass spectrometry (MS), where the aminium ion of the reagent enhances the sensitivity for high mass accuracy. We also outline a protocol for treatment of the AMAH-carbonyl adducts with poly(4-vinylpyridine) to afford the corresponding volatile carbonyl adducts that now can be analyzed by gas chromatography-mass spectrometry (GC-MS). This convenient protocol imparts flexibility for the identification and quantification of isomeric VOCs using both FT-ICR-MS and GC-MS. Representative breath analyses are given to illustrate this applicability of AMAH.
\end{abstract}




\title{
Quantitative Analysis of Carbonyl Compounds in Exhaled Breath
}

\author{
Mingxiao Li ${ }^{1}$, Ralph Knipp ${ }^{2}$, Michael H. Nantz ${ }^{2}$, Michael Bousamra ${ }^{3,4}$, Xiao-An Fu ${ }^{* 1}$ \\ ${ }^{1}$ Department of Chemical Engineering, ${ }^{2}$ Department of Chemistry, ${ }^{3}$ Department of \\ ${ }^{3}$ Department of Cardiovascular and Thoracic Surgery, ${ }^{4}$ James Graham Brown Cancer \\ Center, University of Louisville, Louisville, Kentucky 40292, United States
}

\begin{abstract}
Quantitative analysis of trace volatile carbonyl compounds in human breath is reported. The approach is based on microreactor chips fabricated from silicon wafers. The microreactors have thousands of micropillars in microfluidic channels for uniformly distributing a gaseous breath sample flowing through the channels. The surfaces of the micropillars are functionalized with a quaternary ammonium aminooxy salt, [2(aminooxy)ethyl]- $N, N, N$-trimethylammonium iodide (ATM), for chemoselective trapping of trace carbonyl compounds by means of oximation reactions. ATM adducts and unreacted ATM are eluted from the microreactor with $50 \mu \mathrm{L}$ of methanol and directly analyzed by nanospray Fourier transform ion cyclotron resonance (FTICR) mass spectrometry (MS). The capture efficiencies of formaldehyde, acetaldehyde, acetone and 2-butanone have been determined to be above 95\%. The levels of these and other carbonyl compounds in exhaled breath samples from 45 non-smokers and 45 current smokers were compared. Formaldehyde, acetaldehyde and acetone were present in significantly higher concentrations in the current smokers group, while 2-butanone and 3hydroxy-2-butanone were not affected by smoking cigarettes.
\end{abstract}

\section{INTRODUCTION}

Analysis of volatile organic compounds (VOCs) at trace levels has attracted much interest because of demanding applications in non-invasive disease diagnosis and monitoring environment air quality. Human exhaled breath primarily consists of atmospheric gases, water vapor, and trace level metabolic VOCs. ${ }^{1,2}$ Breath analysis could potentially provide a non-invasive and painless means to detect disease, and the sampling of breath does not require skilled medical staff. ${ }^{3-7}$ Breath analysis challenges existing analytical methods because VOC concentrations in breath are beyond instrument limits of detection. ${ }^{3,7}$ This problem is further exacerbated by other interfering gases that are mixed in large concentration with the VOCs. Gas chromatography coupled with a mass spectrometer detector (GC-MS) is currently the dominant technique for analysis of trace VOCs. A complex preconcentration process consisting of cryogenic adsorption and thermal desorption is typically required before trace VOC samples can enter into a GC column. ${ }^{8-14}$ Solid-phase microextraction (SPME) is a popular preconcentration method and a rapid extraction technique for analysis of VOCs from a variety of matrixes by GCMS. ${ }^{15-18}$ However, SPME is only semiquantitative due to the inefficiency inherent with physical adsorption on the SPME fiber. Other methods including selected ion flow tube mass spectrometry (SIFT-MS), ${ }^{19-20}$ proton transfer reaction mass spectrometry (PTR$\mathrm{MS})^{21-23}$ and electronic nose methods ${ }^{24-25}$ also have been used for analysis of breath. 


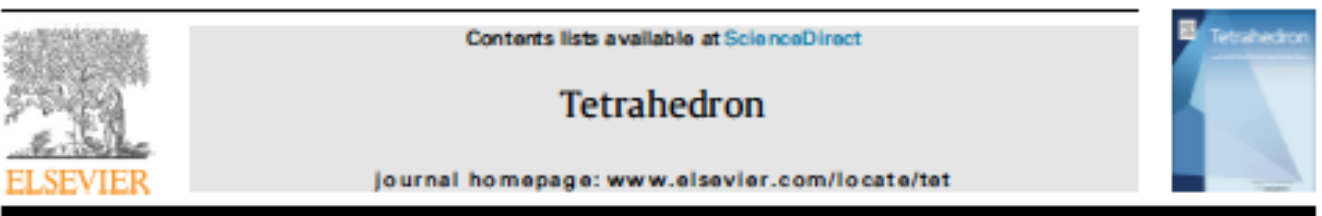

Thermally induced substrate release via intramolecular cyclizations of Amino esters and Amino carbonates

Ralph J. Knipp ${ }^{2}$, Rosendo Estrada ${ }^{\text {b }}$, Palaniappan Sethu ${ }^{\text {b }}$, Michael H. Nantz ${ }^{2,}$

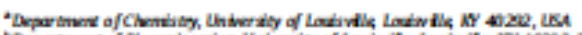

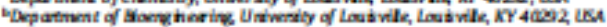

A R TICLE IN F O

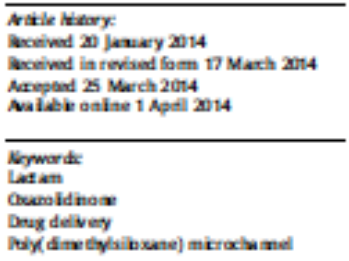

A BSTRACT

The relathe cleava of an a loohol from a p anel of a mino estrs and amino cartonats va in trandecular cyclizatbn was examined as a mechanism for substrate releas. Thermal stablilty at 37 "C waG observed only for the seven-membered ing proznibors. Applicability $d$ the approach was illustrated by s-lactam

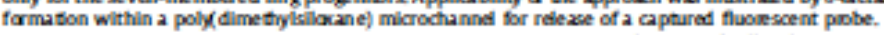

e 2014 Poevier Lat. Nil ights reserved.

\section{Introduction}

We disclase here a brief study on the propers ity of anino ester and amino carbonate substrates of the type indicated in Fig. 1 to undergo intramoleaular cydization for purposes of releasing substrate $H O-R^{\prime}$.Similar intramolecul ar cyd ization modes of substrate relexe previously have been exploited as mechanisms for drug delivery. ${ }^{-1}$ In these cases, the released subs trate is eithera phenol $\left(\mathrm{R}^{\prime}-\mathrm{Ar}\right)$ or an aniline derivative so that the intranoleaular cycli. zation ocaurs rapidly at physiological temperature, generally with a half-life from minutes to $1 \mathrm{~h} .{ }^{6}$ Intramolecular cydizations of this type also have been used to unmask aromatic hydroxyl, amine" or thiol "moieties as mech anisms to initiateelectron cascade reactions or for release of polymer-bound drugr ${ }^{\mathrm{D}} \mathrm{Ag}$ in the focus was to use the intramolecular cydization for rapid drug relexe at physiological temperature, and this required $\mathrm{R}^{\prime}$ to bearomatic. Few ox anples have used this approach to rdese non-aromatic alcohols, ${ }^{1112}$ the most common being for the delivery of 5-halo decaxyuridine analogs. ${ }^{13-15}$

Our interest in control over heat induced intramolecular cyclization as a mechunism for substrate release led us to investigate linkers that would expel $H \mathrm{O}-\mathrm{R}^{\prime}$ at temperstures above $37^{\circ} \mathrm{C}$, such $\mathbf{z}$ in response to an externally trigg ered local hyper thermis. Con sequently, we prepared ${ }^{16}$ a panel of amino carboryl substrates to

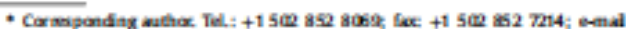

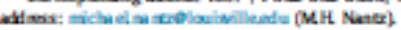

(a)

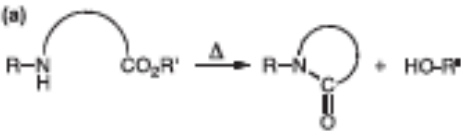

(b)

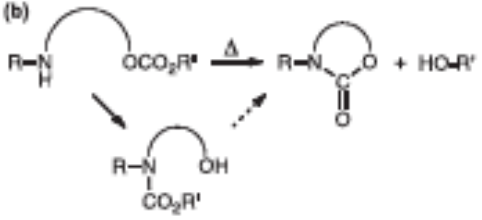

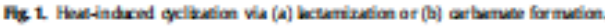

determine factors that would minimize the rate of cyclization at $37^{\circ} \mathrm{C}$ while allowing cyclization to proceed at higher temperature (Fig. 2)

\section{Results and discussion}

21. Cycliation panel (Fig. 2)

All substrates were prepared to contain an $\mathrm{N}$-allyl moiety for onverient functionalization such as hydrosilylation or thiol incorporation, for eventual covalent attachment to various supports. 


\section{NANOPARTICLES FOR DRUG DELIVERY}

\section{Cross Reference to Related Applications}

This patent application claims the benefit of priority of U.S. application serial No. 61/762,832 filed February 8, 2013 and U.S. application serial No. 61/773,663 filed March 6,2013 , which applications are hereby incorporated by reference.

\section{Statement of Government Support}

This invention was made with state government support under Grant number 2190-RDE-013 awarded by the Kentucky Science and Engineering Foundation. The Commonwealth of Kentucky has certain rights in the invention.

\section{Background}

An important attribute of a drug delivery system is its ability to allow for spatial and temporal regulated drug release, thereby minimizing side effects and improving therapeutic efficacy of conventional pharmaceuticals. Iron oxide nanoparticles (NPs), specifically $\mathrm{Fe}_{3} \mathrm{O}_{4}$ nanoparticles, possess many appropriate qualities that make them a viable choice for drug delivery. $\mathrm{Fe}_{3} \mathrm{O}_{4}$ NPs are biocompatible (Kievit, F. M., et al., Accounts of Chemical Research 2011, 44 (10), 853-862), have low cytotoxicity (Bulte, J. W. M., et al., NMR in Biomedicine 2004, 17, 484-499), and provide multiple means for surface modification. Though these attributes are needed in a drug delivery vehicle, there are multiple different NPs that possess similar qualities including gold and silica. $\mathrm{Fe}_{3} \mathrm{O}_{4}$ is set apart from these NPs due to its paramagnetic or superparamagnetic (SPM) qualities (Yang, C., et al., Chemical Communications 2011, 47, 5130-5141). The SPM properties of $\mathrm{Fe}_{3} \mathrm{O}_{4}$ NPs have been used for a variety of applications. A basic utilization of SPM capability is to induce non-invasive hyperthermia within cancer cells. Alternating electromagnetic field (AMF)-induced $\mathrm{Fe}_{3} \mathrm{O}_{4}$ NPs heat body tissue to temperatures as high as $45^{\circ} \mathrm{C}$, and this causes cell death. In addition, when functionalized either by ionic interactions or through entrapment via a polymer gel coating, drugs can be guided to tumor regions through the use of a magnet, as first demonstrated by Meyers in 1963 (Meyers, P.H., et al., American Journal of Roentgenology, Radium Therapy, and Nuclear Medicine 1963, 90, 1068-1077). Through more advanced methods, $\mathrm{Fe}_{3} \mathrm{O}_{4}$ NPs are now extensively functionalized with complex delivery mechanisms and can be directed by taking advantage of tumor folate receptors (Kim, J., et al., Advanced Materials 2008, 20, 
$17541.082 \mathrm{PV} 1$

\section{NANOPARTICLES FOR DRUG DELIVERY}

\section{Background}

An important attribute of a drug delivery system is its ability to allow for spatial and 5 temporal regulated drug release, thereby minimizing side effects and improving therapeutic efficacy of conventional pharmaceuticals. Iron oxide nanoparticles (NPs), specifically $\mathrm{Fe}_{3} \mathrm{O}_{4}$ nanoparticles, possess many appropriate qualities that make them a viable choice for drug delivery. $\mathrm{Fe}_{3} \mathrm{O}_{4}$ NPs are biocompatible (Kievit, F. M. et al., Accounts of Chemical Research 2011, 44 (10), 853-862), have low cytotoxicity (Bulte, J. W. M., et al., NMR in Biomedicine 2004, 17, 484-499), and provide multiple means for surface modification. Though these attributes are needed in a drug delivery vehicle, there are multiple different NPs that possess similar qualities including gold and silica. $\mathrm{Fe}_{3} \mathrm{O}_{4}$ is set apart from these NPs due to its paramagnetic or superparamagnetic (SPM) qualities (Yang, $\mathrm{C}_{4}$ et al., Chemical Communications 2011, 47, 5130-5141). The SPM properties of $\mathrm{Fe}_{3} \mathrm{O}_{4} \mathrm{NPs}$ have been used

15 for a variety of applications. A basic utilization of SPM capability is to induce non-invasive hyperthermia within cancer cells. Alternating electromagnetic field ( $\mathrm{AMF}$ )-induced $\mathrm{Fe}_{3} \mathrm{O}_{4}$ NPs heat body tissue to temperatures as high as $45^{\circ} \mathrm{C}$, and this causes cell death. In addition, when functionalized either by ionic interactions or through entrapment via a polymer gel coating, drugs can be guided to tumor regions through the use of a magnet, as first demonstrated by Meyers in 1963 (Meyers, P.H., et al., American Journal of Roentgenology, Radium Therapy, and Nuclear Medicine 1963, 90, 1068-1077). Through more advanced methods, $\mathrm{Fe}_{3} \mathrm{O}_{4} \mathrm{NPs}$ are now extensively functionalized with complex delivery mechanisms and can be directed by taking advantage of tumor folate receptors (Kim, J., et al., Advanced Materials 2008, 20, 478-483, Zhang. Z,, et al., Biomaterials 2007, 28 (10), 1889-1899, Zhang, L., et al., International Journal of Pharmaceutics 2004, 287 (1-2), 155-162). Finally, iron oxide also can be used as a magnetic resonance imaging contrast agent, so delivery systems based on this material can be visualized (Lee, J. E; et al., Journal of the American Chemical Saciety 2010, 132, 552-557).

Some of the most common methods of functionalization or attachment of drug 30 payloads to $\mathrm{Fe}_{3} \mathrm{O}_{4}$ NPs involve the use of ionic attraction (Nantz, M. H., et al., PCT Int. Appl. 2011, WO 2011049972 A1 20110428), the addition of a mesoporous silica shell around the $\mathrm{Fe}_{3} \mathrm{O}_{4} \mathrm{NPs}$ followed by further functionalization of the silica (Meng, H., et al., A. E., ACS Nano 2010, 4 (8), 4539-4550, Lin, Meng M., et al., Nano Reviews 2010, I, 4883) or the use 
DOI: 10.1002/ ((please add manuscript number))

Article type: Full Paper

\title{
Magnetic-Field Induced Hydrolysis as a Mechanism for Rapid Release of Nanoparticle-Bound Substrates \\ Ralph J. Knipp, Stephanie J. Mattingly and Michael H. Nantz*
}

R. J. Knipp, S. J. Mattingly, Professor M. H. Nantz, Department of Chemistry, University of Louisville, 2320 S. Brook St., Louisville, Kentucky 40292, United States

E-mail: michael.nantz@louisville.edu

Keywords: AMF, carbamates, covalent, hydrolysis, nanoparticles

\begin{abstract}
Application of an alternating magnetic field (AMF) to magnetic core-shell iron oxide-silica nanoparticles fitted with short carbonate- or carbamate-functionalized chains that covalently link a fluorophore results in rapid functional group hydrolysis with attendant release of the fluorophore. This first example of AMF-induced carbamate hydrolysis resulted in $c a$. 10-fold greater release of fluorophore substrate in comparison to the control at $37{ }^{\circ} \mathrm{C}$ in the absence of an AMF.
\end{abstract}

\section{Introduction}

An important attribute of a drug delivery system is regulated spatial and temporal drug release to minimize side effects as well as to improve the therapeutic efficacy of conventional pharmaceuticals. Iron oxide nanoparticles (NPs) possess many features that make them a viable choice for drug delivery formulations. Magnetite $\left(\mathrm{Fe}_{3} \mathrm{O}_{4}\right)$ NPs are biocompatible,${ }^{[1]}$ have low cytotoxicity, ${ }^{[2]}$ can be readily surface-modified and have inherent paramagnetic or superparamagnetic properties. ${ }^{[3]}$ As a result of their magnetism, one highly-studied feature of $\mathrm{Fe}_{3} \mathrm{O}_{4} \mathrm{NPs}$ is their ability to heat a surrounding environment 
CURRICULUM VITAE 


\title{
Ralph J. Knipp
}

\author{
1007 Grande Ln \\ Kernersville, NC 27284 \\ (614) 920-9239 \\ linkedin.com/pub/ralph-knipp/1b/4ba/69 \\ ralph.knipp@louisville.edu
}

\section{EDUCATION}

Ph.D. University of Louisville, Department of Chemistry, Louisville, KY 2009-2014 Organic Chemistry (expected December 2014)

Thesis: Triggered Release of Small Molecules from Solid Supports

Using Heat or an Applied Magnetic Field

B.S. Ohio Dominican University, Columbus, $\mathrm{OH}$

2003-2007

Chemistry (cumulative GPA: 3.786; degree awarded May 2007)

\section{ACADEMIC AND INDUSTRIAL RESEARCH EXPERIENCE}

University of Louisville, Department of Chemistry

2009-Present

Advisor: Dr. Michael H. Nantz

- Engineered and synthesized thermally labile linkers for substrate/drug release via intramolecular cyclization. Applied this technology to the chemoselective capture of carbonyls (aldehydes and ketones) utilizing aminooxy click-chemistry from a metabolite stream for FT-ICR-MS detection.

- Developed a drug delivery mechanism to release an active drug from iron oxide $\left(\mathrm{Fe}_{3} \mathrm{O}_{4}\right)$ nanoparticles on the application of an external alternating magnetic field.

- Designed and optimized aminooxy probes for the early detection of lung cancer from exhaled breath using direct injection FT-ICR-MS, LC-MS and GC-MS conjunctively and without liquid-liquid extraction.

- Successfully planned and completed numerous multi-step syntheses, purifications and characterizations. 
GFS Chemicals, Inc., Columbus, Ohio

Organic Chemist: Kilo Lab

- Specialized in synthesis of acetylenic, olefinic, silane and Grignard reagent building blocks on a $500 \mathrm{~mL}$ to $72 \mathrm{~L}$ multi-molar scale for multiple applications.

- Delivered products meeting customer specifications for custom syntheses, internal and external pilot plant development, as well as maintaining supplies of specific catalog items.

- Implemented several process improvements that remain incorporated into production activities today.

- Provided fast and accurate procedures and notes that were used to establish large-scale production methodologies and yields.

\section{COMMUNICATION SKILLS}

Coauthor \& Co-inventor of 9 publications and 8 presentations

RJ Knipp, SJ Mattingly and MH Nantz. Magnetic-Field Induced Hydrolysis as a Mechanism for Rapid Release of Nanoparticle-Bound Substrates. Adv. Mater. Interfaces, In Review.

M Li, RJ Knipp, MH Nantz, M Bousara and X-A Fu. Analysis of Exhaled Breath for Identification of Lung Cancer Biomarkers. Clin Chim. Acta, In Review.

RJ Knipp, M Li, X-A Fu and MH Nantz. A Versatile Probe for Chemoselective Capture and Analysis of Carbonyl Compounds in Exhaled Breath. Anal. Chem., In Review.

MH Nantz and RJ Knipp. Nanoparticles for Drug Delivery; US Provisional Application 62/034,059; filed Ausust 6, 2014.

M Bousamra, E Schumer, M Li, RJ Knipp, MH Nantz, VH van Berkel and X-A $\mathrm{Fu}$. Quantitative Analysis of Exhaled Carbonyl Compounds Distinguishes Benign From Malignant Pulmonary Disease. J. Thorac. Cardiovasc. Surg. 2014, 148, 10741081.

RJ Knipp, R Estrada, P Sethu and MH Nantz. Thermally-Induced Substrate Release Via Intramolecular Cyclizations of Amino Esters and Amino Carbonates. Tetrahedron 2014, 70, 3422-3429.

X-A Fu, M Li, RJ Knipp, MH Nantz and M Bousamra. Noninvasive Detection of Lung Cancer Using Exhaled Breath. Cancer Medicine 2014, 3, 174-181.

RJ Knipp and MH Nantz. Nanoparticles for Drug Delivery; US Patent Application US2014015413; filed February 7, 2014; Priority: US Provisional Application 61/762,832; Filed February 8, 2013. 
S Biswas, RJ Knipp, LE Gordon, SR Nandula, SU Gorr, GJ Clark and MH Nantz. Hydrophobic Oxime Ethers: A Versitile Class of pDNA and siRNA Transfection Lipids. ChemMedChem 2011, 6, 2063-2069.

\section{PRESENTATIONS}

MH Nantz, RJ Knipp and J Jasinski. AMF-Induced Release of Iron Oxide-Bound Substrates via Cyclization of Thermally Responsive Amino-Carbonates and Carbamates. Poster No. 146, $10^{\text {th }}$ International Conference on the Scientific and Clinical Applications of Magnetic Carriers, Dresden, Germany; June 10, 2014.

RJ Knipp and MH Nantz. AMF-Induced Drug Release via Cyclization of Thermally Labile Amino-Esters and -Carbonates Bound to Iron Oxide Nanoparticles. Oral, IMD $^{3}$ FallFest, Louisville, KY; November 8, 2013.

RJ Knipp and MH Nantz. Thermally Labile Amino-Ester and Amino-Carbonate Linkers for AMF-Induced Drug Release from Iron Oxide Nanoparticles. Oral No. 43, $246^{\text {th }}$ ACS National Meeting and Exposition, Indianapolis, IN; September 8, 2013.

RJ Knipp and MH Nantz. AMF-Induced Cyclization of Thermally Labile AminoEsters and -Carbonates Bound to Iron Oxide Nanoparticles. Oral, Kentucky nanoSymposium, Louisville, KY; August, 172013.

XA Fu, M Li, RJ Knipp, M Bousamra, MH Nantz and RM Higashi. A Microreactor Chip for Analysis of Trace Volatile Organic Compounds. Oral, Kentucky nanoSymposium, Louisville, KY; August, 162013.

J Bergman, K Gupta, K Afonin, M Viard, RJ Knipp, MH Nantz, A Puri and B Shapiro. Preparation of Oxime-Ether Lipoplexes for siRNA Delivery in Breast Cancer Cells. Poster, Frederick, MD; August 9, 2013.

K Nelson, RJ Knipp and MH Nantz. Thermally-Induced Intramolecular Cyclizations for Release of Chemoselectively Bound Carbonyl Metabolites. Poster, Summer Research Opportunities for Undergraduates, University of Louisville, Louisville, KY; July 31, 2013.

RJ Knipp, O Uradu and MH Nantz. Magnetic-Field Induced of Amino-Esters and Carbonates Bound to Iron Oxide Nanoparticles. Oral No. 212, Nanotech, Washington, D.C.; May 15, 2013.

\section{TEACHING SKILLS}

Teaching Assistant: Organic Spectroscopy and Separations Lab (CHEM 527) Department of Chemistry, University of Louisville; Fall 2012 


\section{FELLOWSHIPS and AWARDS}

- Graduate Dean's Citation, University of Louisville, School of Interdisciplinary Graduate Studies (SIGS), 2014

- Travel Award, University of Louisville, Institute for Molecular Diversity and Drug Design to attend Nanotech, Washington, D.C., 2013

- Research Fellowship, Bill and Melinda Gates Foundation, 2012-2013

- University Graduate Student Fellowship, University of Louisville, School of Interdisciplinary Graduate Studies (SIGS), 2009-2011

- Outstanding Senior in Chemistry Award, Ohio Dominican University, Division of Math, Computer and Natural Sciences, 2007

- CRC Press Freshman Chemistry Award, Ohio Dominican University, Division of Math, Computer and Natural Sciences, 2004

\section{PROFESSIONAL ACTIVITIES}

- Member, Kentucky Academy of Science (2013)

- Presenter, Woodland Elementary School Career Day, Hardin County Schools (2013)

- Treasurer Elect, Columbus American Chemical Society (2009); Active Member

\section{REFERENCES}

Professor Michael H. Nantz

Department of Chemistry

University of Louisville, KY 40208

(502) 852-8069

michael.nantz@louisville.edu

Professor Christopher T. Burns

Department of Chemistry

University of Louisville, KY 40208

(502) 852-5977

christopher.burns@louisville.edu

Professor Francis P. Zamborini

Department of Chemistry

University of Louisville, KY 40208

(502) 852-6550

f.zamborini@louisville.edu
Professor Palaniappan Sethu

Department of Cardiovascular Disease

University of Alabama, AL 35294

(205) 975-4185

psethu@uab.edu

David Baust

General Manager

Organic Chemical Division

GFS Chemicals, Inc.

Columbus, OH 43222

(614) $224-5013 \times 310$

david@gfschemicals.com 